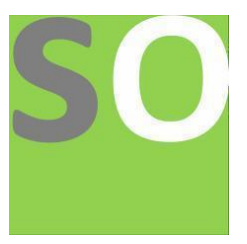

Article title: Combinations of drugs might increase the survival chances of COVID-19 patients: Literature review till date

Authors: bose Karthik[1]

Affiliations: India[1]

Orcid ids: 0000-0003-1992-461X[1]

Contact e-mail: sarscov2analysis33@gmail.com

License information: This work has been published open access under Creative Commons Attribution License http://creativecommons.org/licenses/by/4.0/, which permits unrestricted use, distribution, and reproduction in any medium, provided the original work is properly cited. Conditions, terms of use and publishing policy can be found at https://www.scienceopen.com/.

Preprint statement: This article is a preprint and has not been peer-reviewed, under consideration and submitted to ScienceOpen Preprints for open peer review.

Funder: No funder

DOI: 10.14293/S2199-1006.1.SOR-.PPY8CIF.v1

Preprint first posted online: 13 October 2021

Keywords: COVID-19, SARS-COV-2, Medicines 


\title{
Combinations of drugs might increase the Survival chances of COVID-19 patients: Literature review till date
}

\author{
Bose Karthik \\ Indian Institute of Technology, Bhubaneswar, ( IIT-BBSR ), Odisha, INDIA \\ Indian Institute of Technology, Madras - Biomedical, ( IIT-M ), Tamil Nadu, INDIA
}

\section{Introduction:}

SARS-COV-2 explores every possible vulnerability in human body and uses it against the host. To treat this SARS-COV-2 induced COVID-19, we should target the multiple factors virus is targeting and use the drugs in a strategical way. This approach can save the patients from severe state of illness and damage associated with the disease.

\section{SARS-COV-2 induces delayed humoral responses and accelerated innate immune response mediated damages:}

COVID-19 has been associated with hyper inflammation [10-18] and delayed humoral immune respo-nses $[19,20,21,22,23,24]$. Most of the complications with COVID-19 patients have been associated with early hypoxia, ARDS, pneumonia [60,77,35-39] and Acute Lung Injury [30-34]. Viral load has been found to be associated directly with incidence of lung injury/epithelial injury or remote organ injury in COVID-19 patients [25-30].

\section{Delayed Humoral Responses in Severe COVID-19 Patients:}

Hasan et.al though their studies have attributed the severe form of Covid-19 to a dysfunctional innate immune response, such as a delayed and/or deficient type I/III interferon response, coupled with an exaggerated and/or a dysfunctional adaptive immunity [21]. Differences in T-cell (including CD4+ Tcells, CD8+ T-cells, T follicular helper cells, $\gamma \delta$-T-cells, and regulatory T-cells) and B-cell (transitional cells, double-negative 2 cells, antibody-secreting cells) responses have been identified in patients with severe disease compared to mild cases [21]. Moreover, differences in the kinetic/titer of neutralizing antibody responses have been described in severe COVID-19 disease, which may be confounded by antibody-dependent enhancements [21].

Lucas et.al observed a correlation between anti-spike (S) immunoglobulin G (IgG) levels, length of hospitalization and clinical parameters associated with worse clinical progression [19]. Although high anti-S IgG levels correlated with worse disease severity, such correlation was reported to be time dependent [19]. Lucas et.al reported that deceased patients did not have higher overall humoral response than discharged patients [19]. However, they mounted a robust, yet delayed, response, measured by anti-S, anti-receptor-binding domain IgG and neutralizing antibody (NAb) levels compared to survivors [19]. Delayed seroconversion kinetics correlated with impaired viral control in deceased patients [19]. Finally, although sera from $85 \%$ of patients displayed some neutralization capacity during their disease course, NAb generation before 14 day of disease onset emerged as a key factor for recovery [19]. These data indicate that COVID-19 mortality does not correlate with the cross-sectional antiviral antibody levels per se but, rather, with the delayed kinetics of NAb production [19]. 
respectively [23]. The highest IgA+ frequency was observed at weeks 2 and 3 and the highest IgG+ at weeks 3 and 4 [23]. It is important to note that patients who died presented lower IgA titers in the first two weeks however, a significant increase in IgA levels was observed in the subsequent weeks [32]. Thalia et al identified that significant correlations between Ct values and immunoglobulins levels, both IgA and IgG were correlated with Ct N2 in patients who died [23].

Thalia et.al though their study and results concluded that lower IgA titers in early Covid-19, which is associated with lower Ct values, may indicate patients at higher risk for death [23].

Fraser et.al reported that Peak serological responses for each Ig isotype occurred on different ICU days (IgM day $13>\operatorname{IgA}$ day $17>\operatorname{IgG}$ persistently increased), with the total Ig peaking at approximately ICU day 18 [24]. Fraser et.al found that COVID-19+ patients who died had earlier/similar peaks in IgA and total Ig in their ICU stay when compared with the patients who have survived [24]. Critically ill COVID-19 patients exhibit anti-SARS-CoV-2 serological responses, including those COVID-19 patients who ultimately died, suggesting that blunted serological responses did not contribute to mortality [24].

\section{To Reduce early Viral Load as much as possible is the key to controlling SARS- COV-2 mediated damage:}

Supportively, SARS-COV-2 Viral load has been associated directly with lung injury, endothelial damage and other organ injury in COVID-19 patients [25-30]. After Lung Injury or other systemic organ injury independent of Virus the situation can be treated as systemic inflammatory response syndrome (SIRS) which ultimately leads to Sepsis $[441,472,473]$.

\section{In order to observe better results in treatment, it's critical to slow down the viral growth because:}

1. Virus promotes the recruitment of more Neutrophils and induces more damage to infected organs especially lungs. Virus mediated damage in AT-II lung cells induces collapse of alveolar chambers due to the lack of surfactant and associated surface tension. This leads to Hypoxia in patients.

2. More virus can bind to more LPS in systemic blood and lead to elevated inflammatory response in initial stage and elevated immunosuppressive response in the late phase of systemic Injury inflammatory response mediated Sepsis.

3. More viral burden leads to increased Cellular death and subsequent release of elevated HMGB-1 and other DAMPs.

Conclusively, Delayed Viral entry and reduced subsequent viral load can reduce the immune system mediated damage and might give sufficient amount of time for humoral responses, debris clearance and might delay the possibility of Injury and subsequent onset of Inflammation induced Sepsis.

In this study we will briefly understand the benefits of different drugs and their combinations reported by different authors though their analysis, trials or experiments which were reported to be useful in COVID-19 treatment. 


\section{Overview of the studies that found different combinations of drugs to be effective in COVID-19 till date:}

\section{Povidone-lodine (PVP-I) Solution Gargles and Nasal spray/ swabs:}

1. Recent evidence has confirmed that $0.5 \%$ povidone iodine (PVP-I) mouthrinse/gargle for $30 \mathrm{~s}$ can reduce SARS-CoV-2 virus infectivity to below detectable levels $[148,149,151,152,153,154]$.

2. Hassandarvish et.al showed that $1 \%$ PVP-I achieved $>5$ logs 10 reductions in the SARS CoV-2 virus titre at 15, 30 and 60 seconds [150].

3. PVP-I can even interrupt SARS-CoV-2 attachment to oral and nasopharyngeal tissues and lower the viral particles in the saliva and respiratory droplets [148].

4. PVP-I kills SARS-COV-2 at surface, reduces viral burden and prevents SARS-COV-2 early entry into the systemic blood through damaged endothelial cells in Oral and Nasal compartments.

5. PVP-I can kill fungal and bacterial infection [156,157].

6. Periodontitis and associated bacterial can drive inflammation in COVID-19 patients $[96,97,112]$.

7. Periodontitis and associated bacterial LPS can bind to SARS-COV-2 [834] and induces elevated proinflammatory immune responses. PVP-I can kill this bacteria $[158,159,160]$.

8. Prevents dangerous Secondary Infections through mouth and Nose during Immuno compromised condition established during the usage of dexamethasone, a immunosuppressant. PVP-I Gargle and spray might provide protection against Mucormycosis and secondary bacterial infections.

9. Mohamed et.al conducted a pilot trial and reported that a 3 times/day gargling usage of $1 \%$ povidone-iodine (PVP-I) in five COVID-19 confirmed Asymptomatic Stage 1 patients including two comorbid patients, when monitored on day 4,6 and 12 showed a higher viral clearance from as early as day 4 [155].

\section{Nebulized-NAC (N-Acetyl- Cysteine) and TMPRSS-2 Inhibitor:}

1. Li et.al concluded that Camostat and nafamostat inhibits SARS-CoV-2 infection in well differentiated human airway epithelial cells [140].

2. Li et.al observed that the effectiveness of nafamostat administration through intranasal channel increased as the time interval between nafamostat delivery and viral inoculation decreased [140]. Although intranasal nafamostat effectiveness is humans is not yet known fully [140].

3. Mccord et.al reported that interaction of SARS-COV-2 spike protein with ACE-2 was blocked by clinically proven protease inhibitors of the TMPRSS2, Camostat mesylate $[145,146,137]$ and Nafamostat mesylate [137].

4. Nebulized NAC Disrupts disulfides at the surface of ACE-2 receptor and reduces SARS-COV-2 confirmational change mediated host cellular entry [133].

5. Akhter et.al through their experiments reported that Acetylcysteine was able to reduce only $58 \%$ of the disulfide linkages [133].

6. Sagar et.al reported that Bromelain reduced the expression of TMPRSS2 in Calu-3 and ACE-2 negative normal bronchial epithelial (BEAS-2B) and lung adenocarcinoma (A549) cells [136].

7. Akhter et .al showed that treatment with Bromelain and Acetylcysteine alone each independently did not show any viral inhibition whereas BromAc a combination of both Bromelain and Acetylcysteine (NAC) displayed complete SARS-COV-2 virus inactivation in a concentration dependent manner [133]. 
8. Manček et.al found that NAC alone did not inhibit cell fusion but its derivative NACA [138], with more potent antioxidant properties, strongly inhibited the formation of syncytia[135]. This is in agreement with the other authors results in showing that using NAC alone cannot inhibit viral entry but can help reducing disulfides [133].

9. Manček et.al proposed that NACA, L-ascorbic acid, and JTT-705 alone efficiently inhibited the interaction between SARS-COV-2 spike and ACE2 binding independent of each other, implying that this group of compounds each alone can affect the direct interaction between SARS-COV2 spike and ACE2 when observed in lung-derived A549 cells [135].

10. Several other studies have also opined that Disulfide confirmation perturbations at ACE-2 surface can inhibit SARS-COV-2 cellular entry [130,1392,124].

11. ACE-2 expression is upregulated along with TLR-4 during pro-inflammatory medium in lungs of COVID-19 patients [2029,1394].

12. Nebulized NAC deceases ACE-2 expression at the targeted organ i.e airway epithelial cells and lungs [125,126].

13. Nebulized NAC reduces ROS directly at the target site and reduces cell death and HMGB-1 mediated subsequent inflammatory responses.

14. Acts as an Anti-oxidant directly at lung epithelial cells.

\section{Intranasal / IV-Tempol:}

Manček et.al found that NAC alone did not inhibit cell fusion but its derivative NACA [138] which has more potent antioxidant properties, strongly inhibited the formation of syncytia [135]. Tempol a super antioxidant might be as well a good choice [5].

Tempol is an powerful antioxidant $[2,4,58,73]$. Tempol relieves lung injury in a rat model of chronic intermittent hypoxia via suppression of inflammation and oxidative stress [3]. Tempol is protective against Hypoxia-induced Oxidative Stress and Apoptosis [1]. Maio et.al reported that Tempol can inhibit the viral replication by potentially inhibiting the RdRp [7]. Kavita et.al through their experiments concluded that Tempol as a novel antioxidant might inhibit both activated $\mathrm{T}$ cell and antigen presenting cell derived cytokines production in-vitro from COVID-19 patients [6].

\section{Tempol in COVID-19:}

Peter et.al reported that early administration of Intranasal Tempol treatment in a single COVID-19 patients reduced nasal congestion by day 4-5 and was found to be asymptomatic by day 8 [76]. Although it's a single case report taken into account that powerful antioxidant can inhibit viral entry and taken that Tempol inhibits viral replication of SARS-COV-2 and taken into account the SARS-COV-2 induced Oxidative stress and associated cell death, Intranasal/Nebulized Tempol administration along with standard care can increase the out come drastically [76].

\section{NAC-IV (Intravenous NAC (N-Acetyl-Cysteine)):}

1. Acts as anti-oxidant $[127,129]$.

2. NAC deceases ACE-2 expression [125,126].

3. NAC might upregulate TLR-7 expression [131].

4. Reduces confirmational change for SARS-COV-2 at systemic organ level.

5. Suppresses Pro-inflammatory Cytokines in Severe COVID-19 patients when administered continuously [9].

6. IV-NAC provides positive results in COVID-19 patients [1003, 143,142,141,146].

7. Glutathione therapy has also shown positive results in some COVID-19 patients $[128,146]$. 


\section{Dexamethasone:}

Dexamethasone suppresses Pro-inflammatory Cytokines in Severe COVID-19 patients. Dexamethasone and was found to be beneficial in patients who were receiving either invasive mechanical ventilation or oxygen alone, but not among those who were not receiving any respiratory support $[161,9,1003]$.

\section{Ulinastatin:}

1. Ulinastatin inactivates the extracellular elastase excreted from neutrophils and also suppresses the production of activated elastase [178]. Ulinastatin inhibits (polymorphonuclear neutrophils) PMNs activity and reduces the systemic inflammatory responses [183].

2. Ulinastatin has a confirmed powerful efficacy in inhibiting the release of inflammatory factors, removing oxygen free radicals, improving microcirculation and tissue perfusion, and alleviating endothelial injuries $[180,173,181,182]$.

3. Ulinastatin decreases proinflammatory cytokines during systemic inflammatory response syndrome (SIRS) and associated sepsis [169,174,2032].

4. Ulinastatin inhibits the production of inflammatory markers like Serum $C$ reactive protein(CRP), tumor necrosis factor-alpha (TNF-alpha), interleukin-6 (IL-6), and enhances the anti-inflammatory cytokines in the treatment of SIRS [171].

5. Ulinastatin ameliorates Sepsis, associated multiple organ dysfunction syndrome (MODS) and reduces mortality $[182,187,162]$.

6. Ulinastatin reduces LPS induced inflammatory responses [188,172].

7. Ulinastatin inhibits coagulation and endothelial leakage $[178,180]$.

8. Zhao et.al demonstrated that ulinastatin inhibited the hepatic hypoxia/reoxygenation (H/R) injury in Chang liver cells, and concluded that it might be due to autophagy activation [192].

9. Ulinastatin reduces HMGB-1 expression [190,191,192].

10. Neutrophils and associated NET's have been implicated with SARS-COV-2 mediated damage in COVID-19 patients $[598,597,1448,1449]$.

11. Huang et.al reported that Ulinastatin showed better results on 7th day in COVID-19 patients by preventing lymphocyte decrease, CRP and improving oxygen saturation [2031].

\section{Aerosol/ Nebulized Ribavirin Therapy :}

1. Aerosol Ribavirin has been reported to be reversing parenchymal thickening and multiple ground glass opacity associated areas in lungs of COVID-19 patients when used early [39].

2. Aerosol Ribavirin has been reported to be safe in COVID-19 patients [39].

\section{CD24:}

1. Inhibits NF-KB mediated pro-inflammatory responses [752,753,732].

2. Enhances self and non-self discrimination and promotes CD8+ T cell activity $[754,670,729]$.

3. Binds to HMGB-1 and reduces HMGB-1 and MDSC mediated immunosuppression on CD8+ T cells $[488,685,755]$.

4. One stop solution for preventing Lymphopenia and Sepsis in severe COVID-19 patients. 


\section{Anti-HMGB-1:}

1. Anti-HMGB-1 treatment might reduces HMGB-1 mediated pro-inflammatory responses and reduces subsequent HMGB-1 mediated MDSC recruitment during viral infections especially IAV [351,352, 353, 409,261].

2. Anti-HMGB-1 treatment prevents Lymphopenia [425,517].

3. Anti-HMGB-1 treatment provides protection against systemic inflammatory response syndrome (SIRS) induced Sepsis [504,535].

\section{Remdesivir-IV:}

1. Reduces SARS-COV-2 Viral replication and viral load during early stages $[50,51,52,53]$.

\section{Monoclonal Antibodies Therapy:}

1. Neutralizing monoclonal antibodies (mAb) therapy is useful only if administered at early stage in COVID-19 patients with mild/moderate symptoms [2056,2057,2048-2051].

\section{Nebulised Interferon -I and III therapy:}

1. Nebulised Interferon Therapy at earlier stages is reported to be successful for COVID-19 patients while late stages might not be helpful [1115-1119, 2043-2047].

\section{Nebulized Unfractionated Heparin (UFH):}

1. Nebulized Unfractionated Heparin might target SARS-COV-2 mediated Lung Injury, fibrin deposition, Thrombosis, dead-spaces and hypoxaemia, Ventillation mediated injury [2094-2133].

2. Nebulized Unfractionated Heparin is useful in treating ARDS [2131].

3. Nebulized Unfractionated inhibits confirmational change required by SARS-COV-2 and prevents entry into host cell and subsequent viral replication by destabilizing RBD [2101-2121].

\section{Doxycycline:}

1. Usage of Doxycycline might be effective only in patients with confirmed secondary bacterial infections especially in critical and aged patients [2059, 2060,2061,2062,2063, 2064,2065].

2. Antibiotics are found to be dysregulating gut microbiota independent of the delivery Route I.e: administered either through oral or Intravenous ways [2058]. Antibiotics are known for dysregulating gut microbiota [1728] and gut microbiota is related to TLR-7 expression at mucosal surfaces [1704] and reduction in TLR-7 is associated with higher mortality in COVID-19 patients [616]. Wu et.al reported that TLR-7 ligands rescued the immune impairment in antibiotic-treated mice during Influenza virus (IAV) [1704]. 


\section{TLR-3 and TLR-7 agonist:}

1. In absence of TLR-7 MDSCs get recruited to Lung very quickly during Lung injury [615].

2. TLR-3 is essential for protection against Coronavirus family [830].

3. In, STAT-1-/- mice, MDSCs get recruited swiftly during acute inflammation [896, 368].

\section{Vitamin-D:}

1. Daily dosage of Vitamin-D administration reduces susceptibility to respiratory virus by half but not a single high dose administration [2135].

2. Daily high dose of Vitamin-D administration reduces Inflammatory Cytokines in COVID-19 patients [2148].

3. Daily high dose of Vitamin-D administration reduces fibrinogen in COVID-19 patients [2149].

4. Vitamin-D upregulates cellular Glutathione (GSH) levels and reduces ROS levels [2158].

\section{Flavanoids:}

1. Lot of studies recommend the usage of flavanoids in COVID-19 treatment [2076, 2077, 2078, 2079, 2080].

2. Zhu and Xie et.al though their docking studies and in-vitro studies showed that Flavanoids have the potential in reducing SARS-COV-2 viral replication and associated cell death [2076, 2081].

\section{Accidental causes in COVID-19 :}

\section{- Abuse of Steroids:}

1. Dexamethasone treatment strongly enhances MDSC expansion through upregulation of miR-155 and miR-21 expression [1630, 368]. Dexamethasone or any immunosuppressants administration during early stages of COVID-19 compromises their immune systems ability to stop the virus [161]. As a result the virus can directly reach lungs and induce Alveolar type -II epithelial cell damage and subsequent collapse due to lack of Surface tension. Supportively enough in a COVID-19 case report Dexamethasone misuse has been showed to be associated with delayed viral clearance leading to occurrence of severe COVID-19 pneumonia [2086].

2. Although Steroids usage in elderly critically ill COVID-19 patients has been reported to be showing positive outcomes [2087, 161] it's misuse might show detrimental effects on the patients [2088].

3. Over usage of Steroids in COVID-19 patients especially in patients with Co-morbidities can make them susceptible to dangerous secondary infections like Mucormycosis [2089-2093].

\section{- Abuse of Antibiotics:}

1. Antibiotics are found to be dysregulating gut microbiota independent of the delivery Route l.e: administered either through oral or Intravenous ways [2058]. Antibiotics are known for dysregulating gut microbiota [1728] and gut microbiota is related to TLR-7 expression at mucosal surfaces [1704] and reduction in TLR-7 is associated with higher mortality in COVID-19 patients [617]. Wu et.al reported that TLR-7 ligands rescued the immune impairment in antibiotic-treated mice during Influenza virus (IAV) [1704]. 
2. Abuse of Antibiotics has been reported to be a key factor in worsening/aggravating the diseased condition in COVID-19 patients [2082,2083,2084,2085].

- Damage due to Improper usage of Nasal swabs:

1. While using Nasal swabs, excess usage of force and incorrect sampling techniques might create an injury in the nasal cavity [2066,2075] especially in people who underwent Sinus Surgery [2067]. This injury might pave way for SARS-COV-2 to escape into the blood stream directly. This can lead to systemic inflammation and multi-organ failure independent of the tissue damage in lungs or airways. If injury left unchecked might lead to blood clots and subsequent consequences.

2. There have been reported cases of Nasal swab induced skull base injuries and CSF leakages [2069, 2070,2071,2072,2073]. In one case Traumatic Cribriform Plate Defect have been observed following Self-administration of COVID-19 Nasal Swab Test [2074]. 


\section{Description:}

\section{SARS-COV-2 uses disulfide confirmation:}

SARS-COV-2 attaches to Nose airway epithelial cells [1102,1103]. Host TMRSS-2 helps in cleaving the SARS-COV-2 Spike S protein [1207,1208]. SARS-COV-2 trimeric Spike S glycoprotein along with it's Receptor Binding Domain (RBD) attaches to Angiotensin-Converting Enzyme 2 (ACE2) receptor of the host epithelial cells [1204, 1207,1208]. SARS-COV-2 requires the confirmational change of disulfide bonds present at the surface of host ACE2 receptor to exhibit favourable binding conditions and to facilitate further entry into the host epithelial cells [1104, 1209].

\section{TMPRSS2 accelerates SARS-COV-2 entry:}

The spike (S) protein of coronaviruses facilitates viral entry into target cells. Additionally, Hoffmann and coworkers found that infection by SARS-CoV-2, the virus responsible for COVID-19, may depend almost exclusively on the additional host cell factors like TMPRSS2 [145,137].

Manček et.al through their experiments showed that SARS-COV-2 Spike protein and ACE2 alone are sufficient for the formation of syncytia, this group also observed that TMPRSS2 (transmembrane protease serine 2) co-expression on ACE2 cells further promotes the syncytia formation, thus increasing the sensitivity of the system [135].

\section{In-Silico/Computational analysis of SARS-COV-2 and ACE-2 interactions through disulfide confirmation:}

Hati et.al through their computational studies reported that under severe oxidative stress the cell surface receptor ACE2 and RBD of the SARS-COV-2 Spike S proteins are likely to be present in oxidized form with predominantly more disulfide linkages because reduction of all these disulfides into sulfydryl groups completely impaired the binding of SARS-COV-2 spike S protein with ACE2 receptor [1104]. Pre existing Oxidative stress has been one of the factors in COVID-19 pathogenesis. Additionally, Extracellular thiol-disulfide balance is affected in age dependent manner and it has been implicated to be important in COVID-19 pathogenesis [1203,1104].

Efficiency of different strains of virus in infecting humans depends upon the binding free energy of interaction between SARS-COV-2 RBD and human ACE2 receptor $[1205,1104]$. SARS-COV-2 Spike S protein has higher affinity for human ACE2 receptor when compared with SARS-COV-1 [1206,1104].

Additionally more disulfides at ACE2 receptor protein surface means faster confirmational change and faster interaction between host cell and virus thus faster entry into the host cell. This can be accounted for more transmissible nature of SARS-COV-2. Hati et.al reported that reducing disulfides on SARS-COV-1 RBD didn't reduce the interaction of SARS-COV-1 with ACE2, but reducing disulfides on SARS-COV-2 RBD reduced the interaction of SARS-COV-2 with ACE2 receptor binding by reducing/preventing the binding to certain level [1104]. Hati et.al also reported that reducing disulfide bonds at both RBD and ACE-2 receptor might block the viral entry into the host cell [1104]. SARS-COV-2 also uses PH based confirmational changes inside the cell for replication [1104]. 


\section{Role of Oxidative stress induced disulfides in accelerating SARS-COV-2 and ACE-2 interactions:}

Redox environment at the cell surface receptors in extracellular regions are maintained by thioldisulfide equilibrium [1105, 1201, 1202]. More Oxidative Stress results in more disulfide formation at the surface of the ACE2 receptors (extra cellular) [1105, 1202].

\section{Previous and SARS-COV-2 induced Oxidative Stress and related compounds further accelerates SARS-COV-2 entry into cells and promotes swift cellular damage:}

SARS-COV-2 has been associated with manifesting lot of immune related pathways. One of them includes inducing severe oxidative stress and subsequent increase in ROS levels in the infected cells [144].

Additionally, The ORF-8 protein of SARS-CoV-2 induces endoplasmic reticulum stress and mediates immune evasion by antagonizing production of interferon [1600]. SARS-CoV-2 caused ER-stressactivated unfolded protein response leads to cell death [1601,1600,1602].

Endogenous deficiency of Glutathione has been implied as the most likely cause of serious manifestations and death in COVID-19 Patients [128]. Polonikov et.al hypothesized that in severe COVID-19 cases, SARS-COV-2 would probably manifest and lower the GSH levels by mediating the induction of higher ROS levels and greater redox status (ROS/GSH ratio) than milder cases [128].

Supportively enough, Glutathione therapy has been implied to show efficiently positive outcome in a case study of 2-(two) COVID-19 patients with SARS-COV-2 induced pneumonia [146].

\section{ACE-2 and TMPRSS2 inhibitors (Bromelain) in human airway cells:}

Sagar et.al reported that Bromelain reduces the expression of TMPRSS2 in Calu-3 and ACE-2 in normal bronchial epithelial (BEAS-2B) and lung adenocarcinoma (A549) cells[136]. Cysteine protease inhibitor (E-64) treatment further confirmed that Bromelain's cysteine protease activity could cleave/reduce the expression of ACE-2 and TMPRSS2 [136].

\section{TMPRSS2 inhibitors also restrict SARS-COV-2 entry into the host cells:}

\section{Both NAC and Bromelain in Vero cells:}

Akhter et.al through their experiments reported that Acetylcysteine was able to reduce only $58 \%$ of the disulfide linkages [133]. Akhter et.al showed that the treatment with Bromelain and Acetylcysteine alone each independently did not show complete viral inhibition but BromAc a combination of both Bromelain and Acetylcysteine displayed complete virus inactivation in a concentration dependent manner [133]. Conclusively, In in-vitro whole virus culture of both wild-type and spike mutants, SARS-CoV-2 demonstrated a concentration dependent inactivation from BromAc treatment but not from any of the single agents alone [133]. SARS-COV-2 uses both TMPRSS2 mediated disulfide confirmation outside the cell and $\mathrm{PH}$-dependent confirmation inside the cells as discussed already [1104]. The disadvantage with this study is that author used Vero cells in their experiment which is Cathepsin L-dependent, and thus pH-dependent [139], this leaves room for experiments in the human pulmonary Epithelial cells as virus entry is TMPRSS2-dependent as well [133,1104]. 


\section{The TMPRSS2 Inhibitors reduces SARS-CoV-2 Pulmonary Infection in mouse models:}

Li et.al showed that the TMPRSS2 Inhibitor Nafamostat reduces SARS-CoV-2 Pulmonary Infection in Mouse Models of COVID-19 [140].

Mccord et.al reported that priming of SARS-COV-2 spike protein with ACE-2 was blocked by clinically proven protease inhibitors of the TMPRSS2, Camostat mesylate $[145,146,137]$ and Nafamostat mesylate [137].

Li et.al assessed and showed that Nafamostat was protective against SARS-CoV-2 in vivo using two mouse models[140]. In mice sensitized to SARS-CoV-2 infection by transduction with human ACE2, intranasal nafamostat treatment prior to or shortly after SARS-CoV-2 infection significantly reduced weight loss and lung tissue titers [140].

Li et.al tested the same two serine protease inhibitors, camostat mesylate and nafamostat mesylate, for their ability to inhibit entry of SARS-CoV-2 [140]. Both camostat and nafamostat reduced infection in primary human airway epithelial and in the Calu-3 2B4 cell line, with nafamostat exhibiting greater potency [140]. Both intraperitoneal and intranasal nafamostat inhibited SARS-CoV-2 infection in vivo [140]. Li et.al concluded that among camostat and Nafamostat, Nafamostat showed relatively greater potency against MERS-CoV and SARS-CoV-2 [140].

Li et.al suggested that nafamostat pretreatment significantly reduced viral loads over the course of SARS-CoV-2 infection further highlighting the importance of timing for the in vivo efficacy of nafamostat.

\section{Intra-Nasal Nafamostat in SARS-COV-2 mice model:}

Li et.al observed that the intranasal nafamostat was effective in mice model treatment. Li et.al concluded that the effectiveness of intranasal nafamostat might be due to decreased time interval between nafamostat delivery and viral inoculation [140]. Li et.al opined that currently, there is no data regarding nafamostat stability in respiratory secretions following i.n.(Intra Nasal) administration [140]. It is possible that the fate of nafamostat is different in human airway secretions than that in plasma, potentially contributing to the different outcomes observed via i.n. or i.p. routes in their study [140]. It is also unknown how efficiently nafamostat is transported into airway secretions when delivered systemically, which may influence outcomes following i.n. versus i.p. administration[140]. Conclusively there is no proven data for effective use of inhalation mediated delivery of nafamostat to respiratory tracts in COVID-19 patients which might depend on lot of factors but it was found to show better results when compared with other routes at-least in mice models .

\section{Disulfide inhibitors restrict SARS-COV-2 entry into human airway cells:}

Manček et.al tested the hypothesis that disruption of disulfides within RBD of SARS-CoV-2 spike protein prevents the fusion, subsequent viral entry into the host cells and found it to be true in airway epithelial cells [135]. This is in confirmation with the earlier author Hati et.al's invitro and insilico analysis [1104]. In their experiments Manček et.al used different thiol-reactive registered drugs that can target the reduction of disulfide bonds [135]. Manček et.al found that NAC alone did not inhibit cell fusion between SARS-COV-2 and host cell but its derivative NACA [138], with more potent antioxidant properties, strongly inhibited the formation of syncytia [135]. This is in agreement with the other results that showed that using NAC alone cannot inhibit viral entry but can only help reduce disulfides and delay the entry process [133]. These results show that there is huge potential for super antioxidant to prevent the viral entry. Tempol a super antioxidant which might as well be a good choice [5]. 
Manček et.al also found that L-ascorbic acid effectively inhibited cell fusion [135]. Manček et.al also reported that glutathione failed to inhibit the entry of SARS-COV-2 [135]. Manček et.al proposed that NACA, L-ascorbic acid, and JTT-705 efficiently inhibited the interaction in spike-ACE2 binding assay, implying that this group of compounds each seperately have the potential to affect the direct interaction between SARS-COV-2 spike and ACE2 [135].

Manček et.al through their experiments showed that SARS-COV-2 Spike protein and ACE2 alone are sufficient for the formation of syncytia, this group also observed that TMPRSS2 (transmembrane protease serine 2) co-expression on ACE2 cells further promotes the syncytia formation, thus increasing the sensitivity of the system [135]. They further validated this observation by using camostat mesylate, a TMPRSS2 inhibitor [135]. Conclusively, SARS-COV-2 Spike protein and ACE2 alone are sufficient for the syncytia formation, and TMPRSS2 (transmembrane protease serine 2) coexpression on ACE2 cells further accelerates the syncytia formation. Conclusively, In order to inhibit the entry of SARS-COV-2 into host cell effectively, it's important to target both disulfides and TMPRSS2 at the same time.

Manček et.al observed efficient inhibition of viral entry after 24 hours of exposure to NACA and auranofin without cytotoxicity independently [135]. Manček et.al team also proved the Inhibitory activity of selected compounds in lung-derived A549 cells by infecting them with SARS-CoV-2 virus and by maintaining a stable expressing of ACE2 at an $\mathrm{MOI}$ of 1 , which were pre-treated for 3 hours by the indicated concentrations of the proposed compounds (NACA) [135].

\section{Tempol:}

Manček et.al found that NAC alone did not inhibit cell fusion but its derivative NACA [138] which has more potent antioxidant properties, strongly inhibited the formation of syncytia [135]. Tempol a super antioxidant might be as well a good choice [5].

Tempol is an powerful antioxidant $[2,4,58,73]$. Tempol relieves lung injury in a rat model of chronic intermittent hypoxia via suppression of inflammation and oxidative stress [3]. Tempol is protective against Hypoxia-induced Oxidative Stress and Apoptosis [1].

Maio et.al reported that Tempol can inhibit the viral replication by potentially inhibiting the RdRp [7]. Kavita et.al through their experiments concluded that Tempol as a novel antioxidant might inhibit both activated T cell and antigen presenting cell derived cytokines production in-vitro from COVID-19 patients [6].

\section{Tempol in COVID-19:}

Peter et.al reported that early administration of Intranasal Tempol treatment in a single COVID-19 patients reduced nasal congestion by day 4-5 and was found to be asymptomatic by day 8 . Although it's a single case report taken into account that powerful antioxidant can inhibit viral entry and taken that Tempol inhibits viral replication of SARS-COV-2 and taken into account the SARS-COV-2 induced Oxidative stress and associated cell death, Intranasal/Nebulized Tempol administration along with standard care can increase the out come drastically.

Conclusively Bromelain as TMPRSS2 inhibitor can reduce both TMPRSS2 and ACE-2 expression in airway epithelial cells and in combination with NAC can provide even better results. Whereas NAC can only reduce the disulfides at the surfaces but NAC cannot alone inhibit the viral entry completely, which subsequently might delay the entry as discussed earlier. Adding a powerful antioxidant (super oxide) like NACA or Tempol to this combination can completely inhibit the viral entry. Tempol might also be a good choice. 


\section{NAC and good results in COVID-19 patients:}

Cases with severe COVID-19-associated respiratory failure who have received standard of care and routine treatment along with NAC have been associated with encouraging results and positive outcome in COVID-19 treatment [1003]. Puyo et.al reported that IV- NAC has been found to be producing positive results [143].

Liu et.al reported that inhalation of $\mathrm{N}$-acetylcysteine (NAC) solution through respiratory airway in a single patient with mechanical ventillation showed positive outcome [142].

In another case report, IV and oral Glutathione and alpha-lipoic acid are reported to be associated with successful treated in two COVID-19 patients with dyspnea [146].

Horowitz et.al proposed that Oral and IV glutathione, glutathione precursors ( $\mathrm{N}$-acetyl-cysteine) and alpha lipoic acid may represent a novel treatment approach for blocking NF-KB and addressing "cytokine storm syndrome" and respiratory distress in patients with COVID-19 pneumonia [146].

In another report by Alamdari et al., the combination of methylene blue-vitamin C-NAC (1 mg/kg methylene blue, $1500 \mathrm{mg} / \mathrm{kg}$ vitamin C, $1500 \mathrm{mg} / \mathrm{kg} \mathrm{NAC}$ ), as last resort therapy, was administrated to five critically ill COVID-19 patients with high serum level of nitrite, methemoglobin, and oxidative stress. Results of this small case serious trials also proved to be promising, as four out of five patients responded well to the treatment and recovered [141].

\section{1. (Intravenous N-Acetyl-Cysteine) IV- NAC when administered properly might enhance COVID-19 patient's condition by reducing inflammatory cytokines:}

IV-NAC has long been used to safely treat patients with acetaminophen overdose $[119,120]$, and ARDS (Acute Respiratory distress syndrome) [121,122]. NAC was also found to reduce CRP (C-reactive protein) levels in several controlled clinical trials $[115,116]$. CRP elevation is a prominent risk factor for disease progression in patients infected with COVID-19 $[117,118]$.

Ibrahim et.al showed that IV (Intravenous) NAC (N-Acetyl-Cysteine) administration was useful in the treatment of critical COVID-19 patients with elevated parameters. Ex: Elevated CRP, ferritin etc...[9]. Ibrahim and their group administered IV- NAC multiple times whenever the inflammatory makers were elevated in COVID-19 patients [9]. After admission with NAC they have observed subsequent decrease in the inflammatory markers rapidly [9]. Their NAC admission protocol shows different NAC administration durations (Number of days) for different patients depending on their inflammatory levels with the same dose of approx $600 \mathrm{mg}$ NAC for every 12 hours in 8 patients (actual data can be found in Ibrahim et.al publication-[9]) [9]. The number of days NAC was administered in most of the COVID-19 patients ranged from as short as 2 days to as long as 9 days [9]. Although Two patients with an amount of 20,000 to $30,000 \mathrm{mg} \mathrm{NAC}$ administration were reported to be recovered in two days [9]. This can be considered as an NAC shock treatment [9]. From the above observations we can conclude that there is no generalized time within which NAC should be administered for observing favourable results in COVID-19 patients, but rather we can conclude that IV-NAC should be administered as advised by physician from patient to patient or case to case basis either continuously in long run or should be administered as a shock treatment in non-responding ARDS patients or in a discrete manner whenever required to suppress the pro-inflammatory cytokines or whenever the proinflammatory cytokines are elevated in the absence of IV-NAC administration [9].

Taher et.al conversely reported that IV-NAC administration showed positive impact but did not show significant results in the treatment of critical COVID-19 patients [1003]. Taher et.al in their study of 92 patients administered 47 patients with IV-NAC and treated the other 45 people as placebo. These 
patients were followed up for 28 days [1003]. But in the study Taher et.al[1003] have followed only a 3-day treatment protocol [1003]. We have already seen in the above successful study of Ibrahim et.al [9] that temporary/single time administration of NAC can only produce temporary results by suppressing inflammatory responses temporarily when administered in critical COVID-19 patient [1003]. Supportively enough, Taher et.al also reported that temporary betterment of the COVID-19 patients was observed after following a 3-day treatment protocol just after 3 days but not in the final outcome [1003].

Conclusively enough, the difference in results might most likely be due to the fact that Ibrahim et.al [9] followed a protocol where the number of days NAC was administered in most of the patients ranged from as low as 2 days to as long as 9 days depending on the patients subsequent inflammatory response in the absence of NAC treatment [9].

Both studies have specified that some of the patients have used dexamethasone along with NAC during the course of their study. So dexamethasone might be working synergically along with IV-NAC as well. Using dexamethasone has already been proved to be useful in treating critical COVID-19 patients but not in mild cases [161]. Using dexamethasone at safer levels in combination with IV-NAC can provide much more better results rather than higher dosages of only dexamethasone alone as higher doses of dexamethasone are associated with higher risk of susceptibility to dangerous secondary infections. Early administration of Dexamethasone might not be useful for the patients as it might suppress the early inflammatory response that kills the infected cells and put the patient at risk of Virus mediated alveolar collapse [161]. 
The below images have been taken from Ibrahim et.al [9], the whole credit of this research goes to them and their group, if any data is required please refer to their publication or consult them [9]. The purpose of using this image is to show that NAC when administered continuously can show proper results in COVID-19 patients as proved by Ibrahim et.al [9].

B

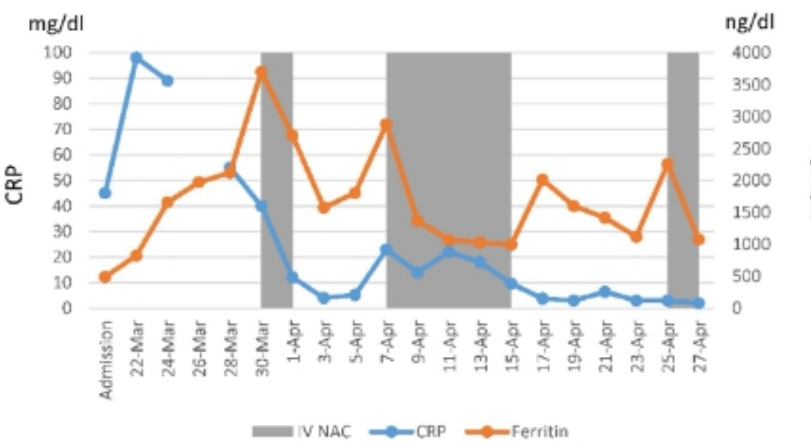

c

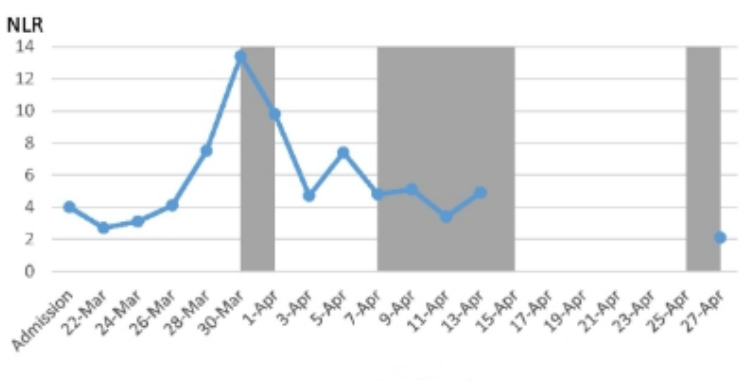

Fig. 1. Effect of IV NAC on clinical and laboratory outcomes in a G6PD-deficient patient infected by COVID-19. Gray shaded areas represent intervals of IV NAC administration. Initiation and termination of CC-ECMO are indicated along the horizontal axis with yellow and blue dots, respectively. A) Display of total and direct bilirubin levels. B) Tracking of CRP and ferritin levels. C) Monitoring of neutrophil/lymphocyte ratio (NLR). (For interpretation of the references to colour in this figure legend, the reader is referred to the web version of this article.)

Effect of IV NAC on inflammation assessed by serum levels of CRP (mg/ml) and ferritin (ng/ml) and clinical outcome of COVID-19 infection in 9 patients without G6PD deficiency. ${ }^{*}, p=.0022 ;{ }^{\star \star}, p=.0301$, using two-tailed paired $t$-test.

\begin{tabular}{|c|c|c|c|c|c|c|c|c|}
\hline $\begin{array}{l}\text { Patient } \\
\text { (Age/Gender) }\end{array}$ & $\begin{array}{l}\text { CRP } \\
\text { before NAC }\end{array}$ & $\begin{array}{l}\text { CRP } \\
\text { after NAC }\end{array}$ & Ferritin before NAC & Ferritin after NAC & NAC duration & NAC dose (mg) & ECMO & Outcome \\
\hline $1(44 / \mathrm{M})$ & 89 & 14 & 3700 & 1500 & 2 days & 30,000 & Yes & Dlscharged home \\
\hline $2(44 / \mathrm{M})$ & 90 & 13 & 9000 & 2000 & 2 days & 20,000 & Yes & Discharged Home \\
\hline $3(48 / \mathrm{M})$ & 243 & 72 & 5900 & 2700 & 7 days & 600 every $12 \mathrm{~h}$ & Yes & Dascharged Home \\
\hline $4(38 / \mathrm{M})$ & 280 & 26 & 4900 & 900 & 9 days & 600 every $12 \mathrm{~h}$ & Yes & Hospitallzed \\
\hline $5(38 / \mathrm{M})$ & 46 & 5 & 1100 & 800 & 4 days & 600 every $12 \mathrm{~h}$ & Yes & Discharged home \\
\hline $6(42 / \mathrm{M})$ & 235 & 31 & 4000 & 2500 & 5 days & 600 every $12 \mathrm{~h}$ & Yes & Hosptaltized \\
\hline $7(48 / 7)$ & 99 & 45 & 300 & 330 & 4 days & 600 every $12 \mathrm{~h}$ & Yes & Discharged Home \\
\hline $8(48 / \mathrm{M})$ & 307 & 23 & 2700 & 1100 & 6 days & 600 every $12 \mathrm{~h}$ & Yes & Discharged Home \\
\hline $9(71 / \mathrm{M})$ & 145 & 71 & 2200 & 1800 & 5 days & 600 every $12 \mathrm{~h}$ & No & Discharged home \\
\hline $10(65 / \mathrm{M})$ & 63 & 11 & 2800 & 1800 & 4 days & 600 every $12 \mathrm{~h}$ & Yes & Discharged home \\
\hline Mean \pm SD & $160 \pm 97$ & $31 \pm 24^{*}$ & $3630 \pm 2526$ & $1543 \pm 762^{* *}$ & & & & \\
\hline
\end{tabular}

Original author of the below images is [lbrahim et.al- [9] with title as: "Therapeutic blockade of inflammation in severe COVID-19 infection with intravenous $\mathrm{N}$-acetylcysteine.", Clin Immunol. 2020 ]-[9] 


\section{Dexamethasone in COVID-19:}

Horby et.al reported that, In patients hospitalized with Covid-19, the use of dexamethasone resulted in lower 28-day mortality among those who were receiving either invasive mechanical ventilation or oxygen alone at randomization but not among those receiving no respiratory support [161]. Conclusively, Dexamethasone or associated immunosuppressants should not be used at earlier stages in COVID-19 unless the inflammatory markers are elevated.

These above studies collectively suggest that using IV NAC in severe COVID-19 patients along with Dexamethasone (only in critical patients) can provide good results.

\section{Additional uses of NAC usage in Viral infections:}

NAC can decreases ACE-II expression [125,126]. High-doses of $\mathrm{N}$-acetylcysteine therapy for novel H1N1 influenza pneumonia has been found to be beneficial [127]. NAC was shown to inhibit NF-KB activation in an in-vitro influenza ( $A$ and $B$ ) model [127]. In the context of influenza virus infection, NAC administration ( $100 \mathrm{mg} / \mathrm{kg}$ continuous iv. infusion daily for 3 days) was reported to promote clinical improvement in a woman with H1N1 influenza pneumonia; Oseltamivir was also employed during treatment [127]. N-acetyl-L-cysteine (NAC) inhibits mucin synthesis and pro-inflammatory mediators in alveolar type-Il epithelial cells infected with influenza virus $A$ and $B$ and with respiratory syncytial virus (RSV) [129]. NAC might increase TLR-7 as, well [131].

\section{Ribavirin:}

Reem et.al treated COVID-19 patients with a combination of Darunavir/ Cobicistat and Ribavirin [40]. His group found that this combination of Darunavir/ Cobicistat and Ribavirin although showed faster viral clearance by day 7, 14 and 28 compared to darunavir/cobicistat alone, but could not show any statistical difference [40].

Reem et.al report of faster viral clearance at day 7 which can be credited to both Darunavir/ Cobicistat and Ribavirin combination but the viral clearance at day 14 can be credited to Ribavirin alone [40]. Since this study has been conducted in Middle east we can assume that there might be some immune dysregulations due to excessive smoking of people [40, 45]. This immune dysregulation might have suppressed the advantages of Ribavirin in final outcome of severe patients.

\section{Aerosole Ribavirin Therapy:}

Ribavirin inhibits RNA synthesis by disrupting the activity of viral RNA-dependent RNA polymerases (RdRp), crucial enzymes in the life cycle of coronaviruses, and also inhibits mRNA capping $[61,62,63,39]$. Ribavirin has been used against RdRp of the hepatitis C virus [63], and orally administered ribavirin (in combination with other medications) is approved by the US Food and Drug Administration (FDA) and European Medicines Agency for the treatment of chronic hepatitis $C$ infection $[64,65,39]$. Additionally Powdered Ribavirin has been shown to be efficiently delivered to lungs in patients with COPD and subsequent viral induced exacerbations [49]. Ribavirin for inhalation solution (ribavirin aerosol) is approved by the FDA and Health Canada for the treatment of infants and young children with severe lower respiratory tract infections due to respiratory syncytial virus $[66,67]$. Aerosol administration of ribavirin has been shown to be effective against multiple variants of influenza $[68,69,70]$. Ribavirin $100 \mathrm{mg} / \mathrm{mL}$ administered using a more efficient nebulizer was effective in reducing mortality in a lethal influenza A virus mouse model [71]. Administration of ribavirin aerosol as recommended in the treatment of respiratory syncytial virus (20 mg/mL over $12 \mathrm{~h}$ ) [66]. The results of a randomized, double-blind, placebo-controlled study support the feasibility and efficacy of using ribavirin aerosol treatment in infants requiring mechanical ventilation for respiratory failure caused by respiratory syncytial virus (RSV) infection [72]. 


\section{a. Ribavirin and SARS-COV-2:}

In order to evaluate the effectiveness of ribavirin and other anti-polymerase drugs against SARS-CoV2, a 2020 study used homology modeling to build the Wuhan SARS-CoV-2 RdRp and then assessed the binding properties of different antiviral compounds using molecular docking methods [63]. Authors concluded that ribavirin demonstrated tight binding to the SARS-CoV-2 RdRp and proposed that it could potentially interfere with protein synthesis, leading to viral eradication [63].

\section{b. Ribavirin in COVID-19:}

Aerosol Ribavirin has been made available in Italy for patients with COVID-19 as part of a compassionate use program and has been demonstrated to be effective by Messina et.al without any significant side effects [39].

Prior to patient's enrollment in the compassionate use program, the diagnosis of COVID-19 was confirmed by positive tests for SARS-CoV-2 and laboratory testing ruled out other bacterial, viral, and fungal etiologies including Legionella, pneumococcus, Chlamydia pneumoniae, Mycoplasma pneumoniae, tuberculosis, cytomegalovirus, human immunodeficiency virus, and aspergillosis [39].

Messina et.al in their study enrolled COVID-19 patients with SARS-COV-2 induced parenchymal thickening and multiple ground glass areas in lungs [39]. The $\operatorname{CRP}(9,75,90,7.8,45 \mathrm{mg} / \mathrm{L})$, Ferritin levels $(301 \mathrm{ng} / \mathrm{mL}, 617,779,420,-)$, IL-6 $(0.6,13.8,31.8,18.2,17.3 \mathrm{ng} / \mathrm{mL})$ in all these patients are high but not too much elevated [39]. One of the patient has been reported to be with obesity. Chest CT scans of all five patients showed to be present with multiple areas of parenchymal thickening and ground glass opacities [39].

Messina et.al (published on June 28, 2021) in their study of four (4) men and (1) one women with ages ranging from 29-72 years reported a successful clinical outcomes in administration of Aerosol Ribavirin for the treatment of SARS-COV-2 [39]. Their group administered the patients with $100 \mathrm{mg} / \mathrm{ml}$ of Ribavirin aerosol for 30 min twice daily for 6 days. (I.e: 12 doses) (For more information and data Please refer to original data from original author's publication -Messina et.al-[39]) [39].

Messina et.al reported that Aerosol Ribavirin either reversed the multiple areas of parenchymal thickening and ground glass opacities in the lungs of almost all COVID-19 patients and prevented their disease condition from becoming worser [39]. Messina et.al has further confirmed this through respective CT-scans images of all the enrolled and Aerosol Ribavirin administered patients.

Aerosol Ribavirin prevented the patients from getting admitted to ICU's. [39]. All patients were observed to be recovered fully, and nasopharyngeal swabs obtained after hospital discharge tested negative for SARS-CoV-2 [39]. Ribavirin aerosol appears to be efficacious in the treatment of patients with COVID-19. Messina et.al did not observe any adverse reactions to Ribavirin treatment in any of the five patients [39].

Administration of ribavirin Aerosol $100 \mathrm{mg} / \mathrm{mL}$ for $30 \mathrm{~min}$ is estimated to deliver $1760 \mathrm{lg} / \mathrm{mL}$ to the alveolar lining fluid, which is approximately 64 times the half maximal response (EC50) of $26.7 \mathrm{lg} / \mathrm{mL}$ observed against a clinical isolate of SARS-CoV-2 in vitro (for data, please check the original publication by Messina et.al-[39]) [39].

There are other studies that confirmed and demonstrated the effectiveness of Ribavirin treatment in COVID-19. In vitro research has demonstrated the antiviral efficacy for lopinavir and ribavirin against SARS-associated coronavirus, and a clinical study showed that patients with probable SARS-CoV-2 treated with a combination of lopinavir/ritonavir, ribavirin (oral or intra- venous), and corticosteroids $(n=41)$ had a significantly lower rate of adverse clinical outcomes (i.e., acute respiratory distress syndrome or patient mortality) than a historical control group treated with ribavirin and corticosteroids alone $(n=111 ; P \backslash 0.001)[79,39]$. 
Supportively enough, Zhou et.al reported the successful use of intravenously administered Ribavirin (3 times daily for 14 days) in a 38-year-old man diagnosed with COVID-19 provided positive outcome [77]. After the treatment Zhou et.al reported that Chest CT scans revealed resolution of previous pneumomediastinum and a reduction of parenchymal consolidation with pulmonary fibrosis and pneumatocele in the inferior left lower lobe of the patient [77]. RT-PCR was negative on day 30, and the patient was found to be discharged for outpatient follow-up [77]. Zhou et.al reported that intravenously administered Ribavirin was used as one of the component of medical therapy that also included antibiotics, antitussives, bronchodilators, and interferon-a1b [77,39].

A 2020 open-label, randomized, phase 2 trial in hospitalized patients with COVID-19 evaluated 14-day combination therapy $(\mathrm{n}=86)$ with orally administered lopinavir/ ritonavir, orally administered ribavirin, and subcutaneously administered interferon-b1b (in the subset of 52 patients admitted less than 7 days from symptom onset) compared with a control group that received only orally administered lopinavir/ritonavir $(n=41)[78,39]$. That study found that the combination treatment was significantly better for alleviating symptoms, reducing viral load, and shortening the duration of hospitalization [78].

Although side effects associated with Ribavirin must be taken into account as it happens to be that side effects might occur in different people with different drugs and their combinations. Scientists and Physicians should look into the safety profile of drugs while administering them.

\section{c. Ribavirin and Pre-cautious measures to be taken :}

Health care workers should be careful when using an aerosol treatment in patients infected with SARS-CoV-2 because of increased risk of healthcare providers being exposed to the virus. It is known that the drug can disperse into the bedside area during treatment with ribavirin aerosol [66, 39], and the extent to which treatment may also impact virus dispersal into open air is unclear. In addition to the standard precautions taken when treating patients with COVID-19, it is recommended that healthcare providers should wear a facemask (as well as eye protection, gloves, and a gown), close the door to the patient room, and remain at a safe distance (possibly outside the door) during all types of nebulizer used treatments $[80,39]$.

\section{Ulinastatin for rescuing from SIRS, Inflammation mediated Injury and Sepsis:}

Urinary trypsin inhibitor (Ulinastatin, UTI) is a nonspecific and multivalent Kunitz-type serine protease inhibitor purified from human urine [176]. It is capable of suppressing various serine proteases such as trypsin, plasmin, neutrophil elastase and chymotrypsin $[177,178]$, and can also effectively stabilize lysosomal and cellular membranes [179]. Ulinastatin has confirmed powerful efficacy in inhibiting the release of inflammatory factors, removing oxygen free radicals, improving microcirculation and tissue perfusion, and alleviating endothelial injuries $[180,173,181,182]$.

Sepsis is a common cause of morbidity and mortality in critically ill patients, and its incidence is increasing worldwide annually $[163,164,162]$. The pathogenesis of sepsis is complex and is believed to be initiated by the interaction between pathogen-associated molecular patterns and pattern recognition receptors on host immune cells $[165,166,162]$. This process sets off a series of proinflammatory mechanisms including synthesis and release of cytokines and complement, chemotaxis and activation of neutrophils, and initiation of coagulation [165-167,162]. Current scientific opinion suggests that the systemic inflammatory response syndrome (SIRS) that characterizes severe sepsis results from an excessive activation of pro-inflammatory mediators, which have pleiotropic effects that overwhelm the body's anti-inflammatory mechanisms, leading to widespread vascular, endothelial, and organ dysfunction that is often fatal [165-168,162,181]. 


\section{a. Ulinastatin usage in SIRS, endothelial damage, Multi organ dysfunction Syndrome and Sepsis:}

Ulinastatin improves the survival of septic mice by suppressing pro-inflammatory responses and lymphocyte apoptosis [169, 174,2032]. Ulinastatin provides protective effect against murine models of sepsis by inhibiting TNF- $\alpha$ and IL-6 $[170,181]$. Ulinastatin is protective against LPS mediated inflammatory responses [172]. Urinary trypsin inhibitor suppresses excessive generation of superoxide anion radical, systemic inflammation, oxidative stress, and endothelial injury in endotoxemic rats [173].

Zhang et.al conducted a trial on sixty patients and reported that Ulinastatin significantly improves the inflammatory symptom and signs of (systemic inflammatory response syndrome) SIRS, such as Temperature $(T)$, heart rate $(H R)$, respiration rate (RR) and white blood cell $(W B C)$ count and inhibits the production of inflammatory markers like Serum $C$ reactive protein $(C R P)$, tumor necrosis factoralpha (TNF- $\alpha$ ), interleukin-6 (IL-6), and enhances the anti-inflammatory cytokines in the treatment of SIRS [171].

Ulinastatin can also effectively prevent the progression of SIRS towards multiple organ dysfunction syndrome (MODS) [171].

In their pilot study, Karnad et.al showed that intravenous administration of Ulinastatin reduced mortality in patients with severe sepsis [162].

Wang et.al conducted a trial to evaluate the effects of ulinastatin on mortality and related outcomes in sepsis patients [187]. Thirteen randomized controlled trials and two prospective studies were published by their team. This study included 1358 patients with sepsis, severe sepsis, or septic shock and were evaluated for the outcome when administered with Ulinastatin [187].

Wang et.al reported that Ulinastatin significantly decreased the all-cause mortality, Acute Physiology and reduced the incidence of multiple organ dysfunction syndrome (MODS). Wang et.al reported that Ulinastatin also decreased the serum levels of IL-6, TNF- $\alpha$ and increased the serum levels of IL-10. Wang et.al found that Ulinastatin administration did not lead to any difference in the occurrence of adverse events [187]. Wang et.al concluded that Ulinastatin improved all cause mortality and other related outcomes in patients with sepsis or septic shock [187].

The results of this meta analysis suggested that ulinastatin may be an effective treatment for sepsis and septic shock [187].

Li et.al found that Ulinastatin attenuates LPS-induced inflammation in mouse macrophage RAW264.7 cells by inhibiting the JNK/NF-KB signaling pathway and activating the PI3K/Akt/Nrf2 pathway [188].

\section{b. Ulinastatin downregulates HMGB-1 expression and pro- inflammatory cytokines:}

Ying et.al reported that Ulinastatin preconditioning attenuates Inflammatory reaction of Hepatic Ischemia Reperfusion Injury in Rats via High Mobility Group Box 1 (HMGB-1) Inhibition [189]. Ying et.al through their experiments showed that pretreatment with ulinastatin attenuated liver ischemia reperfusion (IR) injury by reducing HMGB1 expression through its anti-inflammatory effects [189]. Wang et.al showed that Ulinastatin treatment significantly downregulates HMGB1, TNF- $\alpha$ and IL-6 expression [190].

Such effects of Ulinastatin inhibiting HMGB-1 expression and HMGB-1 mediated inflammatory responses has been reported by other authors [190,191]. Zhao et.al demonstrated that ulinastatin inhibited the hepatic hypoxia/reoxygenation $(H / R)$ injury in Chang liver cells, and concluded that it might be due to autophagy activation [192]. 
Additionally, Sun et.al observed that Glutamine combined with ulinastatin treatment can alleviate damage to intestine after severe burn injury, lower the serum level of inflammatory cytokines, promote wound healing, and reduce the incidence of burn sepsis [193]. Sun et.al reported that Ulinastatin can weaken the immunosuppressive function mediated by splenic CD4(+) CD25(+) Tregs in severely burned rats, and improve proliferative function and secretory function of splenic CD4(+) T lymphocytes, and concluded that this effect may be attributed to the inhibiting effect of ulinastatin on the release of HMGB1 in large amount [193].

Ulinastatin ameliorates Pulmonary Capillary Endothelial permeability induced by Sepsis through protection of endothelial-tight Junctions via the inhibition of TNF- $\alpha$ and related pathways [182]. Ulinastatin may also protect endothelial cells from neutrophil-mediated injury not only by inactivating the extracellular elastase excreted from Neutrophils, but also by suppressing the production of activated elastase [178]. These pathways ultimately lowered the degrees of endothelial injuries and high permeability [178]. Ulinastatin might also modulate Tregs induction [186].

\section{c. Ulinastatin reduces Neutrophil mediated damage:}

In Kawasaki disease (KD), circulating neutrophils proliferate and are functionally activated as levels of reactive oxygen species and elastase increase [184]. This suggests that neutrophil-mediated injury of endothelial cells is involved in the pathogenesis of KD vasculitis. Takeshita et.al proposed that Ulinastatin (urinary trypsin inhibitor; UTI) inhibits neutrophil-mediated injury of endothelial cells in vitro, mainly by inactivating neutrophil elastase [184].

Ulinastatin inhibits (poly-morphonuclear neutrophils) PMNs activity and reduces the systemic inflammatory response [183]. Ulinastatin is an Elastase Inhibitor [185]. Ulinastatin reduces Neutrophils elastase [175].

Serine protease inhibitors induce their Inhibitory effect on neutrophil-mediated endothelial cell injury [178]. Neutrophils have been associated with devastating damage in viral immune responses $[1399,1424,1029,1036]$. Neutrophils mediate the Lung damage in ARDS and Acute Lung Injury $[1013,1014,1028,1030-1036,1044,1045]$. Pulmonary endothelial activation caused by extracellular histones contribute to Neutrophils Activation in ARDS [1015-1020,1026]. Ulinastatin also improves microcirculation during excessive hemorrhage [180].

Neutrophils are reported to be elevated in severe COVID-19 patients [194-199]. Elastase and exacerbation of neutrophil innate immunity are involved in multi visceral manifestations of COVID-19 [2033]. Additionally, Proteinase has been observed to be released from activated neutrophils in mechanically ventilated patients in both non-COVID-19 and COVID-19 pneumonia [2034]. Activated Neutrophils are associated with subsequent endothelial or Lung injury in COVID-19 [598,597,1448,1449,1451,1452,1453,1455,1419,1420,1421,1422,1012,]. Neutrophils associated Neutrophil extracellular traps (NET's) have been associated with thrombosis in COVID-19 pathogenesis [2035]. Neutrophils associated Neutrophil extracellular traps (NET's) have been associated with other negative outcomes in COVID-19 patients [2036, 2037,2038]. Mohamed et.al hypothesized that Ulinastatin administration at the earliest stage in COVID-19 patients might prevent Lymphopenia [2039].

\section{d. Ulinastatin in COVID-19 Patients:}

Huang et.al reported that high doses of Ulinastatin showed promising results in COVID-19 patients [2031]. Huang et.al treated Twelve hospitalized patients with confirmed SARS-CoV-2 infection, with high doses of ulinastatin [2031]. The average age of the patients in this study was $68.0 \pm 11.9$ years. Nine of the 12 patients (75.0\%) had been reported to have one or more comorbidities [2031]. All 5 out 12 patients have been associated with decreased lymphocytes and 7 out of 12 patients have been associated with elevated C-reactive protein (CRP) levels (mean, $49.70 \pm 77.70 \mathrm{mg} / \mathrm{L}$ ) (for original data please look into original authors publication-Huang et.al - [2031]) [2031]. 
Huang et.al reported that the white blood cell levels and the percentage of lymphocytes returned to normal in all of the patients, and CRP decreased significantly and returned to normal in 10 out of 12 patients (mean, $6.87 \pm 6.63 \mathrm{mg} / \mathrm{L}$ ) on the seventh day after high dose Ulinastatin treatment (for original data please look into original authors publication Huang et.al - [2031]) [2031]. Huang et.al also reported that in 8 out of 12 patients the peripheral oxygen saturation was improved after treatment and prevented them from the requirement of mechanical ventillation or ICU admission [2031].

\section{CD-24 for inhibiting pro-inflammatory responses and Lymphopenia:}

CD24 acts as a rheostat of the cell and modulates the cell surface receptor signaling of diverse receptors [544,740]. CD24 is very important in regulating the cell fate [669]. CD24, Siglec-G/10 interactions are very important to discriminate between DAMPs and PAMPs [751]. CD24 Expression on T Cells is required for the Optimal Proliferation of T Cells in lymphopenic Host [729]. CD24 plays a very important role in inducing immune tolerance towards inflammation, CD24-Siglec-10 selectively repress tissue damage induced during inflammatory immune responses [752,753,732]. Additionally, reduced CD24 expression has been indicated as a marker of failure in Immune Tolerance [741, 735].

CD-24 will be a promising treatment given the fact that it has vital role in controlling the proinflammatory immune responses through inhibiting NF-KB. CD-24 can suppress cytokine storm and inhibit MDSCs immunosuppressive response mediated Lymphopenia.

CD-24 can sense and bind to HMGB-1 [488]. CD24-Siglec-G/10 plays a very important role by discriminating between self and non-self during both innate and adaptive immune system induced responses [754].

\section{a. CD24 in COVID-19 Patients:}

CD-24 treatment in COVID-19 patients has been reported to be effective and is undergoing final phase trials $[2040,2041]$.

\section{Anti-HMGB-1:}

\section{a. Anti-HMGB-1 in Influenza:}

High-mobility group box 1 (HMGB-1) protein is associated with negative outcome in the patient with severe influenza H5N1 virus infection [352]. Anti high mobility group box-1 monoclonal antibody treatment has been found to provide protection against influenza A virus (H1N1) induced pneumonia in mice [351,353]. Anti-high mobility group box-1 monoclonal antibody treatment is effective in ameliorating influenza infection and lipopolysaccharide induced brain edema [535].

Anti HMGB1 treatment is associated with reduced inflammation in models of experimental autoimmunity [409]. Anti-HMGB1 Neutralizing Antibody has been showed to attenuates Periodontal Inflammation in a Murine Periodontitis Model [261].

\section{b. Anti-HMGB-1 in Sepsis:}

Systemic T-Cell exhaustion dynamics is linked to Early High Mobility Group Box Protein 1 (HMGB1) driven Hyper-Inflammation in a Polytrauma Rat Model [425].

During peripheral tissue trauma HMGB-1 attenuates T-lymphocyte response and increases splenic CD11b (+) Gr-1 (+) myeloid-derived suppressor cells [517]. 
Anti-high-mobility group box protein 1 antibodies have been found to improve the survival chances of rats with sepsis [504].

Anti-HMGB1 monoclonal antibody ameliorates the immunosuppression after peripheral tissue trauma by suppressing myeloid-derived suppressor cells [517].

\section{Monoclonal Therapy in COVID-19 patients:}

Webb et.al conducted a trial of administering (Neutralizing monoclonal antibodies) mAb in 594 patients out of 7404 patients and reported that MAb treatment of high-risk ambulatory patients with early COVID-19 was well tolerated and likely effective at preventing the need for subsequent emergency department or hospital care [2048].

Piccicacco et.al reported that among high-risk COVID-19 patients with mild/moderate symptoms, early administration of mAbs potentially reduced the illness [2049]. The mortality rate was $0 \%$ in the mAb group compared with $3.5 \%$ in the control group. Patients treated with mAbs were significantly less likely to be hospitalized or visit the (emergency department) ED compared to patients not treated with mAb [2049].

Ganesh et.al through their observational study of 3596 patients reported that the use of bamlanivimab and casirivimab-imdevimab / mAbs in high-risk COVID-19 patients showed lower rates of hospitalization [2050].

Bariola et.al reported that the use of bamlanivimab monotherapy for outpatients with mild to moderate COVID-19 infection was associated with reductions in hospitalizations and mortality within 28 days [2051].

Latest Review regarding mAb therapy in COVID19 patients can be found here [2053]. Guidelines for mAb therapy can be found here [2054, 2055].

\section{Nebulized Unfractionated Heparin (UFH):}

Nebulized Heparin is useful in the treatment of inhalation induced injury in lungs [2094,2095,2096]. Glas et.al conducted a systemic search and found that Nebulized heparin was useful for patients under mechanical ventilation [2100] as an anticoagulant [2100]. The number of ventilator days and mortality at day 28 were observed to be reduced in patients treated with nebulized heparin when compared to patients in the control group [2100, 2128].

Juschten et.al conducted a systemic search of medical research and found that nebulized anticoagulants attenuates the pulmonary coagulopathy in preclinical studies with various models of lung injury, but their effects on inflammation was found to be less consistent [2129]. Viral induced damage in lungs or any other parts usually leads to thrombotic complications [2130]. SARS-COV-2 also induces such manifestations [2130]. Heparin is reported to be useful in the treatment of pulmonary microvascular thrombosis and hyaline membranes [2109]. Heparin has been observed to be widely used in the management of COVID-19 [2130]. A review presented that Heparin can be helpful as a multi targeting drug in the treatment in COVID-19 [2118].

COVID-19 is reported to be associated with the development of ARDS displaying the typical features of diffuse alveolar damage with extensive pulmonary coagulation activation resulting in fibrin deposition in the microvasculature and formation of hyaline membranes in the air sacs [2109]. The anti-coagulant actions of nebulised heparin may limit fibrin deposition and progression of lung injury and thrombosis [2109].

Pulmonary thrombosis is frequently seen in patients with COVID-19 pneumonia [2132,2133] and is associated with increased dead space and subsequent onset of severe hypoxaemia [2128]. Trials in patients with acute lung injury and related conditions found that inhaled UFH reduces the pulmonary dead spaces, coagulation activation, microvascular thrombosis and clinical deterioration, resulting in increased time free of ventilatory support [2103, 2097]. 


\section{a. UFH inhibits SARS-COV-2 entry by destabilizing RBD:}

Additionally, unfractionated heparin (UFH) is found to inactivate the SARS-CoV-2 virus and prevents its entry into host cells [2109]. Nebulisation of heparin may therefore limit both fibrin-mediated lung injury and inhibit pulmonary infection by SARS-CoV-2 [2109].

A number of studies found heparin competes with heparan sulphate for bacterial and viral adhesion and may therefore limit pathogen invasion. Heparin limits adhesion of a number of pathogens including Pseudomonas aeruginosa, Burkholderia cenocepacia, Burkholderia pseudomallei, Legionella pneumophila, Staph aureus, Strep pyogenes, Strep pneumonia, Respiratory syncytial virus and Influenza A virus (IAV) [2109-2116].

Various studies have demonstrated that UFH (Unfractionated Heparin) prevents SARS-associated coronavirus from attaching to and invading the host cells [2103,2099-2108]. Lan et.al showed that the treatment of cells with heparinase or exogenous heparin prevented the binding of spike protein to host cell ACE-2 receptor and inhibited SARS pseudovirus infection, demonstrating that cell-surface (heparan sulfate proteoglycans) HSPGs provide the binding sites for SARS-CoV invasion at the early attachment phase [2099]. Vicenzi et.al showed that heparin (100 microg/mL) inhibited infection of SARS-COV HSR1 strain in Vero cells by $50 \%$ [2101].

The antiviral activity of heparin is based on the affinity of viral glycoproteins to negatively charged glycosaminoglycans such as sulfated heparan, which are ubiquitously expressed on the surface of mammalian cells [2122].

Suryawanshi et.al through their In silico binding affinity studies revealed the possible binding sites of G1 and G2 peptides on (Heparan Sulfate) HS and ACE2, which are required for the SARS-COV-2 spikeHS and SARS-COV-2 spike-ACE2 interactions [2119].

A recent study by Courtney et.al demonstrated that the SARS-CoV-2 Spike S1 protein receptor-binding domain (RBD) attaches to UFH and undergoes conformational changes which may prevent SARS-COV2 from binding to ACE-2 receptor and subsequent entry into the host cell [2098,2103].

Kim et.al reported that Glycosaminoglycan binding motif at S1/S2 proteolytic cleavage site on spike glycoprotein may facilitate novel coronavirus (SARS-CoV-2) host cell entry [2102]. Heparan sulfate interacts with the GAG-binding motif at the S1/S2 site on each monomer interface in the trimeric SARS-CoV-2 SGP, and at one another site when the receptor-binding domain is in an open conformation [2102].

Importantly, the binding of heparin to the receptor- binding domain (RBD) of the SARS-CoV-2 Spike S1 protein was found to be stronger for full-chain length heparin than low molecular weight heparins (LMWHs) [2102,2103].

Tandon et.al through their experiments using pseudotyped SARS-CoV-2 (spike glycoprotein) SGP on a third-generation lentiviral ( $\mathrm{pLV}$ ) vector showed that unfractionated heparin (UFH) and Enoxaparin Derivatives effectively Inhibits SARS-CoV-2 entry in a concentration dependent manner [2120].

Tree et.al observed that heparin binds and destabilizes the RBD protein of SARS-COV-2 and furthermore, they showed that heparin directly inhibits the binding of RBD of SARS-COV-2 to the human ACE2 protein receptor [2121].

Carina et.al conducted a viral plaque reduction assay in Vero E6 cells inoculated with a Dutch SARSCoV-2 isolate in the absence/presence of various concentrations of heparin. Carina et.al observed that Heparin not only decreased the number of plaques but also decreased the size of plaques [2122]. Carina et.al reported that in the presence of $500-1,000 \mu \mathrm{g} / \mathrm{ml}$ heparin, viral replication is almost completely inhibited while at $125-250 \mu \mathrm{g} / \mathrm{ml}$ viral replication was suppressed by more than $60 \%$ [2122]. 


\section{a. Nebulised UFH in ARDS Patients:}

A trial was conducted by Dixon et.al in 252 patients with or at risk of acute respiratory distress syndrome (ARDS) while on Mechanical ventilation in intensive care unity [2131]. Dixon et.al wanted to see if Nebulised heparin can target fibrin deposition and limit the lung injury. Dixon et.al reported that compared with the placebo group, the heparin group had fewer cases of ARDS development to day 5 among the at-risk patients [2131]. Dixon et.al concluded that in patients with or at risk of ARDS, nebulised heparin administration showed less progression of lung injury and was well tolerated with earlier return to home but was not associated with improvement in self-reported performance of daily physical activities [2131].

Inhaled heparin has been associated with proven broad distribution in the respiratory tract including the alveolar space and useful during Acute Lung Injury [2123,2124,2125,2122].

Inhaled UFH reduces the pulmonary dead spaces, coagulation activation, microvascular thrombosis and clinical deterioration, results in increased time free of ventilatory support $[2103,2097]$. In addition, UFH has anti-inflammatory, mucolytic and anti-viral properties and, specifically, has been shown to inactivate the SARS-CoV-2 virus and prevent its entry into mammalian cells, thereby inhibiting pulmonary infection by SARS-CoV-2 [1103,2097].

Taken together the multi-range advantages offered by Nebulized UFH in terms of antiviral effects, anti-Coagulopaty and other advantages it might be useful in the treatment of severe COVID-19 patients [2127].

\section{Vitamin-D:}

Various studies have emphasized on the usage of Vitamin-D to see positive outcomes in COVID-19 patients. Moreover, Vitamin D administration has been associated with protection against respiratory viral infections including IAV.

Martineau et.al conducted an individual participant data meta-analysis of trials in more than a dozen countries, including the U.S., Canada, and the U.K. and found that daily or weekly supplementation had the greatest benefit for individuals with the most significant vitamin D deficiency (blood levels below $10 \mathrm{mg} / \mathrm{dl}$ ) [2135]. Martineau et.al observed that regular vitamin D supplementation in these participants helped in cutting down the risk of respiratory infection by half and that all participants experienced some beneficial effects [2135]. Martineau et.al also observed that occasional administering of high doses of vitamin $D$ in these participants did not produce any significant benefits [2134].

Goncalves et.al in their trial observed that the serum Vitamin-D levels quickly decrease after supplementation ended, suggesting a short-lived efficacy [2135]. Goncalves et.al reported that Vitamin-D supplementation in Elderly People with Influenza Vaccine administration significantly reduced the plasma level of TNF $\alpha$ and IL-6. Goncalves et.al also noted a significant decrease in the Th1/Th2 ratio in link with TNF $\alpha$ and IL-6 reduced levels which is in accordance with Penna et.al [2136] showing that Vitamin-D can inhibit Th1 differentiation (via expression of IFN- $\gamma$ ) and increase the Th2 response by stimulating IL-5 production [2136]. Vitamin-D supplementation was reported to be associated with an increase in TGF- $\beta$ plasma levels after influenza vaccination, while no change in the Treg cell sub-population was observed [2135]. Martineau et.al reported that Vitamin-D supplementation has no affect on the humoral responses as there was no effect on Ab production in either seroprotection or seroconversion [2135]. This is consistent with other trials reports who showed that Vitamin-D has no effect on the humoral responses during Influenza vaccination $[2137,2138]$. Penna et.al reported that Vitamin-D inhibited NF-kappaB p65 phosphorylation and nuclear translocation in (myeloid dendritic cells) M-DCs but not in (plasmacytoid dendritic cells) P-DCs, suggesting a mechanism for the ability of Vitamin-D to modulate tolerogenic properties in M-DCs [2136]. 
Conclusively, Vitamin-D offers protection against respiratory viral infections [2139]. Vitamin-D deficiency is reported to be a risk factor for COVID-19 patients [2153,2155,2156,2157].

\section{a. Vitamin-D usage in COVID-19 Patients:}

There is an accumulating evidence that suggests that Vitamin-D supplementation might provide beneficial results in COVID-19 patients [2140,2141,2142,2143,2144,2147,2151,2152 ].

Jain et.al conducted analysis of vitamin D level among asymptomatic and critically ill COVID-19 patients and its correlation with inflammatory markers [2154]. Jain et.al reported that the fatality rate was high in vitamin D deficient (21\% vs $3.1 \%$ ) and Vitamin D levels are markedly lower in severe COVID-19 patients [2154]. Jain et.al concluded that Inflammatory responses were higher in vitamin D deficient COVID-19 patients [2154]. This all translates into increased mortality in vitamin D deficient COVID-19 patients.

Güven et.al conducted a study that included 175 COVID-19 patients with vitamin D deficiency and were hospitalized in the ICU. Vitamin D3 group included patients who received a single dose of 300,000 IU vitamin D3 intramuscularly. Vitamin D3 was not administered to the control group. Güven et.al concluded that Vitamin-D3 administration was not associated with improved hospital mortality results [2145].

Murai et.al in their randomized clinical trial that involved 240 hospitalized patients with moderate to severe COVID-19 patients, administered a single dose of 2,00,000 IU of vitamin D3 and compared them with the placebo. Murai et.al concluded that vitamin D3 administration did not significantly reduce the hospital length of stay [2146].

Rastogi et.al in their trial administered Vitamin-D in symptomatic or mildly symptomatic SARS-CoV-2 positive patients with vitamin D deficiency [2148]. Patients requiring invasive ventilation or with significant comorbidities were excluded in this trial [2148]. Intervention Participants were randomized to receive daily $60,000 \mathrm{IU}$ of cholecalciferol (oral nano-liquid droplets) for 7 days in comparison with placebo (control group [2148]). Vitamin-D levels were assessed at day 7, and cholecalciferol supplementation was continued for those with less Vitamin-D in the intervention arm. SARS-CoV-2 RNA and inflammatory markers fibrinogen, D-dimer, procalcitonin and (CRP), ferritin were measured periodically [2148]. Rastogi et.al concluded that greater proportion of vitamin D-deficient individuals with SARS-CoV-2 infection turned SARS-CoV-2 negative with a significant decrease in inflammatory markers and fibrinogen at the end of high-dose cholecalciferol supplementation [2148].

Lakkireddy et.al through their randomized trial in 130 patients reported that daily supplementation of 60,000 IUs of vitamin D for 8 or 10 days depending upon their BMI in addition to the standard treatment showed positive results in mild to moderate COVID-19 patients [2149]. Lakkireddy et.al observed that Vitamin-D therapy significantly reduced the inflammatory markers without any side effects in COVID-19 patients [2149]. In this trial it has been reported that no patient have received any drugs like Remdesivir, Favipiravir, Ivermectin or Dexamethasone [2149]. Lakkireddy et.al concluded that mortality of COVID patients is almost zero if Vitamin D level was $60 \mathrm{ng} / \mathrm{ml}$ and it is very high if the levels are less than $30 \mathrm{ng} / \mathrm{ml}$ [2149]. Lakkireddy et.al reported that no adverse effects have been observed in the patients [2149].

De Carvalho et al. reported that mega doses $(6,00,000$ IU) of vitamin D administered through intramuscular route even in cases of nephrolithiasis are safe [2150].

Conclusively, Guven et.al administered single time higher dosage of 3,00,000 IU, Vitamin-D in Critical COVID-19 patients in ICU [2145] and Murai et.al administered single time higher dosage of 2,00,000 IU, Vitamin-D in moderate to severe COVID-19 patients [2146]. Both of these studies reported no positive outcomes. 
Rastogi et.al administered daily 60,000 IU of Vitamin-D in symptomatic or mildly symptomatic COVID19 patients [2148] and Lakkireddy et.al administered daily 60,000 IUs of Vitamin D for 8 or 10 days in mild to moderate COVID-19 patients [2149]. Both of these studies reported positive outcomes but no negative outcomes.

Previously, Martineau et.al has reported that occasional/single time administering of high doses of vitamin $D$ in respiratory viral infection vulnerable candidates did not produce any significant benefits but regular vitamin D supplementation in the participants helped them in cutting down the risk of respiratory infection by half [2135]. Goncalves et.al in their trial observed that the serum Vitamin-D levels quickly decreased after supplementation ended, suggesting a short-lived efficacy of Vitamin-D supplementation [2135].

We can conclude that high doses of Vitamin-D theraphy is not useful if administered in single dose but will be beneficial to every COVID-19 patient if administered daily until inflammatory markers were reduced (above studies indicate 8-10 days). Prolonged usage might create problems which should be taken care.

Irrespective of SARS-COV-2, Vitamin-D plays very important role as Vitamin D is important for the prevention of cardiovascular disease, diabetes, autoimmune diseases, and some cancers [2158]. Using a monocyte cell model, Jain et.al examined the hypothesis that vitamin $D$ upregulates glutamate cysteine ligase (GCLC) and glutathione reductase (GR), which catalyzes Glutathione (GSH) biosynthesis. Jain et.al reported that vitamin-D supplementation improves the cellular Glutathione (GSH) levels in cell culture studies [2158]. Jain et.al concluded that Vitamin-D upregulates glutamate cysteine ligase and glutathione reductase, and GSH formation, and decreases ROS and MCP-1 and IL-8 secretion in high-glucose exposed U937 monocytes [2158].

As there are lot of factors drive immune response in critical/severe COVID-19 patients the results of Vitamin-D supplementation might not be evident but taken the importance of Vitamin-D it has a beneficial role.

\section{Remdesivir and COVID-19:}

Although Remdesivir treatment in COVID-19 patients has shown some positive results [51,52,53] it might lead to negative outcomes/damaging effects in the patients if administered lately $[54,55,56,57]$. Remdesivir is effective and beneficial to COVID-19 patients in mainly reducing viral loads, slowing down the progression of pneumonia/ (Acute Respiratory Distress Syndrome) ARDS only if administered earlier/within the first 10 days of symptoms [50].

Simeprevir Potently Suppresses SARS-CoV-2 Replication and Synergizes with Remdesivir [46]. Remdesivir and Ledipasvir among the FDA-Approved Antiviral Drugs Have Potential to Inhibit SARSCoV-2 Replication [47]. A prepint by lart et.al reported that cobicistat synergizes with Remdesivir to inhibit SARS-CoV-2 replication [48].

Darunavir/ Cobicistat combination was found to be not effective in treating COVID-19 patients [41, 42]. Milic et.al reported that Darunavir/Cobicistat treatment is associated with negative outcome in HIV-Negative Patients with Severe COVID-19 Pneumonia [43]. Contrarily kim et.al reported a positive outcome in COVID-19 patients with usage of Darunavir/Cobicistat. But it's worth noting that kim et.al in his paper reported that their trial group has been comprised of patients with milder illness. This might lead a conclusion that Darunavir/Cobicistat is useful only at earlier stages of disease.

So we can conclude that administration of viral replication inhibitors are effective only if administered at an early stage just like Remdesivir. 


\section{Nebulised / IV- Interferon -I and III therapy:}

Busnadiego et.al reported that antiviral activity of type I, II, and III Interferons counterbalances ACE2 inducibility and restricts SARS-CoV-2 [808].

Bessière et.al through their SARS-CoV-2 infected Syrian hamster model provided evidence that early type I IFN (IFN- $\alpha$ ) treatment is beneficial, while late interventions are ineffective [1118]. Nakhlband et.al through their systemic review based study reported that early administration of INF- $\alpha$ may be accounted as a promising treatment of COVID-19 in association with viral clearance and lower number of hospitalized days [1115].

\section{a. IFN- $\beta$ 1a in COVID-19 Patients:}

Peiffer et.al conducted a small trial and reported that COVID-19 patients who received Nebulised (interferon) IFN- $\beta$ 1a had significantly greater odds of clinical improvement [1116]. Higher or lower doses of IFN- $\beta$ 1a in moderate to severe COVID-19 patients didn't show any improvement in terms of reducing mortality [1117]. Monk et.al in their trial administered Nebulized (interferon) IFN- $\beta$ 1a. The median duration of COVID-19 symptoms before initiation of IFN- $\beta$ 1a treatment in this study was reported to be approximately 10 days. The Interferon therapy administered group has both Comorbid and more number of severe patients receiving oxygen therapy when compared to Control COVID-19 group. Monk et.al concluded that patients receiving IFN- $\beta$ 1a (SNG001) had greater odds of improvement on the (WHO Ordinal Scale for Clinical Improvement) OSCl scale on day 15 or 16 and were more likely than those receiving placebo to recover [1119].

\section{b. IFN- $\lambda$ in COVID-19 Patients:}

Jordan et.al reported that Peginterferon lambda IFN- $\lambda$ accelerates viral decline in outpatients with COVID-19, increasing the proportion of patients with viral clearance by day 7 , particularly in those with high baseline viral load [2046]. Peginterferon lambda has potential to prevent clinical deterioration and shorten duration of viral shedding [2046].

Jagannathan et.al in their study of a single dose of subcutaneous Peginterferon Lambda-1a ( IFN- $\lambda 1 \mathrm{a}$ ) administration in uncomplicated COVID-19 patients reported that IFN- $\lambda 1$ a neither shortened the duration of SARS-CoV-2 viral shedding nor improved symptoms in outpatients with uncomplicated COVID-19 patients [2047].

\section{c. IFN- $\gamma$ in COVID-19 Patients:}

Lower Circulating Interferon-Gamma has been implicated as a Risk Factor for Lung Fibrosis in COVID19 Patients [2043]. Myasnikov et.al conducted an open-label, randomized, low-interventional study, it included patients with moderate new coronavirus infection COVID-19 over 18 years of age of both sexes [2044]. In their study Myasnikov et.al administered IFN- $\gamma$ at 500,000 IU, daily, once a day, during 5 days [2044] (for more details please refer to original authors article- Myasnikov et.al [2044]).

Myasnikov et.al concluded that IFN- $\gamma$ in addition to complex therapy of the disease resulted in more favorable changes in the stabilization of vital signs, as well as in reduced length of fever and hospital stay by 2 days what allows suggesting a positive effect of this substance on the recovery processes in patients with moderate COVID-19 [2044]. Myasnikov et.al also reported that the patients who received recombinant IFN- $\gamma$ experienced no progression of respiratory failure and required no transfer to intensive care unit [2044]. 


\section{Accidental causes in COVID-19 :}

\section{Abuse of Steroids:}

1. Dexamethasone treatment strongly enhances MDSC expansion through upregulation of miR-155 and miR-21 expression [1630, 368]. Dexamethasone or any other immunosuppressants administration during early stages of COVID-19 compromises their immune systems ability to stop the virus [161]. As a result the virus can directly reach lungs and induce Alveolar type -II epithelial cell damage and subsequent collapse due to lack of Surface tension. Supportively enough in a Single COVID-19 case report, Dexamethasone misuse has been showed to be associated with delayed viral clearance leading to the occurrence of severe COVID-19 pneumonia [2086].

2. Although Steroids usage in elderly critically ill COVID-19 patients has been reported to be showing positive outcomes [2087, 161] it's misuse might show detrimental effects on the patients [2088].

3. Over usage of Steroids in COVID-19 patients especially in patients with Co-morbidities can make them susceptible to dangerous secondary infections like Mucormycosis [2089-2093].

\section{Abuse of Antibiotics:}

1. Antibiotics are found to be dysregulating gut microbiota independent of the delivery Route i.e: administered either through oral or Intravenous ways [2058]. Antibiotics are known for dysregulating gut microbiota [1728] and gut microbiota is related to TLR-7 expression at mucosal surfaces [1704] and reduction in TLR-7 is associated with higher mortality in COVID-19 patients [618]. Wu et.al reported that TLR-7 ligands rescued the immune impairment in antibiotic-treated mice during Influenza virus (IAV) [1704].

2. Abuse of Antibiotics has been reported to be a key factor in worsening/aggravating the diseased condition in COVID-19 patients [2082,2083,2084,2085].

\section{Damage due to Improper usage of Nasal swabs:}

1. While using Nasal swabs, excess usage of force and incorrect sampling technique might create an injury in the nasal cavity $[2066,2075]$ especially in people who underwent Sinus Surgery [2067]. This injury might pave way for SARS-COV-2 to escape into the blood stream directly. This can lead to systemic inflammation and multi-organ failure independent of the tissue damage in lungs or airways. If injury left unchecked might lead to blood clots and subsequent consequences.

2. There have been reported cases of Nasal swab induced skull base injuries and CSF leakages [2069, 2070,2071,2072,2073]. In one case Traumatic Cribriform Plate Defect have been observed following Self-administration of COVID-19 Nasal Swab Test [2074].

\section{Flavanoids:}

3. Lot of studies recommend the usage of flavanoids in COVID-19 treatment [2076, 2077, 2078, 2079, 2080].

4. Zhu and Xie et.al though their docking studies and in-vitro studies showed that Flavanoids have the potential in reducing SARS-COV-2 viral replication and associated cell death [2076, 2081]. 


\section{Conclusions:}

These above studies suggest that using combinations of several drugs either for inhibiting viral entry or reducing cellular death in severe COVID-19 patients along with their delivery through intranasal channel if possible can provide best results. This delayed viral burden and delayed time in damage induction can give enough time for the adaptive immune response to produce effective results.

This article is made as a part of the literature review and should not be used as a guide for the treatment of COVID-19 patients until approved by concerned authorities or until published in an renowned journal. 
1. Jing, Linlin et al. "Protective Effect of Tempol Against Hypoxia-Induced Oxidative Stress and Apoptosis in H9c2 Cells." Medical science monitor basic research vol. 23 159-165. 21 Apr. 2017, doi:10.12659/MSMBR.903764.

2. Sainz J, Wangensteen R, Rodríguez Gómez I, Moreno JM, Chamorro V, Osuna A, Bueno P, Vargas F. Antioxidant enzymes and effects of tempol on the development of hypertension induced by nitric oxide inhibition. Am J Hypertens. 2005 Jun;18(6):871-7. doi: 10.1016/j.amjhyper.2004.12.022. PMID: 15925750.

3. Wang, Y., Hai, B., Ai, L., Cao, Y., Li, R., Li, H., \& Li, Y. (2018). Tempol relieves lung injury in a rat model of chronic intermittent hypoxia via suppression of inflammation and oxidative stress. Iranian Journal of Basic Medical Sciences, 21(12), 1238-1244. https://doi.org/10.22038/ijbms.2018.31716.7714.

4. Francischetti, I. M., Gordon, E., Bizzarro, B., Gera, N., Andrade, B. B., Oliveira, F., Ma, D., Assumpção, T. C., Ribeiro, J. M. C., Pena, M., Qi, C.-F., Diouf, A., Moretz, S. E., Long, C. A., Ackerman, H. C., Pierce, S. K., Sá-Nunes, A., \& Waisberg, M. (2014). Tempol, an intracellular antioxidant, inhibits tissue factor expression, attenuates dendritic cell function, and is partially protective in a murine model of cerebral malaria. PLOS ONE, 9(2), Article e87140. https://doi.org/10.1371/journal.pone.0087140

5. DTrnka, Jan et al. "Antioxidant properties of MitoTEMPOL and its hydroxylamine." Free radical research vol. 43,1 (2009): 4-12. doi:10.1080/10715760802582183.

6. Mathi K, Rosenberg-Hasson Y, Maecker H, Carlo DJ, Moss RB. Brief report: Tempol, a novel antioxidant, inhibits both activated T cell and antigen presenting cell derived cytokines in-vitro from COVID-19 patients. Clin Immunol. 2021 Aug 20:108828. doi: 10.1016/j.clim.2021.108828. Epub ahead of print. PMID: 34425240; PMCID: PMC8378988.

7. Maio N, Lafont BAP, Sil D, Li Y, Bollinger JM Jr, Krebs C, Pierson TC, Linehan WM, Rouault TA. Fe-S cofactors in the SARS-CoV-2 RNA-dependent RNA polymerase are potential antiviral targets. Science. 2021 Jul 9;373(6551):236-241. doi: 10.1126/science.abi5224. Epub 2021 Jun 3. PMID: 34083449

8. Zuin R, Palamidese A, Negrin R, Catozzo L, Scarda A, Balbinot M. High-dose N-acetylcysteine in patients with exacerbations of chronic obstructive pulmonary disease. Clin Drug Investig. 2005;25(6):401-8. doi: 10.2165/00044011-200525060-00005. PMID: 17532680 .

9. Ibrahim H, Perl A, Smith D, Lewis T, Kon Z, Goldenberg R, Yarta K, Staniloae C, Williams M. Therapeutic blockade of inflammation in severe COVID-19 infection with intravenous N-acetylcysteine. Clin Immunol. 2020 Oct;219:108544. doi: 10.1016/j.clim.2020.108544. Epub 2020 Jul 22. PMID: 32707089; PMCID: PMC7374140.

10. Gustine JN, Jones D. Immunopathology of Hyperinflammation in COVID-19. Am J Pathol. 2021 Jan;191(1):4-17. doi: 10.1016/j.ajpath.2020.08.009. Epub 2020 Sep 11. PMID: 32919977; PMCID: PMC7484812.

11. Mishra KP, Singh AK, Singh SB. Hyperinflammation and Immune Response Generation in COVID-19. Neuroimmunomodulation 2020;27(2):80-86. doi: 10.1159/000513198. Epub 2020 Dec 18. PMID: 33341814; PMCID: PMC7801965.

12. Wong RSY. Inflammation in COVID-19: from pathogenesis to treatment. Int J Clin Exp Pathol. 2021 Jul 15;14(7):831-844. PMID: 34367415; PMCID: PMC8339720.

13. Tay, M.Z., Poh, C.M., Rénia, L. et al. The trinity of COVID-19: immunity, inflammation and intervention. Nat Rev Immunol 20, 363-374 (2020). https://doi.org/10.1038/s41577-020-0311-8.

14. Jordan SC. Innate and adaptive immune responses to SARS-CoV-2 in humans: relevance to acquired immunity and vaccine responses. Clin Exp Immunol. 2021 Jun;204(3):310-320. doi: 10.1111/cei.13582. Epub 2021 Mar 4. PMID: 33534923; PMCID: PMC8013613.

15. Ni L, Cheng ML, Feng Y, Zhao H, Liu J, Ye F, Ye Q, Zhu G, Li X, Wang P, Shao J, Deng YQ, Wei P, Chen F, Qin CF, Wang G, Li F, Zeng H, Dong C. Impaired Cellular Immunity to SARS-CoV-2 in Severe COVID-19 Patients. Front Immunol. 2021 Feb 2;12:603563. doi: 10.3389/fimmu.2021.603563. PMID: 33603759; PMCID: PMC7884325.

16. Ragab D, Salah Eldin H, Taeimah M, Khattab R, Salem R. The COVID-19 Cytokine Storm; What We Know So Far. Front Immunol. 2020 Jun 16;11:1446. doi: 10.3389/fimmu.2020.01446. PMID: 32612617; PMCID: PMC7308649.

17. Catanzaro, M., Fagiani, F., Racchi, M. et al. Immune response in COVID-19: addressing a pharmacological challenge by targeting pathways triggered by SARS-CoV-2. Sig Transduct Target Ther 5, 84 (2020). https://doi.org/10.1038/s41392-020-0191-1.

18. Peddapalli A, Gehani M, Kalle AM, Peddapalli SR, Peter AE, Sharad S. Demystifying Excess Immune Response in COVID-19 to Reposition an Orphan Drug for Down-Regulation of NF-KB: A Systematic Review. Viruses. 2021 Feb 27;13(3):378. doi: 10.3390/v13030378. PMID: 33673529; PMCID: PMC7997247.

19. Lucas, C., Klein, J., Sundaram, M.E. et al. Delayed production of neutralizing antibodies correlates with fatal COVID-19. Nat Med 27, 1178-1186 (2021). https://doi.org/10.1038/s41591-021-01355-0

20. Wu, J., Liang, B., Chen, C. et al. SARS-CoV-2 infection induces sustained humoral immune responses in convalescent patients following symptomatic COVID-19. Nat Commun 12, 1813 (2021). https://doi.org/10.1038/s41467-021-22034-1

21. Hasan A, Al-Ozairi E, Al-Baqsumi Z, Ahmad R, Al-Mulla F. Cellular and Humoral Immune Responses in Covid-19 and Immunotherapeutic Approaches. Immunotargets Ther. 2021;10:63-85, https://doi.org/10.2147/ITT.S280706

22. Galipeau Y, Greig M, Liu G, Driedger M, Langlois MA. Humoral Responses and Serological Assays in SARS-CoV-2 Infections. Front Immunol. 2020 Dec 18;11:610688. doi: 10.3389/fimmu.2020.610688. PMID: 33391281; PMCID: PMC7775512.

23. Thalia et al. Timeline analysis of IgA and IgG levels in Covid-19 hospitalized patients according to the clinical outcome. Jornal Brasileiro de Patologia e Medicina Laboratorial [online]. 2021, v. 57 [Accessed 23 August 2021] , e4022021. Available from: <https://doi.org/10.5935/1676-2444.20210021>. Epub 09 July 2021. ISSN 1678-4774. https://doi.org/10.5935/16762444.20210021.

24. Fraser DD, Cepinskas G, Slessarev M, Martin CM, Daley M, Patel MA, Miller MR, Patterson EK, O'Gorman DB, Gill SE, Higgins I, John JPP, Melo C, Nini L, Wang X, Zeidler J, Cruz-Aguado JA. Critically III COVID-19 Patients Exhibit Anti-SARS-CoV-2 Serological Responses. Pathophysiology. 2021; 28(2):212-223. https://doi.org/10.3390/pathophysiology28020014

25. Fajnzylber, J., Regan, J., Coxen, K. et al. SARS-CoV-2 viral load is associated with increased disease severity and mortality. Nat Commun 11, 5493 (2020). https://doi.org/10.1038/s41467-020-19057-5

26. Argyropoulos KV, Serrano A, Hu J, Black M, Feng X, Shen G, Call M, Kim MJ, Lytle A, Belovarac B, Vougiouklakis T, Lin LH, Moran U, Heguy A, Troxel A, Snuderl M, Osman I, Cotzia P, Jour G. Association of Initial Viral Load in Severe Acute Respiratory Syndrome Coronavirus 2 (SARS-CoV-2) Patients with Outcome and Symptoms. Am J Pathol. 2020 Sep;190(9):1881-1887. doi: 10.1016/j.ajpath.2020.07.001. Epub 2020 Jul 3. PMID: 32628931; PMCID: PMC7332909.

27. Manuel A. Torres Acosta, Benjamin D. Singer, Pathogenesis of COVID-19-induced ARDS: implications for an aging population, European Respiratory Journal Jan 2020, 2002049; DOI: 10.1183/13993003.02049-2020

28. Zheng S, Fan J, Yu F, Feng B, Lou B, Zou Q et al. Viral load dynamics and disease severity in patients infected with SARS-CoV-2 in Zhejiang province, China, January-March 2020: retrospective cohort study BMJ 2020; 369 :m1443 doi:10.1136/bmj.m1443

29. Pujadas E, Chaudhry F, McBride R, Richter F, Zhao S, Wajnberg A, Nadkarni G, Glicksberg BS, Houldsworth J, Cordon-Cardo C. SARS-CoV-2 viral load predicts COVID-19 mortality. Lancet Respir Med. 2020 Sep;8(9):e70. doi: 10.1016/S2213-2600(20)30354-4. Epub 2020 Aug 6. PMID: 32771081; PMCID: PMC7836878. 
30. Gallelli, Luca et al. "Severe Acute Lung Injury Related to COVID-19 Infection: A Review and the Possible Role for Escin." Journal of clinical pharmacology vol. 60,7 (2020): 815-825. doi:10.1002/jcph.1644

31. Leng, L., Cao, R., Ma, J. et al. Pathological features of COVID-19-associated lung injury: a preliminary proteomics report based on clinical samples. Sig Transduct Target Ther 5, 240 (2020). https://doi.org/10.1038/s41392-020-00355-9

32. Borczuk, A.C., Salvatore, S.P., Seshan, S.V. et al. COVID-19 pulmonary pathology: a multi-institutional autopsy cohort from Italy and New York City. Mod Pathol 33, 2156-2168 (2020). https://doi.org/10.1038/s41379-020-00661-1

33. Mauad, T., Duarte-Neto, A.N., da Silva, L.F.F. et al. Tracking the time course of pathological patterns of lung injury in severe COVID-19. Respir Res 22, 32 (2021). https://doi.org/10.1186/s12931-021-01628-9

34. Weaver, L., Das, A., Saffaran, S. et al. High risk of patient self-inflicted lung injury in COVID-19 with frequently encountered spontaneous breathing patterns: a computational modelling study. Ann. Intensive Care 11, 109 (2021). https://doi.org/10.1186/s13613-021-00904-7

35. Jafari, D. et al. (2021). Trajectories of Hypoxemia \& Respiratory System Mechanics of COVID-19 ARDS in the NorthCARDS dataset. medRxiv. https://doi.org/10.1101/2021.01.26.21250492,https://www.medrxiv.org/content/10.1101/2021.01.26.21250492v1

36. Nitsure, Mohana et al. "Mechanisms of Hypoxia in COVID-19 Patients: A Pathophysiologic Reflection." Indian journal of critical care medicine : peer-reviewed, official publication of Indian Society of Critical Care Medicine vol. 24,10 (2020): $967-970$. doi:10.5005/jp-journals-10071-23547

37. Li, X., Ma, X. Acute respiratory failure in COVID-19: is it "typical" ARDS?. Crit Care 24, 198 (2020). https://doi.org/10.1186/s13054-020-02911-9

38. Swenson KE, Ruoss SJ, Swenson ER. The Pathophysiology and Dangers of Silent Hypoxemia in COVID-19 Lung Injury. Ann Am Thorac Soc. 2021 Jul;18(7):1098-1105. doi: 10.1513/AnnalsATS.202011-1376CME. PMID: 33621159; PMCID: PMC8328372.

39. Messina E, Danise A, Ferrari G, Andolina A, Chiurlo M, Razanakolona M, Barakat M, Israel RJ, Castagna A. Ribavirin Aerosol in the Treatment of SARS-CoV-2: A Case Series. Infect Dis Ther. 2021 Jul 23:1-14. doi: 10.1007/s40121-021-00493-9. Epub ahead of print. PMID: 34302258; PMCID: PMC8302211.

40. Elajez, Reem \& Abdallah, Oraib \& Alnaimi, Shaikha \& Elmekaty, Eman \& Abuyousef, Safae \& Arafa, Eiman \& Barazi, Raja \& Ghasoub, Rola \& Khattab, Mohammed \& Al Hail, Moza. (2021). Exploring Potential Synergism Between Darunavir/ Cobicistat and Ribavirin for the Treatment of Covid-19 Patients: A Retrospective Comparison Study. Open Access Journal of Biomedical Science. 3. 10.38125/OAJBS.000262.

41. Chen J, Xia L, Liu L, Xu Q, Ling Y, Huang D, Huang W, Song S, Xu S, Shen Y, Lu H. Antiviral Activity and Safety of Darunavir/Cobicistat for the Treatment of COVID-19. Open Forum Infect Dis. 2020 Jun 21;7(7):ofaa241. doi: 10.1093/ofid/ofaa241. PMID: 32671131; PMCID: PMC7337805.

42. De Meyer S, Bojkova D, Cinatl J, Van Damme E, Buyck C, Van Loock M, Woodfall B, Ciesek S. Lack of antiviral activity of darunavir against SARS-CoV-2. Int J Infect Dis. 2020 Aug;97:7-10. doi: 10.1016/j.ijid.2020.05.085. Epub 2020 May 29. PMID: 32479865; PMCID: PMC7258847.

43. Milic J, Novella A, Meschiari M, Menozzi M, Santoro A, Bedini A, Cuomo G, Franceschini E, Digaetano M, Carli F, Ciusa G, Volpi S, Bacca E, Franceschi G, Yaacoub D, Rogati C, Tutone M, Burastero G, Faltoni M, ladisernia V, Dolci G, Cossarizza A, Mussini C, Pasina L, Guaraldi G. Darunavir/Cobicistat Is Associated with Negative Outcomes in HIV-Negative Patients with Severe COVID-19 Pneumonia. AIDS Res Hum Retroviruses. 2021 Apr;37(4):283-291. doi: 10.1089/AID.2020.0305. PMID: 33619997.

44. Kim EJ, Choi SH, Park JS, Kwon YS, Lee J, Kim Y, Lee SY, Choi EY. Use of Darunavir-Cobicistat as a Treatment Option for Critically III Patients with SARS-CoV-2 Infection. Yonsei Med J. 2020 Sep;61(9):826-830. doi: 10.3349/ymj.2020.61.9.826. PMID: 32882767; PMCID: PMC7471078.

45. Adel Khattab, Arshad Javaid, Ghali Iraqi, Ashraf Alzaabi, Ali Ben Kheder, Marie-Louise Koniski, Naem Shahrour, Samya Taright, Magdy Idrees, Mehmet Polatli, Nauman Rashid, Abdelkader El Hasnaoui, Smoking habits in the Middle East and North Africa: Results of the BREATHE study, Respiratory Medicine,2012, https://doi.org/10.1016/S0954-6111(12)70011-2.

46. Lo, Ho Sing \& Hui, Kenrie \& Hei Ming, Lai \& He, Xu \& Khan, Khadija \& Kaur, Simranjeet \& Huang, Junzhe \& Li, Zhongqi \& Chan, Anthony \& Cheung, Hayley \& Ng, Ka-Chun \& Ho, John \& Chen, Yu Wai \& Ma, Bowen \& Cheung, Peter \& Shin, Donghyuk \& Wang, Kaidao \& Lee, Meng-Hsuan \& Selisko, Barbara \& Ng, Wai-Lung. (2021). Simeprevir Potently Suppresses SARS-CoV-2 Replication and Synergizes with Remdesivir. ACS Central Science. XXXX. 10.1021/acscentsci.0c01186.

47. Pirzada RH, Haseeb M, Batool M, Kim M, Choi S. Remdesivir and Ledipasvir among the FDA-Approved Antiviral Drugs Have Potential to Inhibit SARS-CoV-2 Replication. Cells. 2021 Apr 29;10(5):1052. doi: 10.3390/cells10051052. PMID: 33946869; PMCID: PMC8146643.

48. Iart Luca Shytaj, Mohamed Fares, Bojana Lucic, Lara Gallucci, Mahmoud M. Tolba, Liv Zimmermann, Ahmed Taha Ayoub, Mirko Cortese, Christopher J. Neufeldt, Vibor Laketa, Petr Chlanda, Oliver T. Fackler, Steeve Boulant, Ralf Bartenschlager, Megan Stanifer, Andrea Savarino, Marina Lusic The FDA-approved drug cobicistat synergizes with remdesivir to inhibit SARS-CoV-2 replication, bioRxiv 2021.03.09.434219; doi:https://doi.org/10.1101/2021.03.09.434219

49. Dumont EF, Oliver AJ, loannou C, Billiard J, Dennison J, van den Berg F, Yang S, Chandrasekaran V, Young GC, Lahiry A, Starbuck DC, Harrell AW, Georgiou A, Hopchet N, Gillies A, Baker SJ. A Novel Inhaled Dry-Powder Formulation of Ribavirin Allows for Efficient Lung Delivery in Healthy Participants and Those with Chronic Obstructive Pulmonary Disease in a Phase 1 Study. Antimicrob Agents Chemother. 2020 Apr 21;64(5):e02267-19. doi: 10.1128/AAC.02267-19. PMID: 32071044; PMCID: PMC7179635.

50. Singh AK, Singh A, Singh R, Misra A. Remdesivir in COVID-19: A critical review of pharmacology, pre-clinical and clinical studies. Diabetes Metab Syndr. 2020 Jul-Aug;14(4):641-648. doi: 10.1016/j.dsx.2020.05.018. Epub 2020 May 12. PMID: 32428865; PMCID: PMC7214279.

51. Beigel JH, Tomashek KM, Dodd LE, Mehta AK, Zingman BS, Kalil AC, Hohmann E, Chu HY, Luetkemeyer A, Kline S, Lopez de Castilla D, Finberg RW, Dierberg K, Tapson V, Hsieh L, Patterson TF, Paredes R, Sweeney DA, Short WR, Touloumi G, Lye DC, Ohmagari N, Oh MD, Ruiz-Palacios GM, Benfield T, Fätkenheuer G, Kortepeter MG, Atmar RL, Creech CB, Lundgren J, Babiker AG, Pett S, Neaton JD, Burgess TH, Bonnett T, Green M, Makowski M, Osinusi A, Nayak S, Lane HC; ACTT-1 Study Group Members. Remdesivir for the Treatment of Covid-19 - Final Report. N Engl J Med. 2020 Nov 5;383(19):1813-1826. doi: 10.1056/NEJMoa2007764. Epub 2020 Oct 8. PMID: 32445440; PMCID: PMC7262788.

52. Wang Y, Zhang D, Du G, Du R, Zhao J, Jin Y, Fu S, Gao L, Cheng Z, Lu Q, Hu Y, Luo G, Wang K, Lu Y, Li H, Wang S, Ruan S, Yang C, Mei C, Wang Y, Ding D, Wu F, Tang X, Ye X, Ye Y, Liu B, Yang J, Yin W, Wang A, Fan G, Zhou F, Liu Z, Gu X, Xu J, Shang L, Zhang Y, Cao L, Guo T, Wan Y, Qin H, Jiang Y, Jaki T, Hayden FG, Horby PW, Cao B, Wang C. Remdesivir in adults with severe COVID-19: a randomised, double-blind, placebo-controlled, multicentre trial. Lancet. 2020 May 16;395(10236):1569-1578. doi: 
10.1016/S0140-6736(20)31022-9. Epub 2020 Apr 29. Erratum in: Lancet. 2020 May 30;395(10238):1694. PMID: 32423584; PMCID: PMC7190303.

53. Spinner CD, Gottlieb RL, Criner GJ, Arribas López JR, Cattelan AM, Soriano Viladomiu A, Ogbuagu O, Malhotra P, Mullane KM, Castagna A, Chai LYA, Roestenberg M, Tsang OTY, Bernasconi E, Le Turnier P, Chang SC, SenGupta D, Hyland RH, Osinusi AO, Cao H, Blair C, Wang H, Gaggar A, Brainard DM, McPhail MJ, Bhagani S, Ahn MY, Sanyal AJ, Huhn G, Marty FM; GS-US-540-5774 Investigators. Effect of Remdesivir vs Standard Care on Clinical Status at 11 Days in Patients With Moderate COVID-19: A Randomized Clinical Trial. JAMA. 2020 Sep 15;324(11):1048-1057. doi: 10.1001/jama.2020.16349. PMID: 32821939; PMCID: PMC7442954.

54. Piscoya A, Ng-Sueng LF, Parra Del Riego A, Cerna-Viacava R, Pasupuleti V, Roman YM, Thota P, White CM, Hernandez AV. Efficacy and harms of remdesivir for the treatment of COVID-19: A systematic review and meta-analysis. PLoS One. 2020 Dec 10;15(12):e0243705. doi: 10.1371/journal.pone.0243705. PMID: 33301514; PMCID: PMC7728272.

55. Gupta, Anupam \& Parker, Barbara \& Priyadarshi, Vikash \& Parker, John. (2020). Cardiac Adverse Events With Remdesivir in COVID-19 Infection. Cureus. 12. 10.7759/cureus.11132.

56. Zampino R, Mele F, Florio LL, Bertolino L, Andini R, Galdo M, De Rosa R, Corcione A, Durante-Mangoni E. Liver injury in remdesivir-treated COVID-19 patients. Hepatol Int. 2020 Sep;14(5):881-883. doi: 10.1007/s12072-020-10077-3. Epub 2020 Jul 28. PMID: 32725454; PMCID: PMC7386240.

57. WHO recommends against the use of remdesivir in COVID-19 patients

https://www.who.int/news-room/feature-stories/detail/who-recommends- against-the-use-of-remdesivir-in-covid-19-patients 58. Rosales MA, Silva KC, Lopes de Faria JB, Lopes de Faria JM. Exogenous SOD mimetic tempol ameliorates the early retinal changes reestablishing the redox status in diabetic hypertensive rats. Invest Ophthalmol Vis Sci. 2010 Aug;51(8):4327-36. doi: 10.1167/iovs.09-4690. Epub 2010 Mar 24. PMID: 20335612.

59. Li H, Xiong N, Li C, Gong Y, Liu L, Yang H, Tan X, Jiang N, Zong Q, Wang J, Lu Z, Yin X. Efficacy of ribavirin and interferon- $\alpha$ therapy for hospitalized patients with COVID-19: A multicenter, retrospective cohort study. Int J Infect Dis. 2021 Mar;104:641648. doi: 10.1016/j.ijid.2021.01.055. Epub 2021 Jan 28. PMID: 33515771; PMCID: PMC7840408.

60. 36. Archer SL, Sharp WW, Weir EK. Differentiating COVID-19 Pneumonia From Acute Respiratory Distress Syndrome and High Altitude Pulmonary Edema: Therapeutic Implications. Circulation. 2020 Jul 14;142(2):101-104. doi: 10.1161/CIRCULATIONAHA.120.047915. Epub 2020 May 5. PMID: 32369390; PMCID: PMC7363563.

61. Artese A, Svicher V, Costa G, Salpini R, Di Maio VC, Alkhatib M, Ambrosio FA, Santoro MM, Assaraf YG, Alcaro S, CeccheriniSilberstein F. Current status of antivirals and druggable targets of SARS CoV-2 and other human pathogenic coronaviruses. Drug Resist Updat. 2020 Dec;53:100721. doi: 10.1016/j.drup.2020.100721. Epub 2020 Aug 26. PMID: 33132205; PMCID: PMC7448791. 62. Hedstrom L. IMP dehydrogenase: structure, mechanism, and inhibition. Chem Rev. 2009 Jul;109(7):2903-28. doi: 10.1021/cr900021w. PMID: 19480389; PMCID: PMC2737513.

63. Elfiky AA. Anti-HCV, nucleotide inhibitors, repurposing against COVID-19. Life Sci. 2020 May 1;248:117477. doi: 10.1016/j.Ifs.2020.117477. Epub 2020 Feb 28. PMID: $32119961 ;$ PMCID: PMC7089605.

64. Copegus (ribavirin, USP) Tablets [package insert]. Nutley, NJ: Roche Laboratories Inc.; 2005

65. Ribavirin 200 mg film-coated tablets [package insert]. South Ruislip, UK: Aurobindo Pharma-Milpharm Ltd.; 2020.

66. Virazole (ribavirin for inhalation solution, USP) prescribing information [package insert]. Bridgewater, NJ: Bausch Health US, LLC; 2019.

67. Virazole (ribavirin for inhalation) Solution, USP [product monograph]. Leval, Quebec, Canada: Bausch Health Canada Inc; 2020. 68. Knight V, McClung HW, Wilson SZ, Waters BK, Quarles JM, Cameron RW, Greggs SE, Zerwas JM, Couch RB. Ribavirin smallparticle aerosol treatment of influenza. Lancet. 1981 Oct 31;2(8253):945-9. doi: 10.1016/s0140-6736(81)91152-1. PMID: 6117726. 69. Wilson SZ, Gilbert BE, Quarles JM, Knight V, McClung HW, Moore RV, Couch RB. Treatment of influenza A (H1N1) virus infection with ribavirin aerosol. Antimicrob Agents Chemother. 1984 Aug;26(2):200-3. doi: 10.1128/AAC.26.2.200. PMID: 6486761; PMCID: PMC284119.

70. Gilbert BE, Wilson SZ, Knight V, Couch RB, Quarles JM, Dure L, Hayes N, Willis G. Ribavirin small-particle aerosol treatment of infections caused by influenza virus strains A/Victoria/7/83 (H1N1) and B/Texas/1/84. Antimicrob Agents Chemother. 1985 Mar;27(3):309-13. doi: 10.1128/AAC.27.3.309. PMID: 3888102; PMCID: PMC176267.

71. Gilbert BE, McLeay MT. MegaRibavirin aerosol for the treatment of influenza A virus infections in mice. Antiviral Res. 2008 Jun;78(3):223-9. doi: 10.1016/j.antiviral.2008.01.005. Epub 2008 Feb 4. PMID: 18281104; PMCID: PMC2405943.

72. Smith DW, Frankel LR, Mathers LH, Tang AT, Ariagno RL, Prober CG. A controlled trial of aerosolized ribavirin in infants receiving mechanical ventilation for severe respiratory syncytial virus infection. N Engl J Med. 1991 Jul 4;325(1):24-9. doi: 10.1056/NEJM199107043250105. PMID: 1904551.

73. Crupi R, Impellizzeri D, Gugliandolo E, Cordaro M, Siracusa R, Britti D, Cuzzocrea S, Di Paola R. Effect of Tempol, a MembranePermeable Free Radical Scavenger, on In Vitro Model of Eye Inflammation on Rabbit Corneal Cells. J Ocul Pharmacol Ther. 2019 Dec;35(10):571-577. doi: 10.1089/jop.2019.0016. PMID: 31825758.

74. $\mathrm{H}$

75. $\mathrm{h}$

76. Proctor, Peter. (2020). Tempol for COVID-19.

77. Zhou C, Gao C, Xie Y, Xu M. COVID-19 with spontaneous pneumomediastinum. Lancet Infect Dis. 2020 Apr;20(4):510. doi: 10.1016/S1473-3099(20)30156-0. Epub 2020 Mar 9. PMID: 32164830; PMCID: PMC7128610.

78. Hung IF, Lung KC, Tso EY, Liu R, Chung TW, Chu MY, Ng YY, Lo J, Chan J, Tam AR, Shum HP, Chan V, Wu AK, Sin KM, Leung WS, Law WL, Lung DC, Sin S, Yeung P, Yip CC, Zhang RR, Fung AY, Yan EY, Leung KH, Ip JD, Chu AW, Chan WM, Ng AC, Lee R, Fung K, Yeung A, Wu TC, Chan JW, Yan WW, Chan WM, Chan JF, Lie AK, Tsang OT, Cheng VC, Que TL, Lau CS, Chan KH, To KK, Yuen KY. Triple combination of interferon beta-1b, lopinavir-ritonavir, and ribavirin in the treatment of patients admitted to hospital with COVID-19: an open-label, randomised, phase 2 trial. Lancet. 2020 May 30;395(10238):1695-1704. doi: 10.1016/S01406736(20)31042-4. Epub 2020 May 10. PMID: 32401715; PMCID: PMC7211500.

79. Chu CM, Cheng VC, Hung IF, Wong MM, Chan KH, Chan KS, Kao RY, Poon LL, Wong CL, Guan Y, Peiris JS, Yuen KY; HKU/UCH SARS Study Group. Role of lopinavir/ritonavir in the treatment of SARS: initial virological and clinical findings. Thorax. 2004 Mar;59(3):252-6. doi: 10.1136/thorax.2003.012658. PMID: 14985565; PMCID: PMC1746980.

80. Minnesota Department of Health. Aerosol-generating procedures and patients with suspected or confirmed COVID-19. St. Paul: Minnesota Department of Health; 2020.

81. $\mathrm{H}$

82. $\mathrm{H}$

83. $\mathrm{H}$ 
91. Pfützner A, Lazzara M, Jantz J. Why Do People With Diabetes Have a High Risk for Severe COVID-19 Disease?-A Dental Hypothesis and Possible Prevention Strategy. J Diabetes Sci Technol. 2020;14(4):769-71. https://doi. org/10.1177/1932296820930287

92. Hui DS, Madani EIA, Ntoumi TA, Kock F, Dar R. O, et al. The continuing 2019-nCoV epidemic threat of novel coronaviruses to global health - The latest 2019 novel coronavirus outbreak in Wuhan, China. Int J Infect Dis. 2020;91:264-6.

93. Rathi M, Jeloka T, Prasad N, Bansal S, Agarwal SK, Bhalla AK, et al. Chronic kidney disease and hypertension with reference to COVID-19. Indian J Nephrol. 2020;30(3):155-7

94. Rapp JL, Lieberman-Cribbin W, Tuminello S, Taioli E. Male Sex, Severe Obesity, Older Age, and Chronic Kidney Disease Are Associated With COVID-19 Severity and Mortality in New York City. Chest. 2021;159(1):112-15. https://doi.org/10.1016/j.chest.2020.08.2065.

95. Pedrosa MDS, Neves Nogueira F. COVID-19 and Diabetes: What Should We Expect? J Diabetes Sci Technol. 2020;14(6):1133-4. https://doi.org/10.1177/1 932296820948041.

96. Campisi G, Bizzoca ME, Lo Muzio L. COVID-19 and periodontitis: reflecting on a possible association. Head Face Med. 2021 May 11;17(1):16. doi: 10.1186/s13005-021-00267-1. PMID: 33975613; PMCID: PMC8110692

97. Sahni V, Gupta S. COVID-19 \& Periodontitis: the cytokine connection. Med Hypotheses. 2020;144:109908.

98. Sukumar K, Tadepalli A. Nexus between COVID-19 and periodontal disease. J Int Med Res. 2021;49(3):3000605211002695. 99. Madapusi Balaji T, Varadarajan S, Rao USV, Raj AT, Patil S, Arakeri G, et al. Oral cancer and periodontal disease increase the risk of COVID 19? A mechanism mediated through furin and cathepsin overexpression. Med Hypotheses. 2020;144:109936. 100. 15Zhou P, Yang XL, Wang XG, Hu B, Zhang L, Zhang W, et al. A pneumonia outbreak associated with a new coronavirus of probable bat origin. Nature. 2020;579(7798):270-3.https://doi.org/10.1038/s41586-020-2012-7

101. Xu H, Zhong L, Deng J, Peng J, Dan H, Zeng X, et al. High expression of ACE2 receptor of 2019-nCoV on the epithelial cells of oral mucosa. Int J Oral Sci. 2020;12(1):8.

102. Badran Z, Gaudin A, Struillou X, Amador G, Soueidan A. Periodontal pockets: A potential reservoir for SARS-CoV-2? Med Hypotheses. 2020;143:109907.

103. Descamps G, Verset L, Trelcat A, et al. ACE2 Protein Landscape in the Head and Neck Region: The Conundrum of SARS-CoV-2 Infection. Biology (Basel).2020;9(8):235. https://doi.org/10.3390/biology9080235.

104. Pascolo L, Zupin L, Melato M, Tricarico PM, Crovella S. TMPRSS2 and ACE2 coexpression in SARS-CoV-2 salivary glands infection. J Dent Res. 2020; 99(10):1120-1.

105. Hoffmann M, Kleine-Weber H, Schroeder S, Kruger N, Herrler T, Erichsen S, et al. SARS-CoV-2 cell entry depends on ACE2 and TMPRSS2 and is blocked by a clinically proven protease inhibitor. Cell. 2020;181(2):271-80. e8.

106. Wang K, Chen W, Zhang Z, Deng Y, et.al. CD147-spike protein is a novel route for SARS-CoV-2 infection to host cells. Signal Transduct Target Ther. 2020 Dec 4;5(1):283. doi: 10.1038/s41392-020-00426-x. PMID: 33277466; PMCID: PMC7714896. 107. Fenizia C, Galbiati S, Vanetti C, Vago R, Clerici M, Tacchetti C, Daniele T. SARS-CoV-2 Entry: At the Crossroads of CD147 and ACE2. Cells. 2021 Jun 8;10(6):1434. doi: 10.3390/cells10061434. PMID: 34201214; PMCID: PMC8226513.

108. Pizzo G, Guiglia R, Lo Russo L, Campisi G. Dentistry and internal medicine: from the focal infection theory to the periodontal medicine concept. Eur J Intern Med. 2010 Dec;21(6):496-502. doi: 10.1016/j.ejim.2010.07.011. PMID: 21111933.

109. Guo W, Li M, Dong Y, Zhou H, Zhang Z, Tian C, Qin R, Wang H, Shen Y, Du K, Zhao L, Fan H, Luo S, Hu D. Diabetes is a risk factor for the progression and prognosis of COVID-19. Diabetes Metab Res Rev. 2020;e3319. https:// doi.org/10.1002/dmrr.3319. 110. Yang LC, Suen YJ, Wang YH, Lin TC, Yu HC, Chang YC. The Association of Periodontal Treatment and Decreased Pneumonia: A Nationwide Population-Based Cohort Study. Int J Environ Res Public Health. 2020;17(1): 356.

https://doi.org/10.3390/ijerph17010356.

111. Botros N, lyer P, Ojcius DM. Is there an association between oral health and severity of COVID-19 complications? Biomed J. 2020;43(4):325-7. https://doi. org/10.1016/j.bj.2020.05.016.

112. Giuseppina Campisi, Maria Eleonora Bizzoca, Lorenzo Lo Muzio. A new exciting hypothesis: direct correlation between periodontitis and clinical evolution of COVID-19 patients. Qeios. 2020. https://doi.org/10.32388/ WK61AM.3.

113. Yamasaki K, Kawanami T, Yatera K, Fukuda K, Noguchi S, Nagata S, et al. Significance of anaerobes and oral bacteria in community-acquired pneumonia. PLoS One. 2013;8(5):e63103.

114. Ioannidou E. The Sex and Gender Intersection in Chronic Periodontitis. Front Public Health. 2017;5:189.

115. Porcu M, Urbano MR, Verri WA Jr, Barbosa DS, Baracat M, Vargas HO, Machado RCBR, Pescim RR, Nunes SOV. Effects of adjunctive $\mathrm{N}$-acetylcysteine on depressive symptoms: Modulation by baseline high-sensitivity C-reactive protein. Psychiatry Res. 2018 May;263:268-274. doi: 10.1016/j.psychres.2018.02.056. Epub 2018 Mar 13. PMID: 29605103.

116. Zuin R, Palamidese A, Negrin R, Catozzo L, Scarda A, Balbinot M. High-dose N-acetylcysteine in patients with exacerbations of chronic obstructive pulmonary disease. Clin Drug Investig. 2005;25(6):401-8. doi: 10.2165/00044011-200525060-00005. PMID: 17532680.

117. Hou W, Zhang W, Jin R, Liang L, Xu B, Hu Z. Risk factors for disease progression in hospitalized patients with COVID-19: a retrospective cohort study. Infect Dis (Lond). 2020 Jul;52(7):498-505. doi: 10.1080/23744235.2020.1759817. Epub 2020 May 6. PMID: 32370577; PMCID: PMC7212540.

118. Zhang J, Yu M, Tong S, Liu LY, Tang LV. Predictive factors for disease progression in hospitalized patients with coronavirus disease 2019 in Wuhan, China. J Clin Virol. 2020 Jun;127:104392. doi: 10.1016/j.jcv.2020.104392. Epub 2020 Apr 28. PMID: 32361327; PMCID: PMC7187844.

119. Wong A, McNulty R, Taylor D, Sivilotti M, Greene S, Gunja N, Koutsogiannis Z, Graudins A. The NACSTOP Trial: A Multicenter, Cluster-Controlled Trial of Early Cessation of Acetylcysteine in Acetaminophen Overdose. Hepatology. 2019 Feb;69(2):774-784. doi: 10.1002/hep.30224. Epub 2019 Jan 19. PMID: 30125376.

120. Smilkstein MJ, Bronstein AC, Linden C, Augenstein WL, Kulig KW, Rumack BH. Acetaminophen overdose: a 48-hour intravenous N-acetylcysteine treatment protocol. Ann Emerg Med. 1991 Oct;20(10):1058-63. doi: 10.1016/s0196-0644(05)813526. PMID: 1928874.

121. Suter PM, Domenighetti G, Schaller MD, Laverrière MC, Ritz R, Perret C. N-acetylcysteine enhances recovery from acute lung injury in man. A randomized, double-blind, placebo-controlled clinical study. Chest. 1994 Jan;105(1):190-4. doi:

10.1378/chest.105.1.190. PMID: 8275731. 
122. Ortolani O, Conti A, De Gaudio AR, Masoni M, Novelli G. Protective effects of N-acetylcysteine and rutin on the lipid peroxidation of the lung epithelium during the adult respiratory distress syndrome. Shock. 2000 Jan;13(1):14-8. doi: 10.1097/00024382-200013010-00003. PMID: 10638663.

123. 130.

124. José Fábio Santos Duarte Lana, Anna Vitória Santos Duarte Lana, Quézia Souza Rodrigues, Gabriel Silva Santos, Riya Navani, Annu Navani, Lucas Furtado da Fonseca, Gabriel Ohana Marques Azzini, Thiago Setti, Tomas Mosaner, Claudio Lopes Simplicio, Taís Mazzini Setti, Nebulization of glutathione and N-Acetylcysteine as an adjuvant therapy for COVID-19 onset,Advances in Redox Research,2021,https://doi.org/10.1016/j.arres.2021.100015.

125. Boesgaard S, Aldershvile J, Poulsen HE, Christensen S, Dige-Petersen H, Giese J. N-acetylcysteine inhibits angiotensin converting enzyme in vivo. J Pharmacol Exp Ther. 1993 Jun;265(3):1239-44. PMID: 8389858.

126. Ullian ME, Gelasco AK, Fitzgibbon WR, Beck CN, Morinelli TA. N-acetylcysteine decreases angiotensin II receptor binding in vascular smooth muscle cells. J Am Soc Nephrol. 2005 Aug;16(8):2346-53. doi: 10.1681/ASN.2004060458. Epub 2005 Jun 8. PMID: 15944340.

127. Lai KY, Ng WY, Osburga Chan PK, Wong KF, Cheng F. High-dose N-acetylcysteine therapy for novel H1N1 influenza pneumonia. Ann Intern Med. 2010 May 18;152(10):687-8. doi: 10.7326/0003-4819-152-10-201005180-00017. PMID: 20479037. 128. Polonikov A. Endogenous Deficiency of Glutathione as the Most Likely Cause of Serious Manifestations and Death in COVID19 Patients. ACS Infect Dis. 2020 Jul 10;6(7):1558-1562. doi: 10.1021/acsinfecdis.0c00288. Epub 2020 May 28. PMID: 32463221; PMCID: PMC7263077.

129. Mata M, Morcillo E, Gimeno C, Cortijo J. N-acetyl-L-cysteine (NAC) inhibit mucin synthesis and pro-inflammatory mediators in alveolar type II epithelial cells infected with influenza virus A and B and with respiratory syncytial virus (RSV). Biochem Pharmacol. 2011 Sep 1;82(5):548-55. doi: 10.1016/j.bcp.2011.05.014. Epub 2011 May 25. PMID: 21635874.

130. Andrey M. Grishin, Nataliya V. Dolgova, Shelby Harms, Ingrid J. Pickering, Graham N. George, Darryl Falzarano, Miroslaw Cygler, Spike protein disulfide disruption as a potential treatment for SARS-CoV-2, bioRxiv 2021.01.02.425099; doi: https://doi.org/10.1101/2021.01.02.425099.

131. McCarty, Mark F, and James J DiNicolantonio. "Nutraceuticals have potential for boosting the type 1 interferon response to RNA viruses including influenza and coronavirus." Progress in cardiovascular diseases vol. 63,3 (2020): 383-385.

doi:10.1016/j.pcad.2020.02.007

132. Wong, Kon Ken et al. "N-Acetylcysteine as Adjuvant Therapy for COVID-19 - A Perspective on the Current State of the Evidence." Journal of inflammation research vol. 14 2993-3013. 6 Jul. 2021, doi:10.2147/JIR.S306849

133. Akhter J, Quéromès G, Pillai K, Kepenekian V, Badar S, Mekkawy AH, Frobert E, Valle SJ, Morris DL. The Combination of Bromelain and Acetylcysteine (BromAc) Synergistically Inactivates SARS-CoV-2. Viruses. 2021 Mar 6;13(3):425. doi: 10.3390/v13030425. PMID: 33800932; PMCID: PMC7999995.

134. Stukalov, A., Girault, V., Grass, V. et al. Multilevel proteomics reveals host perturbations by SARS-CoV-2 and SARS-CoV. Nature 594, 246-252 (2021). https://doi.org/10.1038/s41586-021-03493-4

135. Manček-Keber M, Hafner-Bratkovič I, Lainšček D, Benčina M, Govednik T, Orehek S, Plaper T, Jazbec V, Bergant V, Grass V, Pichlmair A, Jerala R. Disruption of disulfides within RBD of SARS-CoV-2 spike protein prevents fusion and represents a target for viral entry inhibition by registered drugs. FASEB J. 2021 Jun;35(6):e21651. doi: 10.1096/fj.202100560R. PMID: 34004056; PMCID: PMC8206760.

136. Sagar, S., Rathinavel, A.K., Lutz, W.E., Struble, L.R., Khurana, S., Schnaubelt, A.T., Mishra, N.K., Guda, C., Palermo, N.Y., Broadhurst, M.J., Hoffmann, T., Bayles, K.W., Reid, S.P.M., Borgstahl, G.E.O. and Radhakrishnan, P. (2021), Bromelain inhibits SARS-CoV-2 infection via targeting ACE-2, TMPRSS2, and spike protein. Clin. Transl. Med., 11: e281.

https://doi.org/10.1002/ctm2.281

137. Mccord, Joe \& Hybertson, Brooks \& Cota-Gomez, Adela \& Gao, Bifeng. (2020). Nrf2 Activator PB125 ${ }^{\circledR}$ as a Potential Therapeutic Agent Against COVID-19. bioRxiv : the preprint server for biology. 10.1101/2020.05.16.099788.

138. Penugonda S, Mare S, Goldstein G, Banks WA, Ercal N. Effects of N-acetylcysteine amide (NACA), a novel thiol antioxidant against glutamate-induced cytotoxicity in neuronal cell line PC12. Brain Res. 2005 Sep 21;1056(2):132-8. doi: 10.1016/j.brainres.2005.07.032. PMID: 16120436.

139. Hoffmann M, Mösbauer K, Hofmann-Winkler H, Kaul A, Kleine-Weber H, Krüger N, Gassen NC, Müller MA, Drosten C, Pöhlmann S. Chloroquine does not inhibit infection of human lung cells with SARS-CoV-2. Nature. 2020 Sep;585(7826):588-590. doi: 10.1038/s41586-020-2575-3. Epub 2020 Jul 22. PMID: 32698190.

140. Li K, Meyerholz DK, Bartlett JA, McCray PB Jr. The TMPRSS2 Inhibitor Nafamostat Reduces SARS-CoV-2 Pulmonary Infection in Mouse Models of COVID-19. mBio. 2021 Aug 3:e0097021. doi: 10.1128/mBio.00970-21. Epub ahead of print. PMID: 34340553. 141. Alamdari DH, Moghaddam AB, Amini S, Keramati MR, Zarmehri AM, Alamdari AH, Damsaz M, Banpour H, Yarahmadi A, Koliakos G. Application of methylene blue -vitamin C - N-acetyl cysteine for treatment of critically ill COVID-19 patients, report of a phase-I clinical trial. Eur J Pharmacol. 2020 Oct 15;885:173494. doi: 10.1016/j.ejphar.2020.173494. Epub 2020 Aug 20. PMID: 32828741; PMCID: PMC7440159.

142. Liu Y, Wang M, Luo G, Qian X, Wu C, Zhang Y, Chen B, Leung EL, Tang Y. Experience of N-acetylcysteine airway management in the successful treatment of one case of critical condition with COVID-19: A case report. Medicine (Baltimore). 2020 Oct 16;99(42):e22577. doi: 10.1097/MD.0000000000022577. PMID: 33080692; PMCID: PMC7571913.

143. Puyo C, Kreig D, Saddi V et al. Case Report: Use of hydroxychloroquine and N-acetylcysteine for treatment of a COVID-19 patient [version 2; peer review: 2 not approved]. F1000Research 2020, 9:491 (https://doi.org/10.12688/f1000research.23995.2) 144. Nasi A, McArdle S, Gaudernack G, Westman G, Melief C, Rockberg J, Arens R, Kouretas D, Sjölin J, Mangsbo S. Reactive oxygen species as an initiator of toxic innate immune responses in retort to SARS-CoV-2 in an ageing population, consider Nacetylcysteine as early therapeutic intervention. Toxicol Rep. 2020 Jun 18;7:768-771. doi: 10.1016/j.toxrep.2020.06.003. PMID: 32632359; PMCID: PMC7301821.

145. Hoffmann M, Kleine-Weber H, Schroeder S, Krüger N, Herrler T, Erichsen S, Schiergens TS, Herrler G, Wu NH, Nitsche A, Müller MA, Drosten C, Pöhlmann S. SARS-CoV-2 Cell Entry Depends on ACE2 and TMPRSS2 and Is Blocked by a Clinically Proven Protease Inhibitor. Cell. 2020 Apr 16;181(2):271-280.e8. doi: 10.1016/j.cell.2020.02.052. Epub 2020 Mar 5. PMID: 32142651; PMCID: PMC7102627.

146. Horowitz RI, Freeman PR, Bruzzese J. Efficacy of glutathione therapy in relieving dyspnea associated with COVID-19 pneumonia: A report of 2 cases. Respir Med Case Rep. 2020 Apr 21;30:101063. doi: 10.1016/j.rmcr.2020.101063. PMID: 32322478; PMCID: PMC7172740.

147. Zhou, Z., Huang, C., Zhou, Z., Huang, Z., Su, L., Kang, S., Chen, X., Chen, Q., He, S., Rong, X., et al. (2020). Structural Insight Reveals SARS-CoV-2 Orf7a as an Immunomodulating Factor for Human CD14+ Monocytes. SSRN Electronic Journal. DOI: $10.2139 /$ ssrn. 3699795. 
148. Aditi Chopra, Karthik Sivaraman, Raghu Radhakrishnan, Dhanasekar Balakrishnan, Aparna Narayana, Can povidone iodine gargle/mouthrinse inactivate SARS-CoV-2 and decrease the risk of nosocomial and community transmission during the COVID-19 pandemic? An evidence-based update, Japanese Dental Science Review, 2021, https://doi.org/10.1016/j.jdsr.2021.03.001. 149. Naqvi, S.H.S., Citardi, M.J., Cattano, D. et al. Povidone-iodine solution as SARS-CoV-2 prophylaxis for procedures of the upper aerodigestive tract a theoretical framework. J of Otolaryngol - Head \& Neck Surg 49, 77 (2020). https://doi.org/10.1186/s40463020-00474-x

150. Hassandarvish, P., Tiong, V., Mohamed, N. et al. In vitro virucidal activity of povidone iodine gargle and mouthwash against SARS-CoV-2: implications for dental practice. Br Dent J (2020). https://doi.org/10.1038/s41415-020-2402-0

151. Anderson DE, Sivalingam V, Kang AEZ, Ananthanarayanan A, Arumugam H, Jenkins TM, Hadjiat Y, Eggers M. Povidone-lodine Demonstrates Rapid In Vitro Virucidal Activity Against SARS-CoV-2, The Virus Causing COVID-19 Disease. Infect Dis Ther. 2020 Sep;9(3):669-675. doi: 10.1007/s40121-020-00316-3. Epub 2020 Jul 8. PMID: 32643111; PMCID: PMC7341475.

152. Pelletier JS, Tessema B, Frank S, Westover JB, Brown SM, Capriotti JA. Efficacy of Povidone-lodine Nasal and Oral Antiseptic Preparations Against Severe Acute Respiratory Syndrome-Coronavirus 2 (SARS-CoV-2). Ear Nose Throat J. 2021

Apr;100(2_suppl):192S-196S. doi: 10.1177/0145561320957237. Epub 2020 Sep 21. Erratum in: Ear Nose Throat J. 2020 Dec 8;:145561320977784. PMID: 32951446

153. Bidra AS, Pelletier JS, Westover JB, Frank S, Brown SM, Tessema B. Rapid In-Vitro Inactivation of Severe Acute Respiratory Syndrome Coronavirus 2 (SARS-CoV-2) Using Povidone-lodine Oral Antiseptic Rinse. J Prosthodont. 2020 Jul;29(6):529-533. doi: 10.1111/jopr.13209. Epub 2020 Jun 16. PMID: 32511851; PMCID: PMC7300649.

154. Frank S, Capriotti J, Brown SM, Tessema B. Povidone-lodine Use in Sinonasal and Oral Cavities: A Review of Safety in the COVID-19 Era. Ear Nose Throat J. 2020 Nov;99(9):586-593. doi: 10.1177/0145561320932318. Epub 2020 Jun 10. PMID: 32520599. 155. Mohamed, Nurul \& Baharom, Nizam \& Shahida, Wan \& Rashid, Zetti \& Kon Ken, Wong \& Ali, Umi \& Othman, Siti \& SAMAD, MUTTAQILLAH \& Kori, Najma \& Periyasamy, Petrick \& ZAKARIA, NOR \& SUGUMAR, AGNI \& Mohammad Kazmin, Nur Ezzaty \& Xiong Khee, Cheong \& SANIMAN, SITI \& Isahak, llina. (2020). EARLY VIRAL CLEARANCE AMONG COVID-19 PATIENTS WHEN GARGLING WITH POVIDONE-IODINE AND ESSENTIAL OILS: A PILOT CLINICAL TRIAL. 10.1101/2020.09.07.20180448.

156. Capriotti, Kara \& Pelletier, Jesse \& S, Barone \& Capriotti, Joseph. (2018). Efficacy of Dilute Povidone-lodine against MultiDrug Resistant Bacterial Biofilms, Fungal Biofilms and Fungal Spores. Journal of Clinical Research in Dermatology. 5. 1-5. 10.15226/2378-1726/5/1/00174.

157. Kanagalingam, J., Feliciano, R., Hah, J.H., Labib, H., Le, T.A. and Lin, J.-C. (2015), Practical use of povidone-iodine antiseptic in the maintenance of oral health and in the prevention and treatment of common oropharyngeal infections. Int J Clin Pract, 69: 1247-1256. https://doi.org/10.1111/ijcp.12707

158. Cigana F, Kerebel B, David J, Doumenjou F, Da Costa Noble R. Etude clinique et histologique de l'efficacité de la bétadine sur I'inflammation gingivale [A clinical and histological study of the efficacy of betadine on gingival inflammation]. J Biol Buccale. 1991 Jun;19(2):173-84. French. PMID: 1939041.

159. Greenstein G. Povidone-iodine's effects and role in the management of periodontal diseases: a review. J Periodontol. 1999 Nov;70(11):1397-405. doi: 10.1902/jop.1999.70.11.1397. PMID: 10588505.

160. Sindhura H, Harsha RH, Shilpa RH. Efficacy of subgingival irrigation with $10 \%$ povidone-iodine as an adjunct to scaling and root planing: A clinical and microbiological study. Indian J Dent Res. 2017 Sep-Oct;28(5):514-518. doi: 10.4103/ijdr.IJDR_497_15. PMID: 29072213.

161. RECOVERY Collaborative Group, Horby P, Lim WS, Emberson JR, Mafham M, Bell JL, Linsell L, Staplin N, Brightling C,

Ustianowski A, Elmahi E, Prudon B, Green C, Felton T, Chadwick D, Rege K, Fegan C, Chappell LC, Faust SN, Jaki T, Jeffery K, Montgomery A, Rowan K, Juszczak E, Baillie JK, Haynes R, Landray MJ. Dexamethasone in Hospitalized Patients with Covid-19. N Engl J Med. 2021 Feb 25;384(8):693-704. doi: 10.1056/NEJMoa2021436. Epub 2020 Jul 17. PMID: 32678530; PMCID: PMC7383595.

162. Karnad, D.R., Bhadade, R., Verma, P.K. et al. Intravenous administration of ulinastatin (human urinary trypsin inhibitor) in severe sepsis: a multicenter randomized controlled study. Intensive Care Med 40, 830-838 (2014).

https://doi.org/10.1007/s00134-014-3278-8

163. Angus DC, Linde-Zwirble WT, Lidicker J, Clermont G, Carcillo J, Pinsky MR. Epidemiology of severe sepsis in the United States: analysis of incidence, outcome, and associated costs of care. Crit Care Med. 2001 Jul;29(7):1303-10. doi: 10.1097/00003246200107000-00002. PMID: 11445675.

164. Vincent JL, Rello J, Marshall J, Silva E, Anzueto A, Martin CD, Moreno R, Lipman J, Gomersall C, Sakr Y, Reinhart K; EPIC II Group of Investigators. International study of the prevalence and outcomes of infection in intensive care units. JAMA. 2009 DeC 2;302(21):2323-9. doi: 10.1001/jama.2009.1754. PMID: 19952319.

165. Rittirsch D, Flierl MA, Ward PA. Harmful molecular mechanisms in sepsis. Nat Rev Immunol. 2008 Oct;8(10):776-87. doi: 10.1038/nri2402. PMID: 18802444; PMCID: PMC2786961.

166. van der Poll T, Opal SM. Host-pathogen interactions in sepsis. Lancet Infect Dis. 2008 Jan;8(1):32-43. doi: 10.1016/S14733099(07)70265-7. Epub 2007 Dec 11. PMID: 18063412.

167. Levi M, van der Poll T. Endothelial injury in sepsis. Intensive Care Med. 2013 Oct;39(10):1839-42. doi: 10.1007/s00134-0133054-1. Epub 2013 Aug 8. PMID: 23925547.

168. Angus DC, van der Poll T. Severe sepsis and septic shock. N Engl J Med. 2013 Aug 29;369(9):840-51. doi:

10.1056/NEJMra1208623. Erratum in: N Engl J Med. 2013 Nov 21;369(21):2069. PMID: 23984731.

169. Huang N, Wang F, Wang Y, Hou J, Li J, Deng X. Ulinastatin improves survival of septic mice by suppressing inflammatory response and lymphocyte apoptosis. J Surg Res. 2013 Jun 15;182(2):296-302. doi: 10.1016/j.jss.2012.10.043. Epub 2012 Nov 9. PMID: 23158408.

170. Cao YZ, Tu YY, Chen X, Wang BL, Zhong YX, Liu MH. Protective effect of Ulinastatin against murine models of sepsis: inhibition of TNF- $\alpha$ and IL-6 and augmentation of IL-10 and IL-13. Exp Toxicol Pathol. 2012 Sep;64(6):543-7. doi: 10.1016/j.etp.2010.11.011. Epub 2010 Dec 14. PMID: 21159497.

171. Shao YM, Zhang LQ, Deng LH, Yao HG. [Clinical study on effects of ulinastatin on patients with systemic inflammatory response syndrome]. Zhongguo Wei Zhong Bing Ji Jiu Yi Xue. 2005 Apr;17(4):228-30. Chinese. PMID: 15836828. 172. Ueki M, Taie S, Chujo K, Asaga T, Iwanaga Y, Ono J, Maekawa N. Urinary trypsin inhibitor reduces inflammatory response in kidney induced by lipopolysaccharide. J Biosci Bioeng. 2007 Oct;104(4):315-20. doi: 10.1263/jbb.104.315. PMID: 18023806. 173. Tanaka R, Fujita M, Tsuruta R, Fujimoto K, Aki HS, Kumagai K, Aoki T, Kobayashi A, Izumi T, Kasaoka S, Yuasa M, Maekawa T. Urinary trypsin inhibitor suppresses excessive generation of superoxide anion radical, systemic inflammation, oxidative stress, and endothelial injury in endotoxemic rats. Inflamm Res. 2010 Aug;59(8):597-606. doi: 10.1007/s00011-010-0166-8. Epub 2010 Feb 11. PMID: 20148283.

174. Shu H, Liu K, He Q, Zhong F, Yang L, Li Q, Liu W, Ye F, Huang W. Ulinastatin, a protease inhibitor, may inhibit allogeneic blood transfusion-associated pro-inflammatory cytokines and systemic inflammatory response syndrome and improve postoperative 
recovery. Blood Transfus. 2014 Jan;12 Suppl 1(Suppl 1):s109-18. doi: 10.2450/2013.0224-12. Epub 2013 May 8. PMID: 23736923; PMCID: PMC3934215.

175. Meng, Wen-Tao et al. "Ulinastatin: A Potential Alternative to Glucocorticoid in the Treatment of Severe Decompression Sickness." Frontiers in physiology vol. 11 273. 26 Mar. 2020, doi:10.3389/fphys.2020.00273

176. Yano T, Anraku S, Nakayama R, Ushijima K. Neuroprotective effect of urinary trypsin inhibitor against focal cerebral ischemia-reperfusion injury in rats. Anesthesiology. 2003 Feb;98(2):465-73. doi: 10.1097/00000542-200302000-00028. PMID: 12552207.

177. Nishiyama T, Aibiki M, Hanaoka K. The effect of ulinastatin, a human protease inhibitor, on the transfusion-induced increase of plasma polymorphonuclear granulocyte elastase. Anesth Analg. 1996 Jan;82(1):108-12. doi: 10.1097/00000539-199601000 00019. PMID: 8712383.

178. Nakatani K, Takeshita S, Tsujimoto H, Kawamura Y, Sekine I. Inhibitory effect of serine protease inhibitors on neutrophilmediated endothelial cell injury. J Leukoc Biol. 2001 Feb;69(2):241-7. PMID: 11272274.

179. Nakahama H, Kuribayashi K, Sugita M, Shinkawa T. Cytoprotective effect of ulinastatin, a Kunitz-type protease inhibitor, on hypoxic injury in L2 cells treated with antimycin A via stabilization of lysosomal fragility. Pulm Pharmacol Ther. 1999;12(1):1-6. doi: 10.1006/pupt.1998.9998. PMID: 10208830.

180. Takada K, Komori M, Notoya A, Tomizawa Y, Ozaki M. Effect of ulinastatin on microcirculation during excessive hemorrhage using fluid therapy. In Vivo. 2003 Mar-Apr;17(2):129-35. PMID: 12792973.

181. Wei F, Liu S, Luo L, Gu N, Zeng Y, Chen X, Xu S, Zhang D. Anti-inflammatory mechanism of ulinastatin: Inhibiting the hyperpermeability of vascular endothelial cells induced by TNF- $\alpha$ via the RhoA/ROCK signal pathway. Int Immunopharmacol. 2017 May;46:220-227. doi: 10.1016/j.intimp.2017.03.007. Epub 2017 Mar 19. PMID: 28329735.

182. Fang M, Zhong WH, Song WL, Deng YY, Yang DM, Xiong B, Zeng HK, Wang HD. Ulinastatin Ameliorates Pulmonary Capillary Endothelial Permeability Induced by Sepsis Through Protection of Tight Junctions via Inhibition of TNF- $\alpha$ and Related Pathways. Front Pharmacol. 2018 Aug 13;9:823. doi: 10.3389/fphar.2018.00823. PMID: 30150933; PMCID: PMC6099086.

183. Song JE, Kang WS, Kim DK, Yoon TG, Kim TY, Bang YS, Kim SH. The effect of ulinastatin on postoperative blood loss in patients undergoing open heart surgery with cardiopulmonary bypass. J Int Med Res. 2011;39(4):1201-10. doi: 10.1177/147323001103900408. PMID: 21986122

184. Takeshita, Seiichiro \& Kanai, Takashi \& Kawamura, Yoichi. (2017). Ulinastatin. 10.1007/978-4-431-56039-5_27.

185. Masafumi Zaitsu, Yuhei Hamasaki, Katsuya Tashiro, Muneaki Matsuo, Tomohiro Ichimaru, Ichiro Fujita, Hakaru Tasaki, Sumio Miyazaki, Ulinastatin, an Elastase Inhibitor, Inhibits the Increased mRNA Expression of Prostaglandin H2 Synthase-Type 2 in Kawasaki Disease, The Journal of Infectious Diseases, Volume 181, Issue 3, March 2000, Pages 1101-1109, https://doi.org/10.1086/315332

186. Pan, Y., Fang, H., Lu, F. et al. Ulinastatin ameliorates tissue damage of severe acute pancreatitis through modulating regulatory T cells. J Inflamm 14, 7 (2017). https://doi.org/10.1186/s12950-017-0154-7

187. Wang H, Liu B, Tang Y, Chang P, Yao L, Huang B, Lodato RF, Liu Z. Improvement of Sepsis Prognosis by Ulinastatin: A Systematic Review and Meta-Analysis of Randomized Controlled Trials. Front Pharmacol. 2019 Nov 26;10:1370. doi: 10.3389/fphar.2019.01370. Erratum in: Front Pharmacol. 2020 Feb 21;10:1697. PMID: 31849646; PMCID: PMC6893897. 188. Li, St., Dai, Q., Zhang, Sx. et al. Ulinastatin attenuates LPS-induced inflammation in mouse macrophage RAW264.7 cells by inhibiting the JNK/NF-KB signaling pathway and activating the PI3K/Akt/Nrf2 pathway. Acta Pharmacol Sin 39, 1294-1304 (2018) https://doi.org/10.1038/aps.2017.143

189. Tong, Ying et al. "Ulinastatin preconditioning attenuates inflammatory reaction of hepatic ischemia reperfusion injury in rats via high mobility group box 1(HMGB1) inhibition." International journal of medical sciences vol. 11,4 337-43. 11 Feb. 2014, doi:10.7150/ijms.7861

190. Wang, Wen-Ke et al. "Ulinastatin attenuates diabetes-induced cardiac dysfunction by the inhibition of inflammation and apoptosis." Experimental and therapeutic medicine vol. 14,3 (2017): 2497-2504. doi:10.3892/etm.2017.4824

191. Wang, Y., Tao, T., Dong, Y., Zhang, J., \& Qin, Z. (2015). Effect of ulinastatin on the expression and distribution of high mobility group box 1 in human colon carcinoma cells in vitro. Molecular Medicine Reports, 11, 2041-2047.

https://doi.org/10.3892/mmr.2014.2921

192. Zhao Y, Cai H, Zhou P, Lin S, Pan Y, Liang X. Protective effect of ulinastatin on hepatic ischemia reperfusion injury through autophagy activation in Chang liver cells. J Cell Biochem. 2019 Sep;120(9):14960-14970. doi: 10.1002/jcb.28758. Epub 2019 Apr 24. PMID: 31020701.

193. Sun Y, Wang LX, Zhou YF, Sun SG, Mao XF, Deng XD, Zhang P. [Effects of glutamine combined with ulinastatin on inflammatory response of patients with severe burn injury]. Zhonghua Shao Shang Za Zhi. 2013 Aug;29(4):349-54. Chinese. PMID: 24351534.L

194. Ye, W., Chen, G., Li, X. et al. Dynamic changes of D-dimer and neutrophil-lymphocyte count ratio as prognostic biomarkers in COVID-19. Respir Res 21, 169 (2020). https://doi.org/10.1186/s12931-020-01428-7

195. Liu, J., Liu, Y., Xiang, P. et al. Neutrophil-to-lymphocyte ratio predicts critical illness patients with 2019 coronavirus disease in the early stage. J Transl Med 18, 206 (2020). https://doi.org/10.1186/s12967-020-02374-0

196. Alkhatip AAAMM, Kamel MG, Hamza MK, Farag EM, Yassin HM, Elayashy M, Naguib AA, Wagih M, Abd-Elhay FA, Algameel HZ, Yousef MA, Purcell A, Helmy M. The diagnostic and prognostic role of neutrophil-to-lymphocyte ratio in COVID-19: a systematic review and meta-analysis. Expert Rev Mol Diagn. 2021 May;21(5):505-514. doi: 10.1080/14737159.2021.1915773. Epub 2021 Apr 25. PMID: 33840351; PMCID: PMC8074650.

197. Yang, Ai-Ping et al. "The diagnostic and predictive role of NLR, d-NLR and PLR in COVID-19 patients." International immunopharmacology vol. 84 (2020): 106504. doi:10.1016/j.intimp.2020.106504

198. Man MA, Rajnoveanu RM, Motoc NS, Bondor Cl, Chis AF, et al. (2021) Neutrophil-to-lymphocyte ratio, platelets-tolymphocyte ratio, and eosinophils correlation with high-resolution computer tomography severity score in COVID-19 patients. PLOS ONE 16(6): e0252599. https://doi.org/10.1371/journal.pone.0252599

199. Zhang, Bicheng et al. "Immune Phenotyping Based on the Neutrophil-to-Lymphocyte Ratio and IgG Level Predicts Disease Severity and Outcome for Patients With COVID-19." Frontiers in molecular biosciences vol. 7 157. 3 Jul. 2020, doi:10.3389/fmolb.2020.00157

200. Lu M, Yu S, Xu W, Gao B, Xiong S. HMGB1 Promotes Systemic Lupus Erythematosus by Enhancing Macrophage Inflammatory Response. J Immunol Res. 2015;2015:946748. doi: 10.1155/2015/946748. Epub 2015 May 19. PMID: 26078984; PMCID: PMC4452473.

201. Li B, Yue Y, Dong C, Shi Y, Xiong S. Blockade of macrophage autophagy ameliorates activated lymphocytes-derived DNA induced murine lupus possibly via inhibition of proinflammatory cytokine production. Clin Exp Rheumatol. 2014 SepOct;32(5):705-14. Epub 2014 Aug 15. PMID: 25151985. 
202. Wirestam L, Schierbeck H, Skogh T, Gunnarsson I, Ottosson L, Erlandsson-Harris H, Wetterö J, Sjöwall C. Antibodies against High Mobility Group Box protein-1 (HMGB1) versus other anti-nuclear antibody fine-specificities and disease activity in systemic lupus erythematosus. Arthritis Res Ther. 2015 Nov 23;17:338. doi: 10.1186/s13075-015-0856-2. PMID: 26596890; PMCID: PMC4657231.

203. Li X, Yue Y, Zhu Y, Xiong S. Extracellular, but not intracellular HMGB1, facilitates self-DNA induced macrophage activation via promoting DNA accumulation in endosomes and contributes to the pathogenesis of lupus nephritis. Mol Immunol. 2015 May;65(1):177-88. doi: 10.1016/j.molimm.2015.01.023. Epub 2015 Feb 6. PMID: 25660970.

204. Abdulahad DA, Westra J, Limburg PC, Kallenberg CG, Bijl M. HMGB1 in systemic lupus Erythematosus: Its role in cutaneous lesions development. Autoimmun Rev. 2010 Aug;9(10):661-5. doi: 10.1016/j.autrev.2010.05.015. Epub 2010 May 21. PMID: 20546955.

205. Pan HF, Wu GC, Li WP, Li XP, Ye DQ. High Mobility Group Box 1: a potential therapeutic target for systemic lupus erythematosus. Mol Biol Rep. 2010 Mar;37(3):1191-5. doi: 10.1007/s11033-009-9485-7. Epub 2009 Feb 27. PMID: 19247800. 206. Watanabe H, Son M. The Immune Tolerance Role of the HMGB1-RAGE Axis. Cells. 2021 Mar 5;10(3):564. doi: 10.3390/cells10030564. PMID: 33807604; PMCID: PMC8001022.

207. Ibrahim ZA, Armour CL, Phipps S, Sukkar MB. RAGE and TLRs: relatives, friends or neighbours? Mol Immunol. 2013 Dec;56(4):739-44. doi: 10.1016/j.molimm.2013.07.008. Epub 2013 Aug 14. PMID: 23954397.

208. Musumeci D, Roviello GN, Montesarchio D. An overview on HMGB1 inhibitors as potential therapeutic agents in HMGB1related pathologies. Pharmacol Ther. 2014 Mar;141(3):347-57. doi: 10.1016/j.pharmthera.2013.11.001. Epub 2013 Nov 9. PMID: 24220159.

209. Bangert A, Andrassy M, Müller AM, Bockstahler M, Fischer A, Volz CH, Leib C, Göser S, Korkmaz-Icöz S, Zittrich S, Jungmann A, Lasitschka F, Pfitzer G, Müller OJ, Katus HA, Kaya Z. Critical role of RAGE and HMGB1 in inflammatory heart disease. Proc Natl Acad Sci U S A. 2016 Jan 12;113(2):E155-64. doi: 10.1073/pnas.1522288113. Epub 2015 Dec 29. PMID: 26715748; PMCID: PMC4720305.

210. Kim H, Mun D, Kang JY, Lee SH, Yun N, Joung B. Improved cardiac-specific delivery of RAGE siRNA within small extracellular vesicles engineered to express intense cardiac targeting peptide attenuates myocarditis. Mol Ther Nucleic Acids. 2021 May 1;24:1024-1032. doi: 10.1016/j.omtn.2021.04.018. PMID: 34141457; PMCID: PMC8167198.

211. Tian Y, Charles EJ, Yan Z, Wu D, French BA, Kron IL, Yang Z. The myocardial infarct-exacerbating effect of cell-free DNA is mediated by the high-mobility group box 1-receptor for advanced glycation end products-Toll-like receptor 9 pathway. J Thorac Cardiovasc Surg. 2019 Jun;157(6):2256-2269.e3. doi: 10.1016/j.jtcvs.2018.09.043. Epub 2018 Oct 5. PMID: 30401529; PMCID: PMC6450791.

212. Tian Y, Pan D, Chordia MD, French BA, Kron IL, Yang Z. The spleen contributes importantly to myocardial infarct exacerbation during post-ischemic reperfusion in mice via signaling between cardiac HMGB1 and splenic RAGE. Basic Res Cardiol. 2016 Nov;111(6):62. doi: 10.1007/s00395-016-0583-0. Epub 2016 Sep 19. PMID: 27645145; PMCID: PMC5385142.

213. Wang X, Wang J, Tu T, Iyan Z, Mungun D, Yang Z, Guo Y. Remote Ischemic Postconditioning Protects against Myocardia Ischemia-Reperfusion Injury by Inhibition of the RAGE-HMGB1 Pathway. Biomed Res Int. 2018 Jan 23;2018:4565630. doi: 10.1155/2018/4565630. PMID: 29789792; PMCID: PMC5896327.

214. Zhang S, Feng Z, Gao W, Duan Y, Fan G, Geng X, Wu B, Li K, Liu K, Peng C. Aucubin Attenuates Liver Ischemia-Reperfusion Injury by Inhibiting the HMGB1/TLR-4/NF-KB Signaling Pathway, Oxidative Stress, and Apoptosis. Front Pharmacol. 2020 Sep 8;11:544124. doi: 10.3389/fphar.2020.544124. PMID: 33013386; PMCID: PMC7506056.

215. Andrassy M, Volz HC, Igwe JC, Funke B, Eichberger SN, Kaya Z, Buss S, Autschbach F, Pleger ST, Lukic IK, Bea F, Hardt SE, Humpert PM, Bianchi ME, Mairbäurl H, Nawroth PP, Remppis A, Katus HA, Bierhaus A. High-mobility group box-1 in ischemiareperfusion injury of the heart. Circulation. 2008 Jun 24;117(25):3216-26. doi: 10.1161/CIRCULATIONAHA.108.769331. PMID: 18574060.

216. Volz HC, Seidel C, Laohachewin D, Kaya Z, Müller OJ, Pleger ST, Lasitschka F, Bianchi ME, Remppis A, Bierhaus A, Katus HA Andrassy M. HMGB1: the missing link between diabetes mellitus and heart failure. Basic Res Cardiol. 2010 Nov;105(6):805-20. doi: 10.1007/s00395-010-0114-3. Epub 2010 Aug 12. PMID: 20703492.

217. van Beijnum JR, Buurman WA, Griffioen AW. Convergence and amplification of toll-like receptor (TLR) and receptor for advanced glycation end products (RAGE) signaling pathways via high mobility group B1 (HMGB1). Angiogenesis. 2008;11(1):91-9. doi: 10.1007/s10456-008-9093-5. Epub 2008 Feb 9. PMID: 18264787.

218. Volz HC, Kaya Z, Katus HA, Andrassy M. The role of HMGB1/RAGE in inflammatory cardiomyopathy. Semin Thromb Hemost. 2010 Mar;36(2):185-94. doi: 10.1055/s-0030-1251503. Epub 2010 Apr 22. PMID: 20414834.

219. Chong AJ, Shimamoto A, Hampton CR, Takayama H, Spring DJ, Rothnie CL, Yada M, Pohlman TH, Verrier ED. Toll-like receptor 4 mediates ischemia/reperfusion injury of the heart. J Thorac Cardiovasc Surg. 2004 Aug;128(2):170-9. doi: 10.1016/j.jtcvs.2003.11.036. PMID: 15282452

220. Ding HS, Yang J, Chen P, Yang J, Bo SQ, Ding JW, Yu QQ. The HMGB1-TLR4 axis contributes to myocardial ischemia/reperfusion injury via regulation of cardiomyocyte apoptosis. Gene. 2013 Sep 15;527(1):389-93. doi: 10.1016/j.gene.2013.05.041. Epub 2013 May 30. PMID: 23727604.

221. Behl T, Sharma E, Sehgal A, Kaur I, Kumar A, Arora R, Pal G, Kakkar M, Kumar R, Bungau S. Expatiating the molecular approaches of HMGB1 in diabetes mellitus: Highlighting signalling pathways via RAGE and TLRs. Mol Biol Rep. 2021 Feb;48(2):1869-1881. doi: 10.1007/s11033-020-06130-x. Epub 2021 Jan 21. PMID: 33479829.

222. Yang WI, Lee D, Lee DL, Hong SY, Lee SH, Kang SM, Choi DH, Jang Y, Kim SH, Park S. Blocking the receptor for advanced glycation end product activation attenuates autoimmune myocarditis. Circ J. 2014;78(5):1197-205. doi: 10.1253/circj.cj-13-1235. Epub 2014 Mar 6. PMID: 24599045.

223. Song X, Zhang H, Zhao Y, Lin Y, Tang Q, Zhou X, Zhong X. HMGB1 Activates Myeloid Dendritic Cells by Up-Regulating mTOR Pathway in Systemic Lupus Erythematosus. Front Med (Lausanne). 2021 Jun 7;8:636188. doi: 10.3389/fmed.2021.636188. PMID: 34164408; PMCID: PMC8215142.

224. Ding J, Su S, You T, Xia T, Lin X, Chen Z, Zhang L. Serum interleukin-6 level is correlated with the disease activity of systemic lupus erythematosus: a meta-analysis. Clinics (Sao Paulo). 2020 Oct 19;75:e1801. doi: 10.6061/clinics/2020/e1801. PMID: 33084768; PMCID: PMC7536892.

225. Guan SY, Cai HY, Wang P, Lv TT, Liu LN, Mao YM, Zhao CN, Wu Q, Dan YL, Sam NB, Wang DG, Pan HF. Association between circulating 25-hydroxyvitamin D and systemic lupus erythematosus: A systematic review and meta-analysis. Int J Rheum Dis. 2019 Oct;22(10):1803-1813. doi: 10.1111/1756-185X.13676. Epub 2019 Aug 30. PMID: 31468723.

226. Ma L, Zeng A, Chen B, Chen Y, Zhou R. Neutrophil to lymphocyte ratio and platelet to lymphocyte ratio in patients with systemic lupus erythematosus and their correlation with activity: A meta-analysis. Int Immunopharmacol. 2019 Nov;76:105949. doi: 10.1016/j.intimp.2019.105949. Epub 2019 Oct 18. PMID: 31634817. 
227. Zamani, B., Moeini Taba, SM. \& Shayestehpour, M. Systemic lupus erythematosus manifestation following COVID-19: a case report. J Med Case Reports 15, 29 (2021). https://doi.org/10.1186/s13256-020-02582-8

228. Florindo, H.F., Kleiner, R., Vaskovich-Koubi, D. et al. Immune-mediated approaches against COVID-19. Nat. Nanotechnol. 15, 630-645 (2020). https://doi.org/10.1038/s41565-020-0732-3

229. Halima A. Shuwa, Tovah N. Shaw, Sean B. Knight et.al Alterations in T and B cell function persist in convalescent COVID-19 patients, Med, 2021, https://doi.org/10.1016/j.medj.2021.03.013.

230. Su KY, Pisetsky DS. The role of extracellular DNA in autoimmunity in SLE. Scand J Immunol. 2009 Sep; 70(3):175-83. doi: 10.1111/j.1365-3083.2009.02300.x. PMID: 19703007.

231. Pisetsky DS. DNA as a marker of cell death in systemic lupus erythematosus. Rheum Dis Clin North Am. 2004 Aug;30(3):575-

87, x. doi: 10.1016/j.rdc.2004.04.009. PMID: 15261342.

232. Pisetsky DS. The immune response to cell death in SLE. Autoimmun Rev. 2004 Nov;3(7-8):500-4. doi:

10.1016/j.autrev.2004.07.010. PMID: 15546797.

233. Pisetsky DS, Jiang N. The generation of extracellular DNA in SLE: the role of death and sex. Scand J Immunol. 2006 Sep;64(3):200-4. doi: 10.1111/j.1365-3083.2006.01822.x. PMID: 16918687.

234. Andrade $H$, Lin W, Zhang Y. Specificity from nonspecific interaction: regulation of tumor necrosis factor- $\alpha$ activity by DNA. J Biol Chem. 2019 Apr 19;294(16):6397-6404. doi: 10.1074/jbc.RA119.007586. Epub 2019 Feb 27. PMID: 30814250; PMCID: PMC6484132.

235. Pisetsky DS, Fairhurst AM. The origin of extracellular DNA during the clearance of dead and dying cells. Autoimmunity. 2007 Jun;40(4):281-4. doi: 10.1080/08916930701358826. PMID: 17516210

236. Storci G, Bonifazi F, Garagnani P, Olivieri F, Bonafè M. The role of extracellular DNA in COVID-19: Clues from inflamm-aging. Ageing Res Rev. 2021;66:101234. doi:10.1016/j.arr.2020.101234

237. Bonafè M, Prattichizzo F, Giuliani A, Storci G, Sabbatinelli J, Olivieri F. Inflamm-aging: Why older men are the most susceptible to SARS-CoV-2 complicated outcomes. Cytokine Growth Factor Rev. 2020 Jun;53:33-37. doi:

10.1016/j.cytogfr.2020.04.005. Epub 2020 May 3. PMID: 32389499; PMCID: PMC7252014.

238. Storci G, De Carolis S, Olivieri F, Bonafè M. Changes in the biochemical taste of cytoplasmic and cell-free DNA are major fuels for inflamm-aging. Semin Immunol. 2018 Dec;40:6-16. doi: 10.1016/j.smim.2018.08.003. Epub 2018 Sep 15. PMID: 30227944.

239. Thierry, A.; ROCH, B. NETs By-products and Extracellular DNA May Play a Key Role in COVID-19 Pathogenesis: Incidence on Patient Monitoring and Therapy. Preprints 2020, 2020040238 (doi: 10.20944/preprints202004.0238.v1).

240. Arcanjo, A., Logullo, J., Menezes, C.C.B. et al. The emerging role of neutrophil extracellular traps in severe acute respiratory syndrome coronavirus 2 (COVID-19). Sci Rep 10, 19630 (2020). https://doi.org/10.1038/s41598-020-76781-0

241. Monticolo F, Palomba E, Termolino P, Chiaiese P, de Alteriis E, Mazzoleni S, Chiusano ML. The Role of DNA in the

Extracellular Environment: A Focus on NETs, RETs and Biofilms. Front Plant Sci. 2020 Dec 17;11:589837. doi:

10.3389/fpls.2020.589837. PMID: 33424885; PMCID: PMC7793654.

242. Witte T. IgM antibodies against dsDNA in SLE. Clin Rev Allergy Immunol. 2008 Jun;34(3):345-7. doi: 10.1007/s12016-0078046-x. PMID: 18097774.

243. Dema B, Charles N. Autoantibodies in SLE: Specificities, Isotypes and Receptors. Antibodies (Basel). 2016 Jan 4;5(1):2. doi: 10.3390/antib5010002. PMID: 31557984; PMCID: PMC6698872.

244. Villalta D, Bizzaro N, Bassi N, Zen M, Gatto M, Ghirardello A, laccarino L, Punzi L, Doria A. Anti-dsDNA antibody isotypes in systemic lupus erythematosus: IgA in addition to IgG anti-dsDNA help to identify glomerulonephritis and active disease. PLoS One. 2013 Aug 12;8(8):e71458. doi: 10.1371/journal.pone.0071458. PMID: 23951169; PMCID: PMC3741383.

245. Wang, E.Y., Mao, T., Klein, J. et al. Diverse functional autoantibodies in patients with COVID-19. Nature 595, 283-288 (2021). https://doi.org/10.1038/s41586-021-03631-y

246. Khamsi R. Rogue antibodies could be driving severe COVID-19. Nature. 2021 Feb;590(7844):29-31. doi: 10.1038/d41586021-00149-1. PMID: 33469204.

247. Halpert G, Shoenfeld Y. SARS-CoV-2, the autoimmune virus. Autoimmun Rev. 2020 Dec;19(12):102695. doi: 10.1016/j.autrev.2020.102695. Epub 2020 Oct 28. PMID: 33130000; PMCID: PMC7598743.

248. Zhang J, Wu H, Yao X, Zhang D, Zhou Y, Fu B, Wang W, Li H, Wang Z, Hu Z, Ren Y, Sun R, Tian Z, Bian X, Wei H. Pyroptotic macrophages stimulate the SARS-CoV-2-associated cytokine storm. Cell Mol Immunol. 2021 May;18(5):1305-1307. doi: 10.1038/s41423-021-00665-0. Epub 2021 Mar 19. PMID: 33742186; PMCID: PMC7976727.

249. Gao ZW, Zhang HZ, Liu C, Dong K. Autoantibodies in COVID-19: frequency and function. Autoimmun Rev. 2021;20(3):102754. doi:10.1016/j.autrev.2021.102754

250. Xu C, Fan J, Luo Y, Zhao Z, Tang P, Yang G, Pan Y, Guo S, Liu Y, Xiong Y, Xie W, Long X. Prevalence and Characteristics of Rheumatoid-Associated Autoantibodies in Patients with COVID-19. J Inflamm Res. 2021;14:3123-3128 https://doi.org/10.2147/JIR.S312090

251. Spihlman AP, Gadi N, Wu SC, Moulton VR. COVID-19 and Systemic Lupus Erythematosus: Focus on Immune Response and Therapeutics. Front Immunol. 2020 Oct 30;11:589474. doi: 10.3389/fimmu.2020.589474. PMID: 33193418; PMCID: PMC7661632. 252. Farris, A.D., Guthridge, J.M. Overlapping B cell pathways in severe COVID-19 and lupus. Nat Immunol 21, 1478-1480 (2020). https://doi.org/10.1038/s41590-020-00822-z

253. Najafi, Sahar et al. "The potential similarities of COVID-19 and autoimmune disease pathogenesis and therapeutic options: new insights approach." Clinical rheumatology vol. 39,11 (2020): 3223-3235. doi:10.1007/s10067-020-05376-x 254. https://www.lupusresearch.org/Ira-supported-researcher-finds-similarity-between-covid-19-and-lupus-flares/ 255. Zhou T, Su TT, Mudianto T, Wang J. Immune asynchrony in COVID-19 pathogenesis and potential immunotherapies. J Exp Med. 2020 Oct 5;217(10):e20200674. doi: 10.1084/jem.20200674. PMID: 32910820; PMCID: PMC7481961. 256. 302.

257. Andersson, Ulf \& Ottestad, William \& Tracey, Kevin. (2020). Extracellular HMGB1: A therapeutic target in severe pulmonary inflammation including COVID-19?. Molecular Medicine. 26. 10.1186/s10020-020-00172-4.

258. Land, W.G. Role of DAMPs in respiratory virus-induced acute respiratory distress syndrome-with a preliminary reference to SARS-CoV-2 pneumonia. Genes Immun 22, 141-160 (2021). https://doi.org/10.1038/s41435-021-00140-w 259. Fan, Xing et al. "Changes of Damage Associated Molecular Patterns in COVID-19 Patients." Infectious Diseases \& Immunity vol. 1,1 20-27. 20 Apr. 2021, doi:10.1097/01.ID9.0000733572.40970.6c 260. 257.

261. Yoshihara-Hirata C, Yamashiro K, Yamamoto T, Aoyagi H, Ideguchi H, Kawamura M, Suzuki R, Ono M, Wake H, Nishibori M, Takashiba S. Anti-HMGB1 Neutralizing Antibody Attenuates Periodontal Inflammation and Bone Resorption in a Murine Periodontitis Model. Infect Immun. 2018 Apr 23;86(5):e00111-18. doi: 10.1128/IAI.00111-18. PMID: 29531138; PMCID: PMC5913859. 
262. Vomero M, Barbati C, Colasanti T, Celia Al, Speziali M, Ucci FM, Ciancarella C, Conti F, Alessandri C. Autophagy Modulation in Lymphocytes From COVID-19 Patients: New Therapeutic Target in SARS-COV-2 Infection. Front Pharmacol. 2020 Nov 19;11:569849. doi: 10.3389/fphar.2020.569849. PMID: 33424586; PMCID: PMC7794008.

263. Hui X, Zhang L, Cao L, Huang K, Zhao Y, Zhang Y, Chen X, Lin X, Chen M, Jin M. SARS-CoV-2 promote autophagy to suppress type I interferon response. Signal Transduct Target Ther. 2021 May 8;6(1):180. doi: 10.1038/s41392-021-00574-8. PMID: 33966045; PMCID: PMC8105701.

264. Conti P, Caraffa A, Gallenga CE, Ross R, Kritas SK, Frydas I, Younes A, Ronconi G. Coronavirus-19 (SARS-CoV-2) induces acute severe lung inflammation via IL-1 causing cytokine storm in COVID-19: a promising inhibitory strategy. J Biol Regul Homeost Agents. 2020 Nov-Dec;34(6):1971-1975. doi: 10.23812/20-1-E. PMID: 33016027.

265. Wang H, Yang H, Tracey KJ. Extracellular role of HMGB1 in inflammation and sepsis. J Intern Med. 2004 Mar;255(3):320-31. doi: 10.1111/j.1365-2796.2003.01302.x. PMID: 14871456.

266. Rapoport, Bernardo L et al. "High Mobility Group Box 1 in Human Cancer." Cells vol. 9,7 1664. 10 Jul. 2020, doi:10.3390/cells9071664

267. Tang D, Loze MT, Zeh HJ, Kang R. The redox protein HMGB1 regulates cell death and survival in cancer treatment. Autophagy. 2010 Nov;6(8):1181-3. doi: 10.4161/auto.6.8.13367. PMID: 20861675.

268. Tang, D., Kang, R., Cheh, CW. et al. HMGB1 release and redox regulates autophagy and apoptosis in cancer cells. Oncogene 29, 5299-5310 (2010). https://doi.org/10.1038/onc.2010.261

269. Yu Y, Tang D, Kang R. Oxidative stress-mediated HMGB1 biology. Front Physiol. 2015 Apr 7;6:93. doi:

10.3389/fphys.2015.00093. PMID: 25904867; PMCID: PMC4387954.

270. Julie Gauley \& David S. Pisetsky (2009) The translocation of HMGB1 during cell activation and cell death, Autoimmunity, 42:4, 299-301, DOI: 10.1080/08916930902831522

271. Magna, M., Pisetsky, D.S. The Role of HMGB1 in the Pathogenesis of Inflammatory and Autoimmune Diseases. Mol Med 20, 138-146 (2014). https://doi.org/10.2119/molmed.2013.00164

272. Vande Walle, Lieselotte et al. "HMGB1 release by inflammasomes." Virulence vol. 2,2 (2011): 162-5.

doi:10.4161/viru.2.2.15480

273. Yang H, Wang H, Chavan SS, Andersson U. High Mobility Group Box Protein 1 (HMGB1): The Prototypical Endogenous Danger Molecule. Mol Med. 2015 Oct 27;21 Suppl 1(Suppl 1):S6-S12. doi: 10.2119/molmed.2015.00087. PMID: 26605648; PMCID: PMC4661054.

274. Yang H, Hreggvidsdottir HS, Palmblad K, Wang H, Ochani M, Li J, Lu B, Chavan S, Rosas-Ballina M, Al-Abed Y, Akira S, Bierhaus A, Erlandsson-Harris $\mathrm{H}$, Andersson U, Tracey KJ. A critical cysteine is required for HMGB1 binding to Toll-like receptor 4 and activation of macrophage cytokine release. Proc Natl Acad Sci U S A. 2010 Jun 29;107(26):11942-7. doi:

10.1073/pnas.1003893107. Epub 2010 Jun 14. PMID: $20547845 ;$ PMCID: PMC2900689.

275. Li LC, Gao J, Li J. Emerging role of HMGB1 in fibrotic diseases. J Cell Mol Med. 2014 Dec;18(12):2331-9. doi: 10.1111/jcmm.12419. Epub 2014 Oct 6. PMID: 25284457; PMCID: PMC4302638.

276. Hamada N, Maeyama T, Kawaguchi T, Yoshimi M, Fukumoto J, Yamada M, Yamada S, Kuwano K, Nakanishi Y. The role of high mobility group box1 in pulmonary fibrosis. Am J Respir Cell Mol Biol. 2008 Oct;39(4):440-7. doi: 10.1165/rcmb.2007-03300C. Epub 2008 Apr 25. PMID: 18441281.

277. Morris, Gerwyn et al. "The cytokine storms of COVID-19, H1N1 influenza, CRS and MAS compared. Can one sized treatment fit all?." Cytokine vol. 144 (2021): 155593. doi:10.1016/j.cyto.2021.155593

278. Bolourani S, Brenner M, Wang P. The interplay of DAMPs, TLR4, and proinflammatory cytokines in pulmonary fibrosis. J Mol Med (Berl). 2021 Jul 13:1-12. doi: 10.1007/s00109-021-02113-y. Epub ahead of print. PMID: 34258628; PMCID: PMC8277227.

279. Rojas A, Lindner C, Gonzàlez I, Morales MA. Advanced-glycation end-products axis: A contributor to the risk of severe illness from COVID-19 in diabetes patients. World J Diabetes. 2021 May 15;12(5):590-602. doi: 10.4239/wjd.v12.i5.590. PMID: 33995847; PMCID: PMC8107984.

280. Sundén-Cullberg J, Norrby-Teglund A, Rouhiainen A, Rauvala H, Herman G, Tracey KJ, Lee ML, Andersson J, Tokics L, Treutiger CJ. Persistent elevation of high mobility group box-1 protein (HMGB1) in patients with severe sepsis and septic shock. Crit Care Med. 2005 Mar;33(3):564-73. doi: 10.1097/01.ccm.0000155991.88802.4d. PMID: 15753748.

281. Sama AE, D'Amore J, Ward MF, Chen G, Wang H. Bench to bedside: HMGB1-a novel proinflammatory cytokine and potential therapeutic target for septic patients in the emergency department. Acad Emerg Med. 2004 Aug;11(8):867-73. doi: 10.1197/j.aem.2004.03.011. PMID: 15289194.

282. Yoo H, Im Y, Ko RE, Lee JY, Park J, Jeon K. Association of plasma level of high-mobility group box-1 with necroptosis and sepsis outcomes. Sci Rep. 2021 May 4;11(1):9512. doi: 10.1038/s41598-021-88970-6. PMID: 33947887; PMCID: PMC8097071. 283. Eriksson M. Should high mobility group box-1 protein (HMGB1) be measured in patients with severe sepsis and septic shock? If so, when, where, and how? Crit Care Med. 2005 Mar;33(3):682-3. doi: 10.1097/01.ccm.0000155995.62327.93. PMID: 15753771.

284. Gibot S, Massin F, Cravoisy A, Barraud D, Nace L, Levy B, Bollaert PE. High-mobility group box 1 protein plasma concentrations during septic shock. Intensive Care Med. 2007 Aug;33(8):1347-53. doi: 10.1007/s00134-007-0691-2. Epub 2007 May 25. PMID: 17525840.

285. Czura CJ, Yang H, Amella CA, Tracey KJ. HMGB1 in the immunology of sepsis (not septic shock) and arthritis. Adv Immunol. 2004;84:181-200. doi: 10.1016/S0065-2776(04)84005-7. PMID: 15246253

286. Yang K, Fan M, Wang X, Xu J, Wang Y, Tu F, Gill PS, Ha T, Liu L, Williams DL, Li C. Lactate promotes macrophage HMGB1 lactylation, acetylation, and exosomal release in polymicrobial sepsis. Cell Death Differ. 2021 Aug 6. doi: 10.1038/s41418-02100841-9. Epub ahead of print. PMID: 34363018.

287. Deng C, Zhao L, Yang Z, Shang JJ, Wang CY, Shen MZ, Jiang S, Li T, Di WC, Chen Y, Li H, Cheng YD, Yang Y. Targeting HMGB1 for the treatment of sepsis and sepsis-induced organ injury. Acta Pharmacol Sin. 2021 May 26. doi: 10.1038/s41401-021-00676-7. Epub ahead of print. PMID: 34040166.

288. Peek V, Harden LM, Damm J, Aslani F, Leisengang S, Roth J, Gerstberger R, Meurer M, von Köckritz-Blickwede M, Schulz S, Spengler B, Rummel C. LPS Primes Brain Responsiveness to High Mobility Group Box-1 Protein. Pharmaceuticals (Basel). 2021 Jun 11;14(6):558. doi: 10.3390/ph14060558. PMID: 34208101; PMCID: PMC8230749.

289. Levy RM, Mollen KP, Prince JM, Kaczorowski DJ, Vallabhaneni R, Liu S, Tracey KJ, Lotze MT, Hackam DJ, Fink MP, Vodovotz Y, Billiar TR. Systemic inflammation and remote organ injury following trauma require HMGB1. Am J Physiol Regul Integr Comp Physiol. 2007 Oct;293(4):R1538-44. doi: 10.1152/ajpregu.00272.2007. Epub 2007 Jul 25. PMID: 17652366.

290. Sundnes O, Ottestad W, Schjalm C, Lundbäck P, la Cour Poulsen L, Mollnes TE, Haraldsen G, Eken T. Rapid systemic surge of IL-33 after severe human trauma: a prospective observational study. Mol Med. 2021 Mar 26;27(1):29. doi: 10.1186/s10020-02100288-1. PMID: 33771098; PMCID: PMC8004436. 
291. Xu J, Guardado J, Hoffman R, Xu H, Namas R, Vodovotz Y, Xu L, Ramadan M, Brown J, Turnquist HR, Billiar TR. IL33-mediated ILC2 activation and neutrophil IL5 production in the lung response after severe trauma: A reverse translation study from a human cohort to a mouse trauma model. PLoS Med. 2017 Jul 25;14(7):e1002365. doi: 10.1371/journal.pmed.1002365. PMID: 28742815; PMCID: PMC5526517.

292. Xu H, Xu J, Xu L, Jin S, Turnquist HR, Hoffman R, Loughran P, Billiar TR, Deng M. Interleukin-33 contributes to ILC2 activation and early inflammation-associated lung injury during abdominal sepsis. Immunol Cell Biol. 2018 Oct;96(9):935-947. doi: 10.1111/imcb.12159. Epub 2018 May 17. PMID: 29672927.

293. Zhang K, Jin Y, Lai D, Wang J, Wang Y, Wu X, Scott M, Li Y, Hou J, Billiar T, Wilson M, Shu Q, Fang X, Fan J. RAGE-induced ILC2 expansion in acute lung injury due to haemorrhagic shock. Thorax. 2020 Mar;75(3):209-219. doi: 10.1136/thoraxjnl-2019-213613. Epub 2020 Jan 14. PMID: 31937554; PMCID: PMC7063398.

294. Oczypok EA, Milutinovic PS, Alcorn JF, Khare A, Crum LT, Manni ML, Epperly MW, Pawluk AM, Ray A, Oury TD. Pulmonary receptor for advanced glycation end-products promotes asthma pathogenesis through IL-33 and accumulation of group 2 innate Iymphoid cells. J Allergy Clin Immunol. 2015 Sep;136(3):747-756.e4. doi: 10.1016/j.jaci.2015.03.011. Epub 2015 Apr 28. PMID: 25930197; PMCID: PMC4562894.

295. Xie K, Chen YQ, Chai YS, Lin SH, Wang CJ, Xu F. HMGB1 suppress the expression of IL-35 by regulating Naïve CD4+ T cell differentiation and aggravating Caspase-11-dependent pyroptosis in acute lung injury. Int Immunopharmacol. 2021 Feb;91:107295. doi: 10.1016/j.intimp.2020.107295. Epub 2020 Dec 21. PMID: 33360086.

296. Chai YS, Chen YQ, Lin SH, Xie K, Wang CJ, Yang YZ, Xu F. Curcumin regulates the differentiation of naïve CD4+T cells and activates IL-10 immune modulation against acute lung injury in mice. Biomed Pharmacother. 2020 May;125:109946. doi: 10.1016/j.biopha.2020.109946. Epub 2020 Jan 28. PMID: 32004976.

297. Chen YQ, Chai YS, Xie K, Yu F, Wang CJ, Lin SH, Yang YZ, Xu F. Curcumin Promotes the Expression of IL-35 by Regulating Regulatory T Cell Differentiation and Restrains Uncontrolled Inflammation and Lung Injury in Mice. Inflammation. 2020 Oct;43(5):1913-1924. doi: 10.1007/s10753-020-01265-2. PMID: 32535666.

298. Li K, Yang J, Han X. Ketamine attenuates sepsis-induced acute lung injury via regulation of HMGB1-RAGE pathways. Int Immunopharmacol. 2016 May;34:114-128. doi: 10.1016/j.intimp.2016.01.021. Epub 2016 Mar 2. PMID: 26945830. 299. Zhou M, Fang H, Du M, Li C, Tang R, Liu H, Gao Z, Ji Z, Ke B, Chen XL. The Modulation of Regulatory T Cells via HMGB1/PTEN/ $\beta$-Catenin Axis in LPS Induced Acute Lung Injury. Front Immunol. 2019 Jul 25;10:1612. doi: 10.3389/fimmu.2019.01612. PMID: 31402909; PMCID: PMC6669370.

300. Wang C, Xie K, Li K, Lin S, Xu F. Potential therapeutic effects of interleukin-35 on the differentiation of naïve T cells into Helios+Foxp3+ Tregs in clinical and experimental acute respiratory distress syndrome. Mol Immunol. 2021 Apr;132:236-249. doi: 10.1016/j.molimm.2021.01.009. Epub 2021 Jan 16. PMID: 33494935; PMCID: PMC8058740.

301. Chiappalupi S, Salvadori L, Vukasinovic A, Donato R, Sorci G, Riuzzi F. Targeting RAGE to prevent SARS-CoV-2-mediated multiple organ failure: Hypotheses and perspectives. Life Sci. 2021 May 1;272:119251. doi: 10.1016/j.Ifs.2021.119251. Epub 2021 Feb 23. PMID: 33636175; PMCID: PMC7900755.

302. Chiappalupi S, Salvadori L, Donato R, Riuzzi F, Sorci G. Hyperactivated RAGE in Comorbidities as a Risk Factor for Severe COVID-19-The Role of RAGE-RAS Crosstalk. Biomolecules. 2021 Jun 12;11(6):876. doi: 10.3390/biom11060876. PMID: 34204735; PMCID: PMC8231494.

303. Rojas A, Schneider I, Lindner C, Gonzàlez I, Morales MA. Receptor for advanced glycation end-products axis and coronavirus disease 2019 in inflammatory bowel diseases: A dangerous liaison? World J Gastroenterol. 2021 May 21;27(19):2270-2280. doi: 10.3748/wjg.v27.i19.2270. PMID: 34040321; PMCID: PMC8130044.

304. Roy D, Ramasamy R, Schmidt AM. Journey to a Receptor for Advanced Glycation End Products Connection in Severe Acute Respiratory Syndrome Coronavirus 2 Infection: With Stops Along the Way in the Lung, Heart, Blood Vessels, and Adipose Tissue. Arterioscler Thromb Vasc Biol. 2021 Feb;41(2):614-627. doi: 10.1161/ATVBAHA.120.315527. Epub 2020 Dec 17. PMID: 33327744; PMCID: PMC7837689.

305. Sellegounder D, Zafari P, Rajabinejad M, Taghadosi M, Kapahi P. Advanced glycation end products (AGEs) and its receptor, RAGE, modulate age-dependent COVID-19 morbidity and mortality. A review and hypothesis. Int Immunopharmacol. 2021 May 24;98:107806. doi: 10.1016/j.intimp.2021.107806. Epub ahead of print. PMID: 34352471; PMCID: PMC8141786.

306. Zhong WJ, Duan JX, Liu T, Yang HH, Guan XX, Zhang CY, Yang JT, Xiong JB, Zhou Y, Guan CX, Li Q. Activation of NLRP3 inflammasome up-regulates TREM-1 expression in murine macrophages via HMGB1 and IL-18. Int Immunopharmacol. 2020 Dec;89(Pt A):107045. doi: 10.1016/j.intimp.2020.107045. Epub 2020 Oct 9. PMID: 33045564; PMCID: PMC7545267. 307. Qu M, Zhang H, Chen Z, Sun X, Zhu S, Nan K, Chen W, Miao C. The Role of Ferroptosis in Acute Respiratory Distress Syndrome. Front Med (Lausanne). 2021 May 7;8:651552. doi: 10.3389/fmed.2021.651552. PMID: 34026785; PMCID: PMC8137978.

308. Liu T, Zhou Y, Li P, Duan JX, Liu YP, Sun GY, Wan L, Dong L, Fang X, Jiang JX, Guan CX. Blocking triggering receptor expressed on myeloid cells-1 attenuates lipopolysaccharide-induced acute lung injury via inhibiting NLRP3 inflammasome activation. Sci Rep. 2016 Dec 22;6:39473. doi: 10.1038/srep39473. PMID: 28004759; PMCID: PMC5177963.

309. Yang JW, Mao B, Tao RJ, Fan LC, Lu HW, Ge BX, Xu JF. Corticosteroids alleviate lipopolysaccharide-induced inflammation and lung injury via inhibiting NLRP3-inflammasome activation. J Cell Mol Med. 2020 Nov;24(21):12716-12725. doi: 10.1111/jcmm.15849. Epub 2020 Sep 25. PMID: 32977368; PMCID: PMC7686976.

310. Billiar IM, Guardado J, Abdul-Malak O, Vodovotz Y, Billiar TR, Namas RA. Elevations in Circulating sST2 Levels Are Associated With In-Hospital Mortality and Adverse Clinical Outcomes After Blunt Trauma. J Surg Res. 2019 Dec;244:23-33. doi: 10.1016/j.jss.2019.05.057. Epub 2019 Jul 3. PMID: 31279260; PMCID: PMC6815699.

311. Erenler AK, Baydin A. Interleukin-33 (IL-33) as a Diagnostic and Prognostic Factor in Traumatic Brain Injury. Emerg 312. Prince JM, Levy RM, Yang R, Mollen KP, Fink MP, Vodovotz Y, Billiar TR. Toll-like receptor-4 signaling mediates hepatic injury and systemic inflammation in hemorrhagic shock. J Am Coll Surg. 2006 Mar;202(3):407-17. doi: 10.1016/j.jamcollsurg.2005.11.021. Epub 2006 Jan 18. PMID: 16500244. Med Int. 2020 Jan 10;2020:1832345. doi: 10.1155/2020/1832345. PMID: 32399302; PMCID: PMC7199602.

313. Ghali R, Altara R, Louch WE, Cataliotti A, Mallat Z, Kaplan A, Zouein FA, Booz GW. IL-33 (Interleukin 33)/sST2 Axis in Hypertension and Heart Failure. Hypertension. 2018 Oct;72(4):818-828. doi: 10.1161/HYPERTENSIONAHA.118.11157. Erratum in: Hypertension. 2019 Mar;73(3):e12. PMID: 30354724

314. Gebhard F, Pfetsch H, Steinbach G, Strecker W, Kinzl L, Brückner UB. Is interleukin 6 an early marker of injury severity following major trauma in humans? Arch Surg. 2000 Mar;135(3):291-5. doi: 10.1001/archsurg.135.3.291. PMID: 10722030. 315. Levy RM, Prince JM, Yang R, Mollen KP, Liao H, Watson GA, Fink MP, Vodovotz Y, Billiar TR. Systemic inflammation and remote organ damage following bilateral femur fracture requires Toll-like receptor 4. Am J Physiol Regul Integr Comp Physiol. 2006 Oct;291(4):R970-6. doi: 10.1152/ajpregu.00793.2005. Epub 2006 May 4. PMID: 16675630. 
316. Wang J, He GZ, Wang YK, Zhu QK, Chen W, Guo T. TLR4-HMGB1-, MyD88- and TRIF-dependent signaling in mouse intestinal ischemia/reperfusion injury. World J Gastroenterol. 2015 Jul 21;21(27):8314-25. doi: 10.3748/wjg.v21.i27.8314. PMID: 26217083; PMCID: PMC4507101.

317. Dehbi M, Uzzaman T, Baturcam E, Eldali A, Ventura W, Bouchama A. Toll-like receptor 4 and high-mobility group box 1 are critical mediators of tissue injury and survival in a mouse model for heatstroke. PLoS One. 2012;7(9):e44100. doi: 10.1371/journal.pone.0044100. Epub 2012 Sep 4. PMID: $22962600 ;$ PMCID: PMC3433483.

318. Faraco G, Fossati S, Bianchi ME, Patrone M, Pedrazzi M, Sparatore B, Moroni F, Chiarugi A. High mobility group box 1 protein is released by neural cells upon different stresses and worsens ischemic neurodegeneration in vitro and in vivo. J Neurochem. 2007 Oct;103(2):590-603. doi: 10.1111/j.1471-4159.2007.04788.x. Epub 2007 Jul 31. PMID: 17666052.

319. Abraham E, Arcaroli J, Carmody A, Wang H, Tracey KJ. HMG-1 as a mediator of acute lung inflammation. J Immunol. 2000 Sep 15;165(6):2950-4. doi: 10.4049/jimmunol.165.6.2950. PMID: 10975801.

320. Zhou M, Zhang Y, Tang R, Liu H, Du M, Gao Z, Ji Z, Fang H. HMGB1/TLR4 Signaling Affects Regulatory T Cells in Acute Lung Injury. J Inflamm Res. 2021 Apr 19;14:1551-1561. doi: 10.2147/JIR.S302967. PMID: 33907436; PMCID: PMC8064684.

321. Wang H, Bloom O, Zhang M, Vishnubhakat JM, Ombrellino M, Che J, Frazier A, Yang H, Ivanova S, Borovikova L, Manogue KR, Faist E, Abraham E, Andersson J, Andersson U, Molina PE, Abumrad NN, Sama A, Tracey KJ. HMG-1 as a late mediator of endotoxin lethality in mice. Science. 1999 Jul 9;285(5425):248-51. doi: 10.1126/science.285.5425.248. PMID: 10398600. 322. Andersson U, Wang H, Palmblad K, Aveberger AC, Bloom O, Erlandsson-Harris H, Janson A, Kokkola R, Zhang M, Yang $H$, Tracey KJ. High mobility group 1 protein (HMG-1) stimulates proinflammatory cytokine synthesis in human monocytes. J Exp Med. 2000 Aug 21;192(4):565-70. doi: 10.1084/jem.192.4.565. PMID: 10952726; PMCID: PMC2193240.

323. Yang H, Wang H, Tracey KJ. HMG-1 rediscovered as a cytokine. Shock. 2001 Apr;15(4):247-53. doi: 10.1097/00024382200115040-00001. PMID: 11303722.

324. Lutz W, Stetkiewicz J. High mobility group box 1 protein as a late-acting mediator of acute lung inflammation. Int J Occup Med Environ Health. 2004;17(2):245-54. PMID: 15387080.

325. Taniguchi N, Kawahara K, Yone K, Hashiguchi T, Yamakuchi M, Goto M, Inoue K, Yamada S, Ijiri K, Matsunaga S, Nakajima T, Komiya S, Maruyama I. High mobility group box chromosomal protein 1 plays a role in the pathogenesis of rheumatoid arthritis as a novel cytokine. Arthritis Rheum. 2003 Apr;48(4):971-81. doi: 10.1002/art.10859. PMID: 12687539.

326. Sundberg E, Fasth AE, Palmblad K, Harris HE, Andersson U. High mobility group box chromosomal protein 1 acts as a proliferation signal for activated T lymphocytes. Immunobiology. 2009;214(4):303-9. doi: 10.1016/j.imbio.2008.09.006. Epub 2008 Oct 25. PMID: 19201506

327. Lamkanfi M, Sarkar A, Vande Walle L, Vitari AC, Amer AO, Wewers MD, Tracey KJ, Kanneganti TD, Dixit VM. Inflammasomedependent release of the alarmin HMGB1 in endotoxemia. J Immunol. 2010 Oct 1;185(7):4385-92. doi:

10.4049/jimmunol.1000803. Epub 2010 Aug 27. PMID: 20802146; PMCID: PMC3428148.

328. Scaffidi P, Misteli T, Bianchi ME. Release of chromatin protein HMGB1 by necrotic cells triggers inflammation. Nature. 2002 Jul 11;418(6894):191-5. doi: 10.1038/nature00858. Erratum in: Nature. 2010 Sep 30;467(7315):622. PMID: 12110890.

329. Rovere-Querini P, Capobianco A, Scaffidi P, Valentinis B, Catalanotti F, Giazzon M, Dumitriu IE, Müller S, lannacone M,

Traversari C, Bianchi ME, Manfredi AA. HMGB1 is an endogenous immune adjuvant released by necrotic cells. EMBO Rep. 2004 Aug;5(8):825-30. doi: 10.1038/sj.embor.7400205. Epub 2004 Jul 23. PMID: 15272298; PMCID: PMC1299116.

330. Ge Y, Huang M, Yao YM. The Effect and Regulatory Mechanism of High Mobility Group Box-1 Protein on Immune Cells in Inflammatory Diseases. Cells. 2021 Apr 28;10(5):1044. doi: 10.3390/cells10051044. PMID: 33925132; PMCID: PMC8145631. 331. Hosakote YM, Brasier AR, Casola A, Garofalo RP, Kurosky A. Respiratory Syncytial Virus Infection Triggers Epithelial HMGB1 Release as a Damage-Associated Molecular Pattern Promoting a Monocytic Inflammatory Response. J Virol. 2016 Oct 14;90(21):9618-9631. doi: 10.1128/JVI.01279-16. PMID: 27535058; PMCID: PMC5068515.

332. Xu J, Jiang Y, Wang J, Shi X, Liu Q, Liu Z, Li Y, Scott MJ, Xiao G, Li S, Fan L, Billiar TR, Wilson MA, Fan J. Macrophage endocytosis of high-mobility group box 1 triggers pyroptosis. Cell Death Differ. 2014 Aug;21(8):1229-39. doi:

10.1038/cdd.2014.40. Epub 2014 Apr 25. PMID: 24769733; PMCID: PMC4085529.

333. Meihong Deng, Yiting Tang, Wenbo Li, Xiangyu Wang, et.al The Endotoxin Delivery Protein HMGB1 Mediates Caspase-11Dependent Lethality in Sepsis, Immunity,2018,https://doi.org/10.1016/j.immuni.2018.08.016.

334. Bell CW, Jiang W, Reich CF 3rd, Pisetsky DS. The extracellular release of HMGB1 during apoptotic cell death. Am J Physiol Cell Physiol. 2006 Dec;291(6):C1318-25. doi: 10.1152/ajpcell.00616.2005. Epub 2006 Jul 19. PMID: 16855214.

335. Donlan, Alexandra N et al. "IL-13 is a driver of COVID-19 severity." medRxiv : the preprint server for health sciences 2020.06.18.20134353. 1 Mar. 2021, doi:10.1101/2020.06.18.20134353. Preprint.

336. Vaz de Paula, C.B., de Azevedo, M.L.V., Nagashima, S. et al. IL-4/IL-13 remodeling pathway of COVID-19 lung injury. Sci Rep 10, 18689 (2020). https://doi.org/10.1038/s41598-020-75659-5

337. de la Rica R, Borges M, Gonzalez-Freire M. COVID-19: In the Eye of the Cytokine Storm. Front Immunol. 2020 Sep

24;11:558898. doi: 10.3389/fimmu.2020.558898. PMID: 33072097; PMCID: PMC7541915.

338. Arcavi L, Benowitz NL. Cigarette smoking and infection. Arch Intern Med. 2004 Nov 8;164(20):2206-16. doi: 10.1001/archinte.164.20.2206. PMID: 15534156.

339. Tollerud DJ, Clark JW, Brown LM, et al. The effects of cigarette smoking on T cell subsets: a population-based survey of healthy caucasians. Am Rev Respir Dis. 1989;139:1446-1451.

340. Hughes DA, Haslam PL, Townsend PJ, Turner-Warwick M. Numerical and functional alterations in circulatory lymphocytes in cigarette smokers. Clin Exp Immunol. 1985;61:459-466

341. Ferson M, Edwards A, Lind A, Milton GW, Hersey P. Low natural killer-cell activity and immunoglobulin levels associated with smoking in human subjects. Int J Cancer. 1979;23:603-609.

342. Nair MP, Kronfol ZA, Schwartz SA. Effects of alcohol and nicotine on cytotoxic functions of human lymphocytes. Clin Immunol Immunopathol. 1990;54:395-409

343. Tollerud DJ, Clark JW, Brown LM, et al. Association of cigarette smoking with decreased numbers of circulating natural killer cells. Am Rev Respir Dis. 1989;139:194-198

344. Miller LG, Goldstein G, Murphy M, Ginns LC. Reversible alterations in immunoregulatory T cells in smoking: analysis by monoclonal antibodies and flow cytometry. Chest. 1982;82:526-529

345. Hersey P, Prendergast D, Edwards A. Effects of cigarette smoking on the immune system: follow-up studies in normal subjects after cessation of smoking. Med J Aust. 1983;2:425-429.

346. Silverman NA, Potvin C, Alexander JC Jr, Chretien PB. In vitro lymphocyte reactivity and T-cell levels in chronic cigarette smokers. Clin Exp Immunol. 1975;22:285-292.

347. Herberman RB, Holden HT. Natural cell mediated immunity. Adv Cancer Res. 1978; 27:305-377.

348. Herberman RB. Natural Cell-Mediated Immunity Against Tumors. New York, NY: Academic Press; 1980 349. L 
350. Chorny A, Delgado M. Neuropeptides rescue mice from lethal sepsis by down-regulating secretion of the late-acting inflammatory mediator high mobility group box 1. Am J Pathol. 2008 May;172(5):1297-307. doi: 10.2353/ajpath.2008.070969. Epub 2008 Apr 1. PMID: 18385521; PMCID: PMC2329838.

351. Nosaka, Nobuyuki et al. "Anti-high mobility group box-1 monoclonal antibody treatment provides protection against influenza A virus (H1N1)-induced pneumonia in mice." Critical care (London, England) vol. 19,1 249. 11 Jun. 2015, doi:10.1186/s13054-015-0983-9

352. Hou XQ, Qin JL, Zheng XX, Wang L, Yang ST, Gao YW, Xia XZ. Potential role of high-mobility group box 1 protein in the pathogenesis of influenza H5N1 virus infection. Acta Virol. 2014;58(1):69-75. doi: 10.4149/av_2014_01_69. PMID: 24717031. 353. Hatayama K, Nosaka N, Yamada M, Yashiro M, Fujii Y, Tsukahara H, Liu K, Nishibori M, Matsukawa A, Morishima T. Combined effect of anti-high-mobility group box-1 monoclonal antibody and peramivir against influenza $A$ virus-induced pneumonia in mice. J Med Virol. 2019 Mar;91(3):361-369. doi: 10.1002/jmv.25330. Epub 2018 Oct 22. PMID: 30281823. 354. N 351

355. Honore PM, Jacobs R, Hendrickx I, De Waele E, Van Gorp V, Spapen HD. To counteract or to clear high-mobility group box-1 protein in influenza A (H1N1) infection? That may become the question. Crit Care. 2015 Nov 23;19:401. doi: 10.1186/s13054-0151126-z. PMID: 26594030; PMCID: PMC4655500.

356. Yashiro M, Tsukahara H, Matsukawa A, Yamada M, Fujii Y, Nagaoka Y, Tsuge M, Yamashita N, Ito T, Yamada M, Masutani H, Yodoi J, Morishima T. Redox-active protein thioredoxin-1 administration ameliorates influenza A virus (H1N1)-induced acute lung injury in mice. Crit Care Med. 2013 Jan;41(1):171-81. doi: 10.1097/CCM.0b013e3182676352. PMID: 23222257.

357. Bantia S, Kellogg D, Parker C, Upshaw R, llyushina NA, Babu YS. A single intramuscular injection of neuraminidase inhibitor peramivir demonstrates antiviral activity against novel pandemic A/California/04/2009 (H1N1) influenza virus infection in mice. Antiviral Res. 2011 Apr;90(1):17-21. doi: 10.1016/j.antiviral.2011.02.001. Epub 2011 Feb 21. PMID: 21316393.

358. Tanaka A, Nakamura S, Seki M, Iwanaga N, Kajihara T, Kitano M, Homma T, Kurihara S, Imamura Y, Miyazaki T, Izumikawa K, Kakeya H, Yanagihara K, Kohno S. The effect of intravenous peramivir, compared with oral oseltamivir, on the outcome of postinfluenza pneumococcal pneumonia in mice. Antivir Ther. 2015;20(1):11-9. doi: 10.3851/IMP2744. Epub 2014 Feb 12. PMID: 24517996.

359. Sidwell RW, Smee DF. Peramivir (BCX-1812, RWJ-270201): potential new therapy for influenza. Expert Opin Investig Drugs. 2002 Jun;11(6):859-69. doi: 10.1517/13543784.11.6.859. PMID: 12036429.

360. Mancuso CE, Gabay MP, Steinke LM, Vanosdol SJ. Peramivir: an intravenous neuraminidase inhibitor for the treatment of 2009 H1N1 influenza. Ann Pharmacother. 2010 Jul-Aug;44(7-8):1240-9. doi: 10.1345/aph.1P031. Epub 2010 Jun 1. PMID: 20516360.

361. Prantner D, Nallar S, Vogel SN. The role of RAGE in host pathology and crosstalk between RAGE and TLR4 in innate immune signal transduction pathways. FASEB J. 2020 Dec;34(12):15659-15674. doi: 10.1096/fj.202002136R. Epub 2020 Nov 1. PMID: 33131091; PMCID: PMC8121140.

362. L (258)

363. Li Y, Wang S, Liu J, Li X, Lu M, Wang X, Ren Y, Li X, Xiang M. Induced Pluripotent Stem Cells Attenuate Acute Lung Injury Induced by Ischemia Reperfusion via Suppressing the High Mobility Group Box-1. Dose Response. 2020 Oct 30;18(4):1559325820969340. doi: 10.1177/1559325820969340. PMID: 33192202; PMCID: PMC7607776.

364. Lee J, Park JH, Jwa H, Kim YH. Comparison of Efficacy of Intravenous Peramivir and Oral Oseltamivir for the Treatment of Influenza: Systematic Review and Meta-Analysis. Yonsei Med J. 2017 Jul;58(4):778-785. doi: 10.3349/ymj.2017.58.4.778. PMID: 28540991; PMCID: PMC5447109.

365. Kohno S, Yen MY, Cheong HJ, Hirotsu N, Ishida T, Kadota J, Mizuguchi M, Kida H, Shimada J; S-021812 Clinical Study Group. Phase III randomized, double-blind study comparing single-dose intravenous peramivir with oral oseltamivir in patients with seasonal influenza virus infection. Antimicrob Agents Chemother. 2011 Nov;55(11):5267-76. doi: 10.1128/AAC.00360-11. Epub 2011 Aug 8. PMID: 21825298; PMCID: PMC3195028.

366. Yoo JW, Choi SH, Huh JW, Lim CM, Koh Y, Hong SB. Peramivir is as effective as oral oseltamivir in the treatment of severe seasonal influenza. J Med Virol. 2015 Oct;87(10):1649-55. doi: 10.1002/jmv.24232. Epub 2015 May 6. PMID: 25946636.

367. Rosenberg J, Huang J. CD8+ T Cells and NK Cells: Parallel and Complementary Soldiers of Immunotherapy. Curr Opin Chem Eng. 2018;19:9-20. doi:10.1016/j.coche.2017.11.006

368. Ray A, Chakraborty K, Ray P. Immunosuppressive MDSCs induced by TLR signaling during infection and role in resolution of inflammation. Front Cell Infect Microbiol. 2013 Sep 18;3:52. doi: 10.3389/fcimb.2013.00052. PMID: 24066282; PMCID: PMC3776133.

369. De Santo, C., Salio, M., Masri, S. H., Lee, L. Y., Dong, T., Speak, A. O., et al. (2008). Invariant NKT cells reduce the immunosuppressive activity of influenza A virus induced myeloid-derived suppressor cells in mice and humans. J. Clin. Invest. 118, 4036-4048. doi: 10.1172/JCI36264

370. Stegemann-Koniszewski S, Behrens S, Boehme JD, Hochnadel I, Riese P, Guzmán CA, Kröger A, Schreiber J, Gunzer M, Bruder D. Respiratory Influenza A Virus Infection Triggers Local and Systemic Natural Killer Cell Activation via Toll-Like Receptor 7. Front Immunol. 2018 Feb 13;9:245. doi: 10.3389/fimmu.2018.00245. PMID: 29497422; PMCID: PMC5819576.

371. Frank K, Paust S. Dynamic Natural Killer Cell and T Cell Responses to Influenza Infection. Front Cell Infect Microbiol. 2020 Aug 18;10:425. doi: 10.3389/fcimb.2020.00425. PMID: 32974217; PMCID: PMC7461885.

372. Kandasamy M, Furlong K, Perez JT, Manicassamy S, Manicassamy B. Suppression of Cytotoxic T Cell Functions and Decreased Levels of Tissue-Resident Memory T Cells during H5N1 Infection. J Virol. 2020 Apr 16;94(9):e00057-20. doi: 10.1128/JVI.00057-20. PMID: 32075925; PMCID: PMC7163117.

373. Hatta Y, Hershberger K, Shinya K, Proll SC, Dubielzig RR, Hatta M, Katze MG, Kawaoka Y, Suresh M. Viral replication rate regulates clinical outcome and CD8 T cell responses during highly pathogenic H5N1 influenza virus infection in mice. PLoS Pathog. 2010 Oct 7;6(10):e1001139. doi: 10.1371/journal.ppat.1001139. PMID: 20949022; PMCID: PMC2951384.

374. Lupfer CR, Stokes KL, Kuriakose T, Kanneganti TD. Deficiency of the NOD-Like Receptor NLRC5 Results in Decreased CD8+ T Cell Function and Impaired Viral Clearance. J Virol. 2017 Aug 10;91(17):e00377-17. doi: 10.1128/JVI.00377-17. PMID: 28615208; PMCID: PMC5553185.

375. Sun T, Ferrero RL, Girardin SE, Gommerman JL, Philpott DJ. NLRC5 deficiency has a moderate impact on immunodominant CD8+ T-cell responses during rotavirus infection of adult mice. Immunol Cell Biol. 2019 Jul;97(6):552-562. doi: 10.1111/imcb.12244. Epub 2019 Mar 29. PMID: 30768806.

376. Chelbi ST, Dang AT, Guarda G. Emerging Major Histocompatibility Complex Class I-Related Functions of NLRC5. Adv Immunol. 2017;133:89-119. doi: 10.1016/bs.ai.2016.11.003. Epub 2017 Jan 3. PMID: 28215281.

377. Lupfer C, Thomas PG, Kanneganti TD. Nucleotide oligomerization and binding domain 2-dependent dendritic cell activation is necessary for innate immunity and optimal CD8+ T Cell responses to influenza A virus infection. J Virol. 2014 Aug;88(16):8946-55. doi: 10.1128/JVI.01110-14. Epub 2014 May 28. PMID: 24872587; PMCID: PMC4136245. 
378. Yao $Y$, Wang $Y$, Chen F, Huang Y, Zhu S, Leng $Q$, Wang H, Shi Y, Qian Y. NLRC5 regulates MHC class I antigen presentation in host defense against intracellular pathogens. Cell Res. 2012 May;22(5):836-47. doi: 10.1038/cr.2012.56. Epub 2012 Apr 10. PMID: 22491475; PMCID: PMC3346158.

379. Littera R, Chessa L, Deidda S, Angioni G, Campagna M, Lai S, Melis M, Cipri S, Firinu D, Santus S, Lai A, Porcella R, Rassu S, Meloni F, Schirru D, Cordeddu W, Kowalik MA, Ragatzu P, Vacca M, Cannas F, Alba F, Carta MG, Del Giacco S, Restivo A, Deidda S, Palimodde A, Congera P, Perra R, Orrù G, Pes F, Loi M, Murru C, Urru E, Onali S, Coghe F, Giglio S, Perra A. Natural killer-cell immunoglobulin-like receptors trigger differences in immune response to SARS-CoV-2 infection. PLoS One. 2021 Aug 5;16(8):e0255608. doi: 10.1371/journal.pone.0255608. PMID: 34352002; PMCID: PMC8341547.

380. Hart OM, Athie-Morales V, O'Connor GM, Gardiner CM. TLR7/8-mediated activation of human NK cells results in accessory cell-dependent IFN-gamma production. J Immunol. 2005 Aug 1;175(3):1636-42. doi: 10.4049/jimmunol.175.3.1636. PMID: 16034103.

381. Girart MV, Fuertes MB, Domaica Cl, Rossi LE, Zwirner NW. Engagement of TLR3, TLR7, and NKG2D regulate IFN-gamma secretion but not NKG2D-mediated cytotoxicity by human NK cells stimulated with suboptimal doses of IL-12. J Immunol. 2007 Sep 15;179(6):3472-9. doi:10.4049/jimmunol.179.6.3472. PMID: 17804388.

382. Schmidt KN, Leung B, Kwong M, Zarember KA, Satyal S, Navas TA, Wang F, Godowski PJ. APC-independent activation of NK cells by the Toll-like receptor 3 agonist double-stranded RNA. J Immunol. 2004 Jan 1;172(1):138-43. doi:

10.4049/jimmunol.172.1.138. PMID: 14688319.

383. Dong Z, Tian Z. APC-independent NK cell activation. J Immunol. 2005 Aug 15;175(4):2041; author reply 2041. doi: 10.4049/jimmunol.175.4.2041. PMID: 16081766.

384. Lieberman LA, Hunter CA. Regulatory pathways involved in the infection-induced production of IFN-gamma by NK cells. Microbes Infect. 2002 Dec;4(15):1531-8. doi: 10.1016/s1286-4579(02)00036-9. PMID: 12505525.

385. Stegemann-Koniszewski S, Gereke M, Orrskog S, Lienenklaus S, Pasche B, Bader SR, Gruber AD, Akira S, Weiss S, HenriquesNormark B, Bruder D, Gunzer M. TLR7 contributes to the rapid progression but not to the overall fatal outcome of secondary pneumococcal disease following influenza A virus infection. J Innate Immun. 2013;5(1):84-96. doi: 10.1159/000345112. Epub 2012 Nov 15. PMID: 23154432; PMCID: PMC6741512.

386. Ray P, Arora M, Poe SL, Ray A. Lung myeloid-derived suppressor cells and regulation of inflammation. Immunol Res. 2011 Aug;50(2-3):153-8. doi: 10.1007/s12026-011-8230-1. PMID: 21717065.

387. Ahmed, Faria et al. "Can Natural Killer Cells Be a Principal Player in Anti-SARS-CoV-2 Immunity?." Frontiers in immunology vol. 11 586765. 7 Dec. 2020, doi:10.3389/fimmu.2020.586765

388. Vietzen, H., Zoufaly, A., Traugott, M. et al. Deletion of the NKG2C receptor encoding KLRC2 gene and HLA-E variants are risk factors for severe COVID-19. Genet Med 23, 963-967 (2021). https://doi.org/10.1038/s41436-020-01077-7

389. van Eeden, Charmaine et al. "Natural Killer Cell Dysfunction and Its Role in COVID-19." International journal of molecular sciences vol. 21,17 6351. 1 Sep. 2020, doi:10.3390/ijms21176351

390. Market M, Angka L, Martel AB, Bastin D, Olanubi O, Tennakoon G, Boucher DM, Ng J, Ardolino M, Auer RC. Flattening the COVID-19 Curve With Natural Killer Cell Based Immunotherapies. Front Immunol. 2020 Jun 23;11:1512. doi:

10.3389/fimmu.2020.01512. PMID: 32655581; PMCID: PMC7324763.

391. Manickam C, Sugawara S, Reeves RK. Friends or foes? The knowns and unknowns of natural killer cell biology in COVID-19 and other coronaviruses in July 2020. PLoS Pathog. 2020 Aug 26;16(8):e1008820. doi:10.1371/journal.ppat.1008820. PMID: 32845937; PMCID: PMC7449465.

392. Bortolotti D, Gentili V, Rizzo S, Rotola A, Rizzo R. SARS-CoV-2 Spike 1 Protein Controls Natural Killer Cell Activation via the HLA-E/NKG2A Pathway. Cells. 2020 Aug 26;9(9):1975. doi: 10.3390/cells9091975. PMID: 32859121; PMCID: PMC7563485.

393. Jeyaraman, Madhan et al. "Bracing NK cell based therapy to relegate pulmonary inflammation in COVID-19." Heliyon vol. 7,7 (2021): e07635. doi:10.1016/j.heliyon.2021.e07635

394. Masselli E, Vaccarezza M, Carubbi C, Pozzi G, Presta V, Mirandola P, Vitale M. NK cells: A double edge sword against SARSCoV-2. Adv Biol Regul. 2020 Aug;77:100737. doi: 10.1016/j.jbior.2020.100737. Epub 2020 Jun 13. PMID: 32773100; PMCID: PMC7292949.

395. Varchetta, S., Mele, D., Oliviero, B. et al. Unique immunological profile in patients with COVID-19. Cell Mol Immunol $18,604-$ 612 (2021). https://doi.org/10.1038/s41423-020-00557-9

396. Bao, C., Tao, X., Cui, W. et al. Natural killer cells associated with SARS-CoV-2 viral RNA shedding, antibody response and mortality in COVID-19 patients. Exp Hematol Oncol 10, 5 (2021). https://doi.org/10.1186/s40164-021-00199-1 397. Niklas K Björkström, Andrea Ponzetta,Natural killer cells and unconventional T cells in COVID-19, Current Opinion in Virology,2021, https://doi.org/10.1016/j.coviro.2021.06.005.

398. Osman M, Faridi RM, Sligl W, Shabani-Rad MT, Dharmani-Khan P, Parker A, Kalra A, Tripathi MB, Storek J, Cohen Tervaert JW, Khan FM. Impaired natural killer cell counts and cytolytic activity in patients with severe COVID-19. Blood Adv. 2020 Oct 27;4(20):5035-5039. doi:10.1182/bloodadvances.2020002650. PMID: 33075136; PMCID:PMC7594380.

399. Maucourant C, Filipovic I, Ponzetta A, Aleman S, Cornillet M, Hertwig L, Strunz B, Lentini A, Reinius B, Brownlie D, Cuapio A, Ask EH, Hull RM, Haroun-Izquierdo A, Schaffer M, Klingström J, Folkesson E, Buggert M, Sandberg JK, Eriksson LI, Rooyackers O, Ljunggren HG, Malmberg KJ, Michaëlsson J, Marquardt N, Hammer Q, Strålin K, Björkström NK; Karolinska COVID-19 Study Group. Natural killer cell immunotypes related to COVID-19 disease severity. Sci Immunol. 2020 Aug 21;5(50):eabd6832. doi: 10.1126/sciimmunol.abd6832. PMID: 32826343; PMCID: PMC7665314.

400. Magna M, Pisetsky DS. The role of HMGB1 in the pathogenesis of inflammatory and autoimmune diseases. Mol Med. 2014 Mar 24;20(1):138-46. doi: 10.2119/molmed.2013.00164. PMID: 24531836; PMCID: PMC3966993.

401. Li G, Liang X, Lotze MT. HMGB1: The Central Cytokine for All Lymphoid Cells. Front Immunol. 2013 Mar 20;4:68. doi: 10.3389/fimmu.2013.00068. PMID: 23519706; PMCID: PMC3602962.

402. Andersson U, Tracey KJ. HMGB1 is a therapeutic target for sterile inflammation and infection. Annu Rev Immunol. 2011;29:139-62. doi: 10.1146/annurev-immunol-030409-101323. PMID: 21219181; PMCID: PMC4536551.

403. Klune JR, Dhupar R, Cardinal J, Billiar TR, Tsung A. HMGB1: endogenous danger signaling. Mol Med. 2008 Jul-Aug;14(78):476-84. doi: 10.2119/2008-00034.Klune. PMID: 18431461; PMCID: PMC2323334.

404. Liu Y, Zhuang GB, Zhou XZ. HMBG1 as a Driver of Inflammatory and Immune Processes in the Pathogenesis of Ocular Diseases. J Ophthalmol. 2018 Oct 24;2018:5195290. doi: 10.1155/2018/5195290. PMID: 30473885; PMCID: PMC6220384. 405. Sims GP, Rowe DC, Rietdijk ST, Herbst R, Coyle AJ. HMGB1 and RAGE in inflammation and cancer. Annu Rev Immunol. 2010;28:367-88. doi: 10.1146/annurev.immunol.021908.132603. PMID: 20192808.

406. Tang, Daolin et al. "High-mobility group box 1 and cancer." Biochimica et biophysica acta vol. 1799,1-2 (2010): 131-40. doi:10.1016/j.bbagrm.2009.11.014

407. Yang H, Wang H, Andersson U. Targeting Inflammation Driven by HMGB1. Front Immunol. 2020 Mar 20;11:484. doi: 10.3389/fimmu.2020.00484. PMID: 32265930; PMCID: PMC7099994. 
408. Harris HE, Andersson U, Pisetsky DS. HMGB1: a multifunctional alarmin driving autoimmune and inflammatory disease. Nat Rev Rheumatol. 2012 Jan 31;8(4):195-202. doi: 10.1038/nrrheum.2011.222. PMID: 22293756.

409. Lundbäck, Peter \& Klevenvall, Lena \& Ottosson, L. \& Schierbeck, Hanna \& Palmblad, Karin \& Andersson, U. \& Harris, Helena. (2012). Anti HMGB1 treatment reduces inflammation in models of experimental autoimmunity. Annals of the Rheumatic Diseases. 71. A79-A80. 10.1136/annrheumdis-2011-201238.18.

410. Tsung A, Tohme S, Billiar TR. High-mobility group box-1 in sterile inflammation. J Intern Med. 2014 Nov;276(5):425-43. doi: 10.1111/joim.12276. PMID: 24935761.

411. Venereau E, Schiraldi M, Uguccioni M, Bianchi ME. HMGB1 and leukocyte migration during trauma and sterile inflammation. Mol Immunol. 2013 Aug;55(1):76-82. doi: 10.1016/j.molimm.2012.10.037. Epub 2012 Dec 1. PMID: 23207101.

412. Yang H, Wang H, Andersson U. Targeting Inflammation Driven by HMGB1. Front Immunol. 2020 Mar 20;11:484. doi: 10.3389/fimmu.2020.00484. PMID: 32265930; PMCID: PMC7099994.

413. Yang H, Wang H, Ju Z, Ragab AA, Lundbäck P, Long W, Valdes-Ferrer SI, He M, Pribis JP, Li J, Lu B, Gero D, Szabo C, Antoine DJ, Harris HE, Golenbock DT, Meng J, Roth J, Chavan SS, Andersson U, Billiar TR, Tracey KJ, Al-Abed Y. MD-2 is required for disulfide HMGB1-dependent TLR4 signaling. J Exp Med. 2015 Jan 12;212(1):5-14. doi: 10.1084/jem.20141318. Epub 2015 Jan 5. PMID: 25559892; PMCID: PMC4291531.

414. Sun S, He M, Wang Y, Yang H, Al-Abed Y. Folic acid derived-P5779 mimetics regulate DAMP-mediated inflammation through disruption of HMGB1:TLR4:MD-2 axes. PLoS One. 2018 Feb 15;13(2):e0193028. doi: 10.1371/journal.pone.0193028. PMID: $29447234 ;$ PMCID: PMC5814057.

415. Cai J, Yuan H, Wang Q, Yang H, Al-Abed Y, Hua Z, Wang J, Chen D, Wu J, Lu B, Pribis JP, Jiang W, Yang K, Hackam DJ, Tracey KJ, Billiar TR, Chen AF. HMGB1-Driven Inflammation and Intimal Hyperplasia After Arterial Injury Involves Cell-Specific Actions Mediated by TLR4. Arterioscler Thromb Vasc Biol. 2015 Dec;35(12):2579-93. doi: 10.1161/ATVBAHA.115.305789. Epub 2015 Oct 29. PMID: 26515416; PMCID: PMC4880018.

416. Tachibana M. [The Immunosuppressive Function of Myeloid-derived Suppressor Cells Is Regulated by the HMGB1-TLR4 Axis]. Yakugaku Zasshi. 2018;138(2):143-148. Japanese. doi: 10.1248/ yakushi.17-00158. PMID: 29386427.

417. Su Z, Zhang Y, Ni P, Wang J. [Recombinant HMGB1 induces the differentiation of mouse myeloid cells into myeloid-derived suppressor cells in vitro]. Xi Bao Yu Fen Zi Mian Yi Xue Za Zhi. 2016 Oct;32(10):1362-1365. Chinese. PMID: 27667463.

418. Parker KH, Sinha P, Horn LA, Clements VK, Yang H, Li J, Tracey KJ, Ostrand-Rosenberg S. HMGB1 enhances immune suppression by facilitating the differentiation and suppressive activity of myeloid-derived suppressor cells. Cancer Res. 2014 Oct 15;74(20):5723-33. doi: 10.1158/0008-5472.CAN-13-2347. Epub 2014 Aug 27. PMID: 25164013; PMCID: PMC4199911.

419. Liu Z, Falo LD Jr, You Z. Knockdown of HMGB1 in tumor cells attenuates their ability to induce regulatory T cells and uncovers naturally acquired CD8 T cell-dependent antitumor immunity. J Immunol. 2011 Jul 1;187(1):118-25. doi:

10.4049/jimmunol.1003378. Epub 2011 Jun 3. PMID: 21642542; PMCID: PMC3119781.

420. Nam S, Kang K, Cha JS, Kim JW, Lee HG, Kim Y, Yang Y, Lee MS, Lim JS. Interferon regulatory factor 4 (IRF4) controls myeloidderived suppressor cell (MDSC) differentiation and function. J Leukoc Biol. 2016 Dec;100(6):1273-1284. doi: 10.1189/jlb.1A0215068RR. Epub 2016 Sep 6. PMID: 27601624.

421. Hu X, Li B, Li X, Zhao X, Wan L, Lin G, Yu M, Wang J, Jiang X, Feng W, Qin Z, Yin B, Li Z. Transmembrane TNF- $\alpha$ promotes suppressive activities of myeloid-derived suppressor cells via TNFR2. J Immunol. 2014 Feb 1;192(3):1320-31. doi: 10.4049/jimmunol.1203195. Epub 2013 Dec 30. PMID: 24379122.

422. Kapor S, Santibanez JF. Myeloid-Derived Suppressor Cells and Mesenchymal Stem/Stromal Cells in Myeloid Malignancies. J Clin Med. 2021 Jun 24;10(13):2788. doi: 10.3390/jcm10132788. PMID: 34202907; PMCID: PMC8268878.

423. Andersson U, Yang H, Harris H. High-mobility group box 1 protein (HMGB1) operates as an alarmin outside as well as inside cells. Semin Immunol. 2018 Aug;38:40-48. doi: 10.1016/j.smim.2018.02.011. Epub 2018 Mar 9. PMID: 29530410.

424. Paudel YN, Angelopoulou E, Piperi C, Balasubramaniam VRMT, Othman I, Shaikh MF. Enlightening the role of high mobility group box 1 (HMGB1) in inflammation: Updates on receptor signalling. Eur J Pharmacol. 2019 Sep 5;858:172487. doi: 10.1016/j.ejphar.2019.172487. Epub 2019 Jun 20. PMID: 31229535.

425. Muire PJ, Schwacha MG, Wenke JC. Systemic T Cell Exhaustion Dynamics Is Linked to Early High Mobility Group Box Protein 1 (HMGB1) Driven Hyper-Inflammation in a Polytrauma Rat Model. Cells. 2021 Jun 30;10(7):1646. doi: 10.3390/cells10071646. PMID: 34209240; PMCID: PMC8305113.

426. Ullah MA, Loh Z, Gan WJ, Zhang V, Yang H, Li JH, Yamamoto Y, Schmidt AM, Armour CL, Hughes JM, Phipps S, Sukkar MB. Receptor for advanced glycation end products and its ligand high-mobility group box-1 mediate allergic airway sensitization and airway inflammation. J Allergy Clin Immunol. 2014 Aug;134(2):440-50. doi: 10.1016/j.jaci.2013.12.1035. Epub 2014 Feb 4. PMID: 24506934.

427. Yang WS, Kim JJ, Lee MJ, Lee EK, Park SK. Ectodomain Shedding of RAGE and TLR4 as a Negative Feedback Regulation in High-Mobility Group Box 1-Activated Aortic Endothelial Cells. Cell Physiol Biochem. 2018;51(4):1632-1644. doi: 10.1159/000495651. Epub 2018 Nov 29. PMID: 30497069.

428. Falck-Jones S, Vangeti S, Yu M, Falck-Jones R, Cagigi A, Badolati I, Österberg B, Lautenbach MJ, Åhlberg E, Lin A, Lepzien R, Szurgot I, Lenart K, Hellgren F, Maecker H, Sälde J, Albert J, Johansson N, Bell M, Loré K, Färnert A, Smed-Sörensen A. Functional monocytic myeloid-derived suppressor cells increase in blood but not airways and predict COVID-19 severity. J Clin Invest. 2021 Mar 15;131(6):e144734. doi: 10.1172/JCI144734. PMID: 33492309; PMCID: PMC7954608.

429. Agrati, C., Sacchi, A., Bordoni, V. et al. Expansion of myeloid-derived suppressor cells in patients with severe coronavirus disease (COVID-19). Cell Death Differ 27, 3196-3207 (2020). https://doi.org/10.1038

430. Sacchi, A., Grassi, G., Bordoni, V. et al. Early expansion of myeloid-derived suppressor cells inhibits SARS-CoV-2 specific T-cell response and may predict fatal COVID-19 outcome. Cell Death Dis 11, 921 (2020). https://doi.org/10.1038/s41419-020-03125-1 431. Sánchez-Cerrillo I, Landete P, Aldave B, Sánchez-Alonso S, Sánchez-Azofra A, Marcos-Jiménez A, Ávalos E, Alcaraz-Serna A, de Los Santos I, Mateu-Albero T, Esparcia L, López-Sanz C, Martínez-Fleta P, Gabrie L, Del Campo Guerola L, de la Fuente H, Calzada MJ, González-Álvaro I, Alfranca A, Sánchez-Madrid F, Muñoz-Calleja C, Soriano JB, Ancochea J, Martín-Gayo E; REINMUN-COVID and EDEPIMIC groups. COVID-19 severity associates with pulmonary redistribution of CD1C+ DCs and inflammatory transitional and nonclassical monocytes. J Clin Invest. 2020 Dec 1;130(12):6290-6300. doi: 10.1172/JCI140335. PMID: 32784290; PMCID: PMC7685723.

432. Sanchez-Cerrillo I, Landete P, Aldave B, Sanchez-Alonso S, Sanchez-Azofra A, Marcos-Jimenez A, Avalos E, Alcaraz-Serna A, de Los Santos I, Mateu-Albero T, Esparcia L, Lopez-Sanz C, Martinez-Fleta P, Gabrie L, Del Campo Guerola L, Calzada MJ, GonzalezAlvaro I, Alfranca A, Sanchez-Madrid F, Munoz-Calleja C, Soriano JB, Ancochea J, Martin-Gayo E. Differential Redistribution of Activated Monocyte and Dendritic Cell Subsets to the Lung Associates with Severity of COVID-19. medRxiv [Preprint]. 2020 May 16:2020.05.13.20100925. doi: 10.1101/2020.05.13.20100925. PMID: 32511573; PMCID: PMC7274254.

433. D'Alessio FR, Heller NM. COVID-19 and myeloid cells: complex interplay correlates with lung severity. J Clin Invest. 2020 Dec 1;130(12):6214-6217. doi: 10.1172/JCI143361. PMID: 33021506; PMCID: PMC7685713. 
434. Dean MJ, Ochoa JB, Sanchez-Pino MD, Zabaleta J, Garai J, Del Valle L, Wyczechowska D, Baiamonte LB, Philbrook P,

Majumder R, Vander Heide RS, Dunkenberger L, Thylur RP, Nossaman B, Roberts WM, Chapple AG, Wu J, Hicks C, Collins J, Luke B, Johnson R, Koul HK, Rees CA, Morris CR, Garcia-Diaz J, Ochoa AC. Severe COVID-19 Is Characterized by an Impaired Type I Interferon Response and Elevated Levels of Arginase Producing Granulocytic Myeloid Derived Suppressor Cells. Front Immunol. 2021 Jul 14;12:695972. doi: 10.3389/fimmu.2021.695972. PMID: 34341659; PMCID: PMC8324422.

435. Reizine, F., Lesouhaitier, M., Gregoire, M. et al. SARS-CoV-2-Induced ARDS Associates with MDSC Expansion, Lymphocyte Dysfunction, and Arginine Shortage. J Clin Immunol 41, 515-525 (2021). https://doi.org/10.1007/s10875-020-00920-5 436. Stevens, N.E., Chapman, M.J., Fraser, C.K. et al. Therapeutic targeting of HMGB1 during experimental sepsis modulates the inflammatory cytokine profile to one associated with improved clinical outcomes. Sci Rep 7, 5850 (2017).

https://doi.org/10.1038/s41598-017-06205-z

437. Bosmann, Markus, and Peter A Ward. "The inflammatory response in sepsis." Trends in immunology vol. 34,3 (2013): 129-36. doi:10.1016/j.it.2012.09.004

438. Drifte G, Dunn-Siegrist I, Tissières $P$, Pugin J. Innate immune functions of immature neutrophils in patients with sepsis and severe systemic inflammatory response syndrome. Crit Care Med. 2013 Mar;41(3):820-32. doi: 10.1097/CCM.0b013e318274647d. PMID: 23348516.

439. Alshammary AF, AI-Sulaiman AM. The journey of SARS-CoV-2 in human hosts: a review of immune responses,

immunosuppression, and their consequences. Virulence. 2021 Dec;12(1):1771-1794. doi: 10.1080/21505594.2021.1929800. PMID: 34251989; PMCID: PMC8276660.

440. Hotchkiss RS, Coopersmith CM, McDunn JE, Ferguson TA. The sepsis seesaw: tilting toward immunosuppression. Nat Med. 2009 May;15(5):496-7. doi: 10.1038/nm0509-496. PMID: 19424209; PMCID: PMC3786779.

441. Wang, Haichao et al. "Targeting HMGB1 in the treatment of sepsis." Expert opinion on therapeutic targets vol. 18,3 (2014): 257-68. doi:10.1517/14728222.2014.863876

442. Gentile LF, Moldawer LL. HMGB1 as a therapeutic target for sepsis: it's all in the timing! Expert Opin Ther Targets. 2014 Mar;18(3):243-5. doi: 10.1517/14728222.2014.883380. Epub 2014 Jan 30. PMID: 24479494; PMCID: PMC5119516.

443. Li L, Lu YQ. The Regulatory Role of High-Mobility Group Protein 1 in Sepsis-Related Immunity. Front Immunol. 2021 Jan 22;11:601815. doi: 10.3389/fimmu.2020.601815. PMID: 33552058; PMCID: PMC7862754.

444. Ruochan Chen, Yan Huang, Jun Quan, Jiao Liu, Haichao Wang, Timothy R. Billiar, Michael T. Lotze, Herbert J. Zeh, Rui Kang, Daolin Tang, HMGB1 as a potential biomarker and therapeutic target for severe COVID-19, Heliyon, 2020, https://doi.org/10.1016/j.heliyon.2020.e05672.

445. Yan, B., Chen, F., Xu, L. et al. HMGB1-TLR4-IL23-IL17A axis promotes paraquat-induced acute lung injury by mediating neutrophil infiltration in mice. Sci Rep 7, 597 (2017). https://doi.org/10.1038/s41598-017-00721-8

446. Yurong Qu, Yuan Zhan, Shen Yang, Shanhui Ren, Xusheng Qiu, Zaib Ur Rehamn, Lei Tan, Yingjie Sun, Chunchun Meng, Cuiping Song, Shengqing Yu, Chan Ding, Newcastle disease virus infection triggers HMGB1 release to promote the inflammatory response,Virology,2018,https://doi.org/10.1016/j.virol.2018.09.001.

447. Ebina, Masahito \& Taniguchi, Hiroyuki \& Miyasho, Taku \& Yamada, Shingo \& Shibata, Naoko \& Ohta, Hiromitsu \& Hisata, Shu \& Ohkouchi, Shinya \& Tamada, Tsutomu \& Nishimura, Hidekazu \& Ishizaka, Akitoshi \& Maruyama, Ikuro \& Okada, Yoshinori \& Takashi, Kondo \& Nukiwa, Toshihiro. (2011). Gradual Increase of High Mobility Group Protein B1 in the Lungs after the Onset of Acute Exacerbation of Idiopathic Pulmonary Fibrosis. Pulmonary medicine. 2011. 916486. 10.1155/2011/916486.

448. Yang, Z., Deng, Y., Su, D. et al. TLR4 as receptor for HMGB1-mediated acute lung injury after liver ischemia/reperfusion injury. Lab Invest 93, 792-800 (2013). https://doi.org/10.1038/labinvest.2013.66

449. Tolle LB, Standiford TJ. Danger-associated molecular patterns (DAMPs) in acute lung injury. J Pathol. 2013 Jan;229(2):145-56. doi: 10.1002/path.4124. PMID: 23097158.

450. Kim JY, Park JS, Strassheim D, Douglas I, Diaz del Valle F, Asehnoune K, Mitra S, Kwak SH, Yamada S, Maruyama I, Ishizaka A, Abraham E. HMGB1 contributes to the development of acute lung injury after hemorrhage. Am J Physiol Lung Cell Mol Physiol. 2005 May;288(5):L958-65. doi: 10.1152/ajplung.00359.2004. Epub 2005 Jan 7. PMID: 15640285.

451. Gowda P, Patrick S, Joshi SD, Kumawat RK, Sen E. Glycyrrhizin prevents SARS-CoV-2 S1 and Orf3a induced high mobility group box 1 (HMGB1) release and inhibits viral replication. Cytokine. 2021 Jun;142:155496. doi: 10.1016/j.cyto.2021.155496. Epub 2021 Mar 12. PMID: 33773396; PMCID: PMC7953444.

452. Zheng M, Karki R, Williams EP, Yang D, Fitzpatrick E, Vogel P, Jonsson CB, Kanneganti TD. TLR2 senses the SARS-CoV-2 envelope protein to produce inflammatory cytokines. Nat Immunol. 2021 Jul;22(7):829-838. doi: 10.1038/s41590-021-00937-x. Epub 2021 May 7. PMID: 33963333.

453. Zhao Y, Kuang M, Li J, Zhu L, Jia Z, Guo X, Hu Y, Kong J, Yin H, Wang X, You F. Publisher Correction: SARS-CoV-2 spike protein interacts with and activates TLR4. Cell Res. 2021 Jul;31(7):825. doi: 10.1038/s41422-021-00501-0. Erratum for: Cell Res. 2021 Jul;31(7):818-820. PMID: 33907310; PMCID: PMC8077189.

454. Tsung A, Sahai R, Tanaka H, Nakao A, Fink MP, Lotze MT, Yang H, Li J, Tracey KJ, Geller DA, Billiar TR. The nuclear factor HMGB1 mediates hepatic injury after murine liver ischemia-reperfusion. J Exp Med. 2005 Apr 4;201(7):1135-43. doi: 10.1084/jem.20042614. Epub 2005 Mar 28. PMID: 15795240; PMCID: PMC2213120.

455. Qiu J, Nishimura M, Wang Y, Sims JR, Qiu S, Savitz SI, Salomone S, Moskowitz MA. Early release of HMGB-1 from neurons after the onset of brain ischemia. J Cereb Blood Flow Metab. 2008 May;28(5):927-38. doi: 10.1038/sj.jcbfm.9600582. Epub 2007 Nov 14. PMID: 18000511.

456. Cohen MJ, Brohi K, Calfee CS, Rahn P, Chesebro BB, Christiaans SC, Carles M, Howard M, Pittet JF. Early release of high mobility group box nuclear protein 1 after severe trauma in humans: role of injury severity and tissue hypoperfusion. Crit Care. 2009;13(6):R174. doi: 10.1186/cc8152. Epub 2009 Nov 4. PMID: 19887013; PMCID: PMC2811903.

457. Peltz ED, Moore EE, Eckels PC, Damle SS, Tsuruta Y, Johnson JL, Sauaia A, Silliman CC, Banerjee A, Abraham E. HMGB1 is markedly elevated within 6 hours of mechanical trauma in humans. Shock. 2009 Jul;32(1):17-22. doi: 10.1097/shk.0b013e3181997173. PMID: 19533845; PMCID: PMC4097145.

458. Manganelli V, Signore M, Pacini I, Misasi R, Tellan G, Garofalo T, Lococo E, Chirletti P, Sorice M, Delogu G. Increased HMGB1 expression and release by mononuclear cells following surgical/anesthesia trauma. Crit Care. 2010;14(6):R197. doi: 10.1186/cc9316. Epub 2010 Nov 2. PMID: 21044333; PMCID: PMC3220009.

459. Zhou RR, Liu HB, Peng JP, Huang Y, Li N, Xiao MF, Wang H, Fan XG. High mobility group box chromosomal protein 1 in acuteon-chronic liver failure patients and mice with ConA-induced acute liver injury. Exp Mol Pathol. 2012 Oct;93(2):213-9. doi: 10.1016/j.yexmp.2012.05.006. Epub 2012 May 17. PMID: 22609241.

460. Takano K, Shinoda M, Tanabe M, Miyasho T, Yamada S, Ono S, Masugi Y, Suda K, Fukunaga K, Hayashida T, Hibi T, Obara H, Takeuchi H, Kawachi S, Kawasako K, Okamoto M, Yokota H, Maruyama I, Kitagawa Y. Protective effect of high-mobility group box 1 blockade on acute liver failure in rats. Shock. 2010 Dec;34(6):573-9. doi: 10.1097/SHK.0b013e3181df0433. PMID: 20351624. 
461. Yang R, Zhang S, Cotoia A, Oksala N, Zhu S, Tenhunen J. High mobility group B1 impairs hepatocyte regeneration in acetaminophen hepatotoxicity. BMC Gastroenterol. 2012 May 8;12:45. doi: 10.1186/1471-230X-12-45. PMID: 22569100; PMCID: PMC3444430.

462. Antoine DJ, Dear JW, Lewis PS, Platt V, Coyle J, Masson M, Thanacoody RH, Gray AJ, Webb DJ, Moggs JG, Bateman DN, Goldring CE, Park BK. Mechanistic biomarkers provide early and sensitive detection of acetaminophen-induced acute liver injury at first presentation to hospital. Hepatology. 2013 Aug;58(2):777-87. doi: 10.1002/hep.26294. Epub 2013 Jul 2. PMID: 23390034 PMCID: PMC3842113.

463. Seo YS, Kwon JH, Yaqoob U, Yang L, De Assuncao TM, Simonetto DA, Verma VK, Shah VH. HMGB1 recruits hepatic stellate cells and liver endothelial cells to sites of ethanol-induced parenchymal cell injury. Am J Physiol Gastrointest Liver Physiol. 2013 Dec;305(11):G838-48. doi: 10.1152/ajpgi.00151.2013. Epub 2013 Oct 3. PMID: 24091596; PMCID: PMC3882432.

464. Wang, Haichao et al. "Potential role of high mobility group box 1 in viral infectious diseases." Viral immunology vol. 19,1 (2006): 3-9. doi:10.1089/vim.2006.19.3

465. Niu, M., Jiang, Z., Xin, X., Zhu, J., Yang, J., Diao, M. ... Qi, B. (2021). Effect of HMGB1 on monocyte immune function in respiratory syncytial virus bronchiolitis. Experimental and Therapeutic Medicine, 21, 75. https://doi.org/10.3892/etm.2020.9507 466. Wang, H., Zhu, S., Zhou, R., Li, W., \& Sama, A. (2008). Therapeutic potential of HMGB1-targeting agents in sepsis. Expert Reviews in Molecular Medicine, 10, E32. doi:10.1017/S1462399408000884

467. Manti, S., Harford, T., Salpietro, C. et al. Induction of high-mobility group Box-1 in vitro and in vivo by respiratory syncytia virus. Pediatr Res 83, 1049-1056 (2018). https://doi.org/10.1038/pr.2018.6

468. Ong SP, Lee LM, Leong YFI, Ng ML, Chu JJH (2012) Dengue Virus Infection Mediates HMGB1 Release from Monocytes Involving PCAF Acetylase Complex and Induces Vascular Leakage in Endothelial Cells. PLOS ONE 7(7): e41932. https://doi.org/10.1371/journal.pone.0041932

469. Patel MC, Shirey KA, Boukhvalova MS, Vogel SN, Blanco JCG. Serum High-Mobility-Group Box 1 as a Biomarker and a Therapeutic Target during Respiratory Virus Infections. mBio. 2018 Mar 13;9(2):e00246-18. doi: 10.1128/mBio.00246-18. PMID: 29535197; PMCID: PMC5850323.

470. Samy RP, Lim LH. DAMPs and influenza virus infection in ageing. Ageing Res Rev. 2015 Nov;24(Pt A):83-97. doi: 10.1016/j.arr.2015.07.005. Epub 2015 Jul 19. PMID: 26200296

471. Zhu S, Li W, Ward MF, Sama AE, Wang H. High mobility group box 1 protein as a potential drug target for infection- and injury-elicited inflammation. Inflamm Allergy Drug Targets. 2010 Mar;9(1):60-72. doi: 10.2174/187152810791292872. PMID 19906009; PMCID: PMC2866444.

472. Sursal T, Stearns-Kurosawa DJ, Itagaki K, Oh SY, Sun S, Kurosawa S, Hauser CJ. Plasma bacterial and mitochondrial DNA distinguish bacterial sepsis from sterile systemic inflammatory response syndrome and quantify inflammatory tissue injury in nonhuman primates. Shock. 2013 Jan;39(1):55-62. doi: 10.1097/SHK.0b013e318276f4ca. PMID: 23247122; PMCID: PMC3537509. 473. Vincent JL, Opal SM, Marshall JC, Tracey KJ. Sepsis definitions: time for change. Lancet. 2013 Mar 2;381(9868):774-5. doi: 10.1016/S0140-6736(12)61815-7. PMID: 23472921; PMCID: PMC4535310.

474. Whilding LM, Archibald KM, Kulbe H, Balkwill FR, Öberg D, McNeish IA. Vaccinia virus induces programmed necrosis in ovarian cancer cells. Mol Ther. 2013 Nov;21(11):2074-86. doi: 10.1038/mt.2013.195. Epub 2013 Sep 24. PMID: 23985697; PMCID: PMC3831043.

475. Hofner P, Seprényi G, Miczák A, Buzás K, Gyulai Z, Medzihradszky KF, Rouhiainen A, Rauvala H, Mándi Y. High mobility group box 1 protein induction by Mycobacterium bovis BCG. Mediators Inflamm. 2007;2007:53805. doi: 10.1155/2007/53805. PMID: 18288272; PMCID: PMC2233895.

476. Grover A, Taylor J, Troudt J, Keyser A, Sommersted K, Schenkel A, Izzo AA. Mycobacterial infection induces the secretion of high-mobility group box 1 protein. Cell Microbiol. 2008 Jun;10(6):1390-404. doi: 10.1111/j.1462-5822.2008.01135.x. Epub 2008 Mar 5. PMID: 18331466.

477. Zhang Q, Raoof M, Chen Y, Sumi Y, Sursal T, Junger W, Brohi K, Itagaki K, Hauser CJ. Circulating mitochondrial DAMPs cause inflammatory responses to injury. Nature. 2010 Mar 4;464(7285):104-7. doi: 10.1038/nature08780. PMID: 20203610; PMCID: PMC2843437.

478. Zhu S, Ashok M, Li J, Li W, Yang H, Wang P, Tracey KJ, Sama AE, Wang H. Spermine protects mice against lethal sepsis partly by attenuating surrogate inflammatory markers. Mol Med. 2009 Jul-Aug;15(7-8):275-82. doi: 10.2119/molmed.2009.00062. Epub 2009 May 1. PMID: 19593412; PMCID: PMC2707519.

479. Yang $D$, Chen $Q$, Yang $H$, et al. High mobility group box-1 protein induces the migration and activation of human dendritic cells and acts as an alarmin. J Leukoc Biol. 2007;81:59-66. These authors first reported the chemokine activities of extracellular HMGB

480. Dumitriu IE, Bianchi ME, Bacci M, et al. The secretion of HMGB1 is required for the migration of maturing dendritic cells. $J$ Leukoc Biol. 2007:81:84-91

481. Orlova VV, Choi EY, Xie C, et al. A novel pathway of HMGB1-mediated inflammatory cell recruitment that requires Mac-1integrin. EMBO J. 2007;26:1129-1139

482. Degryse B, Bonaldi T, Scaffidi $P$, et al. The high mobility group (HMG) boxes of the nuclear protein HMG1 induce chemotaxis and cytoskeleton reorganization in rat smooth muscle cells. J Cell Biol. 2001;152:1197-1206

483. Degryse B, de Virgilio M. The nuclear protein HMGB1, a new kind of chemokine? FEBS Lett. 2003 Oct 9;553(1-2):11-7. doi: 10.1016/s0014-5793(03)01027-5. PMID: 14550538.

484. Tian J, Avalos AM, Mao SY, Chen B, Senthil K, Wu H, Parroche P, Drabic S, Golenbock D, Sirois C, Hua J, An LL, Audoly L, La Rosa G, Bierhaus A, Naworth P, Marshak-Rothstein A, Crow MK, Fitzgerald KA, Latz E, Kiener PA, Coyle AJ. Toll-like receptor 9dependent activation by DNA-containing immune complexes is mediated by HMGB1 and RAGE. Nat Immunol. 2007 May;8(5):487-96. doi: 10.1038/ni1457. Epub 2007 Apr 8. Erratum in: Nat Immunol. 2007 Jul;8(7):780. PMID: 17417641. 485. Yu M, Wang H, Ding A, Golenbock DT, Latz E, Czura CJ, Fenton MJ, Tracey KJ, Yang H. HMGB1 signals through toll-like receptor (TLR) 4 and TLR2. Shock. 2006 Aug;26(2):174-9. doi: 10.1097/01.shk.0000225404.51320.82. PMID: 16878026. 486. Ha T, Xia Y, Liu X, Lu C, Liu L, Kelley J, Kalbfleisch J, Kao RL, Williams DL, Li C. Glucan phosphate attenuates myocardial HMGB1 translocation in severe sepsis through inhibiting NF-KB activation. Am J Physiol Heart Circ Physiol. 2011 Sep;301(3):H848 55. doi: 10.1152/ajpheart.01007.2010. Epub 2011 Jun 3. PMID: 21642503; PMCID: PMC3191099.

487. Xiang M, Shi X, Li Y, Xu J, Yin L, Xiao G, Scott MJ, Billiar TR, Wilson MA, Fan J. Hemorrhagic shock activation of NLRP3 inflammasome in lung endothelial cells. J Immunol. 2011 Nov 1;187(9):4809-17. doi: 10.4049/jimmunol.1102093. Epub 2011 Sep 21. PMID: 21940680; PMCID: PMC3197874.

488. Chen GY, Tang J, Zheng P, Liu Y. CD24 and Siglec-10 selectively repress tissue damage-induced immune responses. Science. 2009 Mar 27;323(5922):1722-5. doi: 10.1126/science.1168988. Epub 2009 Mar 5. PMID: 19264983; PMCID: PMC2765686. 489. Abeyama K, Stern DM, Ito Y, Kawahara K, Yoshimoto Y, Tanaka M, Uchimura T, Ida N, Yamazaki Y, Yamada S, Yamamoto Y, Yamamoto H, lino S, Taniguchi N, Maruyama I. The N-terminal domain of thrombomodulin sequesters high-mobility group-B1 
protein, a novel antiinflammatory mechanism. J Clin Invest. 2005 May;115(5):1267-74. doi: 10.1172/JCI22782. Epub 2005 Apr 14. PMID: 15841214; PMCID: PMC1077171.

490. Salmivirta M, Rauvala H, Elenius K, Jalkanen M. Neurite growth-promoting protein (amphoterin, p30) binds syndecan. Exp Cell Res. 1992 Jun;200(2):444-51. doi: 10.1016/0014-4827(92)90194-d. PMID: 1369684.

491. Park JS, Svetkauskaite D, He Q, Kim JY, Strassheim D, Ishizaka A, Abraham E. Involvement of toll-like receptors 2 and 4 in cellular activation by high mobility group box 1 protein. J Biol Chem. 2004 Feb 27;279(9):7370-7. doi: 10.1074/jbc.M306793200. Epub 2003 Dec 4. PMID: 14660645.

492. Park JS, Gamboni-Robertson F, He Q, Svetkauskaite D, Kim JY, Strassheim D, Sohn JW, Yamada S, Maruyama I, Banerjee A Ishizaka A, Abraham E. High mobility group box 1 protein interacts with multiple Toll-like receptors. Am J Physiol Cell Physiol. 2006 Mar;290(3):C917-24. doi: 10.1152/ajpcell.00401.2005. Epub 2005 Nov 2. PMID: 16267105.

493. Kokkola R, Andersson A, Mullins G, Ostberg T, Treutiger CJ, Arnold B, Nawroth P, Andersson U, Harris RA, Harris HE. RAGE is the major receptor for the proinflammatory activity of HMGB1 in rodent macrophages. Scand J Immunol. 2005 Jan;61(1):1-9. doi: 10.1111/j.0300-9475.2005.01534.x. PMID: 15644117.

494. Pedrazzi M, Patrone M, Passalacqua M, Ranzato E, Colamassaro D, Sparatore B, Pontremoli S, Melloni E. Selective proinflammatory activation of astrocytes by high-mobility group box 1 protein signaling. J Immunol. 2007 Dec 15;179(12):8525-32. doi: 10.4049/jimmunol.179.12.8525. PMID: 18056400.

495. Fiuza C, Bustin M, Talwar S, Tropea M, Gerstenberger E, Shelhamer JH, Suffredini AF. Inflammation-promoting activity of HMGB1 on human microvascular endothelial cells. Blood. 2003 Apr 1;101(7):2652-60. doi: 10.1182/blood-2002-05-1300. Epub 2002 Nov 27. PMID: 12456506

496. Treutiger CJ, Mullins GE, Johansson AS, Rouhiainen A, Rauvala HM, Erlandsson-Harris H, Andersson U, Yang H, Tracey KJ, Andersson J, Palmblad JE. High mobility group 1 B-box mediates activation of human endothelium. J Intern Med. 2003 Oct;254(4):375-85. doi: 10.1046/j.1365-2796.2003.01204.x. PMID: 12974876.

497. Kazama H, Ricci JE, Herndon JM, Hoppe G, Green DR, Ferguson TA. Induction of immunological tolerance by apoptotic cells requires caspase-dependent oxidation of high-mobility group box-1 protein. Immunity. 2008 Jul 18;29(1):21-32. doi: 10.1016/j.immuni.2008.05.013. PMID: 18631454; PMCID: PMC2704496.

498. Venereau E, Casalgrandi M, Schiraldi M, Antoine DJ, Cattaneo A, De Marchis F, Liu J, Antonelli A, Preti A, Raeli L, Shams SS, Yang H, Varani L, Andersson U, Tracey KJ, Bachi A, Uguccioni M, Bianchi ME. Mutually exclusive redox forms of HMGB1 promote cell recruitment or proinflammatory cytokine release. J Exp Med. 2012 Aug 27;209(9):1519-28. doi: 10.1084/jem.20120189. Epub 2012 Aug 6. PMID: 22869893; PMCID: PMC3428943.

499. Tang D, Billiar TR, Lotze MT. A Janus tale of two active high mobility group box 1 (HMGB1) redox states. Mol Med. 2012 Dec 20;18(1):1360-2. doi: 10.2119/molmed.2012.00314. PMID: 23073660; PMCID: PMC3533642.

500. Wichterman KA, Baue AE, Chaudry IH. Sepsis and septic shock--a review of laboratory models and a proposal. J Surg Res. 1980 Aug;29(2):189-201. doi: 10.1016/0022-4804(80)90037-2. PMID: 6997619.

501. Yang H, Ochani M, Li J, Qiang X, Tanovic M, Harris HE, Susarla SM, Ulloa L, Wang H, DiRaimo R, Czura CJ, Wang H, Roth J, Warren HS, Fink MP, Fenton MJ, Andersson U, Tracey KJ. Reversing established sepsis with antagonists of endogenous highmobility group box 1. Proc Natl Acad Sci U S A. 2004 Jan 6;101(1):296-301. doi: 10.1073/pnas.2434651100. Epub 2003 Dec 26. PMID: 14695889; PMCID: PMC314179.

502. Wang H, Yang H, Czura CJ, Sama AE, Tracey KJ. HMGB1 as a late mediator of lethal systemic inflammation. Am J Respir Crit Care Med. 2001 Nov 15;164(10 Pt 1):1768-73. doi: 10.1164/ajrccm.164.10.2106117. PMID: 11734424.

503. Qin S, Wang H, Yuan R, Li H, Ochani M, Ochani K, Rosas-Ballina M, Czura CJ, Huston JM, Miller E, Lin X, Sherry B, Kumar A, Larosa G, Newman W, Tracey KJ, Yang H. Role of HMGB1 in apoptosis-mediated sepsis lethality. J Exp Med. 2006 Jul 10;203(7):1637-42. doi: 10.1084/jem.20052203. Epub 2006 Jul 3. PMID: 16818669; PMCID: PMC2118346.

504. Suda K, Kitagawa Y, Ozawa S, Saikawa Y, Ueda M, Ebina M, Yamada S, Hashimoto S, Fukata S, Abraham E, Maruyama I, Kitajima M, Ishizaka A. Anti-high-mobility group box chromosomal protein 1 antibodies improve survival of rats with sepsis. World J Surg. 2006 Sep;30(9):1755-62. doi: 10.1007/s00268-005-0369-2. PMID: 16850155.

505. Ivanov S, Dragoi AM, Wang X, Dallacosta C, Louten J, Musco G, Sitia G, Yap GS, Wan Y, Biron CA, Bianchi ME, Wang H, Chu WM. A novel role for HMGB1 in TLR9-mediated inflammatory responses to CpG-DNA. Blood. 2007 Sep 15;110(6):1970-81. doi: 10.1182/blood-2006-09-044776. Epub 2007 Jun 4. PMID: 17548579; PMCID: PMC1976374.

506. Jin, S., Yang, Z., Hao, X. et al. Roles of HMGB1 in regulating myeloid-derived suppressor cells in the tumor microenvironment. Biomark Res 8, 21 (2020). https://doi.org/10.1186/s40364-020-00201-8

507. Parker, Katherine \& Sinha, Pratima \& Horn, Lucas \& Clements, Virginia \& Yang, Huan \& Li, Jianhua \& Tracey, Kevin \&

Ostrand-Rosenberg, S.. (2014). HMGB1 Enhances Immune Suppression by Facilitating the Differentiation and Suppressive Activity of Myeloid-Derived Suppressor Cells. Cancer research. 74. 10.1158/0008-5472.CAN-13-2347.

508. Gorgulho CM, Romagnoli GG, Bharthi R, Lotze MT. Johnny on the Spot-Chronic Inflammation Is Driven by HMGB1. Front Immunol. 2019 Jul 11;10:1561. doi: 10.3389/fimmu.2019.01561. PMID: 31379812; PMCID: PMC6660267.

509. Hubert P, Roncarati P, Demoulin S, et al Extracellular HMGB1 blockade inhibits tumor growth through profoundly remodeling immune micro environment and enhances checkpoint inhibitor-based immuno therapy Journal for ImmunoTherapy of Cancer 2021;9:e001966. doi: 10.1136/jitc-2020-001966

510. Gabrilovich, Dmitry I, and Srinivas Nagaraj. "Myeloid-derived suppressor cells as regulators of the immune system." Nature reviews. Immunology vol. 9,3 (2009): 162-74. doi:10.1038/nri2506

511. Yang Y, Li C, Liu T, Dai X, Bazhin AV. Myeloid-Derived Suppressor Cells in Tumors: From Mechanisms to Antigen Specificity and Microenvironmental Regulation. Front Immunol. 2020 Jul 22;11:1371. doi: 10.3389/fimmu.2020.01371. PMID: 32793192; PMCID: PMC7387650.

512. Bruno A, Mortara L, Baci D, Noonan DM, Albini A. Myeloid Derived Suppressor Cells Interactions With Natural Killer Cells and Pro-angiogenic Activities: Roles in Tumor Progression. Front Immunol. 2019 Apr 18;10:771. doi: 10.3389/fimmu.2019.00771. PMID: 31057536; PMCID: PMC6482162.

513. Zhang, T., Guan, XW., Gribben, J.G. et al. Blockade of HMGB1 signaling pathway by ethyl pyruvate inhibits tumor growth in diffuse large B-cell lymphoma. Cell Death Dis 10, 330 (2019). https://doi.org/10.1038/s41419-019-1563-8 514. Gabrilovich, Dmitry I. "Myeloid-Derived Suppressor Cells." Cancer immunology research vol. 5,1 (2017): 3-8. doi:10.1158/2326-6066.CIR-16-0297

515. Fleming V, Hu X, Weber R, Nagibin V, Groth C, Altevogt P, Utikal J, Umansky V. Targeting Myeloid-Derived Suppressor Cells to Bypass Tumor-Induced Immunosuppression. Front Immunol. 2018 Mar 2;9:398. doi: 10.3389/fimmu.2018.00398. PMID: 29552012; PMCID: PMC5840207.

516. Fultang N, Li X, Li T, Chen YH. Myeloid-Derived Suppressor Cell Differentiation in Cancer: Transcriptional Regulators and Enhanceosome-Mediated Mechanisms. Front Immunol. 2021 Jan 14;11:619253. doi: 10.3389/fimmu.2020.619253. PMID: 33519825; PMCID: PMC7840597. 
517. Ruan X, Darwiche SS, Cai C, Scott MJ, Pape HC, Billiar TR. Anti-HMGB1 monoclonal antibody ameliorates immunosuppression after peripheral tissue trauma: attenuated T-lymphocyte response and increased splenic CD11b (+) Gr-1 (+) myeloid-derived suppressor cells require HMGB1. Mediators Inflamm. 2015;2015:458626. doi: 10.1155/2015/458626. Epub 2015 Jan 29. PMID: 25709155; PMCID: PMC4325468.

518. Ostrand-Rosenberg S, Fenselau C. Myeloid-Derived Suppressor Cells: Immune-Suppressive Cells That Impair Antitumor Immunity and Are Sculpted by Their Environment. J Immunol. 2018 Jan 15;200(2):422-431. doi: 10.4049/jimmunol.1701019. PMID: 29311384; PMCID: PMC5765878.

519. Li J, Kokkola R, Tabibzadeh S, Yang R, Ochani M, Qiang X, Harris HE, Czura CJ, Wang H, Ulloa L, Wang H, Warren HS, Moldawer LL, Fink MP, Andersson U, Tracey KJ, Yang H. Structural basis for the proinflammatory cytokine activity of high mobility group box 1. Mol Med. 2003 Jan-Feb;9(1-2):37-45. PMID: 12765338; PMCID: PMC1430376.

520. Wang H, Li W, Goldstein R, Tracey KJ, Sama AE. HMGB1 as a potential therapeutic target. Novartis Found Symp. 2007;280:73-85; discussion 85-91, 160-4. PMID: 17380789.

521. Yang H, Wang H, Czura CJ, Tracey KJ. HMGB1 as a cytokine and therapeutic target. J Endotoxin Res. 2002;8(6):469-72. doi: 10.1179/096805102125001091. PMID: 12697092.

522. Tang Y, Lv B, Wang H, Xiao X, Zuo X. PACAP inhibit the release and cytokine activity of HMGB1 and improve the survival during lethal endotoxemia. Int Immunopharmacol. 2008 Dec 10;8(12):1646-51. doi: 10.1016/j.intimp.2008.07.014. Epub 2008 Aug 17. PMID: 18713653.

523. Delgado M, Pozo D, Martinez C, Leceta J, Calvo JR, Ganea D, Gomariz RP. Vasoactive intestinal peptide and pituitary adenylate cyclase-activating polypeptide inhibit endotoxin-induced TNF-alpha production by macrophages: in vitro and in vivo studies. J Immunol. 1999 Feb 15;162(4):2358-67. PMID: 9973516.

524. Tang Y, Li B, Wang N, Xie Y, Wang L, Yuan Q, Zhang F, Qin J, Peng Z, Ning W, Wang L, Hu G, Li J, Tao L. Fluorofenidone protects mice from lethal endotoxemia through the inhibition of TNF-alpha and IL-1beta release. Int Immunopharmacol. 2010 May;10(5):580-3. doi: 10.1016/j.intimp.2010.02.005. Epub 2010 Feb 13. PMID: 20159052.

525. Pavlov VA, Ochani M, Yang LH, Gallowitsch-Puerta M, Ochani K, Lin X, Levi J, Parrish WR, Rosas-Ballina M, Czura CJ, Larosa GJ, Miller EJ, Tracey KJ, Al-Abed Y. Selective alpha7-nicotinic acetylcholine receptor agonist GTS-21 improves survival in murine endotoxemia and severe sepsis. Crit Care Med. 2007 Apr;35(4):1139-44. doi: 10.1097/01.CCM.0000259381.56526.96. PMID: 17334244.

526. Andersson U, Erlandsson-Harris H, Yang H, Tracey KJ. HMGB1 as a DNA-binding cytokine. J Leukoc Biol. 2002 Dec;72(6):108491. PMID: 12488489

527. Czura CJ, Yang H, Tracey KJ. High mobility group box-1 as a therapeutic target downstream of tumor necrosis factor. J Infect Dis. 2003 Jun 15;187 Suppl 2:S391-6. doi: 10.1086/374753. PMID: 12792856.

528. Kalinina N, Agrotis A, Antropova Y, DiVitto G, Kanellakis P, Kostolias G, Ilyinskaya O, Tararak E, Bobik A. Increased expression of the DNA-binding cytokine HMGB1 in human atherosclerotic lesions: role of activated macrophages and cytokines. Arterioscler Thromb Vasc Biol. 2004 Dec;24(12):2320-5. doi: 10.1161/01.ATV.0000145573.36113.8a. Epub 2004 Sep 16. PMID: 15374849. 529. Hou LC, Qin MZ, Zheng LN, Lu Y, Wang Q, Peng DR, Yu XP, Xin YC, Ji GL, Xiong LZ. Severity of sepsis is correlated with the elevation of serum high-mobility group box 1 in rats. Chin Med J (Engl). 2009 Feb 20;122(4):449-54. PMID: 19302753.

530. Friedman SG, Czura CJ, Tracey KJ. The gesture life of high mobility group box 1. Curr Opin Clin Nutr Metab Care. 2003 May;6(3):283-7. doi: 10.1097/01.mco.0000068966.34812.b9. PMID: 12690260.

531. 444

532. Street ME. HMGB1: A Possible Crucial Therapeutic Target for COVID-19? Horm Res Paediatr. 2020;93(2):73-75. doi: 10.1159/000508291. Epub 2020 May 6. PMID: 32375153; PMCID: PMC7251586.

533. Chen L, Long X, Xu Q, Tan J, Wang G, Cao Y, Wei J, Luo H, Zhu H, Huang L, Meng F, Huang L, Wang N, Zhou X, Zhao L, Chen X, Mao Z, Chen C, Li Z, Sun Z, Zhao J, Wang D, Huang G, Wang W, Zhou J. Elevated serum levels of S100A8/A9 and HMGB1 at hospital admission are correlated with inferior clinical outcomes in COVID-19 patients. Cell Mol Immunol. 2020 Sep;17(9):992-994. doi: 10.1038/s41423-020-0492-x. Epub 2020 Jul 3. PMID: 32620787; PMCID: PMC7332851.

534. Sivakorn C, Dechsanga J, Jamjumrus L, Boonnak K, Schultz MJ, Dorndorp AM, Phumratanaprapin W, Ratanarat R, Naorungroj T, Wattanawinitchai P, Siripoon T, Duangdee C, Techarang T. High Mobility Group Box 1 and Interleukin 6 at Intensive Care Unit Admission as Biomarkers in Critically III COVID-19 Patients. Am J Trop Med Hyg. 2021 May 3;105(1):73-80. doi: 10.4269/ajtmh.210165. Epub ahead of print. PMID: 33939645; PMCID: PMC8274790.

535. Nosaka N, Hatayama K, Yamada M, Fujii Y, Yashiro M, Wake H, Tsukahara H, Nishibori M, Morishima T. Anti-high mobility group box-1 monoclonal antibody treatment of brain edema induced by influenza infection and lipopolysaccharide. J Med Virol. 2018 Jul;90(7):1192-1198. doi: 10.1002/jmv.25076. Epub 2018 Mar 31. PMID: 29573352.

536. Chen YJ, Wang SF, Weng IC, Hong MH, Lo TH, Jan JT, Hsu LC, Chen HY, Liu FT. Galectin-3 Enhances Avian H5N1 Influenza A Virus-Induced Pulmonary Inflammation by Promoting NLRP3 Inflammasome Activation. Am J Pathol. 2018 Apr;188(4):1031-1042. doi: 10.1016/j.ajpath.2017.12.014. Epub 2018 Jan 31. PMID: 29366678.

537. Boon AC, Finkelstein D, Zheng M, Liao G, Allard J, Klumpp K, Webster R, Peltz G, Webby RJ. H5N1 influenza virus pathogenesis in genetically diverse mice is mediated at the level of viral load. mBio. 2011 Sep 6;2(5):e00171-11. doi: 10.1128/mBio.00171-11. PMID: 21896679; PMCID: PMC3171982.

538. van den Berg DF, Te Velde AA. Severe COVID-19: NLRP3 Inflammasome Dysregulated. Front Immunol. 2020 Jun 26;11:1580 doi: 10.3389/fimmu.2020.01580. PMID: 32670297; PMCID: PMC7332883.

539. Hippensteel JA, LaRiviere WB, Colbert JF, Langouët-Astrié CJ, Schmidt EP. Heparin as a therapy for COVID-19: current evidence and future possibilities. Am J Physiol Lung Cell Mol Physiol. 2020 Aug 1;319(2):L211-L217. doi:

10.1152/ajplung.00199.2020. Epub 2020 Jun 10. PMID: 32519894; PMCID: PMC7381711.

540. Wu MQ, Li C, Zhang LN, Lin J, He K, Niu YW, Che CY, Jiang N, Jiang JQ, Zhao GQ. High-mobility group box1 as an amplifier of immune response and target for treatment in Aspergillus fumigatus keratitis. Int J Ophthalmol. 2020 May 18;13(5):708-717. doi: 10.18240/ijo.2020.05.03. PMID: 32420216; PMCID: PMC7201345.

541. Chen GY, Chen X, King S, Cavassani KA, Cheng J, Zheng X, Cao H, Yu H, Qu J, Fang D, Wu W, Bai XF, Liu JQ, Woodiga SA, Chen C, Sun L, Hogaboam CM, Kunkel SL, Zheng P, Liu Y. Amelioration of sepsis by inhibiting sialidase-mediated disruption of the CD24SiglecG interaction. Nat Biotechnol. 2011 May;29(5):428-35. doi: 10.1038/nbt.1846. Epub 2011 Apr 10. Erratum in: Nat Biotechnol. 2012 Feb;30(2):193. PMID: 21478876; PMCID: PMC4090080.

542. Bertheloot D, Latz E. HMGB1, IL-1 $\alpha$, IL-33 and S100 proteins: dual-function alarmins. Cell Mol Immunol. 2017 Jan;14(1):43-64. doi: 10.1038/cmi.2016.34. Epub 2016 Aug 29. PMID: $27569562 ;$ PMCID: PMC5214941.

543. Kwak MS, Kim HS, Lee B, Kim YH, Son M, Shin JS. Immunological Significance of HMGB1 Post-Translational Modification and Redox Biology. Front Immunol. 2020 Jun 10;11:1189. doi: 10.3389/fimmu.2020.01189. PMID: 32587593; PMCID: PMC7297982. 544. Ayre DC, Christian SL. CD24: A Rheostat That Modulates Cell Surface Receptor Signaling of Diverse Receptors. Front Cell Dev Biol. 2016 Dec 27;4:146. doi: 10.3389/fcell.2016.00146. PMID: 28083532; PMCID: PMC5186806. 
545. Wyganowska-Swiatkowska, Marzena et al. "Influence of Herbal Medicines on HMGB1 Release, SARS-CoV-2 Viral Attachment, Acute Respiratory Failure, and Sepsis. A Literature Review." International journal of molecular sciences vol. 21,13 4639. 30 Jun. 2020, doi:10.3390/ijms21134639

546. Wen Z, Xu L, Chen X, Xu W, Yin Z, Gao X, Xiong S. Autoantibody induction by DNA-containing immune complexes requires HMGB1 with the TLR2/microRNA-155 pathway. J Immunol. 2013 Jun 1;190(11):5411-22. doi: 10.4049/jimmunol.1203301. Epub 2013 Apr 24. PMID: 23616573.

547. Dietz S, Schwarz J, Rühle J, Schaller M, Fehrenbacher B, Marmé A, Schmid E, Peter A, Poets CF, Gille C, Köstlin-Gille N. Extracellular vesicles released by myeloid-derived suppressor cells from pregnant women modulate adaptive immune responses. Cell Immunol. 2021 Mar;361:104276. doi: 10.1016/j.cellimm.2020.104276. Epub 2020 Dec 24. PMID: 33517124.

548. Denning NL, Aziz M, Gurien SD, Wang P. DAMPs and NETs in Sepsis. Front Immunol. 2019 Oct 30;10:2536. doi: 10.3389/fimmu.2019.02536. PMID: 31736963; PMCID: PMC6831555.

549. Reizine F, Lesouhaitier M, Gregoire M, Pinceaux K, et.al. SARS-CoV-2-Induced ARDS Associates with MDSC Expansion, Lymphocyte Dysfunction, and Arginine Shortage. J Clin Immunol. 2021 Apr;41(3):515-525. doi: 10.1007/s10875-020-00920-5. Epub 2021 Jan 2. PMID: 33387156; PMCID: PMC7775842.

550. Ren Y, Shu T, Wu D, Mu J, Wang C, Huang M, Han Y, Zhang XY, Zhou W, Qiu Y, Zhou X. The ORF3a protein of SARS-CoV-2 induces apoptosis in cells. Cell Mol Immunol. 2020 Aug;17(8):881-883. doi: 10.1038/s41423-020-0485-9. Epub 2020 Jun 18. PMID: 32555321; PMCID: PMC7301057.

551. Chen S, Ding R, Hu Z, Yin X, Xiao F, Zhang W, Yan S, Lv C. MicroRNA-34a Inhibition Alleviates Lung Injury in Cecal Ligation and Puncture Induced Septic Mice. Front Immunol. 2020 Aug 13;11:1829. doi: 10.3389/fimmu.2020.01829. PMID: 32903604; PMCID: PMC7438583.

552. Hart M, Walch-Rückheim B, Friedmann KS, Rheinheimer S, Tänzer T, Glombitza B, Sester M, Lenhof HP, Hoth M, Schwarz EC, Keller A, Meese E. miR-34a: a new player in the regulation of T cell function by modulation of NF-KB signaling. Cell Death Dis. 2019 Jan 18;10(2):46. doi: 10.1038/s41419-018-1295-1. PMID: 30718475; PMCID: PMC6362007.

553. Lei, Guang-Sheng et al. "Myeloid-derived suppressor cells impair alveolar macrophages through PD-1 receptor ligation during Pneumocystis pneumonia." Infection and immunity vol. 83,2 (2015): 572-82. doi:10.1128/IAI.02686-14

554. Xin Huang, Fabienne Venet, Yvonne L. Wang et.al. PD-1 expression by macrophages plays a pathologic role in altering microbial clearance and the innate inflammatory response to sepsis Proceedings of the National Academy of Sciences Apr 2009, 106 (15) 6303-6308; DOI: 10.1073/pnas.0809422106.

555. Gu X, Zhou F, Wang Y, Fan G, Cao B. Respiratory viral sepsis: epidemiology, pathophysiology, diagnosis and treatment. Eur Respir Rev. 2020 Jul 21;29(157):200038. doi: 10.1183/16000617.0038-2020. PMID: 32699026.

556. Matsuyama, Toshifumi \& Kubli, Shawn \& Yoshinaga, Steven \& Pfeffer, Klaus \& Mak, Tak. (2020). An aberrant STAT pathway is central to COVID-19. Cell death and differentiation. 27. 10.1038/s41418-020-00633-7.

557. O'Connor MA, Rastad JL, Green WR. The Role of Myeloid-Derived Suppressor Cells in Viral Infection. Viral Immunol. 2017 Mar;30(2):82-97. doi: 10.1089/vim.2016.0125. Epub 2017 Jan 4. PMID: 28051364; PMCID: PMC5346953.

558. Schrijver, Irene T et al. "Myeloid-Derived Suppressor Cells in Sepsis." Frontiers in immunology vol. 10 327. 27 Feb. 2019, doi:10.3389/fimmu.2019.00327

559. Waeckel, Louis et al. "Delayed persistence of elevated monocytic MDSC associates with deleterious outcomes in septic shock: a retrospective cohort study." Critical care (London, England) vol. 24,1 132. 7 Apr. 2020, doi:10.1186/s13054-020-02857-y 560. Marais, C., Claude, C., Semaan, N. et al. Myeloid phenotypes in severe COVID-19 predict secondary infection and mortality: a pilot study. Ann. Intensive Care 11, 111 (2021). https://doi.org/10.1186/s13613-021-00896-4

561. Musolino C, Allegra A, Pioggia G, Gangemi S. Immature myeloid-derived suppressor cells: A bridge between inflammation and cancer (Review). Oncol Rep. 2017 Feb;37(2):671-683. doi: 10.3892/or.2016.5291. Epub 2016 Dec 5. PMID: 27922687. 562. Bei C, Liu S, Yu X, Qiu M, Tang B, Liao W, He S, Yu H. Single Nucleotide Polymorphisms in miR-122 Are Associated with the Risk of Hepatocellular Carcinoma in a Southern Chinese Population. Biomed Res Int. 2018 Dec 19;2018:1540201. doi: 10.1155/2018/1540201. PMID: 30662901; PMCID: PMC6313975.

563. Tang D, Kang R, Livesey KM, Cheh CW, Farkas A, Loughran P, Hoppe G, Bianchi ME, Tracey KJ, Zeh HJ 3rd, Lotze MT. Endogenous HMGB1 regulates autophagy. J Cell Biol. 2010 Sep 6;190(5):881-92. doi: 10.1083/jcb.200911078. PMID: 20819940; PMCID: PMC2935581.

564. Geoffrey Bellingan, Inflammatory cell activation in sepsis, British Medical Bulletin, Volume 55, Issue 1, 1999, Pages 12-29, https://doi.org/10.1258/0007142991902277

565. Yang Y, Sun D, Zhou J, Tan C, Zhang H, Chen Z, Hao C, Zhang J. LPS expands MDSCs by inhibiting apoptosis through the regulation of the GATA2/let-7e axis. Immunol Cell Biol. 2019 Feb;97(2):142-151. doi: 10.1111/imcb.12204. Epub 2018 Oct 21. PMID: 30221399.

566. McClure C, Brudecki L, Ferguson DA, Yao ZQ, Moorman JP, McCall CE, El Gazzar M. MicroRNA 21 (miR-21) and miR-181b couple with NFI-A to generate myeloid-derived suppressor cells and promote immunosuppression in late sepsis. Infect Immun. 2014 Sep;82(9):3816-25. doi: 10.1128/IAI.01495-14. Epub 2014 Jun 30. PMID: 24980967; PMCID: PMC4187818.

567. Malek E, de Lima M, Letterio JJ, Kim BG, Finke JH, Driscoll JJ, Giralt SA. Myeloid-derived suppressor cells: The green light for myeloma immune escape. Blood Rev. 2016 Sep;30(5):341-8. doi: 10.1016/j.blre.2016.04.002. Epub 2016 Apr 12. PMID: 27132116; PMCID: PMC6411302.

568. Sanchez-Pino MD, Dean MJ, Ochoa AC. Myeloid-derived suppressor cells (MDSC): When good intentions go awry. Cell Immunol. 2021 Apr;362:104302. doi: 10.1016/j.cellimm.2021.104302. Epub 2021 Feb 4. PMID: 33592540; PMCID: PMC8054443. 569. Köstlin N, Vogelmann M, Spring B, Schwarz J, Feucht J, Härtel C, Orlikowsky TW, Poets CF, Gille C. Granulocytic myeloidderived suppressor cells from human cord blood modulate T-helper cell response towards an anti-inflammatory phenotype. Immunology. 2017 Sep;152(1):89-101. doi: 10.1111/imm.12751. Epub 2017 Jun 8. PMID: 28464218; PMCID: PMC5543463. 570. Huang A, Zhang H, Chen S, Xia F, Yang Y, Dong F, Sun D, Xiong S, Zhang J. miR-34a expands myeloid-derived suppressor cells via apoptosis inhibition. Exp Cell Res. 2014 Aug 15;326(2):259-66. doi: 10.1016/j.yexcr.2014.04.010. Epub 2014 Apr 26. PMID: 24780820.

571. Srivastava, Minu K et al. "Myeloid-derived suppressor cells inhibit T-cell activation by depleting cystine and cysteine." Cancer research vol. 70,1 (2010): 68-77. doi:10.1158/0008-5472.CAN-09-2587

572. Loftus TJ, Mohr AM, Moldawer LL. Dysregulated myelopoiesis and hematopoietic function following acute physiologic insult. Curr Opin Hematol. 2018 Jan;25(1):37-43. doi: 10.1097/MOH.0000000000000395. PMID: 29035909; PMCID: PMC5733709. 573. Shvedova AA, Kisin ER, Yanamala N, Tkach AV, Gutkin DW, Star A, Shurin GV, Kagan VE, Shurin MR. MDSC and TGF $\beta$ Are Required for Facilitation of Tumor Growth in the Lungs of Mice Exposed to Carbon Nanotubes. Cancer Res. 2015 Apr 15;75(8):1615-23. doi: 10.1158/0008-5472.CAN-14-2376. Epub 2015 Mar 5. PMID: 25744719; PMCID: PMC4401633. 574. Shapiro SD. End-stage chronic obstructive pulmonary disease: the cigarette is burned out but inflammation rages on. Am J Respir Crit Care Med. 2001 Aug 1;164(3):339-40. doi: 10.1164/ajrccm.164.3.2105072c. PMID: 11500330. 
575. Scrimini S, Pons J, Sauleda J. Myeloid-Derived Suppressor Cells: Possible Link Between Chronic Obstrucive Pulmonary Disease and Lung Cancer. Arch Bronconeumol. 2016 Jan;52(1):29-35. English, Spanish. doi: 10.1016/j.arbres.2015.07.003. Epub 2015 Aug 29. PMID: 26321390.

576. Ortiz, Myrna L et al. "Myeloid-derived suppressor cells in the development of lung cancer." Cancer immunology research vol. 2,1 (2014): 50-8. doi:10.1158/2326-6066.CIR-13-0129

577. Foronjy, R F et al. "Protein tyrosine phosphatase 1B negatively regulates S100A9-mediated lung damage during respiratory syncytial virus exacerbations." Mucosal immunology vol. 9,5 (2016): 1317-29. doi:10.1038/mi.2015.138

578. Foronjy RF, Dabo AJ, Taggart CC, Weldon S, Geraghty P. Respiratory syncytial virus infections enhance cigarette smoke induced COPD in mice. PLoS One. 2014 Feb 28;9(2):e90567. doi: 10.1371/journal.pone.0090567. PMID: 24587397; PMCID: PMC3938768.

579. Sikkel MB, Quint JK, Mallia P, Wedzicha JA, Johnston SL. Respiratory syncytial virus persistence in chronic obstructive pulmonary disease. Pediatr Infect Dis J. 2008 Oct;27(10 Suppl):S63-70. doi: 10.1097/INF.0b013e3181684d67. PMID: 18820581. 580. Railwah C, Lora A, Zahid K, Goldenberg H, Campos M, Wyman A, Jundi B, Ploszaj M, Rivas M, Dabo A, Majka SM, Foronjy R, El Gazzar M, Geraghty P. Cigarette smoke induction of S100A9 contributes to chronic obstructive pulmonary disease. Am J Physiol Lung Cell Mol Physiol. 2020 Dec 1;319(6):L1021-L1035. doi: 10.1152/ajplung.00207.2020. Epub 2020 Sep 23. PMID: 32964723; PMCID: PMC7938777.

581. Sattar, Zeeshan et al. "The S100 Protein Family as Players and Therapeutic Targets in Pulmonary Diseases." Pulmonary medicine vol. 2021 5488591. 18 Jun. 2021, doi:10.1155/2021/5488591

582. Xia, Qi \& Wei, Li \& Zhang, Yuntao \& Sheng, Jifang \& Wu, Wei \& Zhang, Yi. (2018). Immune Checkpoint Receptors Tim-3 and PD-1 Regulate Monocyte and T Lymphocyte Function in Septic Patients. Mediators of Inflammation. 2018. 1-8. $10.1155 / 2018 / 1632902$

583. Erickson JJ, Gilchuk P, Hastings AK, Tollefson SJ, Johnson M, Downing MB, Boyd KL, Johnson JE, Kim AS, Joyce S, Williams JV. Viral acute lower respiratory infections impair CD8+ T cells through PD-1. J Clin Invest. 2012 Aug;122(8):2967-82. doi: 10.1172/JCI62860. Epub 2012 Jul 17. PMID: 22797302; PMCID: PMC3408742.

584. Meftahi, Gholam Hossein et al. "The possible pathophysiology mechanism of cytokine storm in elderly adults with COVID-19 infection: the contribution of "inflame-aging"." Inflammation research : official journal of the European Histamine Research Society ... [et al.] vol. 69,9 (2020): 825-839. doi:10.1007/s00011-020-01372-8

585. Conti P, Caraffa A, Tetè G, Gallenga CE, Ross R, Kritas SK, Frydas I, Younes A, Di Emidio P, Ronconi G. Mast cells activated by SARS-CoV-2 release histamine which increases IL-1 levels causing cytokine storm and inflammatory reaction in COVID-19. J Biol Regul Homeost Agents. 2020 Sep-Oct,;34(5):1629-1632. doi: 10.23812/20-2EDIT. PMID: 32945158.

586. Liu J, Haddad EK, Marceau J, Morabito KM, Rao SS, Filali-Mouhim A, Sekaly RP, Graham BS. A Numerically Subdominant CD8 T Cell Response to Matrix Protein of Respiratory Syncytial Virus Controls Infection with Limited Immunopathology. PLoS Pathog. 2016 Mar 4;12(3):e1005486. doi: 10.1371/journal.ppat.1005486. PMID: 26943673; PMCID: PMC4778879.

587. Drewry, Anne M et al. "Persistent lymphopenia after diagnosis of sepsis predicts mortality." Shock (Augusta, Ga.) vol. 42,5 (2014): 383-91. doi:10.1097/SHK.0000000000000234

588. Lin GL, McGinley JP, Drysdale SB, Pollard AJ. Epidemiology and Immune Pathogenesis of Viral Sepsis. Front Immunol. 2018 Sep 27;9:2147. doi: 10.3389/fimmu.2018.02147. PMID: 30319615; PMCID: PMC6170629.

589. Roe, M F E et al. "Lymphocyte apoptosis in acute respiratory syncytial virus bronchiolitis." Clinical and experimental immunology vol. 137,1 (2004): 139-45. doi:10.1111/j.1365-2249.2004.02512.x

590. Troy NM, Bosco A. Respiratory viral infections and host responses; insights from genomics. Respir Res. 2016 Nov 21;17(1):156. doi: 10.1186/s12931-016-0474-9. PMID: 27871304; PMCID: PMC5117516.

591. Erickson JJ, Lu P, Wen S, Hastings AK, Gilchuk P, Joyce S, Shyr Y, Williams JV. Acute Viral Respiratory Infection Rapidly Induces a CD8+ T Cell Exhaustion-like Phenotype. J Immunol. 2015 Nov 1;195(9):4319-30. doi: 10.4049/jimmunol.1403004. Epub 2015 Sep 23. PMID: 26401005; PMCID: PMC4733528.

592. Wyllie DH, Bowler IC, Peto TE. Relation between lymphopenia and bacteraemia in UK adults with medical emergencies. J Clin Pathol. 2004 Sep;57(9):950-5. doi: 10.1136/jcp.2004.017335. PMID: 15333656; PMCID: PMC1770434.

593. Schönrich G, Raftery MJ, Samstag Y. Devilishly radical NETwork in COVID-19: Oxidative stress, neutrophil extracellular traps (NETs), and T cell suppression. Adv Biol Regul. 2020 Aug;77:100741. doi: 10.1016/j.jbior.2020.100741. Epub 2020 Jul 4. PMID: 32773102; PMCID: PMC7334659.

594. Chen S, Xie J, Zhao K, Ren L, Deng Y, Xie X, Chen S, Xu H, Long X, Liu E. LPS aggravates lung inflammation induced by RSV by promoting the ERK-MMP-12 signaling pathway in mice. Respir Res. 2020 Jul 21;21(1):193. doi: 10.1186/s12931-020-01453-6. PMID: 32693803; PMCID: PMC7372760.

595. Zou Y, Chen X, Xiao J, Bo Zhou D, Xiao Lu X, Li W, Xie B, Kuang X, Chen Q. Neutrophil extracellular traps promote lipopolysaccharide-induced airway inflammation and mucus hypersecretion in mice. Oncotarget. 2018 Jan 8;9(17):13276-13286. doi: 10.18632/oncotarget.24022. PMID: 29568356; PMCID: PMC5862577.

596. Funchal, Giselle A et al. "Respiratory syncytial virus fusion protein promotes TLR-4-dependent neutrophil extracellular trap formation by human neutrophils." PloS one vol. 10,4 e0124082. 9 Apr. 2015, doi:10.1371/journal.pone.0124082 597. Li, Haosi et al. "Neutrophil Extracellular Traps Are Pathogenic in Ventilator-Induced Lung Injury and Partially Dependent on TLR4." BioMed research international vol. 2017 (2017): 8272504. doi:10.1155/2017/8272504

598. Cicco S, Cicco G, Racanelli V, Vacca A. Neutrophil Extracellular Traps (NETs) and Damage-Associated Molecular Patterns (DAMPs): Two Potential Targets for COVID-19 Treatment. Mediators Inflamm. 2020 Jul 16;2020:7527953. doi: 10.1155/2020/7527953. PMID: 32724296; PMCID: PMC7366221.

599. Tavakolpour, Soheil et al. "Lymphopenia during the COVID-19 infection: What it shows and what can be learned." Immunology letters vol. 225 (2020): 31-32. doi:10.1016/j.imlet.2020.06.013

600. Atmatzidis S, Koutelidakis IM, Chatzimavroudis G, Kotsaki A, Louis K, Pistiki A, Savva A, Antonopoulou A, Atmatzidis K, Giamarellos-Bourboulis EJ. Detrimental effect of apoptosis of lymphocytes at an early time point of experimental abdominal sepsis. BMC Infect Dis. 2011 Nov 20;11:321. doi: 10.1186/1471-2334-11-321. PMID: 22099496; PMCID: PMC3247197. 601. Perrin-Cocon L, Aublin-Gex A, Sestito SE, Shirey KA, Patel MC, André P, Blanco JC, Vogel SN, Peri F, Lotteau V. TLR4 antagonist FP7 inhibits LPS-induced cytokine production and glycolytic reprogramming in dendritic cells, and protects mice from lethal influenza infection. Sci Rep. 2017 Jan 20;7:40791. doi: 10.1038/srep40791. PMID: 28106157; PMCID: PMC5247753. 602. Jin H, Zhang H, Ma T, Lan H, Feng S, Zhu H, Ji Y. Resveratrol Protects Murine Chondrogenic ATDC5 Cells Against LPS-Induced Inflammatory Injury Through Up-Regulating MiR-146b. Cell Physiol Biochem. 2018;47(3):972-980. doi: 10.1159/000490141. Epub 2018 May 24. PMID: 29843156

603. Olejnik, Judith et al. "Toll-like receptor 4 in acute viral infection: Too much of a good thing." PLoS pathogens vol. 14,12 e1007390. 20 Dec. 2018, doi:10.1371/journal.ppat.1007390 
604. Muraro SP, De Souza GF, Gallo SW, Da Silva BK, De Oliveira SD, Vinolo MAR, Saraiva EM, Porto BN. Respiratory Syncytial Virus induces the classical ROS-dependent NETosis through PAD-4 and necroptosis pathways activation. Sci Rep. 2018 Sep 21;8(1):14166. doi: 10.1038/s41598-018-32576-y. PMID: 30242250; PMCID: PMC6154957.

605. Garofalo RP, Kolli D, Casola A. Respiratory syncytial virus infection: mechanisms of redox control and novel therapeutic opportunities. Antioxid Redox Signal. 2013 Jan 10;18(2):186-217. doi: 10.1089/ars.2011.4307. Epub 2012 Sep 7. PMID: 22799599; PMCID: PMC3513983.

606. Fernandes IG, de Brito CA, Dos Reis VMS, Sato MN, Pereira NZ. SARS-CoV-2 and Other Respiratory Viruses: What Does Oxidative Stress Have to Do with It? Oxid Med Cell Longev. 2020 Dec 21;2020:8844280. doi: 10.1155/2020/8844280. PMID: 33381273; PMCID: PMC7757116.

607. Hotchkiss RS, Osmon SB, Chang KC, Wagner TH, Coopersmith CM, Karl IE. Accelerated lymphocyte death in sepsis occurs by both the death receptor and mitochondrial pathways. J Immunol. 2005 Apr 15;174(8):5110-8. doi: 10.4049/jimmunol.174.8.5110. PMID: 15814742 .

608. Saleh, Jumana et al. "Mitochondria and microbiota dysfunction in COVID-19 pathogenesis." Mitochondrion vol. 54 (2020): 17. doi:10.1016/j.mito.2020.06.008

609. Thompson EA, Cascino K, Ordonez AA, Zhou W, Vaghasia A, Hamacher-Brady A, Brady NR, Sun IH, Wang R, Rosenberg AZ, Delannoy M, Rothman R, Fenstermacher K, Sauer L, Shaw-Saliba K, Bloch EM, Redd AD, Tobian AAR, Horton M, Smith K, Pekosz A, D'Alessio FR, Yegnasubramanian S, Ji H, Cox AL, Powell JD. Metabolic programs define dysfunctional immune responses in severe COVID-19 patients. Cell Rep. 2021 Mar 16;34(11):108863. doi: 10.1016/j.celrep.2021.108863. Epub 2021 Feb 26. PMID: 33691089; PMCID: PMC7908880.

610. Oberkampf M, Guillerey C, Mouriès J, Rosenbaum P, Fayolle C, Bobard A, Savina A, Ogier-Denis E, Enninga J, Amigorena S, Leclerc C, Dadaglio G. Mitochondrial reactive oxygen species regulate the induction of CD8+ T cells by plasmacytoid dendritic cells. Nat Commun. 2018 Jun 8;9(1):2241. doi: 10.1038/s41467-018-04686-8. PMID: 29884826; PMCID: PMC5993805.

611. Lang PA, Xu HC, Grusdat M, Mcllwain DR, Pandyra AA, Harris IS, Shaabani N, Honke N, Maney SK, Lang E, Pozdeev VI, Recher M, Odermatt B, Brenner D, Häussinger D, Ohashi PS, Hengartner H, Zinkernagel RM, Mak TW, Lang KS. Reactive oxygen species delay control of lymphocytic choriomeningitis virus. Cell Death Differ. 2013 Apr;20(4):649-58. doi: 10.1038/cdd.2012.167. Epub 2013 Jan 18. PMID: 23328631; PMCID: PMC3595491.

612. Hildeman, David A et al. "T cell apoptosis and reactive oxygen species." The Journal of clinical investigation vol. 111,5 (2003): 575-81. doi:10.1172/JCl18007

613. Belikov, Aleksey V et al. "T cells and reactive oxygen species." Journal of biomedical science vol. 22 85. 15 Oct. 2015 , doi:10.1186/s12929-015-0194-3

614. Yarosz, Emily L, and Cheong-Hee Chang. "The Role of Reactive Oxygen Species in Regulating T Cell-mediated Immunity and Disease." Immune network vol. 18,1 e14. 22 Feb. 2018, doi:10.4110/in.2018.18.e14

615. Jeisy-Scott, Victoria et al. "Increased MDSC accumulation and Th2 biased response to influenza A virus infection in the absence of TLR7 in mice." PloS one vol. 6,9 (2011): e25242. doi:10.1371/journal.pone.0025242

616. Menezes, Maria Clara Saad et al. "Lower peripheral blood Toll-like receptor 3 expression is associated with an unfavorable outcome in severe COVID-19 patients." Scientific reports vol. 11,1 15223. 27 Jul. 2021, doi:10.1038/s41598-021-94624-4

617. Hüsecken, Yvonne et al. "MDSCs are induced after experimental blunt chest trauma and subsequently alter antigen-specific T cell responses." Scientific reports vol. 7,1 12808. 9 Oct. 2017, doi:10.1038/s41598-017-13019-6

618. Kustermann M, Klingspor M, Huber-Lang M, Debatin KM, Strauss G. Immunostimulatory functions of adoptively transferred MDSCs in experimental blunt chest trauma. Sci Rep. 2019 May 29;9(1):7992. doi: 10.1038/s41598-019-44419-5. PMID: 31142770; PMCID: PMC6541619.

619. Jo, Young-Goun et al. "Natural Killer T Cells Are Numerically and Functionally Deficient in Patients with Trauma." Journal of innate immunity vol. 12,4 (2020): 344-354. doi:10.1159/000504324

620. Jimenez, Rachel V et al. "C-Reactive Protein Promotes the Expansion of Myeloid Derived Cells With Suppressor Functions." Frontiers in immunology vol. 10 2183. 18 Sep. 2019, doi:10.3389/fimmu.2019.02183

621. Schouppe E, Mommer C, Movahedi K, Laoui D, Morias Y, Gysemans C, Luyckx A, De Baetselier P, Van Ginderachter JA. Tumor-induced myeloid-derived suppressor cell subsets exert either inhibitory or stimulatory effects on distinct CD8+ T-cell activation events. Eur J Immunol. 2013 Nov;43(11):2930-42. doi: 10.1002/eji.201343349. Epub 2013 Aug 25. PMID: 23878002. 622. Greifenberg V, Ribechini E, Rössner S, Lutz MB. Myeloid-derived suppressor cell activation by combined LPS and IFN-gamma treatment impairs DC development. Eur J Immunol. 2009 Oct;39(10):2865-76. doi: 10.1002/eji.200939486. PMID: 19637228. 623. Schouppe E, Van Overmeire E, Laoui D, Keirsse J, Van Ginderachter JA. Modulation of CD8(+) T-cell activation events by monocytic and granulocytic myeloid-derived suppressor cells. Immunobiology. 2013 Nov;218(11):1385-91. doi: 10.1016/j.imbio.2013.07.003. Epub 2013 Jul 15. PMID: 23932436.

624. Raber PL, Thevenot P, Sierra R, Wyczechowska D, Halle D, Ramirez ME, Ochoa AC, Fletcher M, Velasco C, Wilk A, Reiss K, Rodriguez PC. Subpopulations of myeloid-derived suppressor cells impair T cell responses through independent nitric oxiderelated pathways. Int J Cancer. 2014 Jun 15;134(12):2853-64. doi: 10.1002/ijc.28622. Epub 2013 Dec 3. PMID: 24259296; PMCID: PMC3980009.

625. Zhu K, Zhang N, Guo N, Yang J, Wang J, Yang C, Yang C, Zhu L, Xu C, Deng Q, Zhu R, Wang H, Chen X, Shi Y, Li Y, Leng Q. SSC(high)CD11b(high)Ly-6C(high)Ly-6G(low) myeloid cells curtail CD4 T cell response by inducible nitric oxide synthase in murine hepatitis. Int J Biochem Cell Biol. 2014 Sep;54:89-97. doi: 10.1016/j.biocel.2014.07.005. Epub 2014 Jul 15. PMID: 25035167. 626. Drabczyk-Pluta M, Werner T, Hoffmann D, Leng Q, Chen L, Dittmer U, Zelinskyy G. Granulocytic myeloid-derived suppressor cells suppress virus-specific CD8+ T cell responses during acute Friend retrovirus infection. Retrovirology. 2017 Aug 23;14(1):42. doi: 10.1186/s12977-017-0364-3. PMID: 28835242; PMCID: PMC5569525.

627. Zelinskyy G, Myers L, Dietze KK, Gibbert K, Roggendorf M, Liu J, Lu M, Kraft AR, Teichgräber V, Hasenkrug KJ, Dittmer U. Virus-specific CD8+ T cells upregulate programmed death-1 expression during acute friend retrovirus infection but are highly cytotoxic and control virus replication. J Immunol. 2011 Oct 1;187(7):3730-7. doi: 10.4049/jimmunol.1101612. Epub 2011 Aug 26. PMID: 21873525; PMCID: PMC3402334.

628. Zelinskyy G, Kraft AR, Schimmer S, Arndt T, Dittmer U. Kinetics of CD8+ effector T cell responses and induced CD4+ regulatory T cell responses during Friend retrovirus infection. Eur J Immunol. 2006 Oct;36(10):2658-70. doi: 10.1002/eji.200636059. PMID: 16981182.

629. Moore TC, Messer RJ, Gonzaga LM, Mather JM, Carmody AB, Bayer W, Littwitz-Salomon E, Dittmer U, Hasenkrug KJ. Effects of Friend Virus Infection and Regulatory T Cells on the Antigen Presentation Function of B Cells. mBio. 2019 Jan 22;10(1):e0257818. doi: 10.1128/mBio.02578-18. PMID: 30670616; PMCID: PMC6343038.

630. Takamura S, Tsuji-Kawahara S, Yagita H, Akiba H, Sakamoto M, Chikaishi T, Kato M, Miyazawa M. Premature terminal exhaustion of Friend virus-specific effector CD8+ T cells by rapid induction of multiple inhibitory receptors. J Immunol. $2010 \mathrm{May}$ 1;184(9):4696-707. doi: 10.4049/jimmunol.0903478. Epub 2010 Mar 29. PMID: 20351188. 
631. David P, Drabczyk-Pluta M, Pastille E, Knuschke T, Werner T, Honke N, Megger DA, Akhmetzyanova I, Shaabani N, EykingSinger A, Cario E, Kershaw O, Gruber AD, Tenbusch M, Dietze KK, Trilling M, Liu J, Schadendorf D, Streeck H, Lang KS, Xie Y, Zimmer L, Sitek B, Paschen A, Westendorf AM, Dittmer U, Zelinskyy G. Combination immunotherapy with anti-PD-L1 antibody and depletion of regulatory $T$ cells during acute viral infections results in improved virus control but lethal immunopathology. PLoS Pathog. 2020 Mar 30;16(3):e1008340. doi: 10.1371/journal.ppat.1008340. PMID: 32226027; PMCID: PMC7105110. 632. Westmeier J, Paniskaki K, Karaköse Z, Werner T, Sutter K, Dolff S, Overbeck M, Limmer A, Liu J, Zheng X, Brenner T, Berger MM, Witzke O, Trilling M, Lu M, Yang D, Babel N, Westhoff T, Dittmer U, Zelinskyy G. Impaired Cytotoxic CD8+ T Cell Response in Elderly COVID-19 Patients. mBio. 2020 Sep 18;11(5):e02243-20. doi: 10.1128/mBio.02243-20. Erratum in: mBio. 2020 Nov 10;11(6): PMID: 32948688; PMCID: PMC7502863.

633. Aghbash PS, Eslami N, Shamekh A, Entezari-Maleki T, Baghi HB. SARS-CoV-2 infection: The role of PD-1/PD-L1 and CTLA-4 axis. Life Sci. 2021 Apr 1;270:119124. doi: 10.1016/j.Ifs.2021.119124. Epub 2021 Jan 27. PMID: 33508291; PMCID: PMC7838580. 634. Darling R, Senapati S, Christiansen J, Liu L, Ramer-Tait AE, Narasimhan B, Wannemuehler M. Polyanhydride Nanoparticles Induce Low Inflammatory Dendritic Cell Activation Resulting in CD8+ T Cell Memory and Delayed Tumor Progression. Int J Nanomedicine. 2020 Sep 7;15:6579-6592. doi: 10.2147/IJN.S261041. PMID: 32982219; PMCID: PMC7490050. 635. Umansky V, Blattner C, Gebhardt C, Utikal J. CCR5 in recruitment and activation of myeloid-derived suppressor cells in melanoma. Cancer Immunol Immunother. 2017 Aug;66(8):1015-1023. doi: 10.1007/s00262-017-1988-9. Epub 2017 Apr 5. PMID: 28382399.

636. Schlecker E, Stojanovic A, Eisen C, Quack C, Falk CS, Umansky V, Cerwenka A. Tumor-infiltrating monocytic myeloid-derived suppressor cells mediate CCR5-dependent recruitment of regulatory T cells favoring tumor growth. J Immunol. $2012 \mathrm{Dec}$ 15;189(12):5602-11. doi: 10.4049/jimmunol.1201018. Epub 2012 Nov 14. PMID: 23152559.

637. El Gazzar M. microRNAs as potential regulators of myeloid-derived suppressor cell expansion. Innate Immun. 2014 Apr;20(3):227-38. doi: 10.1177/1753425913489850. Epub 2013 Jun 11. PMID: 23757323.

638. Paschall AV, Zhang R, Qi CF, Bardhan K, Peng L, Lu G, Yang J, Merad M, McGaha T, Zhou G, Mellor A, Abrams SI, Morse HC 3rd, Ozato $\mathrm{K}$, Xiong $\mathrm{H}$, Liu K. IFN regulatory factor 8 represses GM-CSF expression in T cells to affect myeloid cell lineage differentiation. J Immunol. 2015 Mar 1;194(5):2369-79. doi: 10.4049/jimmunol.1402412. Epub 2015 Feb 2. PMID: 25646302; PMCID: PMC4340766.

639. Salminen A, Kauppinen A, Kaarniranta K. Myeloid-derived suppressor cells (MDSC): an important partner in cellular/tissue senescence. Biogerontology. 2018 Oct;19(5):325-339. doi: 10.1007/s10522-018-9762-8. Epub 2018 Jun 29. PMID: 29959657. 640. Squier TC. Oxidative stress and protein aggregation during biological aging. Exp Gerontol. 2001 Sep;36(9):1539-50. doi: 10.1016/s0531-5565(01)00139-5. PMID: 11525876.

641. Squier TC, Bigelow DJ. Protein oxidation and age-dependent alterations in calcium homeostasis. Front Biosci. 2000 May 1;5:D504-26. doi: 10.2741/squier. PMID: 10799358.

642. Tian H, Gao Z, Wang G, Li H, Zheng J. Estrogen potentiates reactive oxygen species (ROS) tolerance to initiate carcinogenesis and promote cancer malignant transformation. Tumour Biol. 2016 Jan;37(1):141-50. doi: 10.1007/s13277-015-4370-6. Epub 2015 Nov 13. PMID: 26566628.

643. Weyand CM, Shen Y, Goronzy JJ. Redox-sensitive signaling in inflammatory T cells and in autoimmune disease. Free Radic Biol Med. 2018 Sep;125:36-43. doi: 10.1016/j.freeradbiomed.2018.03.004. Epub 2018 Mar 7. PMID: 29524605; PMCID: PMC6128787.

644. Chávez, Miranda D, and Hubert M Tse. "Targeting Mitochondrial-Derived Reactive Oxygen Species in T Cell-Mediated Autoimmune Diseases." Frontiers in immunology vol. 12 703972. 1 Jul. 2021, doi:10.3389/fimmu.2021.703972 645. Imboden JB, Stobo JD. Transmembrane signalling by the T cell antigen receptor. Perturbation of the T3-antigen receptor complex generates inositol phosphates and releases calcium ions from intracellular stores. J Exp Med. 1985 Mar 1;161(3):446-56. doi: 10.1084/jem.161.3.446. PMID: 3919143; PMCID: PMC2187575.

646. Chen J, Ye Y, Liu P, Yu W, Wei F, Li H, Yu J. Suppression of T cells by myeloid-derived suppressor cells in cancer. Hum Immunol. 2017 Feb;78(2):113-119. doi: 10.1016/j.humimm.2016.12.001. Epub 2016 Dec 7. PMID: 27939507.

647. Husain M, Becker EJ Jr, Bone NB, Schmitt A, Pittet JF, Zmijewski JW. NOX2 decoy peptides disrupt trauma-mediated neutrophil immunosuppression and protect against lethal peritonitis. Redox Biol. 2020 Sep;36:101651. doi: 10.1016/j.redox.2020.101651. Epub 2020 Jul 30. PMID: 32771683; PMCID: PMC7415417.

648. Störmann P, Becker N, Künnemeyer L, Wutzler S, Vollrath JT, Lustenberger T, Hildebrand F, Marzi I, Relja B. Contributing factors in the development of acute lung injury in a murine double hit model. Eur J Trauma Emerg Surg. 2020 Feb;46(1):21-30. doi: 10.1007/s00068-019-01121-5. Epub 2019 Apr 1. PMID: 30937460.

649. Kumar V. Pulmonary Innate Immune Response Determines the Outcome of Inflammation During Pneumonia and SepsisAssociated Acute Lung Injury. Front Immunol. 2020 Aug 4;11:1722. doi: 10.3389/fimmu.2020.01722. PMID: 32849610; PMCID: PMC7417316.

650. McVey MJ, Steinberg BE, Goldenberg NM. Inflammasome activation in acute lung injury. Am J Physiol Lung Cell Mol Physiol. 2021 Feb 1;320(2):L165-L178. doi: 10.1152/ajplung.00303.2020. Epub 2020 Dec 9. PMID: 33296269

651. Perl M, Hohmann C, Denk S, Kellermann P, Lu D, Braumüller S, Bachem MG, Thomas J, Knöferl MW, Ayala A, Gebhard F, Huber-Lang MS. Role of activated neutrophils in chest trauma-induced septic acute lung injury. Shock. 2012 Jul;38(1):98-106. doi: 10.1097/SHK.0b013e318254be6a. PMID: 22552016.

652. Appiah SA, Foxx CL, Langgartner D, Palmer A, Zambrano CA, Braumüller S, Schaefer EJ, Wachter U, Elam BL, Radermacher $P$, Stamper CE, Heinze JD, Salazar SN, Luthens AK, Arnold AL, Reber SO, Huber-Lang M, Lowry CA, Halbgebauer R. Evaluation of the gut microbiome in association with biological signatures of inflammation in murine polytrauma and shock. Sci Rep. $2021 \mathrm{Mar}$ 23;11(1):6665. doi: 10.1038/s41598-021-85897-w. PMID: 33758228; PMCID: PMC7988149.

653. Weckbach S, Hohmann C, Denk S, Kellermann P, Huber-Lang MS, Baumann B, Wirth T, Gebhard F, Bachem M, Perl M. Apoptotic and inflammatory signaling via Fas and tumor necrosis factor receptor I contribute to the development of chest trauma-induced septic acute lung injury. J Trauma Acute Care Surg. 2013 Mar;74(3):792-800. doi: 10.1097/TA.0b013e31827a3655. PMID: 23425737.

654. Messer MP, Kellermann P, Weber SJ, Hohmann C, Denk S, Klohs B, Schultze A, Braumüller S, Huber-Lang MS, Perl M. Silencing of fas, fas-associated via death domain, or caspase 3 differentially affects lung inflammation, apoptosis, and development of trauma-induced septic acute lung injury. Shock. 2013 Jan;39(1):19-27. doi: 10.1097/SHK.0b013e318277d856. PMID: 23247118.

655. Niesler U, Palmer A, Radermacher P, Huber-Lang MS. Role of alveolar macrophages in the inflammatory response after trauma. Shock. 2014 Jul;42(1):3-10. doi: 10.1097/SHK.0000000000000167. PMID: 24667621. 656. Chopra M, Reuben JS, Sharma AC. Acute lung injury:apoptosis and signaling mechanisms. Exp Biol Med (Maywood). 2009 Apr;234(4):361-71. doi: 10.3181/0811-MR-318. Epub 2009 Jan 28. PMID: 19176873. 
657. Takano K, Yamamoto S, Tomita K, Takashina M, Yokoo H, Matsuda N, Takano Y, Hattori Y. Successful treatment of acute lung injury with pitavastatin in septic mice: potential role of glucocorticoid receptor expression in alveolar macrophages. J Pharmacol Exp Ther. 2011 Feb;336(2):381-90. doi: 10.1124/jpet.110.171462. Epub 2010 Nov 5. PMID: 21057058.

658. Seitz DH, Perl M, Mangold S, Neddermann A, Braumüller ST, Zhou S, Bachem MG, Huber-Lang MS, Knöferl MW. Pulmonary contusion induces alveolar type 2 epithelial cell apoptosis: role of alveolar macrophages and neutrophils. Shock. 2008 Nov;30(5):537-44. doi: 10.1097/SHK.0b013e31816a394b. PMID: 18317405.

659. Störmann P, Becker N, Vollrath JT, Köhler K, Janicova A, Wutzler S, Hildebrand F, Marzi I, Relja B. Early Local Inhibition of Club Cell Protein 16 Following Chest Trauma Reduces Late Sepsis-Induced Acute Lung Injury. J Clin Med. 2019 Jun 22;8(6):896. doi: 10.3390/jcm8060896. PMID: 31234556; PMCID: PMC6616892.

660. Su VY, Lin CS, Hung SC, Yang KY. Mesenchymal Stem Cell-Conditioned Medium Induces Neutrophil Apoptosis Associated with Inhibition of the NF-KB Pathway in Endotoxin-Induced Acute Lung Injury. Int J Mol Sci. 2019 May 5;20(9):2208. doi: 10.3390/ijms20092208. PMID: 31060326; PMCID: PMC6540353.

661. Vollrath JT, Stoermann P, Becker N, Wutzler S, Hildebrand F, Marzi I, Relja B. Early local neutralization of CC16 in sepsis- induced ALI following blunt chest trauma leads to delayed mortality without benefitting overall survival. Int J Mol Med. 2020 Dec;46(6):2207-2215. doi: 10.3892/ijmm.2020.4767. Epub 2020 Oct 22. PMID: 33125155; PMCID: PMC7595659. 662. Qiao Q, Liu X, Yang T, Cui K, Kong L, Yang C, Zhang Z. Nanomedicine for acute respiratory distress syndrome: The latest application, targeting strategy, and rational design. Acta Pharm Sin B. 2021 May 7. doi: 10.1016/j.apsb.2021.04.023. Epub ahead of print. PMID: 33977080; PMCID: PMC8102084.

663. Relja B, Yang B, Bundkirchen K, Xu B, Köhler K, Neunaber C. Different experimental multiple trauma models induce comparable inflammation and organ injury. Sci Rep. 2020 Nov 19;10(1):20185. doi: 10.1038/s41598-020-76499-z. PMID: 33214576; PMCID: PMC7678855.

664. F

665. F

666. Liu DH, An M, Bao BL, Ren F, Xia P. Nicotine inhibits CD24 expression in Lewis lung carcinoma cells by upregulation of RAS expression. Int J Oncol. 2018 Aug;53(2):815-822. doi: 10.3892/ijo.2018.4415. Epub 2018 May 22. PMID: 29845249.

667. Cavarra E, Bartalesi B, Lucattelli M, Fineschi S, Lunghi B, Gambelli F, Ortiz LA, Martorana PA, Lungarella G. Effects of cigarette smoke in mice with different levels of alpha(1)-proteinase inhibitor and sensitivity to oxidants. Am J Respir Crit Care Med. 2001 Sep 1;164(5):886-90. doi: 10.1164/ajrccm.164.5.2010032. PMID: 11549550.

668. Kristiansen, G., Schlüns, K., Yongwei, Y. et al. CD24 is an independent prognostic marker of survival in nonsmall cell lung cancer patients. Br J Cancer 88, 231-236 (2003). https://doi.org/10.1038/sj.bjc.6600702

669. D. Craig Ayre, Nikitha K. Pallegar, Nicholas A. Fairbridge, Marta Canuti, Andrew S. Lang, Sherri L. Christian, Analysis of the structure, evolution, and expression of CD24, an important regulator of cell fate,

Gene,2016,https://doi.org/10.1016/j.gene.2016.05.038.

670. Niko P. Bretz, Alexei V. Salnikov, Kai Doberstein, Natalio Garbi, Volker Kloess, Safwan Joumaa, Inna Naumov, Louis Boon, Gerhard Moldenhauer, Nadir Arber, Peter Altevogt, Lack of CD24 expression in mice reduces the number of leukocytes in the colon, Immunology Letters, 2014, https://doi.org/10.1016/j.imlet.2014.06.004.

671. Pallegar NK, Ayre DC, Christian SL. Repression of CD24 surface protein expression by oncogenic Ras is relieved by inhibition of Raf but not MEK or PI3K. Front Cell Dev Biol. 2015 Aug 5;3:47. doi: 10.3389/fcell.2015.00047. PMID: 26301220; PMCID: PMC4525067.

672. Dasgupta P, Rastogi S, Pillai S, Ordonez-Ercan D, Morris M, Haura E, Chellappan S. Nicotine induces cell proliferation by betaarrestin-mediated activation of Src and Rb-Raf-1 pathways. J Clin Invest. 2006 Aug;116(8):2208-2217. doi: 10.1172/JCI28164. PMID: 16862215; PMCID: PMC1513051.

673. Chowdhury, Parimal, and Kodetthoor-B Udupa. "Nicotine as a mitogenic stimulus for pancreatic acinar cell proliferation." World journal of gastroenterology vol. 12,46 (2006): 7428-32. doi:10.3748/wjg.v12.i46.7428

674. Chu M, Guo J, Chen CY. Long-term exposure to nicotine, via ras pathway, induces cyclin D1 to stimulate G1 cell cycle transition. J Biol Chem. 2005 Feb 25;280(8):6369-79. doi: 10.1074/jbc.M408947200. Epub 2004 Dec 1. PMID: 15574422. 675. Jull BA, Plummer HK 3rd, Schuller HM. Nicotinic receptor-mediated activation by the tobacco-specific nitrosamine NNK of a Raf-1/MAP kinase pathway, resulting in phosphorylation of c-myc in human small cell lung carcinoma cells and pulmonary neuroendocrine cells. J Cancer Res Clin Oncol. 2001 Dec;127(12):707-17. doi: 10.1007/s004320100289. PMID: 11768610. 676. Schuller HM, Orloff M. Tobacco-specific carcinogenic nitrosamines. Ligands for nicotinic acetylcholine receptors in human lung cancer cells. Biochem Pharmacol. 1998 May 1;55(9):1377-84. doi: 10.1016/s0006-2952(97)00651-5. PMID: 10076528. 677. Schuller HM, Jull BA, Sheppard BJ, Plummer HK. Interaction of tobacco-specific toxicants with the neuronal alpha(7) nicotinic acetylcholine receptor and its associated mitogenic signal transduction pathway: potential role in lung carcinogenesis and pediatric lung disorders. Eur J Pharmacol. 2000 Mar 30;393(1-3):265-77. doi: 10.1016/s0014-2999(00)00094-7. PMID: 10771023. 678. Arredondo J, Chernyavsky AI, Grando SA. Nicotinic receptors mediate tumorigenic action of tobacco-derived nitrosamines on immortalized oral epithelial cells. Cancer Biol Ther. 2006 May;5(5):511-7. doi: 10.4161/cbt.5.5.2601. Epub 2006 May 13. PMID: 16582591.

679. Arredondo J, Chernyavsky AI, Grando SA. The nicotinic receptor antagonists abolish pathobiologic effects of tobacco-derived nitrosamines on BEP2D cells. J Cancer Res Clin Oncol. 2006 Oct;132(10):653-63. doi: 10.1007/s00432-006-0113-9. Epub 2006 Jul 12. PMID: 16835749.

680. Minna JD. Nicotine exposure and bronchial epithelial cell nicotinic acetylcholine receptor expression in the pathogenesis of lung cancer. J Clin Invest. 2003 Jan;111(1):31-3. doi: 10.1172/JCI17492. PMID: 12511585; PMCID: PMC151841.

681. Maneckjee R, Minna JD. Opioid and nicotine receptors affect growth regulation of human lung cancer cell lines. Proc Natl Acad Sci U S A. 1990 May;87(9):3294-8. doi: 10.1073/pnas.87.9.3294. PMID: 2159143; PMCID: PMC53886.

682. Schaal C, Chellappan SP. Nicotine-mediated cell proliferation and tumor progression in smoking-related cancers. Mol Cancer Res. 2014 Jan;12(1):14-23. doi: 10.1158/1541-7786.MCR-13-0541. Epub 2014 Jan 7. PMID: 24398389; PMCID: PMC3915512. 683. Paleari L, Catassi A, Ciarlo M, Cavalieri Z, Bruzzo C, Servent D, Cesario A, Chessa L, Cilli M, Piccardi F, Granone P, Russo P. Role of alpha7-nicotinic acetylcholine receptor in human non-small cell lung cancer proliferation. Cell Prolif. 2008 Dec;41(6):936-59. doi: 10.1111/j.1365-2184.2008.00566.x. Erratum in: Cell Prolif. 2009 Aug;42(4):568. Erratum in: Cell Prolif. 2009 Feb;42(1):122. PMID: 19040571.

684. Schaal C, Chellappan S. Nicotine-Mediated Regulation of Nicotinic Acetylcholine Receptors in Non-Small Cell Lung Adenocarcinoma by E2F1 and STAT1 Transcription Factors. PLoS One. 2016 May 26;11(5):e0156451. doi: 10.1371/journal.pone.0156451. PMID: 27228072; PMCID: PMC4882068. 685. Thaxton, Jessica E et al. "Deletion of CD24 impairs development of heat shock protein gp96-driven autoimmune disease through expansion of myeloid-derived suppressor cells." Journal of immunology (Baltimore, Md. : 1950) vol. 192,12 (2014): 567986. doi:10.4049/jimmunol.1302755 
686. National Center for Chronic Disease Prevention and Health Promotion (US) Office on Smoking and Health. The Health Consequences of Smoking - 50 Years of Progress: A Report of the Surgeon General. Atlanta (GA): Centers for Disease Control and Prevention (US); 2014. PMID: 24455788.

687. Zhang H, Cai B. The impact of tobacco on lung health in China. Respirology. 2003 Mar;8(1):17-21. doi: 10.1046/j.14401843.2003.00433.x. PMID: 12856737.

688. Liu BQ, Peto R, Chen ZM, Boreham J, Wu YP, Li JY, Campbell TC, Chen JS. Emerging tobacco hazards in China: 1 . Retrospective proportional mortality study of one million deaths. BMJ. 1998 Nov 21;317(7170):1411-22. doi: 10.1136/bmj.317.7170.1411. PMID: 9822393; PMCID: PMC28719.

689. Chen Z, Peto R, Zhou M, lona A, Smith M, Yang L, Guo Y, Chen Y, Bian Z, Lancaster G, Sherliker P, Pang S, Wang H, Su H, Wu M, Wu X, Chen J, Collins R, Li L; China Kadoorie Biobank (CKB) collaborative group. Contrasting male and female trends in tobacco-attributed mortality in China: evidence from successive nationwide prospective cohort studies. Lancet. 2015 Oct 10;386(10002):1447-56. doi: 10.1016/S0140-6736(15)00340-2. PMID: 26466050; PMCID: PMC4691901.

690. Malfertheiner P, Schütte K. Smoking--a trigger for chronic inflammation and cancer development in the pancreas. Am J Gastroenterol. 2006 Jan;101(1):160-2. doi: 10.1111/j.1572-0241.2006.00402.x. PMID: 16405549.

691. Wittel UA, Pandey KK, Andrianifahanana M, Johansson SL, Cullen DM, Akhter MP, Brand RE, Prokopczyk B, Batra SK. Chronic pancreatic inflammation induced by environmental tobacco smoke inhalation in rats. Am J Gastroenterol. 2006 Jan;101(1):148-59. doi: 10.1111/j.1572-0241.2006.00405.x. PMID: 16405548.

692. Slattery ML, Edwards S, Curtin K, Schaffer D, Neuhausen S. Associations between smoking, passive smoking, GSTM-1, NAT2, and rectal cancer. Cancer Epidemiol Biomarkers Prev. 2003 Sep;12(9):882-9. PMID: 14504199.

693. Ulrich CM, Bigler J, Whitton JA, Bostick R, Fosdick L, Potter JD. Epoxide hydrolase Tyr113His polymorphism is associated with elevated risk of colorectal polyps in the presence of smoking and high meat intake. Cancer Epidemiol Biomarkers Prev. 2001 Aug;10(8):875-82. PMID: 11489754.

694. Lowenfels AB, Maisonneuve P, Lankisch PG. Chronic pancreatitis and other risk factors for pancreatic cancer. Gastroenterol Clin North Am. 1999 Sep;28(3):673-85, x. doi: 10.1016/s0889-8553(05)70080-7. PMID: 10503143.

695. Heusch WL, Maneckjee R. Signalling pathways involved in nicotine regulation of apoptosis of human lung cancer cells. Carcinogenesis. 1998 Apr;19(4):551-6. doi: 10.1093/carcin/19.4.551. PMID: 9600337.

696. Nakayama H, Numakawa T, Ikeuchi T, Hatanaka H. Nicotine-induced phosphorylation of extracellular signal-regulated protein kinase and CREB in PC12h cells. J Neurochem. 2001 Nov;79(3):489-98. doi: 10.1046/j.1471-4159.2001.00602.x. PMID: 11701752.

697. Nakayama H, Numakawa T, Ikeuchi T. Nicotine-induced phosphorylation of Akt through epidermal growth factor receptor and Src in PC12h cells. J Neurochem. 2002 Dec;83(6):1372-9. doi: 10.1046/j.1471-4159.2002.01248.x. PMID: 12472891.

698. Schuller HM, Porter B, Riechert A, Walker K, Schmoyer R. Neuroendocrine lung carcinogenesis in hamsters is inhibited by green tea or theophylline while the development of adenocarcinomas is promoted: implications for chemoprevention in smokers. Lung Cancer. 2004 Jul;45(1):11-8. doi: 10.1016/j.lungcan.2003.12.007. PMID: 15196729.

699. Tang K, Wu H, Mahata SK, O'Connor DT. A crucial role for the mitogen-activated protein kinase pathway in nicotinic cholinergic signaling to secretory protein transcription in pheochromocytoma cells. Mol Pharmacol. 1998 Jul;54(1):59-69. doi: 10.1124/mol.54.1.59. PMID: 9658190.

700. Wang J, Chen YB, Zhu XN, Chen RZ. Activation of p42/44 mitogen-activated protein kinase pathway in long-term potentiation induced by nicotine in hippocampal CA1 region in rats. Acta Pharmacol Sin. 2001 Aug;22(8):685-90. PMID: 11749838. 701. Cattaneo MG, Codignola A, Vicentini LM, Clementi F, Sher E. Nicotine stimulates a serotonergic autocrine loop in human small-cell lung carcinoma. Cancer Res. 1993 Nov 15;53(22):5566-8. PMID: 8221698.

702. Quik M, Chan J, Patrick J. alpha-Bungarotoxin blocks the nicotinic receptor mediated increase in cell number in a neuroendocrine cell line. Brain Res. 1994 Aug 29;655(1-2):161-7. doi: 10.1016/0006-8993(94)91610-1. PMID: 7812768. 703. Schuller HM. Carbon dioxide potentiates the mitogenic effects of nicotine and its carcinogenic derivative, NNK, in norma and neoplastic neuroendocrine lung cells via stimulation of autocrine and protein kinase C-dependent mitogenic pathways. Neurotoxicology. 1994 Winter;15(4):877-86. PMID: 7715858

704. Jin Z, Gao F, Flagg T, Deng X. Nicotine induces multi-site phosphorylation of Bad in association with suppression of apoptosis. J Biol Chem. 2004 May 28;279(22):23837-44. doi: 10.1074/jbc.M402566200. Epub 2004 Mar 22. PMID: 15037618.

705. Walser, Tonya et al. "Smoking and lung cancer: the role of inflammation." Proceedings of the American Thoracic Society vol. 5,8 (2008): 811-5. doi:10.1513/pats.200809-100TH

706. Engels EA. Inflammation in the development of lung cancer: epidemiological evidence. Expert Rev Anticancer Ther. 2008 Apr;8(4):605-15. doi: 10.1586/14737140.8.4.605. PMID: 18402527.

707. Furrukh, Muhammad. "Tobacco Smoking and Lung Cancer: Perception-changing facts." Sultan Qaboos University medical journal vol. 13,3 (2013): 345-58. doi:10.12816/0003255

708. Yano T, Haro A, Shikada Y, Maruyama R, Maehara Y. Non-small cell lung cancer in never smokers as a representative 'nonsmoking-associated lung cancer': epidemiology and clinical features. Int J Clin Oncol. 2011 Aug;16(4):287-93. doi: 10.1007/s10147-010-0160-8. Epub 2011 May 13. PMID: 21562939.

709. https://www.cancer.org/cancer/lung-cancer/causes-risks-prevention/risk-factors.html

710. Ozlü T, Bülbül Y. Smoking and lung cancer. Tuberk Toraks. 2005;53(2):200-9. PMID: 16100660.

711. Sanner, Tore, and Tom K Grimsrud. "Nicotine: Carcinogenicity and Effects on Response to Cancer Treatment - A Review." Frontiers in oncology vol. 5 196. 31 Aug. 2015, doi:10.3389/fonc.2015.00196

712. Hoffmann D, Brunnemann KD, Prokopczyk B, Djordjevic MV. Tobacco-specific N-nitrosamines and Areca-derived Nnitrosamines: chemistry, biochemistry, carcinogenicity, and relevance to humans. J Toxicol Environ Health. 1994 Jan;41(1):1-52. doi: 10.1080/15287399409531825. PMID: 8277523.

713. Nishioka, T., Luo, LY., Shen, L. et al. Nicotine increases the resistance of lung cancer cells to cisplatin through enhancing Bcl-2 stability. Br J Cancer 110, 1785-1792 (2014). https://doi.org/10.1038/bjc.2014.78

714. Miller DP, De Vivo I, Neuberg D, Wain JC, Lynch TJ, Su L, Christiani DC. Association between self-reported environmental tobacco smoke exposure and lung cancer: modification by GSTP1 polymorphism. Int J Cancer. 2003 May 10;104(6):758-63. doi: 10.1002/ijc.10989. PMID: 12640684.

715. Office on Smoking and Health (US). The Health Consequences of Involuntary Exposure to Tobacco Smoke: A Report of the Surgeon General. Atlanta (GA): Centers for Disease Control and Prevention (US); 2006. Available from: https://www.ncbi.nlm.nih.gov/books/NBK44324/

716. Kim, A-Sol et al. "Exposure to Secondhand Smoke and Risk of Cancer in Never Smokers: A Meta-Analysis of Epidemiologic Studies." International journal of environmental research and public health vol. 15,9 1981. 11 Sep. 2018, doi:10.3390/ijerph15091981 
717. Naeem, Zahid. "Second-hand smoke - ignored implications." International journal of health sciences vol. 9,2 (2015): V-VI. doi:10.12816/0024103

718. Hu J, Yang R, Li DL, Wan YH, Xu HQ, Wang SS, Zhang SC. [Interaction of health literacy and second-hand smoke exposure on psychopathological symptoms of middle school students]. Zhonghua Yu Fang Yi Xue Za Zhi. 2020 Feb 6;54(2):144-148. Chinese. doi: 10.3760/cma.j.issn.0253-9624.2020.02.006. PMID: 32074700

719. Vozoris N, Lougheed MD. Second-hand smoke exposure in Canada: prevalence, risk factors, and association with respiratory and cardiovascular diseases. Can Respir J. 2008 Jul-Aug;15(5):263-9. doi: 10.1155/2008/912354. PMID: 18716689; PMCID: PMC2679549.

720. Miner RN, Crutcher JM. Second-hand tobacco smoke in Oklahoma: a preventable cause of morbidity and mortality and means of reducing exposure. J Okla State Med Assoc. 2002 Mar;95(3):135-41. PMID: 11921863.

721. Kim, S.Y., Sim, S. \& Choi, H.G. Active, passive, and electronic cigarette smoking is associated with asthma in adolescents. Sci Rep 7, 17789 (2017). https://doi.org/10.1038/s41598-017-17958-y

722. Kim, S.Y., Sim, S. \& Choi, H.G. Active and passive smoking impacts on asthma with quantitative and temporal relations: A Korean Community Health Survey. Sci Rep 8, 8614 (2018). https://doi.org/10.1038/s41598-018-26895-3

723. Hess IMR, Lachireddy K, Capon A. A systematic review of the health risks from passive exposure to electronic cigarette vapour. Public Health Res Pract. 2016;26(2):e2621617

724. Bonita R, Duncan J, Truelsen T, Jackson RT, Beaglehole R. Passive smoking as well as active smoking increases the risk of acute stroke. Tob Control. 1999 Summer;8(2):156-60. doi: 10.1136/tc.8.2.156. PMID: 10478399; PMCID: PMC1759715.

725. Johnson KC, Hu J, Mao Y; Canadian Cancer Registries Epidemiology Research Group. Passive and active smoking and breast cancer risk in Canada, 1994-97. Cancer Causes Control. 2000 Mar;11(3):211-21. doi: 10.1023/a:1008906105790. PMID: 10782655. 726. Glantz SA, Parmley WW. Passive smoking and heart disease. Epidemiology, physiology, and biochemistry. Circulation. 1991 Jan;83(1):1-12. doi: 10.1161/01.cir.83.1.1. PMID: 1984876.

727. Mannino DM, Siegel M, Rose D, Nkuchia J, Etzel R. Environmental tobacco smoke exposure in the home and worksite and health effects in adults: results from the 1991 National Health Interview Survey. Tob Control. 1997 Winter;6(4):296-305. doi: 10.1136/tc.6.4.296. PMID: 9583627; PMCID: PMC1759596.

728. Centers for Disease Control and Prevention (US); National Center for Chronic Disease Prevention and Health Promotion (US); Office on Smoking and Health (US). How Tobacco Smoke Causes Disease: The Biology and Behavioral Basis for SmokingAttributable Disease: A Report of the Surgeon General. Atlanta (GA): Centers for Disease Control and Prevention (US); 2010. Available from: https://www.ncbi.nlm.nih.gov/books/NBK53017/

729. Li O, Zheng P, Liu Y. CD24 expression on T cells is required for optimal T cell proliferation in lymphopenic host. J Exp Med. 2004 Oct 18;200(8):1083-9. doi: 10.1084/jem.20040779. Epub 2004 Oct 11. PMID: 15477346; PMCID: PMC2211842.

730. Askew, David, and Clifford V Harding. "Antigen processing and CD24 expression determine antigen presentation by splenic CD4+ and CD8+ dendritic cells." Immunology vol. 123,3 (2008): 447-55. doi:10.1111/j.1365-2567.2007.02711.x

731. Schabath H, Runz S, Joumaa S, Altevogt P. CD24 affects CXCR4 function in pre-B lymphocytes and breast carcinoma cells. J Cell Sci. 2006 Jan 15;119(Pt 2):314-25. doi: 10.1242/jcs.02741. Epub 2006 Jan 3. PMID: 16390867.

732. Lee, Jieun et al. "CD24 enrichment protects while its loss increases susceptibility of juvenile chondrocytes towards inflammation." Arthritis research \& therapy vol. 18,1 292. 12 Dec. 2016, doi:10.1186/s13075-016-1183-y

733. Jackson D, Waibel R, Weber E, Bell J, Stahel RA. CD24, a signal-transducing molecule expressed on human B cells, is a major surface antigen on small cell lung carcinomas. Cancer Res. 1992 Oct 1;52(19):5264-70. PMID: 1327504.

734. Kay R, Rosten PM, Humphries RK. CD24, a signal transducer modulating B cell activation responses, is a very short peptide with a glycosyl phosphatidylinositol membrane anchor. J Immunol. 1991 Aug 15;147(4):1412-6. PMID: 1831224.

735. Sammar M, Siwetz M, Meiri H, Fleming V, Altevogt P, Huppertz B. Expression of CD24 and Siglec-10 in first trimester placenta: implications for immune tolerance at the fetal-maternal interface. Histochem Cell Biol. 2017 May;147(5):565-574. doi: 10.1007/s00418-016-1531-7. Epub 2016 Dec 23. PMID: 28012129.

736. Li D, Zheng L, Jin L, Zhou Y, Li H, Fu J, Shi M, Du P, Wang L, Wu H, Chen GY, Zheng P, Liu Y, Wang FS, Wang S. CD24 polymorphisms affect risk and progression of chronic hepatitis B virus infection. Hepatology. 2009 Sep;50(3):735-42. doi: 10.1002/hep.23047. PMID: 19610054.

737. Wang S, Ma N, Zhao W, Midorikawa K, Kawanishi S, Hiraku Y, Oikawa S, Zhang Z, Huang G, Murata M. Inflammation-Related DNA Damage and Cancer Stem Cell Markers in Nasopharyngeal Carcinoma. Mediators Inflamm. 2016;2016:9343460. doi: 10.1155/2016/9343460. Epub 2016 Aug 28. PMID: 27647953 ; PMCID: PMC5018333.

738. Sheng L, Shui Y. Clusters of differentiation 24 polymorphism and hepatocellular carcinoma. Hepatology. 2011 Dec;54(6):2273; author reply 2273-4. doi: 10.1002/hep.24676. PMID: 21932403.

739. Jia ZF, Wang LZ, Cao XY, Wang C, Cao DH, Wu X, You LL, Jin MS, Wang YP, Zhou BS, Jiang J. CD24 genetic variants contribute to overall survival in patients with gastric cancer. World J Gastroenterol. 2016 Feb 21;22(7):2373-82. doi:

10.3748/wjg.v22.i7.2373. PMID: 26900300; PMCID: PMC4735012.

740. Fang X, Zheng P, Tang J, Liu Y. CD24: from A to Z. Cell Mol Immunol. 2010 Mar;7(2):100-3. doi: 10.1038/cmi.2009.119. Epub 2010 Feb 15. PMID: 20154703; PMCID: PMC4001892.

741. Sammar M, Siwetz M, Meiri H, Sharabi-Nov A, Altevogt P, Huppertz B. Reduced Placental CD24 in Preterm Preeclampsia Is an Indicator for a Failure of Immune Tolerance. Int J Mol Sci. 2021 Jul 28;22(15):8045. doi: 10.3390/ijms22158045. PMID: 34360811; PMCID: PMC8348750.

742. Huang J, Yang Y, Liang Z, Kang M, Kuang Y, Li F. Association between the CD24 Ala57Val polymorphism and risk for multiple sclerosis and systemic lupus erythematosus: a meta-analysis. Sci Rep. 2015 Apr 1;5:9557. doi: 10.1038/srep09557. PMID:

25830931; PMCID: PMC5381688.

743. Lisiansky V, Kraus S, Naumov I, Kazanov D, Nabiochtchikov I, Toledano O, Leshno M, Avivi D, Dotan I, Arber N, Moshkowitz M. Role of CD24 polymorphisms in the susceptibility to inflammatory bowel disease. Int J Biol Markers. 2014 Mar 24;29(1):e62-8. doi: 10.5301/jbm.5000072. PMID: 24557789.

744. Diaz-Gallo LM, Medrano LM, Gómez-García M, Cardeña C, Rodrigo L, Mendoza JL, Taxonera C, Nieto A, Alcain G, Cueto I, López-Nevot MA, Urcelay E, Martin J. Analysis of the influence of two CD24 genetic variants in Crohn's disease and ulcerative colitis. Hum Immunol. 2011 Oct;72(10):969-72. doi: 10.1016/j.humimm.2011.05.028. Epub 2011 Jun 17. PMID: 21684315. 745. Braliou GG, Pantavou KG, Kontou PI, Bagos PG. Polymorphisms of the CD24 Gene Are Associated with Risk of Multiple Sclerosis: A Meta-Analysis. Int J Mol Sci. 2015 Jun 1;16(6):12368-81. doi: 10.3390/ijms160612368. PMID: 26039238; PMCID: PMC4490449.

746. Tan Y, Zhao M, Xiang B, Chang C, Lu Q. CD24: from a Hematopoietic Differentiation Antigen to a Genetic Risk Factor for Multiple Autoimmune Diseases. Clin Rev Allergy Immunol. 2016 Feb;50(1):70-83. doi: 10.1007/s12016-015-8470-2. PMID: 25666875. 
747. Flores-Borja F, Bosma A, Ng D, Reddy V, Ehrenstein MR, Isenberg DA, Mauri C. CD19+CD24hiCD38hi B cells maintain regulatory T cells while limiting TH1 and TH17 differentiation. Sci Transl Med. 2013 Feb 20;5(173):173ra23. doi: 10.1126/scitransImed.3005407. PMID: 23427243.

748. Zheng, Yingxia et al. "miR-155 Regulates IL-10-Producing CD24hiCD27+ B Cells and Impairs Their Function in Patients with Crohn's Disease." Frontiers in immunology vol. 8 914. 3 Aug. 2017, doi:10.3389/fimmu.2017.00914

749. T.

750. 544

751. Liu, Yang et al. “CD24-Siglec G/10 discriminates danger- from pathogen-associated molecular patterns." Trends in immunology vol. 30,12 (2009): 557-61. doi:10.1016/j.it.2009.09.006

752. Chen GY, Tang J, Zheng P, Liu Y. CD24 and Siglec-10 selectively repress tissue damage-induced immune responses. Science. 2009 Mar 27;323(5922):1722-5. doi: 10.1126/science.1168988. Epub 2009 Mar 5. PMID: 19264983; PMCID: PMC2765686.

753. Toubai T, Hou G, Mathewson N, Liu C, Wang Y, Oravecz-Wilson K, Cummings E, Rossi C, Evers R, Sun Y, Wu J, Choi SW, Fang D, Zheng P, Liu Y, Reddy P. Siglec-G-CD24 axis controls the severity of graft-versus-host disease in mice. Blood. 2014 May 29;123(22):3512-23. doi: 10.1182/blood-2013-12-545335. Epub 2014 Apr 2. PMID: 24695850; PMCID: PMC4041170. 754. Chen GY, Brown NK, Zheng P, Liu Y. Siglec-G/10 in self-nonself discrimination of innate and adaptive immunity. Glycobiology. 2014 Sep;24(9):800-6. doi: 10.1093/glycob/cwu068. Epub 2014 Jul 4. PMID: 24996822; PMCID: PMC4116048.

755. Royster, William et al. "The Role of Siglec-G on Immune Cells in Sepsis." Frontiers in immunology vol. 12 621627. 23 Feb. 2021, doi:10.3389/fimmu.2021.621627

756. Millar DG, Garza KM, Odermatt B, Elford AR, Ono N, Li Z, Ohashi PS. Hsp70 promotes antigen-presenting cell function and converts T-cell tolerance to autoimmunity in vivo. Nat Med. 2003 Dec;9(12):1469-76. doi: 10.1038/nm962. Epub 2003 Nov 16. PMID: 14625545.

757. Ospelt C, Camici GG, Engler A, Kolling C, Vogetseder A, Gay RE, Michel BA, Gay S. Smoking induces transcription of the heat shock protein system in the joints. Ann Rheum Dis. 2014 Jul;73(7):1423-6. doi: 10.1136/annrheumdis-2013-204486. Epub 2014 Feb 18. PMID: 24550170.

758. Thyego Mychell Moreira Santos, Irma Godoy, João Paulo Castro Marcondes, Suzana Erico Tanni Minamoto, Thais Garcia, Ilda Godoy, The change of heat shock protein in tobacco users and clinical and laboratory characteristics as markers to evaluate the pathophysiological process promoted by cigarette, European Respiratory Journal Sep 2017, 50 (suppl 61) OA4825; DOI: 10.1183/1393003.congress-2017.0A4825

759. Bobbili, ManojKumar \& Pasupuleti, Swetha \& Manyam, Ravikanth. (2020). "Evaluation of salivary levels of heat shock protein 70 using ELISA among nonsmokers, conventional smokers, and reverse smokers": A prospective study. Biomedical and Biotechnology Research Journal (BBRJ). 4. 220. 10.4103/bbrj.bbrj_47_20.

760. Matsumoto M, Dimayuga PC, Wang C, Kirzner J, Cercek M, Yano J, Chyu KY, Shah PK, Cercek B. Exogenous heat shock protein-70 inhibits cigarette smoke-induced intimal thickening. Am J Physiol Regul Integr Comp Physiol. 2008 Oct;295(4):R1320-7. doi: 10.1152/ajpregu.00624.2007. Epub 2008 Aug 13. PMID: 18703412.

761. Ozawa, Yuichi \& Harutani, Yuhei \& Oyanagi, Jun \& Akamatsu, Hiroaki \& Murakami, Eriko \& Shibaki, Ryota \& Hayata, Atsushi \& Sugimoto, Takeya \& Tanaka, Masanori \& Takakura, Toshiaki \& Furuta, Katsuyuki \& Okuda, Yuka \& Sato, Kouichi \& Teraoka, Shunsuke \& Ueda, Hiroki \& Tokudome, Nahomi \& Kitamura, Yuka \& Fukuoka, Junya \& Nakanishi, Masanori \& Yamamoto, Nobuyuki. (2020). CD24, not CD47, negatively impacts upon response to PD-1/L1 inhibitors in non-small cell lung cancer with PD-L1 TPS. Cancer Science. 112. 10.1111/cas.14705.

762. Fairbridge NA, Southall TM, Ayre DC, Komatsu Y, Raquet PI, Brown RJ, Randell E, Kovacs CS, Christian SL. Loss of CD24 in Mice Leads to Metabolic Dysfunctions and a Reduction in White Adipocyte Tissue. PLoS One. 2015 Nov 4;10(11):e0141966. doi: 10.1371/journal.pone.0141966. PMID: 26536476; PMCID: PMC4633231.

763. Smith, Nicole $\mathrm{C}$ et al. "CD24 is required for regulating gene expression, but not glucose uptake, during adipogenesis." Adipocyte vol. 7,4 (2018): 248-260. doi:10.1080/21623945.2018.1525251

764. Sagiv E, Portman MA. CD24 for Cardiovascular Researchers: A Key Molecule in Cardiac Immunology, Marker of Stem Cells and Target for Drug Development. J Pers Med. 2021 Apr 1;11(4):260. doi: 10.3390/jpm11040260. PMID: 33915986; PMCID: PMC8066264.

765. Wang TW, Chern E, Hsu CW, Tseng KC, Chao HM. SIRT1-Mediated Expression of CD24 and Epigenetic Suppression of Novel Tumor Suppressor miR-1185-1 Increases Colorectal Cancer Stemness. Cancer Res. 2020 Dec 1;80(23):5257-5269. doi: 10.1158/0008-5472.CAN-19-3188. Epub 2020 Oct 12. PMID: 33046442.

766. Shen ZL, Wang B, Jiang KW, Ye CX, Cheng C, Yan YC, Zhang JZ, Yang Y, Gao ZD, Ye YJ, Wang S. Downregulation of miR-199b is associated with distant metastasis in colorectal cancer via activation of SIRT1 and inhibition of CREB/KISS1 signaling. Oncotarget. 2016 Jun 7;7(23):35092-105. doi: 10.18632/oncotarget.9042. PMID: 27145368; PMCID: PMC5085212.

767. Ma W, Xiao GG, Mao J, Lu Y, Song B, Wang L, Fan S, Fan P, Hou Z, Li J, Yu X, Wang B, Wang H, Wang H, Xu F, Li Y, Liu Q, Li L. Dysregulation of the miR-34a-SIRT1 axis inhibits breast cancer stemness. Oncotarget. 2015 Apr 30;6(12):10432-44. doi: 10.18632/oncotarget.3394. PMID: 25826085; PMCID: PMC4496365.

768. Lu RH, Xiao ZQ, Zhou JD, Yin CQ, Chen ZZ, Tang FJ, Wang SH. MiR-199a-5p represses the stemness of cutaneous squamous cell carcinoma stem cells by targeting Sirt1 and CD44ICD cleavage signaling. Cell Cycle. 2020 Jan;19(1):1-14. doi: 10.1080/15384101.2019.1689482. Epub 2019 Dec 6. PMID: 31809227; PMCID: PMC6927716.

769. Altevogt, Peter \& Sammar, Marei \& Hüser, Laura \& Kristiansen, Glen. (2020). Novel insights into the function of CD24: A driving force in cancer. International Journal of Cancer. 148. 10.1002/ijc.33249.

770. Zhang Y, Anoopkumar-Dukie S, Davey AK. SIRT1 and SIRT2 Modulators: Potential Anti-Inflammatory Treatment for Depression? Biomolecules. 2021 Feb 25;11(3):353. doi: 10.3390/biom11030353. PMID: 33669121; PMCID: PMC7996578. 771. Du L, Liu X, Ren Y, Li J, Li P, Jiao Q, Meng P, Wang F, Wang Y, Wang YS, Wang C. Loss of SIRT4 promotes the self-renewal of Breast Cancer Stem Cells. Theranostics. 2020 Jul 25;10(21):9458-9476. doi: 10.7150/thno.44688. PMID: 32863939; PMCID: PMC7449925.

772. Fugle CW, Zhang Y, Hong F, Sun S, Westwater C, Rachidi S, Yu H, Garret-Mayer E, Kirkwood K, Liu B, Li Z. CD24 blunts oral squamous cancer development and dampens the functional expansion of myeloid-derived suppressor cells. Oncoimmunology. 2016 Sep 26;5(10):e1226719. doi: 10.1080/2162402X.2016.1226719. PMID: 27853649; PMCID: PMC5087297.

773. Chen F, Hu SJ. Effect of microRNA-34a in cell cycle, differentiation, and apoptosis: a review. J Biochem Mol Toxicol. 2012 Feb;26(2):79-86. doi: 10.1002/jbt.20412. Epub 2011 Dec 12. PMID: 22162084.

774. Hermeking H. The miR-34 family in cancer and apoptosis. Cell Death Differ. 2010 Feb;17(2):193-9. doi: 10.1038/cdd.2009.56. Epub 2009 May 22. PMID: 19461653.

775. Raver-Shapira N, Marciano E, Meiri E, Spector Y, Rosenfeld N, Moskovits N, Bentwich Z, Oren M. Transcriptional activation of miR-34a contributes to p53-mediated apoptosis. Mol Cell. 2007 Jun 8;26(5):731-43. doi: 10.1016/j.molcel.2007.05.017. Epub 2007 May 31. PMID: 17540598. 
776. Ostrycharz E, Wasik U, Kempinska-Podhorodecka A, Banales JM, Milkiewicz P, Milkiewicz M. Melatonin Protects Cholangiocytes from Oxidative Stress-Induced Proapoptotic and Proinflammatory Stimuli via miR-132 and miR-34. Int J Mol Sci. 2020 Dec 18;21(24):9667. doi: 10.3390/ijms21249667. PMID: 33352965; PMCID: PMC7766218.

777. The PLOS ONE Staff. Correction: CD24 Induces Expression of the Oncomir miR-21 via Src, and CD24 and Src Are Both PostTranscriptionally Downregulated by the Tumor Suppressor miR-34a. PLoS One. 2015 Feb 6;10(2):e0118441. doi: 10.1371/journal.pone.0118441. Erratum for: PLoS One. 2013;8(3):e59563. PMID: 25658602; PMCID: PMC4320079. 778. Khan MJ, Singh P, Dohare R, Jha R, Rahmani AH, Almatroodi SA, Ali S, Syed MA. Inhibition of miRNA-34a Promotes M2 Macrophage Polarization and Improves LPS-Induced Lung Injury by Targeting KIf4. Genes (Basel). 2020 Aug 20;11(9):966. doi: 10.3390/genes11090966. PMID: 32825525; PMCID: PMC7563942.

779. Chen WC, Lai CH, Chuang HC, Lin PY, Chen MF. Inflammation-induced myeloid-derived suppressor cells associated with squamous cell carcinoma of the head and neck. Head Neck. 2017 Feb;39(2):347-355. doi: 10.1002/hed.24595. Epub 2016 Oct 3. PMID: 27696591.

780. Wang, Xin et al. "Twist and miR-34a are involved in the generation of tumor-educated myeloid-derived suppressor cells." International journal of molecular sciences vol. 14,10 20459-77. 14 Oct. 2013, doi:10.3390/ijms141020459

781. Safarzadeh, Elham et al. "MicroRNAs and IncRNAs-A New Layer of Myeloid-Derived Suppressor Cells Regulation." Frontiers in immunology vol. 11 572323. 2 Oct. 2020, doi:10.3389/fimmu.2020.572323

782. Pyzer AR, Cole L, Rosenblatt J, Avigan DE. Myeloid-derived suppressor cells as effectors of immune suppression in cancer. Int J Cancer. 2016 Nov 1;139(9):1915-26. doi: 10.1002/ijc.30232. Epub 2016 Jul 12. PMID: 27299510.

783. Jiang, Jingwei et al. "MicroRNA expression profiles of granulocytic myeloid- derived suppressor cells from mice bearing Lewis lung carcinoma." Molecular medicine reports vol. 14,5 (2016): 4567-4574. doi:10.3892/mmr.2016.5845

784. Liu Y, Hu X, Xia D, Zhang S. MicroRNA-181b is downregulated in non-small cell lung cancer and inhibits cell motility by directly targeting HMGB1. Oncol Lett. 2016 Nov;12(5):4181-4186. doi: 10.3892/ol.2016.5198. Epub 2016 Sep 28. PMID: 27895789; PMCID: PMC5104252.

785. Wang, Yazhen et al. "MicroRNA-181b stimulates inflammation via the nuclear factor-kB signaling pathway in vitro." Experimental and therapeutic medicine vol. 10,4 (2015): 1584-1590. doi:10.3892/etm.2015.2702

786. Iliopoulos D, Jaeger SA, Hirsch HA, Bulyk ML, Struhl K. STAT3 activation of miR-21 and miR-181b-1 via PTEN and CYLD are part of the epigenetic switch linking inflammation to cancer. Mol Cell. 2010 Aug 27;39(4):493-506. doi: 10.1016/j.molcel.2010.07.023. PMID: 20797623; PMCID: PMC2929389.

787. Wegner A, Verhagen J, Wraith DC. Myeloid-derived suppressor cells mediate tolerance induction in autoimmune disease. Immunology. 2017 May;151(1):26-42. doi: 10.1111/imm.12718. Epub 2017 Mar 2. PMID: 28140447; PMCID: PMC5382345. 788. Shen J, Chen X, Wang Z, Zhang G, Chen W. Downregulation of CD40 expression contributes to the accumulation of myeloidderived suppressor cells in gastric tumors. Oncol Lett. 2014 Aug;8(2):775-780. doi: 10.3892/ol.2014.2174. Epub 2014 May 26. PMID: 25009656; PMCID: PMC4081434.

789. Lamping N, Dettmer R, Schröder NW, Pfeil D, Hallatschek W, Burger R, Schumann RR. LPS-binding protein protects mice from septic shock caused by LPS or gram-negative bacteria. J Clin Invest. 1998 May 15;101(10):2065-71. doi: 10.1172/JCI2338. PMID: 9593762; PMCID: PMC508794.

790. Opal, Steven \& Scannon, Patrick J \& Vincent, Jean-Louis \& White, Mark \& Carroll, Stephen F \& Palardy, John \& Parejo, Nicolas A \& Pribble, John \& Lemke, Jon. (1999). Relationship between Plasma Levels of Lipopolysaccharide (LPS) and LPS-Binding Protein in Patients with Severe Sepsis and Septic Shock. The Journal of infectious diseases. 180. 1584-9. 10.1086/315093. 791. Halawa, Amal \& Eladl, Mohamed \& Hamed, Mohamed \& Balboula, Ahmed \& Elmetwally, Mohammed. (2018). Lipopolysaccharide Prompts Oxidative Stress and Apoptosis in Rats' Testicular Tissue. Journal of Veterinary Healthcare. 1. 20-31. 10.14302/issn.2575-1212.jvhc-18-2013.

792. Villar, Jesús et al. "Serum lipopolysaccharide binding protein levels predict severity of lung injury and mortality in patients with severe sepsis." PloS one vol. 4,8 e6818. 31 Aug. 2009, doi:10.1371/journal.pone.0006818

793. Chen KF, Chaou CH, Jiang JY, Yu HW, Meng YH, Tang WC, Wu CC. Diagnostic Accuracy of Lipopolysaccharide-Binding Protein as Biomarker for Sepsis in Adult Patients: A Systematic Review and Meta-Analysis. PLoS One. 2016 Apr 7;11(4):e0153188. doi: 10.1371/journal.pone.0153188. PMID: 27055115; PMCID: PMC4824361.

794. Zhang A, Cai Y, Wang PF, Qu JN, Luo ZC, Chen XD, Huang B, Liu Y, Huang WQ, Wu J, Yin YH. Diagnosis and prognosis of neutrophil gelatinase-associated lipocalin for acute kidney injury with sepsis: a systematic review and meta-analysis. Crit Care. 2016 Feb 16;20:41. doi: 10.1186/s13054-016-1212-x. PMID: 26880194; PMCID: PMC4754917.

795. Sakr Y, Burgett U, Nacul FE, Reinhart K, Brunkhorst F. Lipopolysaccharide binding protein in a surgical intensive care unit: a marker of sepsis? Crit Care Med. 2008 Jul;36(7):2014-22. doi: 10.1097/CCM.0b013e31817b86e3. PMID: 18552695.

796. Şimşek, Özgür et al. "Thiol/Disulfide Balance in Patients with Familial Hypercholesterolemia." Cardiology research and practice vol. 2018 9042461. 12 Jun. 2018, doi:10.1155/2018/9042461

797. Tauman R, Shalitin S, Lavie L. Oxidative stress in obese children and adolescents with and without type 2 diabetes mellitus is not associated with obstructive sleep apnea. Sleep Breath. 2019 Mar;23(1):117-123. doi: 10.1007/s11325-018-1670-2. Epub 2018 May 26. PMID: 29804216

798. Qiu, Chao et al. "Early adaptive humoral immune responses and virus clearance in humans recently infected with pandemic 2009 H1N1 influenza virus." PloS one vol. 6,8 (2011): e22603. doi:10.1371/journal.pone.0022603

799. Wang DY, Shu YL. [Pre-existing immunity is an essential factor for mild pandemic H1N1 2009]. Bing Du Xue Bao. 2011 May;27(3):304-7. Chinese. PMID: 21774259.

800. Zheng S, Fan J, Yu F, Feng B, Lou B, Zou Q, Xie G, Lin S, Wang R, Yang X, Chen W, Wang Q, Zhang D, Liu Y, Gong R, Ma Z, Lu S, Xiao Y, Gu Y, Zhang J, Yao H, Xu K, Lu X, Wei G, Zhou J, Fang Q, Cai H, Qiu Y, Sheng J, Chen Y, Liang T. Viral load dynamics and disease severity in patients infected with SARS-CoV-2 in Zhejiang province, China, January-March 2020: retrospective cohort study. BMJ. 2020 Apr 21;369:m1443. doi: 10.1136/bmj.m1443. PMID: 32317267; PMCID: PMC7190077.

801. Danov, Olga et al. "Cigarette Smoke Affects Dendritic Cell Populations, Epithelial Barrier Function, and the Immune Response to Viral Infection With H1N1." Frontiers in medicine vol. 7 571003. 6 Nov. 2020, doi:10.3389/fmed.2020.571003

802. Hijano DR, Brazelton de Cardenas J, Maron G, Garner CD, Ferrolino JA, Dallas RH, Gu Z, Hayden RT. Clinical correlation of influenza and respiratory syncytial virus load measured by digital PCR. PLoS One. 2019 Sep 3;14(9):e0220908. doi: 10.1371/journal.pone.0220908. PMID: 31479459; PMCID: PMC6720028.

803. Chen, Gang et al. "Cigarette Smoke Disturbs the Survival of CD8+ Tc/Tregs Partially through Muscarinic ReceptorsDependent Mechanisms in Chronic Obstructive Pulmonary Disease." PloS one vol. 11,1 e0147232. 25 Jan. 2016, doi:10.1371/journal.pone.0147232

804. Bermejo-Martin, Jesus F et al. "Host adaptive immunity deficiency in severe pandemic influenza." Critical care (London, England) vol. 14,5 (2010): R167. doi:10.1186/cc9259 
805. To KK, Hung IF, Li IW, Lee KL, Koo CK, Yan WW, Liu R, Ho KY, Chu KH, Watt CL, Luk WK, Lai KY, Chow FL, Mok T, Buckley T, Chan JF, Wong SS, Zheng B, Chen H, Lau CC, Tse H, Cheng VC, Chan KH, Yuen KY. Delayed clearance of viral load and marked cytokine activation in severe cases of pandemic H1N1 2009 influenza virus infection. Clin Infect Dis. 2010 Mar 15;50(6):850-9. doi: 10.1086/650581. PMID: 20136415; PMCID: PMC7107930

806. Cheemarla NR, Watkins TA, Mihaylova VT, Wang B, Zhao D, Wang G, Landry ML, Foxman EF. Dynamic innate immune response determines susceptibility to SARS-CoV-2 infection and early replication kinetics. J Exp Med. 2021 Aug 2;218(8):e20210583. doi: 10.1084/jem.20210583. Epub 2021 Jun 15. PMID: 34128960; PMCID: PMC8210587. 807. Mahallawi WH, Alsamiri AD, Dabbour AF, Alsaeedi H, Al-Zalabani AH. Association of Viral Load in SARS-CoV-2 Patients With Age and Gender. Front Med (Lausanne). 2021 Jan 27;8:608215. doi: 10.3389/fmed.2021.608215. PMID: 33585523; PMCID: PMC7873591.

808. Busnadiego, Idoia et al. "Antiviral Activity of Type I, II, and III Interferons Counterbalances ACE2 Inducibility and Restricts SARS-CoV-2." mBio vol. 11,5 e01928-20. 10 Sep. 2020, doi:10.1128/mBio.01928-20

809. Vanderheiden A, Ralfs P, Chirkova T, Upadhyay AA, Zimmerman MG, Bedoya S, Aoued H, Tharp GM, Pellegrini KL, Manfredi C, Sorscher E, Mainou B, Lobby JL, Kohlmeier JE, Lowen AC, Shi PY, Menachery VD, Anderson L, Grakoui A, Bosinger SE, Suthar MS. Type I and Type III Interferons Restrict SARS-CoV-2 Infection of Human Airway Epithelial Cultures. J Virol. 2020 Sep 15;94(19):e00985-20. doi: 10.1128/JVI.00985-20. PMID: 32699094; PMCID: PMC7495371.

810. Akondy, Rama \& Johnson, Philip \& Nakaya, Helder \& Edupguanti, Sri \& Mulligan, Mark \& Lawson, Benton \& Miller, Joseph \& Pulendran, Bali \& Antia, Rustom \& Ahmed, Rafi. (2015). Initial viral load determines the magnitude of the human CD8 T cell response to yellow fever vaccination. Proceedings of the National Academy of Sciences. 112. 10.1073/pnas.1500475112. 811. Marois I, Cloutier A, Garneau É, Richter MV. Initial infectious dose dictates the innate, adaptive, and memory responses to influenza in the respiratory tract. J Leukoc Biol. 2012 Jul;92(1):107-21. doi: 10.1189/jlb.1011490. Epub 2012 Apr 13. PMID: 22504848.

812. Argyropoulos KV, Serrano A, Hu J, Black M, Feng X, Shen G, Call M, Kim MJ, Lytle A, Belovarac B, Vougiouklakis T, Lin LH, Moran U, Heguy A, Troxel A, Snuderl M, Osman I, Cotzia P, Jour G. Association of Initial Viral Load in Severe Acute Respiratory Syndrome Coronavirus 2 (SARS-CoV-2) Patients with Outcome and Symptoms. Am J Pathol. 2020 Sep;190(9):1881-1887. doi: 10.1016/j.ajpath.2020.07.001. Epub 2020 Jul 3. PMID: 32628931; PMCID: PMC7332909.

813. Paranjpe I, Chaudhary K, Johnson KW, Jaladanki SK, Zhao S, De Freitas JK, Pujdas E, Chaudhry F, Bottinger EP, Levin MA, Fayad ZA, Charney AW, Houldsworth J, Cordon-Cardo C, Glicksberg BS, Nadkarni GN. Association of SARS-CoV-2 viral load at admission with in-hospital acute kidney injury: A retrospective cohort study. PLoS One. 2021 Feb 24;16(2):e0247366. doi: 10.1371/journal.pone.0247366. PMID: 33626098; PMCID: PMC7904214.

814. Involvement of Interferon Regulatory Factor 7 in Nicotine's Suppression of Antiviral Immune Responses

815. Li, Yajuan et al. "A Low Viral Dose in COVID-19 Patient: A Case Report." Frontiers in public health vol. 8339.26 Jun. 2020 , doi:10.3389/fpubh.2020.00339

816. AlQasrawi, Dania, and Saleh A Naser. "Nicotine Modulates MyD88-Dependent Signaling Pathway in Macrophages during Mycobacterial Infection." Microorganisms vol. 8,11 1804. 17 Nov. 2020, doi:10.3390/microorganisms8111804

817. Tacke F, Spehlmann ME, Vucur M, Benz F, Luedde M, Cardenas DV, Roy S, Loosen S, Hippe HJ, Frey N, Trautwein C, Koch A, Roderburg C, Luedde T. miR-155 Predicts Long-Term Mortality in Critically III Patients Younger than 65 Years. Mediators Inflamm. 2019 Feb 24;2019:6714080. doi: 10.1155/2019/6714080. PMID: 30918471; PMCID: PMC6409014.

818. Daveri, Elena et al. "microRNAs Shape Myeloid Cell-Mediated Resistance to Cancer Immunotherapy." Frontiers in immunology vol. 11 1214. 22 Jul. 2020, doi:10.3389/fimmu.2020.01214

819. Martinez-Usatorre A, Sempere LF, Carmona SJ, Carretero-Iglesia L, Monnot G, Speiser DE, Rufer N, Donda A, Zehn D, Jandus C, Romero P. MicroRNA-155 Expression Is Enhanced by T-cell Receptor Stimulation Strength and Correlates with Improved Tumor Control in Melanoma. Cancer Immunol Res. 2019 Jun;7(6):1013-1024. doi: 10.1158/2326-6066.CIR-18-0504. Epub 2019 May 1 PMID: 31043416.

820. Verdon DJ, Mulazzani M, Jenkins MR. Cellular and Molecular Mechanisms of CD8+ T Cell Differentiation, Dysfunction and Exhaustion. Int J Mol Sci. 2020 Oct 5;21(19):7357. doi: 10.3390/ijms21197357. PMID: 33027962; PMCID: PMC7582856.

821. Lam, Jonathan $\mathrm{H}$, and Nicole Baumgarth. "The Multifaceted B Cell Response to Influenza Virus." Journal of immunology (Baltimore, Md. : 1950) vol. 202,2 (2019): 351-359. doi:10.4049/jimmunol.1801208

822. Sun Z, Smyth K, Garcia K, Mattson E, Li L, Xiao Z. Nicotine inhibits memory CTL programming. PLoS One. 2013 Jul 2;8(7):e68183. doi: 10.1371/journal.pone.0068183. PMID: 23844169; PMCID: PMC3699522.

823. Huang, Yongbo et al. "SARS-CoV-2 Viral Load in Clinical Samples from Critically III Patients." American journal of respiratory and critical care medicine vol. 201,11 (2020): 1435-1438. doi:10.1164/rccm.202003-0572LE

824. Xu Y, Zhang Y, Cardell LO. Nicotine exaggerates LPS-induced airway hyperreactivity via JNK-mediated up-regulation of Tolllike receptor 4. Am J Respir Cell Mol Biol. 2014 Sep;51(3):370-9. doi: 10.1165/rcmb.2013-04090C. PMID: 24669857.

825. Fajnzylber J, Regan J, Coxen K, Corry H, Wong C, Rosenthal A, Worrall D, Giguel F, Piechocka-Trocha A, Atyeo C, Fischinger S, Chan A, Flaherty KT, Hall K, Dougan M, Ryan ET, Gillespie E, Chishti R, Li Y, Jilg N, Hanidziar D, Baron RM, Baden L, Tsibris AM, Armstrong KA, Kuritzkes DR, Alter G, Walker BD, Yu X, Li JZ; Massachusetts Consortium for Pathogen Readiness. SARS-CoV-2 viral load is associated with increased disease severity and mortality. Nat Commun. 2020 Oct 30;11(1):5493. doi: 10.1038/s41467-02019057-5. PMID: 33127906; PMCID: PMC7603483.

826. Mirzapoiazova T, Kolosova IA, Moreno L, Sammani S, Garcia JG, Verin AD. Suppression of endotoxin-induced inflammation by taxol. Eur Respir J. 2007 Sep;30(3):429-35. doi: 10.1183/09031936.00154206. Epub 2007 May 30. PMID: 17537765 827. Chen CH, Sheu MT, Chen TF, Wang YC, Hou WC, Liu DZ, Chung TC, Liang YC. Suppression of endotoxin-induced proinflammatory responses by citrus pectin through blocking LPS signaling pathways. Biochem Pharmacol. 2006 Oct 16;72(8):1001-9. doi: 10.1016/j.bcp.2006.07.001. Epub 2006 Aug 22. PMID: 16930561.

828. Schulte-Schrepping, Jonas et al. "Severe COVID-19 Is Marked by a Dysregulated Myeloid Cell Compartment." Cell vol. 182,6 (2020): 1419-1440.e23. doi:10.1016/j.cell.2020.08.001

829. Dhangadamajhi, Gunanidhi, and Ronnaly Rout. "Association of TLR3 functional variant (rs3775291) with COVID-19 susceptibility and death: a population-scale study." Human cell vol. 34,3 (2021): 1025-1027. doi:10.1007/s13577-021-00510-6 830. Totura AL, Whitmore A, Agnihothram S, Schäfer A, Katze MG, Heise MT, Baric RS. Toll-Like Receptor 3 Signaling via TRIF Contributes to a Protective Innate Immune Response to Severe Acute Respiratory Syndrome Coronavirus Infection. $\mathrm{mBio}$. 2015 May 26;6(3):e00638-15. doi: 10.1128/mBio.00638-15. PMID: 26015500; PMCID: PMC4447251.

831. Megjugorac NJ, Young HA, Amrute SB, Olshalsky SL, Fitzgerald-Bocarsly P. Virally stimulated plasmacytoid dendritic cells produce chemokines and induce migration of T and NK cells. J Leukoc Biol. 2004 Mar;75(3):504-14. doi: 10.1189/jlb.0603291. Epub 2004 Jan 23. PMID: 14742635

832. Amitai A. Viral surface geometry shapes influenza and coronavirus spike evolution through antibody pressure. bioRxiv [Preprint]. 2020 Dec 21:2020.10.20.347641. doi: 10.1101/2020.10.20.347641. PMID: 33106808; PMCID: PMC7587782. 
833. Petruk G, Puthia M, Petrlova J, Samsudin F, Strömdahl AC, Cerps S, Uller L, Kjellström S, Bond PJ, Schmidtchen AA. SARS-CoV2 spike protein binds to bacterial lipopolysaccharide and boosts proinflammatory activity. J Mol Cell Biol. 2020 Oct 12;12(12):916932. doi: 10.1093/jmcb/mjaa067. PMID: 33295606; PMCID: PMC7799037.

834. Carissimo G, Ng LFP. A promiscuous interaction of SARS-CoV-2 with bacterial products. J Mol Cell Biol. 2020 Nov 18;12(12):914-915. doi: 10.1093/jmcb/mjaa068. PMID: 33326034; PMCID: PMC7798951.

835. Robbins CS, Dawe DE, Goncharova SI, Pouladi MA, Drannik AG, Swirski FK, Cox G, Stämpfli MR. Cigarette smoke decreases pulmonary dendritic cells and impacts antiviral immune responsiveness. Am J Respir Cell Mol Biol. 2004 Feb;30(2):202-11. doi: 10.1165/rcmb.2003-02590C. Epub 2003 Aug 14. PMID: 12920055.

836. (835).

837. Fusby JS, Kassmeier MD, Palmer VL, Perry GA, Anderson DK, Hackfort BT, Alvarez GK, Cullen DM, Akhter MP, Swanson PC. Cigarette smoke-induced effects on bone marrow B-cell subsets and CD4+:CD8+ T-cell ratios are reversed by smoking cessation: influence of bone mass on immune cell response to and recovery from smoke exposure. Inhal Toxicol. 2010 Aug;22(9):785-96. doi: 10.3109/08958378.2010.483258. PMID: 20482464.

838. Nagarkatti M, Nagarkatti PS, Brooks A. Effect of radon on the immune system: alterations in the cellularity and functions of T cells in lymphoid organs of mouse. J Toxicol Environ Health. 1996 Apr 19;47(6):535-52. doi: 10.1080/009841096161528. PMID: 8614022.

839. Zhao W, Schafer R, Cuff CF, Gandy J, Barnett JB. Changes in primary and secondary lymphoid organ T-cell subpopulations resulting from acute in vivo exposure to propanil. J Toxicol Environ Health. 1995 Oct;46(2):171-81. doi: 10.1080/15287399509532027. PMID: 7563216.

840. Sheil JM, Frankenberry MA, Schell TD, Brundage KM, Barnett JB. Propanil exposure induces delayed but sustained abrogation of cell-mediated immunity through direct interference with cytotoxic T-lymphocyte effectors. Environ Health Perspect. 2006 Jul;114(7):1059-64. doi: 10.1289/ehp.8774. PMID: 16835059; PMCID: PMC1513295.

841. Salazar KD, Ustyugova IV, Brundage KM, Barnett JB, Schafer R. A review of the immunotoxicity of the pesticide 3,4dichloropropionanalide. J Toxicol Environ Health B Crit Rev. 2008 Oct;11(8):630-45. doi: 10.1080/10937400701724386. PMID: 18821422.

842. Singh SP, Razani-Boroujerdi S, Pena-Philippides JC, Langley RJ, Mishra NC, Sopori ML. Early postnatal exposure to cigarette smoke impairs the antigen-specific T-cell responses in the spleen. Toxicol Lett. 2006 Dec 15;167(3):231-7. doi: 10.1016/j.toxlet.2006.10.001. Epub 2006 Oct 10. PMID: 17113252.

843. Kalra R, Singh SP, Savage SM, Finch GL, Sopori ML. Effects of cigarette smoke on immune response: chronic exposure to cigarette smoke impairs antigen-mediated signaling in T cells and depletes IP3-sensitive $\mathrm{Ca}(2+)$ stores. J Pharmacol Exp Ther. 2000 Apr;293(1):166-71. PMID: 10734166.

844. Geng Y, Savage SM, Razani-Boroujerdi S, Sopori ML. Effects of nicotine on the immune response. II. Chronic nicotine treatment induces T cell anergy. J Immunol. 1996 Apr 1;156(7):2384-90. PMID: 8786295.

845. Singh SP, Kalra R, Puttfarcken P, Kozak A, Tesfaigzi J, Sopori ML. Acute and chronic nicotine exposures modulate the immune system through different pathways. Toxicol Appl Pharmacol. 2000 Apr 1;164(1):65-72. doi: 10.1006/taap.2000.8897. PMID: 10739745.

846. Weber GF, Mirza NM, Yunis EJ, Dubey D, Cantor H. Localization and treatment of an oxidation-sensitive defect within the TCR-coupled signalling pathway that is associated with normal and premature immunologic aging. Growth Dev Aging. 1997 FallWinter;61(3-4):191-207. PMID: 9546110.

847. Whelan JP, Shearer WT, Gilliam EB, Hardy KJ. A protein kinase C-activating phorbol ester accelerates the T cell antigen receptor-stimulated phosphatidylinositol cycle in normal human CD4+ T cells. J Immunol. 1992 May 1;148(9):2872-8. PMID: 1349321.

848. Alberola-Ila J, Takaki S, Kerner JD, Perlmutter RM. Differential signaling by lymphocyte antigen receptors. Annu Rev Immunol. 1997;15:125-54. doi: 10.1146/annurev.immunol.15.1.125. PMID: 9143684

849. Zavitz CC, Gaschler GJ, Robbins CS, Botelho FM, Cox PG, Stampfli MR. Impact of cigarette smoke on T and B cell responsiveness. Cell Immunol. 2008 May-Jun;253(1-2):38-44. doi: 10.1016/j.cellimm.2008.04.012. Epub 2008 Jun 3. PMID: 18533139.

850. Yamada Y, Tomaru U, Ishizu A, Ito T, Kiuchi T, Ono A, Miyajima S, Nagai K, Higashi T, Matsuno Y, Dosaka-Akita H, Nishimura M, Miwa S, Kasahara M. Decreased proteasomal function accelerates cigarette smoke-induced pulmonary emphysema in mice. Lab Invest. 2015 Jun;95(6):625-34. doi: 10.1038/labinvest.2015.43. Epub 2015 Apr 27. PMID: 25915723.

851. Bodas M, Vij N. Augmenting autophagy for prognosis based intervention of COPD-pathophysiology. Respir Res. 2017 May 4;18(1):83. doi: 10.1186/s12931-017-0560-7. PMID: 28472967; PMCID: PMC5418861.

852. Bernhard D, Moser C, Backovic A, Wick G. Cigarette smoke--an aging accelerator? Exp Gerontol. 2007 Mar;42(3):160-5. doi: 10.1016/j.exger.2006.09.016. Epub 2006 Nov 3. PMID: 17084574.

853. Tejero JD, Armand NC, Finn CM, Dhume K, Strutt TM, Chai KX, Chen LM, McKinstry KK. Cigarette smoke extract acts directly on CD4 T cells to enhance Th1 polarization and reduce memory potential. Cell Immunol. 2018 Sep;331:121-129. doi: 10.1016/j.cellimm.2018.06.005. Epub 2018 Jun 18. PMID: 29935764; PMCID: PMC6092241.

854. Das A, Ranganathan V, Umar D, Thukral S, George A, Rath S, Bal V. Effector/memory CD4 T cells making either Th1 or Th2 cytokines commonly co-express T-bet and GATA-3. PLoS One. 2017 Oct 31;12(10):e0185932. doi: 10.1371/journal.pone.0185932. PMID: 29088218; PMCID: PMC5663332.

855. Qin Z, Wang PY, Wan JJ, Zhang Y, Wei J, Sun Y, Liu X. MicroRNA124-IL6R Mediates the Effect of Nicotine in Inflammatory Bowel Disease by Shifting Th1/Th2 Balance Toward Th1. Front Immunol. 2020 Feb 21;11:235. doi: 10.3389/fimmu.2020.00235. PMID: 32153570; PMCID: PMC7050625.

856. Bai X, Aerts SL, Verma D, Ordway DJ, Chan ED. Epidemiologic Evidence of and Potential Mechanisms by Which Second-Hand Smoke Causes Predisposition to Latent and Active Tuberculosis. Immune Netw. 2018 Jun 26;18(3):e22. doi: 10.4110/in.2018.18.e22. PMID: 29984040; PMCID: PMC6026693.

857. Kootbodien T, Iyaloo S, Wilson K, Naicker N, Kgalamono S, Haman T, Mathee A, Rees D. Environmental Silica Dust Exposure and Pulmonary Tuberculosis in Johannesburg, South Africa. Int J Environ Res Public Health. 2019 May 27;16(10):1867. doi: 10.3390/ijerph16101867. PMID: 31137851; PMCID: PMC6571666.

858. Patra J, Bhatia M, Suraweera W, Morris SK, Patra C, Gupta PC, Jha P. Exposure to second-hand smoke and the risk of tuberculosis in children and adults: a systematic review and meta-analysis of 18 observational studies. PLoS Med. 2015 Jun 2;12(6):e1001835; discussion e1001835. doi: 10.1371/journal.pmed.1001835. PMID: 26035557; PMCID: PMC4452762. 859. SPLOS Medicine Staff. Correction: Active or Passive Exposure to Tobacco Smoking and Allergic Rhinitis, Allergic Dermatitis, and Food Allergy in Adults and Children: A Systematic Review and Meta-Analysis. PLoS Med. 2016 Feb 2;13(2):e1001939. doi: 10.1371/journal.pmed.1001939. Erratum for: PLoS Med. 2014 Mar;11(3):e1001611. PMID: 27003879; PMCID: PMC4737496. 
860. Lv X, Sun J, Bi Y, Xu M, Lu J, Zhao L, Xu Y. Risk of all-cause mortality and cardiovascular disease associated with secondhand smoke exposure: a systematic review and meta-analysis. Int J Cardiol. 2015 Nov 15;199:106-15. doi: 10.1016/j.ijcard.2015.07.011. Epub 2015 Jul 12. PMID: 26188829.

861. Zhang D, Liu Y, Cheng C, Wang Y, Xue Y, Li W, Li X. Dose-related effect of secondhand smoke on cardiovascular disease in nonsmokers: Systematic review and meta-analysis. Int J Hyg Environ Health. 2020 Jul;228:113546. doi: 10.1016/j.ijheh.2020.113546. Epub 2020 May 6. PMID: 32387882.

862. Eisner MD, Wang Y, Haight TJ, Balmes J, Hammond SK, Tager IB. Secondhand smoke exposure, pulmonary function, and cardiovascular mortality. Ann Epidemiol. 2007 May;17(5):364-73. doi: 10.1016/j.annepidem.2006.10.008. Epub 2007 Feb 14. PMID: 17300955.

863. Wang K, Wang Y, Zhao R, Gong L, Wang L, He Q, Chen L, Qin J. Relationship between childhood secondhand smoke exposure and the occurrence of hyperlipidaemia and coronary heart disease among Chinese non-smoking women: a cross-sectional study. BMJ Open. 2021 Jul 5;11(7):e048590. doi: 10.1136/bmjopen-2020-048590. PMID: 34226229; PMCID: PMC8258554.

864. Sadeghi M, Daneshpour MS, Khodakarim S, Momenan AA, Akbarzadeh M, Soori H. Impact of secondhand smoke exposure in former smokers on their subsequent risk of coronary heart disease: evidence from the population-based cohort of the Tehran Lipid and Glucose Study. Epidemiol Health. 2020;42:e2020009. doi: 10.4178/epih.e2020009. Epub 2020 Mar 8. PMID: 32150674; PMCID: PMC7285426

865. Białas AJ, Sitarek P, Miłkowska-Dymanowska J, Piotrowski WJ, Górski P. The Role of Mitochondria and

Oxidative/Antioxidative Imbalance in Pathobiology of Chronic Obstructive Pulmonary Disease. Oxid Med Cell Longev. 2016;2016:7808576. doi: 10.1155/2016/7808576. Epub 2016 Dec 26. PMID: 28105251; PMCID: PMC5220474.

866. Bodas M, Patel N, Silverberg D, Walworth K, Vij N. Master Autophagy Regulator Transcription Factor EB Regulates Cigarette Smoke-Induced Autophagy Impairment and Chronic Obstructive Pulmonary Disease-Emphysema Pathogenesis. Antioxid Redox Signal. 2017 Jul 20;27(3):150-167. doi: 10.1089/ars.2016.6842. Epub 2017 Feb 1. PMID: 27835930; PMCID: PMC5510670. 867. Demedts IK, Demoor T, Bracke KR, Joos GF, Brusselle GG. Role of apoptosis in the pathogenesis of COPD and pulmonary emphysema. Respir Res. 2006 Mar 30;7(1):53. doi: 10.1186/1465-9921-7-53. PMID: 16571143; PMCID: PMC1501017. 868. Cai S, Chen P, Zhang C, Chen JB, Wu J. Oral N-acetylcysteine attenuates pulmonary emphysema and alveolar septal cell apoptosis in smoking-induced COPD in rats. Respirology. 2009 Apr;14(3):354-9. doi: 10.1111/j.1440-1843.2009.01511.x. PMID: 19341424.

869. Zhang Q, Li W, Ayidaerhan N, Han W, Chen Y, Song W, Yue Y. IP3 R attenuates oxidative stress and inflammation damage in smoking-induced COPD by promoting autophagy. J Cell Mol Med. 2021 May 31;25(13):6174-87. doi: 10.1111/jcmm.16546. Epub ahead of print. PMID: 34060199; PMCID: PMC8256356.

870. Racanelli AC, Kikkers SA, Choi AMK, Cloonan SM. Autophagy and inflammation in chronic respiratory disease. Autophagy. 2018;14(2):221-232. doi: 10.1080/15548627.2017.1389823. Epub 2018 Feb 8. PMID: 29130366; PMCID: PMC5902194.

871. Bodas M, Silverberg D, Walworth K, Brucia K, Vij N. Augmentation of S-Nitrosoglutathione Controls Cigarette Smoke-Induced Inflammatory-Oxidative Stress and Chronic Obstructive Pulmonary Disease-Emphysema Pathogenesis by Restoring Cystic Fibrosis Transmembrane Conductance Regulator Function. Antioxid Redox Signal. 2017 Sep 1;27(7):433-451. doi: 10.1089/ars.2016.6895. Epub 2017 Feb 7. PMID: 28006950; PMCID: PMC5564030.

872. Morse D, Rosas IO. Tobacco smoke-induced lung fibrosis and emphysema. Annu Rev Physiol. 2014;76:493-513. doi: 10.1146/annurev-physiol-021113-170411. Epub 2013 Nov 18. PMID: 24274738.

873. Pehote G, Vij N. Autophagy Augmentation to Alleviate Immune Response Dysfunction, and Resolve Respiratory and COVID19 Exacerbations. Cells. 2020 Aug 24;9(9):1952. doi: 10.3390/cells9091952. PMID: 32847034; PMCID: PMC7565665. 874. Lee JW, Chun W, Lee HJ, Min JH, Kim SM, Seo JY, Ahn KS, Oh SR. The Role of Macrophages in the Development of Acute and Chronic Inflammatory Lung Diseases. Cells. 2021 Apr 14;10(4):897. doi: 10.3390/cells10040897. PMID: 33919784; PMCID: PMC8070705

875. Kosyreva A, Dzhalilova D, Lokhonina A, Vishnyakova P, Fatkhudinov T. The Role of Macrophages in the Pathogenesis of SARSCoV-2-Associated Acute Respiratory Distress Syndrome. Front Immunol. 2021 May 10;12:682871. doi: 10.3389/fimmu.2021.682871. PMID: 34040616; PMCID: PMC8141811.

876. Lupu L, Palmer A, Huber-Lang M. Inflammation, Thrombosis, and Destruction: The Three-Headed Cerberus of Trauma- and SARS-CoV-2-Induced ARDS. Front Immunol. 2020 Sep 25;11:584514. doi: 10.3389/fimmu.2020.584514. PMID: 33101314; PMCID: PMC7546394.

877. Zhang Y, Sun H, Pei R, Mao B, Zhao Z, Li H, Lin Y, Lu K. The SARS-CoV-2 protein ORF3a inhibits fusion of autophagosomes with lysosomes. Cell Discov. 2021 May 4;7(1):31. doi: 10.1038/s41421-021-00268-z. PMID: 33947832; PMCID: PMC8096138.

878. Wang SY, Ni X, Hu KQ, Meng FL, Li M, Ma XL, Meng TT, Wu HH, Ge D, Zhao J, Li Y, Su GH. Cilostazol alleviate nicotine induced cardiomyocytes hypertrophy through modulation of autophagy by CTSB/ROS/p38MAPK/JNK feedback loop. Int J Biol Sci. 2020 Apr 27;16(11):2001-2013. doi: 10.7150/ijbs.43825. PMID: 32398966; PMCID: PMC7211170.

879. Johnson JD, Houchens DP, Kluwe WM, Craig DK, Fisher GL. Effects of mainstream and environmental tobacco smoke on the immune system in animals and humans: a review. Crit Rev Toxicol. 1990;20(5):369-95. doi: 10.3109/10408449009089870. PMID: 2202327.

880. Herrmann M, King K, Weitzman M. Prenatal tobacco smoke and postnatal secondhand smoke exposure and child neuro development. Curr Opin Pediatr. 2008 Apr;20(2):184-90. doi: 10.1097/MOP.0b013e3282f56165. PMID: 18332716.

881. Singh SP, Mishra NC, Rir-Sima-Ah J, Campen M, Kurup V, Razani-Boroujerdi S, Sopori ML. Maternal exposure to secondhand cigarette smoke primes the lung for induction of phosphodiesterase-4D5 isozyme and exacerbated Th2 responses: rolipram attenuates the airway hyperreactivity and muscarinic receptor expression but not lung inflammation and atopy. J Immunol. 2009 Aug 1;183(3):2115-21. doi: 10.4049/jimmunol.0900826. Epub 2009 Jul 13. PMID: 19596983; PMCID: PMC3191864.

882. Majlessi L, Bordenave G. Mechanisms of mouse T lymphocyte-induced suppression of the IgG2ab allotype and T lymphocyte tolerance to IgG2ab. Arch Immunol Ther Exp (Warsz). 2001;49(6):407-15. PMID: 11814234.

883. Lu Y, Di YP, Chang M, Huang X, Chen Q, Hong N, Kahkonen BA, Di ME, Yu C, Keller ET, Zhang J. Cigarette smoke-associated inflammation impairs bone remodeling through NFKB activation. J Transl Med. 2021 Apr 21;19(1):163. doi: 10.1186/s12967-02102836-z. PMID: 33882954; PMCID: PMC8061040.

884. Strzelak A, Ratajczak A, Adamiec A, Feleszko W. Tobacco Smoke Induces and Alters Immune Responses in the Lung Triggering Inflammation, Allergy, Asthma and Other Lung Diseases: A Mechanistic Review. Int J Environ Res Public Health. 2018 May 21;15(5):1033. doi: 10.3390/ijerph15051033. PMID: 29883409; PMCID: PMC5982072.

885. Mortaz E, Kraneveld AD, Smit JJ, Kool M, Lambrecht BN, Kunkel SL, Lukacs NW, Nijkamp FP, Folkerts G. Effect of cigarette smoke extract on dendritic cells and their impact on T-cell proliferation. PLoS One. 2009;4(3):e4946. doi: 10.1371/journal.pone.0004946. Epub 2009 Mar 18. PMID: 19293939; PMCID: PMC2655711.

886. Joffre O, Nolte MA, Spörri R, Reis e Sousa C. Inflammatory signals in dendritic cell activation and the induction of adaptive immunity. Immunol Rev. 2009 Jan;227(1):234-47. doi: 10.1111/j.1600-065X.2008.00718.x. PMID: 19120488. 
887. Danov O, Wolff M, Bartel S, Böhlen S, Obernolte H, Wronski S, Jonigk D, Hammer B, Kovacevic D, Reuter S, Krauss-Etschmann S, Sewald K. Cigarette Smoke Affects Dendritic Cell Populations, Epithelial Barrier Function, and the Immune Response to Viral Infection With H1N1. Front Med (Lausanne). 2020 Nov 6;7:571003. doi: 10.3389/fmed.2020.571003. PMID: 33240904; PMCID: PMC7678748.

888. Gong W, Zheng Y, Chao F, Li Y, XU Z, Huang G, Gao X, Li S, He F. The anti-inflammatory activity of HMGB1 A box is enhanced when fused with C-terminal acidic tail. J Biomed Biotechnol. 2010;2010:915234. doi: 10.1155/2010/915234. Epub 2010 Apr 1. PMID: 20379370; PMCID: PMC2850157.

889. LeBlanc PM, Doggett TA, Choi J, Hancock MA, Durocher Y, Frank F, Nagar B, Ferguson TA, Saleh M. An immunogenic peptide in the A-box of HMGB1 protein reverses apoptosis-induced tolerance through RAGE receptor. J Biol Chem. 2014 Mar 14;289(11):7777-86. doi: 10.1074/jbc.M113.541474. Epub 2014 Jan 28. PMID: 24474694; PMCID: PMC3953289. 890. Wild CA, Bergmann C, Fritz G, Schuler P, Hoffmann TK, Lotfi R, Westendorf A, Brandau S, Lang S. HMGB1 conveys immunosuppressive characteristics on regulatory and conventional T cells. Int Immunol. 2012 Aug;24(8):485-94. doi: 10.1093/intimm/dxs051. Epub 2012 Apr 3. PMID: 22473704.

891. Lee W, Kwon OK, Han MS, Lee YM, Kim SW, Kim KM, Lee T, Lee S, Bae JS. Role of moesin in HMGB1-stimulated severe inflammatory responses. Thromb Haemost. 2015 Aug;114(2):350-63. doi: 10.1160/TH14-11-0969. Epub 2015 May 7. PMID: 25947626.

892. Liu QY, Wang YX, Wu ZS, Shi ZW, Wu X, Chen X, Yang Z, Xu KZ. High Mobility Group Protein 1 Reverses Immune System Paralysis in Late-Phase Sepsis. Infect Immun. 2018 Aug 22;86(9):e00455-18. doi: 10.1128/IAI.00455-18. PMID: 29941462; PMCID: PMC6105879.

893. Kim TH, Ku SK, Bae JS. Persicarin is anti-inflammatory mediator against HMGB1-induced inflammatory responses in HUVECs and in CLP-induced sepsis mice. J Cell Physiol. 2013 Apr;228(4):696-703. doi: 10.1002/jcp.24214. PMID: 22911316.

894. Ved R, Sharouf F, Harari B, Muzaffar M, Manivannan S, Ormonde C, Gray WP, Zaben M. Disulfide HMGB1 acts via TLR2/4 receptors to reduce the numbers of oligodendrocyte progenitor cells after traumatic injury in vitro. Sci Rep. $2021 \mathrm{Mar}$ 17;11(1):6181. doi: 10.1038/s41598-021-84932-0. PMID: 33731757; PMCID: PMC7971069.

895. Jamieson AM, Pasman L, Yu S, Gamradt P, Homer RJ, Decker T, Medzhitov R. Role of tissue protection in lethal respiratory viral-bacterial coinfection. Science. 2013 Jun 7;340(6137):1230-4. doi: 10.1126/science.1233632. Epub 2013 Apr 25. PMID: 23618765; PMCID: PMC3933032.

896. Poe SL, Arora M, Oriss TB, Yarlagadda M, Isse K, Khare A, Levy DE, Lee JS, Mallampalli RK, Chan YR, Ray A, Ray P. STAT1regulated lung MDSC-like cells produce IL-10 and efferocytose apoptotic neutrophils with relevance in resolution of bacteria pneumonia. Mucosal Immunol. 2013 Jan;6(1):189-99. doi: 10.1038/mi.2012.62. Epub 2012 Jul 11. PMID: 22785228; PMCID: PMC3505806.

897. Sander LE, Sackett SD, Dierssen U, Beraza N, Linke RP, Müller M, Blander JM, Tacke F, Trautwein C. Hepatic acute-phase proteins control innate immune responses during infection by promoting myeloid-derived suppressor cell function. J Exp Med. 2010 Jul 5;207(7):1453-64. doi: 10.1084/jem.20091474. Epub 2010 Jun 7. PMID: 20530204; PMCID: PMC2901069.

898. Erlandsson Harris H, Andersson U. Mini-review: The nuclear protein HMGB1 as a proinflammatory mediator. Eur J Immunol. 2004 Jun;34(6):1503-12. doi: 10.1002/eji.200424916. PMID: 15162419.

899. Lotze MT, Deisseroth A, Rubartelli A. Damage associated molecular pattern molecules. Clin Immunol. 2007 Jul;124(1):1-4. doi: 10.1016/j.clim.2007.02.006. Epub 2007 Apr 30. PMID: 17468050; PMCID: PMC2000827.

900. Hreggvidsdottir HS, Ostberg T, Wähämaa H, Schierbeck H, Aveberger AC, Klevenvall L, Palmblad K, Ottosson L, Andersson U, Harris HE. The alarmin HMGB1 acts in synergy with endogenous and exogenous danger signals to promote inflammation. J Leukoc Biol. 2009 Sep;86(3):655-62. doi: 10.1189/jlb.0908548. Epub 2009 Jun 29. PMID: 19564572.

901. van Beijnum JR, Buurman WA, Griffioen AW. Convergence and amplification of toll-like receptor (TLR) and receptor for advanced glycation end products (RAGE) signaling pathways via high mobility group B1 (HMGB1). Angiogenesis. 2008;11(1):91-9. doi: 10.1007/s10456-008-9093-5. Epub 2008 Feb 9. PMID: 18264787.

902. Ren D, Sun R, Wang S. Role of inducible nitric oxide synthase expressed by alveolar macrophages in high mobility group box 1--induced acute lung injury. Inflamm Res. 2006 May;55(5):207-15. doi: 10.1007/s00011-006-0072-2. PMID: 16830108.

903. Park JS, Svetkauskaite D, He Q, Kim JY, Strassheim D, Ishizaka A, Abraham E. Involvement of toll-like receptors 2 and 4 in cellular activation by high mobility group box 1 protein. J Biol Chem. 2004 Feb 27;279(9):7370-7. doi: 10.1074/jbc.M306793200. Epub 2003 Dec 4. PMID: 14660645

904. Hori O, Brett J, Slattery T, Cao R, Zhang J, Chen JX, Nagashima M, Lundh ER, Vijay S, Nitecki D, et al. The receptor for advanced glycation end products (RAGE) is a cellular binding site for amphoterin. Mediation of neurite outgrowth and coexpression of rage and amphoterin in the developing nervous system. J Biol Chem. 1995 Oct 27;270(43):25752-61. doi: 10.1074/jbc.270.43.25752. PMID: 7592757.

905. Huttunen HJ, Fages C, Kuja-Panula J, Ridley AJ, Rauvala H. Receptor for advanced glycation end products-binding $\mathrm{COOH}$ terminal motif of amphoterin inhibits invasive migration and metastasis. Cancer Res. 2002 Aug 15;62(16):4805-11. PMID: 12183440.

906. Huttunen HJ, Fages C, Rauvala H. Receptor for advanced glycation end products (RAGE)-mediated neurite outgrowth and activation of NF-kappaB require the cytoplasmic domain of the receptor but different downstream signaling pathways. J Biol Chem. 1999 Jul 9;274(28):19919-24. doi: 10.1074/jbc.274.28.19919. PMID: 10391939.

907. He M, Kubo H, Ishizawa K, Hegab AE, Yamamoto Y, Yamamoto H, Yamaya M. The role of the receptor for advanced glycation end-products in lung fibrosis. Am J Physiol Lung Cell Mol Physiol. 2007 Dec;293(6):L1427-36. doi: 10.1152/ajplung.00075.2007. Epub 2007 Oct 19. PMID: 17951314.

908. Kong X, Zhang C, Jin X, Wu X, Zhang S, Zhong Z, Feng Q, Liu T, Yuan H. The effect of HMGB1 A box on lung injury in mice with acute pancreatitis. Biofactors. 2011 Jul-Aug;37(4):323-7. doi: 10.1002/biof.177. PMID: 21915938.

909. Fan J, Li Y, Levy RM, Fan JJ, Hackam DJ, Vodovotz Y, Yang H, Tracey KJ, Billiar TR, Wilson MA. Hemorrhagic shock induces NAD(P)H oxidase activation in neutrophils: role of HMGB1-TLR4 signaling. J Immunol. 2007 May 15;178(10):6573-80. doi: 10.4049/jimmunol.178.10.6573. PMID: 17475888.

910. Li Y, Xiang M, Yuan Y, Xiao G, Zhang J, Jiang Y, Vodovotz Y, Billiar TR, Wilson MA, Fan J. Hemorrhagic shock augments lung endothelial cell activation: role of temporal alterations of TLR4 and TLR2. Am J Physiol Regul Integr Comp Physiol. 2009 Dec;297(6):R1670-80. doi: 10.1152/ajpregu.00445.2009. Epub 2009 Oct 14. PMID: 19828841; PMCID: PMC2803625. 911. Ueno H, Matsuda T, Hashimoto S, Amaya F, Kitamura Y, Tanaka M, Kobayashi A, Maruyama I, Yamada S, Hasegawa N, Soejima J, Koh H, Ishizaka A. Contributions of high mobility group box protein in experimental and clinical acute lung injury. Am J Respir Crit Care Med. 2004 Dec 15;170(12):1310-6. doi: 10.1164/rccm.200402-1880C. Epub 2004 Sep 16. PMID: 15374839. 912. Sixt SU, Peters J. Extracellular alveolar proteasome: possible role in lung injury and repair. Proc Am Thorac Soc. 2010 Feb;7(1):91-6. doi: 10.1513/pats.200906-035JS. PMID: 20160154. 
913. Shih CP, Kuo CY, Lin YY, Lin YC, Chen HK, Wang H, Chen HC, Wang CH. Inhibition of Cochlear HMGB1 Expression Attenuates Oxidative Stress and Inflammation in an Experimental Murine Model of Noise-Induced Hearing Loss. Cells. 2021 Apr 5;10(4):810. doi: 10.3390/cells10040810. PMID: 33916471; PMCID: PMC8066810.

914. Kellner M, Noonepalle S, Lu Q, Srivastava A, Zemskov E, Black SM. ROS Signaling in the Pathogenesis of Acute Lung Injury (ALI) and Acute Respiratory Distress Syndrome (ARDS). Adv Exp Med Biol. 2017;967:105-137. doi: 10.1007/978-3-319-63245-2_8. PMID: 29047084; PMCID: PMC7120947.

915. Sitaraman S, Na CL, Yang L, Filuta A, Bridges JP, Weaver TE. Proteasome dysfunction in alveolar type 2 epithelial cells is associated with acute respiratory distress syndrome. Sci Rep. 2019 Aug 29;9(1):12509. doi: 10.1038/s41598-019-49020-4. PMID: 31467330; PMCID: PMC6715642.

916. van Rijt SH, Keller IE, John G, Kohse K, Yildirim AÖ, Eickelberg O, Meiners S. Acute cigarette smoke exposure impairs proteasome function in the lung. Am J Physiol Lung Cell Mol Physiol. 2012 Nov 1;303(9):L814-23. doi:

10.1152/ajplung.00128.2012. Epub 2012 Sep 7. PMID: 22962013.

917. Somborac-Bacura A, van der Toorn M, Franciosi L, Slebos DJ, Zanic-Grubisic T, Bischoff R, van Oosterhout AJ. Cigarette smoke induces endoplasmic reticulum stress response and proteasomal dysfunction in human alveolar epithelial cells. Exp Physiol. 2013 Jan;98(1):316-25. doi: 10.1113/expphysiol.2012.067249. Epub 2012 Jul 30. PMID: 22848082.

918. Nasi A, McArdle S, Gaudernack G, Westman G, Melief C, Rockberg J, Arens R, Kouretas D, Sjölin J, Mangsbo S. Reactive oxygen species as an initiator of toxic innate immune responses in retort to SARS-CoV-2 in an ageing population, consider $\mathrm{N}$ acetylcysteine as early therapeutic intervention. Toxicol Rep. 2020 Jun 18;7:768-771. doi: 10.1016/j.toxrep.2020.06.003. PMID: 32632359; PMCID: PMC7301821.

919. Suhail S, Zajac J, Fossum C, Lowater H, McCracken C, Severson N, Laatsch B, Narkiewicz-Jodko A, Johnson B, Liebau J, Bhattacharyya S, Hati S. Role of Oxidative Stress on SARS-CoV (SARS) and SARS-CoV-2 (COVID-19) Infection: A Review. Protein J. 2020 Dec;39(6):644-656. doi: 10.1007/s10930-020-09935-8. Epub 2020 Oct 26. PMID: 33106987; PMCID: PMC7587547. 920. Poe FL, Corn J. N-Acetylcysteine: A potential therapeutic agent for SARS-CoV-2. Med Hypotheses. 2020 Oct;143:109862. doi: 10.1016/j.mehy.2020.109862. Epub 2020 May 30. PMID: 32504923; PMCID: PMC7261085.

921. Andreou A, Trantza S, Filippou D, Sipsas N, Tsiodras S. COVID-19: The Potential Role of Copper and N-acetylcysteine (NAC) in a Combination of Candidate Antiviral Treatments Against SARS-CoV-2. In Vivo. 2020 Jun;34(3 Suppl):1567-1588. doi: 10.21873/invivo.11946. PMID: 32503814.

922. Jorge-Aarón RM, Rosa-Ester MP. N-acetylcysteine as a potential treatment for COVID-19. Future Microbiol. 2020 Jul;15:959962. doi: 10.2217/fmb-2020-0074. Epub 2020 Jul 14. PMID: 32662664; PMCID: PMC7359418.

923. Tenório MCDS, Graciliano NG, Moura FA, Oliveira ACM, Goulart MOF. N-Acetylcysteine (NAC): Impacts on Human Health. Antioxidants (Basel). 2021 Jun 16;10(6):967. doi: 10.3390/antiox10060967. PMID: 34208683; PMCID: PMC8234027.

924. Sadowska AM, Manuel-Y-Keenoy B, De Backer WA. Antioxidant and anti-inflammatory efficacy of NAC in the treatment of COPD: discordant in vitro and in vivo dose-effects: a review. Pulm Pharmacol Ther. 2007;20(1):9-22. doi: 10.1016/j.pupt.2005.12.007. Epub 2006 Feb 3. PMID: 16458553.

925. Sadowska AM. N-Acetylcysteine mucolysis in the management of chronic obstructive pulmonary disease. Ther Adv Respir Dis. 2012 Jun;6(3):127-35. doi: 10.1177/1753465812437563. Epub 2012 Feb 23. PMID: 22361928.

926. Tse HN, Tseng CZ. Update on the pathological processes, molecular biology, and clinical utility of $\mathrm{N}$-acetylcysteine in chronic obstructive pulmonary disease. Int J Chron Obstruct Pulmon Dis. 2014 Aug 6;9:825-36. doi: 10.2147/COPD.S51057. PMID: 25125976; PMCID: PMC4130719.

927. De Backer J, Vos W, Van Holsbeke C, Vinchurkar S, Claes R, Parizel PM, De Backer W. Effect of high-dose N-acetylcysteine on airway geometry, inflammation, and oxidative stress in COPD patients. Int J Chron Obstruct Pulmon Dis. 2013;8:569-79. doi: 10.2147/COPD.S49307. Epub 2013 Nov 22. PMID: 24293993; PMCID: PMC3842218.

928. Stav D, Raz M. Effect of N-acetylcysteine on air trapping in COPD: a randomized placebo-controlled study. Chest. 2009 Aug;136(2):381-386. doi: 10.1378/chest.09-0421. Epub 2009 May 15. PMID: 19447919.

929. Zhang Q, Ju Y, Ma Y, Wang T. N-acetylcysteine improves oxidative stress and inflammatory response in patients with community acquired pneumonia: A randomized controlled trial. Medicine (Baltimore). 2018 Nov;97(45):e13087. doi: 10.1097/MD.0000000000013087. PMID: 30407312; PMCID: PMC6250560.

930. Gillissen A. Grundlagen der antiinflammatorischen Wirkung von N-Acetylcystein und dessen therapeutische Einsatzmöglichkeiten [Anti-inflammatory efficacy of $\mathrm{N}$-acetylcysteine and therapeutic usefulness]. Pneumologie. 2011 Sep;65(9):549-57. German. doi: 10.1055/s-0030-1256592. Epub 2011 Jul 14. PMID: 21761386.

931. Matera MG, Calzetta L, Cazzola M. Oxidation pathway and exacerbations in COPD: the role of NAC. Expert Rev Respir Med. 2016;10(1):89-97. doi: 10.1586/17476348.2016.1121105. Epub 2015 Dec 15. PMID: 26567752.

932. Sadowska AM, Verbraecken J, Darquennes K, De Backer WA. Role of N-acetylcysteine in the management of COPD. Int J Chron Obstruct Pulmon Dis. 2006;1(4):425-34. doi: 10.2147/copd.2006.1.4.425. PMID: 18044098; PMCID: PMC2707813.

933. Tian H, Zhou Y, Tang L, Wu F, Deng Z, Lin B, Huang P, Wei S, Zhao D, Zheng J, Zhong N, Ran P. High-dose N-acetylcysteine for long-term, regular treatment of early-stage chronic obstructive pulmonary disease (GOLD I-II): study protocol for a multicenter, double-blinded, parallel-group, randomized controlled trial in China. Trials. 2020 Sep 11;21(1):780. doi: 10.1186/s13063-02004701-8. PMID: 32917271; PMCID: PMC7488567.

934. Fowdar K, Chen H, He Z, Zhang J, Zhong X, Zhang J, Li M, Bai J. The effect of N-acetylcysteine on exacerbations of chronic obstructive pulmonary disease: A meta-analysis and systematic review. Heart Lung. 2017 Mar-Apr;46(2):120-128. doi: 10.1016/j.hrtlng.2016.12.004. Epub 2017 Jan 18. PMID: 28109565.

935. Zheng JP, Wen FQ, Bai CX, Wan HY, Kang J, Chen P, Yao WZ, Ma L, Li X, Raiteri L, Sardina M, Gao Y, Wang BS, Zhong NS PANTHEON study group. Twice daily N-acetylcysteine $600 \mathrm{mg}$ for exacerbations of chronic obstructive pulmonary disease (PANTHEON): a randomised, double-blind placebo-controlled trial. Lancet Respir Med. 2014 Mar;2(3):187-94. doi: 10.1016/S2213-2600(13)70286-8. Epub 2014 Jan 30. Erratum in: Lancet Respir Med. 2014 Apr;2(4):e4. PMID: 24621680. 936. Cazzola M, Matera MG. N-acetylcysteine in COPD may be beneficial, but for whom? Lancet Respir Med. 2014 Mar;2(3):166-7. doi: 10.1016/S2213-2600(13)70294-7. Epub 2014 Jan 30. PMID: 24621673.

937. Turner RD, Bothamley GH. N-acetylcysteine for COPD: the evidence remains inconclusive. Lancet Respir Med. 2014 Apr;2(4):e3. doi: 10.1016/S2213-2600(14)70054-2. PMID: 24717631.

938. Victoni T, Barreto E, Lagente V, Carvalho VF. Oxidative Imbalance as a Crucial Factor in Inflammatory Lung Diseases: Could Antioxidant Treatment Constitute a New Therapeutic Strategy? Oxid Med Cell Longev. 2021 Feb 9;2021:6646923. doi: 10.1155/2021/6646923. PMID: 33628371; PMCID: PMC7889360.

939. Kirkham P, Rahman I. Oxidative stress in asthma and COPD: antioxidants as a therapeutic strategy. Pharmacol Ther. 2006 Aug;111(2):476-94. doi: 10.1016/j.pharmthera.2005.10.015. Epub 2006 Feb 3. PMID: 16458359. 
940. Adam-Bonci TI, Bonci EA, Pârvu AE, Herdean Al, Moț A, Taulescu M, Ungur A, Pop RM, Bocșan C, Irimie A. Vitamin D Supplementation: Oxidative Stress Modulation in a Mouse Model of Ovalbumin-Induced Acute Asthmatic Airway Inflammation. Int J Mol Sci. 2021 Jun 30;22(13):7089. doi: 10.3390/ijms22137089. PMID: 34209324; PMCID: PMC8268667.

941. Bao W, Zhang Y, Zhang M, Bao A, Fei X, Zhang X, Zhou X. Effects of ozone repeated short exposures on the airway/lung inflammation, airway hyperresponsiveness and mucus production in a mouse model of ovalbumin-induced asthma. Biomed Pharmacother. 2018 May;101:293-303. doi: 10.1016/j.biopha.2018.02.079. Epub 2018 Feb 27. PMID: 29499403.

942. Busse PJ, Zhang TF, Srivastava K, Schofield B, Li XM. Effect of ageing on pulmonary inflammation, airway

hyperresponsiveness and T and B cell responses in antigen-sensitized and -challenged mice. Clin Exp Allergy. 2007

Sep;37(9):1392-403. doi: 10.1111/j.1365-2222.2007.02775.x. PMID: 17845421; PMCID: PMC2818115.

943. Luu Quoc Q, Cao Thi Bich T, Kim SH, Park HS, Shin YS. Administration of vitamin E attenuates airway inflammation through restoration of Nrf2 in a mouse model of asthma. J Cell Mol Med. 2021 Jul;25(14):6721-6732. doi: 10.1111/jcmm.16675. Epub 2021 Jun 4. PMID: 34089243; PMCID: PMC8278095.

944. Agrawal T, Gupta GK, Agrawal DK. Vitamin D supplementation reduces airway hyperresponsiveness and allergic airway inflammation in a murine model. Clin Exp Allergy. 2013 Jun;43(6):672-83. doi: 10.1111/cea.12102. PMID: 23711130; PMCID: PMC3671499.

945. Easter M, Bollenbecker S, Barnes JW, Krick S. Targeting Aging Pathways in Chronic Obstructive Pulmonary Disease. Int J Mol Sci. 2020 Sep 21;21(18):6924. doi: 10.3390/ijms21186924. PMID: 32967225; PMCID: PMC7555616.

946. Soma T, Uchida Y, Hoshino Y, Katayama K, Kobayashi T, Nakagome K, Nagata M. Relationship between airway inflammation and airflow limitation in elderly asthmatics. Asia Pac Allergy. 2020 Apr 23;10(2):e17. doi: 10.5415/apallergy.2020.10.e17. PMID: 32411582; PMCID: PMC7203439.

947. Crossley D, Stockley R, Sapey E. Alpha-1 Antitrypsin Deficiency and Accelerated Aging: A New Model for an Old Disease? Drugs Aging. 2019 Sep;36(9):823-840. doi: 10.1007/s40266-019-00684-7. PMID: 31179525.

948. Harkema JR, Hotchkiss JA. Ozone- and endotoxin-induced mucous cell metaplasias in rat airway epithelium: novel animal models to study toxicant-induced epithelial transformation in airways. Toxicol Lett. 1993 May;68(1-2):251-63. doi: 10.1016/03784274(93)90136-I. PMID: 8516771.

949. Harkema JR, Wagner JG. Epithelial and inflammatory responses in the airways of laboratory rats coexposed to ozone and biogenic substances: enhancement of toxicant-induced airway injury. Exp Toxicol Pathol. 2005 Jul;57 Suppl 1:129-41. doi: 10.1016/j.etp.2005.05.013. PMID: 16092720.

950. Rodríguez E, Ferrer J, Zock JP, Serra I, Antó JM, de Batlle J, Kromhout H, Vermeulen R, Donaire-González D, Benet M, Balcells E, Monsó E, Gayete A, Garcia-Aymerich J; PAC-COPD Study Group. Lifetime occupational exposure to dusts, gases and fumes is associated with bronchitis symptoms and higher diffusion capacity in COPD patients. PLoS One. 2014 Feb 6;9(2):e88426. doi: 10.1371/journal.pone.0088426. PMID: 24516659; PMCID: PMC3916435.

951. Shore SA. Mechanistic Basis for Obesity-related Increases in Ozone-induced Airway Hyperresponsiveness in Mice. Ann Am Thorac Soc. 2017 Nov;14(Supplement_5):S357-S362. doi: 10.1513/AnnalsATS.201702-140AW. PMID: 29161088; PMCID: PMC5711270.

952. Wiegman CH, Li F, Ryffel B, Togbe D, Chung KF. Oxidative Stress in Ozone-Induced Chronic Lung Inflammation and Emphysema: A Facet of Chronic Obstructive Pulmonary Disease. Front Immunol. 2020 Sep 2;11:1957. doi: 10.3389/fimmu.2020.01957. PMID: 32983127; PMCID: PMC7492639.

953. Mumby S, Chung KF, Adcock IM. Transcriptional Effects of Ozone and Impact on Airway Inflammation. Front Immunol. 2019 Jul 10;10:1610. doi: 10.3389/fimmu.2019.01610. PMID: 31354743; PMCID: PMC6635463.

954. Cho SW, Kim JH, Choi JH, Lim DH. Preventive and therapeutic effects of vitamin D in a mouse model of allergic asthma. Asian Pac J Allergy Immunol. 2019 Sep;37(3):130-137. doi: 10.12932/AP-010218-0248. PMID: 30447649.

955. Wei Y, Luo QL, Sun J, Chen MX, Liu F, Dong JC. Bu-Shen-Yi-Qi formulae suppress chronic airway inflammation and regulate Th17/Treg imbalance in the murine ovalbumin asthma model. J Ethnopharmacol. 2015 Apr 22;164:368-77. doi: 10.1016/j.jep.2015.01.016. Epub 2015 Jan 24. PMID: 25625352.

956. Zheng JP, Wen FQ, Bai CX, Wan HY, Kang J, Chen P, Yao WZ, Ma L, Xia QK, Gao Y, Zhong NS; PANTHEON study committee. High-dose $\mathrm{N}$-acetylcysteine in the prevention of COPD exacerbations: rationale and design of the PANTHEON Study. COPD. 2013 Apr;10(2):164-71. doi: 10.3109/15412555.2012.732628. Epub 2012 Oct 12. PMID: 23061828.

957. van Overveld FJ, Demkow U, Górecka D, de Backer WA, Zielinski J. New developments in the treatment of COPD: comparing the effects of inhaled corticosteroids and N-acetylcysteine. J Physiol Pharmacol. 2005 Sep;56 Suppl 4:135-42. PMID: 16204787. 958. Zhang JQ, Zhang JQ, Liu H, Zhao ZH, Fang LZ, Liu L, Fu WP, Shu JK, Feng JG, Dai LM. Effect of N-acetylcysteine in COPD patients with different microsomal epoxide hydrolase genotypes. Int J Chron Obstruct Pulmon Dis. 2015 May 13;10:917-23. doi: 10.2147/COPD.S79710. PMID: 25999707; PMCID: PMC4437521.

959. Sadowska AM, van Overveld FJ, Górecka D, Zdral A, Filewska M, Demkow UA, Luyten C, Saenen E, Zielinski J, De Backer WA. The interrelationship between markers of inflammation and oxidative stress in chronic obstructive pulmonary disease: modulation by inhaled steroids and antioxidant. Respir Med. 2005 Feb;99(2):241-9. doi: 10.1016/j.rmed.2004.07.005. PMID: 15715193.

960. Lee SF, Harris R, Stout-Delgado HW. Targeted antioxidants as therapeutics for treatment of pneumonia in the elderly. Transl Res. 2020 Jun;220:43-56. doi: 10.1016/j.trsl.2020.03.002. Epub 2020 Mar 16. PMID: 32268130; PMCID: PMC7989851. 961. Oyewole AO, Birch-Machin MA. Mitochondria-targeted antioxidants. FASEB J. 2015 Dec;29(12):4766-71. doi: 10.1096/fj.15275404. Epub 2015 Aug 7. PMID: 26253366

962. Víctor VM, Espulgues JV, Hernández-Mijares A, Rocha M. Oxidative stress and mitochondrial dysfunction in sepsis: a potential therapy with mitochondria-targeted antioxidants. Infect Disord Drug Targets. 2009 Aug;9(4):376-89. doi: 10.2174/187152609788922519. PMID: 19689380.

963. Cecchini R, Cecchini AL. SARS-CoV-2 infection pathogenesis is related to oxidative stress as a response to aggression. Med Hypotheses. 2020 Oct;143:110102. doi: 10.1016/j.mehy.2020.110102. Epub 2020 Jul 13. PMID: 32721799; PMCID: PMC7357498. 964. Rocha M, Herance R, Rovira S, Hernández-Mijares A, Victor VM. Mitochondrial dysfunction and antioxidant therapy in sepsis. Infect Disord Drug Targets. 2012 Apr;12(2):161-78. doi: 10.2174/187152612800100189. PMID: 22420514.

965. Oyewole AO, Birch-Machin MA. Mitochondria-targeted antioxidants. FASEB J. 2015 Dec;29(12):4766-71. doi: 10.1096/fj.15275404. Epub 2015 Aug 7. PMID: 26253366.

966. Zhou WC, Qu J, Xie SY, Sun Y, Yao HW. Mitochondrial Dysfunction in Chronic Respiratory Diseases: Implications for the Pathogenesis and Potential Therapeutics. Oxid Med Cell Longev. 2021 Jul 27;2021:5188306. doi: 10.1155/2021/5188306. PMID: 34354793; PMCID: PMC8331273.

967. Wiegman CH, Michaeloudes C, Haji G, Narang P, Clarke CJ, Russell KE, Bao W, Pavlidis S, Barnes PJ, Kanerva J, Bittner A, Rao N, Murphy MP, Kirkham PA, Chung KF, Adcock IM; COPDMAP. Oxidative stress-induced mitochondrial dysfunction drives 
inflammation and airway smooth muscle remodeling in patients with chronic obstructive pulmonary disease. J Allergy Clin Immunol. 2015 Sep;136(3):769-80. doi: 10.1016/j.jaci.2015.01.046. Epub 2015 Mar 29. PMID: 25828268; PMCID: PMC4559140. 968. Dikalov S. Cross talk between mitochondria and NADPH oxidases. Free Radic Biol Med. 2011 Oct 1;51(7):1289-301. doi: 10.1016/j.freeradbiomed.2011.06.033. Epub 2011 Jul 6. PMID: 21777669; PMCID: PMC3163726.

969. Minov J, Stoleski S, Petrova T, Vasilevska K, Mijakoski D, Karadzinska-Bislimovska J. Effects of a Long-Term Use of Carbocysteine on Frequency and Duration of Exacerbations in Patients with Bronchiectasis. Open Access Maced J Med Sci. 2019 Dec 10;7(23):4030-4035. doi: 10.3889/oamjms.2019.697. PMID: 32165947; PMCID: PMC7061384.

970. Um DJ, Hong SJ, Park JT. Examination of the Ability of N-acetylcysteine Administration during Anesthesia to Prevent Perioperative Deterioration of Pulmonary Function in Patients Undergoing Nephrectomy. J Lifestyle Med. 2014 Sep;4(2):95-100. doi: 10.15280/jlm.2014.4.2.95. Epub 2014 Sep 30. PMID: 26064860; PMCID: PMC4391019.

971. Liu X, Hu Z, Zhou H. N-Acetylcysteine Improves Inflammatory Response in COPD Patients by Regulating Th17/Treg Balance through Hypoxia Inducible Factor-1 $\alpha$ Pathway. Biomed Res Int. 2021 Jun 29;2021:6372128. doi: 10.1155/2021/6372128. PMID: 34258270; PMCID: PMC8260296.

972. Qi Q, Ailiyaer Y, Liu R, Zhang Y, Li C, Liu M, Wang X, Jing L, Li Y. Effect of N-acetylcysteine on exacerbations of bronchiectasis (BENE): a randomized controlled trial. Respir Res. 2019 Apr 11;20(1):73. doi: 10.1186/s12931-019-1042-x. PMID: 30975143; PMCID: PMC6458826.

973. Tse HN, Raiteri L, Wong KY, Yee KS, Ng LY, Wai KY, Loo CK, Chan MH. High-dose N-acetylcysteine in stable COPD: the 1-year, double-blind, randomized, placebo-controlled HIACE study. Chest. 2013 Jul;144(1):106-118. doi: 10.1378/chest.12-2357. PMID: 23348146.

974. Tse HN, Raiteri L, Wong KY, Ng LY, Yee KS, Tseng CZS. Benefits of high-dose N-acetylcysteine to exacerbation-prone patients with COPD. Chest. 2014 Sep;146(3):611-623. doi: 10.1378/chest.13-2784. PMID: 24833327.

975. Jiang C, Zou J, Lv Q, Yang Y. Systematic review and meta-analysis of the efficacy of N-acetylcysteine in the treatment of acute exacerbation of chronic obstructive pulmonary disease. Ann Palliat Med. 2021 Jun;10(6):6564-6576. doi: 10.21037/apm-21-1138. PMID: 34237968.

976. Schwalfenberg GK. N-Acetylcysteine: A Review of Clinical Usefulness (an Old Drug with New Tricks). J Nutr Metab. 2021 Jun 9;2021:9949453. doi: 10.1155/2021/9949453. PMID: 34221501; PMCID: PMC8211525.

977. Atefi N, Behrangi E, Mozafarpoor S, Seirafianpour F, Peighambari S, Goodarzi A. N-acetylcysteine and coronavirus disease 2019: May it work as a beneficial preventive and adjuvant therapy? A comprehensive review study. J Res Med Sci. 2020 Nov 26;25:109. doi: 10.4103/jrms.JRMS 777 20. PMID: 33824674; PMCID: PMC8019127.

978. Rushworth GF, Megson IL. Existing and potential therapeutic uses for N-acetylcysteine: the need for conversion to intracellular glutathione for antioxidant benefits. Pharmacol Ther. 2014 Feb;141(2):150-9. doi:

10.1016/j.pharmthera.2013.09.006. Epub 2013 Sep 28. PMID: 24080471.

979. Gibson KR, Neilson IL, Barrett F, Winterburn TJ, Sharma S, MacRury SM, Megson IL. Evaluation of the antioxidant properties of $\mathrm{N}$-acetylcysteine in human platelets: prerequisite for bioconversion to glutathione for antioxidant and antiplatelet activity. $J$ Cardiovasc Pharmacol. 2009 Oct;54(4):319-26. doi: 10.1097/FJC.0b013e3181b6e77b. PMID: 19668088.

980. Choy KH, Dean O, Berk M, Bush Al, van den Buuse M. Effects of N-acetyl-cysteine treatment on glutathione depletion and a short-term spatial memory deficit in 2-cyclohexene-1-one-treated rats. Eur J Pharmacol. 2010 Dec 15;649(1-3):224-8. doi: 10.1016/j.ejphar.2010.09.035. Epub 2010 Sep 22. PMID: 20868666.

981. Li D, Ding Z, Du K, Ye X, Cheng S. Reactive Oxygen Species as a Link between Antioxidant Pathways and Autophagy. Oxid Med Cell Longev. 2021 Jul 21;2021:5583215. doi: 10.1155/2021/5583215. PMID: 34336103; PMCID: PMC8324391.

982. Paskal W, Paskal AM, Pietruski P, Stachura A, Pełka K, Woessner AE, Quinn KP, Kopka M, Galus R, Wejman J, Włodarski P. NAcetylcysteine Added to Local Anesthesia Reduces Scar Area and Width in Early Wound Healing-An Animal Model Study. Int J Mol Sci. 2021 Jul 14;22(14):7549. doi: 10.3390/ijms22147549. PMID: 34299175; PMCID: PMC8307704.

983. Wang H, Xu Y, Sun J, Sui Z. The Novel Curcumin Derivative 1g Induces Mitochondrial and ER-Stress-Dependent Apoptosis in Colon Cancer Cells by Induction of ROS Production. Front Oncol. 2021 Jun 14;11:644197. doi: 10.3389/fonc.2021.644197. PMID: 34195069; PMCID: PMC8236884.

984. Forcados GE, Muhammad A, Oladipo OO, Makama S, Meseko CA. Metabolic Implications of Oxidative Stress and Inflammatory Process in SARS-CoV-2 Pathogenesis: Therapeutic Potential of Natural Antioxidants. Front Cell Infect Microbiol. 2021 May 26;11:654813. doi: 10.3389/fcimb.2021.654813. PMID: 34123871; PMCID: PMC8188981.

985. Chernyak BV, Popova EN, Prikhodko AS, Grebenchikov OA, Zinovkina LA, Zinovkin RA. COVID-19 and Oxidative Stress. Biochemistry (Mosc). 2020 Dec;85(12):1543-1553. doi: 10.1134/S0006297920120068. PMID: 33705292; PMCID: PMC7768996. 986. Khanmohammadi S, Rezaei N. Role of Toll-like receptors in the pathogenesis of COVID-19. J Med Virol. 2021 May;93(5):2735-2739. doi: 10.1002/jmv.26826. Epub 2021 Feb 9. PMID: 33506952; PMCID: PMC8014260. 987. Root-Bernstein R. Innate Receptor Activation Patterns Involving TLR and NLR Synergisms in COVID-19, ALI/ARDS and Sepsis Cytokine Storms: A Review and Model Making Novel Predictions and Therapeutic Suggestions. Int J Mol Sci. 2021 Feb 20;22(4):2108. doi: 10.3390/ijms22042108. PMID: 33672738; PMCID: PMC7924650.

988. Choudhury A, Das NC, Patra R, Mukherjee S. In silico analyses on the comparative sensing of SARS-CoV-2 mRNA by the intracellular TLRs of humans. J Med Virol. 2021 Apr;93(4):2476-2486. doi: 10.1002/jmv.26776. Epub 2021 Jan 12. PMID: 33404091.

989. Addetia A, Lieberman NAP, Phung Q, Hsiang TY, Xie H, Roychoudhury P, Shrestha L, Loprieno MA, Huang ML, Gale M Jr, Jerome KR, Greninger AL. SARS-CoV-2 ORF6 Disrupts Bidirectional Nucleocytoplasmic Transport through Interactions with Rae1 and Nup98. mBio. 2021 Apr 13;12(2):e00065-21. doi: 10.1128/mBio.00065-21. PMID: 33849972; PMCID: PMC8092196. 990. Miorin L, Kehrer T, Sanchez-Aparicio MT, Zhang K, Cohen P, Patel RS, Cupic A, Makio T, Mei M, Moreno E, Danziger O, White KM, Rathnasinghe R, Uccellini M, Gao S, Aydillo T, Mena I, Yin X, Martin-Sancho L, Krogan NJ, Chanda SK, Schotsaert M, Wozniak RW, Ren Y, Rosenberg BR, Fontoura BMA, García-Sastre A. SARS-CoV-2 Orf6 hijacks Nup98 to block STAT nuclear import and antagonize interferon signaling. Proc Natl Acad Sci U S A. 2020 Nov 10;117(45):28344-28354. doi: 10.1073/pnas.2016650117. Epub 2020 Oct 23. PMID: 33097660; PMCID: PMC7668094.

991. Kato K, Ikliptikawati DK, Kobayashi A, Kondo H, Lim K, Hazawa M, Wong RW. Overexpression of SARS-CoV-2 protein ORF6 dislocates RAE1 and NUP98 from the nuclear pore complex. Biochem Biophys Res Commun. 2021 Jan 15;536:59-66. doi: 10.1016/j.bbrc.2020.11.115. Epub 2020 Dec 13. PMID: 33360543 ; PMCID: PMC7733692.

992. Lapointe CP, Grosely R, Johnson AG, Wang J, Fernández IS, Puglisi JD. Dynamic competition between SARS-CoV-2 NSP1 and mRNA on the human ribosome inhibits translation initiation. Proc Natl Acad Sci U S A. 2021 Feb 9;118(6):e2017715118. doi: 10.1073/pnas.2017715118. PMID: 33479166; PMCID: PMC8017934.

993. Yuan S, Peng L, Park JJ, Hu Y, Devarkar SC, Dong MB, Shen Q, Wu S, Chen S, Lomakin IB, Xiong Y. Nonstructural Protein 1 of SARS-CoV-2 Is a Potent Pathogenicity Factor Redirecting Host Protein Synthesis Machinery toward Viral RNA. Mol Cell. 2020 Dec 17;80(6):1055-1066.e6. doi: 10.1016/j.molcel.2020.10.034. Epub 2020 Oct 29. PMID: 33188728; PMCID: PMC7833686. 
994. Thoms M, Buschauer R, Ameismeier M, Koepke L, Denk T, Hirschenberger M, Kratzat H, Hayn M, Mackens-Kiani T, Cheng J, Straub JH, Stürzel CM, Fröhlich T, Berninghausen O, Becker T, Kirchhoff F, Sparrer KMJ, Beckmann R. Structural basis for translational shutdown and immune evasion by the Nsp1 protein of SARS-CoV-2. Science. 2020 Sep 4;369(6508):1249-1255. doi: 10.1126/science.abc8665. Epub 2020 Jul 17. PMID: 32680882 ; PMCID: PMC7402621.

995. Schubert K, Karousis ED, Jomaa A, Scaiola A, Echeverria B, Gurzeler LA, Leibundgut M, Thiel V, Mühlemann O, Ban N. Author Correction: SARS-CoV-2 Nsp1 binds the ribosomal mRNA channel to inhibit translation. Nat Struct Mol Biol. 2020 Nov;27(11):1094. doi: 10.1038/s41594-020-00533-x. Erratum for: Nat Struct Mol Biol. 2020 Oct;27(10):959-966. PMID: 33082564; PMCID: PMC7574671.

996. Knoll R, Schultze JL, Schulte-Schrepping J. Monocytes and Macrophages in COVID-19. Front Immunol. 2021 Jul 21;12:720109. doi: 10.3389/fimmu.2021.720109. PMID: 34367190; PMCID: PMC8335157.

997. Roychoudhury S, Das A, Jha NK, Kesari KK, Roychoudhury S, Jha SK, Kosgi R, Choudhury AP, Lukac N, Madhu NR, Kumar D, Slama P. Viral pathogenesis of SARS-CoV-2 infection and male reproductive health. Open Biol. 2021 Jan;11(1):200347. doi: 10.1098/rsob.200347. Epub 2021 Jan 20. PMID: 33465325; PMCID: PMC7881178.

998. Daskaya H, Yilmaz S, Uysal H, Calim M, Sümbül B, Yurtsever I, Karaaslan K. Usefulness of oxidative stress marker evaluation at admission to the intensive care unit in patients with COVID-19. J Int Med Res. 2021 Jul;49(7):3000605211027733. doi: 10.1177/03000605211027733. PMID: 34310245; PMCID: PMC8320569.

999. Ducastel M, Chenevier-Gobeaux C, Ballaa Y, Meritet JF, Brack M, Chapuis N, Pene F, Carlier N, Szwebel TA, Roche N, Terrier B, Borderie D. Oxidative Stress and Inflammatory Biomarkers for the Prediction of Severity and ICU Admission in Unselected Patients Hospitalized with COVID-19. Int J Mol Sci. 2021 Jul 12;22(14):7462. doi: 10.3390/ijms22147462. PMID: 34299080; PMCID: PMC8306526.

1000. Filardo S, Di Pietro M, Diaco F, Romano S, Sessa R. Oxidative Stress and Inflammation in SARS-CoV-2- and Chlamydia pneumoniae-Associated Cardiovascular Diseases. Biomedicines. 2021 Jun 24;9(7):723. doi: 10.3390/biomedicines9070723. PMID: 34202515; PMCID: PMC8301438.

1001. Casillas Santana MA, Arreguín Cano JA, Dib Kanán A, Dipp Velázquez FA, Munguía PDCS, Martínez Castañón GA, Castillo Silva BE, Sámano Valencia C, Salas Orozco MF. Should We Be Concerned about the Association of Diabetes Mellitus and Periodontal Disease in the Risk of Infection by SARS-CoV-2? A Systematic Review and Hypothesis. Medicina (Kaunas). 2021 May 13;57(5):493. doi: 10.3390/medicina57050493. PMID: 34068221; PMCID: PMC8153122.

1002. Mrityunjaya M, Pavithra V, Neelam R, Janhavi P, Halami PM, Ravindra PV. Immune-Boosting, Antioxidant and Antiinflammatory Food Supplements Targeting Pathogenesis of COVID-19. Front Immunol. 2020 Oct 7;11:570122. doi: 10.3389/fimmu.2020.570122. PMID: 33117359; PMCID: PMC7575721.

1003. Taher A, Lashgari M, Sedighi L, Rahimi-Bashar F, Poorolajal J, Mehrpooya M. A pilot study on intravenous N-Acetylcysteine treatment in patients with mild-to-moderate COVID19-associated acute respiratory distress syndrome. Pharmacol Rep. 2021 Jun 10:1-10. doi: 10.1007/s43440-021-00296-2. Epub ahead of print. PMID: 34114174; PMCID: PMC8191712.

1004. Qi H, Liu DP, Xiao DW, Tian DC, Su YW, Jin SF. Exosomes derived from mesenchymal stem cells inhibit mitochondrial dysfunction-induced apoptosis of chondrocytes via p38, ERK, and Akt pathways. In Vitro Cell Dev Biol Anim. 2019 Mar;55(3):203210. doi: 10.1007/s11626-019-00330-x. Epub 2019 Feb 19. PMID: 30783864.

1005. Sixt SU, Adamzik M, Spyrka D, Saul B, Hakenbeck J, Wohlschlaeger J, Costabel U, Kloss A, Giesebrecht J, Dahlmann B, Peters J. Alveolar extracellular $20 \mathrm{~S}$ proteasome in patients with acute respiratory distress syndrome. Am J Respir Crit Care Med. 2009 Jun 15;179(12):1098-106. doi: 10.1164/rccm.200802-1990C. Epub 2009 Mar 12. PMID: 19286628.

1006. Majetschak M, Sorell LT, Patricelli T, Seitz DH, Knöferl MW. Detection and possible role of proteasomes in the bronchoalveolar space of the injured lung. Physiol Res. 2009;58(3):363-372. doi: 10.33549/physiolres.931526. Epub 2008 Jul 18. PMID: 18637707.

1007. Kellner M, Noonepalle S, Lu Q, Srivastava A, Zemskov E, Black SM. ROS Signaling in the Pathogenesis of Acute Lung Injury (ALI) and Acute Respiratory Distress Syndrome (ARDS). Adv Exp Med Biol. 2017;967:105-137. doi: 10.1007/978-3-319-63245-2 8. PMID: 29047084; PMCID: PMC7120947.

1008. Sharma A, Chakraborty A, Jaganathan BG. Review of the potential of mesenchymal stem cells for the treatment of infectious diseases. World J Stem Cells. 2021 Jun 26;13(6):568-593. doi: 10.4252/wjsc.v13.i6.568. PMID: 34249228; PMCID: PMC8246252.

1009. Dubina MV, Gomonova VV, Taraskina AE, Vasilyeva NV, Sayganov SA. Pathogenesis-based preexposure prophylaxis associated with a low risk of SARS-CoV-2 infection in healthcare workers at a designated COVID-19 hospital: a pilot study. BMC Infect Dis. 2021 Jun 7;21(1):536. doi: 10.1186/s12879-021-06241-1. PMID: 34098889; PMCID: PMC8182762.

1010. Maniatis NA, Kotanidou A, Catravas JD, Orfanos SE. Endothelial pathomechanisms in acute lung injury. Vascul Pharmacol. 2008 Oct-Dec;49(4-6):119-33. doi: 10.1016/j.vph.2008.06.009. Epub 2008 Jul 29. PMID: 18722553; PMCID: PMC7110599. 1011. Zhou X, Dai Q, Huang X. Neutrophils in acute lung injury. Front Biosci (Landmark Ed). 2012 Jun 1;17:2278-83. doi: 10.2741/4051. PMID: 22652778.

1012. Grommes J, Soehnlein O. Contribution of neutrophils to acute lung injury. Mol Med. 2011 Mar-Apr;17(3-4):293-307. doi: 10.2119/molmed.2010.00138. Epub 2010 Oct 18. PMID: 21046059; PMCID: PMC3060975.

1013. Sercundes MK, Ortolan LS, Debone D, Soeiro-Pereira PV, Gomes E, Aitken EH, Condino-Neto A, Russo M, D' Império Lima MR, Alvarez JM, Portugal S, Marinho CR, Epiphanio S. Targeting Neutrophils to Prevent Malaria-Associated Acute Lung Injury/Acute Respiratory Distress Syndrome in Mice. PLoS Pathog. 2016 Dec 7;12(12):e1006054. doi:

10.1371/journal.ppat.1006054. Erratum in: PLoS Pathog. 2017 Nov 15;13(11):e1006730. PMID: 27926944; PMCID: PMC5142790. 1014. Vassallo A, Wood AJ, Subburayalu J, Summers C, Chilvers ER. The counter-intuitive role of the neutrophil in the acute respiratory distress syndrome. Br Med Bull. 2019 Sep 19;131(1):43-55. doi: 10.1093/bmb/ldz024. PMID: 31504234. 1015. Zhang Y, Guan L, Yu J, Zhao Z, Mao L, Li S, Zhao J. Pulmonary endothelial activation caused by extracellular histones contributes to neutrophil activation in acute respiratory distress syndrome. Respir Res. 2016 Nov 21;17(1):155. doi: 10.1186/s12931-016-0472-y. PMID: 27871277; PMCID: PMC5117496.

1016. Zhang YL, Zhao J, Guan L, Zheng YM, Chen M, Guo LX, Guan XX, Mao L, Li SQ, Zhao JY. [Activation of lung endothelial cells by extracellular histone in mice with acute respiratory distress syndrome]. Zhonghua Lao Dong Wei Sheng Zhi Ye Bing Za Zhi. 2019 Oct 20;37(10):732-736. Chinese. doi: 10.3760/cma.j.issn.1001-9391.2019.10.004. PMID: 31726502.

1017. Zhang Y, Wen Z, Guan L, Jiang P, Gu T, Zhao J, Lv X, Wen T. Extracellular histones play an inflammatory role in acid aspiration-induced acute respiratory distress syndrome. Anesthesiology. 2015 Jan;122(1):127-39. doi: 10.1097/ALN.0000000000000429. PMID: 25188000.

1018. Lv X, Wen T, Song J, Xie D, Wu L, Jiang X, Jiang P, Wen Z. Extracellular histones are clinically relevant mediators in the pathogenesis of acute respiratory distress syndrome. Respir Res. 2017 Sep 2;18(1):165. doi: 10.1186/s12931-017-0651-5. PMID: 28865478; PMCID: PMC5581408. 
1019. Jin $Y$, Sun $M$, Jiang $X$, Zhang $Q$, Feng D, Wen Z. [Extracellular histones aggravate acute respiratory distress syndrome by inducing peripheral blood mononuclear cells pyroptosis]. Zhonghua Wei Zhong Bing Ji Jiu Yi Xue. 2019 Nov;31(11):1357-1362. Chinese. doi: 10.3760/cma.j.issn.2095-4352.2019.11.009. PMID: 31898565.

1020. Sun M, Jiang X, Jin Y, Yang H, Chen C, Lyu X, Wen Z. [Extracellular histones are involved in lipopolysaccharide-induced alveolar macrophage injury by activating the TWIK2-NLRP3 pathway]. Zhonghua Wei Zhong Bing Ji Jiu Yi Xue. 2020 Feb;32(2):194198. Chinese. doi: 10.3760/cma.j.cn121430-20191113-00036. PMID: 32275005.

1021. Peng W, Jiang R, Li Y, Chen J, Shao Q, Qian K, Liu F. [Exosomes derived from human-induced pluripotent mesenchymal stem cells inhibit the pyrolysis of alveolar macrophages]. Zhonghua Wei Zhong Bing Ji Jiu Yi Xue. 2021 Jan;33(1):43-48. Chinese. doi: 10.3760/cma.j.cn121430-20201126-00728. PMID: 33565399.

1022. Tavakoli Dargani Z, Singla DK. Embryonic stem cell-derived exosomes inhibit doxorubicin-induced TLR4-NLRP3-mediated cell death-pyroptosis. Am J Physiol Heart Circ Physiol. 2019 Aug 1;317(2):H460-H471. doi: 10.1152/ajpheart.00056.2019. Epub 2019 Jun 7. PMID: 31172809; PMCID: PMC6732475.

1023. Peng W, Zhao N, Liu Q, Nie C, Qing C, Shao Q, Liu F, Qian K, Ding C. [MicroRNA-155 reduces inflammatory response induced by lipopolysaccharide in alveolar macrophages]. Zhonghua Wei Zhong Bing Ji Jiu Yi Xue. 2018 Nov;30(11):1061-1065. Chinese. doi: 10.3760/cma.j.issn.2095-4352.2018.011.010. PMID: 30541646

1024. Zheng X, Zhong Q, Lin X, Gu X, Ling X, Liang Z, Qin Q, Du X. Transforming growth factor- $\beta 1$-induced podocyte injury is associated with increased microRNA-155 expression, enhanced inflammatory responses and MAPK pathway activation. Exp Ther Med. 2021 Jun;21(6):620. doi: 10.3892/etm.2021.10052. Epub 2021 Apr 14. PMID: 33936277; PMCID: PMC8082603.

1025. Gulifeire T, Yang C, Li X, Wang Y, Yu X. [Effects of resveratrol-mediated inhibition of NOD-like receptor protein 3 inflammasomevia activating silent information regulator 1 on the injury of intestinal mucosal barrier function after sepsis]. Zhonghua Wei Zhong Bing Ji Jiu Yi Xue. 2021 May;33(5):535-540. Chinese. doi: 10.3760/cma.j.cn121430-20210218-00249. PMID: 34112288 .

1026. Xu Z, Huang Y, Mao P, Zhang J, Li Y. Sepsis and ARDS: The Dark Side of Histones. Mediators Inflamm. 2015;2015:205054. doi: 10.1155/2015/205054. Epub 2015 Nov 1. PMID: 26609197 ; PMCID: PMC4644547.

1027. Joshi MB, Kamath A, Nair A, Yt P, Sriranjini SJ, Gangadharan GG, Satyamoorthy K. Modulation of neutrophil (dys)function by Ayurvedic herbs and its potential influence on SARS-CoV-2 infection. J Ayurveda Integr Med. 2021 Mar 16. doi:

10.1016/j.jaim.2021.03.006. Epub ahead of print. PMID: 33746457; PMCID: PMC7962552.

1028. Li H, Zhou X, Tan H, Hu Y, Zhang L, Liu S, Dai M, Li Y, Li Q, Mao Z, Pan P, Su X, Hu C. Neutrophil extracellular traps contribute to the pathogenesis of acid-aspiration-induced ALI/ARDS. Oncotarget. 2017 Nov 28;9(2):1772-1784. doi:

10.18632/oncotarget.22744. PMID: 29416730; PMCID: PMC5788598.

1029. Liu S, Su X, Pan P, Zhang L, Hu Y, Tan H, Wu D, Liu B, Li H, Li H, Li Y, Dai M, Li Y, Hu C, Tsung A. Neutrophil extracellular traps are indirectly triggered by lipopolysaccharide and contribute to acute lung injury. Sci Rep. 2016 Nov 16;6:37252. doi: 10.1038/srep37252. PMID: 27849031; PMCID: PMC5110961.

1030. Liu S, Yue Y, Pan P, Zhang L, Su X, Li H, Li H, Li Y, Dai M, Li Q, Mao Z. IRF-1 Intervention in the Classical ROS-Dependent Release of NETs during LPS-Induced Acute Lung Injury in Mice. Inflammation. 2019 Feb;42(1):387-403. doi: 10.1007/s10753-0180903-7. PMID: 30315525.

1031. Zhang K, He W, Guo X, Zhang Y, Huang C. [Progress in mechanism of formation of neutrophil extracellular traps: Review]. Xi Bao Yu Fen Zi Mian Yi Xue Za Zhi. 2020 Jun;36(6):561-564. Chinese. PMID: 32696748.

1032. Yang C, Song C, Liu Y, Qu J, Li H, Xiao W, Kong L, Ge H, Sun Y, Lv W. Re-Du-Ning injection ameliorates LPS-induced lung injury through inhibiting neutrophil extracellular traps formation. Phytomedicine. 2021 Jun 19;90:153635. doi:

10.1016/j.phymed.2021.153635. Epub ahead of print. PMID: 34229173; PMCID: PMC8213523.

1033. Liu Y, Tang G, Li J. Effect and Mechanism Study of Sodium Houttuyfonate on Ventilator-Induced Lung Injury by Inhibiting ROS and Inflammation. Yonsei Med J. 2021 Jun;62(6):545-554. doi: 10.3349/ymj.2021.62.6.545. PMID: 34027642; PMCID: PMC8149929.

1034. Yang RQ, Guo PF, Ma Z, Chang C, Meng QN, Gao Y, Khan I, Wang XB, Cui ZJ. Effects of simvastatin on iNOS and caspase- 3 levels and oxidative stress following smoke inhalation injury. Mol Med Rep. 2020 Oct;22(4):3405-3417. doi: 10.3892/mmr.2020.11413. Epub 2020 Aug 4. Erratum in: Mol Med Rep. 2020 Dec;22(6):5479. PMID: 32945441; PMCID: PMC7453554.

1035. Zhao J, Zhang G, Cui W, Tian B. [Progress of neutrophil extracellular traps in airway inflammation of acute lung injury/acute respiratory distress syndrome: Review]. Xi Bao Yu Fen Zi Mian Yi Xue Za Zhi. 2020 Jul;36(7):664-670. Chinese. PMID: 32727651. 1036. Rawat S, Vrati S, Banerjee A. Neutrophils at the crossroads of acute viral infections and severity. Mol Aspects Med. 2021 Jul 17:100996. doi: 10.1016/j.mam.2021.100996. Epub ahead of print. PMID: 34284874; PMCID: PMC8286244.

1037. Dushianthan A, Grocott MP, Postle AD, Cusack R. Acute respiratory distress syndrome and acute lung injury. Postgrad Med J. 2011 Sep;87(1031):612-22. doi: 10.1136/pgmj.2011.118398. Epub 2011 Jun 4. PMID: 21642654.

1038. Zhang Y, Zhao J, Guan L, Mao L, Li S, Zhao J. Histone H4 aggravates inflammatory injury through TLR4 in chlorine gasinduced acute respiratory distress syndrome. J Occup Med Toxicol. 2020 Oct 8;15:31. doi: 10.1186/s12995-020-00282-z. PMID: 33062035; PMCID: PMC7545935.

1039. Zhang Y, Zhao Z, Guan L, Mao L, Li S, Guan X, Chen M, Guo L, Ding L, Cong C, Wen T, Zhao J. N-acetyl-heparin attenuates acute lung injury caused by acid aspiration mainly by antagonizing histones in mice. PLoS One. 2014 May 9;9(5):e97074. doi: 10.1371/journal.pone.0097074. PMID: 24816808; PMCID: PMC4016230.

1040. Chambers E, Rounds S, Lu Q. Pulmonary Endothelial Cell Apoptosis in Emphysema and Acute Lung Injury. Adv Anat Embryol Cell Biol. 2018;228:63-86. doi: 10.1007/978-3-319-68483-3_4. PMID: 29288386; PMCID: PMC5930489.

1041. Chen H, Bai C, Wang X. The value of the lipopolysaccharide-induced acute lung injury model in respiratory medicine. Expert Rev Respir Med. 2010 Dec;4(6):773-83. doi: 10.1586/ers.10.71. PMID: 21128752.

1042. Puegge J, Wang Y, Roller J, Zhang S, Luo L, Vollmar B, Thorlacius H. Adhesive Mechanisms of Histone-Induced NeutrophilEndothelium Interactions in the Muscle Microcirculation. Eur Surg Res. 2016;56(1-2):19-31. doi: 10.1159/000441778. Epub 2015 Nov 18. PMID: 26575178.

1043. Wang L, Wang T, Li H, Liu Q, Zhang Z, Xie W, Feng Y, Socorburam T, Wu G, Xia Z, Wu Q. Receptor Interacting Protein 3Mediated Necroptosis Promotes Lipopolysaccharide-Induced Inflammation and Acute Respiratory Distress Syndrome in Mice. PLoS One. 2016 May 19;11(5):e0155723. doi: 10.1371/journal.pone.0155723. PMID: 27195494; PMCID: PMC4873150. 1044. Williams AE, José RJ, Mercer PF, Brealey D, Parekh D, Thickett DR, O'Kane C, McAuley DF, Chambers RC. Evidence for chemokine synergy during neutrophil migration in ARDS. Thorax. 2017 Jan;72(1):66-73. doi: 10.1136/thoraxjnl-2016-208597 Epub 2016 Aug 5. PMID: 27496101; PMCID: PMC5329051.

1045. Zhang W, Wang Y, Li W, Wang G. The Association Between the Baseline and the Change in Neutrophil-to-Lymphocyte Ratio and Short-Term Mortality in Patients With Acute Respiratory Distress Syndrome. Front Med (Lausanne). 2021 May 14;8:636869. doi: 10.3389/fmed.2021.636869. PMID: 34055826; PMCID: PMC8160236. 
1046. Liu B, He R, Zhang L, Hao B, Jiang W, Wang W, Geng Q. Inflammatory Caspases Drive Pyroptosis in Acute Lung Injury. Front Pharmacol. 2021 Feb 5;12:631256. doi: 10.3389/fphar.2021.631256. PMID: 33613295; PMCID: PMC7892432.

1047. Strieter RM, Kunkel SL. Acute lung injury: the role of cytokines in the elicitation of neutrophils. J Investig Med. 1994 Dec;42(4):640-51. Erratum in: J Investig Med 1995 Apr;43(2):204. PMID: 8521027.

1048. Orfanos SE, Mavrommati I, Korovesi I, Roussos C. Pulmonary endothelium in acute lung injury: from basic science to the critically ill. Intensive Care Med. 2004 Sep;30(9):1702-14. doi: 10.1007/s00134-004-2370-x. Epub 2004 Jul 16. PMID: 15258728. 1049. Li Y, Xiang M, Yuan Y, Xiao G, Zhang J, Jiang Y, Vodovotz Y, Billiar TR, Wilson MA, Fan J. Hemorrhagic shock augments lung endothelial cell activation: role of temporal alterations of TLR4 and TLR2. Am J Physiol Regul Integr Comp Physiol. 2009 Dec;297(6):R1670-80. doi: 10.1152/ajpregu.00445.2009. Epub 2009 Oct 14. PMID: 19828841; PMCID: PMC2803625. 1050. Reino DC, Palange D, Feketeova E, Bonitz RP, Xu DZ, Lu Q, Sheth SU, Peña G, Ulloa L, De Maio A, Feinman R, Deitch EA. Activation of toll-like receptor 4 is necessary for trauma hemorrhagic shock-induced gut injury and polymorphonuclear neutrophil priming. Shock. 2012 Jul;38(1):107-14. doi: 10.1097/SHK.0b013e318257123a. PMID: 22575992; PMCID: PMC3378823. 1051. McGhan $\amalg$, Jaroszewski DE. The role of toll-like receptor-4 in the development of multi-organ failure following traumatic haemorrhagic shock and resuscitation. Injury. 2012 Feb;43(2):129-36. doi: 10.1016/j.injury.2011.05.032. PMID: 21689818. 1052. McGhan $\sqcup$, Jaroszewski DE. The role of toll-like receptor-4 in the development of multi-organ failure following traumatic haemorrhagic shock and resuscitation. Injury. 2012 Feb;43(2):129-36. doi: 10.1016/j.injury.2011.05.032. PMID: 21689818. 1053. Feng Y, Yang Q, Xu J, Qian G, Liu Y. Effects of HMGB1 on PMN apoptosis during LPS-induced acute lung injury. Exp Mol Pathol. 2008 Dec;85(3):214-22. doi: 10.1016/j.yexmp.2008.09.002. Epub 2008 Oct 8. PMID: 18952078.

1054. Huang XL, ma HJ, Zhou XH, Fan YM, Xian XH, Cao H. [Effect of exogenous hydrogen sulfide on polymorphonuclear neutrophil accumulation in acute lung injury rat induced by lipopolysaccharides and its mechanism]. Zhongguo Ying Yong Sheng $\mathrm{Li}$ Xue Za Zhi. 2010 Nov;26(4):477-80. Chinese. PMID: 21328993.

1055. Xie K, Yu Y, Huang Y, Zheng L, Li J, Chen H, Han H, Hou L, Gong G, Wang G. Molecular hydrogen ameliorates lipopolysaccharide-induced acute lung injury in mice through reducing inflammation and apoptosis. Shock. 2012 May;37(5):54855. doi: 10.1097/SHK.0b013e31824ddc81. PMID: 22508291.

1056. Xu X, Chen H, Zhu X, Ma Y, Liu Q, Xue Y, Chu H, Wu W, Wang J, Zou H. S100A9 promotes human lung fibroblast cells activation through receptor for advanced glycation end-product-mediated extracellular-regulated kinase 1/2, mitogen-activated protein-kinase and nuclear factor-kB-dependent pathways. Clin Exp Immunol. 2013 Sep;173(3):523-35. doi: 10.1111/cei.12139. PMID: 23682982; PMCID: PMC3949640.

1057. Vezza T, de Marañón AM, Canet F, Díaz-Pozo P, Marti M, D'Ocon P, Apostolova N, Rocha M, Víctor VM. MicroRNAs and Oxidative Stress: An Intriguing Crosstalk to Be Exploited in the Management of Type 2 Diabetes. Antioxidants (Basel). 2021 May 19;10(5):802. doi: 10.3390/antiox10050802. PMID: 34069422; PMCID: PMC8159096.

1058. G

1059. G

1060. G

1061. G

1062. G

1063. G

1064. G

1065. G

1066. G

1067. G

1068. G

1069. G

1070. G

1071. G

1072. G

1073. G

1074. G

1075. G

1076. G

1077. G

1078. G

1079. G

1080. G

1081. G

1082. G

1083. G

1084. G

1085. G

1086. G

1087. G

1088. G

1089. G

1090. G

1091. Gg

1092. G

1093. G

1094. G

1095. G

1096. G

1097. Gg

1098. G

1099. G

1100. $\mathrm{g}$

1101. G 
1102. Sungnak, W., Huang, N., Bécavin, C. et al. SARS-CoV-2 entry factors are highly expressed in nasal epithelial cells together with innate immune genes. Nat Med 26, 681-687 (2020). https://doi.org/10.1038/s41591-020-0868-6

1103. Gallo, O., Locatello, L.G., Mazzoni, A. et al. The central role of the nasal microenvironment in the transmission, modulation, and clinical progression of SARS-CoV-2 infection. Mucosal Immunol 14, 305-316 (2021). https://doi.org/10.1038/s41385-02000359-2

1104. Hati S, Bhattacharyya S. Impact of Thiol-Disulfide Balance on the Binding of Covid-19 Spike Protein with AngiotensinConverting Enzyme 2 Receptor. ACS Omega. 2020 Jun 23;5(26):16292-16298. doi: 10.1021/acsomega.0c02125. PMID: 32656452; PMCID: PMC7346263.

1105. Yi MC, Khosla C. Thiol-Disulfide Exchange Reactions in the Mammalian Extracellular Environment. Annual Review of Chemical and Biomolecular Engineering. 2016 Jun;7:197-222. DOI: 10.1146/annurev-chembioeng-080615-033553.

1106. Poltorak A, He X, Smirnova I, Liu MY, Van Huffel C, Du X, Birdwell D, Alejos E, Silva M, Galanos C, Freudenberg M, RicciardiCastagnoli P, Layton B, Beutler B. Defective LPS signaling in C3H/HeJ and C57BL/10ScCr mice: mutations in T/r4 gene. Science. 1998 Dec 11;282(5396):2085-8. doi: 10.1126/science.282.5396.2085. PMID: 9851930.

1107. Bhattacharya M, Sharma AR, Mallick B, Sharma G, Lee SS, Chakraborty C. Immunoinformatics approach to understand molecular interaction between multi-epitopic regions of SARS-CoV-2 spike-protein with TLR4/MD-2 complex. Infect Genet Evol. 2020;85:104587. doi:10.1016/j.meegid.2020.104587

1108. Choudhury A, Mukherjee S. In silico studies on the comparative characterization of the interactions of SARS-CoV-2 spike glycoprotein with ACE-2 receptor homologs and human TLRs. J Med Virol. 2020 Oct;92(10):2105-2113. doi: 10.1002/jmv.25987. Epub 2020 May 17. PMID: 32383269; PMCID: PMC7267663.

1109. Zhao Y, Kuang M, Li J, Zhu L, Jia Z, Guo X, Hu Y, Kong J, Yin H, Wang X, You F. SARS-CoV-2 spike protein interacts with and activates TLR4. Cell Res. 2021 Jul;31(7):818-820. doi: 10.1038/s41422-021-00495-9. Epub 2021 Mar 19. Erratum in: Cell Res. 2021 Apr 27;: PMID: 33742149; PMCID: PMC7975240.

1110. Pino P, Kint J, Kiseljak D, Agnolon V, Corradin G, Kajava AV, Rovero P, Dijkman R, den Hartog G, McLellan JS, Byrne PO, Wurm MJ, Wurm FM. Trimeric SARS-CoV-2 Spike Proteins Produced from CHO Cells in Bioreactors Are High-Quality Antigens. Processes. 2020; 8(12):1539. https://doi.org/10.3390/pr8121539

1111. Bevan DE, Martinko AJ, Loram LC, et al. Selection, Preparation, and Evaluation of Small- Molecule Inhibitors of Toll-Like Receptor 4. ACS Medicinal Chemistry Letters. 2010 Apr;1(5):194-198. DOI: 10.1021/ml100041f.

1112. Sanui, Terukazu, et al. "Anti-CD14 Antibody-treated Neutrophils Respond to LPS: Possible Involvement of CD14

Upregulated By Anti-CD14 Antibody Binding." Immunological Investigations, vol. 46, no. 2, 2017, pp. 190-200.

1113. J(615)(REMOVED)

1114. Choudhury A, Mukherjee S. In silico studies on the comparative characterization of the interactions of SARS-CoV-2 spike glycoprotein with ACE-2 receptor homologs and human TLRs. J Med Virol. 2020 Oct;92(10):2105-2113. doi: 10.1002/jmv.25987. Epub 2020 May 17. PMID: 32383269; PMCID: PMC7267663.

1115. Nakhlband, A, Fakhari, A, Azizi, H. Interferon-alpha position in combating with COVID-19: A systematic review. J Med Virol. 2021; 93: 5277- 5284. https://doi.org/10.1002/jmv.27072

1116. Peiffer-Smadja N, Yazdanpanah Y. Nebulised interferon beta-1a for patients with COVID-19. Lancet Respir Med. 2021 Feb;9(2):122-123. doi: 10.1016/S2213-2600(20)30523-3. Epub 2020 Nov 12. PMID: 33189160; PMCID: PMC7833737.

1117. Alavi Darazam I, Hatami F, Mahdi Rabiei M, Amin Pourhoseingholi M, Shabani M, Shokouhi S, Mardani M, Moradi O, Javandoust Gharehbagh F, Mirtalaee N, Negahban H, Amirdosara M, Zangi M, Hajiesmaeili M, Kazempour M, Shafigh N. An investigation into the beneficial effects of high-dose interferon beta 1-a, compared to low-dose interferon beta 1-a in severe COVID-19: The COVIFERON II randomized controlled trial. Int Immunopharmacol. 2021 Jun 29;99:107916. doi:

10.1016/j.intimp.2021.107916. Epub ahead of print. PMID: 34224994; PMCID: PMC8238656.

1118. Bessière $P$, Wasniewski M, Picard-Meyer E, Servat A, Figueroa T, Foret-Lucas C, Coggon A, Lesellier S, Boué F, Cebron N, Gausserès B, Trumel C, Foucras G, Salguero FJ, Monchatre-Leroy E, Volmer R. Intranasal type I interferon treatment is beneficial only when administered before clinical signs onset in the SARS-CoV-2 hamster model. PLoS Pathog. 2021 Aug 9;17(8):e1009427. doi: 10.1371/journal.ppat.1009427. PMID: 34370799; PMCID: PMC8376007.

1119. Monk PD, Marsden RJ, Tear VJ, Brookes J, Batten TN, Mankowski M, Gabbay FJ, Davies DE, Holgate ST, Ho LP, Clark T, Djukanovic R, Wilkinson TMA; Inhaled Interferon Beta COVID-19 Study Group. Safety and efficacy of inhaled nebulised interferon beta-1a (SNG001) for treatment of SARS-CoV-2 infection: a randomised, double-blind, placebo-controlled, phase 2 trial. Lancet Respir Med. 2021 Feb;9(2):196-206. doi: 10.1016/S2213-2600(20)30511-7. Epub 2020 Nov 12. PMID: 33189161; PMCID: PMC7836724.

1120. Kumar A, Ishida R, Strilets T, Cole J, Lopez-Orozco J, Fayad N, Felix-Lopez A, Elaish M, Evseev D, Magor KE, Mahal LK, Nagata LP, Evans DH, Hobman TC. SARS-CoV-2 Nonstructural Protein 1 Inhibits the Interferon Response by Causing Depletion of Key Host Signaling Factors. J Virol. 2021 Jun 10;95(13):e0026621. doi: 10.1128/JVI.00266-21. Epub 2021 Jun 10. PMID: 34110264.

1121. Miorin, L., Kehrer, T., Sanchez-Aparicio, M.T., Zhang, K., Cohen, P., Patel, R.S., Cupic, A., Makio, T., Mei, M., Moreno, E., et al. (2020). SARS-CoV-2 Orf6 hijacks Nup98 to block STAT nuclear import and antagonize interferon signaling. Proceedings of the National Academy of Sciences. 117(45), 28344-28354. DOI: 10.1073/pnas.2016650117.

1122. [22] 451.

1123. Nemudryi A, Nemudraia A, Wiegand T, Nichols J, Snyder DT, Hedges JF, Cicha C, Lee H, Vanderwood KK, Bimczok D, Jutila MA, Wiedenheft B. SARS-CoV-2 genomic surveillance identifies naturally occurring truncation of ORF7a that limits immune suppression. Cell Rep. 2021 Jun 1;35(9):109197. doi: 10.1016/j.celrep.2021.109197. Epub 2021 May 14. PMID: 34043946; PMCID: PMC8118641.

1124. Young BE, Fong SW, Chan YH, et al. Effects of a major deletion in the SARS-CoV-2 genome on the severity of infection and the inflammatory response: an observational cohort study. Lancet. 2020;396(10251):603-611. doi:10.1016/S01406736(20)31757-8

1125. Yiwen Zhang, Yingshi Chen, Yuzhuang Li, Feng Huang, Baohong Luo, Yaochang Yuan, Baijin Xia, Xiancai Ma, Tao Yang, Fei Yu, Jun Liu, Bingfeng Liu, Zheng Song, Jingliang Chen, Shumei Yan, Liyang Wu, Ting Pan, Xu Zhang, Rong Li, Wenjing Huang, Xin He, Fe i Xiao, Junsong Zhang, Hui Zhang. The ORF8 protein of SARS-CoV-2 mediates immune evasion through down-regulating MHC-I, Proceedings of the National Academy of Sciences Jun 2021, 118 (23) e2024202118; DOI: 10.1073/pnas.2024202118

1126. Konno, Y., Kimura, I., Uriu, K., Fukushi, M., Irie, T., Koyanagi, Y., Sauter, D., Gifford, R.J., Nakagawa, S., and Sato, K. (2020). SARS-CoV-2 ORF3b Is a Potent Interferon Antagonist Whose Activity Is Increased by a Naturally Occurring Elongation Variant. Cell Reports. 32(12)DOI: 10.1016/j.celrep.2020.108185.

1127. Lei, X., Dong, X., Ma, R., Wang, W., Xiao, X., Tian, Z., Wang, C., Wang, Y., Li, L., Ren, L., et al. (2020). Activation and evasion of type I interferon responses by SARS-CoV-2. Nat Commun. 11(1), 3810. Published online 2020/08/01 DOI: 10.1038/s41467-020$17665-9$ 
1128. Xia, H., Cao, Z., Xie, X., Zhang, X., Chen, J.Y.-C., Wang, H., Menachery, V.D., Rajsbaum, R., and Shi, P.-Y. (2020). Evasion of Type I Interferon by SARS-CoV-2. Cell Reports. 33(1). DOI: 10.1016/j.celrep.2020.108234

1129. Martin-Sancho L, Lewinski MK, Pache L, et al. Functional Landscape of SARS-CoV-2 Cellular Restriction. Preprint. bioRxiv. 2020;2020.09.29.319566. Published 2020 Sep 30. doi:10.1101/2020.09.29.319566

1130. Hachim, A., Kavian, N., Cohen, C.A., Chin, A.W.H., Chu, D.K.W., Mok C.K.P., Tsang, O.T.Y., Yeung, Y.C., Perera, R.A.P.M.,

Poon, L.L.M., et al. (2020). ORF8 and ORF3b antibodies are accurate serological markers of early and late SARSCoV-2 infection. Nature Immunology. 21(10), 1293-1301. DOI: 10.1038/s41590- 020-0773-7

1131. Su, Y. C. F. et al. Discovery and genomic characterization of a 382-nucleotide deletion in ORF7b and ORF8 during the early evolution of SARS-CoV-2. Mbio https://doi.org/10.1128/mBio.01610-20 (2020).

1132. Keng, C. T. et al. Te human severe acute respiratory syndrome coronavirus (SARS-CoV) $8 \mathrm{~b}$ protein is distinct from its counterpart in animal SARS-CoV and down-regulates the expression of the envelope protein in infected cells. Virology 354, 132142 (2006).

1133. McBride, R. \& Fielding, B. C. The role of severe acute respiratory syndrome (SARS)-coronavirus accessory proteins in virus pathogenesis. Viruses 4, 2902-2923 (2012).

1134. Hongye Wang, X. H. et al. SARS-CoV-2 proteome microarray for mapping COVID-19 antibody interactions at amino acid resolution. Preprint at bioRxiv https://doi.org/10.1101/2020.03.26.994756 (2020)

1135. Zeng, W. et al. Biochemical characterization of SARS-CoV-2 nucleocapsid protein. Biochem. Biophys. Res. Commun. 527, 618-623 (2020).

1136. Jin, X., Zhang, B., Zhou, X., Qiu, Y., Song, Y., Feng, F., Feng, J., Song, Q., Jia, Q., and Wang, J. (2020). Clinical characteristics of 82 cases of death from COVID-19. Plos One. 15(7). DOI: 10.1371/journal.pone.0235458

1137. Zhang, Yiwen \& Zhang, Junsong \& Chen, Yingshi \& Luo, Baohong \& Yuan, Yaochang \& Huang, Feng \& Yang, Tao \& Yu, Fei \& Liu, Jun \& Liu, Bingfeng \& Song, Zheng \& Chen, Jingliang \& Pan, Ting \& zhang, xu \& Li, Yuzhuang \& Li, Rong \& Huang, Wenjing \& Xiao, Fei \& Zhang, Hui. (2020). The ORF8 protein of SARS-CoV-2 mediates immune evasion through potently downregulating MHC-I. 10.1101/2020.05.24.111823.

1138. 147.

1139. McNab, F., Mayer-Barber, K., Sher, A., Wack, A., and O'Garra, A. (2015). Type I interferons in infectious disease. Nature Reviews Immunology. 15(2), 87-103. DOI: 10.1038/nri3787

1140. [40]Mantlo, E., Bukreyeva, N., Maruyama, J., Paessler, S., and Huang, C. (2020). Antiviral activities of type I interferons to SARS-CoV-2 infection. Antiviral Research. 179. DOI: 10.1016/j.antiviral.2020.104811.

1141. Arunachalam, P.S., Wimmers, F., Mok, C.K.P., Perera, R., Scott, M., Hagan, T., Sigal, N., Feng, Y., Bristow, L., Tak-Yin Tsang, O., et al. (2020). Systems biolog ical assessment of immunity to mild versus severe COVID-19 infection in humans. Science. 369(6508), 1210-1220. Published online 2020/08/14 DOI: 10.1126/ science.abc6261.

1142. Hadjadj, J., Yatim, N., Barnabei, L., Corneau, A., Boussier, J., Smith, N., Pere, H., Charbit, B., Bondet, V., Chenevier-Gobeaux, C., et al. (2020). Impaired type I interferon activity and inflflammatory responses in severe COVID-19 patients. Science. 369(6504), 718-724. Published online 2020/07/15 DOI: 10.1126/science.abc6027

1143. Lucas, C., Wong, P., Klein, J., Castro, T.B.R., Silva, J., Sundaram, M., Ellingson, M.K., Mao, T., Oh, J.E., Israelow, B., et al. (2020). Longitudinal analyses reveal immunological misfifiring in severe COVID-19. Nature. 584(7821), 463-469. Published online 2020/07/28 DOI: 10.1038/s41586-020-2588-y.

1144. Tay, M.Z., Poh, C.M., Renia, L., MacAry, P.A., and Ng, L.F.P. (2020). The trinity of COVID-19: immunity, inflammation and intervention. Nat Rev Immunol. 20(6), 363-374. Published online 2020/04/30 DOI: 10.1038/s41577-020-0311-8. 1145. Andolfo I, Russo R, Lasorsa VA, Cantalupo S, Rosato BE, Bonfiglio F, Frisso G, Abete P, Cassese GM, Servillo G, Esposito G, Gentile I, Piscopo C, Villani R, Fiorentino G, Cerino P, Buonerba C, Pierri B, Zollo M, Iolascon A, Capasso M. Common variants at 21q22.3 locus influence MX1 and TMPRSS2 gene expression and susceptibility to severe COVID-19. iScience. $2021 \mathrm{Apr}$ 23;24(4):102322. doi: 10.1016/j.isci.2021.102322. Epub 2021 Mar 17. PMID: 33748697; PMCID: PMC7968217.

1146. Zhang Y, Qin L, Zhao Y, Zhang P, Xu B, Li K, Liang L, Zhang C, Dai Y, Feng Y, Sun J, Hu Z, Xiang H, Knight JC, Dong T, Jin R. Interferon-Induced Transmembrane Protein 3 Genetic Variant rs12252-C Associated With Disease Severity in Coronavirus Disease 2019. J Infect Dis. 2020 Jun 16;222(1):34-37. doi: 10.1093/infdis/jiaa224. PMID: 32348495; PMCID: PMC7197559. 1147. Pati A, Padhi S, Suvankar S, Panda AK. Minor Allele of Interferon-Induced Transmembrane Protein 3 Polymorphism (rs12252) Is Covered Against Severe Acute Respiratory Syndrome Coronavirus 2 Infection and Mortality: A Worldwide Epidemiological Investigation. J Infect Dis. 2021;223(1):175-178. doi:10.1093/infdis/jiaa630

1148. Schoggins JW. Interferon-Stimulated Genes: What Do They All Do? Annu Rev Virol. 2019 Sep 29;6(1):567-584. doi: 10.1146/annurev-virology-092818-015756. Epub 2019 Jul 5. PMID: 31283436.

1149. Shi, G., Kenney, A.D., Kudryashova, E., Zani, A., Zhang, L., Lai, K.K., Hall Stoodley, L., Robinson, R.T., Kudryashov, D.S., Compton, A.A., et al. (2020). Opposing activities of IFITM proteins in SARS-CoV-2 infection. The EMBO Journal. DOI: 10.15252/embj.2020106501

1150. Zang, R., Case, J.B., Yutuc, E., Ma, X., Shen, S., Gomez Castro, M.F., Liu, Z., Zeng, Q., Zhao, H., Son, J., et al. (2020). Cholesterol 25-hydroxylase suppresses SARS-CoV-2 replication by blocking membrane fusion. Proceedings of the National Academy of Sciences. 117(50), 32105-32113. DOI: 10.1073/pnas.2012197117.

1151. Addetia, A., Xie, H., Roychoudhury, P., Shrestha, L., Loprieno, M., Huang, M.-L., Jerome, K.R., and Greninger, A.L. (2020) Identification of multiple large deletions in ORF7a resulting in in-frame gene fusions in clinical SARS-CoV-2 isolates. Journal of Clinical Virology. 129. DOI: 10.1016/j.jcv.2020.104523.

1152. Holland, L.A., Kaelin, E.A., Maqsood, R., Estifanos, B., Wu, L.I., Varsani, A., Halden, R.U., Hogue, B.G., Scotch, M., Lim, E.S., et al. (2020). An 81-Nucleotide Deletion in SARS-CoV-2 ORF7a Identifified from Sentinel Surveillance in Arizona (January to March 2020). Journal of Virology. 94(14). DOI: 10.1128/jvi.00711-20

1153. Rosenthal, S.H., Kagan, R.M., Gerasimova, A., Anderson, B., Grover, D., Hua, M., Liu, Y., Owen, R., and Lacbawan, F. (2020) Identifification of eight SARS-CoV- 2 ORF7a deletion variants in 2,726 clinical specimens. bioRxiv. DOI: 10.1101/ 2020.12.10.418855.

1154. Rosenthal, S.H., Kagan, R.M., Gerasimova, A., Anderson, B., Grover, D., Hua, M., Liu, Y., Owen, R., and Lacbawan, F. (2020). Identifification of eight SARS-CoV- 2 ORF7a deletion variants in 2,726 clinical specimens. bioRxiv. DOI: 10.1101/ 2020.12.10.418855.

1155. Su, Y.C.F., Anderson, D.E., Young, B.E., Linster, M., Zhu, F., Jayakumar, J., Zhuang, Y., Kalimuddin, S., Low, J.G.H., Tan, C.W., et al. (2020). Discovery and Genomic Characterization of a 382-Nucleotide Deletion in ORF7b and ORF8 during the Early Evolution of SARS-CoV-2. mBio. 11(4). Published online 2020/07/23 DOI: 10.1128/mBio.01610-20.

1156. Su CM, Wang L, Yoo D. Activation of NF-KB and induction of proinflammatory cytokine expressions mediated by ORF7a protein of SARS-CoV-2. Sci Rep. 2021;11(1):13464. Published 2021 Jun 29. doi:10.1038/s41598-021-92941-2 
1157. Chen G, Wu D, Guo W, Cao Y, Huang D, Wang H, Wang T, Zhang X, Chen H, Yu H, Zhang X, Zhang M, Wu S, Song J, Chen T, Han M, Li S, Luo X, Zhao J, Ning Q. Clinical and immunological features of severe and moderate coronavirus disease 2019. J Clin Invest. 2020 May 1;130(5):2620-2629. doi: 10.1172/JCI137244. PMID: 32217835; PMCID: PMC7190990.

1158. Giamarellos-Bourboulis EJ, Netea MG, Rovina N, Akinosoglou K, Antoniadou A, Antonakos N, Damoraki G, Gkavogianni T, Adami ME, Katsaounou P, Ntaganou M, Kyriakopoulou M, Dimopoulos G, Koutsodimitropoulos I, Velissaris D, Koufargyris P, Karageorgos A, Katrini K, Lekakis V, Lupse M, Kotsaki A, Renieris G, Theodoulou D, Panou V, Koukaki E, Koulouris N, Gogos C, Koutsoukou A. Complex Immune Dysregulation in COVID-19 Patients with Severe Respiratory Failure. Cell Host Microbe. 2020 Jun 10;27(6):992-1000.e3. doi: 10.1016/j.chom.2020.04.009. Epub 2020 Apr 21. PMID: 32320677; PMCID: PMC7172841.

1159. Zhou, Z. et al. Heightened innate immune responses in the respiratory tract of COVID-19 patients. Cell Host Microbe 27, 883-890 (2020)

1160. Hojyo S, Uchida M, Tanaka K, Hasebe R, Tanaka Y, Murakami M, Hirano T. How COVID-19 induces cytokine storm with high mortality. Inflamm Regen. 2020 Oct 1;40:37. doi: 10.1186/s41232-020-00146-3. PMID: 33014208; PMCID: PMC7527296. 1161. Giannakodimos I, Gkountana GV, Lykouras D, Karkoulias K, Tsakas S. The role of Interleukin-6 in the pathogenesis, prognosis and treatment of severe COVID-19. Curr Med Chem. 2020 Dec 8. doi: 10.2174/0929867328666201209100259. Epub ahead of print. PMID: 33297909.

1162. Hariharan A, Hakeem AR, Radhakrishnan S, Reddy MS, Rela M. The Role and Therapeutic Potential of NF-kappa-B Pathway in Severe COVID-19 Patients. Inflammopharmacology. 2021 Feb;29(1):91-100. doi: 10.1007/s10787-020-00773-9. Epub 2020 Nov 7. PMID: 33159646; PMCID: PMC7648206.

1163. Carcaterra M, Caruso C. Alveolar epithelial cell type II as main target of SARS-CoV-2 virus and COVID-19 development via NF-Kb pathway deregulation: A physio-pathological theory. Med Hypotheses. 2021 Jan;146:110412. doi: 10.1016/j.mehy.2020.110412. Epub 2020 Nov 23. PMID: 33308936; PMCID: PMC7681037.

1164. Han K, Blair RV, Iwanaga N, Liu F, Russell-Lodrigue KE, Qin Z, Midkiff CC, Golden NA, Doyle-Meyers LA, Kabir ME, Chandler KE, Cutrera KL, Ren M, Monjure CJ, Lehmicke G, Fischer T, Beddingfield B, Wanek AG, Birnbaum A, Maness NJ, Roy CJ, Datta PK, Rappaport J, Kolls JK, Qin X. Lung Expression of Human Angiotensin-Converting Enzyme 2 Sensitizes the Mouse to SARS-CoV-2 Infection. Am J Respir Cell Mol Biol. 2021 Jan;64(1):79-88. doi: 10.1165/rcmb.2020-03540C. Erratum in: Am J Respir Cell Mol Biol. 2021 Jul;65(1):121. PMID: 32991819; PMCID: PMC7781002.

1165. Bakhshandeh B, Sorboni SG, Javanmard AR, Mottaghi SS, Mehrabi MR, Sorouri F, Abbasi A, Jahanafrooz Z. Variants in ACE2; potential influences on virus infection and COVID-19 severity. Infect Genet Evol. 2021 Jun;90:104773. doi: 10.1016/j.meegid.2021.104773. Epub 2021 Feb 17. PMID: 33607284; PMCID: PMC7886638.

1166. Torre-Fuentes L, Matías-Guiu J, Hernández-Lorenzo L, Montero-Escribano P, Pytel V, Porta-Etessam J, Gómez-Pinedo U, Matías-Guiu JA. ACE2, TMPRSS2, and Furin variants and SARS-CoV-2 infection in Madrid, Spain. J Med Virol. 2021 Feb;93(2):863869. doi: 10.1002/jmv.26319. Epub 2020 Jul 28. PMID: 32691890; PMCID: PMC7404937.

1167. Hörnich BF, Großkopf AK, Schlagowski S, Tenbusch M, Kleine-Weber H, Neipel F, Stahl-Hennig C, Hahn AS. SARS-CoV-2 and SARS-CoV Spike-Mediated Cell-Cell Fusion Differ in Their Requirements for Receptor Expression and Proteolytic Activation. J Virol. 2021 Apr 12;95(9):e00002-21. doi: 10.1128/JVI.00002-21. PMID: 33608407; PMCID: PMC8104116.

1168. Wang X, Chen CH, Badeti S, Cho JH, Naghizadeh A, Wang Z, Liu D. Deletion of ER-retention motif on SARS-CoV-2 spike protein reduces cell hybrid during cell-cell fusion. Cell Biosci. 2021 Jun 23;11(1):114. doi: 10.1186/s13578-021-00626-0. PMID: 34162440; PMCID: PMC8220125.

1169. Yang Q, Hughes TA, Kelkar A, Yu X, Cheng K, Park S, Huang WC, Lovell JF, Neelamegham S. Inhibition of SARS-CoV-2 viral entry upon blocking $\mathrm{N}$ - and O-glycan elaboration. Elife. 2020 Oct 26;9:e61552. doi:10.7554/eLife.61552. PMID: 33103998; PMCID: PMC7685702.

1170. Nguyen K, Chakraborty S, Mansbach RA, Korber B, Gnanakaran S. Exploring the Role of Glycans in the Interaction of SARSCoV-2 RBD and Human Receptor ACE2. Viruses. 2021 May 17;13(5):927. doi: 10.3390/v13050927. PMID: 34067878; PMCID: PMC8156887.

1171. Davies DA, Adlimoghaddam A, Albensi BC. The Effect of COVID-19 on NF-kB and Neurological Manifestations of Disease. Mol Neurobiol. 2021 Aug;58(8):4178-4187. doi: 10.1007/s12035-021-02438-2. Epub 2021 Jun 1. PMID: 34075562; PMCID: PMC8169418.

1172. Humphries F, Shmuel-Galia L, Jiang Z, Wilson R, Landis P, Ng SL, Parsi KM, Maehr R, Cruz J, Morales-Ramos A, Ramanjulu JM, Bertin J, Pesiridis GS, Fitzgerald KA. A diamidobenzimidazole STING agonist protects against SARS-CoV-2 infection. Sci Immunol. 2021 May 18;6(59):eabi9002. doi:10.1126/sciimmunol.abi9002. PMID: 34010139; PMCID: PMC8158975.

1173. Coperchini F, Chiovato L, Rotondi M. Interleukin-6, CXCL10 and Infiltrating Macrophages in COVID-19-Related Cytokine Storm: Not One for All But All for One! Front Immunol. 2021 Apr 26;12:668507. doi: 10.3389/fimmu.2021.668507. PMID: 33981314; PMCID: PMC8107352.

1174. Oliviero A, de Castro F, Coperchini F, Chiovato L, Rotondi M. COVID-19 Pulmonary and Olfactory Dysfunctions: Is the Chemokine CXCL10 the Common Denominator? Neuroscientist. 2021 Jun;27(3):214-221. doi: 10.1177/1073858420939033. Epub 2020 Jul 13. PMID: 32659199.

1175. Adas G, Cukurova Z, Yasar KK, Yilmaz R, Isiksacan N, Kasapoglu P, Yesilbag Z, Koyuncu ID, Karaoz E. The Systematic Effect of Mesenchymal Stem Cell Therapy in Critical COVID-19 Patients: A Prospective Double Controlled Trial. Cell Transplant. 2021 JanDec;30:9636897211024942. doi: 10.1177/09636897211024942. PMID: 34180719; PMCID: PMC8243094.

1176. Conti P, Caraffa A, Gallenga CE, Ross R, Kritas SK, Frydas I, Younes A, Ronconi G. Coronavirus-19 (SARS-CoV-2) induces acute severe lung inflammation via IL-1 causing cytokine storm in COVID-19: a promising inhibitory strategy. J Biol Regul Homeost Agents. 2020 Nov-Dec;34(6):1971-1975. doi: 10.23812/20-1-E. PMID: 33016027.

1177. Islam H, Chamberlain TC, Mui AL, Little JP. Elevated Interleukin-10 Levels in COVID-19: Potentiation of Pro-Inflammatory Responses or Impaired Anti-Inflammatory Action? Front Immunol. 2021 Jun 21;12:677008. doi: 10.3389/fimmu.2021.677008. PMID: 34234779; PMCID: PMC8255680.

1178. Jafarzadeh A, Chauhan P, Saha B, Jafarzadeh S, Nemati M. Contribution of monocytes and macrophages to the local tissue inflammation and cytokine storm in COVID-19: Lessons from SARS and MERS, and potential therapeutic interventions. Life Sci. 2020 Sep 15;257:118102. doi: 10.1016/j.Ifs.2020.118102. Epub 2020 Jul 18. PMID: 32687918; PMCID: PMC7367812. 1179. Conti P, Caraffa A, Gallenga CE, Ross R, Kritas SK, Frydas I, Younes A, Di Emidio P, Ronconi G, Toniato E. IL-1 induces throboxane-A2 (TXA2) in COVID-19 causing inflammation and micro-thrombi: inhibitory effect of the IL-1 receptor antagonist (IL1Ra). J Biol Regul Homeost Agents. 2020 Sep-Oct,;34(5):1623-1627. doi: 10.23812/20-34-4EDIT-65. PMID: 32744052. 1180. Grobler C, Maphumulo SC, Grobbelaar LM, Bredenkamp JC, Laubscher GJ, Lourens PJ, Steenkamp J, Kell DB, Pretorius E. Covid-19: The Rollercoaster of Fibrin(Ogen), D-Dimer, Von Willebrand Factor, P-Selectin and Their Interactions with Endothelial Cells, Platelets and Erythrocytes. Int J Mol Sci. 2020 Jul 21;21(14):5168. doi: 10.3390/ijms21145168. PMID: 32708334; PMCID: PMC7403995. 
1181. Venter C, Bezuidenhout JA, Laubscher GJ, Lourens PJ, Steenkamp J, Kell DB, Pretorius E. Erythrocyte, Platelet, Serum Ferritin, and P-Selectin Pathophysiology Implicated in Severe Hypercoagulation and Vascular Complications in COVID-19. Int J Mol Sci. 2020 Nov 3;21(21):8234. doi: 10.3390/ijms21218234. PMID: 33153161; PMCID: PMC7662625.

1182. Manne BK, Denorme F, Middleton EA, Portier I, Rowley JW, Stubben C, Petrey AC, Tolley ND, Guo L, Cody M, Weyrich AS, Yost CC, Rondina MT, Campbell RA. Platelet gene expression and function in patients with COVID-19. Blood. 2020 Sep 10;136(11):1317-1329. doi: 10.1182/blood.2020007214. PMID: 32573711; PMCID: PMC7483430.

1183. Kaushik D, Bhandari R, Kuhad A. TLR4 as a therapeutic target for respiratory and neurological complications of SARS-CoV-2. Expert Opin Ther Targets. 2021 Apr 28:1-18. doi: 10.1080/14728222.2021.1918103. Epub ahead of print. PMID: 33857397; PMCID: PMC8095161.

1184. Sabaka P, Koščálová A, Straka I, Hodosy J, Lipták R, Kmotorková B, Kachlíková M, Kušnírová A. Role of interleukin 6 as a predictive factor for a severe course of Covid-19: retrospective data analysis of patients from a long-term care facility during Covid-19 outbreak. BMC Infect Dis. 2021 Mar 29;21(1):308. doi: 10.1186/s12879-021-05945-8. PMID: 33781216; PMCID: PMC8006112.

1185. Sayah W, Berkane I, Guermache I, Sabri M, Lakhal FZ, Yasmine Rahali S, Djidjeli A, Lamara Mahammed L, Merah F, Belaid B, Berkani L, Lazli NZ, Kheddouci L, Kadi A, Ouali M, Khellafi R, Mekideche D, Kheliouen A, Hamidi RM, Ayoub S, Raaf NB, Derrar F, Gharnaout M, Allam I, Djidjik R. Interleukin-6, procalcitonin and neutrophil-to-lymphocyte ratio: Potential immune-inflammatory parameters to identify severe and fatal forms of COVID-19. Cytokine. 2021 May;141:155428. doi: 10.1016/j.cyto.2021.155428. Epub 2021 Jan 15. PMID: 33550165; PMCID: PMC7834734.

1186. Liu L, Zheng Y, Cai L, Wu W, Tang S, Ding Y, Liu W, Kou G, Xiong Z, Wang S, Zheng S. Neutrophil-to-lymphocyte ratio, a critical predictor for assessment of disease severity in patients with COVID-19. Int J Lab Hematol. 2021 Apr;43(2):329-335. doi: 10.1111/ijlh.13374. Epub 2020 Oct 25. PMID: 33099889.

1187. Singh K, Mittal S, Gollapudi S, Butzmann A, Kumar J, Ohgami RS. A meta-analysis of SARS-CoV-2 patients identifies the combinatorial significance of D-dimer, C-reactive protein, lymphocyte, and neutrophil values as a predictor of disease severity. Int J Lab Hematol. 2021 Apr;43(2):324-328. doi: 10.1111/ijlh.13354. Epub 2020 Oct 3. PMID: 33010111; PMCID: PMC7675731. 1188. Lapić I, Brenčić T, Rogić D, Lukić M, Lukić I, Kovačić M, Honović L, Šerić V. Cell population data: Could a routine hematology analyzer aid in the differential diagnosis of COVID-19? Int J Lab Hematol. 2021 Apr;43(2):e64-e67. doi: 10.1111/ijlh.13368. Epub 2020 Oct 20. PMID: 33079479.

1189. Hartog NL, Davis AT, Prokop JW, Walls A, Rajasekaran S. Monitoring neutrophil-to-lymphocyte ratio in patients with coronavirus disease 2019 receiving tocilizumab. Ann Allergy Asthma Immunol. 2021 Mar;126(3):306-308. doi: 10.1016/j.anai.2020.12.014. Epub 2020 Dec 30. PMID: 33387615; PMCID: PMC7834747.

1190. Shen Y, Cheng C, Zheng X, Jin Y, Duan G, Chen M, Chen S. Elevated Procalcitonin Is Positively Associated with the Severity of COVID-19: A Meta-Analysis Based on 10 Cohort Studies. Medicina (Kaunas). 2021 Jun 9;57(6):594. doi: 10.3390/medicina57060594. PMID: 34207689; PMCID: PMC8227321.

1191. [91]Zhang P, Shi L, Xu J, Wang Y, Yang H. Elevated interleukin-6 and adverse outcomes in COVID-19 patients: a metaanalysis based on adjusted effect estimates. Immunogenetics. 2020 Oct;72(8):431-437. doi: 10.1007/s00251-020-01179-1. Epub 2020 Oct 17. PMID: 33067641; PMCID: PMC7567652.

1192. Abate BB, Kassie AM, Kassaw MW, Aragie TG, Masresha SA. Sex difference in coronavirus disease (COVID-19): a systematic review and meta-analysis. BMJ Open. 2020 Oct 6;10(10):e040129. doi: 10.1136/bmjopen-2020-040129. PMID: 33028563; PMCID: PMC7539579.

1193. Ramírez-Soto MC, Arroyo-Hernández H, Ortega-Cáceres G. Sex differences in the incidence, mortality, and fatality of COVID-19 in Peru. PLoS One. 2021 Jun 14;16(6):e0253193. doi: 10.1371/journal.pone.0253193. PMID: 34125851; PMCID: PMC8202928.

1194. Yang W, Kandula S, Huynh M, Greene SK, Van Wye G, Li W, Chan HT, McGibbon E, Yeung A, Olson D, Fine A, Shaman J. Estimating the infection-fatality risk of SARS-CoV-2 in New York City during the spring 2020 pandemic wave: a model-based analysis. Lancet Infect Dis. 2021 Feb;21(2):203-212. doi: 10.1016/S1473-3099(20)30769-6. Epub 2020 Oct 19. Erratum in: Lancet Infect Dis. 2021 Jan;21(1):e1. PMID: 33091374; PMCID: PMC7572090.

1195. Sales-Peres SHC, de Azevedo-Silva L, Bonato RCS, Sales-Peres MC, Pinto ACDS, Santiago Junior JF. Coronavirus (SARS-CoV-2) and the risk of obesity for critically illness and ICU admitted: Meta-analysis of the epidemiological evidence. Obes Res Clin Pract. 2020 Sep-Oct;14(5):389-397. doi: 10.1016/j.orcp.2020.07.007. Epub 2020 Aug 3. PMID: 32773297; PMCID: PMC7396969. 1196. Roncon L, Zuin M, Rigatelli G, Zuliani G. Diabetic patients with COVID-19 infection are at higher risk of ICU admission and poor short-term outcome. J Clin Virol. 2020 Jun;127:104354. doi: 10.1016/j.jcv.2020.104354. Epub 2020 Apr 9. PMID: 32305882; PMCID: PMC7195018.

1197. Alcocer-Gamba MA, Gutiérrez-Fajardo P, Sosa-Caballero A, Cabrera-Rayo A, Faradji-Hazan RN, Padilla-Padilla FG, GarnicaCuellar JC, Hernández-Arispe LM, Reyes-Cianeros FA, León-Suárez A, de-Jesús-Rivera J, Mancillas-Adame L, Gaxiola-Macias M, Márquez-Rodríguez E, Miranda-Malpica E, Sanchez-Pedraza V, Lara-Martínez DS, Segovia-Palomo A, Nava-Hernández A, RiveraReyes R. Recommendations for the care of patients with diabetes mellitus with risk factors or established cardiovascular disease and SARS-CoV-2mellitus con factores de riesgo o enfermedad cardiovascular establecida y SARS-CoV-2. Arch Cardiol Mex. 2020;90(Supl):77-83. English. doi: 10.24875/ACM.M20000074. PMID: 32523151.

1198. Saed Aldien A, Ganesan GS, Wahbeh F, Al-Nassr N, Altarawneh H, Al Theyab L, Saed Aldien S, Tomerak S, Naveed H, Elshazly $M B$, Zakaria D. Systemic inflammation may induce cardiac injury in COVID-19 patients including children and adolescents without underlying cardiovascular diseases: A systematic review. Cardiovasc Revasc Med. 2021 Apr 15:S1553-8389(21)00195-0. doi: 10.1016/j.carrev.2021.04.007. Epub ahead of print. PMID: 33952432; PMCID: PMC8046745.

1199. Sharma YP, Agstam S, Yadav A, Gupta A, Gupta A. Cardiovascular manifestations of COVID-19: An evidence-based narrative review. Indian J Med Res. 2020 Dec 7. doi: 10.4103/ijmr.IJMR 2450_20. Epub ahead of print. PMID: 33281125.

1200. Lima RS, Rocha LPC, Moreira PR. Genetic and epigenetic control of ACE2 expression and its possible role in COVID-19. Cell Biochem Funct. 2021 Jun 1:10.1002/cbf.3648. doi: 10.1002/cbf.3648. Epub ahead of print. PMID: 34075603; PMCID: PMC8239811.

1201. Bechtel, T. J.; Weerapana, E. From structure to redox: The diverse functional roles of disulfides and implications in disease. Proteomics 2017, 17, No. 1600391.

1202. slund F, Beckwith J. Bridge over troubled waters: sensing stress by disulfide bond formation. Cell. 1999 Mar;96(6):751-753. DOI: 10.1016/s0092-8674(00)80584-x.

1203. Daniela Giustarini, Annalisa Santucci, Desirée Bartolini, Francesco Galli, Ranieri Rossi, The age-dependent decline of the extracellular thiol-disulfide balance and its role in SARS-CoV-2 infection, Redox Biology, Volume 41, 2021, 101902,ISSN 22132317,https://doi.org/10.1016/j.redox.2021.101902.

1204. [104]Z(100 (removed) 
1205. [105]Jiahui Chen, Rui Wang, Menglun Wang, Guo-Wei Wei, Mutations Strengthened SARS-CoV-2 Infectivity, Journal of Molecular Biology,Volume 432, Issue 19,2020, Pages 5212-5226,ISSN 0022-2836, https://doi.org/10.1016/j.jmb.2020.07.009. 1206. [106]R. Yan, et al., Structural basis for the recognition of SARS-CoV-2 by full-length human ACE2, Science 367 (6485) (2020) 1444-1448.

1207. [107]M. Hoffmann, et al., SARS-CoV-2 cell entry depends on ACE2 and TMPRSS2 and is blocked by a clinically proven protease inhibitor, Cell 181 (2) (2020) 271-280.e8

1208. [108]Mercurio, I., Tragni, V., Busto, F. et al. Protein structure analysis of the interactions between SARS-CoV-2 spike protein and the human ACE2 receptor: from conformational changes to novel neutralizing antibodies. Cell. Mol. Life Sci. 78, 1501-1522 (2021). https://doi.org/10.1007/s00018-020-03580-1

1209. Dimitri Lavillette, Rym Barbouche, Yongxiu Yao, Bertrand Boson, François-Loïc Cosset, Ian M. Jones, Emmanuel Fenouillet, Significant Redox Insensitivity of the Functions of the SARS-CoV Spike Glycoprotein: COMPARISON WITH HIV ENVELOPE*, Journa of Biological Chemistry,2006,ISSN 0021-9258,https://doi.org/10.1074/jbc.M512529200.

1210. Jaspers I. Cigarette smoke effects on innate immune mechanisms in the nasal mucosa. Potential effects on the microbiome. Ann Am Thorac Soc. 2014 Jan;11 Suppl 1:S38-42. doi: 10.1513/AnnalsATS.201306-154MG. PMID: 24437404.

1211. Lee J, Taneja V, Vassallo R. Cigarette smoking and inflammation: cellular and molecular mechanisms. J Dent Res. 2012 Feb;91(2):142-9. doi: 10.1177/0022034511421200. Epub 2011 Aug 29. PMID: 21876032; PMCID: PMC3261116.

1212. Barker BR, Taxman DJ, Ting JP. Cross-regulation between the IL-1B/IL-18 processing inflammasome and other inflammatory cytokines. Curr Opin Immunol. 2011 Oct;23(5):591-7. doi: 10.1016/j.coi.2011.07.005. Epub 2011 Aug 10. PMID: 21839623; PMCID: PMC3380339.4

1213. Wu W, Tian L, Zhang W, Booth JL, Ainsua-Enrich E, Kovats S, Brown BR, Metcalf JP. Long-term cigarette smoke exposure dysregulates pulmonary T cell response and IFN- $\gamma$ protection to influenza virus in mouse. Respir Res. 2021 Apr 20;22(1):112. doi: 10.1186/s12931-021-01713-z. PMID: 33879121; PMCID: PMC8056367.

1214. Wu W, Patel KB, Booth JL, Zhang W, Metcalf JP. Cigarette smoke extract suppresses the RIG-I-initiated innate immune response to influenza virus in the human lung. Am J Physiol Lung Cell Mol Physiol. 2011 Jun;300(6):L821-30. doi: 10.1152/ajplung.00267.2010. Epub 2011 Feb 18. PMID: 21335520; PMCID: PMC3119130.

1215. Wang X, Wu W, Zhang W, Leland Booth J, Duggan ES, Tian L, More S, Zhao YD, Sawh RN, Liu L, Zou MH, Metcalf JP. RIG-I overexpression decreases mortality of cigarette smoke exposed mice during influenza A virus infection. Respir Res. 2017 Sep 2;18(1):166. doi: 10.1186/s12931-017-0649-z. PMID: 28865477; PMCID: PMC5581920.

1216. Wu W, Zhang W, More S, Booth JL, Duggan ES, Liu L, Zhao YD, Metcalf JP. Cigarette smoke attenuates the RIG-I-initiated innate antiviral response to influenza infection in two murine models. Am J Physiol Lung Cell Mol Physiol. 2014 Dec 1;307(11):L848-58. doi: 10.1152/ajplung.00158.2014. Epub 2014 Sep 26. PMID: 25260755; PMCID: PMC4254961. 1217. Wu J, Xu H, Wong PF, Xia S, Xu J, Dong J. Icaritin attenuates cigarette smoke-mediated oxidative stress in human lung epithelial cells via activation of PI3K-AKT and Nrf2 signaling. Food Chem Toxicol. 2014 Feb;64:307-13. doi: 10.1016/j.fct.2013.12.006. Epub 2013 Dec 8. PMID: 24333105.

1218. Faux SP, Tai T, Thorne D, Xu Y, Breheny D, Gaca M. The role of oxidative stress in the biological responses of lung epithelial cells to cigarette smoke. Biomarkers. 2009 Jul;14 Suppl 1:90-6. doi: 10.1080/13547500902965047. PMID: 19604067.

1219. Dechanet C, Brunet C, Anahory T, Hamamah S, Hedon B, Dechaud H. Effet du tabagisme sur l'implantation embryonnaire et la placentation précoce et facteurs influençant la toxicité tabagique sur la reproduction (Partie II) [Effects of cigarette smoking on embryo implantation and placentation and analysis of factors interfering with cigarette smoke effects (Part II)]. Gynecol Obstet Fertil. 2011 Oct;39(10):567-74. French. doi: 10.1016/j.gyobfe.2011.07.023. Epub 2011 Sep 19. PMID: 21937253.

1220. 885

1221. Danov O, Wolff M, Bartel S, Böhlen S, Obernolte H, Wronski S, Jonigk D, Hammer B, Kovacevic D, Reuter S, KraussEtschmann S, Sewald K. Cigarette Smoke Affects Dendritic Cell Populations, Epithelial Barrier Function, and the Immune Response to Viral Infection With H1N1. Front Med (Lausanne). 2020 Nov 6;7:571003. doi: 10.3389/fmed.2020.571003. PMID: 33240904 PMCID: PMC7678748.

1222. Duffney PF, McCarthy CE, Nogales A, Thatcher TH, Martinez-Sobrido L, Phipps RP, Sime PJ. Cigarette smoke dampens antiviral signaling in small airway epithelial cells by disrupting TLR3 cleavage. Am J Physiol Lung Cell Mol Physiol. 2018 Mar 1;314(3):L505-L513. doi: 10.1152/ajplung.00406.2017. Epub 2017 Dec 14. PMID: 29351447; PMCID: PMC5900359.

1223. Modestou MA, Manzel L, El-Mahdy S, Look DC. Inhibition of IFN-gamma-dependent antiviral airway epithelial defense by cigarette smoke. Respir Res. 2010 May 26;11(1):64. doi: 10.1186/1465-9921-11-64. PMID: 20504369; PMCID: PMC2890646.

1224. Nyunoya T, Mebratu Y, Contreras A, Delgado M, Chand HS, Tesfaigzi Y. Molecular processes that drive cigarette smokeinduced epithelial cell fate of the lung. Am J Respir Cell Mol Biol. 2014 Mar;50(3):471-82. doi: 10.1165/rcmb.2013-0348TR. PMID: 24111585; PMCID: PMC4068939.

1225. Eddleston J, Lee RU, Doerner AM, Herschbach J, Zuraw BL. Cigarette smoke decreases innate responses of epithelial cells to rhinovirus infection. Am J Respir Cell Mol Biol. 2011 Jan;44(1):118-26. doi: 10.1165/rcmb.2009-02660C. Epub 2010 Mar 11. PMID: 20224072; PMCID: PMC3028255.

1226. Pang Y, Zheng B, Fan LW, Rhodes PG, Cai Z. IGF-1 protects oligodendrocyte progenitors against TNFalpha-induced damage by activation of PI3K/Akt and interruption of the mitochondrial apoptotic pathway. Glia. 2007 Aug 15;55(11):1099-107. doi: 10.1002/glia.20530. PMID: 17577243.

1227. Li Y, Xie J, Wu S, Xia J, Zhang P, Liu C, Zhang P, Huang X. Protein kinase regulated by dsRNA downregulates the interferon production in dengue virus- and dsRNA-stimulated human lung epithelial cells. PLoS One. 2013;8(1):e55108. doi: 10.1371/journal.pone.0055108. Epub 2013 Jan 25. PMID: 23372823; PMCID: PMC3555826.

1228. Bannerman DD, Goldblum SE. Direct effects of endotoxin on the endothelium: barrier function and injury. Lab Invest. 1999 Oct;79(10):1181-99. PMID: 10532583.

1229. Agod Z, Fekete T, Budai MM, Varga A, Szabo A, Moon H, Boldogh I, Biro T, Lanyi A, Bacsi A, Pazmandi K. Regulation of type I interferon responses by mitochondria-derived reactive oxygen species in plasmacytoid dendritic cells. Redox Biol. 2017 Oct;13:633-645. doi: 10.1016/j.redox.2017.07.016. Epub 2017 Jul 29. PMID: 28818792; PMCID: PMC5558471.

1230. Szabo A, Magyarics Z, Pazmandi K, Gopcsa L, Rajnavolgyi E, Bacsi A. TLR ligands upregulate RIG-I expression in human plasmacytoid dendritic cells in a type I IFN-independent manner. Immunol Cell Biol. 2014 Sep;92(8):671-8. doi: 10.1038/icb.2014.38. Epub 2014 May 20. PMID: 24839978.

1231. Bao M, Liu YJ. Regulation of TLR7/9 signaling in plasmacytoid dendritic cells. Protein Cell. 2013 Jan;4(1):40-52. doi: 10.1007/s13238-012-2104-8. Epub 2012 Nov 7. PMID: 23132256; PMCID: PMC3667388.

1232. Fekete T, Bencze D, Szabo A, Csoma E, Biro T, Bacsi A, Pazmandi K. Regulatory NLRs Control the RLR-Mediated Type I Interferon and Inflammatory Responses in Human Dendritic Cells. Front Immunol. 2018 Oct 5;9:2314. doi: 10.3389/fimmu.2018.02314. PMID: 30344524; PMCID: PMC6182093. 
1233. Fekete T, Ágics B, Bencze D, Bene K, Szántó A, Tarr T, Veréb Z, Bácsi A, Pázmándi K. Regulation of RLR-Mediated Antiviral Responses of Human Dendritic Cells by mTOR. Front Immunol. 2020 Sep 11;11:572960. doi: 10.3389/fimmu.2020.572960. PMID: 33013932; PMCID: PMC7516067.

1234. Swiecki M, Colonna M. Unraveling the functions of plasmacytoid dendritic cells during viral infections, autoimmunity, and tolerance. Immunol Rev. 2010 Mar;234(1):142-62. doi: 10.1111/j.0105-2896.2009.00881.x. PMID: 20193017; PMCID: PMC3507434.

1235. Ali S, Mann-Nüttel R, Schulze A, Richter L, Alferink J, Scheu S. Sources of Type I Interferons in Infectious Immunity: Plasmacytoid Dendritic Cells Not Always in the Driver's Seat. Front Immunol. 2019 Apr 12;10:778. doi: 10.3389/fimmu.2019.00778. PMID: 31031767; PMCID: PMC6473462.

1236. Hirsch I, Caux C, Hasan U, Bendriss-Vermare N, Olive D. Impaired Toll-like receptor 7 and 9 signaling: from chronic viral infections to cancer. Trends Immunol. 2010 Oct;31(10):391-7. doi: 10.1016/j.it.2010.07.004. Epub 2010 Sep 9. PMID: 20832362 1237. Onodi F, Bonnet-Madin L, Meertens L, Karpf L, Poirot J, Zhang SY, Picard C, Puel A, Jouanguy E, Zhang Q, Le Goff J, Molina JM, Delaugerre C, Casanova JL, Amara A, Soumelis V. SARS-CoV-2 induces human plasmacytoid predendritic cell diversification via UNC93B and IRAK4. J Exp Med. 2021 Apr 5;218(4):e20201387. doi: 10.1084/jem.20201387. PMID: 33533916; PMCID: PMC7849819.

1238. Busnadiego I, Fernbach S, PohI MO, Karakus U, Huber M, Trkola A, Stertz S, Hale BG. Antiviral Activity of Type I, II, and III Interferons Counterbalances ACE2 Inducibility and Restricts SARS-CoV-2. mBio. 2020 Sep 10;11(5):e01928-20. doi: 10.1128/mBio.01928-20. PMID: 32913009; PMCID: PMC7484541.

1239. Quarleri J, Delpino MV. Type I and III IFN-mediated antiviral actions counteracted by SARS-CoV-2 proteins and host inherited factors. Cytokine Growth Factor Rev. 2021 Apr;58:55-65. doi: 10.1016/j.cytogfr.2021.01.003. Epub 2021 Feb 9. PMID: 33608189; PMCID: PMC7871890.

1240. Guiducci C, Coffman RL, Barrat FJ. Signalling pathways leading to IFN-alpha production in human plasmacytoid dendritic cell and the possible use of agonists or antagonists of TLR7 and TLR9 in clinical indications. J Intern Med. 2009 Jan;265(1):43-57. doi: 10.1111/j.1365-2796.2008.02050.x. PMID: 19093959.

1241. Glaum MC, Narula S, Song D, Zheng Y, Anderson AL, Pletcher CH, Levinson Al. Toll-like receptor 7-induced naive human Bcell differentiation and immunoglobulin production. J Allergy Clin Immunol. 2009 Jan;123(1):224-230.e4. doi: 10.1016/j.jaci.2008.09.018. Epub 2008 Nov 7. PMID: 18995892.

1242. Giordani L, Sanchez M, Libri I, Quaranta MG, Mattioli B, Viora M. IFN-alpha amplifies human naive B cell TLR-9-mediated activation and Ig production. J Leukoc Biol. 2009 Aug;86(2):261-71. doi: 10.1189/jlb.0908560. Epub 2009 Apr 28. PMID: 19401392. 1243. Tsukamoto Y, Nagai Y, Kariyone A, Shibata T, Kaisho T, Akira S, Miyake K, Takatsu K. Toll-like receptor 7 cooperates with IL-4 in activated $B$ cells through antigen receptor or CD38 and induces class switch recombination and IgG1 production. Mol Immunol. 2009 Apr;46(7):1278-88. doi: 10.1016/j.molimm.2008.11.022. Epub 2009 Jan 20. PMID: 19157556.

1244. Mehrotra PT, Grant AJ, Siegel JP. Synergistic effects of IL-7 and IL-12 on human T cell activation. J Immunol. 1995 May 15;154(10):5093-102. PMID: 7730615.

1245. Castro SM, Chakraborty K, Guerrero-Plata A. Cigarette smoke suppresses TLR-7 stimulation in response to virus infection in plasmacytoid dendritic cells. Toxicol In Vitro. 2011 Aug;25(5):1106-13. doi: 10.1016/j.tiv.2011.03.011. Epub 2011 Mar 22. PMID: 21435390.

1246. Megjugorac NJ, Young HA, Amrute SB, Olshalsky SL, Fitzgerald-Bocarsly P. Virally stimulated plasmacytoid dendritic cells produce chemokines and induce migration of T and NK cells. J Leukoc Biol. 2004 Mar;75(3):504-14. doi: 10.1189/jlb.0603291. Epub 2004 Jan 23. PMID: 14742635.

1247. Schlender J, Hornung V, Finke S, Günthner-Biller M, Marozin S, Brzózka K, Moghim S, Endres S, Hartmann G, Conzelmann KK. Inhibition of toll-like receptor 7- and 9-mediated alpha/beta interferon production in human plasmacytoid dendritic cells by respiratory syncytial virus and measles virus. J Virol. 2005 May;79(9):5507-15. doi: 10.1128/JVI.79.9.5507-5515.2005. PMID: 15827165; PMCID: PMC1082779.

1248. Ito T, Wang YH, Liu YJ. Plasmacytoid dendritic cell precursors/type I interferon-producing cells sense viral infection by Tolllike receptor (TLR) 7 and TLR9. Springer Semin Immunopathol. 2005 Jan;26(3):221-9. doi: 10.1007/s00281-004-0180-4. Epub 2004 Nov 13. PMID: 15592841.

1249. Hoshino K, Sugiyama T, Matsumoto M, Tanaka T, Saito M, Hemmi H, Ohara O, Akira S, Kaisho T. IkappaB kinase-alpha is critical for interferon-alpha production induced by Toll-like receptors 7 and 9. Nature. 2006 Apr 13;440(7086):949-53. doi: 10.1038/nature04641. PMID: 16612387.

1250. Izaguirre A, Barnes BJ, Amrute S, Yeow WS, Megjugorac N, Dai J, Feng D, Chung E, Pitha PM, Fitzgerald-Bocarsly P. Comparative analysis of IRF and IFN-alpha expression in human plasmacytoid and monocyte-derived dendritic cells. J Leukoc Biol. 2003 Dec;74(6):1125-38. doi: 10.1189/jlb.0603255. Epub 2003 Sep 2. PMID: 12960254.

1251. Lichtenegger FS, Schnorfeil FM, Rothe M, Deiser K, Altmann T, Bücklein VL, Köhnke T, Augsberger C, Konstandin NP, Spiekermann K, Moosmann A, Boehm S, Boxberg M, Heemskerk MH, Goerlich D, Wittmann G, Wagner B, Hiddemann W, Schendel DJ, Kvalheim G, Bigalke I, Subklewe M. Toll-like receptor 7/8-matured RNA-transduced dendritic cells as post-remission therapy in acute myeloid leukaemia: results of a phase I trial. Clin Transl Immunology. 2020 Mar 3;9(3):e1117. doi: 10.1002/cti2.1117. PMID: 32153780; PMCID: PMC7053229.

1252. Xu S, Koldovsky U, Xu M, Wang D, Fitzpatrick E, Son G, Koski G, Czerniecki BJ. High-avidity antitumor T-cell generation by toll receptor 8-primed, myeloid- derived dendritic cells is mediated by IL-12 production. Surgery. 2006 Aug;140(2):170-8. doi: 10.1016/j.surg.2006.03.006. PMID: 16904966.

1253. Gierlich P, Lex V, Technau A, Keupp A, Morper L, Glunz A, Sennholz H, Rachor J, Sauer S, Marcu A, Grigoleit GU, Wölfl M, Schlegel PG, Eyrich M. Prostaglandin E2 in a TLR3- and 7/8-agonist-based DC maturation cocktail generates mature, cytokineproducing, migratory DCs but impairs antigen cross-presentation to CD8+ T cells. Cancer Immunol Immunother. 2020 Jun;69(6):1029-1042. doi: 10.1007/s00262-019-02470-1. Epub 2020 Feb 25. PMID: 32100075; PMCID: PMC7223547. 1254. Pufnock JS, Cigal M, Rolczynski LS, Andersen-Nissen E, Wolfl M, McElrath MJ, Greenberg PD. Priming CD8+ T cells with dendritic cells matured using TLR4 and TLR7/8 ligands together enhances generation of CD8+ T cells retaining CD28. Blood. 2011 Jun 16;117(24):6542-51. doi: 10.1182/blood-2010-11-317966. Epub 2011 Apr 14. PMID: 21493800; PMCID: PMC3123022. 1255. Fehres CM, Bruijns SC, van Beelen AJ, Kalay H, Ambrosini M, Hooijberg E, Unger WW, de Gruijl TD, van Kooyk Y. Topical rather than intradermal application of the TLR7 ligand imiquimod leads to human dermal dendritic cell maturation and CD8+ Tcell cross-priming. Eur J Immunol. 2014 Aug;44(8):2415-24. doi: 10.1002/eji.201344094. Epub 2014 Jun 24. PMID: 24825342. 1256. Miller SM, Cybulski V, Whitacre M, Bess LS, Livesay MT, Walsh L, Burkhart D, Bazin HG, Evans JT. Novel Lipidated Imidazoquinoline TLR7/8 Adjuvants Elicit Influenza-Specific Th1 Immune Responses and Protect Against Heterologous H3N2 Influenza Challenge in Mice. Front Immunol. 2020 Mar 10;11:406. doi: 10.3389/fimmu.2020.00406. PMID: 32210973; PMCID: PMC7075946. 
1257. Sachin Bhagchandani, Jeremiah A. Johnson, Darrell J. Irvine, Evolution of Toll-like receptor $7 / 8$ agonist therapeutics and their delivery approaches: From antiviral formulations to vaccine adjuvants, Advanced Drug Delivery Reviews, Volume 175,2021,113803,ISSN 0169-409X, https://doi.org/10.1016/j.addr.2021.05.013.

1258. Tomić Sergej, Joksimović Bojan, Bekić Marina, Vasiljević Miloš, Milanović Marijana, Čolić Miodrag, Vučević Dragana Prostaglanin-E2 Potentiates the Suppressive Functions of Human Mononuclear Myeloid-Derived Suppressor Cells and Increases Their Capacity to Expand IL-10-Producing Regulatory T Cell Subsets, Frontiers in Immunology,P,10,

2019,10.3389/fimmu.2019.00475

1259. Marshall JD, Heeke DS, Gesner ML, Livingston B, Van Nest G. Negative regulation of TLR9-mediated IFN-alpha induction by a small-molecule, synthetic TLR7 ligand. J Leukoc Biol. 2007 Sep;82(3):497-508. doi: 10.1189/jlb.0906575. Epub 2007 Jun 12. PMID: 17565046.

1260. Vreća M, Zeković A, Damjanov N, Andjelković M, Ugrin M, Pavlović S, Spasovski V. Expression of TLR7, TLR9, JAK2, and STAT3 genes in peripheral blood mononuclear cells from patients with systemic sclerosis. J Appl Genet. 2018 Feb;59(1):59-66. doi: 10.1007/s13353-017-0415-4. Epub 2017 Nov 16. PMID: 29147913.

1261. Wang J, Lau KY, Jung J, Ravindran P, Barrat FJ. Bruton's tyrosine kinase regulates TLR9 but not TLR7 signaling in human plasmacytoid dendritic cells. Eur J Immunol. 2014 Apr;44(4):1130-6. doi: 10.1002/eji.201344030. Epub 2014 Jan 20. PMID: 24375473.

1262. Guiducci C, Coffman RL, Barrat FJ. Signalling pathways leading to IFN-alpha production in human plasmacytoid dendritic cell and the possible use of agonists or antagonists of TLR7 and TLR9 in clinical indications. J Intern Med. 2009 Jan;265(1):43-57. doi: 10.1111/j.1365-2796.2008.02050.x. PMID: 19093959.

1263. Mubarak RA, Roberts N, Mason RJ, Alper S, Chu HW. Comparison of pro- and anti-inflammatory responses in paired human primary airway epithelial cells and alveolar macrophages. Respir Res. 2018 Jun 25;19(1):126. doi: 10.1186/s12931-018-0825-9. PMID: 29940963; PMCID: PMC6020222.

1264. Hamon R, Tran HB, Roscioli E, Ween M, Jersmann H, Hodge S. Bushfire smoke is pro-inflammatory and suppresses macrophage phagocytic function. Sci Rep. 2018 Sep 7;8(1):13424. doi: 10.1038/s41598-018-31459-6. PMID: 30194323; PMCID: PMC6128914

1265. Murugan V, Peck MJ. Signal transduction pathways linking the activation of alveolar macrophages with the recruitment of neutrophils to lungs in chronic obstructive pulmonary disease. Exp Lung Res. 2009 Aug;35(6):439-85. doi: 10.1080/01902140902759290. PMID: 19842832.

1266. Heulens N, Korf H, Cielen N, De Smidt E, Maes K, Gysemans C, Verbeken E, Gayan-Ramirez G, Mathieu C, Janssens W. Vitamin D deficiency exacerbates COPD-like characteristics in the lungs of cigarette smoke-exposed mice. Respir Res. 2015 Sep 16;16(1):110. doi: 10.1186/s12931-015-0271-x. PMID: $26376849 ;$ PMCID: PMC4574263.

1267. Toledo-Arruda AC, Vieira RP, Guarnier FA, Suehiro CL, Caleman-Neto A, Olivo CR, Arantes PMM, Almeida FM, Lopes FDTQS, Ramos EMC, Cecchini R, Lin CJ, Martins MA. Time-course effects of aerobic physical training in the prevention of cigarette smokeinduced COPD. J Appl Physiol (1985). 2017 Sep 1;123(3):674-683. doi: 10.1152/japplphysiol.00819.2016. Epub 2017 Jul 20. PMID: 28729393.

1268. John G, Kohse K, Orasche J, Reda A, Schnelle-Kreis J, Zimmermann R, Schmid O, Eickelberg O, Yildirim AÖ. The composition of cigarette smoke determines inflammatory cell recruitment to the lung in COPD mouse models. Clin Sci (Lond). 2014 Feb;126(3):207-21. doi: 10.1042/CS20130117. PMID: 23875733; PMCID: PMC3906955.

1269. Higaki M, Wada H, Mikura S, Yasutake T, Nakamura M, Niikura M, Kobayashi F, Kamma H, Kamiya S, Ito K, Barnes PJ, Goto H, Takizawa H. Interleukin-10 modulates pulmonary neutrophilic inflammation induced by cigarette smoke exposure. Exp Lung Res. 2015;41(10):525-34. doi: 10.3109/01902148.2015.1096315. PMID: 26651880.

1270. Sato A, Hoshino Y, Hara T, Muro S, Nakamura H, Mishima M, Yodoi J. Thioredoxin-1 ameliorates cigarette smoke-induced lung inflammation and emphysema in mice. J Pharmacol Exp Ther. 2008 May;325(2):380-8. doi: 10.1124/jpet.107.134007. Epub 2008 Feb 6. PMID: 18256171.

1271. astro P, Nasser H, Abrahão A, Dos Reis LC, Riça I, Valença SS, Rezende DC, Quintas LE, Cavalcante MC, Porto LC, Koatz VL. Aspirin and indomethacin reduce lung inflammation of mice exposed to cigarette smoke. Biochem Pharmacol. $2009 \mathrm{Mar}$ 15;77(6):1029-39. doi: 10.1016/j.bcp.2008.12.012. Epub 2008 Dec 31. PMID: 19161990

1272. Metcalfe HJ, Lea S, Hughes D, Khalaf R, Abbott-Banner K, Singh D. Effects of cigarette smoke on Toll-like receptor (TLR) activation of chronic obstructive pulmonary disease (COPD) macrophages. Clin Exp Immunol. 2014 Jun;176(3):461-72. doi: 10.1111/cei.12289. PMID: 24528166; PMCID: PMC4008991.

1273. Lee KM, Renne RA, Harbo SJ, Clark ML, Johnson RE, Gideon KM. 3-week inhalation exposure to cigarette smoke and/or lipopolysaccharide in AKR/J mice. Inhal Toxicol. 2007 Jan;19(1):23-35. doi: 10.1080/08958370600985784. PMID: 17127640. 1274. Bozinovski S, Seow HJ, Chan SP, Anthony D, McQualter J, Hansen M, Jenkins BJ, Anderson GP, Vlahos R. Innate cellular sources of interleukin-17A regulate macrophage accumulation in cigarette- smoke-induced lung inflammation in mice. Clin Sci (Lond). 2015 Nov;129(9):785-96. doi: 10.1042/CS20140703. Epub 2015 Jul 1. PMID: 26201093; PMCID: PMC4613531 1275. Chen Z, Yan Q, Zhang Z, Lan T, Liu P, Han S, Lin Y, Lin J. Immunomodulatory Effects of Hydrolyzed Seawater Pearl Tablet (HSPT) on Th1/Th2 Functionality in a Mice Model of Chronic Obstructive Pulmonary Disease (COPD) Induced by Cigarette Smoke. Evid Based Complement Alternat Med. 2020 Nov 18;2020:5931652. doi: 10.1155/2020/5931652. PMID: 33281913; PMCID: PMC7688355

1276. Liang P, Peng S, Zhang M, Ma Y, Zhen X, Li H. Huai Qi Huang corrects the balance of Th1/Th2 and Treg/Th17 in an ovalbumin-induced asthma mouse model. Biosci Rep. 2017 Dec 22;37(6):BSR20171071. doi: 10.1042/BSR20171071. PMID: 29162668; PMCID: PMC5741832.

1277. Bracke KR, D'hulst AI, Maes T, Moerloose KB, Demedts IK, Lebecque S, Joos GF, Brusselle GG. Cigarette smoke-induced pulmonary inflammation and emphysema are attenuated in CCR6-deficient mice. J Immunol. 2006 Oct 1;177(7):4350-9. doi: 10.4049/jimmunol.177.7.4350. PMID: 16982869.

1278. Barnes PJ. Alveolar macrophages in chronic obstructive pulmonary disease (COPD). Cell Mol Biol (Noisy-le-grand). 2004;50 Online Pub:OL627-37. PMID: 15579256.

1279. Hristova M, Spiess PC, Kasahara DI, Randall MJ, Deng B, van der Vliet A. The tobacco smoke component, acrolein, suppresses innate macrophage responses by direct alkylation of c-Jun N-terminal kinase. Am J Respir Cell Mol Biol. 2012 Jan;46(1):23-33. doi: 10.1165/rcmb.2011-0134OC. PMID: $21778411 ;$ PMCID: PMC3262655.

1280. Endale M, Park SC, Kim S, Kim SH, Yang Y, Cho JY, Rhee MH. Quercetin disrupts tyrosine-phosphorylated phosphatidylinositol 3-kinase and myeloid differentiation factor-88 association, and inhibits MAPK/AP-1 and IKK/NF-KB-induced inflammatory mediators production in RAW 264.7 cells. Immunobiology. 2013 Dec;218(12):1452-67. doi: 10.1016/j.imbio.2013.04.019. Epub 2013 May 9. PMID: 23735482.

1281. Birrell MA, Wong S, Catley MC, Belvisi MG. Impact of tobacco-smoke on key signaling pathways in the innate immune response in lung macrophages. J Cell Physiol. 2008 Jan;214(1):27-37. doi: 10.1002/jcp.21158. PMID: 17541958. 
1282. Kim TH, Lee HK. Differential roles of lung dendritic cell subsets against respiratory virus infection. Immune Netw. 2014 Jun;14(3):128-37. doi: 10.4110/in.2014.14.3.128. Epub 2014 Jun 19. PMID: 24999309; PMCID: PMC4079819.

1283. Bauer CM, Zavitz CC, Botelho FM, Lambert KN, Brown EG, Mossman KL, Taylor JD, Stämpfli MR. Treating viral exacerbations of chronic obstructive pulmonary disease: insights from a mouse model of cigarette smoke and H1N1 influenza infection. PLoS One. 2010 Oct 12;5(10):e13251. doi: 10.1371/journal.pone.0013251. PMID: 20967263; PMCID: PMC2953496.

1284. Wang W, Yin Y, Xu L, Su J, Huang F, Wang Y, Boor PPC, Chen K, Wang W, Cao W, Zhou X, Liu P, van der Laan LW, Kwekkeboom J, Peppelenbosch MP, Pan Q. Unphosphorylated ISGF3 drives constitutive expression of interferon-stimulated genes to protect against viral infections. Sci Signal. 2017 Apr 25;10(476):eaah4248. doi: 10.1126/scisignal.aah4248. PMID: 28442624.

1285. Blaszczyk K, Olejnik A, Nowicka H, Ozgyin L, Chen YL, Chmielewski S, Kostyrko K, Wesoly J, Balint BL, Lee CK, Bluyssen HA STAT2/IRF9 directs a prolonged ISGF3-like transcriptional response and antiviral activity in the absence of STAT1. Biochem J. 2015 Mar 15;466(3):511-24. doi: 10.1042/BJ20140644. PMID: 25564224; PMCID: PMC4403947.

1286. Michalska A, Blaszczyk K, Wesoly J, Bluyssen HAR. A Positive Feedback Amplifier Circuit That Regulates Interferon (IFN)Stimulated Gene Expression and Controls Type I and Type II IFN Responses. Front Immunol. 2018 May 28;9:1135. doi: 10.3389/fimmu.2018.01135. PMID: 29892288; PMCID: PMC5985295.

1287. Pahan K. A Broad Application of CRISPR Cas9 in Infectious, Inflammatory and Neurodegenerative Diseases. J Neuroimmune Pharmacol. 2019 Dec;14(4):534-536. doi: 10.1007/s11481-019-09889-4. Epub 2019 Nov 28. PMID: 31782056; PMCID: PMC6944276.

1288. Mian MF, Stämpfli MR, Mossman KL, Ashkar AA. Cigarette smoke attenuation of poly I:C-induced innate antiviral responses in human PBMC is mainly due to inhibition of IFN-beta production. Mol Immunol. 2009 Feb;46(5):821-9. doi: 10.1016/j.molimm.2008.09.007. Epub 2008 Oct 18. PMID: 18930547.

1289. uljic R, Benediktus E, Plater-Zyberk C, Baeuerle PA, Szelenyi S, Brune K, Pahl A. Lipopolysaccharide-induced lung inflammation is inhibited by neutralization of GM-CSF. Eur J Pharmacol. 2007 Feb 28;557(2-3):230-5. doi: 10.1016/j.ejphar.2006.11.023. Epub 2006 Nov 14. PMID: 17174948

1290. Bozinovski S, Seow HJ, Crack PJ, Anderson GP, Vlahos R. Glutathione peroxidase-1 primes pro-inflammatory cytokine production after LPS challenge in vivo. PLoS One. 2012;7(3):e33172. doi: 10.1371/journal.pone.0033172. Epub 2012 Mar 6. PMID: 22412999; PMCID: PMC3295802.

1291. 82161.Duong C, Seow HJ, Bozinovski S, Crack PJ, Anderson GP, Vlahos R. Glutathione peroxidase-1 protects against cigarette smoke-induced lung inflammation in mice. Am J Physiol Lung Cell Mol Physiol. 2010 Sep;299(3):L425-33. doi: 10.1152/ajplung.00038.2010. Epub 2010 May 28. PMID: 20511341.

1292. Zhang Y, Sun H, Pei R, Mao B, Zhao Z, Li H, Lin Y, Lu K. The SARS-CoV-2 protein ORF3a inhibits fusion of autophagosomes with lysosomes. Cell Discov. 2021 May 4;7(1):31. doi: 10.1038/s41421-021-00268-z. PMID: 33947832; PMCID: PMC8096138. 1293. Hudy MH, Traves SL, Proud D. Transcriptional and epigenetic modulation of human rhinovirus-induced CXCL10 production by cigarette smoke. Am J Respir Cell Mol Biol. 2014 Mar;50(3):571-82. doi: 10.1165/rcmb.2013-01290C. PMID: 24127910. 1294. Zaheer RS, Koetzler R, Holden NS, Wiehler S, Proud D. Selective transcriptional down-regulation of human rhinovirusinduced production of CXCL10 from airway epithelial cells via the MEK1 pathway. J Immunol. 2009 Apr 15;182(8):4854-64. doi: 10.4049/jimmunol.0802401. PMID: 19342664

1295. Ghosh TK, Mickelson DJ, Solberg JC, Lipson KE, Inglefield JR, Alkan SS. TLR-TLR cross talk in human PBMC resulting in synergistic and antagonistic regulation of type-1 and 2 interferons, IL-12 and TNF-alpha. Int Immunopharmacol. 2007 Aug;7(8):1111-21. doi: 10.1016/j.intimp.2007.04.006. Epub 2007 May 4. PMID: 17570328.

1296. Sawaki J, Tsutsui H, Hayashi N, Yasuda K, Akira S, Tanizawa T, Nakanishi K. Type 1 cytokine/chemokine production by mouse NK cells following activation of their TLR/MyD88-mediated pathways. Int Immunol. 2007 Mar;19(3):311-20. doi: 10.1093/intimm/dxl148. Epub 2007 Feb 7. PMID: 17289654.

1297. Levy O. Innate immunity of the human newborn: distinct cytokine responses to LPS and other Toll-like receptor agonists. J Endotoxin Res. 2005;11(2):113-6. doi: 10.1179/096805105X37376. PMID: 15949138.

1298. Ghosh TK, Mickelson DJ, Fink J, Solberg JC, Inglefield JR, Hook D, Gupta SK, Gibson S, Alkan SS. Toll-like receptor (TLR) 2-9 agonists-induced cytokines and chemokines: I. Comparison with T cell receptor-induced responses. Cell Immunol. 2006 Sep;243(1):48-57. doi: 10.1016/j.cellimm.2006.12.002. Epub 2007 Jan 23. PMID: 17250816.

1299. Olliver M, Spelmink L, Hiew J, Meyer-Hoffert U, Henriques-Normark B, Bergman P. Immunomodulatory effects of vitamin D on innate and adaptive immune responses to Streptococcus pneumoniae. J Infect Dis. 2013 Nov 1;208(9):1474-81. doi: 10.1093/infdis/jit355. Epub 2013 Aug 6. PMID: 23922371

1300. Martin TR, Frevert CW. Innate immunity in the lungs. Proc Am Thorac Soc. 2005;2(5):403-11. doi: 10.1513/pats.200508090JS. PMID: 16322590; PMCID: PMC2713330.

1301. Ayranci E, Hoda N. Adsorption of bentazon and propanil from aqueous solutions at the high area activated carbon-cloth Chemosphere. 2004 Nov;57(8):755-62. doi: 10.1016/j.chemosphere.2004.08.042. PMID: 15488566.

1302. UTILIZATION OF ACTIVATED CARBON CLOTH FOR CIGARATTE SMOKE FILTRATION Firdevs MERT [1] , Numan HODA [2] , Ayhan TOPUZ [3] , İsmail TONTUL [4]

1303. Wu W, Zhang W, Booth JL, Hutchings DC, Wang X, White VL, Youness H, Cross CD, Zou MH, Burian D, Metcalf JP. Human primary airway epithelial cells isolated from active smokers have epigenetically impaired antiviral responses. Respir Res. 2016 Sep 7;17(1):111. doi: 10.1186/s12931-016-0428-2. PMID: 27604339 ; PMCID: PMC5013564.

1304. Deng X, Rui W, Zhang F, Ding W. PM2.5 induces Nrf2-mediated defense mechanisms against oxidative stress by activating PIK3/AKT signaling pathway in human lung alveolar epithelial A549 cells. Cell Biol Toxicol. 2013 Jun;29(3):143-57. doi: 10.1007/s10565-013-9242-5. Epub 2013 Mar 24. PMID: 23525690.

1305. Gualano RC, Hansen MJ, Vlahos R, Jones JE, Park-Jones RA, Deliyannis G, Turner SJ, Duca KA, Anderson GP. Cigarette smoke worsens lung inflammation and impairs resolution of influenza infection in mice. Respir Res. 2008 Jul 15;9(1):53. doi: 10.1186/1465-9921-9-53. PMID: 18627612; PMCID: PMC2483272

1306. Radan M, Dianat M, Badavi M, Mard SA, Bayati V, Ahmadizadeh M. The Association of Cigarette Smoke Exposure with Lung Cellular Toxicity and Oxidative Stress: the Protective Role of Crocin. Inflammation. 2020 Feb;43(1):135-145. doi: 10.1007/s10753019-01102-1. PMID: 31659585.

1307. Herrmann M, King K, Weitzman M. Prenatal tobacco smoke and postnatal secondhand smoke exposure and child neurodevelopment. Curr Opin Pediatr. 2008 Apr;20(2):184-90. doi: 10.1097/MOP.0b013e3282f56165. PMID: 18332716. 1308. Merianos AL, Nabors LA, Fiser KA, Mahabee-Gittens EM. Exposure to Tobacco Smoke and Temperament among U.S. Children 0-5 Years Old. J Pediatr Psychol. 2021 Apr 16;46(4):454-464. doi: 10.1093/jpepsy/jsaa123. PMID: 33355348; PMCID PMC8056209.

1309. Arnson Y, Shoenfeld Y, Amital H. Effects of tobacco smoke on immunity, inflammation and autoimmunity. J Autoimmun. 2010 May;34(3):J258-65. doi: 10.1016/j.jaut.2009.12.003. Epub 2009 Dec 29. PMID: 20042314. 
1310. Profita M, Bonanno A, Siena L, Bruno A, Ferraro M, Montalbano AM, Albano GD, Riccobono L, Casarosa P, Pieper MP, Gjomarkaj M. Smoke, choline acetyltransferase, muscarinic receptors, and fibroblast proliferation in chronic obstructive pulmonary disease. J Pharmacol Exp Ther. 2009 May;329(2):753-63. doi: 10.1124/jpet.108.145888. Epub 2009 Feb 3. PMID: 19190237.

1311. Han L, Zhuang MW, Deng J, Zheng Y, Zhang J, Nan ML, Zhang XJ, Gao C, Wang PH. SARS-CoV-2 ORF9b antagonizes type I and III interferons by targeting multiple components of the RIG-I/MDA-5-MAVS, TLR3-TRIF, and cGAS-STING signaling pathways. J Med Virol. 2021 Apr 29:10.1002/jmv.27050. doi: 10.1002/jmv.27050. Epub ahead of print. PMID: 33913550; PMCID: PMC8242602.

1312. Yang D, Geng T, Harrison AG, Wang P. Differential roles of RIG-I-like receptors in SARS-CoV-2 infection. bioRxiv [Preprint]. 2021 Feb 11:2021.02.10.430677. doi: 10.1101/2021.02.10.430677. PMID: 33594370; PMCID: PMC7885922.

1313. Oh SJ, Shin OS. SARS-CoV-2 Nucleocapsid Protein Targets RIG-I-Like Receptor Pathways to Inhibit the Induction of Interferon Response. Cells. 2021 Mar 2;10(3):530. doi: 10.3390/cells10030530. PMID: 33801464; PMCID: PMC7999926. 1314. Wu W, Zhang W, Duggan ES, Booth JL, Zou MH, Metcalf JP. RIG-I and TLR3 are both required for maximum interferon induction by influenza virus in human lung alveolar epithelial cells. Virology. 2015 Aug;482:181-8. doi: 10.1016/j.virol.2015.03.048. Epub 2015 Apr 11. PMID: 25880109; PMCID: PMC4461467.

1315. Castanier, Céline \& Garcin, Dominique \& Vazquez, Aimé \& Arnoult, Damien. (2009). Mitochondrial dynamics regulate the RIG-I-like receptor antiviral pathway. EMBO reports. 11. 133-8. 10.1038/embor.2009.258.

1316. 6.Wu W, Metcalf JP. The Role of Type I IFNs in Influenza: Antiviral Superheroes or Immunopathogenic Villains? J Innate Immun. 2020;12(6):437-447. doi: 10.1159/000508379. Epub 2020 Jun 19. PMID: 32564033; PMCID: PMC7747089.

1317. Sharma A, Lal SK. Is tetherin a true antiviral: The influenza a virus controversy. Rev Med Virol. 2019 May;29(3):e2036. doi: 10.1002/rmv.2036. Epub 2019 Jan 31. PMID: 30706579

1318. 12.Stewart H, Johansen KH, McGovern N, Palmulli R, Carnell GW, Heeney JL, Okkenhaug K, Firth AE, Peden AA, Edgar JR. SARS-CoV-2 spike downregulates tetherin to enhance viral spread. bioRxiv [Preprint]. 2021 Jan 6:2021.01.06.425396. doi: 10.1101/2021.01.06.425396. PMID: 33442692; PMCID: PMC7805449.

1319. Daniel Blanco-Melo, Benjamin E. Nilsson-Payant, Wen-Chun Liu, Skyler Uhl, Daisy Hoagland, Rasmus Møller, Tristan X. Jordan, Kohei Oishi, Maryline Panis, David Sachs, Taia T. Wang, Robert E. Schwartz, Jean K. Lim, Randy A. Albrecht, Benjamin R. tenOever,Imbalanced Host Response to SARS-CoV-2 Drives Development of COVID-19,Cell,Volume 181, Issue 5,2020,Pages 10361045.e9,ISSN 0092-8674,https://doi.org/10.1016/j.cell.2020.04.026.

1320. Bastard $P$, Rosen LB, Zhang $Q$, Michailidis $E$,et.al. 14.Autoantibodies against type I IFNs in patients with life-threatening COVID-19. Science. 2020 Oct 23;370(6515):eabd4585. doi: 10.1126/science.abd4585. Epub 2020 Sep 24. PMID: 32972996; PMCID: PMC7857397.

1321. Costenbader KH, Karlson EW. Cigarette smoking and autoimmune disease: what can we learn from epidemiology? Lupus. 2006;15(11):737-45. doi: 10.1177/0961203306069344. PMID: 17153844

1322. Strzelak A, Ratajczak A, Adamiec A, Feleszko W. Tobacco Smoke Induces and Alters Immune Responses in the Lung Triggering Inflammation, Allergy, Asthma and Other Lung Diseases: A Mechanistic Review. Int J Environ Res Public Health. 2018 May 21;15(5):1033. doi: 10.3390/ijerph15051033. PMID: 29883409 ; PMCID: PMC5982072.

1323. Harel-Meir M, Sherer Y, Shoenfeld Y. Tobacco smoking and autoimmune rheumatic diseases. Nat Clin Pract Rheumatol. 2007 Dec;3(12):707-15. doi: 10.1038/ncprheum0655. PMID: 18037930.

1324. Klareskog L, Padyukov L, Alfredsson L. Smoking as a trigger for inflammatory rheumatic diseases. Curr Opin Rheumatol. 2007 Jan;19(1):49-54. doi: 10.1097/BOR.0b013e32801127c8. PMID: 17143096.

1325. Leslie D, Lipsky P, Notkins AL. Autoantibodies as predictors of disease. J Clin Invest. 2001 Nov;108(10):1417-22. doi: 10.1172/JCI14452. PMID: 11714731; PMCID: PMC209428.

1326. 20.Elkon K, Casali P. Nature and functions of autoantibodies. Nat Clin Pract Rheumatol. 2008 Sep;4(9):491-8. doi: 10.1038/ncprheum0895. PMID: 18756274; PMCID: PMC2703183.

1327. 21.Fabrizio Maggi, Alfredo Rosellini, Pietro Giorgio Spezia, Daniele Focosi, Lisa Macera, Michele Lai, Mauro Pistello, Antonio de lure, Carlo Tomino, Stefano Bonassi, Nicotine upregulates ACE2 expression and increases competence for SARS-CoV-2 in human pneumocytes, Patrizia Russo ERJ Open Research Apr 2021, 7 (2) 00713-2020; DOI: 10.1183/23120541.00713-2020. 1328. 22.Yuyang Lei, Jiao Zhang, Cara R. Schiavon, Ming He, Lili Chen, Hui Shen, Yichi Zhang, Qian Yin, Yoshitake Cho, Leonardo Andrade, Gerald S. Shadel, Mark Hepokoski, Ting Lei, Hongliang Wang, Jin Zhang, Jason X.-J. Yuan, Atul Malhotra, Uri Manor, Shengpeng Wang, Zu-Yi Yuan, John Y-J. Shyy, SARS-CoV-2 Spike Protein Impairs Endothelial Function via Downregulation of ACE 2, https://doi.org/10.1161/CIRCRESAHA.121.318902

1329. 14.Vlahos R, Stambas J, Bozinovski S, Broughton BR, Drummond GR, Selemidis S. Inhibition of Nox2 oxidase activity ameliorates influenza A virus-induced lung inflammation. PLoS Pathog. 2011 Feb 3;7(2):e1001271. doi: 10.1371/journal.ppat.1001271. PMID: 21304882; PMCID: PMC3033375

1330. To EE, Broughton BR, Hendricks KS, Vlahos R, Selemidis S. Influenza A virus and TLR7 activation potentiate NOX2 oxidasedependent ROS production in macrophages. Free Radic Res. 2014 Aug;48(8):940-7.

1331. Ushio-Fukai M. Localizing NADPH oxidase-derived ROS. Sci STKE. 2006 Aug 22;2006(349):re8. doi: 10.1126/stke.3492006re8. PMID: 16926363.

1332. Strengert M, Jennings R, Davanture $S$, Hayes $P$, Gabriel G, Knaus UG. Mucosal reactive oxygen species are required for antiviral response: role of Duox in influenza a virus infection. Antioxid Redox Signal. 2014 Jun 10;20(17):2695-709. doi: 10.1089/ars.2013.5353. Epub 2013 Oct 15. PMID: 24128054.

1333. Vlahos R, Stambas J, Selemidis S. Suppressing production of reactive oxygen species (ROS) for influenza A virus therapy. Trends Pharmacol Sci. 2012 Jan;33(1):3-8. doi: 10.1016/j.tips.2011.09.001. Epub 2011 Oct 1. PMID: 21962460. 1334. Yoneyama M, Jogi M, Onomoto K. Regulation of antiviral innate immune signaling by stress-induced RNA granules. J Biochem. 2016 Mar;159(3):279-86. doi: 10.1093/jb/mvv122. Epub 2016 Jan 8. PMID: 26748340; PMCID: PMC4763080. 1335. Eiermann N, Haneke K, Sun Z, Stoecklin G, Ruggieri A. Dance with the Devil: Stress Granules and Signaling in Antiviral Responses. Viruses. 2020 Sep 4;12(9):984. doi: 10.3390/v12090984. PMID: 32899736; PMCID: PMC7552005.

1336. Chen KK, Minakuchi M, Wuputra K, Ku CC, Pan JB, Kuo KK, Lin YC, Saito S, Lin CS, Yokoyama KK. Redox control in the pathophysiology of influenza virus infection. BMC Microbiol. 2020 Jul 20;20(1):214. doi: 10.1186/s12866-020-01890-9. PMID: 32689931; PMCID: PMC7370268.

1337. Amatore D, Sgarbanti R, Aquilano K, Baldelli S, Limongi D, Civitelli L, Nencioni L, Garaci E, Ciriolo MR, Palamara AT. Influenza virus replication in lung epithelial cells depends on redox-sensitive pathways activated by NOX4-derived ROS. Cell Microbiol. 2015 Jan;17(1):131-45. doi: 10.1111/cmi.12343. Epub 2014 Oct 7. PMID: 25154738; PMCID: PMC4311438.

1338. Jung KI, Ko DH, Shin N, Pyo CW, Choi SY. Endoplasmic reticulum-associated degradation potentiates the infectivity of influenza A virus by regulating the host redox state. Free Radic Biol Med. 2019 May 1;135:293-305. doi: 10.1016/j.freeradbiomed.2019.03.021. Epub 2019 Mar 21. PMID: 30905731. 
1339. Jung KI, Pyo CW, Choi SY. Influenza A virus-induced autophagy contributes to enhancement of virus infectivity by SOD1 downregulation in alveolar epithelial cells. Biochem Biophys Res Commun. 2018 Apr 15;498(4):960-966. doi: 10.1016/j.bbrc.2018.03.089. Epub 2018 Mar 17. PMID: 29548827.

1340. Haque MM, Murale DP, Lee JS. Role of microRNA and Oxidative Stress in Influenza A Virus Pathogenesis. Int J Mol Sci. 2020 Nov 25;21(23):8962. doi: 10.3390/ijms21238962. PMID: 33255826; PMCID: PMC7728370.

1341. 28.Kawai T, Akira S. Toll-like receptor and RIG-I-like receptor signaling. Ann N Y Acad Sci. 2008 Nov;1143:1-20. doi: 10.1196/annals.1443.020. PMID: 19076341

1342. Liu G, Zhou Y. Cytoplasm and Beyond: Dynamic Innate Immune Sensing of Influenza A Virus by RIG-I. J Virol. 2019 Apr 3;93(8):e02299-18. doi: 10.1128/JVI.02299-18. PMID: $30760567 ;$ PMCID: PMC6450113.

1343. 30.Malik G, Zhou Y. Innate Immune Sensing of Influenza A Virus. Viruses. 2020 Jul 14;12(7):755. doi: 10.3390/v12070755. PMID: 32674269; PMCID: PMC7411791.

1344. 31.Wu W, Wang X, Zhang W, Tian L, Booth JL, Duggan ES, More S, Liu L, Dozmorov M, Metcalf JP. RIG-I Signaling via MAVS Is Dispensable for Survival in Lethal Influenza Infection In Vivo. Mediators Inflamm. 2018 Nov 8;2018:6808934. doi: 10.1155/2018/6808934. PMID: 30532653; PMCID: PMC6250004.

1345. 32.Rehwinkel J, Reis e Sousa C. RIGorous detection: exposing virus through RNA sensing. Science. 2010 Jan 15;327(5963):284-6. doi: 10.1126/science.1185068. PMID: 20075242.

1346. Onomoto K, Onoguchi K, Yoneyama M. Regulation of RIG-I-like receptor-mediated signaling: interaction between host and viral factors. Cell Mol Immunol. 2021 Mar;18(3):539-555. doi: 10.1038/s41423-020-00602-7. Epub 2021 Jan 18. PMID: 33462384; PMCID: PMC7812568

1347. 34.Ramos HJ, Gale M Jr. RIG-I like receptors and their signaling crosstalk in the regulation of antiviral immunity. Curr Opin Virol. 2011 Sep;1(3):167-76. doi: 10.1016/j.coviro.2011.04.004. PMID: 21949557; PMCID: PMC3177754.

1348. 35.Jabłońska A, Paradowska E. Rola receptorów RIG-I-podobnych w odpowiedzi przeciwwirusowej [Role of the RIG-I-like receptors in antiviral response]. Postepy Hig Med Dosw (Online). 2014 Jan 2;68:541-56. Polish. doi: 10.5604/17322693.1102281. PMID: 24864105.

1349. Onomoto K, Yoneyama M, Fujita T. Regulation of antiviral innate immune responses by RIG-I family of RNA helicases. Curr Top Microbiol Immunol. 2007;316:193-205. doi: 10.1007/978-3-540-71329-6 10. PMID: 17969449.

1350. 37.Takeuchi O, Akira S. MDA5/RIG-I and virus recognition. Curr Opin Immunol. 2008 Feb;20(1):17-22. doi:

10.1016/j.coi.2008.01.002. Epub 2008 Feb 12. PMID: 18272355

1351. 38.Esser-Nobis K, Hatfield LD, Gale M Jr. Spatiotemporal dynamics of innate immune signaling via RIG-I-like receptors. Proc Natl Acad Sci U S A. 2020 Jul 7;117(27):15778-15788. doi: 10.1073/pnas.1921861117. Epub 2020 Jun 22. PMID: 32571931; PMCID: PMC7354926.

1352. Min YQ, Ning YJ, Wang H, Deng F. A RIG-I-like receptor directs antiviral responses to a bunyavirus and is antagonized by virus-induced blockade of TRIM25-mediated ubiquitination. J Biol Chem. 2020 Jul 10;295(28):9691-9711. doi: 10.1074/jbc.RA120.013973. Epub 2020 May 29. PMID: $32471869 ;$ PMCID: PMC7363118.

1353. 40.Kell AM, Gale M Jr. RIG-I in RNA virus recognition. Virology. 2015 May;479-480:110-21. doi: 10.1016/j.virol.2015.02.017. Epub 2015 Mar 5. PMID: 25749629; PMCID: PMC4424084.

1354. 41.Lee NR, Ban J, Lee NJ, Yi CM, Choi JY, Kim H, Lee JK, Seong J, Cho NH, Jung JU, Inn KS. Activation of RIG-I-Mediated Antiviral Signaling Triggers Autophagy Through the MAVS-TRAF6-Beclin-1 Signaling Axis. Front Immunol. 2018 Sep 12;9:2096. doi: 10.3389/fimmu.2018.02096. PMID: 30258449; PMCID: PMC6143786.

1355. 42.Wang Y, Ye L, Wang X, Li J, Song L, Ho W. Retinoic acid inducible gene-I (RIG-I) signaling of hepatic stellate cells inhibits hepatitis C virus replication in hepatocytes. Innate Immun. 2013;19(2):193-202. doi: 10.1177/1753425912460414. Epub 2012 Oct 11. PMID: 23060457; PMCID: PMC3935722.

1356. 44.Quicke KM, Diamond MS, Suthar MS. Negative regulators of the RIG-I-like receptor signaling pathway. Eur J Immunol. 2017 Apr;47(4):615-628. doi: 10.1002/eji.201646484. PMID: 28295214; PMCID: PMC5554756.

1357. 45.Yan K, Zhu W, Yu L, Li N, Zhang X, Liu P, Chen Q, Chen Y, Han D. Toll-like receptor 3 and RIG-I-like receptor activation induces innate antiviral responses in mouse ovarian granulosa cells. Mol Cell Endocrinol. 2013 Jun 15;372(1-2):73-85. doi: 10.1016/j.mce.2013.03.027. Epub 2013 Apr 6. PMID: 23567548.

1358. 46.Eisenächer K, Krug A. Regulation of RLR-mediated innate immune signaling--it is all about keeping the balance. Eur J Cell Biol. 2012 Jan;91(1):36-47. doi: 10.1016/j.ejcb.2011.01.011. Epub 2011 Apr 9. PMID: 21481967.

1359. 47.Belgnaoui SM, Paz S, Hiscott J. Orchestrating the interferon antiviral response through the mitochondrial antiviral signaling (MAVS) adapter. Curr Opin Immunol. 2011 Oct;23(5):564-72. doi: 10.1016/j.coi.2011.08.001. Epub 2011 Aug 22. PMID: 21865020.

1360. 49.Onomoto K, Yoneyama M, Fujita T. [Recognition of viral nucleic acids and regulation of type I IFN expression]. Nihon Rinsho. 2006 Jul;64(7):1236-43. Japanese. PMID: 16838638.

1361. 50.Li J, Ye L, Wang X, Hu S, Ho W. Induction of interferon- $\gamma$ contributes to Toll-like receptor 3-mediated herpes simplex virus type 1 inhibition in astrocytes. J Neurosci Res. 2012 Feb;90(2):399-406. doi: 10.1002/jnr.22758. Epub 2011 Nov 4. PMID: 22057682; PMCID: PMC3411314.

1362. 51.Zhang SY, Jouanguy E, Sancho-Shimizu V, von Bernuth H, Yang K, Abel L, Picard C, Puel A, Casanova JL. Human Toll-like receptor-dependent induction of interferons in protective immunity to viruses. Immunol Rev. 2007 Dec;220(1):225-36. doi: 10.1111/j.1600-065X.2007.00564.x. PMID: 17979850; PMCID: PMC7165931.

1363. 52.Blaszczyk K, Nowicka H, Kostyrko K, Antonczyk A, Wesoly J, Bluyssen HA. The unique role of STAT2 in constitutive and IFN-induced transcription and antiviral responses. Cytokine Growth Factor Rev. 2016 Jun;29:71-81. doi: 10.1016/j.cytogfr.2016.02.010. Epub 2016 Mar 18. PMID: 27053489.

1364. 53.Takeuchi O, Akira S. Innate immunity to virus infection. Immunol Rev. 2009 Jan;227(1):75-86. doi: 10.1111/j.1600065X.2008.00737.x. PMID: 19120477; PMCID: PMC5489343.

1365. 55.Andersen JM, Al-Khairy D, Ingalls RR. Innate immunity at the mucosal surface: role of toll-like receptor 3 and toll-like receptor 9 in cervical epithelial cell responses to microbial pathogens. Biol Reprod. 2006 May;74(5):824-31. doi: 10.1095/biolreprod.105.048629. Epub 2006 Jan 18. PMID: 16421230.

1366. Negishi H, Osawa T, Ogami K, Ouyang X, Sakaguchi S, Koshiba R, Yanai H, Seko Y, Shitara H, Bishop K, Yonekawa H, Tamura T, Kaisho T, Taya C, Taniguchi T, Honda K. A critical link between Toll-like receptor 3 and type II interferon signaling pathways in antiviral innate immunity. Proc Natl Acad Sci U S A. 2008 Dec 23;105(51):20446-51. doi: 10.1073/pnas.0810372105. Epub 2008 Dec 11. PMID: 19074283; PMCID: PMC2629334.

1367. 57. Khvalevsky E, Rivkin L, Rachmilewitz J, Galun E, Giladi H. TLR3 signaling in a hepatoma cell line is skewed towards apoptosis. J Cell Biochem. 2007 Apr 1;100(5):1301-12. doi: 10.1002/jcb.21119. PMID: 17243100.

1368. 58. Yang K, Puel A, Zhang S, Eidenschenk C, Ku CL, Casrouge A, Picard C, von Bernuth H, Senechal B, Plancoulaine S, Al-Hajjar S, Al-Ghonaium A, Maródi L, Davidson D, Speert D, Roifman C, Garty BZ, Ozinsky A, Barrat FJ, Coffman RL, Miller RL, Li X, Lebon P, 
Rodriguez-Gallego C, Chapel H, Geissmann F, Jouanguy E, Casanova JL. Human TLR-7-, -8-, and -9-mediated induction of IFNalpha/beta and -lambda Is IRAK-4 dependent and redundant for protective immunity to viruses. Immunity. 2005 Nov;23(5):46578. doi: 10.1016/j.immuni.2005.09.016. PMID: 16286015; PMCID: PMC7111074.

1369. 59.Lozhkov AA, Klotchenko SA, Ramsay ES, Moshkoff HD, Moshkoff DA, Vasin AV, Salvato MS. The Key Roles of Interferon Lambda in Human Molecular Defense against Respiratory Viral Infections. Pathogens. 2020 Nov 26;9(12):989. doi: 10.3390/pathogens9120989. PMID: 33255985; PMCID: PMC7760417.

1370. Suprunenko T, Hofer MJ. The emerging role of interferon regulatory factor 9 in the antiviral host response and beyond. Cytokine Growth Factor Rev. 2016 Jun;29:35-43. doi: 10.1016/j.cytogfr.2016.03.002. Epub 2016 Mar 4. PMID: 26987614 1371. i Z, Lu X, Zhu Y, Cheng P, Liu S, Zhang Y, Tang J, Yang S, Zhou L. Lambda-Interferons Inhibit Herpes Simplex Virus Type 2 Replication in Human Cervical Epithelial Cells by Activating the JAK/STAT Pathway. Jpn J Infect Dis. 2017 Jul 24;70(4):416-422. doi: 10.7883/yoken.JJID.2016.465. Epub 2017 Feb 28. PMID: 28250263.

1372. LZhang SY, Boisson-Dupuis S, Chapgier A, Yang K, Bustamante J, Puel A, Picard C, Abel L, Jouanguy E, Casanova JL. Inborn errors of interferon (IFN)-mediated immunity in humans: insights into the respective roles of IFN-alpha/beta, IFN-gamma, and IFN-lambda in host defense. Immunol Rev. 2008 Dec;226:29-40. doi: 10.1111/j.1600-065X.2008.00698.x. PMID: 19161414 1373. 63.Mordstein M, Kochs G, Dumoutier L, Renauld JC, Paludan SR, Klucher K, Staeheli P. Interferon-lambda contributes to innate immunity of mice against influenza A virus but not against hepatotropic viruses. PLoS Pathog. 2008 Sep 12;4(9):e1000151. doi: 10.1371/journal.ppat.1000151. PMID: 18787692; PMCID: PMC2522277.

1374. Komal A, Noreen M, El-Kott AF. TLR3 agonists: RGC100, ARNAX, and poly-IC: a comparative review. Immunol Res. 2021 Jun 19:1-11. doi: 10.1007/s12026-021-09203-6. Epub ahead of print. PMID: 34145551; PMCID: PMC8213534.

1375. 67.Yu J, Sun X, Goie JYG, Zhang Y. Regulation of Host Immune Responses against Influenza A Virus Infection by MitogenActivated Protein Kinases (MAPKs). Microorganisms. 2020 Jul 17;8(7):1067. doi: 10.3390/microorganisms8071067. PMID: 32709018; PMCID: PMC7409222.

1376. 69.Chen CY, Hung YF, Tsai CY, Shih YC, Chou TF, Lai MZ, Wang TF, Hsueh YP. Transcriptomic Analysis and C-Terminal Epitope Tagging Reveal Differential Processing and Signaling of Endogenous TLR3 and TLR7. Front Immunol. 2021 Jun 15;12:686060. doi: 10.3389/fimmu.2021.686060. PMID: 34211474; PMCID: PMC8240634.

1377. 70.Zhou L, Li JL, Zhou Y, Liu JB, Zhuang K, Gao JF, Liu S, Sang M, Wu JG, Ho WZ. Induction of interferon- $\lambda$ contributes to TLR3 and RIG-I activation-mediated inhibition of herpes simplex virus type 2 replication in human cervical epithelial cells. Mol Hum Reprod. 2015 Dec;21(12):917-29. doi: 10.1093/molehr/gav058. Epub 2015 Oct 26. PMID: 26502803; PMCID: PMC4664393. 1378. Gao D, Ciancanelli MJ, Zhang P, Harschnitz O, Bondet V, Hasek M, Chen J, Mu X, Itan Y, Cobat A, Sancho-Shimizu V, Bigio B, Lorenzo L, Ciceri G, McAlpine J, Anguiano E, Jouanguy E, Chaussabel D, Meyts I, Diamond MS, Abel L, Hur S, Smith GA, Notarangelo L, Duffy D, Studer L, Casanova JL, Zhang SY. TLR3 controls constitutive IFN- $\beta$ antiviral immunity in human fibroblasts and cortical neurons. J Clin Invest. 2021 Jan 4;131(1):e134529. doi: 10.1172/JCI134529. PMID: 33393505; PMCID: PMC7773389. 1379. Forbester JL, Clement M, Wellington D, Yeung A, Dimonte S, Marsden M, Chapman L, Coomber EL, Tolley C, Lees E, Hale C, Clare S, Udalova I, Dong T, Dougan G, Humphreys IR. IRF5 Promotes Influenza Virus-Induced Inflammatory Responses in Human Induced Pluripotent Stem Cell-Derived Myeloid Cells and Murine Models. J Virol. 2020 Apr 16;94(9):e00121-20. doi: 10.1128/JVI.00121-20. PMID: 32075938; PMCID: PMC7163152.

1380. 73.Ghosh TK, Mickelson DJ, Fink J, Solberg JC, Inglefield JR, Hook D, Gupta SK, Gibson S, Alkan SS. Toll-like receptor (TLR) 2 9 agonists-induced cytokines and chemokines: I. Comparison with T cell receptor-induced responses. Cell Immunol. 2006 Sep;243(1):48-57. doi: 10.1016/j.cellimm.2006.12.002. Epub 2007 Jan 23. PMID: 17250816.

1381. 74.Ramaswamy M, Shi L, Monick MM, Hunninghake GW, Look DC. Specific inhibition of type I interferon signal transduction by respiratory syncytial virus. Am J Respir Cell Mol Biol. 2004 Jun;30(6):893-900. doi: 10.1165/rcmb.2003-04100C. Epub 2004 Jan 12. PMID: 14722224.

1382. 75.Ramaswamy M, Shi L, Varga SM, Barik S, Behlke MA, Look DC. Respiratory syncytial virus nonstructural protein 2 specifically inhibits type I interferon signal transduction. Virology. 2006 Jan 20;344(2):328-39. doi: 10.1016/j.virol.2005.09.009. Epub 2005 Oct 10. Erratum in: Virology. 2007 Sep 30;366(2):453. PMID: 16216295.

1383. 76. Chattoraj SS, Ganesan S, Faris A, Comstock A, Lee WM, Sajjan US. Pseudomonas aeruginosa suppresses interferon response to rhinovirus infection in cystic fibrosis but not in normal bronchial epithelial cells. Infect Immun. 2011 Oct;79(10):413145. doi: 10.1128/IAI.05120-11. Epub 2011 Aug 8. PMID: 21825067; PMCID: PMC3187241.

1384. Vareille M, Kieninger E, Alves MP, Kopf BS, Möller A, Geiser T, Johnston SL, Edwards MR, Regamey N. Impaired type I and type III interferon induction and rhinovirus control in human cystic fibrosis airway epithelial cells. Thorax. 2012 Jun;67(6):517-25. doi: 10.1136/thoraxjnl-2011-200405. Epub 2012 Jan 2. Retraction in: Thorax. 2013 Sep;68(9):886. PMID: 22213737.

1385. 1.Kawai T, Akira S. Antiviral signaling through pattern recognition receptors. J Biochem. 2007 Feb;141(2):137-45. doi: 10.1093/jb/mvm032. Epub 2006 Dec 26. PMID: 17190786.

1386. 2.Campbell LK, Magor KE. Pattern Recognition Receptor Signaling and Innate Responses to Influenza A Viruses in the Mallard Duck, Compared to Humans and Chickens. Front Cell Infect Microbiol. 2020 May 12;10:209. doi: 10.3389/fcimb.2020.00209. PMID: 32477965; PMCID: PMC7236763.

1387. 3.Laghlali G, Lawlor KE, Tate MD. Die Another Way: Interplay between Influenza A Virus, Inflammation and Cell Death. Viruses. 2020 Apr 4;12(4):401. doi: 10.3390/v12040401. PMID: 32260457; PMCID: PMC7232208.

1388. 4. Harpur CM, Le Page MA, Tate MD. Too young to die? How aging affects cellular innate immune responses to influenza virus and disease severity. Virulence. 2021 Dec;12(1):1629-1646. doi: 10.1080/21505594.2021.1939608. PMID: 34152253; PMCID: PMC8218692.

1389. Proud PC, Tsitoura D, Watson RJ, Chua BY et.al. Prophylactic intranasal administration of a TLR2/6 agonist reduces upper respiratory tract viral shedding in a SARS-CoV-2 challenge ferret model. EBioMedicine. 2021 Jan;63:103153. doi: 10.1016/j.ebiom.2020.103153. Epub 2020 Dec 3. PMID: 33279857; PMCID: PMC7711201.

1390. Iqbal, Muhammad \& Sardar, Nimra \& Akmal, Wajiha \& Sultan, Rabia \& Abdullah, Humaira \& Qindeel, Maimoona \& Dhama, Kuldeep \& Bilal, Muhammad. (2020). ROLE OF TOLL-LIKE RECEPTORS IN CORONAVIRUS INFECTION AND IMMUNE RESPONSE. Journal of Experimental Biology and Agricultural Sciences. 8. 66-78. 10.18006/2020.8(Spl-1-SARS-CoV-2).S66.S78.

1391. 60.Miao Zhao, Hao-Tao Zeng, Gui Yang, Xiao-Rui Geng, Yuan-Yi Zhang, Fei Ma, Jiang-Qi Liu, Zhi-Qiang Liu, Mei-Zhen Zhao, Li-Hua Mo, Xiang-Qian Luo, Xiao-Wen Zhang, Da-Bo Liu, Ping-Chang Yang, Toll-like receptor signal is required in maintenance of immune suppressive capacity of regulatory T cells, Immunology Letters, 10.1016/j.imlet.2019.04.005, 210, (47-54), (2019). 1392. Debnath, Utsab \& Dewaker, Varun \& Prabhakar, Yenamandra \& Bhattacharyya, Parthasarathi \& Mandal, Amit. (2020). Conformational Perturbation of SARS-CoV-2 Spike Protein Using N-Acetyl Cysteine, a Molecular Scissor: A Probable Strategy to Combat COVID-19. 10.26434/chemrxiv.12687923.

1393. Miettinen M, Sareneva T, Julkunen I, Matikainen S. IFNs activate toll-like receptor gene expression in viral infections. Genes Immun. 2001 Oct;2(6):349-55. doi: 10.1038/sj.gene.6363791. PMID: 11607792. 
1394. Aboudounya, Mohamed \& Heads, Richard. (2021). COVID-19 and Toll-Like Receptor 4 (TLR4): SARS-CoV-2 May Bind and Activate TLR4 to Increase ACE2 Expression, Facilitating Entry and Causing Hyperinflammation. Mediators of Inflammation. 2021. 1-18. 10.1155/2021/8874339.

1395. Droemann D, Goldmann T, Tiedje T, Zabel P, Dalhoff K, Schaaf B. Toll-like receptor 2 expression is decreased on alveolar macrophages in cigarette smokers and COPD patients. Respir Res. 2005 Jul 8;6(1):68. doi: 10.1186/1465-9921-6-68. PMID: 16004610; PMCID: PMC1187924.

1396. Segovia J, Sabbah A, Mgbemena V, Tsai SY, Chang TH, Berton MT, Morris IR, Allen IC, Ting JP, Bose S. TLR2/MyD88/NF-KB pathway, reactive oxygen species, potassium efflux activates NLRP3/ASC inflammasome during respiratory syncytial virus infection. PLoS One. 2012;7(1):e29695. doi: 10.1371/journal.pone.0029695. Epub 2012 Jan 25. PMID: 22295065; PMCID: PMC3266238.

1397. Dou, Ying \& Zhao, Yao \& Zhang, Zhi-Yong \& Mao, Hua-Wei \& Tu, Wenwei \& Zhao, Xiao-Dong. (2013). Respiratory Syncytial Virus Infection Induces Higher Toll-Like Receptor-3 Expression and TNF- $\alpha$ Production Than Human Metapneumovirus Infection. PloS one. 8. e73488. 10.1371/journal.pone.0073488.

1398. Caballero MT, Serra ME, Acosta PL, Marzec J, Gibbons L, et.al. TLR4 genotype and environmental LPS mediate RSV bronchiolitis through Th2 polarization. J Clin Invest. 2015 Feb;125(2):571-82. doi: 10.1172/JCI75183. Epub 2015 Jan 2. PMID: 25555213; PMCID: PMC4319428.

1399. Sebina I, Phipps S. The Contribution of Neutrophils to the Pathogenesis of RSV Bronchiolitis. Viruses. 2020 Jul 27;12(8):808. doi: 10.3390/v12080808. PMID: 32726921; PMCID: PMC7472258.

1400. Haynes, Lia \& Moore, Deborah \& Kurt-Jones, Evelyn \& Finberg, Robert \& Anderson, Larry \& Tripp, Ralph. (2001). Involvement of Toll-Like Receptor 4 in Innate Immunity to Respiratory Syncytial Virus. Journal of virology. 75. 10730-7. 10.1128/JVI.75.22.10730-10737.2001.

1401. Pace, Elisabetta \& Ferraro, Maria \& Siena, Liboria \& Melis, Mario \& Montalbano, A. Marina \& Johnson, Malcolm \& Bonsignore, Maria \& Bonsignore, Giovanni \& Gjomarkaj, Mark. (2008). Cigarette smoke increases TLR4 and modifies LPS mediated responses in airway epithelial cells. Immunology. 124. 401-11. 10.1111/j.1365-2567.2007.02788.x.

1402. Arora, Shweta \& Ahmad, Shaniya \& Irshad, Rasha \& Goyal, Yamini \& Rafat, Sahar \& Siddiqui, Neha \& Dev, Kapil \& Husain, Mohammad \& Ali, Shakir \& Mohan, Anant \& Ali, Syed. (2019). TLRs in pulmonary diseases. Life Sciences. 233. 116671. 10.1016/j.Ifs.2019.116671

1403. Sidletskaya K, Vitkina T, Denisenko Y. The Role of Toll-Like Receptors 2 and 4 in the Pathogenesis of Chronic Obstructive Pulmonary Disease. Int J Chron Obstruct Pulmon Dis. 2020 Jun 23;15:1481-1493. doi: 10.2147/COPD.S249131. PMID: 32606656; PMCID: PMC7320879.

1404. Tengroth L, Millrud CR, Kvarnhammar AM, Kumlien Georén S, Latif L, Cardell LO. Functional effects of Toll-like receptor (TLR)3, 7, 9, RIG-I and MDA-5 stimulation in nasal epithelial cells. PLoS One. 2014 Jun 2;9(6):e98239. doi:

10.1371/journal.pone.0098239. PMID: 24886842; PMCID: PMC4041746.

1405. Haw TJ, Starkey MR, Pavlidis S, Fricker M, Arthurs AL, Nair PM, Liu G, Hanish I, Kim RY, Foster PS, Horvat JC, Adcock IM, Hansbro PM. Toll-like receptor 2 and 4 have opposing roles in the pathogenesis of cigarette smoke-induced chronic obstructive pulmonary disease. Am J Physiol Lung Cell Mol Physiol. 2018 Feb 1;314(2):L298-L317. doi: 10.1152/ajplung.00154.2017. Epub 2017 Oct 12. PMID: 29025711; PMCID: PMC5866502.

1406. Arpaia N, Barton GM. Toll-like receptors: key players in antiviral immunity. Curr Opin Virol. 2011 Dec;1(6):447-54. doi: 10.1016/j.coviro.2011.10.006. Epub 2011 Oct 28. PMID: 22440908; PMCID: PMC3311989.

1407. 1406

1408. Nadigel J, Préfontaine D, Baglole CJ, Maltais F, Bourbeau J, Eidelman DH, Hamid Q. Cigarette smoke increases TLR4 and TLR9 expression and induces cytokine production from CD8(+) T cells in chronic obstructive pulmonary disease. Respir Res. 2011 Nov 9;12(1):149. doi: 10.1186/1465-9921-12-149. PMID: 22070100; PMCID: PMC3260101.

1409. Foronjy RF, Salathe MA, Dabo AJ, Baumlin N, Cummins N, Eden E, Geraghty P. TLR9 expression is required for the development of cigarette smoke-induced emphysema in mice. Am J Physiol Lung Cell Mol Physiol. 2016 Jul 1;311(1):L154-66. doi: 10.1152/ajplung.00073.2016. Epub 2016 Jun 10. PMID: 27288485; PMCID: PMC4967186.

1410. Piccinini AM, Midwood KS. DAMPening inflammation by modulating TLR signalling. Mediators Inflamm. 2010;2010:672395. doi: 10.1155/2010/672395. Epub 2010 Jul 13. PMID: 20706656; PMCID: PMC2913853.

1411. Grassin-Delyle S, Abrial C, Salvator H, Brollo M, Naline E, Devillier P. The Role of Toll-Like Receptors in the Production of Cytokines by Human Lung Macrophages. J Innate Immun. 2020;12(1):63-73. doi: 10.1159/000494463. Epub 2018 Dec 17. PMID: 30557876; PMCID: PMC6959095.

1412. Park BS, Lee JO. Recognition of lipopolysaccharide pattern by TLR4 complexes. Exp Mol Med. 2013 Dec 6;45(12):e66. doi: 10.1038/emm.2013.97. PMID: 24310172; PMCID: PMC3880462.

1413. Yokota S, Okabayashi T, Fujii N. The battle between virus and host: modulation of Toll-like receptor signaling pathways by virus infection. Mediators Inflamm. 2010;2010:184328. doi: 10.1155/2010/184328. Epub 2010 Jun 16. PMID: 20672047; PMCID: PMC2903949.

1414. Patel MC, Shirey KA, Pletneva LM, Boukhvalova MS, Garzino-Demo A, Vogel SN, Blanco JC. Novel drugs targeting Toll-like receptors for antiviral therapy. Future Virol. 2014 Sep;9(9):811-829. doi: 10.2217/fvl.14.70. PMID: 25620999; PMCID: PMC4303062.

1415. Cizmecioglu A, Akay Cizmecioglu H, Goktepe MH, Emsen A, Korkmaz C, Esenkaya Tasbent F, Colkesen F, Artac H. Apoptosisinduced T-cell lymphopenia is related to COVID-19 severity. J Med Virol. 2021 May;93(5):2867-2874. doi: 10.1002/jmv.26742. Epub 2021 Feb 23. PMID: 33331657.

1416. Kahan SM, Wherry EJ, Zajac AJ. T cell exhaustion during persistent viral infections. Virology. 2015 May;479-480:180-93. doi: 10.1016/j.virol.2014.12.033. Epub 2015 Jan 22. PMID: 25620767; PMCID: PMC4424083.

1417. Inoue, Shigeaki \& Suzuki, Kodai \& Komori, Yukako \& Morishita, Yukiko \& Suzuki-Utsunomiya, Kyoko \& Hozumi, Katsuto \& Inokuchi, Sadaki \& Sato, Takehito. (2014). Persistent inflammation and T cell exhaustion in severe sepsis in the elderly. Critical care (London, England). 18. R130. 10.1186/cc13941.

1418. Liu L, Ren W, Chen K. MiR-34a Promotes Apoptosis and Inhibits Autophagy by Targeting HMGB1 in Acute Myeloid Leukemia Cells. Cell Physiol Biochem. 2017;41(5):1981-1992. doi: 10.1159/000475277. Epub 2017 Apr 13. PMID: 28478444

1419. Zou Y, Chen X, Xiao J, Bo Zhou D, Xiao Lu X, Li W, Xie B, Kuang X, Chen Q. Neutrophil extracellular traps promote lipopolysaccharide-induced airway inflammation and mucus hypersecretion in mice. Oncotarget. 2018 Jan 8;9(17):13276-13286. doi: 10.18632/oncotarget.24022. PMID: 29568356; PMCID: PMC5862577.

1420. Zou Y, Chen X, Xiao J, Bo Zhou D, Xiao Lu X, Li W, Xie B, Kuang X, Chen Q. Neutrophil extracellular traps promote lipopolysaccharide-induced airway inflammation and mucus hypersecretion in mice. Oncotarget. 2018 Jan 8;9(17):13276-13286. doi: 10.18632/oncotarget.24022. PMID: 29568356; PMCID: PMC5862577. 
1421. Hu, Z., Murakami, T., Tamura, H., Reich, J., Kuwahara- Arai, K., Iba, T. ... Nagaoka, I. (2017). Neutrophil extracellular traps induce IL-1 $\beta$ production by macrophages in combination with lipopolysaccharide. International Journal of Molecular Medicine, 39 , 549-558. https://doi.org/10.3892/ijmm.2017.2870

1422. Chen, L., Zhao, Y., Lai, D. et al. Neutrophil extracellular traps promote macrophage pyroptosis in sepsis. Cell Death Dis 9 , 597 (2018). https://doi.org/10.1038/s41419-018-0538-5

1423. Farrera C, Fadeel B. Macrophage clearance of neutrophil extracellular traps is a silent process. J Immunol. $2013 \mathrm{Sep}$ 1;191(5):2647-56. doi: 10.4049/jimmunol.1300436. Epub 2013 Jul 31. PMID: 23904163

1424. Funchal GA, Jaeger N, Czepielewski RS, Machado MS, Muraro SP, Stein RT, Bonorino CB, Porto BN. Respiratory syncytial virus fusion protein promotes TLR-4-dependent neutrophil extracellular trap formation by human neutrophils. PLoS One. 2015 Apr 9;10(4):e0124082. doi: 10.1371/journal.pone.0124082. PMID: 25856628; PMCID: PMC4391750.

1425. Liu T, Zhang L, Joo D, Sun SC. NF-kB signaling in inflammation. Signal Transduct Target Ther. 2017;2:17023-. doi: 10.1038/sigtrans.2017.23. Epub 2017 Jul 14. PMID: 29158945; PMCID: PMC5661633.

1426. Azkur AK, Akdis M, Azkur D, Sokolowska M, van de Veen W, Brüggen MC, O'Mahony L, Gao Y, Nadeau K, Akdis CA. Immune response to SARS-CoV-2 and mechanisms of immunopathological changes in COVID-19. Allergy. 2020 Jul;75(7):1564-1581. doi: 10.1111/all.14364. PMID: 32396996; PMCID: PMC7272948.

1427. Jozwik, Agnieszka \& Habibi, Maximillian \& Paras, Allan \& Zhu, Jie \& Guvenel, et.al. (2015). RSV-specific airway resident memory CD8+ T cells and differential disease severity after experimental human infection. Nature Communications. 6. 10224. $10.1038 /$ ncomms 10224

1428. Ascough S, Paterson S, Chiu C. Induction and Subversion of Human Protective Immunity: Contrasting Influenza and Respiratory Syncytial Virus. Front Immunol. 2018 Mar 2;9:323. doi: 10.3389/fimmu.2018.00323. PMID: 29552008; PMCID: PMC5840263.

1429. Lolachi S, Morin S, Coen M, Samii K, Calmy A, Serratrice J. Macrophage activation syndrome as an unusual presentation of paucisymptomatic severe acute respiratory syndrome coronavirus 2 infection: A case report. Medicine (Baltimore). 2020 Aug 7;99(32):e21570. doi: 10.1097/MD.0000000000021570. PMID: 32769902; PMCID: PMC7593078.

1430. Schönrich G, Raftery MJ. The PD-1/PD-L1 Axis and Virus Infections: A Delicate Balance. Front Cell Infect Microbiol. 2019 Jun 13;9:207. doi: 10.3389/fcimb.2019.00207. PMID: 31263684; PMCID: PMC6584848.

1431. Shirato K, Kizaki T. SARS-CoV-2 spike protein S1 subunit induces pro-inflammatory responses via toll-like receptor 4 signaling in murine and human macrophages. Heliyon. 2021 Feb 2;7(2):e06187. doi: 10.1016/j.heliyon.2021.e06187. PMID: 33644468; PMCID: PMC7887388.

1432. Yongzhi X. COVID-19-associated cytokine storm syndrome and diagnostic principles: an old and new Issue. Emerg Microbes Infect. 2021 Dec;10(1):266-276. doi: 10.1080/22221751.2021.1884503. PMID: 33522893; PMCID: PMC7894425.

1433. Li H, Liu L, Zhang D, Xu J, Dai H, Tang N, Su X, Cao B. SARS-CoV-2 and viral sepsis: observations and hypotheses. Lancet 2020 May 9;395(10235):1517-1520. doi: 10.1016/S0140-6736(20)30920-X. Epub 2020 Apr 17. PMID: 32311318; PMCID: PMC7164875.

1434. Letarov AV, Babenko VV, Kulikov EE. Free SARS-CoV-2 Spike Protein S1 Particles May Play a Role in the Pathogenesis of COVID-19 Infection. Biochemistry (Mosc). 2021 Mar;86(3):257-261. doi: 10.1134/S0006297921030032. PMID: 33838638; PMCID: PMC7772528.

1435. Patra T, Meyer K, Geerling L, Isbell TS, Hoft DF, Brien J, Pinto AK, Ray RB, Ray R. SARS-CoV-2 spike protein promotes IL-6 trans-signaling by activation of angiotensin II receptor signaling in epithelial cells. PLoS Pathog. 2020 Dec 7;16(12):e1009128. doi: 10.1371/journal.ppat.1009128. PMID: 33284859; PMCID: PMC7746263.

1436. Hojyo S, Uchida M, Tanaka K, Hasebe R, Tanaka Y, Murakami M, Hirano T. How COVID-19 induces cytokine storm with high mortality. Inflamm Regen. 2020 Oct 1;40:37. doi: 10.1186/s41232-020-00146-3. PMID: 33014208; PMCID: PMC7527296. 1437. Rayan Khaddaj-Mallat, Natija Aldib, Anne-Sophie Paquette, Aymeric Ferreira, et.al. SARS-CoV-2 spike protein induces brain pericyte immunoreactivity in absence of productive viral infection bioRxiv 2021.04.30.442194; doi:

https://doi.org/10.1101/2021.04.30.442194

1438. Fuke N, Nagata N, Suganuma H, Ota T. Regulation of Gut Microbiota and Metabolic Endotoxemia with Dietary Factors. Nutrients. 2019 Sep 23;11(10):2277. doi: 10.3390/nu11102277. PMID: 31547555; PMCID: PMC6835897.

1439. Panahipour L, Nasserzare S, Amer Z, Brücke F, Stähli A, Kreissl A, Haiden N, Gruber R. The anti-inflammatory effect of milk and dairy products on periodontal cells: an in vitro approach. Clin Oral Investig. 2019 Apr;23(4):1959-1966. doi: 10.1007/s00784 018-2642-4. Epub 2018 Sep 20. PMID: 30238412.

1440. Yuan M, Singer MR, Moore LL. Yogurt Consumption Is Associated with Lower Levels of Chronic Inflammation in the Framingham Offspring Study. Nutrients. 2021 Feb 4;13(2):506. doi: 10.3390/nu13020506. PMID: 33557067; PMCID: PMC7913863. 1441. Pei R, DiMarco DM, Putt KK, Martin DA, Gu Q, Chitchumroonchokchai C, White HM, Scarlett CO, Bruno RS, Bolling BW. Lowfat yogurt consumption reduces biomarkers of chronic inflammation and inhibits markers of endotoxin exposure in healthy premenopausal women: a randomised controlled trial. Br J Nutr. 2017 Dec;118(12):1043-1051. doi:

10.1017/S0007114517003038. Epub 2017 Nov 28. PMID: 29179781

1442. Karakike E, Giamarellos-Bourboulis EJ. Macrophage Activation-Like Syndrome: A Distinct Entity Leading to Early Death in Sepsis. Front Immunol. 2019 Jan 31;10:55. doi: 10.3389/fimmu.2019.00055. PMID: 30766533; PMCID: PMC6365431.

1443. Schnaubelt S, Tihanyi D, Strassl R, Schmidt R, Anders S, Laggner AN, Agis H, Domanovits H. Hemophagocytic lymphohistiocytosis in COVID-19: Case reports of a stepwise approach. Medicine (Baltimore). 2021 Mar 26;100(12):e25170. doi: 10.1097/MD.0000000000025170. PMID: 33761694.

1444. Meng M, Chen L, Zhang S, Dong X, Li W, Li R, Deng Y, Wang T, Xu Y, Liu J, Huang Y, Chen Y, Huang S, Wen Z, Zhang L, Du H, Liu Y, Annane D, Qu J, Chen D. Risk factors for secondary hemophagocytic lymphohistiocytosis in severe coronavirus disease 2019 adult patients. BMC Infect Dis. 2021 Apr 29;21(1):398. doi: 10.1186/s12879-021-06094-8. PMID: 33926377; PMCID: PMC8084265. 1445. Crayne CB, Albeituni S, Nichols KE, Cron RQ. The Immunology of Macrophage Activation Syndrome. Front Immunol. 2019 Feb 1;10:119. doi: 10.3389/fimmu.2019.00119. PMID: 30774631; PMCID: PMC6367262.

1446. Núñez-Torrón C, Ferrer-Gómez A, Moreno Moreno E, Pérez-Mies B, Villarrubia J, Chamorro S, López-Jiménez J, Palacios J, Piris-Villaespesa M, García-Cosío M. Secondary haemophagocytic lymphohistiocytosis in COVID-19: correlation of the autopsy findings of bone marrow haemophagocytosis with HScore. J Clin Pathol. 2021 Mar 15:jclinpath-2020-207337. doi: 10.1136/jclinpath-2020-207337. Epub ahead of print. PMID: 33722841; PMCID: PMC7970658.

1447. Lichtenberger LM, Vijayan KV. Is COVID-19-Induced Platelet Activation a Cause of Concern for Patients with Cancer? Cancer Res. 2021 Mar 1;81(5):1209-1211. doi: 10.1158/0008-5472.CAN-20-3691. Epub 2020 Dec 21. PMID: 33355186; PMCID: PMC8026656.

1448. Borges, Leandro \& Pithon-Curi, Tania \& Curi, Rui \& Hatanaka, Elaine. (2020). COVID-19 and Neutrophils: The Relationship between Hyperinflammation and Neutrophil Extracellular Traps. Mediators of Inflammation. 2020. 10.1155/2020/8829674. 
1449. Veras FP, Pontelli MC, Silva CM, Toller-Kawahisa JE, de Lima M, et.al. SARS-CoV-2-triggered neutrophil extracellular traps mediate COVID-19 pathology. J Exp Med. 2020 Dec 7;217(12):e20201129. doi: 10.1084/jem.20201129. PMID: 32926098; PMCID: PMC7488868.

1450. Ackermann M, Anders HJ, Bilyy R, Bowlin GL, Daniel C, De Lorenzo R, Egeblad M et.al. Patients with COVID-19: in the darkNETs of neutrophils. Cell Death Differ. 2021 May 24:1-15. doi: 10.1038/s41418-021-00805-z. Epub ahead of print. PMID: 34031543; PMCID: PMC8142290.

1451. Bautista-Becerril B, Campi-Caballero R, Sevilla-Fuentes S, Hernández-Regino LM, Hanono A, Flores-Bustamante A, GonzálezFlores J, García-Ávila CA, Aquino-Gálvez A, Castillejos-López M, Juárez-Cisneros A, Camarena A. Immunothrombosis in CoVID-19: Implications of Neutrophil Extracellular Traps. Biomolecules. 2021 May 6;11(5):694. doi: 10.3390/biom11050694. PMID: 34066385; PMCID: PMC8148218.

1452. Thierry AR, Roch B. Neutrophil Extracellular Traps and By-Products Play a Key Role in COVID-19: Pathogenesis, Risk Factors, and Therapy. J Clin Med. 2020 Sep 11;9(9):2942. doi: 10.3390/jcm9092942. PMID: 32933031; PMCID: PMC7565044.

1453. Thierry, A.; ROCH, B. NETs By-products and Extracellular DNA May Play a Key Role in COVID-19 Pathogenesis: Incidence on Patient Monitoring and Therapy. Preprints 2020, 2020040238 (doi: 10.20944/preprints202004.0238.v1).

1454. Grobbelaar LM, Venter C, Vlok M, Ngoepe M, Laubscher GJ, Lourens PJ, Steenkamp J, Kell D, Pretorius E. SARS-CoV-2 spike protein S1 induces fibrin(ogen) resistant to fibrinolysis: Implications for microclot formation in COVID-19. Biosci Rep. 2021 Jul 30:BSR20210611. doi: 10.1042/BSR20210611. Epub ahead of print. PMID: 34328172.

1455. Fuchs TA, Brill A, Wagner DD. Neutrophil extracellular trap (NET) impact on deep vein thrombosis. Arterioscler Thromb Vasc Biol. 2012 Aug;32(8):1777-83. doi: 10.1161/ATVBAHA.111.242859. Epub 2012 May 31. PMID: 22652600; PMCID: PMC3495595.

1456. Dayang EZ, Plantinga J, Ter Ellen B, van Meurs M, Molema G, Moser J. Identification of LPS-Activated Endothelial Subpopulations With Distinct Inflammatory Phenotypes and Regulatory Signaling Mechanisms. Front Immunol. 2019 May 24;10:1169. doi: 10.3389/fimmu.2019.01169. PMID: 31178871; PMCID: PMC6543489.

1457. Sharifi-Rad M, Anil Kumar NV, Zucca P, Varoni EM, Dini L, Panzarini E, Rajkovic J, Tsouh Fokou PV, Azzini E, Peluso I, Prakash Mishra A, Nigam M, El Rayess Y, Beyrouthy ME, Polito L, Iriti M, Martins N, Martorell M, Docea AO, Setzer WN, Calina D, Cho WC Sharifi-Rad J. Lifestyle, Oxidative Stress, and Antioxidants: Back and Forth in the Pathophysiology of Chronic Diseases. Front Physiol. 2020 Jul 2;11:694. doi: 10.3389/fphys.2020.00694. PMID: 32714204; PMCID: PMC7347016.

1458. Zweigner J, Gramm HJ, Singer OC, Wegscheider K, Schumann RR. High concentrations of lipopolysaccharide-binding protein in serum of patients with severe sepsis or septic shock inhibit the lipopolysaccharide response in human monocytes. Blood. 2001 Dec 15;98(13):3800-8. doi: 10.1182/blood.v98.13.3800. PMID: 11739189.

1459. Zhang S, Liu Y, Wang X, Yang L, Li H, Wang Y, Liu M, Zhao X, Xie Y, Yang Y, Zhang S, Fan Z, Dong J, Yuan Z, Ding Z, Zhang Y, Hu L. SARS-CoV-2 binds platelet ACE2 to enhance thrombosis in COVID-19. J Hematol Oncol. 2020 Sep 4;13(1):120. doi: 10.1186/s13045-020-00954-7. PMID: 32887634; PMCID: PMC7471641.

1460. Maiuolo J, Mollace R, Gliozzi M, Musolino V, Carresi C, Paone S, Scicchitano M, Macrì R, Nucera S, Bosco F, Scarano F, Zito MC, Ruga S, Tavernese A, Mollace V. The Contribution of Endothelial Dysfunction in Systemic Injury Subsequent to SARS-Cov-2 Infection. Int J Mol Sci. 2020 Dec 6;21(23):9309. doi: 10.3390/ijms21239309. PMID: 33291346; PMCID: PMC7730352.

1461. Bongiovanni D, Klug M, Lazareva O, Weidlich S, Biasi M, Ursu S, Warth S, Buske C, Lukas M, Spinner CD, Scheidt MV, Condorelli G, Baumbach J, Laugwitz KL, List M, Bernlochner I. SARS-CoV-2 infection is associated with a pro-thrombotic platelet phenotype. Cell Death Dis. 2021 Jan 5;12(1):50. doi: 10.1038/s41419-020-03333-9. PMID: 33414384; PMCID: PMC7790351. 1462. Binet Q, Goffinet C, Etogo-Asse FE, Shaza L. Nonbacterial thrombotic endocarditis in a patient with gastric cancer and SARSCoV-2 infection. Clin J Gastroenterol. 2021 Aug;14(4):1031-1035. doi: 10.1007/s12328-021-01412-8. Epub 2021 Apr 9. PMID: 33835416; PMCID: PMC8032844.

1463. Khatri I, Staal FJT, van Dongen JJM. Blocking of the High-Affinity Interaction-Synapse Between SARS-CoV-2 Spike and Human ACE2 Proteins Likely Requires Multiple High-Affinity Antibodies: An Immune Perspective. Front Immunol. 2020 Sep 17;11:570018. doi: 10.3389/fimmu.2020.570018. Erratum in: Front Immunol. 2021 Apr 14;12:659375. PMID: 33042151; PMCID: PMC7527437.

1464. Ni W, Yang X, Yang D, Bao J, Li R, Xiao Y, Hou C, Wang H, Liu J, Yang D, Xu Y, Cao Z, Gao Z. Role of angiotensin-converting enzyme 2 (ACE2) in COVID-19. Crit Care. 2020 Jul 13;24(1):422. doi: 10.1186/s13054-020-03120-0. PMID: 32660650; PMCID: PMC7356137.

1465. Jafarinejad-Farsangi S, Jazi MM, Rostamzadeh F, Hadizadeh M. High affinity of host human microRNAs to SARS-CoV-2 genome: An in silico analysis. Noncoding RNA Res. 2020 Dec;5(4):222-231. doi: 10.1016/j.ncrna.2020.11.005. Epub 2020 Nov 21. PMID: 33251388; PMCID: PMC7680021.

1466. Kabat AM, Pott J, Maloy KJ. The Mucosal Immune System and Its Regulation by Autophagy. Front Immunol. 2016 Jun 22;7:240. doi: 10.3389/fimmu.2016.00240. PMID: 27446072; PMCID: PMC4916208.

1467. Nur Husna SM, Tan HT, Md Shukri N, Mohd Ashari NS, Wong KK. Nasal Epithelial Barrier Integrity and Tight Junctions Disruption in Allergic Rhinitis: Overview and Pathogenic Insights. Front Immunol. 2021 May 21;12:663626. doi: 10.3389/fimmu.2021.663626. PMID: 34093555; PMCID: PMC8176953.

1468. Qiu F, Liang CL, Liu H, Zeng YQ, Hou S, Huang S, Lai X, Dai Z. Impacts of cigarette smoking on immune responsiveness: Up and down or upside down? Oncotarget. 2017 Jan 3;8(1):268-284. doi: 10.18632/oncotarget.13613. PMID: 27902485; PMCID: PMC5352117.

1469. Gordon DE, Jang GM, Bouhaddou M, Xu J, Obernier K, White KM, O'Meara MJ et.al. A SARS-CoV-2 protein interaction map reveals targets for drug repurposing. Nature. 2020 Jul;583(7816):459-468. doi: 10.1038/s41586-020-2286-9. Epub 2020 Apr 30. PMID: 32353859; PMCID: PMC7431030.

1470. Kim SY, Shin JS, Chung KS, Han HS, Lee HH, Lee JH, Kim SY, Ji YW, Ha Y, Kang J, Rhee YK, Lee KT. Immunostimulatory Effects of Live Lactobacillus sakei K040706 on the CYP-Induced Immunosuppression Mouse Model. Nutrients. 2020 Nov 22;12(11):3573. doi: 10.3390/nu12113573. PMID: 33266362; PMCID: PMC7700367.

1471. Hachimura S, Totsuka M, Hosono A. Immunomodulation by food: impact on gut immunity and immune cell function. Biosci Biotechnol Biochem. 2018 Apr;82(4):584-599. doi: 10.1080/09168451.2018.1433017. Epub 2018 Feb 16. PMID: 29448897. 1472. Kruglikov IL, Scherer PE. Preexisting and inducible endotoxemia as crucial contributors to the severity of COVID-19 outcomes. PLoS Pathog. 2021 Feb 18;17(2):e1009306. doi: 10.1371/journal.ppat.1009306. PMID: 33600486; PMCID: PMC7891777

1473. Kim YH, Kang MK, Lee EJ, Kim DY, Oh H, Kim SI, Oh SY, Kim KH, Park SJ, Choi YJ, Kang YH. Dried Yeast Extracts Curtails Pulmonary Oxidative Stress, Inflammation and Tissue Destruction in a Model of Experimental Emphysema. Antioxidants (Basel). 2019 Sep 1;8(9):349. doi: 10.3390/antiox8090349. PMID: 31480536; PMCID: PMC6769699. 
1474. Askarova S, Umbayev B, Masoud AR, Kaiyrlykyzy A, Safarova Y, Tsoy A, Olzhayev F, Kushugulova A. The Links Between the Gut Microbiome, Aging, Modern Lifestyle and Alzheimer's Disease. Front Cell Infect Microbiol. 2020 Mar 18;10:104. doi: 10.3389/fcimb.2020.00104. PMID: 32257964; PMCID: PMC7093326.

1475. Brandão SCS, Ramos JOX, Dompieri LT, Godoi ETAM, Figueiredo JL, Sarinho ESC, Chelvanambi S, Aikawa M. Is Toll-like receptor 4 involved in the severity of COVID-19 pathology in patients with cardiometabolic comorbidities? Cytokine Growth Factor Rev. 2021 Apr;58:102-110. doi: 10.1016/j.cytogfr.2020.09.002. Epub 2020 Sep 21. PMID: 32988728; PMCID: PMC7505161. 1476. Poulas K, Farsalinos K, Zanidis C. Activation of TLR7 and Innate Immunity as an Efficient Method Against COVID-19 Pandemic: Imiquimod as a Potential Therapy. Front Immunol. 2020 Jun 11;11:1373. doi: 10.3389/fimmu.2020.01373. PMID: 32612613; PMCID: PMC7307572.

1477. McNamara PS, Smyth RL. The pathogenesis of respiratory syncytial virus disease in childhood. Br Med Bull. 2002;61:13-28. doi: 10.1093/bmb/61.1.13. PMID: 11997296.

1478. Lee YT, Kim KH, Hwang HS, Lee Y, Kwon YM, Ko EJ, Jung YJ, Lee YN, Kim MC, Kang SM. Innate and adaptive cellular phenotypes contributing to pulmonary disease in mice after respiratory syncytial virus immunization and infection. Virology. 2015 Nov;485:36-46. doi: 10.1016/j.virol.2015.07.001. Epub 2015 Jul 18. PMID: 26196232; PMCID: PMC4619147. 1479. Bohmwald K, Gálvez NMS, Canedo-Marroquín G, Pizarro-Ortega MS, Andrade-Parra C, Gómez-Santander F, Kalergis AM. Contribution of Cytokines to Tissue Damage During Human Respiratory Syncytial Virus Infection. Front Immunol. 2019 Mar 18;10:452. doi: 10.3389/fimmu.2019.00452. PMID: 30936869; PMCID: PMC6431622.

1480. Tang PS, Mura M, Seth R, Liu M. Acute lung injury and cell death: how many ways can cells die? Am J Physiol Lung Cell Mol Physiol. 2008 Apr;294(4):L632-41. doi: 10.1152/ajplung.00262.2007. Epub 2008 Jan 18. PMID: 18203816.

1481. Proctor, Peter. “Tempol Treatment of Covid-19." Free Radical Biology \& Medicine vol. 159 (2020): S95. doi:10.1016/j.freeradbiomed.2020.10.245

1482. Kavya Srinivasan, Ashutosh Pandey, Sundararajan Venkatesh. Roles of host mitochondria in the development of COVID-19 pathology. Authorea. January 12, 2021. DOI: 10.22541/au.161047974.41668545/v1

1483. Singh KK, Chaubey G, Chen JY, Suravajhala P. Decoding SARS-CoV-2 hijacking of host mitochondria in COVID-19 pathogenesis. Am J Physiol Cell Physiol. 2020 Aug 1;319(2):C258-C267. doi: 10.1152/ajpcell.00224.2020. Epub 2020 Jun 8. PMID: 32510973; PMCID: PMC7381712.

1484. Aghapour M, Remels AHV, Pouwels SD, Bruder D, Hiemstra PS, Cloonan SM, Heijink IH. Mitochondria: at the crossroads of regulating lung epithelial cell function in chronic obstructive pulmonary disease. Am J Physiol Lung Cell Mol Physiol. 2020 Jan 1;318(1):L149-L164. doi: 10.1152/ajplung.00329.2019. Epub 2019 Nov 6. PMID: 31693390; PMCID: PMC6985875.

1485. Galeas-Pena M, McLaughlin N, Pociask D. The role of the innate immune system on pulmonary infections. Biol Chem. 2019 Mar 26;400(4):443-456. doi: 10.1515/hsz-2018-0304. PMID: 29604208.

1486. Morris GF, Danchuk S, Wang Y, Xu B, Rando RJ, Brody AR, Shan B, Sullivan DE. Cigarette smoke represses the innate immune response to asbestos. Physiol Rep. 2015 Dec;3(12):e12652. doi: 10.14814/phy2.12652. PMID: 26660560; PMCID PMC4760433.

1487. Lin HY, Chuang JH, Wang PW, Lin TK, Wu MT, Hsu WM, Chuang HC. 5-aza-2'-Deoxycytidine Induces a RIG-I-Related Innate Immune Response by Modulating Mitochondria Stress in Neuroblastoma. Cells. 2020 Aug 19;9(9):1920. doi:

10.3390/cells9091920. PMID: 32824929; PMCID: PMC7564572.

1488. Mehta M, Paudel KR, Shukla SD, Shastri MD, Singh SK, Gulati M, Dureja H, Gupta G, Satija S, Hansbro PM, Chellappan DK, Dua K. Interferon therapy for preventing COPD exacerbations. EXCLI J. 2020 Nov 4;19:1477-1480. doi: 10.17179/excli2020-2997. PMID: 33312108; PMCID: PMC7726488.

1489. Agarwal R, Aggarwal AN, Gupta D, Jindal SK. Inhaled corticosteroids vs placebo for preventing COPD exacerbations: a systematic review and metaregression of randomized controlled trials. Chest. 2010 Feb;137(2):318-25. doi: 10.1378/chest.091305. Epub 2009 Sep 25. PMID: 19783669.

1490. Simin Nikbin Meydani, Woel-Kyu Ha, Immunologic effects of yogurt, The American Journal of Clinical Nutrition, Volume 71 , Issue 4, April 2000, Pages 861-872, https://doi.org/10.1093/ajcn/71.4.861

1491. Rabelo LA, Alenina N, Bader M. ACE2-angiotensin-(1-7)-Mas axis and oxidative stress in cardiovascular disease. Hypertens Res. 2011 Feb;34(2):154-60. doi: 10.1038/hr.2010.235. Epub 2010 Dec 2. PMID: 21124322.

1492. De Flora S, Balansky R, La Maestra S. Rationale for the use of N-acetylcysteine in both prevention and adjuvant therapy of COVID-19. FASEB J. 2020 Oct;34(10):13185-13193. doi: 10.1096/fj.202001807. Epub 2020 Aug 11. PMID: 32780893; PMCID: PMC7436914.

1493. Gioannini TL, Zhang D, Teghanemt A, Weiss JP. An essential role for albumin in the interaction of endotoxin with lipopolysaccharide-binding protein and SCD14 and resultant cell activation. J Biol Chem. 2002 Dec 6;277(49):47818-25. doi: 10.1074/jbc.M206404200. Epub 2002 Oct 7. PMID: 12372833.

1494. Sun K, Yajjala VK, Bauer C, Talmon GA, Fischer KJ, Kielian T, Metzger DW. Nox2-derived oxidative stress results in inefficacy of antibiotics against post-influenza S. aureus pneumonia. J Exp Med. 2016 Aug 22;213(9):1851-64. doi: 10.1084/jem.20150514. Epub 2016 Aug 15. PMID: 27526712; PMCID: PMC4995072.

1495. Yamaoka-Tojo M. Vascular Endothelial Glycocalyx Damage in COVID-19. Int J Mol Sci. 2020 Dec 19;21(24):9712. doi: 10.3390/ijms21249712. PMID: 33352699; PMCID: PMC7766512.

1496. Butler MJ, Down CJ, Foster RR, Satchell SC. The Pathological Relevance of Increased Endothelial Glycocalyx Permeability. Am J Pathol. 2020 Apr;190(4):742-751. doi: 10.1016/j.ajpath.2019.11.015. Epub 2020 Feb 6. PMID: 32035881; PMCID: PMC7163249.

1497. Yamaoka-Tojo M. Endothelial glycocalyx damage as a systemic inflammatory microvascular endotheliopathy in COVID-19. Biomed J. 2020 Oct;43(5):399-413. doi: 10.1016/j.bj.2020.08.007. Epub 2020 Aug 24. PMID: 33032965; PMCID: PMC7443638. 1498. Fosse JH, Haraldsen G, Falk K, Edelmann R. Endothelial Cells in Emerging Viral Infections. Front Cardiovasc Med. 2021 Feb 24;8:619690. doi: 10.3389/fcvm.2021.619690. PMID: 33718448; PMCID: PMC7943456.

1499. Dayang EZ, Plantinga J, Ter Ellen B, van Meurs M, Molema G, Moser J. Identification of LPS-Activated Endothelial Subpopulations With Distinct Inflammatory Phenotypes and Regulatory Signaling Mechanisms. Front Immunol. 2019 May 24;10:1169. doi: 10.3389/fimmu.2019.01169. PMID: 31178871; PMCID: PMC6543489.

1500. Ehrentraut S, Frede S, Stapel H, Mengden T, Grohé C, Fandrey J, Meyer R, Baumgarten G. Antagonism of

lipopolysaccharide-induced blood pressure attenuation and vascular contractility. Arterioscler Thromb Vasc Biol. 2007 Oct;27(10):2170-6. doi: 10.1161/ATVBAHA.107.146100. Epub 2007 Jul 26. PMID: 17656666.

1501. Grylls A, Seidler K, Neil J. Link between microbiota and hypertension: Focus on LPS/TLR4 pathway in endothelial dysfunction and vascular inflammation, and therapeutic implication of probiotics. Biomed Pharmacother. 2021 May;137:111334. doi: 10.1016/j.biopha.2021.111334. Epub 2021 Feb 5. PMID: 33556874.

1502. Kaur, Savneet et al. "The Enigma of Endothelium in COVID-19." Frontiers in physiology vol. 11 989. 4 Aug. 2020, doi:10.3389/fphys.2020.00989 
1503. Ferreira AC, Soares VC, de Azevedo-Quintanilha IG, Dias SDSG, Fintelman-Rodrigues N, et.al. SARS-CoV-2 engages inflammasome and pyroptosis in human primary monocytes. Cell Death Discov. 2021 Mar 1;7(1):43. doi: 10.1038/s41420-021 00428-w. Erratum in: Cell Death Discov. 2021 May 19;7(1):116. PMID: 33649297; PMCID: PMC7919254.

1504. Java A, Apicelli AJ, Liszewski MK, Coler-Reilly A, Atkinson JP, Kim AH, Kulkarni HS. The complement system in COVID-19: friend and foe? JCl Insight. 2020 Aug 6;5(15):e140711. doi: 10.1172/jci.insight.140711. PMID: 32554923; PMCID: PMC7455060. 1505. Hemmat N, Asadzadeh Z, Ahangar NK, Alemohammad H, Najafzadeh B, Derakhshani A, Baghbanzadeh A, Baghi HB, Javadrashid D, Najafi S, Ar Gouilh M, Baradaran B. The roles of signaling pathways in SARS-CoV-2 infection; lessons learned from SARS-CoV and MERS-CoV. Arch Virol. 2021 Mar;166(3):675-696. doi: 10.1007/s00705-021-04958-7. Epub 2021 Jan 18. PMID: 33462671; PMCID: PMC7812983.

1506. Gómez-Pastora J, Weigand M, Kim J, Wu X, Strayer J, Palmer AF, Zborowski M, Yazer M, Chalmers JJ. Hyperferritinemia in critically ill COVID-19 patients - Is ferritin the product of inflammation or a pathogenic mediator? Clin Chim Acta. 2020 Oct;509:249-251. doi: 10.1016/j.cca.2020.06.033. Epub 2020 Jun 21. PMID: 32579952; PMCID: PMC7306200.

1507. Vargas-Vargas, Manuel, and Christian Cortés-Rojo. "Ferritin levels and COVID-19." Revista panamericana de salud publica = Pan American journal of public health vol. 44 e72. 1 Jun. 2020, doi:10.26633/RPSP.2020.72

1508. Silvagno F, Vernone A, Pescarmona GP. The Role of Glutathione in Protecting against the Severe Inflammatory Response Triggered by COVID-19. Antioxidants (Basel). 2020 Jul 16;9(7):624. doi: 10.3390/antiox9070624. PMID: 32708578; PMCID: PMC7402141.

1509. Khanfar A, Al Qaroot B. Could glutathione depletion be the Trojan horse of COVID-19 mortality? Eur Rev Med Pharmacol Sci. 2020 Dec;24(23):12500-12509. doi: 10.26355/eurrev_202012_24046. PMID: 33336769.

1510. Shi, Zhongcheng, and Carlos A Puyo. "N-Acetylcysteine to Combat COVID-19: An Evidence Review." Therapeutics and clinical risk management vol. 16 1047-1055. 2 Nov. 2020, doi:10.2147/TCRM.S273700

1511. Yan F, Gao H, Zhao H, Bhatia M, Zeng Y. Roles of airway smooth muscle dysfunction in chronic obstructive pulmonary disease. J Transl Med. 2018 Sep 26;16(1):262. doi: 10.1186/s12967-018-1635-z. PMID: 30257694; PMCID: PMC6158847.

1512. Chung KF, Adcock IM. Multifaceted mechanisms in COPD: inflammation, immunity, and tissue repair and destruction. Eur Respir J. 2008 Jun;31(6):1334-56. doi: 10.1183/09031936.00018908. PMID: 18515558.

1513. Maggio, Roberto, and Giovanni U Corsini. "Repurposing the mucolytic cough suppressant and TMPRSS2 protease inhibitor bromhexine for the prevention and management of SARS-CoV-2 infection." Pharmacological research vol. 157 (2020): 104837. doi:10.1016/j.phrs.2020.104837

1514. Habtemariam S, Nabavi SF, Ghavami S, Cismaru CA, Berindan-Neagoe I, Nabavi SM. Possible use of the mucolytic drug bromhexine hydrochloride, as a prophylactic agent against SARS-CoV-2 infection based on its action on the Transmembrane Serine Protease 2. Pharmacol Res. 2020 Jul;157:104853. doi: 10.1016/j.phrs.2020.104853. Epub 2020 Apr 30. PMID: 32360584; PMCID: PMC7192109.

1515. Yue G, Shi G, Azaro MA, Yang Q, Hu G, Luo M, Yin K, Nagele RG, Fine DH, Yang JM, Li H. Lipopolysaccharide (LPS) potentiates hydrogen peroxide toxicity in T98G astrocytoma cells by suppression of anti-oxidative and growth factor gene expression. BMC Genomics. 2008 Dec 16;9:608. doi: 10.1186/1471-2164-9-608. PMID: 19087328; PMCID: PMC2631585. 1516. Wang HM, Fu L, Cheng CC, Gao R, Lin MY, Su HL, Belinda NE, Nguyen TH, Lin WH, Lee PC, Hsieh LP. Inhibition of LPSInduced Oxidative Damages and Potential Anti-Inflammatory Effects of Phyllanthus emblica Extract via Down-Regulating NF-KB, COX-2, and iNOS in RAW 264.7 Cells. Antioxidants (Basel). 2019 Aug 2;8(8):270. doi: 10.3390/antiox8080270. PMID: 31382466; PMCID: PMC6721275.

1517. Chen Y, Luo G, Yuan J, Wang Y, Yang X, Wang X, Li G, Liu Z, Zhong N. Vitamin C mitigates oxidative stress and tumor necrosis factor-alpha in severe community-acquired pneumonia and LPS-induced macrophages. Mediators Inflamm. 2014;2014:426740. doi: 10.1155/2014/426740. Epub 2014 Sep 1. PMID: 25253919; PMCID: PMC4165740.

1518. Sindona C, Schepici G, Contestabile V, Bramanti P, Mazzon E. NOX2 Activation in COVID-19: Possible Implications for Neurodegenerative Diseases. Medicina (Kaunas). 2021 Jun 11;57(6):604. doi: 10.3390/medicina57060604. PMID: 34208136; PMCID: PMC8230853.

1519. Sirivongrangson P, Kulvichit W, Payungporn S, Pisitkun T, Chindamporn A, Peerapornratana S, et,al. Endotoxemia and circulating bacteriome in severe COVID-19 patients. Intensive Care Med Exp. 2020 Dec 7;8(1):72. doi: 10.1186/s40635-02000362-8. PMID: 33284413; PMCID: PMC7719737.

1520. Ashraf MZ, Singh M, Hussain ME, Prasad AK, Fahim M. Influence of influenza viral infection on airway smooth muscle activity. Indian J Exp Biol. 2001 Apr;39(4):329-33. PMID: 11491576.

1521. Xin Peng, Yi Wu, Xiao Kong, Yunxiu Chen, Yonglu Tian, Qinyuan Li, Xiaoyin Tian, Guangli Zhang, Luo Ren, Zhengxiu Luo, "Neonatal Streptococcus pneumoniae Pneumonia Induces an Aberrant Airway Smooth Muscle Phenotype and AHR in Mice Model", BioMed Research International, vol. 2019, Article ID 1948519, 8 pages, 2019. https://doi.org/10.1155/2019/1948519 1522. Feng S, Zeng D, Zheng J, Zhao D. MicroRNAs: Mediators and Therapeutic Targets to Airway Hyper Reactivity After Respiratory Syncytial Virus Infection. Front Microbiol. 2018 Sep 11;9:2177. doi: 10.3389/fmicb.2018.02177. PMID: 30254626; PMCID: PMC6141694.

1523. Piedimonte G. Neural mechanisms of respiratory syncytial virus-induced inflammation and prevention of respiratory syncytial virus sequelae. Am J Respir Crit Care Med. 2001 Mar;163(3 Pt 2):S18-21. doi

10.1164/ajrccm.163.supplement_1.2011113. PMID: 11254547.

1524. Robinson PJ, Hegele RG, Schellenberg RR. Allergic sensitization increases airway reactivity in guinea pigs with respiratory syncytial virus bronchiolitis. J Allergy Clin Immunol. 1997 Oct;100(4):492-8. doi: 10.1016/s0091-6749(97)70141-6. PMID: 9338543. 1525. Higham, Andrew et al. "COVID-19 and COPD: a narrative review of the basic science and clinical outcomes." European respiratory review : an official journal of the European Respiratory Society vol. 29,158 200199. 5 Nov. 2020, doi:10.1183/16000617.0199-2020

1526. Ojo AS, Balogun SA, Williams OT, Ojo OS. Pulmonary Fibrosis in COVID-19 Survivors: Predictive Factors and Risk Reduction Strategies. Pulm Med. 2020 Aug 10;2020:6175964. doi: 10.1155/2020/6175964. PMID: 32850151; PMCID: PMC7439160. 1527. Zhang D, Ma Z, Chen H, Lu Y, Chen X. Valinomycin as a potential antiviral agent against coronaviruses: A review. Biomed J. 2020 Oct;43(5):414-423. doi: 10.1016/j.bj.2020.08.006. Epub 2020 Aug 11. PMID: 33012699; PMCID: PMC7417921.

1528. De Virgiliis F, Di Giovanni S. Lung innervation in the eye of a cytokine storm: neuroimmune interactions and COVID-19. Nat Rev Neurol. 2020 Nov;16(11):645-652. doi: 10.1038/s41582-020-0402-y. Epub 2020 Aug 25. PMID: 32843733; PMCID: PMC7446605

1529. Hernandez ML, Lay JC, Harris B, Esther CR Jr, Brickey WJ, Bromberg PA, Diaz-Sanchez D, Devlin RB, Kleeberger SR, Alexis NE, Peden DB. Atopic asthmatic subjects but not atopic subjects without asthma have enhanced inflammatory response to ozone. J Allergy Clin Immunol. 2010 Sep;126(3):537-44.e1. doi: 10.1016/j.jaci.2010.06.043. PMID: 20816188; PMCID: PMC2949284. 
1530. Bauer, Rebecca N et al. "Effects of air pollutants on innate immunity: the role of Toll-like receptors and nucleotide-binding oligomerization domain-like receptors." The Journal of allergy and clinical immunology vol. 129,1 (2012): 14-24; quiz 25-6. doi:10.1016/j.jaci.2011.11.004

1531. Shoenfelt J, Mitkus RJ, Zeisler R, Spatz RO, Powell J, Fenton MJ, Squibb KA, Medvedev AE. Involvement of TLR2 and TLR4 in inflammatory immune responses induced by fine and coarse ambient air particulate matter. J Leukoc Biol. 2009 Aug;86(2):303-12. doi: 10.1189/jlb.1008587. PMID: 19406832; PMCID: PMC2726765.

1532. Ciencewicki J, Brighton L, Wu WD, Madden M, Jaspers I. Diesel exhaust enhances virus- and poly(I:C)-induced Toll-like receptor 3 expression and signaling in respiratory epithelial cells. Am J Physiol Lung Cell Mol Physiol. 2006 Jun;290(6):L1154-63. doi: 10.1152/ajplung.00318.2005. Epub 2006 Jan 6. PMID: 16399790.

1533. Mortaz E, Adcock IM, Ito K, Kraneveld AD, Nijkamp FP, Folkerts G. Cigarette smoke induces CXCL8 production by human neutrophils via activation of TLR9 receptor. Eur Respir J. 2010 Nov;36(5):1143-54. doi: 10.1183/09031936.00062209. Epub 2009 Oct 19. PMID: 19840968.

1534. Doz E, Noulin N, Boichot E, Guénon I, Fick L, Le Bert M, Lagente V, Ryffel B, Schnyder B, Quesniaux VF, Couillin I. Cigarette smoke-induced pulmonary inflammation is TLR4/MyD88 and IL-1R1/MyD88 signaling dependent. J Immunol. 2008 Jan 15;180(2):1169-78. doi: 10.4049/jimmunol.180.2.1169. PMID: 18178857.

1535. Chaudhuri N, Paiva C, Donaldson K, Duffin R, Parker LC, Sabroe I. Diesel exhaust particles override natural injury-limiting pathways in the lung. Am J Physiol Lung Cell Mol Physiol. 2010 Aug;299(2):L263-71. doi: 10.1152/ajplung.00297.2009. Epub 2010 Apr 30. PMID: 20435687; PMCID: PMC2928610.

1536. MacRedmond, R.E., Greene, C.M., Dorscheid, D.R. et al. Epithelial expression of TLR4 is modulated in COPD and by steroids, salmeterol and cigarette smoke. Respir Res 8, 84 (2007). https://doi.org/10.1186/1465-9921-8-84

1537. Suzuki M, Hisamatsu T, Podolsky DK. Gamma interferon augments the intracellular pathway for lipopolysaccharide (LPS) recognition in human intestinal epithelial cells through coordinated up-regulation of LPS uptake and expression of the intracellular Toll-like receptor 4-MD-2 complex. Infect Immun. 2003 Jun;71(6):3503-11. doi: 10.1128/IAI.71.6.3503-3511.2003. PMID: 12761135; PMCID: PMC155722.

1538. Suzuki M, Hisamatsu T, Podolsky DK. Gamma interferon augments the intracellular pathway for lipopolysaccharide (LPS) recognition in human intestinal epithelial cells through coordinated up-regulation of LPS uptake and expression of the intracellular Toll-like receptor 4-MD-2 complex. Infect Immun. 2003 Jun;71(6):3503-11. doi: 10.1128/IAI.71.6.3503-3511.2003. PMID: 12761135; PMCID: PMC155722.

1539. Van den Bosch JM, Westermann CJ, Aumann J, Edsbäcker S, Tönnesson M, Selroos O. Relationship between lung tissue and blood plasma concentrations of inhaled budesonide. Biopharm Drug Dispos. 1993 Jul;14(5):455-9. doi: 10.1002/bdd.2510140511. PMID: 8218963.

1540. Van den Bosch JM, Westermann CJ, Aumann J, Edsbäcker S, Tönnesson M, Selroos O. Relationship between lung tissue and blood plasma concentrations of inhaled budesonide. Biopharm Drug Dispos. 1993 Jul;14(5):455-9. doi: 10.1002/bdd.2510140511. PMID: 8218963.

1541. Nouri-Shirazi M, Tamjidi S, Nourishirazi E, Guinet E. Combination of TLR8 and TLR4 agonists reduces the degrading effects of nicotine on DC-NK mediated effector T cell generation. Int Immunopharmacol. 2018 Aug;61:54-63. doi:

10.1016/j.intimp.2018.05.012. Epub 2018 May 24. PMID: 29803914; PMCID: PMC6050097.

1542. Strzelak, Agnieszka et al. "Tobacco Smoke Induces and Alters Immune Responses in the Lung Triggering Inflammation, Allergy, Asthma and Other Lung Diseases: A Mechanistic Review." International journal of environmental research and public health vol. 15,5 1033. 21 May. 2018, doi:10.3390/ijerph15051033

1543. Semlali A, Witoled C, Alanazi M, Rouabhia M (2012) Whole Cigarette Smoke Increased the Expression of TLRs, HBDs, and Proinflammory Cytokines by Human Gingival Epithelial Cells through Different Signaling Pathways. PLOS ONE 7(12): e52614. https://doi.org/10.1371/journal.pone.0052614

1544. Yin, Y., Hou, G., Li, E. et al. PPAR Gamma agonists regulate tobacco smoke-induced toll like receptor 4 expression in alveolar macrophages. Respir Res 15, 28 (2014). https://doi.org/10.1186/1465-9921-15-28

1545. Keatings VM, Collins PD, Scott DM, Barnes PJ. Differences in interleukin-8 and tumor necrosis factor-alpha in induced sputum from patients with chronic obstructive pulmonary disease or asthma. Am J Respir Crit Care Med. 1996 Feb;153(2):530-4 doi: 10.1164/ajrccm.153.2.8564092. PMID: 8564092.

1546. Hill AT, Bayley D, Stockley RA. The interrelationship of sputum inflammatory markers in patients with chronic bronchitis. Am J Respir Crit Care Med. 1999 Sep;160(3):893-8. doi: 10.1164/ajrccm.160.3.9901091. PMID: 10471615.

1547. Di Stefano A, Capelli A, Lusuardi M, Balbo P, Vecchio C, Maestrelli P, Mapp CE, Fabbri LM, Donner CF, Saetta M. Severity of airflow limitation is associated with severity of airway inflammation in smokers. Am J Respir Crit Care Med. 1998 Oct;158(4):1277-85. doi: 10.1164/ajrccm.158.4.9802078. PMID: 9769292.

1548. Pace E, Giarratano A, Ferraro M, Bruno A, Siena L, Mangione S, Johnson M, Gjomarkaj M. TLR4 upregulation underpins airway neutrophilia in smokers with chronic obstructive pulmonary disease and acute respiratory failure. Hum Immunol. 2011 Jan;72(1):54-62. doi: 10.1016/j.humimm.2010.09.009. Epub 2010 Oct 1. PMID: 20888880.

1549. Givi ME, Folkerts G, Wagenaar GT, Redegeld FA, Mortaz E. Cigarette smoke differentially modulates dendritic cell maturation and function in time. Respir Res. 2015 Oct 24;16:131. doi: 10.1186/s12931-015-0291-6. PMID: 26498483; PMCID: PMC4619524.

1550. Robbins CS, Franco F, Mouded M, Cernadas M, Shapiro SD. Cigarette smoke exposure impairs dendritic cell maturation and T cell proliferation in thoracic lymph nodes of mice. J Immunol. 2008 May 15;180(10):6623-8. doi: 10.4049/jimmunol.180.10.6623. PMID: 18453581; PMCID: PMC2885874.

1551. Kandasamy M, Ying PC, Ho AW, Sumatoh HR, Schlitzer A, Hughes TR, Kemeny DM, Morgan BP, Ginhoux F, Sivasankar B. Complement mediated signaling on pulmonary $\mathrm{CD} 103(+)$ dendritic cells is critical for their migratory function in response to influenza infection. PLoS Pathog. 2013 Jan;9(1):e1003115. doi: 10.1371/journal.ppat.1003115. Epub 2013 Jan 10. PMID: 23326231; PMCID: PMC3542115.

1552. Aicher A, Heeschen C, Mohaupt M, Cooke JP, Zeiher AM, Dimmeler S. Nicotine strongly activates dendritic cell-mediated adaptive immunity: potential role for progression of atherosclerotic lesions. Circulation. 2003 Feb 4;107(4):604-11. doi: 10.1161/01.cir.0000047279.42427.6d. PMID: 12566374.

1553. Vassallo R, Tamada K, Lau JS, Kroening PR, Chen L. Cigarette smoke extract suppresses human dendritic cell function leading to preferential induction of Th-2 priming. J Immunol. 2005 Aug 15;175(4):2684-91. doi: 10.4049/jimmunol.175.4.2684. PMID: 16081845.

1554. Kroening PR, Barnes TW, Pease L, Limper A, Kita H, Vassallo R. Cigarette smoke-induced oxidative stress suppresses generation of dendritic cell IL-12 and IL-23 through ERK-dependent pathways. J Immunol. 2008 Jul 15;181(2):1536-47. doi: 10.4049/jimmunol.181.2.1536. PMID: 18606709; PMCID: PMC2819390. 
1555. Vassallo R, Kroening PR, Parambil J, Kita H. Nicotine and oxidative cigarette smoke constituents induce immune-modulatory and pro-inflammatory dendritic cell responses. Mol Immunol. 2008 Jul;45(12):3321-9. doi: 10.1016/j.molimm.2008.04.014. Epub 2008 Jun 3. PMID: 18533267; PMCID: PMC2857673.

1556. Nouri-Shirazi $M$, Guinet E. Evidence for the immunosuppressive role of nicotine on human dendritic cell functions. Immunology. 2003 Jul;109(3):365-73. doi: 10.1046/j.1365-2567.2003.01655.x. PMID: 12807482; PMCID: PMC1782971. 1557. Nouri-Shirazi M, Tinajero R, Guinet E. Nicotine alters the biological activities of developing mouse bone marrow-derived dendritic cells (DCs). Immunol Lett. 2007 Apr 15;109(2):155-64. doi: 10.1016/j.imlet.2007.02.005. Epub 2007 Mar 9. PMID: 17368810.

1558. Feldman C, Anderson R. Cigarette smoking and mechanisms of susceptibility to infections of the respiratory tract and other organ systems. J Infect. 2013 Sep;67(3):169-84. doi: 10.1016/j.jinf.2013.05.004. Epub 2013 May 21. PMID: 23707875.

1559. Givi ME, Peck MJ, Boon L, Mortaz E. The role of dendritic cells in the pathogenesis of cigarette smoke-induced emphysema in mice. Eur J Pharmacol. 2013 Dec 5;721(1-3):259-66. doi: 10.1016/j.ejphar.2013.09.027. Epub 2013 Oct 9. PMID: 24120403. 1560. Liao SX, Ding T, Rao XM, Sun DS, Sun PP, Wang YJ, Fu DD, Liu XL, Ou-Yang Y. Cigarette smoke affects dendritic cell maturation in the small airways of patients with chronic obstructive pulmonary disease. Mol Med Rep. 2015 Jan;11(1):219-25. doi: 10.3892/mmr.2014.2759. Epub 2014 Oct 23. PMID: 25338516; PMCID: PMC4237095.

1561. Stoll P, Heinz AS, Bratke K, Bier A, Garbe K, Kuepper M, Virchow JC, Lommatzsch M. Impact of smoking on dendritic cell phenotypes in the airway lumen of patients with COPD. Respir Res. 2014 Apr 18;15(1):48. doi: 10.1186/1465-9921-15-48. PMID: 24742278; PMCID: PMC4021430.

1562. Zanini A, Spanevello A, Baraldo S, Majori M, Della Patrona S, Gumiero F, Aiello M, Olivieri D, Saetta M, Chetta A. Decreased maturation of dendritic cells in the central airways of COPD patients is associated with VEGF, TGF- $\beta$ and vascularity. Respiration. 2014;87(3):234-42. doi: 10.1159/000356749. Epub 2014 Jan 14. PMID: 24435103.

1563. Rogers AV, Adelroth E, Hattotuwa K, Dewar A, Jeffery PK. Bronchial mucosal dendritic cells in smokers and ex-smokers with COPD: an electron microscopic study. Thorax. 2008 Feb;63(2):108-14. doi: 10.1136/thx.2007.078253. Epub 2007 Sep 17. PMID: 17875567.

1564. Sopori ML, Kozak W, Savage SM, Geng Y, Soszynski D, Kluger MJ, Perryman EK, Snow GE. Effect of nicotine on the immune system: possible regulation of immune responses by central and peripheral mechanisms. Psychoneuroendocrinology. 1998 Feb;23(2):189-204. doi: 10.1016/s0306-4530(97)00076-0. PMID: 9621398.

1565. Feng $Y$, Kong $Y$, Barnes PF, Huang FF, Klucar P, Wang X, Samten B, Sengupta M, Machona B, Donis R, Tvinnereim AR, Shams $\mathrm{H}$. Exposure to cigarette smoke inhibits the pulmonary T-cell response to influenza virus and Mycobacterium tuberculosis. Infect Immun. 2011 Jan;79(1):229-37. doi: 10.1128/IAI.00709-10. Epub 2010 Oct 25. PMID: 20974820; PMCID: PMC3019896. 1566. Magkouta, Panagiotis Chouvardas, Vassiliki Karavana, Konstantinos Glynos, et.al. Cigarette Smoke-Induced Emphysema Exhausts Early Cytotoxic CD8+ T Cell Responses against Nascent Lung Cancer Cells Dimitra Kerdidani, Sophia The Journal of Immunology July 23, 2018, ji1700700; DOI: 10.4049/jimmunol.1700700

1567. Goud, S.N., Kaplan, A.M. \& Subbarao, B. Effects of cigarette smoke on the antibody responses to thymic independent antigens from different lymphoid tissues of mice. Arch Toxicol 66, 164-169 (1992). https://doi.org/10.1007/BF01974009 1568. Intlekofer AM, Takemoto N, Wherry EJ, Longworth SA, Northrup JT, Palanivel VR, Mullen AC, Gasink CR, Kaech SM, Miller JD, Gapin L, Ryan K, Russ AP, Lindsten T, Orange JS, Goldrath AW, Ahmed R, Reiner SL. Effector and memory CD8+ T cell fate coupled by T-bet and eomesodermin. Nat Immunol. 2005 Dec;6(12):1236-44. doi: 10.1038/ni1268. Epub 2005 Nov 6. Erratum in: Nat Immunol. 2006 Jan;7(1):113. PMID: 16273099.

1569. Guérit D, Brondello JM, Chuchana P, Philipot D, Toupet K, Bony C, Jorgensen C, Noël D. FOXO3A regulation by miRNA-29a Controls chondrogenic differentiation of mesenchymal stem cells and cartilage formation. Stem Cells Dev. 2014 Jun 1;23(11):1195-205. doi: 10.1089/scd.2013.0463. Epub 2014 Mar 21. PMID: 24467486.

1570. Cushing L, Costinean S, Xu W, Jiang Z, Madden L, et al. (2015) Disruption of miR-29 Leads to Aberrant Differentiation of Smooth Muscle Cells Selectively Associated with Distal Lung Vasculature. PLOS Genetics 11(5): e1005238. https://doi.org/10.1371/journal.pgen.1005238.

1571. Ohkura N, Sakaguchi S. Foxo1 and Foxo3 help Foxp3. Immunity. 2010 Dec 14;33(6):835-7. doi: 10.1016/j.immuni.2010.12.004. PMID: 21168774.

1572. Kerdiles YM, Stone EL, Beisner DR, McGargill MA, Ch'en IL, Stockmann C, Katayama CD, Hedrick SM. Foxo transcription factors control regulatory T cell development and function. Immunity. 2010 Dec 14;33(6):890-904. doi:

10.1016/j.immuni.2010.12.002. Erratum in: Immunity. 2011 Jan 28;34(1):135. Beisner, Daniel L [corrected to Beisner, Daniel R]. PMID: 21167754; PMCID: PMC3034255.

1573. Ambrogini E, Almeida M, Martin-Millan M, Paik JH, Depinho RA, Han L, Goellner J, Weinstein RS, Jilka RL, O'Brien CA, Manolagas SC. FoxO-mediated defense against oxidative stress in osteoblasts is indispensable for skeletal homeostasis in mice. Cell Metab. 2010 Feb 3;11(2):136-46. doi: 10.1016/j.cmet.2009.12.009. PMID: 20142101; PMCID: PMC2819984.

1574. Zhu, Wan Long \& Honglian, Tong \& Teh, Jing \& Wang, Mei. (2014). Forkhead Box Protein O3 Transcription Factor Negatively Regulates Autophagy in Human Cancer Cells by Inhibiting Forkhead Box Protein O1 Expression and Cytosolic Accumulation. PloS one. 9. e115087. 10.1371/journal.pone.0115087.

1575. Ouyang W, Beckett O, Ma Q, Paik JH, DePinho RA, Li MO. Foxo proteins cooperatively control the differentiation of Foxp3+ regulatory T cells. Nat Immunol. 2010 Jul;11(7):618-27. doi: 10.1038/ni.1884. Epub 2010 May 13. PMID: 20467422.

1576. Huang, Junxia et al. "miRNAs Alter T Helper 17 Cell Fate in the Pathogenesis of Autoimmune Diseases." Frontiers in immunology vol. 12 593473. 21 Apr. 2021, doi:10.3389/fimmu.2021.593473

1577. Wang, Qiuyuan \& Liu, Yang \& Wu, Yiru \& Wen, Jie \& Man, Chaolai. (2021). Immune function of miR-214 and its application prospects as molecular marker. PeerJ. 9. e10924. 10.7717/peerj.10924.

1578. Namdari, Haideh \& Ghayedi, Mojgan \& Hadjati, Jamshid \& Rezaei, Farhad \& Kalantar, Kurosh \& Rahimzadeh, Parisa \&

Salehi, Eisa. (2017). Effect of MicroRNA-21 Transfection on In-vitro Differentiation of Human Naive CD4+ T Cells to Regulatory T Cells. Iranian Journal of Allergy, Asthma and Immunology. 16. 235-244.

1579. Ragni, Enrico \& Colombini, Alessandra \& Viganò, Marco \& Libonati, Francesca \& Perucca Orfei, Carlotta \& Zagra, Luigi \& de Girolamo, Laura. (2021). Cartilage Protective and Immunomodulatory Features of Osteoarthritis Synovial Fluid-Treated AdiposeDerived Mesenchymal Stem Cells Secreted Factors and Extracellular Vesicles-Embedded miRNAs. Cells. 10.

$10.3390 /$ cells10051072.

1580. Qi Y, Wu H, Mai C, Lin H, Shen J, Zhang X, Gao Y, Mao Y, Xie X. LncRNA-MIAT-Mediated miR-214-3p Silencing Is Responsible for IL-17 Production and Cardiac Fibrosis in Diabetic Cardiomyopathy. Front Cell Dev Biol. 2020 Apr 15;8:243. doi: 10.3389/fcell.2020.00243. PMID: 32351959; PMCID: PMC7174588.

1581. Wang S, Xu J, Guo Y, Cai Y, Ren X, Zhu W, Geng M, Meng L, Jiang C, Lu S. MicroRNA-497 Reduction and Increase of Its Family Member MicroRNA-424 Lead to Dysregulation of Multiple Inflammation Related Genes in Synovial Fibroblasts With Rheumatoid Arthritis. Front Immunol. 2021 Mar 26;12:619392. doi: 10.3389/fimmu.2021.619392. PMID: 33841401; PMCID: PMC8034293. 
1582. Sullivan, Jeremy A et al. "FOXO3 regulates the CD8 T cell response to a chronic viral infection." Journal of virology vol. 86,17 (2012): 9025-34. doi:10.1128/JVI.00942-12

1583. Dingsdag, S.A., Clay, O.K. and Quintero, G.A. (2021), COVID-19 severity, miR-21 targets, and common human genetic variation. Letter regarding the article 'Circulating cardiovascular microRNAs in critically ill COVID-19 patients'. Eur J Heart Fail. https://doi.org/10.1002/ejhf.2317

1584. Keikha, Reza et al. "The relative expression of miR-31, miR-29, miR-126, and miR-17 and their mRNA targets in the serum of COVID-19 patients with different grades during hospitalization." European journal of medical research vol. 26,1 75. 13 Jul. 2021, doi:10.1186/s40001-021-00544-4

1585. Centa A, Fonseca AS, Ferreira SGDS, Azevedo MLV, Vaz de Paula CB, Nagashima S, Machado-Souza C, Miggiolaro AFRDS, Baena CP, de Noronha L, Cavalli LR. Deregulated miRNA expression is associated with endothelial dysfunction in post-mortem lung biopsies of COVID-19 patients. Am J Physiol Lung Cell Mol Physiol. 2020 Dec 2;320(3):L405-12. doi: 10.1152/ajplung.00457.2020. Epub ahead of print. PMID: 33651636; PMCID: PMC7938642.

1586. Ma F, Xu S, Liu X, Zhang Q, Xu X, Liu M, Hua M, Li N, Yao H, Cao X. The microRNA miR-29 controls innate and adaptive immune responses to intracellular bacterial infection by targeting interferonc. Nat Immunol 12: 861-869, 2011. doi:10.1038/ni.2073.

1587. $\mathrm{H}$, Bhandari V. MicroRNA-34a promotes endothelial dysfunction and mitochondrial-mediated apoptosis in murine models of acute lung injury. Am J Respir Cell Mol Biol 60: 465-477, 2019. doi:10.1165/ rcmb.2018-01940C.

1588. Bartoszewski R, Dabrowski M, Jakiela B, Matalon S, Harrod KS, Sanak M, Collawn JF. SARS-CoV-2 may regulate cellular responses through depletion of specific host miRNAs. Am J Physiol Lung Cell Mol Physiol. 2020 Sep 1;319(3):L444-L455. doi: 10.1152/ajplung.00252.2020. Epub 2020 Aug 5. PMID: 32755307 ; PMCID: PMC7473886.

1589. Nagashima S, Mendes MC, Camargo Martins AP, Borges NH, Godoy TM, Miggiolaro AFRDS, da Silva Dezidério F, MachadoSouza C, de Noronha L. Endothelial Dysfunction and Thrombosis in Patients With COVID-19-Brief Report. Arterioscler Thromb Vasc Biol. 2020 Oct;40(10):2404-2407. doi: 10.1161/ATVBAHA.120.314860. Epub 2020 Aug 7. PMID: 32762443; PMCID: PMC7505138.

1590. Azevedo, Marina \& Vaz de Paula, Caroline \& Nagashima, Seigo \& Souza, Cleber \& Miggiolaro, Anna \& Junior, Jarbas \& Malaquias, Mineia \& Raboni, Sonia \& Neto, Plínio \& Souza, Daiane \& Baena, Cristina \& Noronha, Lúcia. (2020). Alveolar Neutrophilic Recruitment in COVID-19 May Not be Mediated by Th17 Response. 10.21203/rs.3.rs-36238/v2.

1591. Vaz de Paula CB, de Azevedo MLV, Nagashima S, Martins APC, Malaquias MAS, Miggiolaro AFRDS, da Silva Motta Júnior J, Avelino G, do Carmo LAP, Carstens LB, de Noronha L. IL-4/IL-13 remodeling pathway of COVID-19 lung injury. Sci Rep. 2020 Oct 29;10(1):18689. doi: 10.1038/s41598-020-75659-5. PMID: 33122784; PMCID: PMC7596721.

1592. Yang X, Liang L, Zhang XF, Jia HL, Qin Y, Zhu XC, Gao XM, Qiao P, Zheng Y, Sheng YY, Wei JW, Zhou HJ, Ren N, Ye QH, Dong QZ, Qin LX. MicroRNA-26a suppresses tumor growth and metastasis of human hepatocellular carcinoma by targeting interleukin6-Stat3 pathway. Hepatology 58: 158-170, 2013. doi:10.1002/hep.26305

1593. Fang J, Hao Q, Liu L, Li Y, Wu J, Huo X, Zhu Y. Epigenetic changes mediated by microRNA miR29 activate cyclooxygenase 2 and lambda-1 interferon production during viral infection. J Virol. 2012 Jan;86(2):1010-20. doi: 10.1128/JVI.06169-11. Epub 2011 Nov 9. PMID: 22072783; PMCID: PMC3255816.

1594. Liu L, Ren W, Chen K. MiR-34a Promotes Apoptosis and Inhibits Autophagy by Targeting HMGB1 in Acute Myeloid Leukemia Cells. Cell Physiol Biochem. 2017;41(5):1981-1992. doi: 10.1159/000475277. Epub 2017 Apr 13. PMID: 28478444.

1595. Bartoszewska S, Collawn JF. Unfolded protein response (UPR) integrated signaling networks determine cell fate during hypoxia. Cell Mol, Biol Lett 25: 18, 2020. doi:10.1186/s11658-020-00212-1.

1596. Bartoszewski R, Gebert M, Janaszak-Jasiecka A, Cabaj A, Królic- zewski J, Bartoszewska S, Sobolewska A, Crossman DK, Ochocka R, Kamysz W, Kalinowski L, Da browski M, Collawn JF. Genome-wide mRNA profiling identifies RCAN1 and GADD45A as regulators of the transitional switch from survival to apoptosis during ER stress. FEBS J 287: 2923-2947, 2020. doi:10.1111/febs.15195

1597. Hetz C. The unfolded protein response: controlling cell fate decisions under ER stress and beyond. Nat Rev Mol Cell Biol 13: 89-102, 2012. doi:10.1038/nrm3270.

1598. Kim R, Emi M, Tanabe K, Murakami S. Role of the unfolded protein response in cell death. Apoptosis 11: 5-13, 2006. doi:10.1007/s10495- 005-3088-0

1599. Walter $P$, Ron D. The unfolded protein response: from stress pathway to homeostatic regulation. Science 334: 1081-1086, 2011. doi:10.1126/ science.1209038.

1600. Rashid, Farooq et al. "The ORF8 protein of SARS-CoV-2 induced endoplasmic reticulum stress and mediated immune evasion by antagonizing production of interferon beta." Virus research vol. 296 (2021): 198350.

doi:10.1016/j.virusres.2021.198350

1601. Livia Rosa-Fernandes, Lucas C. Lazari, Janaina Macedo da Silva et.al SARS-CoV-2 activates ER stress and Unfolded protein response, bioRxiv 2021.06.21.449284; doi: https://doi.org/10.1101/2021.06.21.449284

1602. B Balakrishnan, K Lai, Modulation of SARS-CoV-2 Spike-induced Unfolded Protein Response (UPR) in HEK293T cells by selected small chemical molecules, bioRxiv 2021.02.04.429769; doi: https://doi.org/10.1101/2021.02.04.429769 1603. Fung TS, Liu DX. Coronavirus infection, ER stress, apoptosis and innate immunity. Front Microbiol 5: $296,2014$. doi:10.3389/fmicb.2014.00296

1604. David-Ferreira JF, Manaker RA. An electron microscope study of the development of a mouse hepatitis virus in tissue culture cells. J Cell Biol 24: 57-78, 1965. doi:10.1083/jcb.24.1.57

1605. Knoops K, Kikkert M, Worm SH, Zevenhoven-Dobbe JC, van der Meer Y, Koster AJ, Mommaas AM, Snijder EJ. SARScoronavirus replication is supported by a reticulovesicular network of modified endoplasmic reticulum. PLoS Biol 6: e226, 2008. doi:10.1371/journal. pbio.0060226

1606. Reggiori F, Monastyrska I, Verheije MH, Calì T, Ulasli M, Bianchi S, Bernasconi R, de Haan CAM, Molinari M. Coronaviruses Hijack the LC3-I-positive EDEMosomes, ER-derived vesicles exporting short- lived ERAD regulators, for replication. Cell Host Microbe 7: 500-508, 2010. doi:10.1016/j.chom.2010.05.013.

1607. Dong, Liyang et al. "Decreased expression of microRNA-21 correlates with the imbalance of Th17 and Treg cells in patients with rheumatoid arthritis." Journal of cellular and molecular medicine vol. 18,11 (2014): 2213-24. doi:10.1111/jcmm.12353 1608. Murugaiyan, Gopal et al. "MicroRNA-21 promotes Th17 differentiation and mediates experimental autoimmune encephalomyelitis." The Journal of clinical investigation vol. 125,3 (2015): 1069-80. doi:10.1172/JCI74347 1609. Lee, Gap Ryol. "The Balance of Th17 versus Treg Cells in Autoimmunity." International journal of molecular sciences vol. 19,3 730. 3 Mar. 2018, doi:10.3390/ijms19030730.

1610. Kimura A, Kishimoto T. IL-6: regulator of Treg/Th17 balance. Eur J Immunol. 2010 Jul;40(7):1830-5. doi: 10.1002/eji.201040391. PMID: 20583029. 
1611. Naoya Hoshikawa, Atsushi Sakai, Shinro Takai, Hidenori Suzuki,Targeting Extracellular miR-21-TLR7 Signaling Provides LongLasting Analgesia in Osteoarthritis,Molecular Therapy - Nucleic Acids, Volume 19,2020,https://doi.org/10.1016/j.omtn.2019.11.011.

1612. Wang S, Wan X, Ruan Q. The MicroRNA-21 in Autoimmune Diseases. International Journal of Molecular Sciences. 2016; 17(6):864. https://doi.org/10.3390/ijms17060864

1613. Zanotti S, Gibertini S, Curcio M, Savadori P, Pasanisi B, Morandi L, Cornelio F, Mantegazza R, Mora M. Opposing roles of miR-21 and miR-29 in the progression of fibrosis in Duchenne muscular dystrophy. Biochim Biophys Acta. 2015 Jul;1852(7):145164. doi: 10.1016/j.bbadis.2015.04.013. Epub 2015 Apr 17. PMID: 25892183.

1614. Kunze-Schumacher H, Winter SJ, Imelmann E, Krueger A. miRNA miR-21 Is Largely Dispensable for Intrathymic T-Cel Development. Front Immunol. 2018 Nov 5;9:2497. doi: 10.3389/fimmu.2018.02497. PMID: 30455689; PMCID: PMC6230590. 1615. Hackett, Emer E, and Frederick J Sheedy. "miR-21 alters circulating Treg function in vascular disease-hope for restoring immunoregulatory responses in atherosclerosis?." Annals of translational medicine vol. 5,1 (2017): 21. doi:10.21037/atm.2016.12.72

1616. Rouas R, Fayyad-Kazan H, El Zein N, et al. Human natural Treg microRNA signature: role of microRNA-31 and microRNA-21 in FOXP3 expression. Eur J Immunol 2009;39:1608-18. 10.1002/eji.200838509

1617. Bhairavabhotla R, Kim YC, Glass DD, et al. Transcriptome profiling of human FoxP3+ regulatory T cells. Hum Immunol 2016;77:201-13. 10.1016/j.humimm.2015.12.004

1618. Li S, Fan Q, He S, et al. MicroRNA-21 negatively regulates Treg cells through a TGF- $\beta 1 /$ Smad-independent pathway in patients with coronary heart disease. Cell Physiol Biochem 2015;37:866-78. 10.1159/000430214

1619. Nersisyan S, Engibaryan N, Gorbonos A, Kirdey K, Makhonin A, Tonevitsky A. Potential role of cellular miRNAs in coronavirus-host interplay. PeerJ. 2020 Sep 14;8:e9994. doi: 10.7717/peerj.9994. PMID: 32983652; PMCID: PMC7497610. 1620. He, F., Xiao, Z., Yao, H. et al. The protective role of microRNA-21 against coxsackievirus B3 infection through targeting the MAP2K3/P38 MAPK signaling pathway. J Transl Med 17, 335 (2019). https://doi.org/10.1186/s12967-019-2077-y

1621. Kim C, Hu B, Jadhav RR, Jin J, Zhang H, Cavanagh MM, Akondy RS, Ahmed R, Weyand CM, Goronzy JJ. Activation of miR-21Regulated Pathways in Immune Aging Selects against Signatures Characteristic of Memory T Cells. Cell Rep. 2018 Nov 20;25(8):2148-2162.e5. doi: 10.1016/j.celrep.2018.10.074. PMID: 30463012; PMCID: PMC6371971.

1622. Pace E, Di Vincenzo S, Di Salvo E, Genovese S, Dino P, Sangiorgi C, Ferraro M, Gangemi S. MiR-21 upregulation increases IL-8 expression and tumorigenesis program in airway epithelial cells exposed to cigarette smoke. J Cell Physiol. 2019 Dec;234(12):22183-22194. doi: 10.1002/jcp.28786. Epub 2019 May 3. PMID: 31054160.

1623. Chen Y, Chen J, Wang H, Shi J, Wu K, et al. (2013) HCV-Induced miR-21 Contributes to Evasion of Host Immune System by Targeting MyD88 and IRAK1. PLOS Pathogens 9(4): e1003248. https://doi.org/10.1371/journal.ppat.1003248

1624. Pandey, N., Rastogi, M. \& Singh, S.K. Chandipura virus dysregulates the expression of hsa-miR-21-5p to activate NF-kB in human microglial cells .J Biomed Sci 28, 52 (2021). https://doi.org/10.1186/s12929-021-00748-0

1625. Jianli Shi, Ping Feng, Tingting Gu; MicroRNA-21-3p modulates FGF2 to facilitate influenza A virus H5N1 replication by refraining type I interferon response. Biosci Rep 29 May 2020; 40 (5): BSR20200158. doi: https://doi.org/10.1042/BSR20200158 1626. Jafarinejad-Farsangi S, Jazi MM, Rostamzadeh F, Hadizadeh M. High affinity of host human microRNAs to SARS-CoV-2 genome: An in silico analysis. Noncoding RNA Res. 2020 Dec;5(4):222-231. doi: 10.1016/j.ncrna.2020.11.005. Epub 2020 Nov 21. PMID: 33251388; PMCID: PMC7680021.

1627. Zhang S, Amahong K, Sun X, Lian X, Liu J, Sun H, Lou Y, Zhu F, Qiu Y. The miRNA: a small but powerful RNA for COVID-19. Brief Bioinform. 2021 Mar 22;22(2):1137-1149. doi: 10.1093/bib/bbab062. PMID: 33675361; PMCID: PMC7989616. 1628. Barwari T, Eminaga S, Mayr U, Lu R, Armstrong PC, Chan MV, Sahraei M, Fernández-Fuertes M, Moreau T, BarallobreBarreiro J, Lynch M, Yin X, Schulte C, Baig F, Pechlaner R, Langley SR, Zampetaki A, Santer P, Weger M, Plasenzotti R, Schosserer M, Grillari J, KiechI S, Willeit J, Shah AM, Ghevaert C, Warner TD, Fernández-Hernando C, Suárez Y, Mayr M. Inhibition of profibrotic microRNA-21 affects platelets and their releasate. JCI Insight. 2018 Nov 2;3(21):e123335. doi: 10.1172/jci.insight.123335. PMID: 30385722; PMCID: PMC6238735.

1629. He G, Ding J, Zhang Y, Cai M, Yang J, Cho WC, Zheng Y. microRNA-21: a key modulator in oncogenic viral infections. RNA Biol. 2021 May;18(5):809-817. doi: 10.1080/15476286.2021.1880756. Epub 2021 Mar 22. PMID: 33499700; PMCID: PMC8078529. 1630. Li L, Zhang J, Diao W, Wang D, Wei Y, Zhang CY, Zen K. MicroRNA-155 and MicroRNA-21 promote the expansion of functional myeloid-derived suppressor cells. J Immunol. 2014 Feb 1;192(3):1034-43. doi: 10.4049/jimmunol.1301309. Epub 2014 Jan 3. PMID: 24391219.

1631. Yao R, Ma YL, Liang W, Li HH, Ma ZJ, et al. (2012) MicroRNA-155 Modulates Treg and Th17 Cells Differentiation and Th17 Cell Function by Targeting SOCS1. PLOS ONE 7(10): e46082. https://doi.org/10.1371/journal.pone.0046082

1632. Zhenke Wen, Lin Xu, Xi Chen, Wei Xu, Zhinan Yin, Xiaoming Gao and Sidong Xiong, Autoantibody Induction by DNAContaining Immune Complexes Requires HMGB1 with the TLR2/MicroRNA-155 Pathway, J Immunol April 24, 2013, 1203301; DOI: https://doi.org/10.4049/jimmunol.1203301

1633. Zhang, Yue \& Xie, Yifang \& Zhang, Leifang \& Zhao, Hang. (2020). MicroRNA-155 Participates in Smoke-Inhalation-Induced Acute Lung Injury through Inhibition of SOCS-1. Molecules. 25. 1022. 10.3390/molecules25051022.

1634. Beer, Lucian \& Kalinina, Polina \& Köcher, Martin \& Laggner, Maria \& Jeitler, Markus \& Abbas Zadeh, Salman \& Copic, Dragan \& Tschachler, Erwin \& Mildner, Michael. (2020). miR-155 Contributes to Normal Keratinocyte Differentiation and Is Upregulated in the Epidermis of Psoriatic Skin Lesions. International Journal of Molecular Sciences. 21. 9288. $10.3390 /$ ijms 21239288.

1635. Sheedy FJ. Turning 21: Induction of miR-21 as a Key Switch in the Inflammatory Response. Front Immunol. 2015 Jan $29 ; 6: 19$. doi: 10.3389/fimmu.2015.00019. PMID: 25688245; PMCID: PMC4310327.

1636. Rizkita, Leonny Dwi, and Indwiani Astuti. "The potential of miRNA-based therapeutics in severe acute respiratory syndrome coronavirus 2 (SARS-CoV-2) infection: A review." Journal of pharmaceutical analysis vol. 11,3 (2021): 265-271. doi:10.1016/j.jpha.2021.03.003

1637. de Gonzalo-Calvo, David et al. "Circulating microRNA profiles predict the severity of COVID-19 in hospitalized patients." Translational research : the journal of laboratory and clinical medicine, vol. 236 147-159. 26 May. 2021, doi:10.1016/j.trsl.2021.05.004

1638. Kaur, Taruneet et al. "Tmprss2 specific miRNAs as promising regulators for SARS-CoV-2 entry checkpoint." Virus research vol. 294 (2021): 198275. doi:10.1016/j.virusres.2020.198275

1639. Liu, Xuming. (2015). Regulation of Cellular MicroRNA Expression in Oligodendrocytes during Acute and Persistent Coronavirus Infection. Virology \& Mycology. 04. 10.4172/2161-0517.1000138.

1640. Pandey G, Cohain A, Miller J, Merad M. Decoding dendritic cell function through module and network analysis. J Immunol Methods. 2013 Jan 31;387(1-2):71-80. doi: 10.1016/j.jim.2012.09.012. Epub 2012 Oct 23. PMID: 23098840. 
1641. Alexander \& Steffen (2014) Alexander M, Steffen J. Development and function of dendritic cell subsets. Immunity. 2014;40(5):642-656. doi: 10.1016/j.immuni.2014.04.016

1642. Svajger \& Rozman (2014) Svajger U, Rozman P. Tolerogenic dendritic cells: molecular and cellular mechanisms in transplantation. Journal of Leukocyte Biology. 2014;95(1):53-69. doi: 10.1189/jlb.0613336.

1643. Huan et al. (2017) Huan Y, He Y, Liu B, Li Y, Jia L, Qu C, Lv B, Zhang X, Peng H. Zhenbao Pill reduces the percentage of Treg cells by inducing HSP27 expression. Biomedicine and Pharmacotherapy. 2017;96:818-824. doi: 10.1016/j.biopha.2017.09.133. 1644. Ilarregui et al. (2009) Ilarregui JM, Croci DO, Bianco GA, Toscano MA, Salatino M, Vermeulen ME, Geffner JR, Rabinovich GA. Tolerogenic signals delivered by dendritic cells to $T$ cells through a galectin-1-driven immunoregulatory circuit involving interleukin 27 and interleukin 10. Nature Immunology. 2009;10(9):981-991. doi: 10.1038/ni.1772.

1645. Ezzelarab \& Thomson (2011) Ezzelarab M, Thomson AW. Tolerogenic dendritic cells and their role in transplantation. Seminars in Immunology. 2011;23(4):252-263. doi: 10.1016/j.smim.2011.06.007.

1646. Li \& Shi (2015) Li H, Shi B. Tolerogenic dendritic cells and their applications in transplantation. Cellular \& Molecular Immunology. 2015;12(1):24-30. doi: 10.1038/cmi.2014.52.

1647. Brucklacher-Waldert et al. (2009) Brucklacher-Waldert V, Stuerner K, Kolster M, Wolthausen J, Tolosa E. Phenotypical and functional characterization of T helper 17 cells in multiple sclerosis. Brain. 2009;32(Pt 12):3329-3341. doi: 10.1093/brain/awp289. 1648. Ahmadian-Elmi et al. (2016) Ahmadian-Elmi M, Bidmeshki Pour A, Naghavian R, Ghaedi K, Tanhaei S, Izadi T, Nasr-Esfahani MH. miR-27a and miR-214 exert opposite regulatory roles in Th17 differentiation via mediating different signaling pathways in peripheral blood CD4(+) T lymphocytes of patients with relapsing-remitting multiple sclerosis. Immunogenetics. 2016;68(1):43-54. doi: 10.1007/s00251-015-0881-y

1649. Ying, H., Ebrahimi, M., Keivan, M., Khoshnam, S. E., Salahi, S., \& Farzaneh, M. (2021). miRNAs; a novel strategy for the treatment of COVID-19. Cell Biology International, 1- 9. https://doi.org/10.1002/cbin.11653

1650. Farr, Ryan \& Rootes, Christina \& Rowntree, Louise \& Nguyen, Thi \& Hensen, Luca \& Kedzierski, Lukasz \& Cheng, Allen \& Kedzierska, Katherine \& Au, Gough \& Marsh, Glenn \& Vasan, Seshadri \& Foo, Chwan \& Cowled, Christopher \& Stewart, Cameron. (2021). Altered microRNA expression in COVID-19 patients enables identification of SARS-CoV-2 infection. 10.21203/rs.3.rs253459/v1.

1651. Grehl C, Schultheiß C, Hoffmann K, Binder M, Altmann T, Grosse I, Kuhlmann M. Detection of SARS-CoV-2 Derived Small RNAs and Changes in Circulating Small RNAs Associated with COVID-19. Viruses. 2021; 13(8):1593.

https://doi.org/10.3390/v13081593

1652. Luo Y, Wang H. Effects of Non-Coding RNA on Regulatory T Cells and Implications for Treatment of Immunological Diseases. Front Immunol. 2020 Nov 20;11:612060. doi: 10.3389/fimmu.2020.612060. PMID: 33329608; PMCID: PMC7714939.

1653. Fontenot JD, Gavin MA, Rudensky AY. Foxp3 programs the development and function of CD4+CD25+ regulatory T cells. Nat Immunol (2003) 4(4):330-6. 10.1038/ni904'

1654. Egarnes, Benoit, and Jean Gosselin. "Contribution of Regulatory T Cells in Nucleotide-Binding Oligomerization Domain 2 Response to Influenza Virus Infection." Frontiers in immunology vol. 9 132. 31 Jan. 2018, doi:10.3389/fimmu.2018.00132 1655. He, Q., Ye, A., Ye, W. et al. Cancer-secreted exosomal miR-21-5p induces angiogenesis and vascular permeability by targeting KRIT1. Cell Death Dis 12, 576 (2021). https://doi.org/10.1038/s41419-021-03803-8

1656. Rahman MK, Midtling EH, Svingen PA, Xiong Y, Bell MP, Tung J, et al. The pathogen recognition receptor NOD2 regulates human FOXP3+ T cell survival. J Immunol (2010) 184(12):7247-56.10.4049/jimmunol.0901479

1657. Lupfer C, Thomas PG, Anand PK, Vogel P, Milasta S, Martinez J, et al. Receptor interacting protein kinase 2-mediated mitophagy regulates inflammasome activation during virus infection. Nat Immunol (2013) 14(5):480-8.10.1038/ni.2563 1658. Sabbah A, Chang TH, Harnack R, Frohlich V, Tominaga K, Dube PH, et al. Activation of innate immune antiviral responses by Nod2. Nat Immunol (2009) 10(10):1073-80.10.1038/ni.1782

1659. Eisenstein EM, Williams CB. The T(reg)/Th17 cell balance: a new paradigm for autoimmunity. Pediatr Res (2009) 65(5 Pt 2):26R-31R.10.1203/PDR.0b013e31819e76c7

1660. Jamshidian A, Shaygannejad V, Pourazar A, Zarkesh-Esfahani SH, Gharagozloo M. Biased Treg/Th17 balance away from regulatory toward inflammatory phenotype in relapsed multiple sclerosis and its correlation with severity of symptoms. J Neuroimmunol (2013) 262(1-2):106-12.10.1016/j.jneuroim.2013.06.007

1661. Yang X, Li J, Liu J, Gao M, Zhou L, Lu W. Relationship of Treg/Th17 balance with HBeAg change in HBeAg-positive chronic hepatitis $B$ patients receiving telbivudine antiviral treatment: a longitudinal observational study. Medicine (Baltimore) (2017) 96(23):e7064.10.1097/MD.0000000000007064

1662. Newcomb DC, Peebles RS, Jr. Th17-mediated inflammation in asthma. Curr Opin Immunol (2013) 25(6):75560.10.1016/j.coi.2013.08.002

1663. Guglani L, Khader SA. Th17 cytokines in mucosal immunity and inflammation. Curr Opin HIV AIDS (2010)

1664. Perrone LA, Plowden JK, Garcia-Sastre A, Katz JM, Tumpey TM. H5N1 and 1918 pandemic influenza virus infection results in early and excessive infiltration of macrophages and neutrophils in the lungs of mice. PLoS Pathog (2008)

4(8):e1000115.10.1371/journal.ppat.1000115

1665. Sakai S, Kawamata H, Mantani N, Kogure T, Shimada Y, Terasawa K, et al. Therapeutic effect of anti-macrophage inflammatory protein 2 antibody on influenza virus-induced pneumonia in mice. J Virol (2000) 74(5):24726.10.1128/JVI.74.5.2472-2476.2000

1666. Tumpey TM, Garcia-Sastre A, Taubenberger JK, Palese P, Swayne DE, Pantin-Jackwood MJ, et al. Pathogenicity of influenza viruses with genes from the 1918 pandemic virus: functional roles of alveolar macrophages and neutrophils in limiting virus replication and mortality in mice. J Virol (2005) 79(23):14933-44.10.1128/JVI.79.23.14933-14944.2005

1667. Oliphant S, Lines JL, Hollifield ML, Garvy BA. Regulatory T cells are critical for clearing influenza A virus in neonatal mice. Viral Immunol (2015) 28(10):580-9.10.1089/vim.2015.0039

1668. Moser EK, Hufford MM, Braciale TJ. Late engagement of CD86 after influenza virus clearance promotes recovery in a FoxP3+ regulatory T cell dependent manner. PLoS Pathog (2014) 10(8):e1004315.10.1371/journal.ppat.1004315 1669. Aldhous MC, Soo K, Stark LA, Ulanicka AA, Easterbrook JE, Dunlop MG, Satsangi J. Cigarette smoke extract (CSE) delays NOD2 expression and affects NOD2/RIPK2 interactions in intestinal epithelial cells. PLoS One. 2011;6(9):e24715. doi: 10.1371/journal.pone.0024715. Epub 2011 Sep 12. PMID: 21931826; PMCID: PMC3171477.

1670. Qian YJ, Wang X, Gao YF, Duan N, Huang XF, Sun FF, Han XD, Wang WM. Cigarette Smoke Modulates NOD1 Signal Pathway and Human $\beta$ Defensins Expression in Human Oral Mucosa. Cell Physiol Biochem. 2015;36(2):457-73. doi: 10.1159/000430112. Epub 2015 May 11. PMID: 25968832.

1671. Till A, Rosenstiel P, Brautigam K, et al. A role for membrane-bound CD147 in NOD2-mediated recognition of bacterial cytoinvasion. J Cell Sci. 2008;121(Pt 4):487-495.

1672. Zhou S, Zhou H, Walian PJ, Jap BK. CD147 is a regulatory subunit of the gamma-secretase complex in Alzheimer's disease amyloid beta-peptide production. Proc Natl Acad Sci USA. 2005;102(21):7499-7504. 
1673. Yao H, Teng $Y$, Sun $Q$, et al. Important functional roles of basigin in thymocyte development and T cell activation. Int J Biol Sci. 2013;10(1):43-52.

1674. Ruiz S, Castro-Castro A, Bustelo XR. CD147 inhibits the nuclear factor of activated T-cells by impairing Vav1 and Rac1 downstream signaling. J Biol Chem. 2008;283(9):5554-5566.

1675. Muller MR, Rao A. NFAT, immunity and cancer: a transcription factor comes of age. Nat Rev Immunol. 2010;10(9):645-656 1676. Macian F. NFAT proteins: key regulators of T-cell development and function. Nat Rev Immunol. 2005;5(6):472-484. 1677. Bao W, Min D, Twigg SM, et al. Monocyte CD147 is induced by advanced glycation end products and high glucose concentration: possible role in diabetic complications. Am J Physiol Cell Physiol. 2010;299(5):C1212-1219.

1678. Radzikowska U, Ding M, Tan G, Zhakparov D, Peng Y, Wawrzyniak P, Wang M, Li S, Morita H, Altunbulakli C, Reiger M, Neumann AU, Lunjani N, Traidl-Hoffmann C, Nadeau KC, O'Mahony L, Akdis C, Sokolowska M. Distribution of ACE2, CD147, CD26, and other SARS-CoV-2 associated molecules in tissues and immune cells in health and in asthma, COPD, obesity, hypertension, and COVID-19 risk factors. Allergy. 2020 Nov;75(11):2829-2845. doi: 10.1111/all.14429. Epub 2020 Aug 24. PMID: 32496587; PMCID: PMC7300910.

1679. Pierdomenico M, Cesi V, Cucchiara S, Vitali R, Prete E, Costanzo M, Aloi M, Oliva S, Stronati L. NOD2 Is Regulated By Mir320 in Physiological Conditions but this Control Is Altered in Inflamed Tissues of Patients with Inflammatory Bowel Disease. Inflamm Bowel Dis. 2016 Feb;22(2):315-26. doi: 10.1097/MIB.0000000000000659. PMID: 26752466.

1680. Chuang, Alice Y et al. "NOD2 expression is regulated by microRNAs in colonic epithelial HCT116 cells." Inflammatory bowel diseases vol. 20,1 (2014): 126-35. doi:10.1097/01.MIB.0000436954.70596.9b

1681. Chen Y, Wang C, Liu Y, et al. miR-122 targets NOD2 to decrease intestinal epithelial cell injury in Crohn's disease. Biochem Biophys Res Commun. 2013;438:133-139

1682. Mukhopadhyay D, Mussa BM. Identification of Novel Hypothalamic MicroRNAs as Promising Therapeutics for SARS-CoV-2 by Regulating ACE2 and TMPRSS2 Expression: An In Silico Analysis. Brain Sci. 2020 Sep 25;10(10):666. doi:

10.3390/brainsci10100666. PMID: 32992681; PMCID: PMC7601472

1683. Pierce JB, Simion V, Icli B, Pérez-Cremades D, Cheng HS, Feinberg MW. Computational Analysis of Targeting SARS-CoV-2, Viral Entry Proteins ACE2 and TMPRSS2, and Interferon Genes by Host MicroRNAs. Genes (Basel). 2020 Nov 16;11(11):1354. doi: 10.3390/genes11111354. PMID: 33207533; PMCID: PMC7696723.

1684. Chen, Y., Salem, M., Boyd, M. et al. Relation between NOD2 genotype and changes in innate signaling in Crohn's disease on mRNA and miRNA levels. npj Genomic Med 2, 3 (2017). https://doi.org/10.1038/s41525-016-0001-4

1685. Alam, Tanvir, and Leonard Lipovich. "miRCOVID-19: Potential Targets of Human miRNAs in SARS-CoV-2 for RNA-Based Drug Discovery." Non-coding RNA vol. 7,1 18. 2 Mar. 2021, doi:10.3390/ncrna7010018.

1686. Collison AM, Sokulsky LA, Kepreotes E, Pereira de Siqueira A, Morten M, Edwards MR, Walton RP, Bartlett NW, Yang M, Nguyen TH, Johnston SL, Foster PS, Mattes J. miR-122 promotes virus-induced lung disease by targeting SOCS1. JCI Insight. 2021 Apr 8;6(7):e127933. doi: 10.1172/jci.insight.127933. PMID: 33830082; PMCID: PMC8119205.

1687. Shi, Yucong \& Xu, Huachong \& Xiao, Yike \& Liu, Pei \& Pang, Peng \& Wu, Sizhi \& Deng, Li \& Chen, Xiaoyin. (2019). Gegen Qinlian Decoction Downregulates the TLR7 Signalling Pathway to Control Influenza A Virus Infection. Biomedicine \& pharmacotherapy = Biomedecine \& pharmacotherapie. 121. 109471. 10.1016/j.biopha.2019.109471.

1688. Anz, David \& Koelzer, Viktor \& Moder, Stefan \& Thaler, Raffael \& Schwerd, Tobias \& Lahl, Katharina \& Sparwasser, Tim \& Besch, Robert \& Poeck, Hendrik \& Hornung, Veit \& Hartmann, Gunther \& Rothenfusser, Simon \& Bourquin, Carole \& Endres, Stefan. (2009). Immunostimulatory RNA Blocks Suppression by Regulatory T Cells. Journal of immunology (Baltimore, Md. : 1950). 184. 939-46. 10.4049/jimmunol.0901245

1689. Hackl D, Loschko J, Sparwasser T, Reindl W, Krug AB. Activation of dendritic cells via TLR7 reduces Foxp3 expression and suppressive function in induced Tregs. Eur J Immunol. 2011 May;41(5):1334-43. doi: 10.1002/eji.201041014. PMID: 21469103. 1690. Schön, M., Schön, M. TLR7 and TLR8 as targets in cancer therapy. Oncogene 27, 190-199 (2008). https://doi.org/10.1038/sj.onc.1210913

1691. Kobold S, Wiedemann G, Rothenfußer S, Endres S. Modes of action of TLR7 agonists in cancer therapy. Immunotherapy. 2014;6(10):1085-95. doi: 10.2217/imt.14.75. PMID: 25428647.

1692. Oldstone M.B.A., Rosen H. (2014) Cytokine Storm Plays a Direct Role in the Morbidity and Mortality from Influenza Virus Infection and is Chemically Treatable with a Single Sphingosine-1-Phosphate Agonist Molecule. In: Oldstone M., Rosen H. (eds) Sphingosine-1-Phosphate Signaling in Immunology and Infectious Diseases. Current Topics in Microbiology and Immunology, vo 378. Springer, Cham. https://doi.org/10.1007/978-3-319-05879-5_6

1693. Peter Larson, Tamara A. Kucaba, Zhengming Xiong, Michael Olin, Thomas S. Griffith, and David M. Ferguson, Design and Synthesis of N1-Modified Imidazoquinoline Agonists for Selective Activation of Toll-like Receptors 7 and 8, ACS Medicinal Chemistry Letters 20178 (11), 1148-1152, DOI: 10.1021/acsmedchemlett.7b00256

1694. Conroy, H., Marshall, N. \& Mills, K. TLR ligand suppression or enhancement of Treg cells? A double-edged sword in immunity to tumours. Oncogene 27, 168-180 (2008). https://doi.org/10.1038/sj.onc.1210910

1695. Huang S, Wei W, Yun Y. Upregulation of TLR7 and TLR3 gene expression in the lung of respiratory syncytial virus infected mice. Wei Sheng Wu Xue Bao. 2009 Feb;49(2):239-45. PMID: 19445181

1696. Kubo, T., R. D. Hatton, J. Oliver, X. Liu, C. O. Elson, and C. T. Weaver. 2004. Regulatory T cell suppression and anergy are differentially regulated by proin- flammatory cytokines produced by TLR activated dendritic cells. J. Immunol. 173: 7249-7258 1697. Peng, G., Z. Guo, Y. Kiniwa, K. S. Voo, W. Peng, T. Fu, D. Y. Wang, Y. Li, H. Y. Wang, and R. F. Wang.2005. Toll-like receptor 8-mediated reversal of CD4+, regulatory T cell function. Science 309: 1380-1384

1698. Sutmuller, R. P., M. H. den Brok, M. Kramer, E. J. Bennink, L. W. Toonen, B. J. Kullberg, L. A. Joosten, S. Akira, M. G. Netea, and G. J. Adema. 2006. Toll-like receptor 2 controls expansion and function of regulatory T cells. J. Clin. Invest. 116: 485-494. 1699. Liu, H., M. Komai-Koma, D. Xu, and F. Y. Liew. 2006. Toll-like receptor 2 signaling modulates the functions of CD4+ CD25+ regulatory T cells. Proc. Natl. Acad. Sci. USA 103: 7048-7053

1700. Crellin, N. K., R. V. Garcia, O. Hadisfar, S. E. Allan, T. S. Steiner, and M. K. Levings. 2005. Human CD4+ T cells express TLR5 and its ligand flagellin enhances the suppressive capacity and expression of FOXP3 in CD4+CD25+ T regulatory cells. J. Immunol. 175: 8051-8059

1701. Nicholas W. Lukacs, Joost J. Smit, Sumanta Mukherjee, Susan B. Morris, Gabriel Nunez and Dennis M. Lindell, Respiratory Virus-Induced TLR7 Activation Controls IL-17-Associated Increased Mucus via IL-23 Regulation, J Immunol August 15, 2010, 185 (4) 2231-2239; DOI: https://doi.org/10.4049/jimmunol.1000733

1702. Zhang T, Zhu J, Su B, Cao L, Li Z, Wei H, Huang X, Zheng K, Li A, Chen N, Liu L, Xia W, Wu H, He Q. Effects of TLR7 Polymorphisms on the Susceptibility and Progression of HIV-1 Infection in Chinese MSM Population. Front Immunol. 2020 Oct 26;11:589010. doi: 10.3389/fimmu.2020.589010. PMID: 33193416; PMCID: PMC7649213. 
1703. Honda K, Yanai H, Negishi H, Asagiri M, Sato M, Mizutani T, Shimada N, Ohba Y, Takaoka A, Yoshida N, Taniguchi T. IRF-7 is the master regulator of type-I interferon-dependent immune responses. Nature. 2005 Apr 7;434(7034):772-7. doi: 10.1038/nature03464. Epub 2005 Mar 30. PMID: 15800576.

1704. Wu S, Jiang ZY, Sun YF, Yu B, Chen J, Dai CQ, Wu XL, Tang XL, Chen XY. Microbiota regulates the TLR7 signaling pathway against respiratory tract influenza A virus infection. Curr Microbiol. 2013 Oct;67(4):414-22. doi: 10.1007/s00284-013-0380-z. Epub 2013 May 16. PMID: 23677145.

1705. Ichinohe T, Pang IK, Kumamoto Y, Peaper DR, Ho JH, Murray TS, Iwasaki A. Microbiota regulates immune defense against respiratory tract influenza A virus infection. Proc Natl Acad Sci U S A. 2011 Mar 29;108(13):5354-9. doi:

10.1073/pnas.1019378108. Epub 2011 Mar 14. PMID: $21402903 ;$ PMCID: PMC3069176.

1706. Borey M, Blanc F, Lemonnier G, Leplat JJ, Jardet D, Rossignol MN, Ravon L, Billon Y, Bernard M, Estellé J, Rogel-Gaillard C. Links between fecal microbiota and the response to vaccination against influenza A virus in pigs. NPJ Vaccines. 2021 Jul 22;6(1):92. doi: 10.1038/s41541-021-00351-2. PMID: 34294732; PMCID: PMC8298503.

1707. Yang $M$, Yang $Y$, He Q, Zhu $P$, Liu M, Xu J, Zhao M. Intestinal Microbiota-A Promising Target for Antiviral Therapy? Front Immunol. 2021 May 12;12:676232. doi: 10.3389/fimmu.2021.676232. PMID: 34054866; PMCID: PMC8149780.

1708. Lu W, Fang Z, Liu X, Li L, Zhang P, Zhao J, Zhang H, Chen W. The Potential Role of Probiotics in Protection against Influenza a Virus Infection in Mice. Foods. 2021 Apr 20;10(4):902. doi: 10.3390/foods10040902. PMID: 33924002; PMCID: PMC8073107.

1709. Shi HY, Zhu X, Li WL, Mak JWY, Wong SH, Zhu ST, Guo SL, Chan FKL, Zhang ST, Ng SC. Modulation of gut microbiota protects against viral respiratory tract infections: a systematic review of animal and clinical studies. Eur J Nutr. 2021 Apr 14:1-24. doi: 10.1007/s00394-021-02519-x. Epub ahead of print. PMID: 33852069; PMCID: PMC8044287.

1710. Vignesh R, Swathirajan CR, Tun ZH, Rameshkumar MR, Solomon SS, Balakrishnan P. Could Perturbation of Gut Microbiota Possibly Exacerbate the Severity of COVID-19 via Cytokine Storm? Front Immunol. 2021 Jan 25;11:607734. doi: 10.3389/fimmu.2020.607734. PMID: 33569053; PMCID: PMC7868418.

1711. Saleh, Jumana et al. "Mitochondria and microbiota dysfunction in COVID-19 pathogenesis." Mitochondrion vol. 54 (2020): 1-7. doi:10.1016/j.mito.2020.06.008

1712. Gasmi A, Tippairote T, Mujawdiya PK, Peana M, Menzel A, Dadar M, Benahmed AG, Bjørklund G. The microbiota-mediated dietary and nutritional interventions for COVID-19. Clin Immunol. 2021 May;226:108725. doi: 10.1016/j.clim.2021.108725. Epub 2021 Apr 9. PMID: 33845194; PMCID: PMC8032598.

1713. Zhang Q, Liang Z, Zhang J, Lei T, Dong X, Su H, Chen Y, Zhang Z, Tan L, Zhao Y. Sirt6 Regulates the Development of Medullary Thymic Epithelial Cells and Contributes to the Establishment of Central Immune Tolerance. Front Cell Dev Biol. $2021 \mathrm{Mar}$ 29;9:655552. doi: 10.3389/fcell.2021.655552. PMID: 33869219; PMCID: PMC8044826.

1714. Zheng M, Williams EP, Malireddi RKS, Karki R, Banoth B, Burton A, Webby R, Channappanavar R, Jonsson CB, Kanneganti TD. Impaired NLRP3 inflammasome activation/pyroptosis leads to robust inflammatory cell death via caspase-8/RIPK3 during coronavirus infection. J Biol Chem. 2020 Oct 9;295(41):14040-14052. doi: 10.1074/jbc.RA120.015036. Epub 2020 Aug 6. PMID: 32763970; PMCID: PMC7549031.

1715. Merad, M., Martin, J.C. Pathological inflammation in patients with COVID-19: a key role for monocytes and macrophages. Nat Rev Immunol 20, 355-362 (2020). https://doi.org/10.1038/s41577-020-0331-4

1716. Jeremy K. Y. Yap, Miyu Moriyama and Akiko Iwasaki,Inflammasomes and Pyroptosis as Therapeutic Targets for COVID-19, J Immunol July 15, 2020, 205 (2) 307-312; DOI: https://doi.org/10.4049/jimmunol.2000513

1717. Courjon J, Dufies O, Robert A, Bailly L, Torre C, Chirio D, Contenti J, Vitale S, Loubatier C, Doye A, Pomares-Estran C, Gonfrier G, Lotte R, Munro P, Visvikis O, Dellamonica J, Giordanengo V, Carles M, Yvan-Charvet L, Ivanov S, Auberger P, Jacquel A, Boyer L. Heterogeneous NLRP3 inflammasome signature in circulating myeloid cells as a biomarker of COVID-19 severity. Blood Adv. 2021 Mar 9;5(5):1523-1534. doi: 10.1182/bloodadvances.2020003918. PMID: 33683342; PMCID: PMC7942161. 1718. Lu F, Lan Z, Xin Z, He C, Guo Z, Xia X, Hu T. Emerging insights into molecular mechanisms underlying pyroptosis and functions of inflammasomes in diseases. J Cell Physiol. 2020 Apr;235(4):3207-3221. doi: 10.1002/jcp.29268. Epub 2019 Oct 17. PMID: 31621910.

1719. Todt JC, Freeman CM, Brown JP, Sonstein J, Ames TM, McCubbrey AL, Martinez FJ, Chensue SW, Beck JM, Curtis JL. Smoking decreases the response of human lung macrophages to double-stranded RNA by reducing TLR3 expression. Respir Res. 2013 Mar 9;14(1):33. doi: 10.1186/1465-9921-14-33. PMID: 23497334; PMCID: PMC3599854.

1720. Xu Y, Zhang Y, Cardell LO. Nicotine exaggerates LPS-induced airway hyperreactivity via JNK-mediated up-regulation of Tolllike receptor 4. Am J Respir Cell Mol Biol. 2014 Sep;51(3):370-9. doi: 10.1165/rcmb.2013-0409OC. PMID: 24669857. 1721. Valdez-Miramontes CE, Trejo Martínez LA, Torres-Juárez F, Rodríguez Carlos A, Marin-Luévano SP, de Haro-Acosta JP, Enciso-Moreno JA, Rivas-Santiago B. Nicotine modulates molecules of the innate immune response in epithelial cells and macrophages during infection with M. tuberculosis. Clin Exp Immunol. 2020 Feb;199(2):230-243. doi: 10.1111/cei.13388. Epub 2019 Nov 3. PMID: 31631328; PMCID: PMC6954679.

1722. Todt, J.C., Freeman, C.M., Brown, J.P. et al. Smoking decreases the response of human lung macrophages to doublestranded RNA by reducing TLR3 expression. Respir Res 14, 33 (2013). https://doi.org/10.1186/1465-9921-14-33 1723. Grassin-Delyle S, Abrial C, Salvator H, Brollo M, Naline E, Devillier P: The Role of Toll-Like Receptors in the Production of Cytokines by Human Lung Macrophages. J Innate Immun 2020;12:63-73. doi: 10.1159/000494463

1724. Haiyan Chen, Mark J. Cowan, Jeffrey D. Hasday, Stefanie N. Vogel, Andrei E. Medvedev, Tobacco Smoking Inhibits Expression of Proinflammatory Cytokines and Activation of IL-1R-Associated Kinase, p38, and NF-KB in Alveolar Macrophages Stimulated with TLR2 and TLR4 Agonists, The Journal of Immunology November 1, 2007, 179 (9) 6097-6106; DOI: 10.4049/jimmunol.179.9.6097

1725. Koarai A, Yanagisawa S, Sugiura H, Ichikawa T, Akamatsu K, Hirano T, Nakanishi M, Matsunaga K, Minakata Y, Ichinose M. Cigarette smoke augments the expression and responses of toll-like receptor 3 in human macrophages. Respirology. 2012 Aug;17(6):1018-25. doi: 10.1111/j.1440-1843.2012.02198.x. PMID: 22591330.

1726. Tae-Hoon Kim, So-Jin Kim, Sun-Mee Lee, Stimulation of the a7 Nicotinic Acetylcholine Receptor Protects Against Sepsis by Inhibiting Toll-like Receptor via Phosphoinositide 3-Kinase Activation, The Journal of Infectious Diseases, Volume 209, Issue 10, 15 May 2014, Pages 1668-1677, https://doi.org/10.1093/infdis/jit669

1727. Ramalingam A, Budin SB, Mohd Fauzi N, Ritchie RH, Zainalabidin S. Angiotensin II Type I Receptor Antagonism Attenuates Nicotine-Induced Cardiac Remodeling, Dysfunction, and Aggravation of Myocardial Ischemia-Reperfusion Injury in Rats. Front Pharmacol. 2019 Dec 12;10:1493. doi: 10.3389/fphar.2019.01493. PMID: 31920673; PMCID: PMC6920178.

1728. Zuo T, Zhang F, Lui GCY, Yeoh YK, Li AYL, Zhan H, Wan Y, Chung ACK, Cheung CP, Chen N, Lai CKC, Chen Z, Tso EYK, Fung KSC, Chan V, Ling L, Joynt G, Hui DSC, Chan FKL, Chan PKS, Ng SC. Alterations in Gut Microbiota of Patients With COVID-19 During Time of Hospitalization. Gastroenterology. 2020 Sep;159(3):944-955.e8. doi: 10.1053/j.gastro.2020.05.048. Epub 2020 May 20. PMID: 32442562; PMCID: PMC7237927. 
1729. Peng Y, Zhao J, Tun HM. The New Foe and Old Friends: Are We Ready for Microbiota-Based Therapeutics in Treating COVID19 Patients? Gastroenterology. 2021 May;160(6):2192-2193. doi: 10.1053/j.gastro.2020.08.048. Epub 2020 Aug 30. PMID: 32877709; PMCID: PMC7456457.

1730. Xu K, Cai H, Shen Y, Ni Q, Chen Y, Hu S, Li J, Wang H, Yu L, Huang H, Qiu Y, Wei G, Fang Q, Zhou J, Sheng J, Liang T, Li L. [Management of corona virus disease-19 (COVID-19): the Zhejiang experience]. Zhejiang Da Xue Xue Bao Yi Xue Ban. 2020 Feb 21;49(1):147-157. Chinese. doi: 10.3785/j.issn.1008-9292.2020.02.02. PMID: 32096367.

1731. Dhar D, Mohanty A. Gut microbiota and Covid-19- possible link and implications. Virus Res. 2020 Aug;285:198018. doi 10.1016/j.virusres.2020.198018. Epub 2020 May 13. PMID: 32430279; PMCID: PMC7217790.

1732. Mak JWY, Chan FKL, Ng SC. Probiotics and COVID-19: one size does not fit all. Lancet Gastroenterol Hepatol. 2020 Jul;5(7):644-645. doi: 10.1016/S2468-1253(20)30122-9. Epub 2020 Apr 25. PMID: 32339473; PMCID: PMC7182525. 1733. d'Ettorre G, Ceccarelli G, Marazzato M, Campagna G, Pinacchio C, Alessandri F, Ruberto F, Rossi G, Celani L, Scagnolari C, Mastropietro C, Trinchieri V, Recchia GE, Mauro V, Antonelli G, Pugliese F, Mastroianni CM. Challenges in the Management of SARS-CoV2 Infection: The Role of Oral Bacteriotherapy as Complementary Therapeutic Strategy to Avoid the Progression of COVID-19. Front Med (Lausanne). 2020 Jul 7;7:389. doi: 10.3389/fmed.2020.00389. PMID: 32733907; PMCID: PMC7358304. 1734. Xu, Zhilu et al. "Reply." Gastroenterology vol. 160,6 (2021): 2195-2196. doi:10.1053/j.gastro.2021.01.198 1735. Hanada S, Pirzadeh M, Carver KY, Deng JC. Respiratory Viral Infection-Induced Microbiome Alterations and Secondary Bacterial Pneumonia. Front Immunol. 2018 Nov 16;9:2640. doi: 10.3389/fimmu.2018.02640. PMID: 30505304; PMCID: PMC6250824.

1736. Grayson MH, Camarda LE, Hussain SA, Zemple SJ, Hayward M, Lam V, Hunter DA, Santoro JL, Rohlfing M, Cheung DS, Salzman NH. Intestinal Microbiota Disruption Reduces Regulatory T Cells and Increases Respiratory Viral Infection Mortality Through Increased IFNy Production. Front Immunol. 2018 Jul 10;9:1587. doi: 10.3389/fimmu.2018.01587. PMID: 30042764 PMCID: PMC6048222.

1737. Abt MC, Osborne LC, Monticelli LA, Doering TA, Alenghat T, Sonnenberg GF, Paley MA, Antenus M, Williams KL, Erikson J, Wherry EJ, Artis D. Commensal bacteria calibrate the activation threshold of innate antiviral immunity. Immunity. 2012 Jul 27;37(1):158-70. doi: 10.1016/j.immuni.2012.04.011. Epub 2012 Jun 14. PMID: 22705104; PMCID: PMC3679670.

1738. Bradley KC, Finsterbusch K, Schnepf D, Crotta S, Llorian M, Davidson S, Fuchs SY, Staeheli P, Wack A. Microbiota-Driven Tonic Interferon Signals in Lung Stromal Cells Protect from Influenza Virus Infection. Cell Rep. 2019 Jul 2;28(1):245-256.e4. doi: 10.1016/j.celrep.2019.05.105. PMID: 31269444.

1739. Ahlawat S, Asha, Sharma KK. Immunological co-ordination between gut and lungs in SARS-CoV-2 infection. Virus Res. 2020 Sep;286:198103. doi: 10.1016/j.virusres.2020.198103. Epub 2020 Jul 24. PMID: 32717345; PMCID: PMC7380259.

1740. Vouloumanou EK, Makris GC, Karageorgopoulos DE, Falagas ME. Probiotics for the prevention of respiratory tract infections: a systematic review. Int J Antimicrob Agents. 2009 Sep;34(3):197.e1-10. doi: 10.1016/j.ijantimicag.2008.11.005. Epub 2009 Jan 28. PMID: 19179052.

1741. Lehtoranta L, Latvala S, Lehtinen MJ. Role of Probiotics in Stimulating the Immune System in Viral Respiratory Tract Infections: A Narrative Review. Nutrients. 2020 Oct 16;12(10):3163. doi: 10.3390/nu12103163. PMID: 33081138; PMCID: PMC7602805.

1742. Luoto R, Ruuskanen O, Waris M, Kalliomäki M, Salminen S, Isolauri E. Prebiotic and probiotic supplementation prevents rhinovirus infections in preterm infants: a randomized, placebo-controlled trial. J Allergy Clin Immunol. 2014 Feb;133(2):405-13. doi: 10.1016/j.jaci.2013.08.020. Epub 2013 Oct 13. PMID: 24131826; PMCID: PMC7112326.

1743. Amaral MA, Guedes GHBF, Epifanio M, Wagner MB, Jones MH, Mattiello R. Network meta-analysis of probiotics to prevent respiratory infections in children and adolescents. Pediatr Pulmonol. 2017 Jun;52(6):833-843. doi: 10.1002/ppul.23643. Epub 2017 Jan 3. PMID: 28052594.

1744. de Vos P, Mujagic Z, de Haan BJ, Siezen RJ, Bron PA, Meijerink M, Wells JM, Masclee AAM, Boekschoten MV, Faas MM, Troost FJ. Lactobacillus plantarum Strains Can Enhance Human Mucosal and Systemic Immunity and Prevent Non-steroidal Antiinflammatory Drug Induced Reduction in T Regulatory Cells. Front Immunol. 2017 Aug 23;8:1000. doi: 10.3389/fimmu.2017.01000. PMID: 28878772; PMCID: PMC5572349.

1745. He X, Jing Z, Cheng G. MicroRNAs: new regulators of Toll-like receptor signalling pathways. Biomed Res Int. 2014;2014:945169. doi: 10.1155/2014/945169. Epub 2014 Mar 20. PMID: 24772440; PMCID: PMC3977468.

1746. Pandiyan, Pushpa et al. "Microbiome Dependent Regulation of Tregs and Th17 Cells in Mucosa." Frontiers in immunology vol. 10 426. 8 Mar. 2019, doi:10.3389/fimmu.2019.00426

1747. Cario E. Bacterial interactions with cells of the intestinal mucosa: Toll-like receptors and NOD2. Gut. 2005 Aug;54(8):118293. doi: 10.1136/gut.2004.062794. Epub 2005 Apr 19. PMID: 15840688; PMCID: PMC1774880.

1748. Xu C, Ren G, Cao G, Chen Q, Shou P, Zheng C, Du L, Han X, Jiang M, Yang Q, Lin L, Wang G, Yu P, Zhang X, Cao W, Brewer G, Wang Y, Shi Y. miR-155 regulates immune modulatory properties of mesenchymal stem cells by targeting TAK1-binding protein 2. J Biol Chem. 2013 Apr 19;288(16):11074-9. doi: 10.1074/jbc.M112.414862. Epub 2013 Feb 28. PMID: 23449975; PMCID: PMC3630877.

1749. Yuan G, Zhao Y, Wu D, Gao C, Jiao Z. miRNA-20a upregulates TAK1 and increases proliferation in osteosarcoma cells. Future Oncol. 2018 Feb;14(5):461-469. doi: 10.2217/fon-2017-0490. Epub 2018 Jan 12. PMID: 29327611.

1750. Yao, W., Xu, L., Jia, X., Li, S., \& Wei, L. (2021). MicroRNA- 129 plays a protective role in sepsis- induced acute lung injury through the suppression of pulmonary inflammation via the modulation of the TAK1/NF- KB pathway. International Journal of Molecular Medicine, 48, 139. https://doi.org/10.3892/ijmm.2021.4972

1751. Prakhar, Praveen et al. "Ac2PIM-responsive miR-150 and miR-143 target receptor-interacting protein kinase 2 and transforming growth factor beta-activated kinase 1 to suppress NOD2-induced immunomodulators." The Journal of biological chemistry vol. 290,44 (2015): 26576-86. doi:10.1074/jbc.M115.662817

1752. Su H, Chang R, Zheng W, Sun Y, Xu T. microRNA-210 and microRNA-3570 Negatively Regulate NF-kB-Mediated Inflammatory Responses by Targeting RIPK2 in Teleost Fish. Front Immunol. 2021 Mar 31;12:617753. doi: 10.3389/fimmu.2021.617753. PMID: 33868233; PMCID: PMC8044448.

1753. Xu T, Chu Q, Cui J, Huo R. MicroRNA-216a Inhibits NF-kB-Mediated Inflammatory Cytokine Production in Teleost Fish by Modulating p65. Infect Immun. 2018 May 22;86(6):e00256-18. doi: 10.1128/IAI.00256-18. PMID: 29632247; PMCID: PMC5964508.

1754. Manuel Koch, Hans-Joachim Mollenkopf, Uwe Klemm, Thomas F. Meyer, H. pylori-induced miR-155 in macrophages, Proceedings of the National Academy of Sciences May 2012, 109 (19) E1153-E1162; DOI: 10.1073/pnas.1116125109 1755. Wu SC, Yang JCS, Rau CS, Chen YC, Lu TH, et al. (2013) Profiling Circulating MicroRNA Expression in Experimental Sepsis Using Cecal Ligation and Puncture. PLOS ONE 8(10): e77936. https://doi.org/10.1371/journal.pone.0077936 
1756. Xu J, Feng Y, Jeyaram A, Jay SM, Zou L, Chao W. Circulating Plasma Extracellular Vesicles from Septic Mice Induce Inflammation via MicroRNA- and TLR7-Dependent Mechanisms. J Immunol. 2018 Dec 1;201(11):3392-3400. doi: 10.4049/jimmunol.1801008. Epub 2018 Oct 24. PMID: 30355788; PMCID: PMC6240609.

1757. ong L, Li Y, Han C, et al. miRNA microarray reveals specific expression in

the peripheral blood of glioblastoma patients. Int J Oncol. 2014;45:746-756.

1758. eller JF, Carrouel F, Staquet MJ, et al. Expression of NOD2 is increased in inflamed human dental pulps and lipoteichoic acid-stimulated odonto- blast-like cells. Innate Immun. 2011;17:29-34

1759. Negroni A, Stronati L, Pierdomenico M, et al. Activation of NOD2- mediated intestinal pathway in a pediatric population with Crohn's disease. Inflamm Bowel Dis. 2009;15:1145-1154

1760. Stronati L, Negroni A, Merola P, et al. Mucosal NOD2 expression and NF-kappaB activation in pediatric Crohn's disease. Inflamm Bowel Dis. 2008;14:295-302.

1761. Fujisawa M, Kiyosue M, Hori M, et al. Identification of Card15/Nod2 mRNA in intestinal tissue of experimentally induced colitis in rats. J Vet Med Sci. 2006;68:701-708.

1762. Berrebi D, Maudinas R, Hugot JP, et al. Card15 gene overexpression in mononuclear and epithelial cells of the inflamed Crohn's disease colon. Gut. 2003;52:840-846

1763. Stronati L, Negroni A, Pierdomenico M, et al. Altered expression of innate immunity genes in different intestinal sites of children with ulcerative colitis. Dig Liver Dis. 2010;42:848-853.

1764. Arroyo M, Salka K, Chorvinsky E, Xuchen X, Abutaleb K, et al. (2020) Airway mir-155 responses are associated with TH1 cytokine polarization in young children with viral respiratory infections. PLOS ONE 15(5): e0233352.

https://doi.org/10.1371/journal.pone.0233352

1765. Farr RJ, Rootes CL, Rowntree LC, Nguyen THO, Hensen L, et al. (2021) Altered microRNA expression in COVID-19 patients enables identification of SARS-CoV-2 infection. PLOS Pathogens 17(7): e1009759. https://doi.org/10.1371/journal.ppat.1009759 1766. Zhi Liu and Jianwei Wang and Yuyu Xu and Mengchen Guo et.al, Implications of the virus-encoded miRNA and host miRNA in the pathogenicity of SARS-CoV-2, 2020,2004.04874,arXiv

1767. Abu-Izneid, Tareq et al. "Micro-RNAs in the regulation of immune response against SARS CoV-2 and other viral infections." Journal of advanced research vol. 30 (2021): 133-145. doi:10.1016/j.jare.2020.11.013

1768. Ryan M. O'Connell, Aadel A. Chaudhuri, Dinesh S. Rao, David Baltimore, Inositol phosphatase SHIP1 is a primary target of miR-155, Proceedings of the National Academy of Sciences Apr 2009, 106 (17) 7113-7118; DOI: 10.1073/pnas.0902636106

1769. Jin, Hye Mi et al. "MicroRNA-155 as a proinflammatory regulator via SHIP-1 down-regulation in acute gouty arthritis." Arthritis research \& therapy vol. 16,2 R88. 7 Apr. 2014, doi:10.1186/ar4531

1770. Chen Y, Hu F, Dong X, Zhao M, Wang J, Sun X, Kim TJ, Li Z, Liu W. SHIP-1 Deficiency in AID+ B Cells Leads to the Impaired Function of B10 Cells with Spontaneous Autoimmunity. J Immunol. 2017 Nov 1;199(9):3063-3073. doi: 10.4049/jimmunol.1700138. Epub 2017 Oct 2. PMID: 28972092.

1771. Xue H, Hua LM, Guo M, Luo JM. SHIP1 is targeted by miR-155 in acute myeloid leukemia. Oncol Rep. 2014 Nov;32(5):2253-9. doi: 10.3892/or.2014.3435. Epub 2014 Aug 22. PMID: 25175984.

1772. Kandell WM, Donatelli SS, Trinh TL, Calescibetta AR, So T, et al. (2020) MicroRNA-155 governs SHIP-1 expression and localization in NK cells and regulates subsequent infiltration into murine AT3 mammary carcinoma. PLOS ONE 15(2): e0225820. https://doi.org/10.1371/journal.pone.0225820

1773. Lee, D., Futami, M., Carroll, M. et al. Loss of SHIP-1 protein expression in high-risk myelodysplastic syndromes is associated with miR-210 and miR-155. Oncogene 31, 4085-4094 (2012). https://doi.org/10.1038/onc.2011.579

1774. Chen, Siqi et al. "Host miR155 promotes tumor growth through a myeloid-derived suppressor cell-dependent mechanism." Cancer research vol. 75,3 (2015): 519-31. doi:10.1158/0008-5472.CAN-14-2331

1775. Tuerdi, Baihetinisha et al. "Downregulation of miR-155 attenuates sepsis-induced acute lung injury by targeting SIRT1." International journal of clinical and experimental pathology vol. 11,9 4483-4492. 1 Sep. 2018

1776. Yang P, Xiong W, Chen X, Liu J, Ye Z. Overexpression of miR-129-5p Mitigates Sepsis-Induced Acute Lung Injury by Targeting High Mobility Group Box 1. J Surg Res. 2020 Dec;256:23-30. doi: 10.1016/j.jss.2020.05.101. Epub 2020 Jul 15. PMID: 32682121.

1777. Meng L, Cao H, Wan C, Jiang L. MiR-539-5p alleviates sepsis-induced acute lung injury by targeting ROCK1. Folia Histochem Cytobiol. 2019;57(4):168-178. doi: 10.5603/FHC.a2019.0019. Epub 2019 Dec 11. PMID: 31825519.

1778. Chen L, Xie W, Wang L, Zhang X, Liu E, Kou Q. MiRNA-133a aggravates inflammatory responses in sepsis by targeting SIRT1. Int Immunopharmacol. 2020 Nov;88:106848. doi: 10.1016/j.intimp.2020.106848. Epub 2020 Aug 7. PMID: 32771944. 1779. Ge, Junke et al. "Inhibition of miR-21 ameliorates LPS-induced acute lung injury through increasing B cell lymphoma-2 expression." Innate immunity vol. 26,8 (2020): 693-702. doi:10.1177/1753425920942574

1780. Xie W, Lu Q, Wang K, Lu J, Gu X, Zhu D, Liu F, Guo Z. miR-34b-5p inhibition attenuates lung inflammation and apoptosis in an LPS-induced acute lung injury mouse model by targeting progranulin. J Cell Physiol. 2018 Sep;233(9):6615-6631. doi: 10.1002/jcp.26274. Epub 2018 Mar 25. PMID: 29150939; PMCID: PMC6001482.

1781. Chen, Song et al. "MicroRNA-34a Inhibition Alleviates Lung Injury in Cecal Ligation and Puncture Induced Septic Mice." Frontiers in immunology vol. 11 1829. 13 Aug. 2020, doi:10.3389/fimmu.2020.01829

1782. Podsiad A, Standiford TJ, Ballinger MN, Eakin R, Park P, Kunkel SL, Moore BB, Bhan U. MicroRNA-155 regulates host immune response to postviral bacterial pneumonia via IL-23/IL-17 pathway. Am J Physiol Lung Cell Mol Physiol. 2016 Mar 1;310(5):L465-75. doi: 10.1152/ajplung.00224.2015. Epub 2015 Nov 20. PMID: 26589478; PMCID: PMC4773845. 1783. Shen SM, Jiang H, Zhao JN, Shi Y. Down-regulation of miR-155 inhibits inflammatory response in human pulmonary microvascular endothelial cells infected with influenza A virus by targeting sphingosine-1-phosphate receptor 1. Chin Med J (Engl). 2020 Oct 20;133(20):2429-2436. doi: 10.1097/CM9.0000000000001036. PMID: 32889908; PMCID: PMC7575171.

1784. Guo L, Wang Q, Zhang D. MicroRNA-4485 ameliorates severe influenza pneumonia via inhibition of the STAT3/PI3K/AKT signaling pathway. Oncol Lett. 2020 Nov;20(5):215. doi: 10.3892/ol.2020.12078. Epub 2020 Sep 9. PMID: 32963621; PMCID: PMC7491079.

1785. Sun K, Metzger DW. Inhibition of pulmonary antibacterial defense by interferon-gamma during recovery from influenza infection. Nat Med 14: 558-564, 2008.

1786. Rothberg MB, Haessler SD, Brown RB. Complications of viral influenza. Am J Med. 2008 Apr;121(4):258-64. doi: 10.1016/j.amjmed.2007.10.040. PMID: 18374680; PMCID: PMC7172971.

1787. McCullers JA, Rehg JE. Lethal synergism between influenza virus and Streptococcus pneumoniae: characterization of a mouse model and the role of platelet-activating factor receptor. J Infect Dis. 2002 Aug 1;186(3):341-50. doi: 10.1086/341462. Epub 2002 Jul 10. PMID: 12134230.

1788. Didierlaurent A, Goulding J, Patel S, Snelgrove R, Low L, Bebien M, Lawrence T, van Rijt LS, Lambrecht BN, Sirard JC, Hussell T. Sustained desensitization to bacterial Toll-like receptor ligands after resolution of respiratory influenza infection. J Exp Med 205: 323-329, 2008. 
1789. Sun K, Metzger DW. Inhibition of pulmonary antibacterial defense by interferon-gamma during recovery from influenza infection. Nat Med. 2008 May;14(5):558-64. doi: 10.1038/nm1765. Epub 2008 Apr 27. PMID: 18438414.

1790. Jingyi, Yang \& Zhang, Ejuan \& Zhong, Maohua \& Yang, Qingyu \& Hong, Ke \& Shu, Ting \& Zhou, Dihan \& Xiang, Jie \& Xia, Jianbo \& Zhou, Xi \& Zhang, Dingyu \& Huang, Chaolin \& Shang, You \& Yan, Huimin. (2020). Impaired T Cell Functions Along with Elevated Activated Tregs at the Early Stage of Asymptomatic SARS-CoV-2 Infection. SSRN Electronic Journal.

10.2139/ssrn.3605177.

1791. Mahmoudvand S, Shokri S. Interactions between SARS coronavirus 2 papain-like protease and immune system: A potentia drug target for the treatment of COVID-19. Scand J Immunol. 2021 Apr 19:e13044. doi: 10.1111/sji.13044. Epub ahead of print. PMID: 33872387; PMCID: PMC8250271.

1792. Shin D, Mukherjee R, Grewe D, Bojkova D, Baek K, Bhattacharya A, Schulz L, Widera M, Mehdipour AR, Tascher G, Geurink PP, Wilhelm A, van der Heden van Noort GJ, Ovaa H, Müller S, Knobeloch KP, Rajalingam K, Schulman BA, Cinatl J, Hummer G, Ciesek S, Dikic I. Papain-like protease regulates SARS-CoV-2 viral spread and innate immunity. Nature. 2020 Nov;587(7835):657662. doi: 10.1038/s41586-020-2601-5. Epub 2020 Jul 29. PMID: 32726803; PMCID: PMC7116779.

1793. Lu R, Zhao X, Li J, Niu P, Yang B, Wu H, Wang W, Song H, Huang B, Zhu N, Bi Y, Ma X, Zhan F, Wang L, Hu T, Zhou H, Hu Z, Zhou W, Zhao L, Chen J, Meng Y, Wang J, Lin Y, Yuan J, Xie Z, Ma J, Liu WJ, Wang D, Xu W, Holmes EC, Gao GF, Wu G, Chen W, Shi W, Tan W. Genomic characterisation and epidemiology of 2019 novel coronavirus: implications for virus origins and receptor binding. Lancet. 2020 Feb 22;395(10224):565-574. doi: 10.1016/S0140-6736(20)30251-8. Epub 2020 Jan 30. PMID: 32007145; PMCID: PMC7159086.

1794. Harcourt BH, Jukneliene D, Kanjanahaluethai A, Bechill J, Severson KM, Smith CM, Rota PA, Baker SC. Identification of severe acute respiratory syndrome coronavirus replicase products and characterization of papain-like protease activity. J Virol. 2004 Dec;78(24):13600-12. doi: 10.1128/JVI.78.24.13600-13612.2004. PMID: 15564471; PMCID: PMC533933.

1795. Lim KP, Ng LF, Liu DX. Identification of a novel cleavage activity of the first papain-like proteinase domain encoded by open reading frame 1a of the coronavirus Avian infectious bronchitis virus and characterization of the cleavage products. J Virol. 2000 Feb;74(4):1674-85. doi: 10.1128/jvi.74.4.1674-1685.2000. PMID: 10644337; PMCID: PMC111642.

1796. Zheng Y, Zhuang MW, Han L, Zhang J, Nan ML, Zhan P, Kang D, Liu X, Gao C, Wang PH. Severe acute respiratory syndrome coronavirus 2 (SARS-CoV-2) membrane (M) protein inhibits type I and III interferon production by targeting RIG-I/MDA-5 signaling. Signal Transduct Target Ther. 2020 Dec 28;5(1):299. doi: 10.1038/s41392-020-00438-7. PMID: 33372174; PMCID: PMC7768267. 1797. Chen K, Xiao F, Hu D, Ge W, Tian M, Wang W, Pan P, Wu K, Wu J. SARS-CoV-2 Nucleocapsid Protein Interacts with RIG-I and Represses RIG-Mediated IFN- $\beta$ Production. Viruses. 2020 Dec 30;13(1):47. doi: 10.3390/v13010047. PMID: 33396605; PMCID: PMC7823417.

1798. Mu J, Fang Y, Yang Q, Shu T, Wang A, Huang M, Jin L, Deng F, Qiu Y, Zhou X. SARS-CoV-2 N protein antagonizes type interferon signaling by suppressing phosphorylation and nuclear translocation of STAT1 and STAT2. Cell Discov. 2020 Sep 15;6:65. doi: 10.1038/s41421-020-00208-3. PMID: 32953130; PMCID: PMC7490572.

1799. Yuen, Chun-Kit et al. "SARS-CoV-2 nsp13, nsp14, nsp15 and orf6 function as potent interferon antagonists." Emerging microbes \& infections vol. 9,1 (2020): 1418-1428. doi:10.1080/22221751.2020.1780953

1800. Wang, Y., Zhu, X., Jiang, XM. et al. Decreased inhibition of exosomal miRNAs on SARS-CoV-2 replication underlies poor outcomes in elderly people and diabetic patients. Sig Transduct Target Ther 6, 300 (2021). https://doi.org/10.1038/s41392-02100716-y

1801. Roncarati, Roberta et al. "The Importance of microRNAs in RAS Oncogenic Activation in Human Cancer." Frontiers in oncology vol. 9 988. 27 Sep. 2019, doi:10.3389/fonc.2019.00988

1802. Masliah-Planchon J, Garinet S, Pasmant E. RAS-MAPK pathway epigenetic activation in cancer: miRNAs in action

Oncotarget. 2016 Jun 21;7(25):38892-38907. doi: 10.18632/oncotarget.6476. PMID: 26646588; PMCID: PMC5122439. 1803. Chen X, Guo X, Zhang H, Xiang Y, Chen J, Yin Y, Cai X, Wang K, Wang G, Ba Y, Zhu L, Wang J, Yang R, Zhang Y, Ren Z, Zen K, Zhang J, Zhang CY. Role of miR-143 targeting KRAS in colorectal tumorigenesis. Oncogene. 2009 Mar 12;28(10):1385-92. doi: 10.1038/onc.2008.474. Epub 2009 Jan 12. PMID: 19137007.

1804. Gao JS, Zhang Y, Tang X, Tucker LD, Tarwater PM, Quesenberry PJ, Rigoutsos I, Ramratnam B. The Evi1, microRNA-143, KRas axis in colon cancer. FEBS Lett. 2011 Feb 18;585(4):693-9. doi: 10.1016/j.febslet.2011.01.033. Epub 2011 Jan 26. PMID: 21276449; PMCID: PMC3440864.

1805. Wang L, Shi ZM, Jiang CF, Liu X, Chen QD, Qian X, Li DM, Ge X, Wang XF, Liu LZ, You YP, Liu N, Jiang BH. MiR-143 acts as a tumor suppressor by targeting N-RAS and enhances temozolomide-induced apoptosis in glioma. Oncotarget. $2014 \mathrm{Jul}$ 30;5(14):5416-27. doi: 10.18632/oncotarget.2116. PMID: 24980823; PMCID: PMC4170647.

1806. Michael MZ, O' Connor SM, van Holst Pellekaan NG, Young GP, James RJ. Reduced accumulation of specific microRNAs in colorectal neoplasia. Mol Cancer Res. 2003 Oct;1(12):882-91. PMID: 14573789.

1807. Pagliuca A, Valvo C, Fabrizi E, di Martino S, Biffoni M, Runci D, Forte S, De Maria R, Ricci-Vitiani L. Analysis of the combined action of miR-143 and miR-145 on oncogenic pathways in colorectal cancer cells reveals a coordinate program of gene repression. Oncogene. 2013 Oct;32(40):4806-13. doi: 10.1038/onc.2012.495. Epub 2012 Nov 5. PMID: 23128394.

1808. Zeinali T, Mansoori B, Mohammadi A, Baradaran B. Regulatory mechanisms of miR-145 expression and the importance of its function in cancer metastasis. Biomed Pharmacother. 2019 Jan;109:195-207. doi: 10.1016/j.biopha.2018.10.037. Epub 2018 Nov 2. PMID: 30396077.

1809. Liu, Sha et al. "Effects of miR-145-5p through NRAS on the cell proliferation, apoptosis, migration, and invasion in melanoma by inhibiting MAPK and PI3K/AKT pathways." Cancer medicine vol. 6,4 (2017): 819-833. doi:10.1002/cam4.1030 1810. Liep J, Kilic E, Meyer HA, Busch J, Jung K, et al. (2016) Cooperative Effect of miR-141-3p and miR-145-5p in the Regulation of Targets in Clear Cell Renal Cell Carcinoma. PLOS ONE 11(6): e0157801. https://doi.org/10.1371/journal.pone.0157801 1811. Xin, Z., Tong, Z., Tan, J., \& Liu, C. (2021). MicroRNA-145-5p aggravates cell apoptosis and oxidative stress in tongue squamous cell carcinoma. Experimental and Therapeutic Medicine, 21, 373. https://doi.org/10.3892/etm.2021.9804 1812. Mataki, Hiroko et al. "Dual-strand tumor-suppressor microRNA-145 (miR-145-5p and miR-145-3p) coordinately targeted MTDH in lung squamous cell carcinoma." Oncotarget vol. 7,44 (2016): 72084-72098. doi:10.18632/oncotarget.12290 1813. Cheng, C., Xu, D., Liu, X., Bi, S., \& Zhang, J. (2021). MicroRNA- 145- 5 p inhibits hypoxia/reoxygenation- induced apoptosis in H9c2 cardiomyocytes by targeting ROCK1. Experimental and Therapeutic Medicine, 22, 796. https://doi.org/10.3892/etm.2021.10228 1814. Yu, Y., Nangia-Makker, P., Farhana, L. et al. miR-21 and miR-145 cooperation in regulation of colon cancer stem cells. Mol Cancer 14, 98 (2015). https://doi.org/10.1186/s12943-015-0372-7

1815. Johnson SM, Grosshans H, Shingara J, Byrom M, Jarvis R, Cheng A, Labourier E, Reinert KL, Brown D, Slack FJ. RAS is regulated by the let-7 microRNA family. Cell. 2005 Mar 11;120(5):635-47. doi: 10.1016/j.cell.2005.01.014. PMID: 15766527. 1816. Xie, C., Chen, Y., Luo, D. et al. Therapeutic potential of C1632 by inhibition of SARS-CoV-2 replication and viral-induced inflammation through upregulating let-7. Sig Transduct Target Ther 6, 84 (2021). https://doi.org/10.1038/s41392-021-00497-4 
1817. Fani, Mona et al. "The role of miRNAs in COVID-19 disease." Future Virology (2021): 10.2217/fvl-2020-0389.

doi:10.2217/fvl-2020-0389

1818. Sardar R, Satish D, Birla S, Gupta D. Integrative analyses of SARS-CoV-2 genomes from different geographical locations reveal unique features potentially consequential to host-virus interaction, pathogenesis and clues for novel therapies. Heliyon. 2020 Sep;6(9):e04658. doi: 10.1016/j.heliyon.2020.e04658. Epub 2020 Aug 20. PMID: 32844125; PMCID: PMC7439967. 1819. Chow, Jonathan Tak-Sum, and Leonardo Salmena. "Prediction and Analysis of SARS-CoV-2-Targeting MicroRNA in Human Lung Epithelium." Genes vol. 11,9 1002. 26 Aug. 2020, doi:10.3390/genes11091002.

1820. Nicoletti, Aline \& Visacri, Marília \& Ronda, Carla \& Nascimento Silva Vasconcelos, Pedro Eduardo \& Quintanilha, Júlia \& Souza, Rafael \& Ventura, Deise \& Eguti, Adriana \& Silva, Lilian \& Junior, Mauricio \& Catharino, Rodrigo \& Oliveira Reis, Leonardo \& Santos, Luiz \& Duran, Nelson \& Fávaro, Wagner \& Lancellotti, Marcelo \& Costa, José \& Moriel, Patricia \& Pincinato, Eder. (2021). Differentially expressed circulating microRNAs in Brazilian patients with COVID-19: a preliminary study on potential biomarkers for diagnosis and severity. 10.21203/rs.3.rs-630726/v1.

1821. Fani, Mona \& Zandi, Milad \& Ebrahimi, Saeedeh \& Soltani, Saber \& Abbasi, Samaneh. (2021). The role of miRNAs in COVID19 disease. Future Virology. 16. 10.2217/fvl-2020-0389.

1822. Ying, H., Ebrahimi, M., Keivan, M., Khoshnam, S. E., Salahi, S., \& Farzaneh, M. (2021). miRNAs; a novel strategy for the treatment of COVID-19. Cell Biology International, 1- 9. https://doi.org/10.1002/cbin.11653

1823. Donyavi, Tahereh et al. "Acute and post-acute phase of COVID-19: Analyzing expression patterns of miRNA-29a-3p, 146a3p, 155-5p, and let-7b-3p in PBMC." International immunopharmacology vol. 97 (2021): 107641.

doi:10.1016/j.intimp.2021.107641

1824. Zheng HY, Xu M, Yang CX, Tian RR, Zhang M, Li JJ, Wang XC, Ding ZL, Li GM, Li XL, He YQ, Dong XQ, Yao YG, Zheng YT. Longitudinal transcriptome analyses show robust $T$ cell immunity during recovery from COVID-19. Signal Transduct Target Ther. 2020 Dec 24;5(1):294. doi: 10.1038/s41392-020-00457-4. PMID: 33361761; PMCID: PMC7758413.

1825. Li JY, Liao CH, Wang Q, Tan YJ, Luo R, Qiu Y, Ge XY. The ORF6, ORF8 and nucleocapsid proteins of SARS-CoV-2 inhibit type I interferon signaling pathway. Virus Res. 2020 Sep;286:198074. doi: 10.1016/j.virusres.2020.198074. Epub 2020 Jun 23. PMID: 32589897; PMCID: PMC7309931.

1826. Jiang HW, Zhang HN, Meng QF, Xie J, Li Y, Chen H, Zheng YX, Wang XN, Qi H, Zhang J, Wang PH, Han ZG, Tao SC. SARS-CoV-2 Orf9b suppresses type I interferon responses by targeting TOM70. Cell Mol Immunol. 2020 Sep;17(9):998-1000. doi: 10.1038/s41423-020-0514-8. Epub 2020 Jul 29. PMID: 32728199; PMCID: PMC7387808.

1827. Szretter KJ, Gangappa S, Lu X, Smith C, Shieh WJ, Zaki SR, Sambhara S, Tumpey TM, Katz JM. Role of host cytokine responses in the pathogenesis of avian H5N1 influenza viruses in mice. J Virol. 2007 Mar;81(6):2736-44. doi: 10.1128/JVI.0233606. Epub 2006 Dec 20. PMID: 17182684; PMCID: PMC1866007.

1828. Condamine T, Gabrilovich DI. Molecular mechanisms regulating myeloid-derived suppressor cell differentiation and function. Trends Immunol. 2011 Jan;32(1):19-25. doi: 10.1016/j.it.2010.10.002. Epub 2010 Nov 8. PMID: 21067974; PMCID: PMC3053028.

1829. Arora M, Poe SL, Oriss TB, Krishnamoorthy N, Yarlagadda M, Wenzel SE, Billiar TR, Ray A, Ray P. TLR4/MyD88-induced CD11b+Gr-1 int F4/80+ non-migratory myeloid cells suppress Th2 effector function in the lung. Mucosal Immunol. 2010 Nov;3(6):578-93. doi: 10.1038/mi.2010.41. Epub 2010 Jul 21. Erratum in: Mucosal Immunol. 2011 Jan;4(1):124. PMID: 20664577 PMCID: PMC2958091.

1830. Ribechini E, Greifenberg V, Sandwick S, Lutz MB. Subsets, expansion and activation of myeloid-derived suppressor cells. Med Microbiol Immunol. 2010 Aug;199(3):273-81. doi: 10.1007/s00430-010-0151-4. Epub 2010 Apr 8. PMID: 20376485. 1831. Lin KL, Suzuki Y, Nakano H, Ramsburg E, Gunn MD. CCR2+ monocyte-derived dendritic cells and exudate macrophages produce influenza-induced pulmonary immune pathology and mortality. J Immunol. 2008 Feb 15;180(4):2562-72. doi: 10.4049/jimmunol.180.4.2562. PMID: 18250467.

1832. Tsou CL, Peters W, Si Y, Slaymaker S, Aslanian AM, Weisberg SP, Mack M, Charo IF. Critical roles for CCR2 and MCP-3 in monocyte mobilization from bone marrow and recruitment to inflammatory sites. J Clin Invest. 2007 Apr;117(4):902-9. doi: 10.1172/JCI29919. Epub 2007 Mar 15. PMID: 17364026; PMCID: PMC1810572.

1833. Koyama S, Ishii KJ, Kumar H, Tanimoto T, Coban C, Uematsu S, Kawai T, Akira S. Differential role of TLR- and RLR-signaling in the immune responses to influenza A virus infection and vaccination. J Immunol. 2007 Oct 1;179(7):4711-20. doi: 10.4049/jimmunol.179.7.4711. PMID: 17878370.

1834. López CB, Moltedo B, Alexopoulou L, Bonifaz L, Flavell RA, Moran TM. TLR-independent induction of dendritic cell maturation and adaptive immunity by negative-strand RNA viruses. J Immunol. 2004 Dec 1;173(11):6882-9. doi: 10.4049/jimmunol.173.11.6882. PMID: 15557183.

1835. Guillot L, Le Goffic R, Bloch S, Escriou N, Akira S, Chignard M, Si-Tahar M. Involvement of toll-like receptor 3 in the immune response of lung epithelial cells to double-stranded RNA and influenza A virus. J Biol Chem. 2005 Feb 18;280(7):5571-80. doi: 10.1074/jbc.M410592200. Epub 2004 Dec 3. PMID: 15579900.

1836. Pichlmair A, Schulz O, Tan CP, Näslund TI, Liljeström P, Weber F, Reis e Sousa C. RIG-I-mediated antiviral responses to single-stranded RNA bearing 5'-phosphates. Science. 2006 Nov 10;314(5801):997-1001. doi: 10.1126/science.1132998. Epub 2006 Oct 12. PMID: 17038589.

1837. Seo SU, Kwon HJ, Song JH, Byun YH, Seong BL, Kawai T, Akira S, Kweon MN. MyD88 signaling is indispensable for primary influenza A virus infection but dispensable for secondary infection. J Virol. 2010 Dec;84(24):12713-22. doi: 10.1128/JVI.01675-10. Epub 2010 Oct 13. PMID: 20943980; PMCID: PMC3004294.

1838. 510.

1839. Umemura N, Saio M, Suwa T, Kitoh Y, Bai J, Nonaka K, Ouyang GF, Okada M, Balazs M, Adany R, Shibata T, Takami T. Tumor-infiltrating myeloid-derived suppressor cells are pleiotropic-inflamed monocytes/macrophages that bear M1- and M2type characteristics. J Leukoc Biol. 2008 May;83(5):1136-44. doi: 10.1189/jlb.0907611. Epub 2008 Feb 19. PMID: 18285406. 1840. Heer AK, Shamshiev A, Donda A, Uematsu S, Akira S, Kopf M, Marsland BJ. TLR signaling fine-tunes anti-influenza B cell responses without regulating effector T cell responses. J Immunol. 2007 Feb 15;178(4):2182-91. doi:

10.4049/jimmunol.178.4.2182. PMID: 17277123.

1841. Koyama S, Ishii KJ, Kumar H, Tanimoto T, Coban C, Uematsu S, Kawai T, Akira S. Differential role of TLR- and RLR-signaling in the immune responses to influenza A virus infection and vaccination. J Immunol. 2007 Oct 1;179(7):4711-20. doi: 10.4049/jimmunol.179.7.4711. PMID: 17878370.

1842. Tumpey TM, García-Sastre A, Taubenberger JK, Palese P, Swayne DE, Pantin-Jackwood MJ, Schultz-Cherry S, Solórzano A, Van Rooijen N, Katz JM, Basler CF. Pathogenicity of influenza viruses with genes from the 1918 pandemic virus: functional roles of alveolar macrophages and neutrophils in limiting virus replication and mortality in mice. J Virol. 2005 Dec;79(23):14933-44. doi: 10.1128/JVI.79.23.14933-14944.2005. PMID: 16282492; PMCID: PMC1287592. 
1843. Tate MD, Deng YM, Jones JE, Anderson GP, Brooks AG, Reading PC. Neutrophils ameliorate lung injury and the development of severe disease during influenza infection. J Immunol. 2009 Dec 1;183(11):7441-50. doi: 10.4049/jimmunol.0902497. Epub 2009 Nov 16. PMID: 19917678.

1844. Delano MJ, Scumpia PO, Weinstein JS, Coco D, Nagaraj S, Kelly-Scumpia KM, O'Malley KA, Wynn JL, Antonenko S, Al-Quran SZ, Swan R, Chung CS, Atkinson MA, Ramphal R, Gabrilovich DI, Reeves WH, Ayala A, Phillips J, Laface D, Heyworth PG, ClareSalzler M, Moldawer LL. MyD88-dependent expansion of an immature GR-1(+)CD11b(+) population induces T cell suppression and Th2 polarization in sepsis. J Exp Med. 2007 Jun 11;204(6):1463-74. doi: 10.1084/jem.20062602. Epub 2007 Jun 4. PMID: 17548519; PMCID: PMC2118626.

1845. Haverkamp JM, Crist SA, Elzey BD, Cimen C, Ratliff TL. In vivo suppressive function of myeloid-derived suppressor cells is limited to the inflammatory site. Eur J Immunol. 2011 Mar;41(3):749-59. doi: 10.1002/eji.201041069. Epub 2011 Feb 2. PMID: 21287554; PMCID: PMC3089902.

1846. Sinha P, Clements VK, Bunt SK, Albelda SM, Ostrand-Rosenberg S. Cross-talk between myeloid-derived suppressor cells and macrophages subverts tumor immunity toward a type 2 response. J Immunol. 2007 Jul 15;179(2):977-83. doi: 10.4049/jimmunol.179.2.977. PMID: 17617589.

1847. Corzo CA, Cotter MJ, Cheng P, Cheng F, Kusmartsev S, Sotomayor E, Padhya T, McCaffrey TV, McCaffrey JC, Gabrilovich DI. Mechanism regulating reactive oxygen species in tumor-induced myeloid-derived suppressor cells. J Immunol. 2009 May 1;182(9):5693-701. doi: 10.4049/jimmunol.0900092. PMID: 19380816; PMCID: PMC2833019.

1848. Hayes KS, Bancroft AJ, Grencis RK. The role of TNF-alpha in Trichuris muris infection II: global enhancement of ongoing Th1 or Th2 responses. Parasite Immunol. 2007 Nov;29(11):583-94. doi: 10.1111/j.1365-3024.2007.00980.x. PMID: 17944748. 1849. Hernandez-Pando R, Rook GA. The role of TNF-alpha in T-cell-mediated inflammation depends on the Th1/Th2 cytokine balance. Immunology. 1994 Aug;82(4):591-5. PMID: 7835922; PMCID: PMC1414923.

1850. O'Neill LA, Bowie AG. The family of five: TIR-domain-containing adaptors in Toll-like receptor signalling. Nat Rev Immunol. 2007 May;7(5):353-64. doi: 10.1038/nri2079. PMID: 17457343.

1851. Schmitz N, Kurrer M, Bachmann MF, Kopf M. Interleukin-1 is responsible for acute lung immunopathology but increases survival of respiratory influenza virus infection. J Virol. 2005 May;79(10):6441-8. doi: 10.1128/JVI.79.10.6441-6448.2005. PMID: 15858027; PMCID: PMC1091664.

1852. Mosser DM, Edwards JP. Exploring the full spectrum of macrophage activation. Nat Rev Immunol. 2008 Dec;8(12):958-69. doi: 10.1038/nri2448. Erratum in: Nat Rev Immunol.2010 Jun;10(6):460. PMID: 19029990; PMCID: PMC2724991. 1853. Zoglmeier C, Bauer H, Noerenberg D, Wedekind G, Bittner P, Sandholzer N, Rapp M, Anz D, Endres S, Bourquin C. CpG blocks immunosuppression by myeloid-derived suppressor cells in tumor-bearing mice. Clin Cancer Res. 2011 Apr 1;17(7):176575. doi: 10.1158/1078-0432.CCR-10-2672. Epub 2011 Jan 13. Erratum in: Clin Cancer Res. 2017 Feb 15;23 (4):1117. PMID: 21233400 .

1854. Kumagai Y, Takeuchi O, Kato H, Kumar H, Matsui K, Morii E, Aozasa K, Kawai T, Akira S. Alveolar macrophages are the primary interferon-alpha producer in pulmonary infection with RNA viruses. Immunity. 2007 Aug;27(2):240-52. doi: 10.1016/j.immuni.2007.07.013. PMID: 17723216.

1855. Koerner P, Traeger T, Mehmcke H, Cziupka K, Kessler W, Busemann A, Diedrich S, Hartmann G, Heidecke CD, Maier S. Stimulation of TLR7 prior to polymicrobial sepsis improves the immune control of the inflammatory response in adult mice. Inflamm Res. 2011 Mar;60(3):271-9. doi: 10.1007/s00011-010-0265-6. Epub 2010 Oct 17. PMID: 20953969.

1856. Heer AK, Shamshiev A, Donda A, Uematsu S, Akira S, Kopf M, Marsland BJ. TLR signaling fine-tunes anti-influenza B cell responses without regulating effector T cell responses. J Immunol. 2007 Feb 15;178(4):2182-91. doi:

10.4049/jimmunol.178.4.2182. PMID: 17277123.

1857. Goff PH, Hayashi T, He W, Yao S, Cottam HB, Tan GS, Crain B, Krammer F, Messer K, Pu M, Carson DA, Palese P, Corr M. Synthetic Toll-Like Receptor 4 (TLR4) and TLR7 Ligands Work Additively via MyD88 To Induce Protective Antiviral Immunity in Mice. J Virol. 2017 Sep 12;91(19):e01050-17. doi: 10.1128/JVI.01050-17. PMID: 28724768; PMCID: PMC5599744.

1858. Chothe SK, Nissly RH, Lim L, Bhushan G, Bird I, Radzio-Basu J, Jayarao BM, Kuchipudi SV. NLRC5 Serves as a Pro-viral Factor During Influenza Virus Infection in Chicken Macrophages. Front Cell Infect Microbiol. 2020 May 19;10:230. doi: 10.3389/fcimb.2020.00230. PMID: 32509599; PMCID: PMC7248199.

1859. Staehli F, Ludigs K, Heinz LX, Seguín-Estévez Q, Ferrero I, Braun M, Schroder K, Rebsamen M, Tardivel A, Mattmann C, MacDonald HR, Romero P, Reith W, Guarda G, Tschopp J. NLRC5 deficiency selectively impairs MHC class I- dependent lymphocyte killing by cytotoxic T cells. J Immunol. 2012 Apr 15;188(8):3820-8. doi: 10.4049/jimmunol.1102671. Epub 2012 Mar 12. PMID: 22412192.

1860. Neerincx A, Rodriguez GM, Steimle V, Kufer TA. NLRC5 controls basal MHC class I gene expression in an MHC enhanceosome-dependent manner. J Immunol. 2012 May 15;188(10):4940-50. doi: 10.4049/jimmunol.1103136. Epub 2012 Apr 6. PMID: 22490867.

1861. Xu X, Shi Y, Luan P, Kou W, Li B, Zhai M, You S, Yu Q, Zhuang J, Jian W, Feinberg MW, Peng W. The subcellular redistribution of NLRC5 promotes angiogenesis via interacting with STAT3 in endothelial cells. Theranostics. 2021 Mar 4;11(9):4483-4501. doi: 10.7150/thno.54473. PMID: 33754073; PMCID: PMC7977449.

1862. Jafarzadeh A, Nemati M, Jafarzadeh S. Contribution of STAT3 to the pathogenesis of COVID-19. Microb Pathog. 2021 May;154:104836. doi: 10.1016/j.micpath.2021.104836. Epub 2021 Mar 7. PMID: 33691172; PMCID: PMC7937040.

1863. Neerincx A, Castro W, Guarda G, Kufer TA. NLRC5, at the Heart of Antigen Presentation. Front Immunol. 2013 Nov 22;4:397. doi: 10.3389/fimmu.2013.00397. PMID: 24319445; PMCID: PMC3837245.

1864. Kuenzel S, Till A, Winkler M, Häsler R, Lipinski S, Jung S, Grötzinger J, Fickenscher H, Schreiber S, Rosenstiel P. The nucleotide-binding oligomerization domain-like receptor NLRC5 is involved in IFN-dependent antiviral immune responses. J Immunol. 2010 Feb 15;184(4):1990-2000. doi: 10.4049/jimmunol.0900557. Epub 2010 Jan 8. PMID: 20061403.

1865. Sun T, Ferrero RL, Girardin SE, Gommerman JL, Philpott DJ. NLRC5 deficiency has a moderate impact on immunodominant CD8+ T-cell responses during rotavirus infection of adult mice. Immunol Cell Biol. 2019 Jul;97(6):552-562. doi: 10.1111/imcb.12244. Epub 2019 Mar 29. PMID: 30768806

1866. Yu J, Wu H, Liu ZY, Zhu Q, Shan C, Zhang KQ. Advanced glycation end products induce the apoptosis of and inflammation in mouse podocytes through CXCL9-mediated JAK2/STAT3 pathway activation. Int J Mol Med. 2017 Oct;40(4):1185-1193. doi: 10.3892/ijmm.2017.3098. Epub 2017 Aug 11. PMID: 28849106; PMCID: PMC5593472.

1867. Tong Y, Cui J, Li Q, Zou J, Wang HY, Wang RF. Enhanced TLR-induced NF-KB signaling and type I interferon responses in NLRC5 deficient mice. Cell Res. 2012 May;22(5):822-35. doi: 10.1038/cr.2012.53. Epub 2012 Apr 3. PMID: 22473004; PMCID: PMC3343662

1868. Cui J, Zhu L, Xia X, Wang HY, Legras X, Hong J, Ji J, Shen P, Zheng S, Chen ZJ, Wang RF. NLRC5 negatively regulates the NFkappaB and type I interferon signaling pathways. Cell. 2010 Apr 30;141(3):483-96. doi: 10.1016/j.cell.2010.03.040. PMID: 20434986; PMCID: PMC3150216. 
1869. Jie Chen, Judith Brands, Marta Bueno, Heiko Adler, Ana Mora, Silke Meiners, Ilona Kammerl, Effect of cigarette smoke on immunoproteasome function during virus infection, ERJ Open Research Mar 2019, 5 (suppl 2) PP119; DOI: 10.1183/23120541.lungscienceconference-2019.PP119

1870. Shanze Chen, Ilona Keller, Oliver Vosyka, Anke-Gabriele Lenz, Oliver Eickelberg, Silke Meiners, Tobias Stoeger,Polarization of alveolar macrophages is regulated by the immunoproteasome, European Respiratory Journal Sep 2014, 44 (Suppl 58) P879; 1871. Michael Basler, Marleen, Christ, Heike Goebel, Marcus Groettrup, Immunoproteasome Upregulation Is Not Required to Control Protein Homeostasis during Viral Infection, The Journal of Immunology March 17, 2021, ji2000822; DOI: 10.4049/jimmunol.2000822

1872. Hiroaki Kimura, Tadayoshi Karasawa, Sachiko Watanabe, Koichi Suzuki, Patrizio Caturegli, Masafumi Takahashi, The Journa of Immunology Contribution of the immunoproteasome to the inflammatory phenotype observed in obese mice, May 1, 2017, 198 (1 Supplement) 206.25;

1873. Dietmar M. W. Zaiss, Cornelis P. J. Bekker, Andrea Gröne, Benedicte A. Lie, Alice J. A. M. Sijts, Proteasome Immunosubunits Protect against the Development of CD8 T Cell-Mediated Autoimmune Diseases, The Journal of Immunology September 1, 2011, 187 (5) 2302-2309; DOI: 10.4049/jimmunol.1101003

1874. Philipp Osterloh, Kathrin Linkemann, Stefan Tenzer, Hans-Georg Rammensee, Markus P. Radsak, Dirk H. Busch, and Hansjörg Schild, Proteasomes shape the repertoire of T cells participating in antigen-specific immune responses 1875. Scott T. Kuhns, Michelle D. Tallquist, Aaron J. Johnson, Yanice Mendez-Fernandez, and Larry R. Pease, T cell receptor interactions with class I heavy-chain influence T cell selection

1876. Britta Strehl, Thorsten Joeris, Melanie Rieger, Alexander Visekruna, Kathrin Textoris-Taube, Stefan H. E. Kaufmann, PeterMichael Kloetzel, Ulrike Kuckelkorn and Ulrich Steinhoff, Immunoproteasomes Are Essential for Clearance of Listeria monocytogenes in Nonlymphoid Tissues but Not for Induction of Bacteria-Specific CD8+ T Cells, J Immunol November 1, 2006, 177 (9) 6238-6244; DOI: https://doi.org/10.4049/jimmunol.177.9.6238

1877. Ilona Keller, Angela Dann, Isis Fernandez-Buelvaz, Shinji Takenaka, Andreas Günther, Oliver Eickelberg, Silke Meiners, Immunoproteasome expression in pulmonary fibrosis, European Respiratory Journal Sep 2013, 42 (Suppl 57) P4739; 1878. Xuan He, Francesco Bonella, Miao-tian Cai, Toshio Mori, Josune Guzman, Ulrich Costabel, Expression of immunoproteasome subunits LMP2 and LMP7 by alveolar macrophages is reduced in patients with idiopathic pulmonary fibrosis, European Respiratory Journal Sep 2013, 42 (Suppl 57) 3524;

1879. Faezeh Fathi Aghdam, Alan Hsu, Kristy Parsons, Simon Keely, Lisa Wood, Peter Wark, Oxidative stress impairs mitochondrial function and leads to deficient antiviral responses in primary bronchial epithelial cells, European Respiratory Journal Sep 2014, 44 (Suppl 58) P4216;

1880. Serena Di Vincenzo, Claudia Sangiorgi, Maria Ferraro, Mark Gjomarkaj, Dennis K Ninaber, Pieter S Hiemstra, Elisabetta Pace, Cigarette smoke decreases E-Cadherin expression downregulating FoxO3 in lung epithelial cells, European Respiratory Journal Sep 2019, 54 (suppl 63) PA1678; DOI: 10.1183/13993003.congress-2019.PA1678

1881. Faezeh Fathi Aghdam, Lisa Wood, Alan Hsu, Kristy Parsons, Simon Keely, Peter Wark, Comparing the effects of oxidative stress on mitochondrial function and antiviral responses in primary bronchial epithelial cells of asthmatics and non-asthmatics, European Respiratory Journal Sep 2014, 44 (Suppl 58) P2499;

1882. Peter Wark, Prabuddha Pathinyake, Alan Hsu, Kristy Parsons, Lisa Wood, Effect of oxidative stress and rhinovirus infection on mitochondrial/endoplasmic reticular function in human primary bronchial epithelial cells, European Respiratory Journal Sep 2016, 48 (suppl 60) PA3989; DOI: 10.1183/13993003.congress-2016.PA3989

1883. Ilona Kammerl, Shanze Chen, Martin Irmler, Johannes Beckers, Oliver Eickelberg, Silke Meiners, Tobias Stöger, Deficiency of immunoproteasome subunit LMP2 increases alternative polarization of alveolar macrophages, European Respiratory Journal Sep 2016, 48 (suppl 60) PA1817; DOI: 10.1183/13993003.congress-2016.PA1817

1884. Hardarson HS, Baker JS, Yang Z, Purevjav E, Huang CH, Alexopoulou L, Li N, Flavell RA, Bowles NE, Vallejo JG. Toll-like receptor 3 is an essential component of the innate stress response in virus-induced cardiac injury. Am J Physiol Heart Circ Physiol. 2007 Jan;292(1):H251-8. doi: 10.1152/ajpheart.00398.2006. Epub 2006 Aug 25. PMID: 16936008.

1885. Vij N, Chandramani-Shivalingappa P, Van Westphal C, Hole R, Bodas M. Cigarette smoke-induced autophagy impairment accelerates lung aging, COPD-emphysema exacerbations and pathogenesis. Am J Physiol Cell Physiol. 2018 Jan 1;314(1):C73-C87. doi: 10.1152/ajpcell.00110.2016. Epub 2016 Jul 13. PMID: 27413169; PMCID: PMC5866380.

1886. An $\mathrm{CH}$, Wang XM, Lam HC, Ifedigbo E, Washko GR, Ryter SW, Choi AM. TLR4 deficiency promotes autophagy during cigarette smoke-induced pulmonary emphysema. Am J Physiol Lung Cell Mol Physiol. 2012 Nov 1;303(9):L748-57. doi: 10.1152/ajplung.00102.2012. Epub 2012 Sep 14. PMID: 22983353; PMCID: PMC3517684.

1887. Selcuk Yatmaz, Huei Jiunn Seow, Rosa Gualano, Zi Xin Wong, Peter Crack, Steven Bozinovski, Gary Anderson, Ross Vlahos, Glutathione peroxidase-1 as a therapeutic target in influenza A virus-induced lung disease, European Respiratory Journal Sep 2011, 38 (Suppl 55) p4357.

1888. Kim HP, Wang X, Chen ZH, Lee SJ, Huang MH, Wang Y, Ryter SW, Choi AM. Autophagic proteins regulate cigarette smokeinduced apoptosis: protective role of heme oxygenase-1. Autophagy. 2008 Oct;4(7):887-95. doi: 10.4161/auto.6767. Epub 2008 Oct 12. PMID: 18769149.

1889. Guan SP, Tee W, Ng DS, Chan TK, Peh HY, Ho WE, Cheng C, Mak JC, Wong WS. Andrographolide protects against cigarette smoke-induced oxidative lung injury via augmentation of Nrf2 activity. Br J Pharmacol. 2013 Apr;168(7):1707-18. doi:

10.1111/bph.12054. PMID: 23146110; PMCID: PMC3605877.

1890. Ng DS, Liao W, Tan WS, Chan TK, Loh XY, Wong WS. Anti-malarial drug artesunate protects against cigarette smoke-induced lung injury in mice. Phytomedicine. 2014 Oct 15;21(12):1638-44. doi: 10.1016/j.phymed.2014.07.018. Epub 2014 Sep 1. PMID: 25442271.

1891. Zhu T, Chen Z, Chen G, Wang D, Tang S, Deng H, Wang J, Li S, Lan J, Tong J, Li H, Deng X, Zhang W, Sun J, Tu Y, Luo W, Li C. Curcumin Attenuates Asthmatic Airway Inflammation and Mucus Hypersecretion Involving a PPARY-Dependent NF-KB Signaling Pathway In Vivo and In Vitro. Mediators Inflamm. 2019 Apr 3;2019:4927430. doi: 10.1155/2019/4927430. PMID: 31073274; PMCID: PMC6470457.

1892. Sun X, Feng X, Zheng D, Li A, Li C, Li S, Zhao Z. Ergosterol attenuates cigarette smoke extract-induced COPD by modulating inflammation, oxidative stress and apoptosis in vitro and in vivo. Clin Sci (Lond). 2019 Jul 15;133(13):1523-1536. doi: 10.1042/CS20190331. Erratum in: Clin Sci (Lond). 2019 Nov 15;133(21):2237. PMID: 31270147.

1893. Fujimoto K, Karuppuchamy T, Takemura N, Shimohigoshi M, Machida T, Haseda Y, Aoshi T, Ishii KJ, Akira S, Uematsu S. A new subset of CD103+CD8alpha+ dendritic cells in the small intestine expresses TLR3, TLR7, and TLR9 and induces Th1 response and CTL activity. J Immunol. 2011 Jun 1;186(11):6287-95. doi: 10.4049/jimmunol.1004036. Epub 2011 Apr 27. PMID: 21525388. 1894. Gangaplara A, Martens C, Dahlstrom E, Metidji A, Gokhale AS, Glass DD, Lopez-Ocasio M, Baur R, Kanakabandi K, Porcella $\mathrm{SF}$, Shevach EM. Type I interferon signaling attenuates regulatory T cell function in viral infection and in the tumor 
microenvironment. PLoS Pathog. 2018 Apr 19;14(4):e1006985. doi: 10.1371/journal.ppat.1006985. PMID: 29672594; PMCID: PMC5929570.

1895. Ousman SS, Wang J, Campbell IL. Differential regulation of interferon regulatory factor (IRF)-7 and IRF-9 gene expression in the central nervous system during viral infection. J Virol. 2005 Jun;79(12):7514-27. doi: 10.1128/JVI.79.12.7514-7527.2005. PMID: 15919906; PMCID: PMC1143633

1896. Schultz KLW, Troisi EM, Baxter VK, Glowinski R, Griffin DE. Interferon regulatory factors 3 and 7 have distinct roles in the pathogenesis of alphavirus encephalomyelitis. J Gen Virol. 2019 Jan;100(1):46-62. doi: 10.1099/jgv.0.001174. Epub 2018 Nov 19. PMID: 30451651; PMCID: PMC7011693.

1897. Yang L, Tu L, Zhao P, Wang Y, Wang S, Lu W, Wang Y, Li X, Yu Y, Hua S, Wang L. Attenuation of interferon regulatory factor 7 activity in local infectious sites of trachea and lung for preventing the development of acute lung injury caused by influenza $A$ virus. Immunology. 2019 May;157(1):37-51. doi: 10.1111/imm.13045. Epub 2019 Feb 27. PMID: 30667045; PMCID: PMC6459777. 1898. Pang IK, Pillai PS, Iwasaki A. Efficient influenza A virus replication in the respiratory tract requires signals from TLR7 and RIG-I. Proc Natl Acad Sci U S A. 2013 Aug 20;110(34):13910-5. doi: 10.1073/pnas.1303275110. Epub 2013 Aug 5. PMID: 23918369; PMCID: PMC3752242

1899. Wang X, Wu W, Zhang W, Leland Booth J, Duggan ES, Tian L, More S, Zhao YD, Sawh RN, Liu L, Zou MH, Metcalf JP. RIG-I overexpression decreases mortality of cigarette smoke exposed mice during influenza A virus infection. Respir Res. 2017 Sep 2;18(1):166. doi: 10.1186/s12931-017-0649-z. PMID: 28865477 ; PMCID: PMC5581920.

1900. Hatesuer B, Hoang HT, Riese P, Trittel S, Gerhauser I, Elbahesh H, Geffers R, Wilk E, Schughart K. Deletion of Irf3 and Irf7 Genes in Mice Results in Altered Interferon Pathway Activation and Granulocyte-Dominated Inflammatory Responses to Influenza A Infection. J Innate Immun. 2017;9(2):145-161. doi: 10.1159/000450705. Epub 2016 Nov 4. PMID: 27811478; PMCID: PMC6738875.

1901. Lin HY, Yang YL, Wang PW, Wang FS, Huang YH. The Emerging Role of MicroRNAs in NAFLD: Highlight of MicroRNA-29a in Modulating Oxidative Stress, Inflammation, and Beyond. Cells. 2020 Apr 22;9(4):1041. doi: 10.3390/cells9041041. PMID: 32331364; PMCID: PMC7226429.

1902. Wu W, Wang X, Zhang W, Tian L, Booth JL, Duggan ES, More S, Liu L, Dozmorov M, Metcalf JP. RIG-I Signaling via MAVS Is Dispensable for Survival in Lethal Influenza Infection In Vivo. Mediators Inflamm. 2018 Nov 8;2018:6808934. doi: 10.1155/2018/6808934. PMID: 30532653; PMCID: PMC6250004.

1903. Wu W, Zhang W, Tian L, Brown BR, Walters MS, Metcalf JP. IRF7 Is Required for the Second Phase Interferon Induction during Influenza Virus Infection in Human Lung Epithelia. Viruses. 2020 Mar 29;12(4):377. doi: 10.3390/v12040377. PMID: 32235406; PMCID: PMC7232147.

1904. Steinberg C, Eisenächer K, Gross O, ReindI W, Schmitz F, Ruland J, Krug A. The IFN regulatory factor 7-dependent type I IFN response is not essential for early resistance against murine cytomegalovirus infection. Eur J Immunol. 2009 Apr;39(4):1007-18. doi: 10.1002/eji.200838814. PMID: 19283778.

1905. Kulkarni RR, Rasheed MA, Bhaumik SK, Ranjan P, Cao W, Davis C, Marisetti K, Thomas S, Gangappa S, Sambhara S, MuraliKrishna K. Activation of the RIG-I pathway during influenza vaccination enhances the germinal center reaction, promotes $T$ follicular helper cell induction, and provides a dose-sparing effect and protective immunity. J Virol. 2014 Dec;88(24):13990-4001. doi: 10.1128/JVI.02273-14. Epub 2014 Sep 24. PMID: 25253340; PMCID: PMC4249139.

1906. Forbester JL, Clement M, Wellington D, Yeung A, Dimonte S, Marsden M, Chapman L, Coomber EL, Tolley C, Lees E, Hale C, Clare S, Udalova I, Dong T, Dougan G, Humphreys IR. IRF5 Promotes Influenza Virus-Induced Inflammatory Responses in Human Induced Pluripotent Stem Cell-Derived Myeloid Cells and Murine Models. J Virol. 2020 Apr 16;94(9):e00121-20. doi: 10.1128/JVI.00121-20. PMID: 32075938; PMCID: PMC7163152.

1907. Maarouf M, Chen B, Chen Y, Wang X, Rai KR, Zhao Z, Liu S, Li Y, Xiao M, Chen JL. Identification of IncRNA-155 encoded by MIR155HG as a novel regulator of innate immunity against influenza A virus infection. Cell Microbiol. 2019 Aug;21(8):e13036. doi: 10.1111/cmi.13036. Epub 2019 May 29. PMID: 31045320.

1908. Elton TS, Selemon H, Elton SM, Parinandi NL. Regulation of the MIR155 host gene in physiological and pathological processes. Gene. 2013 Dec 10;532(1):1-12. doi: 10.1016/j.gene.2012.12.009. Epub 2012 Dec 14. PMID: 23246696. 1909. Zhang J, Zhu L, Shi H, Zheng H. Protective effects of miR-155-5p silencing on IFN- $\gamma$-induced apoptosis and inflammation in salivary gland epithelial cells. Exp Ther Med. 2021 Aug;22(2):882. doi: 10.3892/etm.2021.10314. Epub 2021 Jun 15. PMID: 34194560; PMCID: PMC8237265.

1910. He L, Wang Z, Zhou R, Xiong W, Yang Y, Song N, Qian J. Dexmedetomidine exerts cardioprotective effect through miR-146a$3 p$ targeting IRAK1 and TRAF6 via inhibition of the NF-KB pathway. Biomed Pharmacother. 2021 Jan;133:110993. doi: 10.1016/j.biopha.2020.110993. Epub 2020 Nov 18. PMID: 33220608.

1911. Zhang Q, Yu H, Yang Z, Li L, He Y, Zhu S, Li C, Zhang S, Luo B, Gao Y. A Functional Indel Polymorphism Within MIR155HG Is Associated With Sudden Cardiac Death Risk in a Chinese Population. Front Cardiovasc Med. 2021 May 31;8:671168. doi: 10.3389/fcvm.2021.671168. PMID: 34136547; PMCID: PMC8200405.

1912. Laha S, Saha C, Dutta S, Basu M, Chatterjee R, Ghosh S, Bhattacharyya NP. In silico analysis of altered expression of long non-coding RNA in SARS-CoV-2 infected cells and their possible regulation by STAT1, STAT3 and interferon regulatory factors. Heliyon. 2021 Mar;7(3):e06395.doi: 10.1016/j.heliyon.2021.e06395. Epub 2021 Feb 27. PMID: 33688586; PMCID: PMC7914022. 1913. Rahim MMA, Parsons BD, Price EL, Slaine PD, Chilvers BL, Seaton GS, Wight A, Medina-Luna D, Dey S, Grandy SL, Anderson LE, Zamorano Cuervo N, Grandvaux N, Gaglia MM, Kelvin AA, Khaperskyy DA, McCormick C, Makrigiannis AP. Defective Influenza A Virus RNA Products Mediate MAVS-Dependent Upregulation of Human Leukocyte Antigen Class I Proteins. J Virol. 2020 Jun 16;94(13):e00165-20. doi: 10.1128/JVI.00165-20. PMID: 32321802; PMCID: PMC7307169.

1914. Lee NR, Ban J, Lee NJ, Yi CM, Choi JY, Kim H, Lee JK, Seong J, Cho NH, Jung JU, Inn KS. Activation of RIG-I-Mediated Antiviral Signaling Triggers Autophagy Through the MAVS-TRAF6-Beclin-1 Signaling Axis. Front Immunol. 2018 Sep 12;9:2096. doi: 10.3389/fimmu.2018.02096. PMID: 30258449; PMCID: PMC6143786.

1915. Hüsser L, Alves MP, Ruggli N, Summerfield A. Identification of the role of RIG-I, MDA-5 and TLR3 in sensing RNA viruses in porcine epithelial cells using lentivirus-driven RNA interference. Virus Res. 2011 Jul;159(1):9-16. doi:

10.1016/j.virusres.2011.04.005. Epub 2011 Apr 22. PMID: 21539869.

1916. Huang YL, Huang MT, Sung PS, Chou TY, Yang RB, Yang AS, Yu CM, Hsu YW, Chang WC, Hsieh SL. Endosomal TLR3 coreceptor CLEC18A enhances host immune response to viral infection. Commun Biol. 2021 Feb 18;4(1):229. doi: 10.1038/s42003021-01745-7. PMID: 33603190; PMCID: PMC7893028.

1917. Li RF, Zhou XB, Zhou HX, Yang ZF, Jiang HM, Wu X, Li WJ, Qiu JJ, Mi JN, Chen M, Zhong NS, Zhu GY, Jiang ZH. Novel Fatty Acid in Cordyceps Suppresses Influenza A (H1N1) Virus-Induced Proinflammatory Response Through Regulating Innate Signaling Pathways. ACS Omega. 2021 Jan 7;6(2):1505-1515. doi: 10.1021/acsomega.0c05264. PMID: 33490810; PMCID: PMC7818636. 
1918. Cai W, Chen S, Li Y, Zhang A, Zhou H, Chen H, Jin M. 14-Deoxy-11,12-didehydroandrographolide attenuates excessive inflammatory responses and protects mice lethally challenged with highly pathogenic $A(H 5 N 1)$ influenza viruses. Antiviral Res. 2016 Sep;133:95-105. doi: 10.1016/j.antiviral.2016.07.020. Epub 2016 Jul 28. PMID: 27476045.

1919. Jia D, Rahbar R, Chan RW, Lee SM, Chan MC, Wang BX, Baker DP, Sun B, Peiris JS, Nicholls JM, Fish EN. Influenza virus nonstructural protein 1 (NS1) disrupts interferon signaling. PLoS One. 2010 Nov 10;5(11):e13927. doi: 10.1371/journal.pone.0013927. PMID: 21085662; PMCID: PMC2978095.

1920. Wang BX, Fish EN. Global virus outbreaks: Interferons as 1st responders. Semin Immunol. 2019 Jun;43:101300. doi: 10.1016/j.smim.2019.101300. PMID: 31771760; PMCID: PMC7128104.

1921. Zhao J, Wang Y, Huang X, Ma Q, Song J, Wu X, Zhou H, Weng Y, Yang Z, Wang X. Liu Shen Wan inhibits influenza virusinduced secondary Staphylococcus aureus infection in vivo and in vitro. J Ethnopharmacol. 2021 Sep 15;277:114066. doi: 10.1016/j.jep.2021.114066. Epub 2021 Mar 23. PMID: 33766755

1922. Conti P, Caraffa A, Tetè G, Gallenga CE, Ross R, Kritas SK, Frydas I, Younes A, Di Emidio P, Ronconi G. Mast cells activated by SARS-CoV-2 release histamine which increases IL-1 levels causing cytokine storm and inflammatory reaction in COVID-19. J Biol Regul Homeost Agents. 2020 Sep-Oct,;34(5):1629-1632. doi: 10.23812/20-2EDIT. PMID: 32945158.

1923. Zarnegar B, Westin A, Evangelidou S, Hallgren J. Innate Immunity Induces the Accumulation of Lung Mast Cells During Influenza Infection. Front Immunol. 2018 Oct 4;9:2288. doi: 10.3389/fimmu.2018.02288. PMID: 30337928; PMCID: PMC6180200. 1924. Murphy-Schafer AR, Paust S. Divergent Mast Cell Responses Modulate Antiviral Immunity During Influenza Virus Infection. Front Cell Infect Microbiol. 2021 Feb 19;11:580679. doi: 10.3389/fcimb.2021.580679. PMID: 33680987; PMCID: PMC7935524. 1925. Toy R, Keenum MC, Pradhan P, Phang K, Chen P, Chukwu C, Nguyen LAH, Liu J, Jain S, Kozlowski G, Hosten J, Suthar MS, Roy K. TLR7 and RIG-I dual-adjuvant loaded nanoparticles drive broadened and synergistic responses in dendritic cells in vitro and generate unique cellular immune responses in influenza vaccination. J Control Release. 2021 Feb 10;330:866-877. doi: 10.1016/j.jconrel.2020.10.060. Epub 2020 Nov 5. PMID: 33160004; PMCID: PMC7906919.

1926. Rojas-Quintero J, Wang X, Tipper J, Burkett PR, Zuñiga J, Ashtekar AR, Polverino F, Rout A, Yambayev I, Hernández C, Jimenez L, Ramírez G, Harrod KS, Owen CA. Matrix metalloproteinase- 9 deficiency protects mice from severe influenza A viral infection. JCl Insight. 2018 Dec 20;3(24):e99022. doi: 10.1172/jci.insight.99022. PMID: 30568032; PMCID: PMC6338318. 1927. Mishra R, Banerjea AC. SARS-CoV-2 Spike Targets USP33-IRF9 Axis via Exosomal miR-148a to Activate Human Microglia. Front Immunol. 2021 Apr 14;12:656700. doi: 10.3389/fimmu.2021.656700. PMID: 33936086; PMCID: PMC8079643. 1928. Barchet W, Krug A, Cella M, Newby C, Fischer JA, Dzionek A, Pekosz A, Colonna M. Dendritic cells respond to influenza virus through TLR7- and PKR-independent pathways. Eur J Immunol. 2005 Jan;35(1):236-42. doi: 10.1002/eji.200425583. PMID: 15593126.

1929. Diebold SS, Kaisho T, Hemmi H, Akira S, Reis e Sousa C. Innate antiviral responses by means of TLR7-mediated recognition of single-stranded RNA. Science. 2004 Mar 5;303(5663):1529-31. doi: 10.1126/science.1093616. Epub 2004 Feb 19. PMID: 14976261.

1930. Ling LJ, Lu Y, Zhang YY, Zhu HY, Tu P, Li H, Chen DF. Flavonoids from Houttuynia cordata attenuate H1N1-induced acute lung injury in mice via inhibition of influenza virus and Toll-like receptor signalling. Phytomedicine. 2020 Feb;67:153150. doi: 10.1016/j.phymed.2019.153150. Epub 2019 Dec 16. PMID: 31958713.

1931. Burbage M, Amigorena S. Antigen-Primed CD8+ T Cells Call DCs for Back Up. Immunity. 2017 Feb 21;46(2):163-164. doi: 10.1016/j.immuni.2017.02.002. PMID: 28228271.

1932. Castellino F, Huang AY, Altan-Bonnet G, Stoll S, Scheinecker C, Germain RN. Chemokines enhance immunity by guiding naive CD8+ T cells to sites of CD4+ T cell-dendritic cell interaction. Nature. 2006 Apr 13;440(7086):890-5. doi: 10.1038/nature04651. PMID: 16612374

1933. Hackler Y, Siebenhaar F, Löhning M, Maurer M, Muñoz M. Mast Cells Modulate Antigen-Specific CD8+ T Cell Activation During LCMV Infection. Front Immunol. 2021 Jun 14;12:688347. doi: 10.3389/fimmu.2021.688347. PMID: 34194439; PMCID: PMC8236703.

1934. Khanna KM, Aguila CC, Redman JM, Suarez-Ramirez JE, Lefrançois L, Cauley LS. In situ imaging reveals different responses by naïve and memory CD8 T cells to late antigen presentation by lymph node DC after influenza virus infection. Eur J Immunol 2008 Dec;38(12):3304-15. doi: 10.1002/eji.200838602. PMID: 19009527; PMCID: PMC2662394.

1935. Ballesteros-Tato A, León B, Lee BO, Lund FE, Randall TD. Epitope-specific regulation of memory programming by differential duration of antigen presentation to influenza-specific CD8(+) T cells. Immunity. 2014 Jul 17;41(1):127-40. doi:

10.1016/j.immuni.2014.06.007. PMID: 25035957; PMCID: PMC4233138.

1936. Dolfi DV, Duttagupta PA, Boesteanu AC, Mueller YM, Oliai CH, Borowski AB, Katsikis PD. Dendritic cells and CD28

costimulation are required to sustain virus-specific CD8+ T cell responses during the effector phase in vivo. J Immunol. $2011 \mathrm{Apr}$ 15;186(8):4599-608. doi: 10.4049/jimmunol.1001972. Epub 2011 Mar 9. PMID: 21389258.

1937. Seah SG, Carrington EM, Ng WC, Belz GT, Brady JL, Sutherland RM, Hancock MS, La Gruta NL, Brown LE, Turner SJ, Zhan Y, Lew AM. Unlike CD4+ T-cell help, CD28 costimulation is necessary for effective primary CD8+ T-cell influenza-specific immunity. Eur J Immunol. 2012 Jul;42(7):1744-54. doi: 10.1002/eji.201142211. Epub 2012 Jun 14. PMID: 22585421.

1938. Reimann J, Böhm W, Schirmbeck R. Alternative processing pathways for MHC class I-restricted epitope presentation to CD8+ cytotoxic T lymphocytes. Biol Chem Hoppe Seyler. 1994 Nov;375(11):731-6. doi: 10.1515/bchm3.1994.375.11.731. PMID: 7535058 .

1939. Deronic A, Nilsson A, Thagesson M, Werchau D, Enell Smith K, Ellmark P. The human anti-CD40 agonist antibody mitazalimab (ADC-1013; JNJ-64457107) activates antigen-presenting cells, improves expansion of antigen-specific T cells, and enhances anti-tumor efficacy of a model cancer vaccine in vivo. Cancer Immunol Immunother. 2021 May 5. doi: 10.1007/s00262021-02932-5. Epub ahead of print. PMID: 33948686.

1940. Seah SG, Brady JL, Carrington EM, Ng WC, Sutherland RM, Hancock MS, La Gruta NL, Brown LE, Turner SJ, Lew AM, Zhan Y. Influenza-induced, helper-independent CD8+ T cell responses use CD40 costimulation at the late phase of the primary response. J Leukoc Biol. 2013 Jan;93(1):145-54. doi: 10.1189/jlb.0612266. Epub 2012 Oct 29. PMID: 23108101

1941. Van Den Berg TK, Hasbold J, Renardel De Lavalette C, Döpp EA, Dijkstra CD, Klaus GG. Properties of mouse CD40: differential expression of CD40 epitopes on dendritic cells and epithelial cells. Immunology. 1996;88(2):294-300. doi:10.1111/j.1365-2567.1996.tb00018.x

1942. van Kooten C, Banchereau J. Functions of CD40 on B cells, dendritic cells and other cells. Curr Opin Immunol. 1997 Jun;9(3):330-7. doi: 10.1016/s0952-7915(97)80078-7. PMID: 9203418

1943. Oxenius A, Campbell KA, Maliszewski CR, Kishimoto T, Kikutani H, Hengartner H, Zinkernagel RM, Bachmann MF. CD40CD40 ligand interactions are critical in T-B cooperation but not for other anti-viral CD4+ T cell functions. J Exp Med. 1996 May 1;183(5):2209-18. doi: 10.1084/jem.183.5.2209. PMID: 8642330; PMCID: PMC2192545. 
1944. Castro A, Ozturk K, Zanetti M, Carter H. In silico analysis suggests less effective MHC-II presentation of SARS-CoV-2 RBM peptides: Implication for neutralizing antibody responses. PLoS One. 2021 Feb 11;16(2):e0246731. doi: 10.1371/journal.pone.0246731. PMID: 33571241; PMCID: PMC7877779.

1945. Grewal IS, Flavell RA. The role of CD40 ligand in costimulation and T-cell activation. Immunol Rev. 1996 Oct;153:85-106. doi: 10.1111/j.1600-065x.1996.tb00921.x. PMID: 9010720.

1946. Borrow P, Tishon A, Lee S, Xu J, Grewal IS, Oldstone MB, Flavell RA. CD40L-deficient mice show deficits in antiviral immunity and have an impaired memory CD8+ CTL response. J Exp Med. 1996 May 1;183(5):2129-42. doi: 10.1084/jem.183.5.2129. PMID: 8642323; PMCID: PMC2192549.

1947. Huang Q, Hu J, Tang J, Xu L, Ye L. Molecular Basis of the Differentiation and Function of Virus Specific Follicular Helper CD4+ T Cells. Front Immunol. 2019 Feb 15;10:249. doi: 10.3389/fimmu.2019.00249. PMID: 30828337; PMCID: PMC6384271.

1948. Wang M, Huang Y, Gu W, Wang H. Evaluation of T Follicular Helper Cells and Germinal Center Response During Influenza A Virus Infection in Mice. J Vis Exp. 2020 Jun 27;(160). doi: 10.3791/60523. PMID: 32658200.

1949. Andreasen SO, Christensen JE, Marker O, Thomsen AR. Role of CD40 ligand and CD28 in induction and maintenance of antiviral CD8+ effector T cell responses. J Immunol. 2000 Apr 1;164(7):3689-97. doi: 10.4049/jimmunol.164.7.3689. PMID: 10725727.

1950. Borrow P, Tough DF, Eto D, Tishon A, Grewal IS, Sprent J, Flavell RA, Oldstone MB. CD40 ligand-mediated interactions are involved in the generation of memory CD8(+) cytotoxic T lymphocytes (CTL) but are not required for the maintenance of CTL memory following virus infection. J Virol. 1998 Sep;72(9):7440-9. doi: 10.1128/JVI.72.9.7440-7449.1998. PMID: 9696840; PMCID: PMC109974.

1951. Durlanik S, Loyal L, Stark R, Sercan Alp Ö, Hartung A, Radbruch A, von Herrath M, Matzmohr N, Frentsch M, Thiel A. CD40L expression by $\mathrm{CD} 4+$ but not CD8+ T cells regulates antiviral immune responses in acute LCMV infection in mice. Eur J Immunol. 2016 Nov;46(11):2566-2573. doi: 10.1002/eji.201646420. Epub 2016 Sep 20. PMID: 27562840

1952. Bhadra R, Cobb DA, Khan IA. CD40 signaling to the rescue: A CD8 exhaustion perspective in chronic infectious diseases. Crit Rev Immunol. 2013;33(4):361-78. doi: 10.1615/critrevimmunol.2013007444. PMID: 23971530; PMCID: PMC4051158. 1953. Xu A, Wang R, Freywald A, Stewart K, Tikoo S, Xu J, Zheng C, Xiang J. CD40 agonist converting CTL exhaustion via the activation of the mTORC1 pathway enhances PD-1 antagonist action in rescuing exhausted CTLs in chronic infection. Biochem Biophys Res Commun. 2017 Mar 11;484(3):662-667. doi: 10.1016/j.bbrc.2017.01.172. Epub 2017 Jan 31. PMID: 28153727. 1954. Whitmire JK, Slifka MK, Grewal IS, Flavell RA, Ahmed R. CD40 ligand-deficient mice generate a normal primary cytotoxic Tlymphocyte response but a defective humoral response to a viral infection. J Virol. 1996 Dec;70(12):8375-81. doi: 10.1128/JVI.70.12.8375-8381.1996. Erratum in: J Virol 1997 Feb;71(2):1736. PMID: 8970958; PMCID: PMC190926. 1955. Umeshappa CS, Nanjundappa RH, Xie Y, Freywald A, Xu Q, Xiang J. Differential requirements of CD4(+) T-cell signals for effector cytotoxic T-lymphocyte (CTL) priming and functional memory CTL development at higher CD8(+) T-cell precursor frequency. Immunology. 2013 Apr;138(4):298-306. doi: 10.1111/imm.12033. PMID: 23113741; PMCID: PMC3719941. 1956. Diggs LP, Ruf B, Ma C, Heinrich B, Cui L, Zhang Q, McVey JC, Wabitsch S, Heinrich S, Rosato U, Lai W, Subramanyam V, Longerich T, Loosen SH, Luedde T, Neumann UP, Desar S, Kleiner D, Gores G, Wang XW, Greten TF. CD40-mediated immune cell activation enhances response to anti-PD-1 in murine intrahepatic cholangiocarcinoma. J Hepatol. 2021 May;74(5):1145-1154. doi: 10.1016/j.jhep.2020.11.037. Epub 2020 Dec 1. PMID: 33276030.

1957. Bhadra R, Gigley JP, Khan IA. Cutting edge: CD40-CD40 ligand pathway plays a critical CD8-intrinsic and -extrinsic role during rescue of exhausted CD8 T cells. J Immunol. 2011 Nov 1;187(9):4421-5. doi: 10.4049/jimmunol.1102319. Epub 2011 Sep 26. PMID: 21949017; PMCID: PMC3197960.

1958. Clarke SR. The critical role of CD40/CD40L in the CD4-dependent generation of CD8+ T cell immunity. J Leukoc Biol. 2000 May;67(5):607-14. doi: 10.1002/jlb.67.5.607. PMID: 10810999.

1959. Cullen JG, McQuilten HA, Quinn KM, Olshansky M, Russ BE, Morey A, Wei S, Prier JE, La Gruta NL, Doherty PC, Turner SJ. CD4+ T help promotes influenza virus-specific CD8+ T cell memory by limiting metabolic dysfunction. Proc Natl Acad Sci U S A. 2019 Mar 5;116(10):4481-4488. doi: 10.1073/pnas.1808849116. Epub 2019 Feb 20. PMID: 30787194; PMCID: PMC6410826. 1960. Olson MR, Seah SG, Edenborough K, Doherty PC, Lew AM, Turner SJ. CD154+ CD4+ T-cell dependence for effective memory influenza virus-specific CD8+ T-cell responses. Immunol Cell Biol. 2014 Aug;92(7):605-11. doi: 10.1038/icb.2014.28. Epub 2014 Apr 29. PMID: 24777309

1961. Gorbachev AV, Heeger PS, Fairchild RL. CD4+ and CD8+ T cell priming for contact hypersensitivity occurs independently of CD40-CD154 interactions. J Immunol. 2001 Feb 15;166(4):2323-32. doi: 10.4049/jimmunol.166.4.2323. PMID: 11160289. 1962. Gorbachev AV, Fairchild RL. CD40 engagement enhances antigen-presenting langerhans cell priming of IFN-gammaproducing CD4+ and CD8+ T cells independently of IL-12. J Immunol. 2004 Aug 15;173(4):2443-52. doi: 10.4049/jimmunol.173.4.2443. PMID: 15294958.

1963. O'Sullivan B, Thomas R. CD40 and dendritic cell function. Crit Rev Immunol. 2003;23(1-2):83-107. doi:

10.1615/critrevimmunol.v23.i12.50. PMID: 12906261.

1964. O'Sullivan B, Thomas R. Recent advances on the role of CD40 and dendritic cells in immunity and tolerance. Curr Opin Hematol. 2003 Jul;10(4):272-8. doi: 10.1097/00062752-200307000-00004. PMID: 12799532.

1965. Grewal IS, Flavell RA. The role of CD40 ligand in costimulation and T-cell activation. Immunol Rev. 1996 Oct;153:85-106. doi: 10.1111/j.1600-065x.1996.tb00921.x. PMID: 9010720.

1966. Kelleher M, Beverley PC. Lipopolysaccharide modulation of dendritic cells is insufficient to mature dendritic cells to generate CTLs from naive polyclonal CD8+ T cells in vitro, whereas CD40 ligation is essential. J Immunol. 2001 Dec 1;167(11):6247-55. doi: 10.4049/jimmunol.167.11.6247. PMID: 11714787.

1967. Miga AJ, Masters SR, Durell BG, Gonzalez M, Jenkins MK, Maliszewski C, Kikutani H, Wade WF, Noelle RJ. Dendritic cell longevity and T cell persistence is controlled by CD154-CD40 interactions. Eur J Immunol. 2001 Mar;31(3):959-65. doi: 10.1002/1521-4141(200103)31:3<959::aid-immu959>3.0.co;2-a. PMID: 11241301.

1968. 1961

1969. Yellin MJ, Brett J, Baum D, Matsushima A, Szabolcs M, Stern D, Chess L. Functional interactions of T cells with endothelial cells: the role of CD40L-CD40-mediated signals. J Exp Med. 1995 Dec 1;182(6):1857-64. doi: 10.1084/jem.182.6.1857. PMID: 7500031; PMCID: PMC2192229.

1970. van Kooten C, Banchereau J. Functional role of CD40 and its ligand. Int Arch Allergy Immunol. 1997 Aug;113(4):393-9. doi: 10.1159/000237614. PMID: 9250584.

1971. 1956.

1972. 1951.

1973. 1954 
1974. Moseman EA, Wu T, de la Torre JC, Schwartzberg PL, McGavern DB. Type I interferon suppresses virus-specific B cell responses by modulating CD8+ T cell differentiation. Sci Immunol. 2016 Oct;1(4):eaah3565. Epub 2016 Oct 21. PMID: 27812556; PMCID: PMC5089817.

1975. Cook KD, Shpargel KB, Starmer J, Whitfield-Larry F, Conley B, Allard DE, Rager JE, Fry RC, Davenport ML, Magnuson T, Whitmire JK, Su MA. T Follicular Helper Cell-Dependent Clearance of a Persistent Virus Infection Requires T Cell Expression of the Histone Demethylase UTX. Immunity. 2015 Oct 20;43(4):703-14. doi: 10.1016/j.immuni.2015.09.002. Epub 2015 Sep 29. PMID: 26431949; PMCID: PMC4731234.

1976. Hale JS, Youngblood B, Latner DR, Mohammed AU, Ye L, Akondy RS, Wu T, lyer SS, Ahmed R. Distinct memory CD4+ T cells with commitment to $T$ follicular helper- and T helper 1-cell lineages are generated after acute viral infection. Immunity. $2013 \mathrm{Apr}$ 18;38(4):805-17. doi: 10.1016/j.immuni.2013.02.020. Epub 2013 Apr 11. PMID: 23583644; PMCID: PMC3741679.

1977. Mcllwain DR, Grusdat M, Pozdeev VI, Xu HC, Shinde P, Reardon C, Hao Z, Beyer M, Bergthaler A, Häussinger D, Nolan GP Lang KS, Lang PA. T-cell STAT3 is required for the maintenance of humoral immunity to LCMV. Eur J Immunol. 2015 Feb;45(2):418-27. doi: 10.1002/eji.201445060. Epub 2014 Dec 15. PMID: 25393615; PMCID: PMC4383653.

1978. Mitchell JE, Lund MM, Starmer J, Ge K, Magnuson T, Shpargel KB, Whitmire JK. UTX promotes CD8+ T cell-mediated antiviral defenses but reduces T cell durability. Cell Rep. 2021 Apr 13;35(2):108966. doi: 10.1016/j.celrep.2021.108966. PMID: 33852868; PMCID: PMC8112613.

1979. Matloubian M, Concepcion RJ, Ahmed R. CD4+ T cells are required to sustain CD8+ cytotoxic T-cell responses during chronic viral infection. J Virol. 1994 Dec;68(12):8056-63. doi: 10.1128/JVI.68.12.8056-8063.1994. PMID: 7966595; PMCID: PMC237269. 1980. Asano MS, Ahmed R. CD8 T cell memory in B cell-deficient mice. J Exp Med. 1996 May 1;183(5):2165-74. doi: 10.1084/jem.183.5.2165. PMID: 8642326; PMCID: PMC2192575.

1981. Nahill SR, Welsh RM. High frequency of cross-reactive cytotoxic T lymphocytes elicited during the virus-induced polyclonal cytotoxic T lymphocyte response. J Exp Med. 1993 Feb 1;177(2):317-27. doi: 10.1084/jem.177.2.317. PMID: 8093891; PMCID: PMC2190893.

1982. Sears JD, Waldron KJ, Wei J, Chang CH. Targeting metabolism to reverse T-cell exhaustion in chronic viral infections. Immunology. 2021 Feb;162(2):135-144. doi: 10.1111/imm.13238. Epub 2020 Aug 11. PMID: 32681647; PMCID: PMC7808153. 1983. Brooks DG, Tishon A, Oldstone MBA, McGavern DB. Prevention of CD8 T Cell Deletion during Chronic Viral Infection. Viruses. 2021 Jun 22;13(7):1189. doi: 10.3390/v13071189. PMID: 34206262; PMCID: PMC8310272.

1984. Klein S, Ghersi D, Manns MP, Prinz I, Cornberg M, Kraft ARM. PD-L1 Checkpoint Inhibition Narrows the Antigen-Specific T Cell Receptor Repertoire in Chronic Lymphocytic Choriomeningitis Virus Infection. J Virol. 2020 Aug 31;94(18):e00795-20. doi: 10.1128/JVI.00795-20. PMID: 32641478; PMCID: PMC7459548.

1985. Li G, Wulan H, Song Z, Paik PA, Tsao ML, Goodman GM, MacEachern PT, Downey RS, Jankowska AJ, Rabinowitz YM, Learch TB, Song DZ, Yuan JJ, Zheng S, Zheng Z. Regulatory B Cell Function Is Suppressed by Smoking and Obesity in H. pylori-Infected Subjects and Is Correlated with Elevated Risk of Gastric Cancer. PLoS One. 2015 Jul 30;10(7):e0134591. doi: 10.1371/journal.pone.0134591. PMID: 26226399; PMCID: PMC4520600.

1986. Bangaru, S., Ozorowski, G., Turner, H.L., et al. (2020). Structural analysis of full-length SARS-CoV-2 spike protein from an advanced vaccine candidate. Science 370, 10891094.

1987. Rodriguez, P. C., Hernandez, C. P., Quiceno, D., Dubinett, S. M., Zabaleta, J., Ochoa, J. B., et al. (2005). Arginase I in myeloid suppressor cells is induced by COX-2 in lung carcinoma. J. Exp. Med. 202, 931-939. doi: 10.1084/jem.20050715.

1988. Yang, L., Huang, J., Ren, X., Gorska, A. E., Chytil, A., Aakre, M., et al. (2008). Abrogation of TGF beta signaling in mammary carcinomas recruits Gr-1+CD11b+ myeloid cells that promote metastasis. Cancer Cell 13, 23-35. doi:10.1016/j.ccr.2007.12.004 1989. Filipazzi, P., Huber, V., and Rivoltini, L. (2012). Phenotype, function and clinical implications of myeloid-derived suppressor cells in cancer patients. Cancer Immunol. Immunother. 61, 255-263. doi:10.1007/s00262-011-1161-9 1990. Huang, B., Pan, P. Y., Li, Q., Sato, A. I., Levy, D. E., Bromberg, J., et al. (2006). Gr-1+CD115+ immature myeloid suppressor cells mediate the development of tumor-induced T regulatory cells and T-cell anergy in tumor-bearing host. Cancer Res. 66, 1123-1131. doi: 10.1158/0008- 5472.CAN-05-1299

1991. Serafini, P., Mgebroff, S., Noonan, K., and Borrello, I. (2008). Myeloidderived suppressor cells promote cross-tolerance in Bcell lymphoma by expanding regulatory T cells. Cancer Res. 68, 5439-5449. doi: 10.1158/0008-5472.CAN-07-6621

1992. Nagaraj, S., Youn, J. I., and Gabrilovich, D. I. (2013). Reciprocal Relationship between Myeloid-Derived Suppressor Cells and T Cells. J. Immunol. 191, 17-23. doi: 10.4049/jimmunol.1300654

1993. Arora, M., Poe, S. L., Oriss, T. B., Krishnamoorthy, N., Yarlagadda, M., Wenzel, et al. (2010). TLR4/MyD88-induced $\mathrm{CD} 11 \mathrm{~b}+\mathrm{Gr}-1$ int F4/80+ non-migratory myeloid cells suppress Th2 effector function in the lung. Mucosal Immunol. 3, 578-593. doi: $10.1038 / \mathrm{mi} .2010 .41$

1994. Ostrand-Rosenberg, S., and Sinha, P. (2009). Myeloid-derived suppressor cells: linking inflammation and cancer. J. Immunol. 182, 4499-4506. doi: 10.4049/jimmunol.0802740

1995. Peranzoni, E., Zilio, S., Marigo, I., Dolcetti, L., Zanovello, P., Mandruzzato, S., et al. (2010). Myeloid-derived suppressor cell heterogeneity and subset definition. Curr. Opin. Immunol. 22, 238-244. doi: 10.1016/j.coi.2010.01.021

1996. Massberg, S., Schaerli, P., KnezevicMaramica, I., Kollnberger, M., Tubo, N., Moseman, E. A., et al. (2007).

Immunosurveillance by hematopoietic progenitor cells trafficking through blood, lymph, and peripheral tissues. Cell 131, $994-$ 1008. doi: 10.1016/j.cell.2007.09.047

1997. Zheng, W., and Flavell, R. A. (1997). The transcription factor GATA- 3 is necessary and sufficient for Th2 cytokine gene expression in CD4 T cells. Cell 89, 587-596. doi: 10.1016/S0092-8674 (00)80240-8

1998. Zhang, D. H., Cohn, L., Ray, P., Bottomly, K., and Ray, A. (1997). Transcription factor GATA-3 is differentially expressed in murine Th1 and Th2 cells and controls Th2-specific expression of the interleukin-5 gene. J. Biol. Chem. 272, 21597-21603. doi: 10.1074/jbc.272.34.21597

1999. Zhu, J., Cote-Sierra, J., Guo, L., and Paul, W. E. (2003). Stat5 activation plays a critical role in Th2 differentiation. Immunity 19, 739-748. doi: 10.1016/S1074-7613 (03)00292-9

2000. Dean, Matthew J et al. "Transcriptome and Functions of Granulocytic Myeloid-Derived Suppressor Cells Determine their Association with Disease Severity of COVID-19." medRxiv : the preprint server for health sciences 2021.03.26.21254441. 29 Mar. 2021, doi:10.1101/2021.03.26.21254441. Preprint.

2001. Bronte, Vincenzo et al. "Recommendations for myeloid-derived suppressor cell nomenclature and characterization standards." Nature communications vol. 7 12150. 6 Jul. 2016, doi:10.1038/ncomms12150

2002. Veglia F, Perego M, Gabrilovich D. Myeloid-derived suppressor cells coming of age. Nat Immunol. 2018 Feb;19(2):108-119. doi: 10.1038/s41590-017-0022-x. Epub 2018 Jan 18. PMID: 29348500; PMCID: PMC5854158.

2003. Rowlands, Marianna et al. "Myeloid-Derived Suppressor Cells as a Potential Biomarker and Therapeutic Target in COVID-19." Frontiers in immunology vol. 12 697405. 18 Jun. 2021, doi:10.3389/fimmu.2021.697405 
2004. Canè, S., Ugel, S., Trovato, R., Marigo, I., De Sanctis, F., Sartoris, S., \& Bronte, V. (2019). The Endless Saga of Monocyte Diversity. Frontiers in immunology, 10, 1786. https://doi.org/10.3389/fimmu.2019.01786

2005. Wofford, J. A., Wieman, H. L., Jacobs, S. R., Zhao, Y., and Rathmell, J. C. (2008). IL-7 promotes Glut1 trafficking and glucose uptake via STAT5-mediated activation of Akt to support T-cell survival. Blood 111, 2101-2111. doi: 10.1182/blood-2007-06096297

2006. Hand, T. W., Cui, W., Jung, Y. W., Sefik, E., Joshi, N. S., Chandele, A. et al. (2010). Differential effects of STAT5 and PI3K/AKT signaling on effector and memory CD8 T-cell survival. Proc. Natl. Acad. Sci. U.S.A. 107, 16601-16606. doi: 10.1073/pnas.1003457107

2007. Hu, H., Huston, G., Duso, D., Lepak, N., Roman, E., and Swain, S. L. (2001). CD4(+) T cell effectors can become memory cells with high efficiency and without further division. Nat. Immunol. 2, 705-710. doi: 10.1038/90643

2008. De Wilde, V., Van Rompaey, N., Hill, M., Lebrun, J. F., Lemaitre, P., Lhomme, F., et al. (2009). Endotoxin-induced myeloidderived suppressor cells inhibit alloimmune responses via heme oxygenase-1. Am. J. Transplant. 9, 2034-2047. doi: 10.1111/j.1600-6143.2009.02757.x

2009. Bozinovski, S., Jones, J. E., Vlahos, R.,Hamilton, J. A., and Anderson, G. P.(2002). Granulocyte/macrophage colonystimulating factor(GM-CSF) regulates lung innate immunity to lipopolysaccharide through Akt/Erk activation of NF-kappa B and AP-1 in vivo. J. Biol.Chem. 277, 42808-42814. doi:10.1074/jbc.M207840200.

2010. Nefedova, Y., Huang, M., Kusmartsev, S., Bhattacharya, R., Cheng, P., Salup, R., et al. (2004). Hyperactivation of STAT3 is involved in abnormal differentiation of dendritic cells in cancer. J. Immunol. 172, 464-474.

2011. Chalmin, F., Ladoire, S., Mignot, G., Vincent, J., Bruchard, M., Remy-Martin, J. P. et al. (2010). Membrane-associated Hsp72 from tumor-derived exosomes mediates STAT3-dependent immunosuppressive function of mouse and human myeloid-derived suppressor cells. J. Clin. Invest. 120, 457-471. doi: 10.1172/JCl40483

2012. Cheng, P., Corzo, C. A., Luetteke, N.,Yu, B., Nagaraj, S., Bui, M. M., et al. (2008). Inhibition of dendritic cell differentiation and accumulation of myeloid-derived suppressor cells in cancer is regulated by S100A9 protein. J. Exp. Med. 205, 2235-2249.doi: $10.1084 /$ jem. 20080132

2013. Bunt, S. K., Clements, V. K., Hanson, E. M., Sinha, P., and OstrandRosenberg, S. (2009). Inflammation enhances myeloidderived suppressor cell cross-talk by signaling through Toll-like receptor 4. J. Leukoc. Biol. 85, 996-1004. doi: 10.1189/jlb.0708446

2014. Karki, R. et al. Synergism of TNF- $\alpha$ and IFN- $\nu$ triggers infammatory cell death, tissue damage, and mortality in SARS-CoV-2 infection and cytokine shock syndromes. Cell https://doi.org/10.1016/j.cell.2020.11.025 (2020).

2015. Esposito, S., Molteni, C.G., Giliani, S. et al. Toll-like receptor 3 gene polymorphisms and severity of pandemic A/H1N1/2009 influenza in otherwise healthy children. Virol J 9, 270 (2012). https://doi.org/10.1186/1743-422X-9-270

2016. Ding, X., Jin, S., Tong, Y. et al. TLR4 signaling induces TLR3 up-regulation in alveolar macrophages during acute lung injury. Sci Rep 7, 34278 (2017). https://doi.org/10.1038/srep34278.

2017. Shirey, Kari Ann et al. "Targeting TLR4 Signaling to Blunt Viral-Mediated Acute Lung Injury." Frontiers in immunology vol. 12 705080. 2 Jul. 2021, doi:10.3389/fimmu.2021.705080

2018. Yamada H, Odonnell MA, Matsumoto T, Luo Y. Interferon-gamma up-regulates toll-like receptor 4 and cooperates with lipopolysaccharide to produce macrophage-derived chemokine and interferon-gamma inducible protein-10 in human bladder cancer cell line RT4. J Urol. 2005 Sep;174(3):1119-23. doi: 10.1097/01.ju.0000168619.25341.96. PMID: 16094077.

2019. Zheng, W., Xu, Q., Zhang, Y. et al. Toll-like receptor-mediated innate immunity against herpesviridae infection: a current perspective on viral infection signaling pathways. Virol J 17, 192 (2020). https://doi.org/10.1186/s12985-020-01463-2 2020. Deng Y, Yang Z, Gao Y, Xu H, Zheng B, et al. (2013) Toll-Like Receptor 4 Mediates Acute Lung Injury Induced by High Mobility Group Box-1. PLOS ONE 8(5): e64375. https://doi.org/10.1371/journal.pone.0064375

2021. Hongpeng Jia, Chhinder P. Sodhi, Yukihiro Yamaguchi, Peng Lu, Laura Y et.al, Pulmonary Epithelial TLR4 Activation Leads to Lung Injury in Neonatal Necrotizing Enterocolitis, The Journal of Immunology August 1, 2016, 197 (3) 859-871; DOI: 10.4049/jimmunol.1600618

2022. He, X., Qian, Y., Li, Z. et al. TLR4-Upregulated IL-1ß and IL-1RI Promote Alveolar Macrophage Pyroptosis and Lung Inflammation through an Autocrine Mechanism. Sci Rep 6, 31663 (2016). https://doi.org/10.1038/srep31663 2023. Yuxiao Deng,1 Lei Hou,2 Qiaoyi Xu,1 Qi Liu et.al, Cardiopulmonary Bypass Induces Acute Lung Injury via the High-Mobility Group Box 1/Toll-Like Receptor 4 Pathway, https://doi.org/10.1155/2020/8854700

2024. Skinner NA, Maclsaac CM, Hamilton JA, Visvanathan K. Regulation of Toll-like receptor (TLR)2 and TLR4 on CD14dimCD16+ monocytes in response to sepsis-related antigens. Clin Exp Immunol. 2005 Aug;141(2):270-8. doi: 10.1111/j.13652249.2005.02839.x. PMID: 15996191; PMCID: PMC1809439.

2025. Mirlashari MR, Lyberg T. Expression and involvement of Toll-like receptors (TLR)2, TLR4, and CD14 in monocyte TNF-alpha production induced by lipopolysaccharides from Neisseria meningitidis. Med Sci Monit. 2003 Aug;9(8):BR316-24. PMID: 12942028.

2026. Feng JM, Shi JQ, Liu YS. The effect of lipopolysaccharides on the expression of CD14 and TLR4 in rat Kupffer cells. Hepatobiliary Pancreat Dis Int. 2003 May;2(2):265-9. PMID: 14599981.

2027. Zhou M, Wan HY, Huang SG, Li B, Li M. [Expression of toll-like receptor 4 in human alveolar epithelial cells and its role in cellular inflammation]. Zhonghua Yi Xue Za Zhi. 2008 Aug 5;88(30):2112-6. Chinese. PMID: 19080471.

2028. Matsumura T, Ito A, Takii T, Hayashi H, Onozaki K. Endotoxin and cytokine regulation of toll-like receptor (TLR) 2 and TLR4 gene expression in murine liver and hepatocytes. J Interferon Cytokine Res. 2000 Oct;20(10):915-21. doi: 10.1089/10799900050163299. PMID: 11054280.

2029. Mohamed M. Aboudounya, Mark R. Holt, Richard J. Heads, SARS-CoV-2 Spike S1 glycoprotein is a TLR4 agonist, upregulates ACE2 expression and induces pro-inflammatory M1 macrophage polarisation, bioRxiv 2021.08.11.455921; doi:

https://doi.org/10.1101/2021.08.11.455921

2030. Sohn, Kyung Mok et al. "COVID-19 Patients Upregulate Toll-like Receptor 4-mediated Inflammatory Signaling That Mimics Bacterial Sepsis." Journal of Korean medical science vol. 35,38 e343. 28 Sep. 2020, doi:10.3346/jkms.2020.35.e343

2031. Huang, Hai \& Hu, Ping-Fang \& Sun, Liang-Liang \& Guo, Yibin \& Wang, Qiong \& Liu, Zhi-Min \& Yin, Ji-Zhong \& Shi, Pei-Mei \& Yuan, Zong-Li \& Tan, Yu \& Zhou, Chao \& Liu, Ya-Long \& Chen, Cheng \& Song, Hui-Hui \& Xie, weifen. (2020). Treatment of Covid-19 Patients With High Dose of Ulinastatin. 10.21203/rs.3.rs-32627/v1.

2032. Chen, Guochao \& Gao, Yanyan \& Jiang, Yue \& Yang, Fei \& Li, Shuangshuang \& Tan, Di \& Ma, Qun. (2018). Efficacy and Safety of Xuebijing Injection Combined With Ulinastatin as Adjunctive Therapy on Sepsis: A Systematic Review and Meta-Analysis. Frontiers in Pharmacology. 9. 10.3389/fphar.2018.00743.

2033. Guéant JL, Guéant-Rodriguez RM, Fromonot J, Oussalah A, Louis H, Chery C, Gette M, Gleye S, Callet J, Raso J, Blanchecotte F, Lacolley P, Guieu R, Regnault V. Elastase and exacerbation of neutrophil innate immunity are involved in multi-visceral 
manifestations of COVID-19. Allergy. 2021 Jun;76(6):1846-1858. doi: 10.1111/all.14746. Epub 2021 Feb 27. PMID: 33484168; PMCID: PMC8014109.

2034. Seda Seren, Lohann Derian, Irem Keleş, Antoine Guillon, Adam Lesner, Loïc Gonzalez, Thomas Baranek, Mustapha Si-Tahar, Sylvain Marchand-Adam, Dieter E. Jenne, Christophe Paget, Youenn Jouan, Brice Korkmaz, Proteinase release from activated neutrophils in mechanically ventilated patients with non-COVID-19 and COVID-19 pneumonia, European Respiratory Journal Jan 2021, 2003755; DOI: 10.1183/13993003.03755-2020

2035. Iliadi V, Konstantinidou I, Aftzoglou K, Iliadis S, Konstantinidis TG, Tsigalou C. The Emerging Role of Neutrophils in the Pathogenesis of Thrombosis in COVID-19. Int J Mol Sci. 2021 May 20;22(10):5368. doi: 10.3390/ijms22105368. PMID: 34065210; PMCID: PMC8161034.

2036. Ackermann, M., Anders, HJ., Bilyy, R. et al. Patients with COVID-19: in the dark-NETs of neutrophils. Cell Death Differ (2021). https://doi.org/10.1038/s41418-021-00805-z

2037. Leandro Borges, Tania Cristina Pithon-Curi, Rui Curi, Elaine Hatanaka, "COVID-19 and Neutrophils: The Relationship between Hyperinflammation and Neutrophil Extracellular Traps", Mediators of Inflammation, vol. 2020, Article ID 8829674, 7 pages, 2020. https://doi.org/10.1155/2020/8829674

2038. Reusch, Nico et al. "Neutrophils in COVID-19." Frontiers in immunology vol. 12 652470. 25 Mar. 2021, doi:10.3389/fimmu.2021.652470

2039. Mohamed, Mahmoud \& El-Shimy, Ismail \& Abdul Hadi, Muhammad. (2020). Neutrophil Elastase Inhibitors: A potential prophylactic treatment option for SARS-CoV-2-induced respiratory complications?. Critical Care. 24. 10.1186/s13054-020-030230 .

2040. https://clinicaltrials.gov/ct2/show/NCT04747574.

2041. https://www.sciencetimes.com/articles/32746/20210810/covid-19-treatment-israeli-drug-treat-patients-virus-5-days.htm 2042. Abdel-Hamed, E.F., Ibrahim, M.N., Mostafa, N.E. et al. Role of interferon gamma in SARS-CoV-2-positive patients with parasitic infections. Gut Pathog 13, 29 (2021). https://doi.org/10.1186/s13099-021-00427-3

2043. Hu, Zhong-Jie et al. "Lower Circulating Interferon-Gamma Is a Risk Factor for Lung Fibrosis in COVID-19 Patients." Frontiers in immunology vol. 11 585647. 29 Sep. 2020, doi:10.3389/fimmu.2020.585647.

2044. Myasnikov AL, Berns SA, Talyzin PA, Ershov FI. [Interferon gamma in the treatment of patients with moderate COVID-19]. Vopr Virusol. 2021 Mar 7;66(1):47-54. Russian. doi: 10.36233/0507-4088-24. PMID: 33683065.

2045. Lu, Y., Liu, F., Tong, G. et al. Clinical evidence of an interferon-glucocorticoid therapeutic synergy in COVID-19. Sig Transduct Target Ther 6, 107 (2021). https://doi.org/10.1038/s41392-021-00496-5

2046. Feld JJ, Kandel C, Biondi MJ, Kozak RA, Zahoor MA, Lemieux C, Borgia SM, Boggild AK, Powis J, et.al. Peginterferon lambda for the treatment of outpatients with COVID-19: a phase 2, placebo-controlled randomised trial. Lancet Respir Med. 2021 May;9(5):498-510. doi: 10.1016/S2213-2600(20)30566-X. Epub 2021 Feb 5. PMID: 33556319; PMCID: PMC7906707.

2047. Jagannathan, P., Andrews, J.R., Bonilla, H. et al. Peginterferon Lambda-1a for treatment of outpatients with uncomplicated COVID-19: a randomized placebo-controlled trial. Nat Commun 12, 1967 (2021). https://doi.org/10.1038/s41467-021-22177-1 2048. Webb BJ, Buckel W, Vento T, Butler AM, Grisel N, Brown SM, Peltan ID, Spivak ES, Shah M, Sakata T, Wallin A, Stenehjem E, Poulsen G, Bledsoe J. Real-world Effectiveness and Tolerability of Monoclonal Antibody Therapy for Ambulatory Patients With Early COVID-19. Open Forum Infect Dis. 2021 Jun 23;8(7):ofab331. doi: 10.1093/ofid/ofab331. PMID: 34327256; PMCID: PMC8314951.

2049. Piccicacco N, Zeitler K, Montero J, Kumar A, Lakshmi S, Kim K, Wein D, Vasey T, Vasey M, Oxner A. Effectiveness of Severe Acute Respiratory Syndrome Coronavirus 2 Monoclonal Antibody Infusions in High-Risk Outpatients. Open Forum Infect Dis. 2021 Jun 4;8(7):ofab292. doi: 10.1093/ofid/ofab292. PMID: 34258319; PMCID: PMC8195104.

2050. Ganesh R, Philpot LM, Bierle DM, Anderson RJ, Arndt LL, Arndt RF, Culbertson TL, Destro Borgen MJ, Hanson SN, Kennedy BD, Kottke BB, Larsen JJ, Ramar P, Rosedahl JK, Seville MT, Speicher LL, Tulledge-Scheitel SM, Wilker CG, Razonable RR; Monoclonal Antibody Treatment Program. Real-World Clinical Outcomes of Bamlanivimab and Casirivimab-Imdevimab among High-Risk Patients with Mild to Moderate Coronavirus Disease 2019. J Infect Dis. 2021 Jul 19:jiab377. doi: 10.1093/infdis/jiab377. Epub ahead of print. PMID: 34279629; PMCID: PMC8344643.

2051. Bariola JR, McCreary EK, Wadas RJ, Kip KE, Marroquin OC, Minnier T, Koscumb S, Collins K, Schmidhofer M, Shovel JA, Wisniewski MK, Sullivan C, Yealy DM, Nace DA, Huang DT, Haidar G, Khadem T, Linstrum K, Seymour CW, Montgomery SK, Angus DC, Snyder GM. Impact of Bamlanivimab Monoclonal Antibody Treatment on Hospitalization and Mortality Among Nonhospitalized Adults With Severe Acute Respiratory Syndrome Coronavirus 2 Infection. Open Forum Infect Dis. 2021 May 17;8(7):ofab254. doi: 10.1093/ofid/ofab254. PMID: 34250192; PMCID: PMC8241472.

2052. Nathan R, Shawa I, De La Torre I, Pustizzi JM, Haustrup N, Patel DR, Huhn G. A Narrative Review of the Clinical Practicalities of Bamlanivimab and Etesevimab Antibody Therapies for SARS-CoV-2. Infect Dis Ther. 2021 Aug 10:1-15. doi: 10.1007/s40121021-00515-6. Epub ahead of print. PMID: 34374951; PMCID: PMC8353431.

2053. Nathan R, Shawa I, De La Torre I, Pustizzi JM, Haustrup N, Patel DR, Huhn G. A Narrative Review of the Clinical Practicalities of Bamlanivimab and Etesevimab Antibody Therapies for SARS-CoV-2. Infect Dis Ther. 2021 Aug 10:1-15. doi: 10.1007/s40121021-00515-6. Epub ahead of print. PMID: 34374951; PMCID: PMC8353431.

2054. Coronavirus (COVID-19) Update: FDA Authorizes Additional Monoclonal Antibody for Treatment of COVID-19. https://www.fda.gov/news-events/press-announcements/coronavirus-covid-19-update-fda-authorizes-additional-monoclonalantibody-treatment-covid-19.

2055. Anti-SARS-CoV-2 Monoclonal Antibodies.

https://www.covid19treatmentguidelines.nih.gov/therapies/anti-sars-cov-2-antibody-products/anti-sars-cov-2-monoclonalantibodies/

2056. Taylor, P.C., Adams, A.C., Hufford, M.M. et al. Neutralizing monoclonal antibodies for treatment of COVID-19. Nat Rev Immunol 21, 382-393 (2021). https://doi.org/10.1038/s41577-021-00542-x

2057. Mark Jarrett, Warren B. Licht, Kevin Bock, Zenobia Brown, Jamie S. Hirsch, Kevin Coppa, Rajdeep Brar, Steven Bello, Ira S. Nash,Early Experience With Neutralizing Monoclonal Antibody Therapy For COVID-19, medRxiv 2021.04.09.21255219; doi:https://doi.org/10.1101/2021.04.09.21255219

2058. Kelly SA, Nzakizwanayo J, Rodgers AM, Zhao L, Weiser R, Tekko IA, McCarthy HO, Ingram RJ, Jones BV, Donnelly RF, Gilmore BF. Antibiotic Therapy and the Gut Microbiome: Investigating the Effect of Delivery Route on Gut Pathogens. ACS Infect Dis. 2021 May 14;7(5):1283-1296. doi: 10.1021/acsinfecdis.1c00081. Epub 2021 Apr 12. PMID: 33843198.

2059. Malek, Alexandre E et al. "Doxycycline as a potential partner of COVID-19 therapies." IDCases vol. 21 e00864. 6 Jun. 2020 , doi:10.1016/j.idcr.2020.e00864

2060. Gendrot M, Andreani J, Jardot P, Hutter S, Delandre O, Boxberger M, Mosnier J, Le Bideau M, Duflot I, Fonta I, Rolland C, Bogreau H, La Scola B, Pradines B. In Vitro Antiviral Activity of Doxycycline against SARS-CoV-2. Molecules. 2020 Oct 31;25(21):5064. doi: 10.3390/molecules25215064. PMID: 33142770; PMCID: PMC7663271. 
2061. Christopher C Butler, FMedSci, Ly-Mee Yu, DPhil, Jienchi DorwarD et.al, Doxycycline for community treatment of suspected COVID-19 in people at high risk of adverse outcomes in the UK (PRINCIPLE): a randomised, controlled, open-label, adaptive platform trial, ,2021, DOI:https://doi.org/10.1016/S2213-2600(21)00310-6

2062. Yates PA, Newman SA, Oshry L, Glassman RH, Leone AM, Reichel E. Doxycycline treatment of high-risk COVID-19-positive patients with comorbid pulmonary disease. Ther Adv Respir Dis. 2020 Jan-Dec;14:1753466620951053. doi: 10.1177/1753466620951053. PMID: 32873175; PMCID: PMC7476338.

2063. Hoyt JC, Ballering J, Numanami H, Hayden JM, Robbins RA. Doxycycline modulates nitric oxide production in murine lung epithelial cells. J Immunol. 2006 Jan 1;176(1):567-72. doi: 10.4049/jimmunol.176.1.567. PMID: 16365451.

2064. Kim HS, Luo L, Pflugfelder SC, Li DQ. Doxycycline inhibits TGF-beta1-induced MMP-9 via Smad and MAPK pathways in human corneal epithelial cells. Invest Ophthalmol Vis Sci. 2005 Mar;46(3):840-8. doi: 10.1167/iovs.04-0929. PMID: 15728539. 2065. Hsu AT, Barrett CD, DeBusk GM, Ellson CD, Gautam S, Talmor DS, Gallagher DC, Yaffe MB. Kinetics and Role of Plasma Matrix Metalloproteinase-9 Expression in Acute Lung Injury and the Acute Respiratory Distress Syndrome. Shock. 2015 Aug;44(2):128-36. doi: 10.1097/SHK.0000000000000386. PMID: 26009816; PMCID: PMC4830084.

2066. Koskinen A, Tolvi M, Jauhiainen M, Kekäläinen E, Laulajainen-Hongisto A, Lamminmäki S. Complications of COVID-19 Nasopharyngeal Swab Test. JAMA Otolaryngol Head Neck Surg. 2021;147(7):672-674. doi:10.1001/jamaoto.2021.0715 2067. Fish T, Issa K, Levine CG, Jang DW, Chen PG. Assessment of Available Online Information About Nasopharyngeal Swab Testing in Patient Instructions for Sinus and Pituitary Surgery. JAMA Otolaryngol Head Neck Surg. 2021 Jun 1;147(6):572-573. doi: 10.1001/jamaoto.2020.5663. PMID: 33662126; PMCID: PMC7934076.

2068. Nasopharyngeal swabs are not risk-free, https://www.academie-medecine.fr/nasopharyngeal-swabs-are-not-riskfree/?lang=en

2069. Mistry, Sandeep G et al. "COVID-19 swab-related skull base injury." The Medical journal of Australia vol. 214,10 (2021): 457-459.e1. doi:10.5694/mja2.51082

2070. Paquin, Rebecca et al. "CSF Leak After COVID-19 Nasopharyngeal Swab: A Case Report." The Laryngoscope vol. 131,9 (2021): 1927-1929. doi:10.1002/lary.29462

2071. Rajah J, Lee J. CSF rhinorrhoea post COVID-19 swab: A case report and review of literature. J Clin Neurosci. 2021 Apr;86:6-9. doi: 10.1016/j.jocn.2021.01.003. Epub 2021 Jan 14. PMID: 33775348; PMCID: PMC7834700.

2072. Sullivan CB, Schwalje AT, Jensen M, Li L, Dlouhy BJ, Greenlee JD, Walsh JE. Cerebrospinal Fluid Leak After Nasal Swab Testing for Coronavirus Disease 2019. JAMA Otolaryngol Head Neck Surg. 2020 Dec 1;146(12):1179-1181. doi:

10.1001/jamaoto.2020.3579. Erratum in: JAMA Otolaryngol Head Neck Surg. 2020 Dec 1;146(12):1181. PMID: 33022069.

2073. Perneczky, Julian et al. "Rhinorrhea following SARS-CoV-2 nasopharyngeal swab: A case for $\beta 2$-transferrin testing." European journal of neurology, 10.1111/ene.14883. 26 Apr. 2021, doi:10.1111/ene.14883

2074. Clayton F Douglas, MD; Benjamin D White, MD, Traumatic Cribriform Plate Defect Following Self-administered COVID-19 Nasal Swab Test

https://www.appliedradiology.com/articles/traumatic-cribriform-plate-defect-following-self-administered-covid-19-nasal-swabtest

2075. Hobson, Jonathan C, and Jeremy A Lavy. "Use and abuse of cotton buds." Journal of the Royal Society of Medicine vol. 98,8 (2005): 360-1. doi:10.1258/jrsm.98.8.360.

2076. Zhu Y, Xie DY. Docking Characterization and in vitro Inhibitory Activity of Flavan-3-ols and Dimeric Proanthocyanidins Against the Main Protease Activity of SARS-Cov-2. Front Plant Sci. 2020 Nov 30;11:601316. doi: 10.3389/fpls.2020.601316. PMID: 33329667; PMCID: PMC7733993.

2077. Yang L, Wang Z. Natural Products, Alone or in Combination with FDA-Approved Drugs, to Treat COVID-19 and Lung Cancer. Biomedicines. 2021 Jun 18;9(6):689. doi: 10.3390/biomedicines9060689. PMID: 34207313; PMCID: PMC8234041.

2078. Zhang Z, Zhang X, Bi K, He Y, Yan W, Yang CS, Zhang J. Potential protective mechanisms of green tea polyphenol EGCG against COVID-19. Trends Food Sci Technol. 2021 Aug;114:11-24. doi: 10.1016/j.tifs.2021.05.023. Epub 2021 May 25. PMID: 34054222; PMCID: PMC8146271.

2079. Park J, Park R, Jang M, Park YI. Therapeutic Potential of EGCG, a Green Tea Polyphenol, for Treatment of Coronavirus Diseases. Life (Basel). 2021 Mar 4;11(3):197. doi: 10.3390/life11030197. PMID: 33806274; PMCID: PMC8002208.

2080. Liskova, Alena et al. "Flavonoids against the SARS-CoV-2 induced inflammatory storm." Biomedicine \& pharmacotherapy = Biomedecine \& pharmacotherapie vol. 138 (2021): 111430. doi:10.1016/j.biopha.2021.111430

2081. Yue Zhu, Frank Scholle, Samantha C. Kisthardt, De-Yu Xie, Flavonols and dihydroflavonols inhibit the main protease activity of SARS-CoV-2 and the replication of human coronavirus 229E, bioRxiv 2021.07.01.450756; doi: https://doi.org/10.1101/2021.07.01.450756

2082. Sulis G, Batomen B, Kotwani A, Pai M, Gandra S (2021) Sales of antibiotics and hydroxychloroquine in India during the COVID-19 epidemic: An interrupted time series analysis. PLOS Medicine 18(7): e1003682.

https://doi.org/10.1371/journal.pmed.1003682

2083. Garg, Sunil K. “Antibiotic misuse during COVID-19 Pandemic: A Recipe for Disaster." Indian journal of critical care medicine : peer-reviewed, official publication of Indian Society of Critical Care Medicine vol. 25,6 (2021): 617-619. doi:10.5005/jp-journals10071-23862

2084. Elsayed AA, Darwish SF, Zewail MB, Mohammed M, Saeed H, Rabea H. Antibiotic misuse and compliance with infection control measures during COVID-19 pandemic in community pharmacies in Egypt. Int J Clin Pract. 2021 Jun;75(6):e14081. doi: 10.1111/ijcp.14081. Epub 2021 Feb 15. PMID: 33559255; PMCID: PMC7995210.

2085. Manohar, Prasanth1; Loh, Belinda1; Leptihn, Sebastian1,2,3, $\otimes$ Will the Overuse of Antibiotics During the Coronavirus Pandemic Accelerate Antimicrobial Resistance of Bacteria?, Infectious Microbes \& Diseases: September 2020 - Volume 2 - Issue 3 - p 87-88 doi: 10.1097/IM9.0000000000000034

2086. "Dexamethasone misuse: Delayed viral clearance leading to severe COVID-19 pneumonia: case report." Reactions Weekly vol. 1845,1 (2021): 156. doi:10.1007/s40278-021-92039-3

2087. Christian Jung, Bernhard Wernly, Jesper Fjølner, Raphael Romano Bruno, David Dudzinski, Antonio Artigas, Bernardo Bollen Pinto, Joerg C. Schefold, Georg Wolff, et.al. Steroid use in elderly critically ill COVID-19 patients, European Respiratory Journal Jan 2021, 2100979; DOI: 10.1183/13993003.00979-2021

2088. Britt RC, Devine A, Swallen KC, et al. Corticosteroid Use in the Intensive Care Unit: At What Cost? Arch Surg.

2006;141(2):145-149. doi:10.1001/archsurg.141.2.145

2089. Singh Y, Ganesh V, Kumar S, et al. (July 03, 2021) Coronavirus Disease-Associated Mucormycosis from a Tertiary Care Hospital in India: A Case Series. Cureus 13(7): e16152. doi:10.7759/cureus.16152

2090. Akshay Raut, Nguyen Tien Huy, Rising incidence of mucormycosis in patients with COVID-19: another challenge for India amidst the second wave?, 2021DOI:https://doi.org/10.1016/S2213-2600(21)00265-4 
2091. Khushboo Chauhan,Deepak Soni, Deepayan Sarkar, Tadepalli Karuna,Prof Bhavana Sharma, Prof Sarman Singh et al. Mucormycosis after COVID-19 in a patient with diabetes, 2021, DOI:https://doi.org/10.1016/S0140-6736(21)01641-X 2092. Singh, Awadhesh Kumar et al. "Mucormycosis in COVID-19: A systematic review of cases reported worldwide and in India." Diabetes \& metabolic syndrome vol. 15,4 (2021): 102146. doi:10.1016/j.dsx.2021.05.019 2093. Gupta, Amod; Sharma, Aman1; Chakrabarti, Arunaloke2, The emergence of post-COVID-19 mucormycosis in India, Indian Journal of Ophthalmology: July 2021 - Volume 69 - Issue 7 - p 1645-1647 doi: 10.4103/ijo.IJO 139221 2094. Xiaodong Lan, Zhiyong Huang, Ziming Tan, Zhenjia Huang, Dehuai Wang, Yuesheng Huang, Nebulized heparin for inhalation injury in burn patients: a systematic review and meta-analysis, Burns \& Trauma, Volume 8, 2020, tkaa015, https://doi.org/10.1093/burnst/tkaa015 2095. Ashraf, Umair et al. "Nebulized heparin and N-acetylcysteine for smoke inhalational injury: A case report." Medicine vol. 97,19 (2018): e0638. doi:10.1097/MD.0000000000010638

2096. Phelps MK, Olson LM, Patel MAVB, Thompson MJ, Murphy CV. Nebulized Heparin for Adult Patients With Smoke Inhalation Injury: A Review of the Literature. Journal of Pharmacy Technology. 2020;36(4):130-140. doi:10.1177/8755122520925774 2097. Eedara, Basanth Babu et al. "Inhalation Delivery for the Treatment and Prevention of COVID-19 Infection." Pharmaceutics vol. 13,7 1077. 14 Jul. 2021, doi:10.3390/pharmaceutics13071077

2098. Courtney Mycroft-West, Dunhao Su, Stefano Elli, Yong Li, Scott Guimond, Gavin Miller, Jeremy Turnbull, et.al. The 2019 coronavirus (SARS-CoV-2) surface protein (Spike) S1 Receptor Binding Domain undergoes conformational change upon heparin binding, bioRxiv 2020.02.29.971093; doi: https://doi.org/10.1101/2020.02.29.971093

2099. Lang, Jianshe et al. "Inhibition of SARS pseudovirus cell entry by lactoferrin binding to heparan sulfate proteoglycans." PloS one vol. 6,8 (2011): e23710. doi:10.1371/journal.pone.0023710

2100. Glas GJ, Serpa Neto A, Horn J, Cochran A, Dixon B, Elamin EM, Faraklas I, Dissanaike S, Miller AC, Schultz MJ. Nebulized heparin for patients under mechanical ventilation: an individual patient data meta-analysis. Ann Intensive Care. 2016 Dec;6(1):33. doi: 10.1186/s13613-016-0138-4. Epub 2016 Apr 16. PMID: 27083915; PMCID: PMC4833759.

2101. Vicenzi E, Canducci F, Pinna D, Mancini N, Carletti S, Lazzarin A, Bordignon C, Poli G, Clementi M. Coronaviridae and SARSassociated coronavirus strain HSR1. Emerg Infect Dis. 2004 Mar;10(3):413-8. doi: 10.3201/eid1003.030683. PMID: 15109406; PMCID: PMC3322807.

2102. So Young Kim, Weihua Jin, Amika Sood, David W. Montgomery, Oliver C. Grant, Mark M. Fuster et.al. Glycosaminoglycan binding motif at $\mathrm{S} 1 / \mathrm{S} 2$ proteolytic cleavage site on spike glycoprotein may facilitate novel coronavirus (SARS-CoV-2) host cel entry, bioRxiv 2020.04.14.041459; doi: https://doi.org/10.1101/2020.04.14.041459

2103. van Haren, F.M.P., Page, C., Laffey, J.G. et al. Nebulised heparin as a treatment for COVID-19: scientific rationale and a call for randomised evidence. Crit Care 24, 454 (2020). https://doi.org/10.1186/s13054-020-03148-2

2104. de Haan, Cornelis A M et al. "Murine coronavirus with an extended host range uses heparan sulfate as an entry receptor." Journal of virology vol. 79,22 (2005): 14451-6. doi:10.1128/JVI.79.22.14451-14456.2005

2105. Madu IG, Chu VC, Lee H, Regan AD, Bauman BE, Whittaker GR. Heparan sulfate is a selective attachment factor for the avian coronavirus infectious bronchitis virus Beaudette. Avian Dis. 2007 Mar;51(1):45-51. doi: 10.1637/00052086(2007)051[0045:HSIASA]2.0.CO;2. PMID: 17461266.

2106. Cagno V, Tseligka ED, Jones ST, Tapparel C. Heparan Sulfate Proteoglycans and Viral Attachment: True Receptors or Adaptation Bias? Viruses. 2019 Jul 1;11(7):596. doi: 10.3390/v11070596. PMID: 31266258; PMCID: PMC6669472. 2107. Milewska A, Zarebski M, Nowak P, Stozek K, Potempa J, Pyrc K. Human coronavirus NL63 utilizes heparan sulfate proteoglycans for attachment to target cells. J Virol. 2014 Nov;88(22):13221-30. doi: 10.1128/JVI.02078-14. Epub 2014 Sep 3. PMID: 25187545; PMCID: PMC4249106.

2108. Moulard M, Lortat-Jacob H, Mondor I, Roca G, Wyatt R, Sodroski J, Zhao L, Olson W, Kwong PD, Sattentau QJ. Selective interactions of polyanions with basic surfaces on human immunodeficiency virus type 1 gp120. J Virol. 2000 Feb;74(4):1948-60. doi: 10.1128/jvi.74.4.1948-1960.2000. PMID: 10644368; PMCID: PMC111673.

2109. Dixon, Barry \& Smith, Roger \& Artigas, Antonio \& Laffey, John \& Mcnicholas, Bairbre \& Schmidt, Eric \& Nunes, Quentin \& Skidmore, Mark \& Lome, Marcelo \& Moran, John \& Van Haren, Frank \& Doig, Gordon \& Gupta, Sachin \& Ghosh, Angajendra \& Said, Simone \& Santamaria, John. (2020). Can Nebulised Heparin Reduce Time to Extubation in SARS CoV 2 The CHARTER Study Protocol. 10.1101/2020.04.28.20082552.

2110. Thomas R, Brooks T. Common oligosaccharide moieties inhibit the adherence of typical and atypical respiratory pathogens. J Med Microbiol. 2004 Sep;53(Pt 9):833-840. doi: 10.1099/jmm.0.45643-0. PMID: 15314189.

2111. Idänpään-Heikkilä I, Simon PM, Zopf D, Vullo T, Cahill P, Sokol K, Tuomanen E. Oligosaccharides interfere with the establishment and progression of experimental pneumococcal pneumonia. J Infect Dis. 1997 Sep;176(3):704-12. doi: 10.1086/514094. PMID: 9291319.

2112. Bryan R, Feldman M, Jawetz SC, Rajan S, DiMango E, Tang HB, Scheffler L, Speert DP, Prince A. The effects of aerosolized dextran in a mouse model of Pseudomonas aeruginosa pulmonary infection. J Infect Dis. 1999 Jun;179(6):1449-58. doi: 10.1086/314755. PMID: 10228067

2113. Liang OD, Ascencio F, Fransson LA, Wadström T. Binding of heparan sulfate to Staphylococcus aureus. Infect Immun. 1992 Mar;60(3):899-906. doi: 10.1128/iai.60.3.899-906.1992. PMID: 1541563; PMCID: PMC257571.

2114. Tsang KW, Shum DK, Chan S, Ng P, Mak J, Leung R, Shum IH, Ooi GC, Tipoe GL, Lam WK. Pseudomonas aeruginosa adherence to human basement membrane collagen in vitro. Eur Respir J. 2003 Jun;21(6):932-8. doi: 10.1183/09031936.03.00097302. PMID: 12797484

2115. Martínez I, Melero JA. Binding of human respiratory syncytial virus to cells: implication of sulfated cell surface proteoglycans. J Gen Virol. 2000 Nov;81(Pt 11):2715-2722. doi: 10.1099/0022-1317-81-11-2715. PMID: 11038384 2116. Hosoya M, Balzarini J, Shigeta S, De Clercq E. Differential inhibitory effects of sulfated polysaccharides and polymers on the replication of various myxoviruses and retroviruses, depending on the composition of the target amino acid sequences of the viral envelope glycoproteins. Antimicrob Agents Chemother. 1991 Dec;35(12):2515-20. doi: 10.1128/AAC.35.12.2515. PMID: 1725692; PMCID: PMC245423.

2117. Abdellatif AAH, Tawfeek HM, Abdelfattah A, El-Saber Batiha G, Hetta HF. Recent updates in COVID-19 with emphasis on inhalation therapeutics: Nanostructured and targeting systems. J Drug Deliv Sci Technol. 2021 Jun;63:102435. doi: 10.1016/j.jddst.2021.102435. Epub 2021 Feb 19. PMID: 33643448; PMCID: PMC7894098. 2118. Vitiello, Antonio, and Francesco Ferrara. "Low Molecular Weight Heparin, Anti-inflammatory/Immunoregulatory and Antiviral Effects, a Short Update." Cardiovascular drugs and therapy, 1-5. 30 Aug. 2021, doi:10.1007/s10557-021-07251-6 2119. Suryawanshi, Rahul K et al. "Heparan Sulfate Binding Cationic Peptides Restrict SARS-CoV-2 Entry." Pathogens (Basel, Switzerland) vol. 10,7 803. 24 Jun. 2021, doi:10.3390/pathogens 10070803 
2120. Tandon R, Sharp JS, Zhang F, Pomin VH, Ashpole NM, Mitra D, McCandless MG, Jin W, Liu H, Sharma P, Linhardt RJ. Effective Inhibition of SARS-CoV-2 Entry by Heparin and Enoxaparin Derivatives. J Virol. 2021 Jan 13;95(3):e01987-20. doi: 10.1128/JVI.01987-20. PMID: 33173010; PMCID: PMC7925120.

2121. Tree, JA, Turnbull, JE, Buttigieg, KR, et al. Unfractionated heparin inhibits live wild type SARS-CoV-2 cell infectivity at therapeutically relevant concentrations. Br J Pharmacol. 2021; 178: 626- 635. https://doi.org/10.1111/bph.15304 2122. Carina Conzelmann, Janis A Müller, Lukas Perkhofer, Konstantin MJ Sparrer, Alexander N Zelikin, Jan Münch, Alexander Kleger, Inhaled and systemic heparin as a repurposed direct antiviral drug for prevention and treatment of COVID-19, Clinical Medicine Nov 2020, 20 (6) e218-e221; DOI: 10.7861/clinmed.2020-0351

2123. Bendstrup KE, Jensen JI. Inhaled heparin is effective in exacerbations of asthma. Respir Med. 2000 Feb;94(2):174-5. doi: 10.1053/rmed.1999.0677. PMID: 10714425.

2124. Markart P, Nass R, Ruppert C, Hundack L, Wygrecka M, Korfei M, Boedeker RH, Staehler G, Kroll H, Scheuch G, Seeger W Guenther A. Safety and tolerability of inhaled heparin in idiopathic pulmonary fibrosis. J Aerosol Med Pulm Drug Deliv. 2010 Jun;23(3):161-72. doi: 10.1089/jamp.2009.0780. PMID: 20109123.

2125. Monagle K, Ryan A, Hepponstall M, Mertyn E, Monagle P, Ignjatovic V, Newall F. Inhalational use of antithrombotics in humans: Review of the literature. Thromb Res. 2015 Dec;136(6):1059-66. doi: 10.1016/j.thromres.2015.10.011. Epub 2015 Oct 9. PMID: 26475409

2126. Dixon B, Santamaria JD, Campbell DJ. A phase 1 trial of nebulised heparin in acute lung injury. Crit Care. 2008;12(3):R64. doi: 10.1186/cc6894. Epub 2008 May 6. PMID: 18460218; PMCID: PMC2481447.

2127. van Haren, FMP, Richardson, A, Yoon, H-J, et al. INHALEd nebulised unfractionated HEParin for the treatment of hospitalised patients with COVID-19 (INHALE-HEP): Protocol and statistical analysis plan for an investigator-initiated international metatrial of randomised studies. Br J Clin Pharmacol. 2021; 87: 3075- 3091. https://doi.org/10.1111/bcp.14714

2128. Ball L, Schultz MJ, Pelosi P. Nebulised heparin for patients on ventilation: implications for COVID-19 pneumonia. Lancet Respir Med. 2021 Apr;9(4):321-322. doi: 10.1016/S2213-2600(20)30513-0. Epub 2021 Jan 22. PMID: 33493452; PMCID: PMC7826131.

2129. Juschten, Jenny et al. "Nebulized anticoagulants in lung injury in critically ill patients-an updated systematic review of preclinical and clinical studies." Annals of translational medicine vol. 5,22 (2017): 444. doi:10.21037/atm.2017.08.23 2130. Kipshidze N, Dangas G, White CJ, Kipshidze N, Siddiqui F, Lattimer CR, Carter CA, Fareed J. Viral Coagulopathy in Patients With COVID-19: Treatment and Care. Clin Appl Thromb Hemost. 2020 Jan-Dec;26:1076029620936776. doi: 10.1177/1076029620936776. PMID: 32687449; PMCID: PMC7461127.

2131. Dixon B, Smith RJ, Campbell DJ, Moran JL, Doig GS, Rechnitzer T, Maclsaac CM, Simpson N, van Haren FMP, Ghosh AN, Gupta S, Broadfield EJC, Crozier TME, French C, Santamaria JD; CHARLI Study Group. Nebulised heparin for patients with or at risk of acute respiratory distress syndrome: a multicentre, randomised, double-blind, placebo-controlled phase 3 trial. Lancet Respir Med. 2021 Apr;9(4):360-372. doi: 10.1016/S2213-2600(20)30470-7. Epub 2021 Jan 22. PMID: 33493448; PMCID: PMC7826120. 2132. Ackermann M, Verleden SE, Kuehnel M, Haverich A, Welte T, Laenger F, Vanstapel A, Werlein C, Stark H, Tzankov A, Li WW, Li VW, Mentzer SJ, Jonigk D. Pulmonary Vascular Endothelialitis, Thrombosis, and Angiogenesis in Covid-19. N Engl J Med. 2020 Jul 9;383(2):120-128. doi: 10.1056/NEJMoa2015432. Epub 2020 May 21. PMID: 32437596; PMCID: PMC7412750.

2133. Barisione E, Grillo F, Ball L, Bianchi R, Grosso M, Morbini P, Pelosi P, Patroniti NA, De Lucia A, Orengo G, Gratarola A, Verda M, Cittadini G, Mastracci L, Fiocca R. Fibrotic progression and radiologic correlation in matched lung samples from COVID-19 post-mortems. Virchows Arch. 2021 Mar;478(3):471-485. doi: 10.1007/s00428-020-02934-1. Epub 2020 Sep 28. PMID: 32989525; PMCID: PMC7521863.

2134. Martineau AR, Jolliffe DA, Hooper RL, Greenberg L, Aloia JF, Bergman P, Dubnov-Raz G, Esposito S, Ganmaa D, Ginde AA Goodall EC, Grant CC, Griffiths CJ, Janssens W, Laaksi I, Manaseki-Holland S, Mauger D, Murdoch DR, Neale R, Rees JR, Simpson S Jr, Stelmach I, Kumar GT, Urashima M, Camargo CA Jr. Vitamin D supplementation to prevent acute respiratory tract infections: systematic review and meta-analysis of individual participant data. BMJ. 2017 Feb 15;356:i6583. doi: 10.1136/bmj.i6583. PMID: 28202713; PMCID: PMC5310969.

2135. Goncalves-Mendes N, Talvas J, Dualé C, Guttmann A, Corbin V, Marceau G, Sapin V, Brachet P, Evrard B, Laurichesse H, Vasson MP. Impact of Vitamin D Supplementation on Influenza Vaccine Response and Immune Functions in Deficient Elderly Persons: A Randomized Placebo-Controlled Trial. Front Immunol. 2019 Feb 8;10:65. doi: 10.3389/fimmu.2019.00065. PMID: 30800121; PMCID: PMC6375825.

2136. Penna G, Amuchastegui S, Giarratana N, Daniel KC, Vulcano M, Sozzani S, Adorini L. 1,25-Dihydroxyvitamin D3 selectively modulates tolerogenic properties in myeloid but not plasmacytoid dendritic cells. J Immunol. 2007 Jan 1;178(1):145-53. doi: 10.4049/jimmunol.178.1.145. PMID: 17182549.

2137. Kriesel JD, Spruance J. Calcitriol (1,25-dihydroxy-vitamin D3) coadministered with influenza vaccine does not enhance humoral immunity in human volunteers. Vaccine. 1999 Apr 9;17(15-16):1883-8. doi: 10.1016/s0264-410x(98)00476-9. PMID 10217585.

2138. Principi N, Marchisio P, Terranova L, Zampiero A, Baggi E, Daleno C, Tirelli S, Pelucchi C, Esposito S. Impact of vitamin D administration on immunogenicity of trivalent inactivated influenza vaccine in previously unvaccinated children. Hum Vaccin Immunother. 2013 May;9(5):969-74. doi: 10.4161/hv.23540. Epub 2013 Jan 16. PMID: 23324599; PMCID: PMC3899163. 2139. Zhou, Jian MD*; Du, Juan MD*; Huang, Leting MD+; Wang, Youcheng MD¥; Shi, Yimei MD¥; Lin, Hailong MD† Preventive Effects of Vitamin D on Seasonal Influenza A in Infants: A Multicenter, Randomized, Open, Controlled Clinical Trial, The Pediatric Infectious Disease Journal: August 2018 - Volume 37 - Issue 8 - p 749-754, doi: 10.1097/INF.0000000000001890 2140. Vassiliou, Alice G et al. "Vitamin D in infectious complications in critically ill patients with or without COVID-19." Metabolism open vol. 11 (2021): 100106. doi:10.1016/j.metop.2021.100106

2141. Grant, William B et al. "Evidence that Vitamin D Supplementation Could Reduce Risk of Influenza and COVID-19 Infections and Deaths." Nutrients vol. 12,4 988. 2 Apr. 2020, doi:10.3390/nu12040988

2142. Taha, Rbab et al. "The Relationship Between Vitamin D and Infections Including COVID-19: Any Hopes?." International journal of general medicine vol. 14 3849-3870. 24 Jul. 2021, doi:10.2147/IJGM.S317421

2143. Xu Y, Baylink DJ, Chen CS, Reeves ME, Xiao J, Lacy C, Lau E, Cao H. The importance of vitamin d metabolism as a potential prophylactic, immunoregulatory and neuroprotective treatment for COVID-19. J Transl Med. 2020 Aug 26;18(1):322. doi: 10.1186/s12967-020-02488-5. PMID: 32847594; PMCID: PMC7447609.

2144. Quesada-Gomez JM, Entrenas-Castillo M, Bouillon R. Vitamin D receptor stimulation to reduce acute respiratory distress syndrome (ARDS) in patients with coronavirus SARS-CoV-2 infections: Revised Ms SBMB 2020 166. J Steroid Biochem Mol Biol. 2020 Sep;202:105719. doi: 10.1016/j.jsbmb.2020.105719. Epub 2020 Jun 11. PMID: 32535032; PMCID: PMC7289092. 2145. Güven, M., Gültekin, H. The effect of high-dose parenteral vitamin D3 on COVID-19-related inhospital mortality in critical COVID-19 patients during intensive care unit admission: an observational cohort study. Eur J Clin Nutr 75, 1383-1388 (2021). https://doi.org/10.1038/s41430-021-00984-5 
2146. Murai IH, Fernandes AL, Sales LP, et al. Effect of a Single High Dose of Vitamin D3 on Hospital Length of Stay in Patients With Moderate to Severe COVID-19: A Randomized Clinical Trial. JAMA. 2021;325(11):1053-1060. doi:10.1001/jama.2020.26848 2147. Grant WB, Lahore H, McDonnell SL, Baggerly CA, French CB, Aliano JL, Bhattoa HP. Evidence that Vitamin D Supplementation Could Reduce Risk of Influenza and COVID-19 Infections and Deaths. Nutrients. 2020 Apr 2;12(4):988. doi: 10.3390/nu12040988. PMID: 32252338; PMCID: PMC7231123.

2148. Rastogi A, Bhansali A, Khare N, et al, Short term, high-dose vitamin D supplementation for COVID-19 disease: a randomised, placebo-controlled, study (SHADE study), Postgraduate Medical Journal Published Online First: 12 November 2020. doi: 10.1136/postgradmedj-2020-139065

2149. Lakkireddy, M., Gadiga, S.G., Malathi, R.D. et al. Impact of daily high dose oral vitamin D therapy on the inflammatory markers in patients with COVID 19 disease. Sci Rep 11, 10641 (2021). https://doi.org/10.1038/s41598-021-90189-4 2150. de Carvalho, J. F. \& Churilov, L. P. Safety of megadose of vitamin D in patients with nephrolithiasis. Nutrition 12(87-88), 111201. https://doi.org/10.1016/j.nut.2021.111201 (2021)

2151. Boucher BJ. Vitamin D status as a predictor of Covid-19 risk in Black, Asian and other ethnic minority groups in the UK. Diabetes Metab Res Rev. 2020 Nov;36(8):e3375. doi: 10.1002/dmrr.3375. Epub 2020 Jul 31. PMID: 32588937; PMCID: PMC7361214.

2152. Giannini S, Passeri G, Tripepi G, Sella S, Fusaro M, Arcidiacono G, Torres MO, Michielin A, Prandini T, Baffa V, Aghi A, Egan CG, Brigo M, Zaninotto M, Plebani M, Vettor R, Fioretto P, Rossini M, Vignali A, Fabris F, Bertoldo F. Effectiveness of In-Hospital Cholecalciferol Use on Clinical Outcomes in Comorbid COVID-19 Patients: A Hypothesis-Generating Study. Nutrients. 2021 Jan 14;13(1):219. doi: 10.3390/nu13010219. PMID: 33466642; PMCID: PMC7828675.

2153. Maghbooli Z, Sahraian MA, Ebrahimi M, Pazoki M, Kafan S, Tabriz HM, Hadadi A, Montazeri M, Nasiri M, Shirvani A, Holick MF. Vitamin D sufficiency, a serum 25-hydroxyvitamin D at least $30 \mathrm{ng} / \mathrm{mL}$ reduced risk for adverse clinical outcomes in patients with COVID-19 infection. PLoS One. 2020 Sep 25;15(9):e0239799. doi: 10.1371/journal.pone.0239799. PMID: 32976513; PMCID: PMC7518605.

2154. Jain A, Chaurasia R, Sengar NS, Singh M, Mahor S, Narain S. Analysis of vitamin D level among asymptomatic and critically ill COVID-19 patients and its correlation with inflammatory markers. Sci Rep. 2020 Nov 19;10(1):20191. doi: 10.1038/s41598-02077093-z. PMID: 33214648; PMCID: PMC7677378.

2155. Aygun H. Vitamin D can prevent COVID-19 infection-induced multiple organ damage. Naunyn Schmiedebergs Arch Pharmacol. 2020 Jul;393(7):1157-1160. doi: 10.1007/s00210-020-01911-4. Epub 2020 May 25. PMID: 32451597; PMCID PMC7246956.

2156. Kaufman HW, Niles JK, Kroll MH, Bi C, Holick MF. SARS-CoV-2 positivity rates associated with circulating 25-hydroxyvitamin D levels. PLoS One. 2020 Sep 17;15(9):e0239252. doi: 10.1371/journal.pone.0239252. PMID: 32941512; PMCID: PMC7498100. 2157. Merzon E, Tworowski D, Gorohovski A, Vinker S, Golan Cohen A, Green I, Frenkel-Morgenstern M. Low plasma 25(OH) vitamin D level is associated with increased risk of COVID-19 infection: an Israeli population-based study. FEBS J. 2020 Sep;287(17):3693-3702. doi: 10.1111/febs.15495. Epub 2020 Aug 28. PMID: 32700398; PMCID: PMC7404739.

2158. Jain, Sushil K, and David Micinski. "Vitamin D upregulates glutamate cysteine ligase and glutathione reductase, and GSH formation, and decreases ROS and MCP-1 and IL-8 secretion in high-glucose exposed U937 monocytes." Biochemical and biophysical research communications vol. 437,1 (2013): 7-11. doi:10.1016/j.bbrc.2013.06.004 


